ORNL-4585

UC.-8C-Reacto- Technolagy

THE MORSE CODE - A MIILTIGROUP

INEUTRON AND GAMMIA-RA

MONTE CARLO IRANSPOR
E. A. Straker
P. N. Stevens
D. C. Hring
Y. R. Coin

OAK RIDGE NATZONAL LABOEÁORY

$$
\text { sperated } 2 y
$$

UNION CARSIDE CORPORATION

$$
\text { for the }
$$

U. S. ATCMIC EHEPSY COMMISSION 


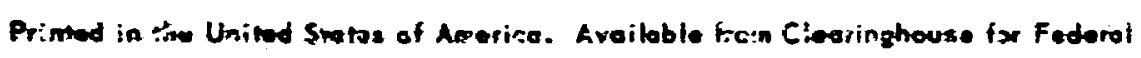
Scientifie and Technical inismatial. Netional Bureas at Standerds, U.S. Jepertment st Ecomeres, Springfie:d, Virginio 22151

Price: Prinied Copr \$3.00; Microfiche $\$ 0.65$

L.

TESAL MUTICE

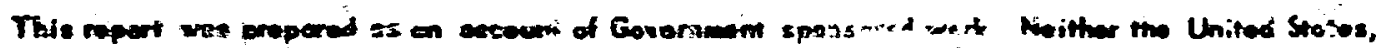

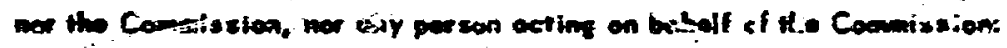

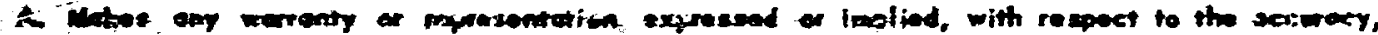

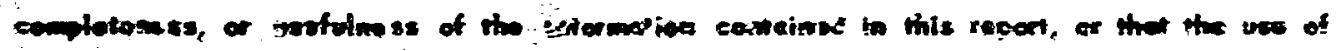

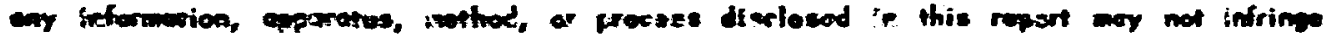

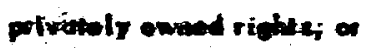

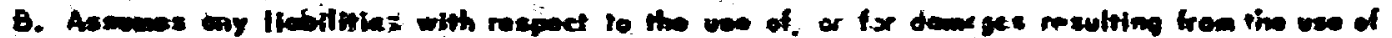
an informotion, opperatios, enthod, or process diselused in tisis mport.

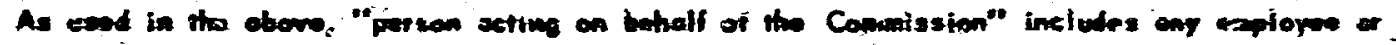

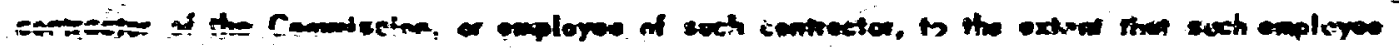

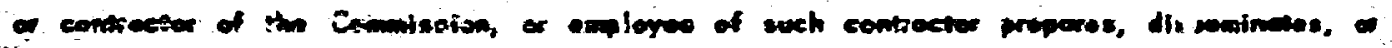

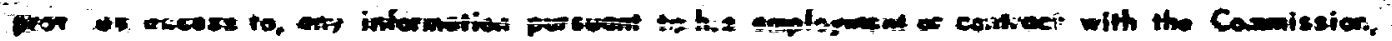
of wiongleymont with euch conwecter. 


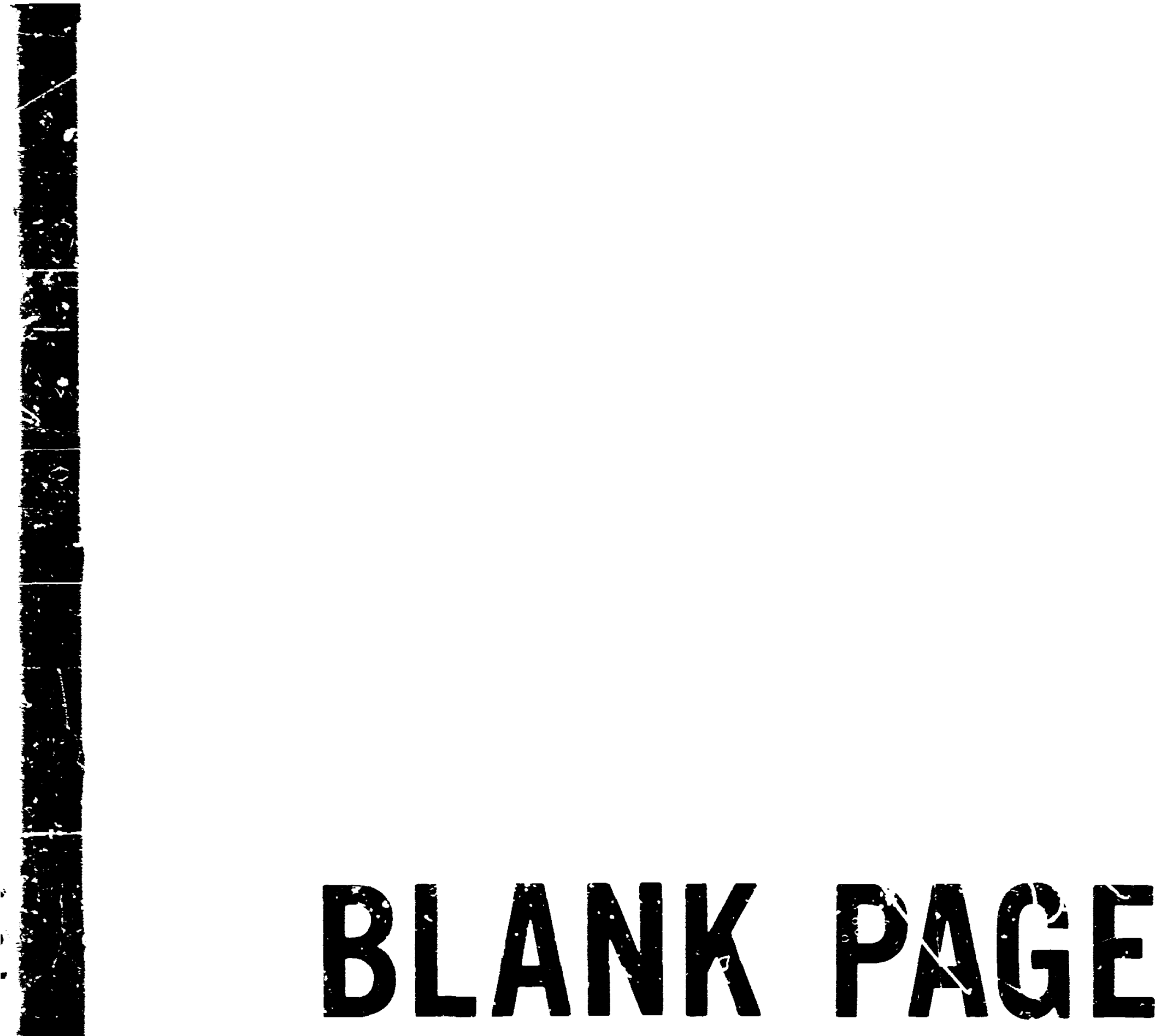


Neutron Physics Division

THE MORSE CODE - A MUTIGROUP NEUTRON AND

GALRLA-RAY MONTE' CARLO TRANSPOPT CODE

E. A. Straker, P. H. Stevens, ,

I. C. Irving, ${ }^{\dagger}$ and V. R. Cain

University of Tennessee, Knoxville, Tennessee.

${ }^{\dagger}$ Fresent address: Savannah River Laboratory, Aiken, South Carolina 29802

\title{
NOTE:
}

This Work Partially Funded by DEFEISE ATOMIC SUPPORT ACEICY Under Subtask PE08001

\section{SEPTEMBER 1970}

\author{
OAK RIDGE HATIONAL LABORATORY \\ Oak Ridge, Tennessee \\ operated by \\ UIION CARBIDE CORPORATIOF \\ fos the \\ i. S. ATCMIC EIJERG! COMISSIOA
}

This nopon wh preped an account of wort

romered by the United Sente: Goremmeat. Neither the United states nor the Uaited Stotes Atomic Energy Comainisa, wor any of their employees, nor any of ind contractors, subcomtractors, of the employees

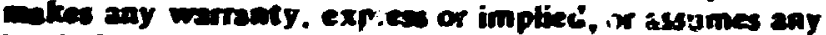

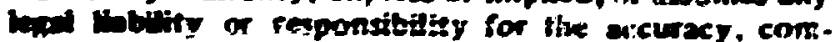

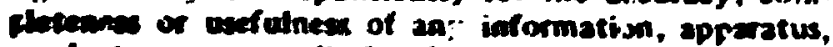
prodect of proces disclosed, or repres nts tinge its use would wot infiriege pots ately owned ripht 
TABLE OF CONTEATS

PAGE

I. INTRODUCTION . . . . . . . . . . . . . . . . 1

II. RANDOM WALK MODULE . . . . . . . . . . . . . 6

Main Frogran .................. 18

Subroutine 20

Subroutine DATE. . . . . . . . . . . . 26

Subroutine EURLID. . . . . . . . . . . . 29

Sutroutine FBARK . . . . . . . . . . . . 32

Random Number Pcckage. . . . . . . . . . . 34

Subrcutine FPR⿴B ............... 36

Subroutine FEqIJR . . . . . . . . . . . . 39

Subroutine GETETA. . ............... 40

Subroutine GETH ................ 44

Subrcutine Gor ................. 47

Subroutine GPRQB . . . . . . . . . . . . 49

Subroutine GSTWRE. ............... 51

Subroutine IRPUT . . . . . . . . . . . 53

Pur.ction IUEEK ................... 64

Subroutine MS申IR . . . . . . . . . . . . 66

Subroutine NXTCLL. . . . . . . . . . . 69

Subroutine UTPR . . . . . . . . . . . . 72

Subroutine quTPT2. . . . . . . . . . . . 74

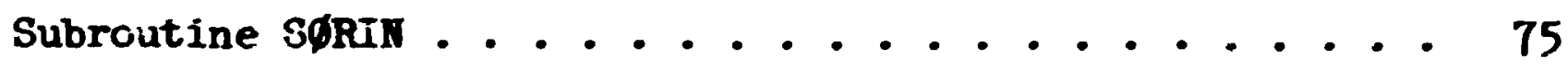

subroutine TEST ................ 80

Subroutine TIMER . . . . . . . . . . . . 82 
III. MLTIGROUP CROSS-SECTIOR MODULE. . . . . . . . . . 85

Subrcutine ALBD . . . . . . . . . . . . 93

Subroutine AllGLES. . . . . . . . . . . . 94

Subroutine BADGM. . . . . . . . . . . . . 99

Subroltine COLISH. . . . . . . . . . . . . 103

Subroutine FIIID. . . . . . . . . . . . 107

Subroutine FISGER. . ............... 111

Stibroutine GAKGEH. . . . . . . . . . . . . 112

Subroutine GEMUS. . . . . . . . . . . 114

Subroutine GITUT. . . . . . . . . . . . 1i8

Subroutine JIPUT . . . . . . . . . . . . 120

Subroutine LEGERD. . . . . . . . . . . . . 129

Subroutine MAYGT. . . . . . . . . . . . 132

Subroutine ISIGTA. . . . . . . . . . . . 135

Subroutine PTHETA. . . . . . . . . . . . 136

Frunction Q................. 139

Suioroutine FEALSG. . . . . . . . . . . . 141

Subrchtine SHRRE ................ 145

subroutine XSEC. . . . . . . . . . . . . 147

IV. DIAGTOSTIC MODULE. . . . . . . . . . . . . 151

Subroutine BNHHLP. . . . . . . . . . . . 152

Subroutine HELP. . . . . . . . . . . . 155

Subroutine HELPER. . . . . . . . . . . . 159

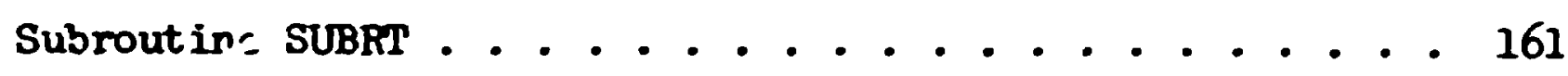

Subrcutine XSCHL!. . . . . . . . . . . . . 162 
V. ATALYSIS INTERFACE AND SATPLE USER ROUTIRES. . . . . . . 165

Sample User Routines . . . . . . . . . . . . 168

Subroutine BANKR .................. 170

Subroutine BDRYX . . . . . . . . . . . 173

Function IIREC ................. 176

Subroutine GTMED . . . . . . . . . . . . 179

Subroutine NBATCH. . . . . . . . . . . . 181

Subroutine NRUN. . . . . . . . . . . . 183

Subroutine SCфRIN. ............... 186

Subroutine SDATA ................. 189

Subroutine SqURCE. . . . . . . . . . . . 192

Subrcutine STBTCH. . . . . . . . . . . . 195

Subroutire STRUI . . . . . . . . . . . . 198

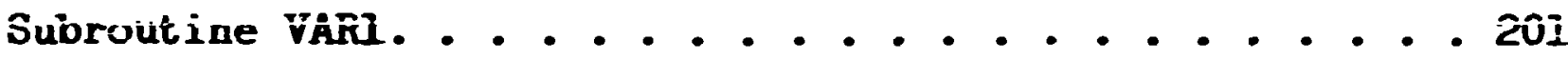

Sample Problen . . . . . . . . . . . . . . 204

VI. GEONETRY MODULE. . . . . . . . . . . . . . . 220

Subroutine JlMrI . . . . . . . . . . . . 220

Subroutine LKZ . . . . . . . . . . . . 220

Subroutine GEM. . . . . . . . . . . . 220

Spherical GEM . . . . . . . . . . . . . 222

Slab GEdM. . . . . . . . . . . . . . . . 223

Cylindricel GEQM . . . . . . . . . . . . . . . 224

General GEM ..................... 225

Changes to Geometry Packages . . . . . . . . . . 227

Additional Parameters in Labelled Common . . . . . . . 227 
PAGE

Subroutine GdMLP. . . . . . . . . . . . 229

Subroutine NøRML ............... 230

APPENDIX A. THE IAANY INTEGRAL FORMS OF THE BOLTZMANN TRANSPORT EQUATIOK AND ITS ADJOINT . . . . . . . . . . . . . A-1

The Boltzmann iransport Equation ... . . . . . . . A-1

Integral Flux Density Equation ........... A-4

Integral Event Density Equation. . . . . . . . . A-7

Integral Emergent Particle Density Equation. . . . . A-8

Operator Notation and Summary of the Forward Equations . A-10

Random Walk Procedure. . . . . . . . . . . A-12

Derivation of the Adjoint Integro-Differential Boltzmann

Transport Equation . . . . . . . . . . A-13

Integral Foint-Value Equation. . . . . . . . . A-18

Integral Erent-Yalue Equation. . . . . . . . . . A-23

Integral Energent Adjuncton Density Equation . . . . A-24

Multiplying Systems. . . . . . . . . . . . A-29

APPEIIDIX B. GETERALIZED GAUSSIA QUADRATURE . . . . . . . . B-1

General Statement of the Problem and Its Solution. . . B-1

Equivalence of Moments and Legendre Coefficients . . . B-2

Generation of Polynomials Orthogoral with Respect

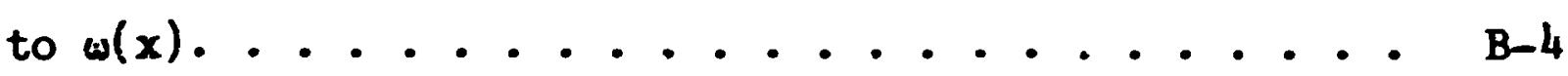

Properties of the Roots of the Orthogonal Polynomials. - B-8 The Meaning of the Two Restrictions Which Replace the Non-Negativity Requirement, $\omega(x) \geq 0$. . . . . B-9 Generation of the Jeneralized Gaussian Quadrature. . . B-10 


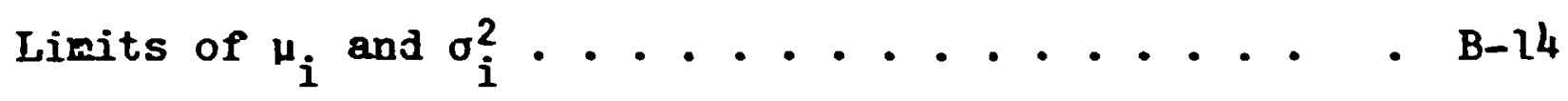
APPENDIX C. MRSE INPUT INSTRUCTIONS ........... . C-1

Geometry Input Data . . . . . . . . . . C-5 XSEC Input Data . . . . . . . . . . . . C-5

Analysis Input Data . . . . . . . . . . C-6

APPENDIX D. GEGMETRY INPUI INSTRUCTIONS. . . . . . . . D D-I

Spherical GEфM. . . . . . . . . . . . . . D-1

slab GEQM ................... D-2

Cylindrical GEфK. . . . . . . . . . . . . D-3

General GEQ................ D-4

APPENDIX E. LIBRARY SUBROUTIKES AND FUNCTIONS. . . . . . . E-1 


\section{ABSTRELTT}

The MORSE code is a multipurpose neutron and gamma-ray iransport Monte Carlo code. Through the use of multigroup cross sections, the solutiun of neutron, gamma-ray, or coupled neutron-ganma-ray problems may he obtained in either the forward or adjoint mode. Time dependence for both shielding end criticality problems is $\bar{s}^{+}$ovided. licheral three-dimensional gecmetry, as well as specialized one-dimensional geometry descriptions, may be used with an albedo option avizilable at any material surface.

Standard multigroup cross sections such es those used in discrete ordinates codes may be used as input; either ANISN or DTF-IV cross-section formats are acceptable. Anisotropic scattering is treated for esch grupto-group transfer by utilizing a generalized Gaussian quadrature technique. The modular form of the code with built-in analysis capability for all types of estimators makes it possible to solve a complete neutron-gammaray problem as one job and without the use of tapes.

A detailed discussion of the relationship between forward and adioint flux and collision densities, as well as a detailed description of the treatment of the angle of scattering, is given in the appendices. Logical flow charts for each subroutine add to the understanding of the code. 
LIST OF TABLES

TABLE

PAGE

I. DEFINITION OF VARIABIES IN COMMON AP $\emptyset L L \dot{\varphi} . \ldots . . . . . . .8$

II. DFFINITION OF VARIABLES IN HUTRQN COMMON . . . . . . . 12

III. DEFINITIONS OF VARIABLES IN BLANK COMMON . . . . . . . . 14

IV. LOCATICN OF BLLANK COMMON ARRAYS. . . . . . . . . . 16

v. DEFINITIONS OF VARIADIES IN COMRON LøCSIG. . . . . . . 88

VT. IOCATION OF PERMANENT CROSS SECTIONS IN BLANK COMMON . • . . 91

VII. BANKR ARGUMENTS. . . . . . . . . . . . . I66

VIII. DEFTNITIUN OF VARIABLES IN COMMON USER . . . . . . . . I67

IX. DEFINITION OF VARIABLEC, IN COMMON DET. . . . . . . . . 169

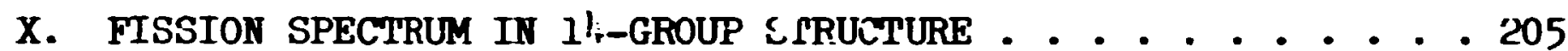

XI. DEFINITIONS OF VARIABLES IN COMAON GEQMC AS FOIND IN SUBROLTINE GEQM. . . . . . . . . . . . . . 221

C-I. VARIABTES THAT MAY BE WRITMTEN ON TAPE. . . . . . . . . C-7

C-II. SAMPLE GROUP INPUT NUMBERS FOR SOME REPRESENTATIVE

PRCBLEMS .................. . . . 


\section{Introduction}

The Multigroup Oak Ridge Stochastic Experiment code (mpRE) is a multipurpose neutron and gamma-ray transport Monte Carlo code. Some of its features include the ability to treat the transport of either neutrons or ganma rays or a coupled neutron and secondary gamna-ray probiem, tine incorporation of multigroup cross sections, an option of sulving either the forward or adjoint problem, modular input-output, cross section, analysis" and geometry modules, debugging routines, time dependence for both shielding and criticality problems, albedo option at any material boundary, one-, two-, and three-dimersional gecmetry packages, and several types of optional importance sampling.

fraditionally, Monte Cario codes for solving neutron and gamma-ray transport problems have been separate coajes. This has been due to the physics of the interaction processes and the corresponding cross-section information required. However, when multigroup cross sections are employed, the energy group to energy group transfers contain the cross sections for all processes. Also, for anisotropic scattering each group-to-group transfer has an associated angular distribution which is a weighted average over the various cross sections involved in the energy transfer process. Thus, these multigroup cross sections have the same format for both neutrons and gamma rays. In addition, the generation of secondary gamma rays may be considered as just another group-to-group transfer. Therefore using multigroup cross sections, the logic of the random walk process (the process of being transported from one collision $: 0$ another) is identical for both neutrons and gamma rays.

The use of multigroup cross sections in a Monte Carlo code means that the effort rsquired to produce cross-section libraries is reduced. (A set of mulitigroup neutron cross sections - 99 group, $\mathrm{P}_{8}$ - based on ENDF/B is available from the Radiation Shielding Information Center; ${ }^{2}$ likewise, some coupled neutron gamma-ray sets are also available from RSIC.)

Cross sections may be read in eithar the DTF-IV ${ }^{3}$ format $u$ r ANISN ${ }^{4}$ and DOT $^{5}$ format. The auxiliary information giving the number of groups, elements

A versatile analysis package, SAMBO, which handles must of the drudgery associated with estimation from raldum walk events is described in ref. 1. 
coefricients, etc., is used to produce the necessary probability tables needed by the randce walk nodule. The possible transport cases that can be treated are neutroa only, gsma ray only, coupled reutron-grama ray, gama ray froa a coupled set, and fission, with all of the above options for either a forward or adjoint case and for isotropic or anisotropic scattering up to a $\mathrm{P}_{16}$ expansion of the angular distribution. The option of storing the Legendre coefficients for use in a next-event estimator is also provided.

The sclution of the forrard or normal transport equation by Mnnte Carlo generally involves a solution for $X(P)$, the density of particles vith phase space crordinates $\underline{P}$ leaving collisions. Quantities of interest are then obtained by suming the contributions over all collisions, and frequentiy over most of phase space. The equations solved are derived in Appendix A and ace written as Bquations (40) and (95).

In sone cases, it is of interest to solve the adjoint probler. This requires solving a transport problem with the detector response as a source. The various relationships between the adjoint and forward quantities are derived in Appendix A. The adjoint equations solved by MORSE are Bquations (93) and (99). In utilizing these adjoint equations, the logic of the random walk is the same as the forward mode.

Input to WRSE is read in five separate modules: (1) walk; (2) cross section: (3) user; (4) source; and (5) geometry. The walk input is read in subroutine IIPUT and includes al. variables needed for the walk process. The crosssection input is read in cross-section module subroutines XSEC, JNPUT, and READSG. The parameters needed to set aside storage are read in XSEC, the mixing parameters are read in JIPUT, and the actual cross sections are read by RRADSG. Input information required for analysis of the histories must be read by a user-written subroutine SC $\emptyset \mathrm{RIN}$ which is called from BANKR. Since the source varies from problem to problem, input may also be read in a user-written subroutine SøRIN for the definition of the sou:ce. The geometry input is read by subroutine JøMTN. 
In general, output of input parameters occurs in the same routine in which the input was read. In addition, there are two routines (बUTPT and (UTPT2) for the output of rezilts of the random walk process. Output of analysis results is generally performed in the user-written routine ARU.

Figure 1 shows the bierarchy of subroutines for MRSE. Fron this diagran, it is possible to see the functions of the modules. The input section takes sare of setting up all variables needed in the transport process. Note that initial calculations by the cross-section module sten from XSEC. The anajysis partion of the code is interfaced vith WORSE through $B$ / JKR vith several uses made of cross-section routines in making estimates of the quantity of interest. Witr, the exception of output from the walk process, the rest of the code consists of subroutine calls by MRSE. The geometry module is interfaced through GousT and the source is interfaced through MSqUR. The diagnostic module is independent and any cart of it r.uy be executed from any routine.

The diagnostic module provides an easy means of printing out, in useful form, the information in the various labelled commons and any part of blank common. A special routine is provided for printing out the particle bank. By loading parts of core vith a junk word, the diagnostic package can determine which variables tave been used. A "repeating line" feature is also included.

The georetry module consists of any of the geometry packages written for $\emptyset \mathrm{SR}, 6,7$ including the general three-dimensional geometry. Slight modifications have been made to include variable input-output units and to include the logic for albedo scattering.

An albedo scattering may be forced to occur at every entry into a specified medium. A sample subroutine is provided for specular reflection and a subroutine call is provided (ALBII, called from XSEC) for reading and storing albedo data of any degree of complexity. Thus transport of particies may be carried out in parts of the problem and an albedo scattering treated for other parts of the problem.

Time dependence is included by keeping track of the chronological arge of the particle. For neutrons the age is incremented by the time needed 


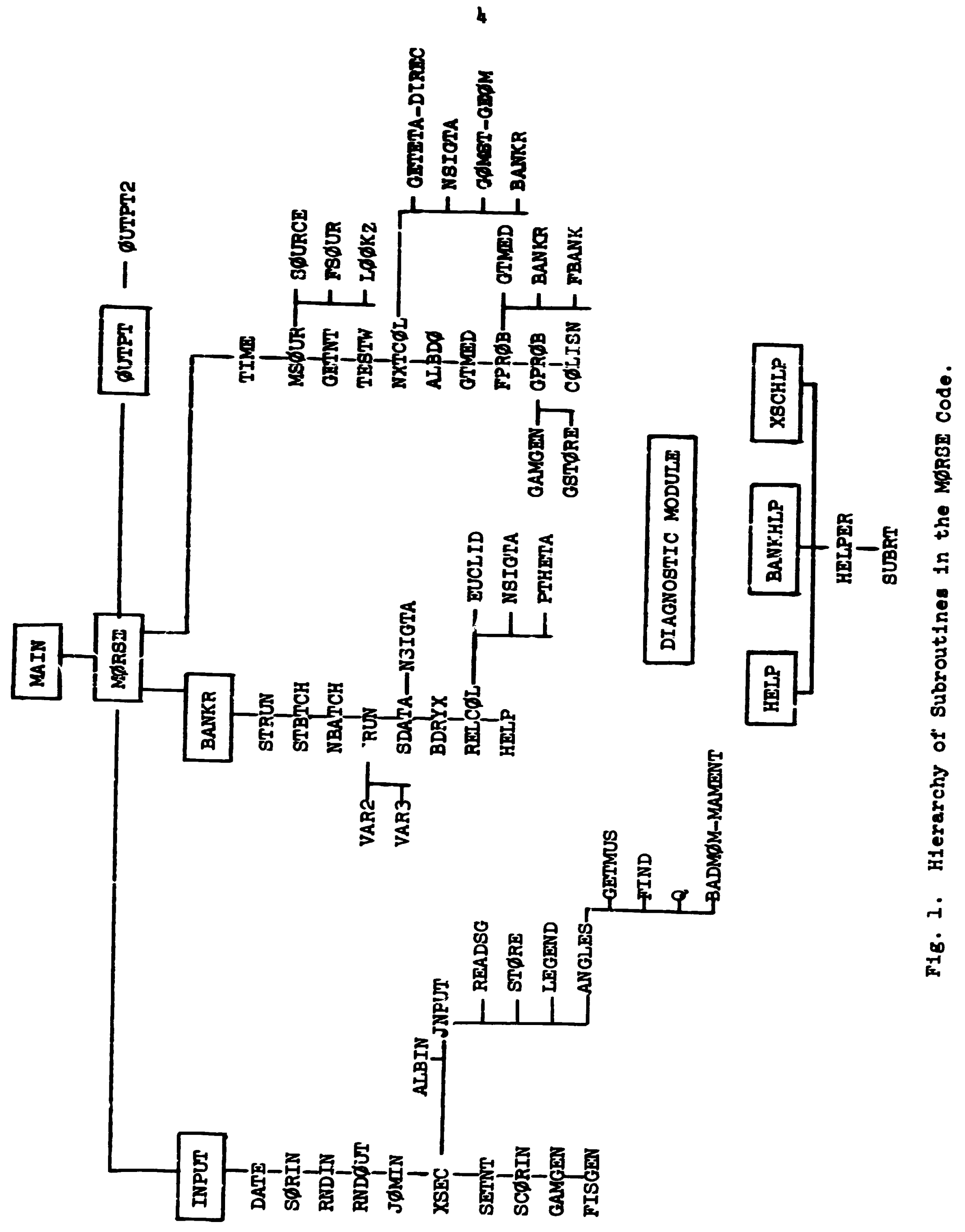


to travel the distarce betveen collisions if it traveled at 3 velocity corresponding to the average energy of the group. Provision is ande for inputting a thernal group velocity separately. Monrelativistic nechanics are assured. The age of secondary gama rays is determined from the reutron age at the collisicn site and is incremented by determinirg the time required to travel between callisions at the speed of light. For fission problens the age of the parent is giren to the daughters at birth.

There are several types of importance samping techniques included in the code. The Russian roulette and splitting logic of $95 R$ is an option in WRSE. Also the exponential transforn is provided vith paraneters allowed as a function of ener $B J$ and region. Source energy biasing is an option as well as enerby biasing at each collision. In fission problens the fission weights was be renornalized as a function of an estimate of $k$ so thet the nuber of histories per generation remin approxinately corstant. If desired, all inportance sapling nay be turned off.

Some ouher general features include the ability to run problens vithout the use of magetic tapes, the ability to terninate a job internally after a set elapsed c.p.u. time and obtain the output based on the number of histories treated up to that time, batch processing for the purpose of determining statistics for groups of particles, and a repeat run feature so that resilts for a time-dependent fission problem may be obtained with statistical estimates. The output of numerous counters permits one to obtain an insight into the physics of the problem.

Detdiled descriptions of the subroutines with the lofical fiow charts are found on the following pages. The appendices contain detailed derivations of various forms of the transport equation, a detailed derivation of the treatment of the anguiar distribution of scattering, and a detailed description of the required input. 


\section{Randa Vell Yodule}

The basic randon walk process of choosing a source particle and then following it taround ite history of events is governed by the routines in this noduie of MORSE. A given problen is perforned by folloring a nuber or batches of particles which then constitute a rin. Wutiple runs are also pernitted. The batch process feature is used so that statistical variations between groups of particles can be deternined. Thus a bateh of scarce particles is generated and stored in the bank. The randon walk for this batch of particles is deternined by picking one particle out of the bank and transporting it from collision to collision, splitting it into tyo particles, killing by fussian roulette, and generating secondary particles (either gana rays or fission neutrons) and storing then in the bank for ruture processing. Ternination of a history when a particie ieais from the system, reaches an enerbo cutoff, reaches an age linit, or is killed by Russian roulette.

The rendon walk nodule periorns the necessary bookkeeping for the bank and the transportation and generation of new perticles and relays this information to the analysis module for estination of the desired quantities. Use is made of the rross-section module and the geonetry module during the randon walk process and the input-output routines for the reading and printing of pertinent information about the problem.

In this module the main program is used to set aside the storage required in blark common and to pass this information to subroutine MQRSE which is the executive routine for the random walk process. After performing the necessary input operations and setting up storage requirements, the walk process consists of three nested loops: one for runs, one for batches, and the inner-most is for particles. After each termination of the batch loop, some bookkeeping is required before the generation of a new batch of source particles. After the termination of a run, a sumary of the particle terminations, scattering counters, and secondary production counters are output, as well as the results of Russian roulette and splitting for each group and region. 


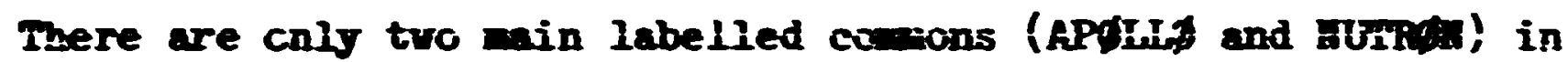
the randon valk routines. Tables I and II list the definitions of the rariables in these two camons. Note that in Table II "current" and "previous" refer to values of paraneters leavirg the current and previous event sites, respectively (WHBC is the exception, being the veight entering the current event site). Also nute that "event" includes boundary crossings, albedo collisions, etc., as vell as real collisions. A description of blank comon is given in Pig. 2, along with definitions in Table III. The locations of the variables are given in Table IV. All the variables used as location labels, except IGES, locate cell zero of an array. Cells MIAST + 1 to ILAST + ILBFT are available to the user for analysis arrays.

A description and a logical flor chart for the subroutines that make up the randon walk nodule are given the folloring pages in this chapter. 
Table I. Definition of Variables in Comon APQII

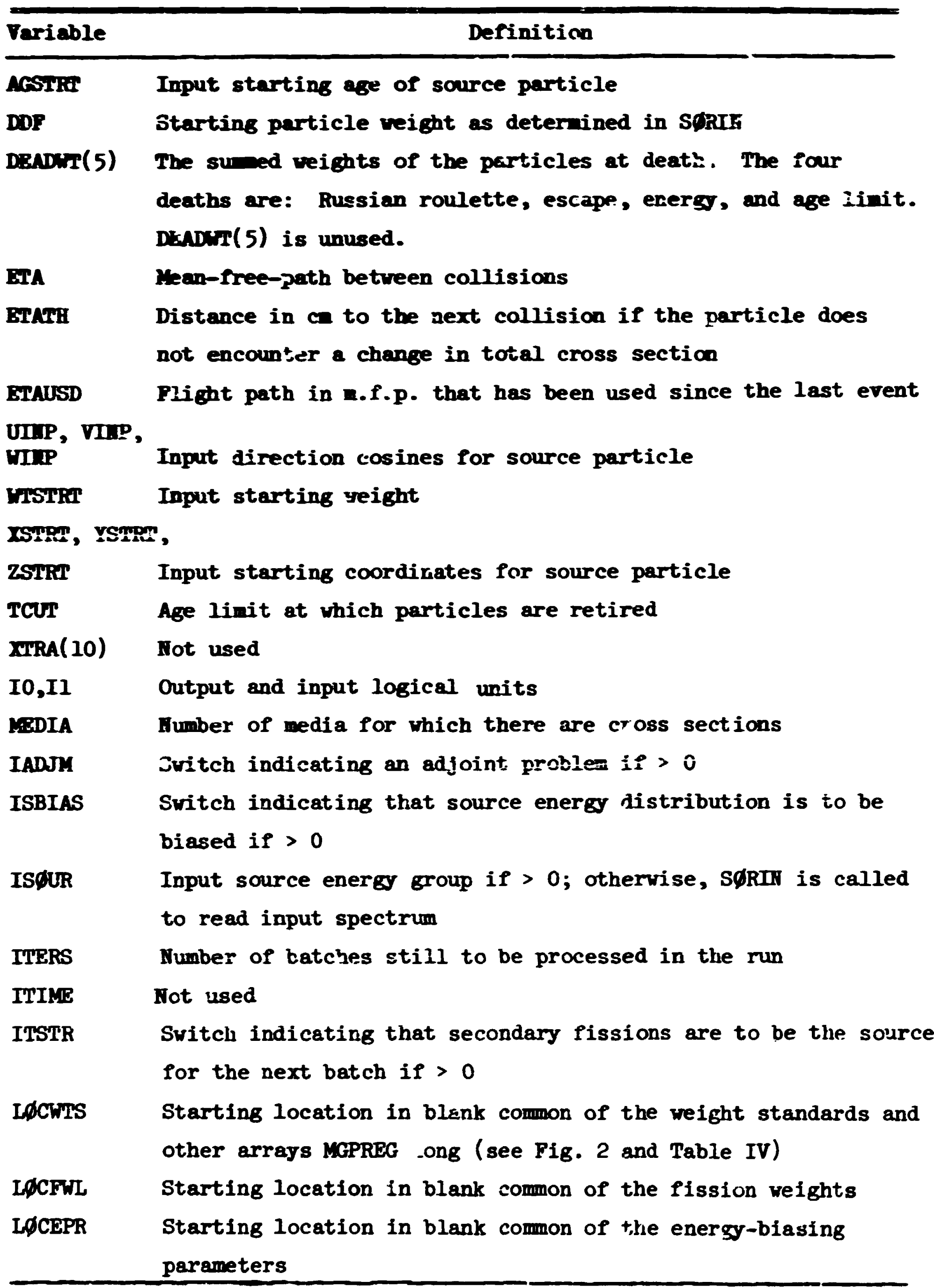


Table I (cont.)

Variable $\quad$ Defiaition

LqCISC Starting location in blank comon of the scattering counters

LCCFS Starting location in klank comon of the fission and gamageneration probailities for each mediu and grouf

MAXCP Maximun nuber of energy groups for which there are weight stardards or path-length stretching paraneters

MAXIIM The elapsed clock time at wich the problea is terminated

MEDALB Nedium number for the slbedo me tium

MGPREG Product of number of veight standard groups (MAXGP) and regions (MXREG)

MXREG Mariman number of regions in the system

MALB An isdex indicating that an albedo scattering has occurred if $>0$

IDEAD(5) Number of deaths of each type (see DEADAT).

IEMM Nane of the last particle in the bank

NGEM Location of first cell of geometry data storage in blank comion

IGPQT1* The lowest energy group (largest group number) for which primary particles are to be followed

HCPQT2* The number of primary particle groups

MGPQT3* The lowest energy group (largest group number) for which any particle is to be followed

NGPQTG* Number of energy groups of secondary particles to be followed NGPQTW* Number of enerEy groups of prinary particles to be followed IITS Number of batches per run

NKCALC The first batch to be used for a $\mathrm{k}$ calculation. If $0, \mathrm{k}$ is not calculated

NKILL An index to indicate that Russian roulette is to be played if $>0$

NLAST The last cell in blanis common that was used by the crosssection st,orage or is set aside for banking

See page 11 for diagram of energy group structure. 
Table I (cont.)

\begin{tabular}{|c|c|}
\hline Variable & Definition \\
\hline MEIBY & $\begin{array}{l}\text { The location of the next particle in the bank to be pro- } \\
\text { cessed }\end{array}$ \\
\hline ILCP & $\begin{array}{l}\text { The number of primary particle groups for wich there are } \\
\text { cr iss sections }\end{array}$ \\
\hline $\begin{array}{l}\text { MrST } \\
\text { HeTG }\end{array}$ & $\begin{array}{l}\text { The maximum number of particles that the bank can hold } \\
\text { The total number of energy groups (ooth primary and secon- } \\
\text { dary) for wich there are cross sections }\end{array}$ \\
\hline IOLEAK & $\begin{array}{l}\text { An index wich indicates that nonleakage path-length selec- } \\
\text { tion is to be used if }>0 \\
\text { An index to indicate that the fission parameters are to be } \\
\text { renormalized if }>0\end{array}$ \\
\hline DPAST & $\begin{array}{l}\text { An index to inaicate that the exponential transform is to } \\
\text { be used if }>0\end{array}$ \\
\hline $\operatorname{IPSCL}(13)$ & $\begin{array}{l}\text { An array of counters of events for each batch: } \\
\text { (1) sources generated } \\
\text { (2) splittings occurring } \\
\text { (3) fissions occurring } \\
\text { (4) ganno rays generated } \\
\text { (5) real collisions } \\
\text { (6) albedo scatterings } \\
\text { (7) boundary crossings } \\
\text { (8) escapes } \\
\text { (9) energy cutoffs } \\
\text { (10) time cutoffs } \\
\text { (11) Russian rculette kills } \\
\text { (12) Russian roulette survivors } \\
\text { (13) gamma rays not generated because bank was full }\end{array}$ \\
\hline $\begin{array}{l}\text { HQUIT } \\
\text { ISIGL } \\
\text { MSQUR }\end{array}$ & $\begin{array}{l}\text { Number of runs still to be processed } \\
\text { Starting location of the bank in blank common } \\
\text { An index input to indicate that fissions are to be the } \\
\text { source for future batches }\end{array}$ \\
\hline
\end{tabular}

See page 11 for diagram of energy group structure. 
Table I (cont.)

\begin{tabular}{|c|c|}
\hline Variable & Definition \\
\hline RSPLT & $\begin{array}{l}\text { An index to indicate that sfitting is to be considered if } \\
>0\end{array}$ \\
\hline $\begin{array}{l}\text { NSTRT } \\
\text { NXTRA (10) }\end{array}$ & $\begin{array}{l}\text { The number of particles to be started in each batch } \\
\text { Not used. }\end{array}$ \\
\hline
\end{tabular}

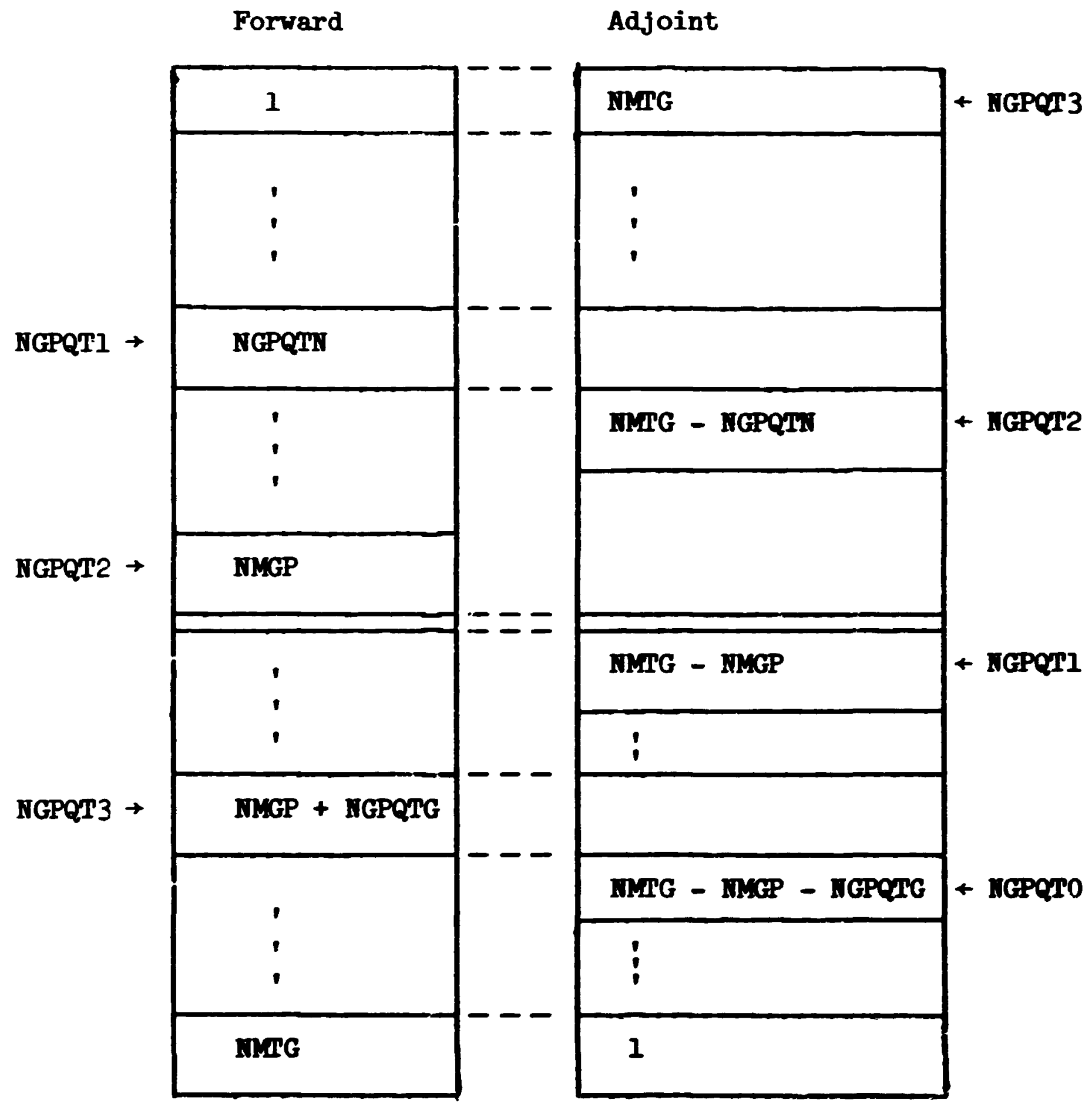

Diagram of Energy Group Structure 
Table II. Definition of Variables in NUTRd Common

\begin{tabular}{|c|c|}
\hline Variable & Definition \\
\hline 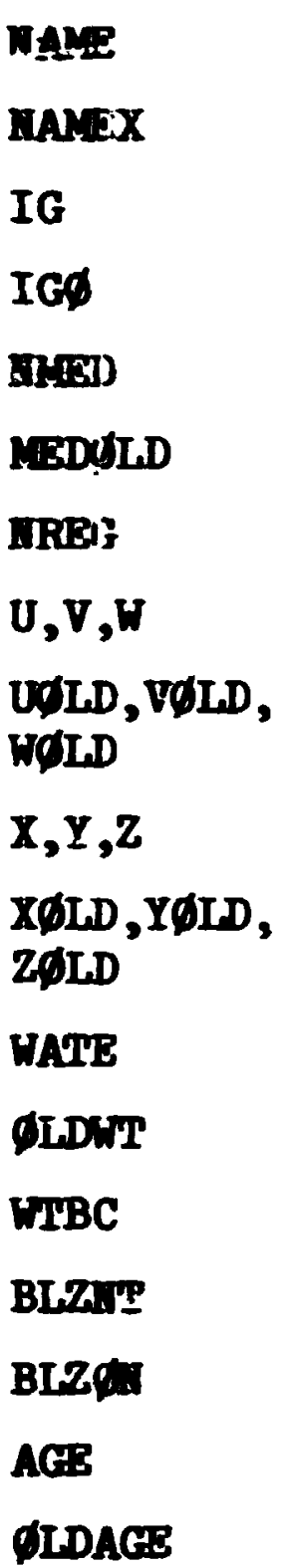 & $\begin{array}{l}\text { Particle's first name. } \\
\text { Particle's family name. (Hote that particles do not marry.) } \\
\text { Current energy group index. } \\
\text { Previous energy group index. } \\
\text { Yedium number at current location. } \\
\text { Yedium number at previous location. } \\
\text { Region number at current location. } \\
\text { Current direction cosine. }\end{array}$ \\
\hline
\end{tabular}




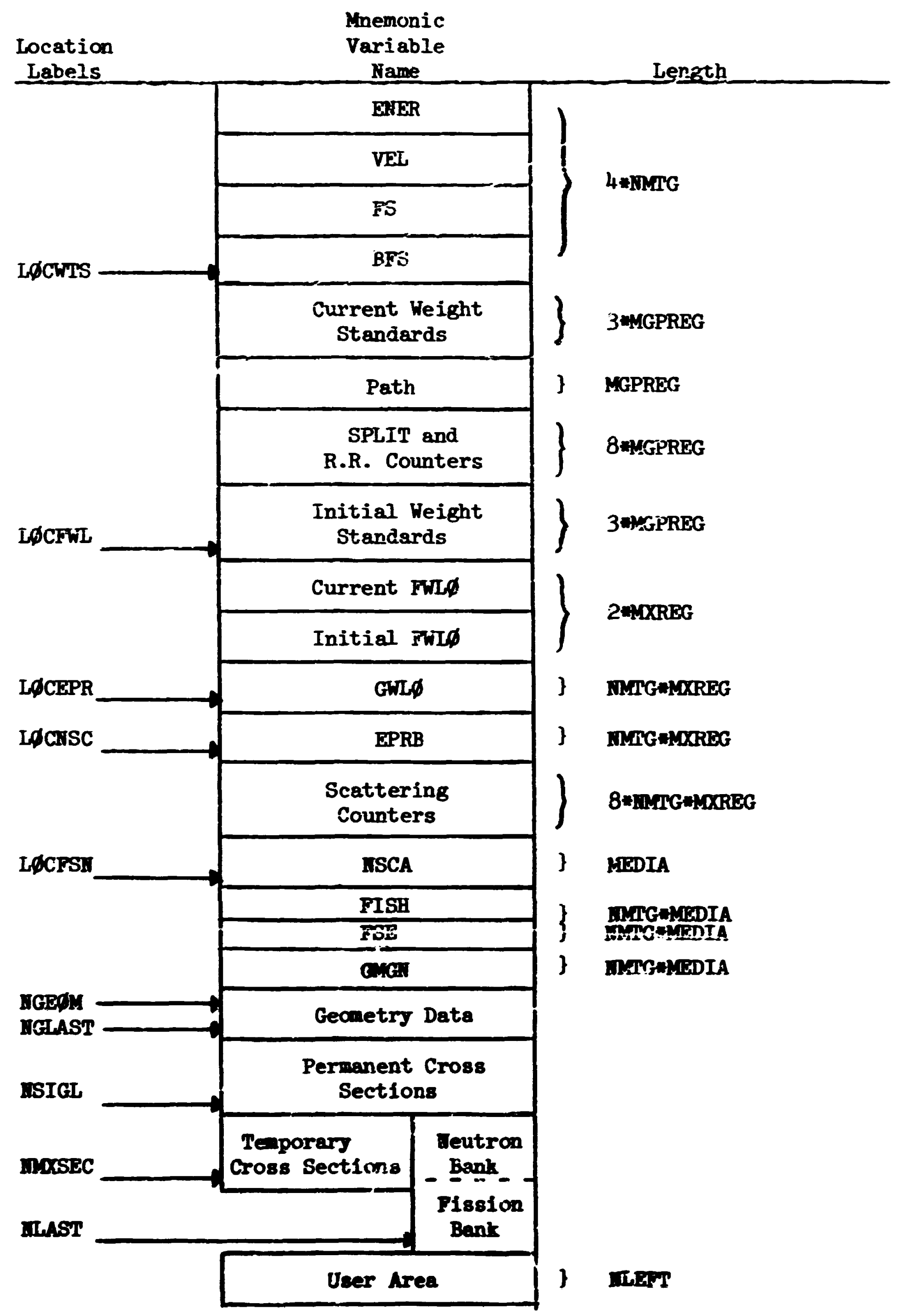

F16. 2. Lagout of Blank Common 
Table III. Definitions of Variables in Blank Common

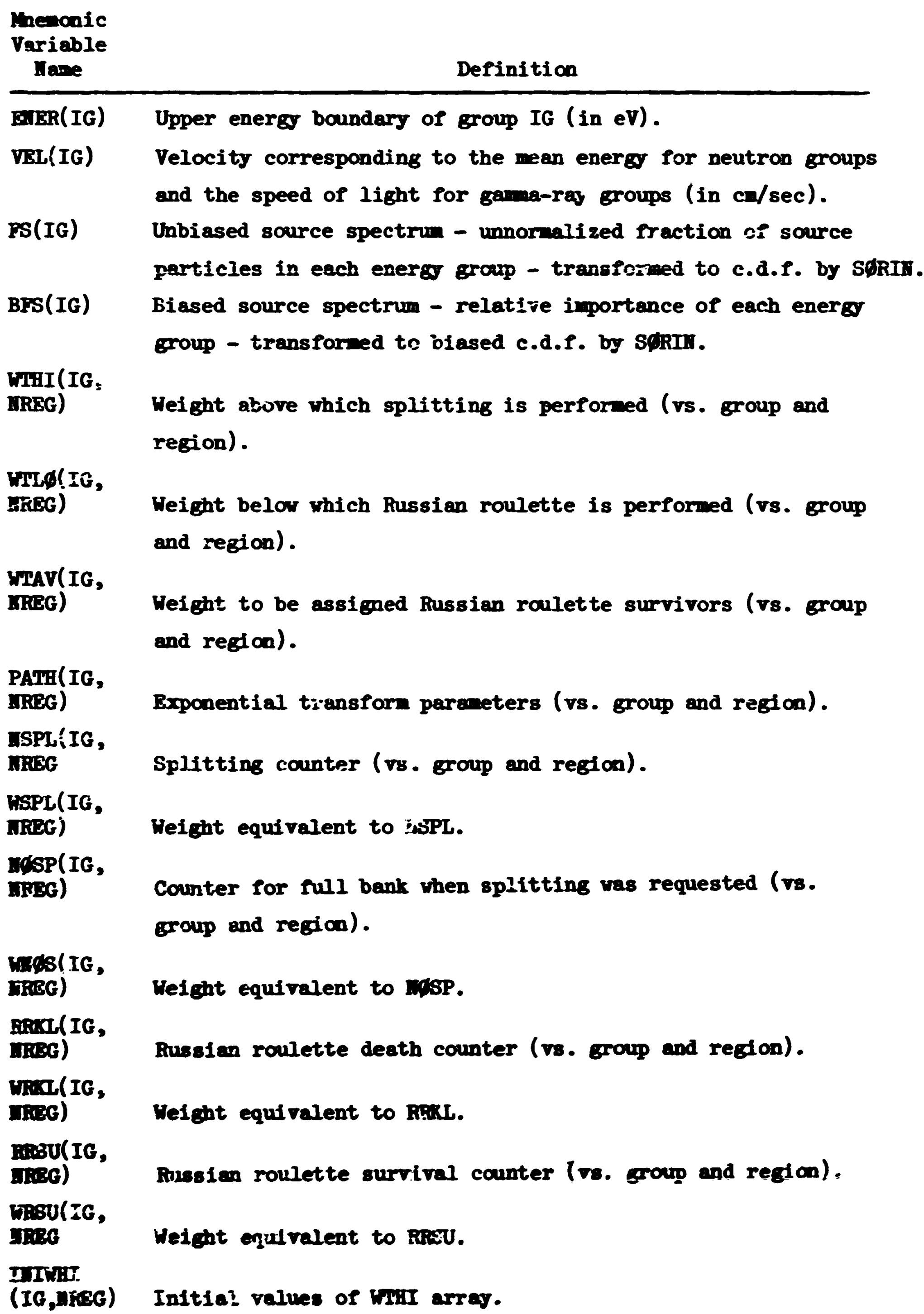


Table III (cont.)

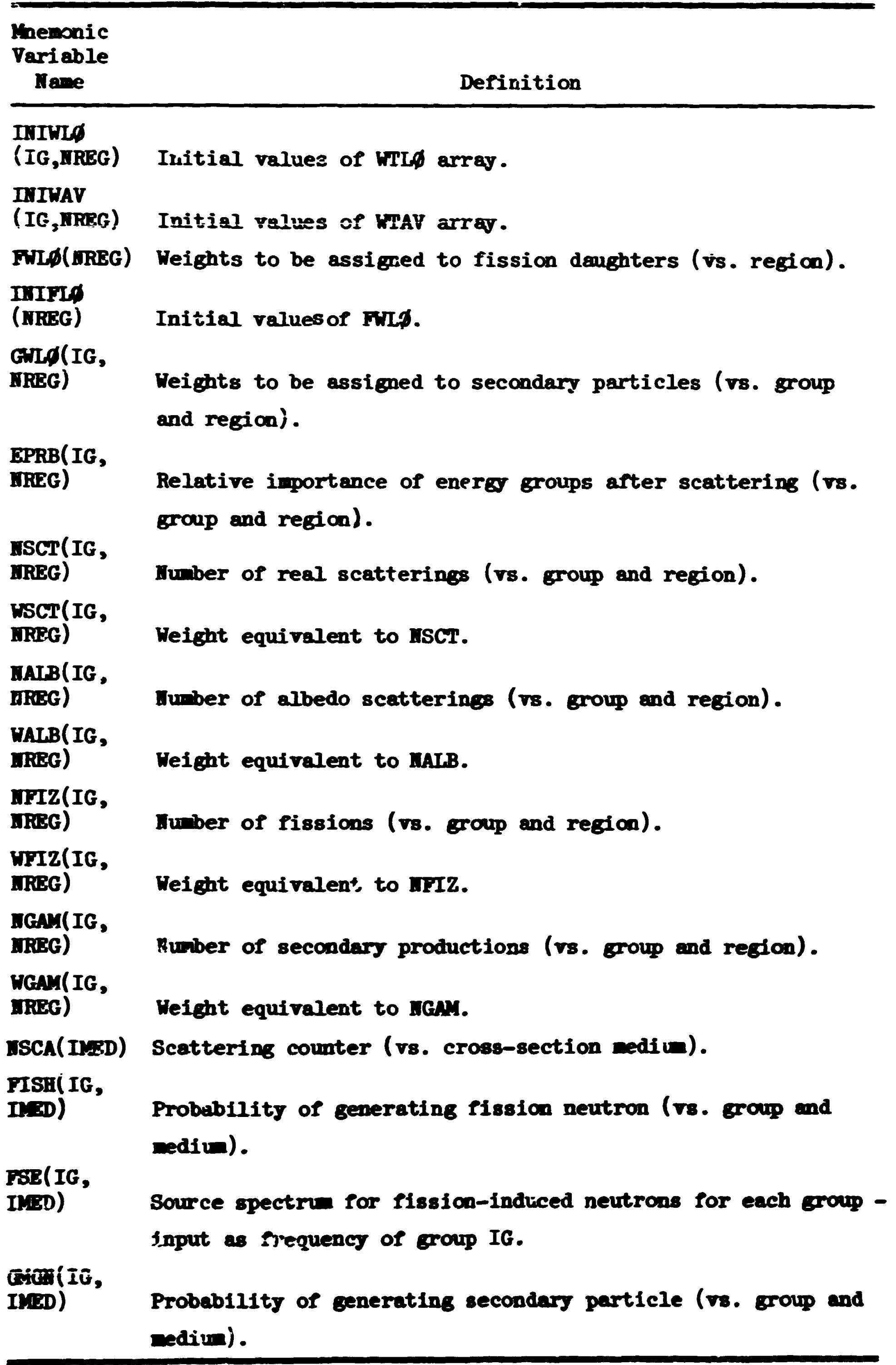


Table IV. Iocatica of Blank Comon Arrays

\begin{tabular}{|c|c|}
\hline $\begin{array}{l}\text { Wemanic } \\
\text { Variable } \\
\text { Iace }\end{array}$ & $\begin{array}{l}\text { Location of Array in Blank Comon } \\
(B C(I) \text { or } \mathrm{BC}(I))\end{array}$ \\
\hline $\begin{array}{l}\text { BGIER(IG) } \\
\operatorname{VE}(I G) \\
\operatorname{PS}(I G) \\
\operatorname{BFS}(I G)\end{array}$ & 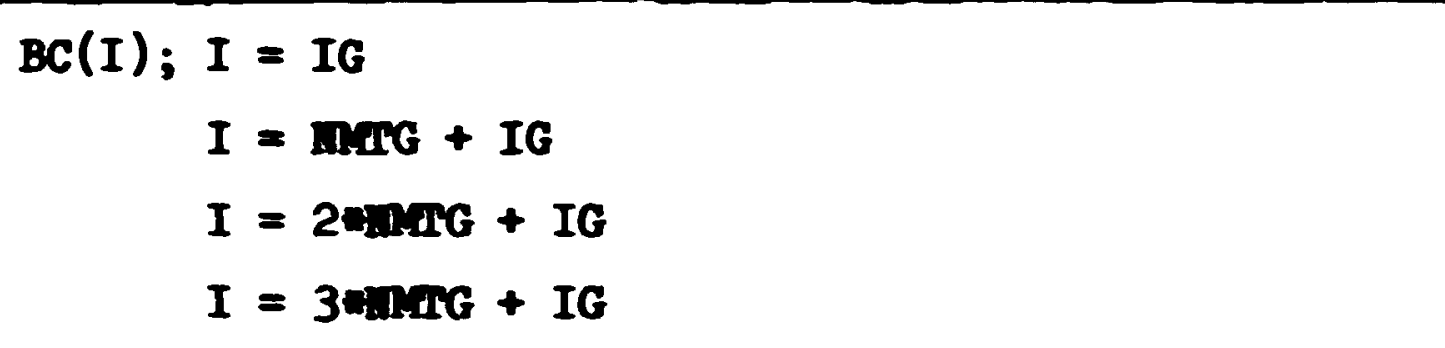 \\
\hline 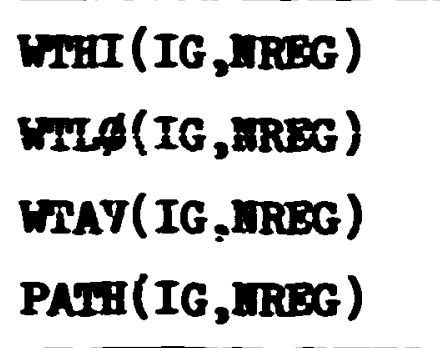 & 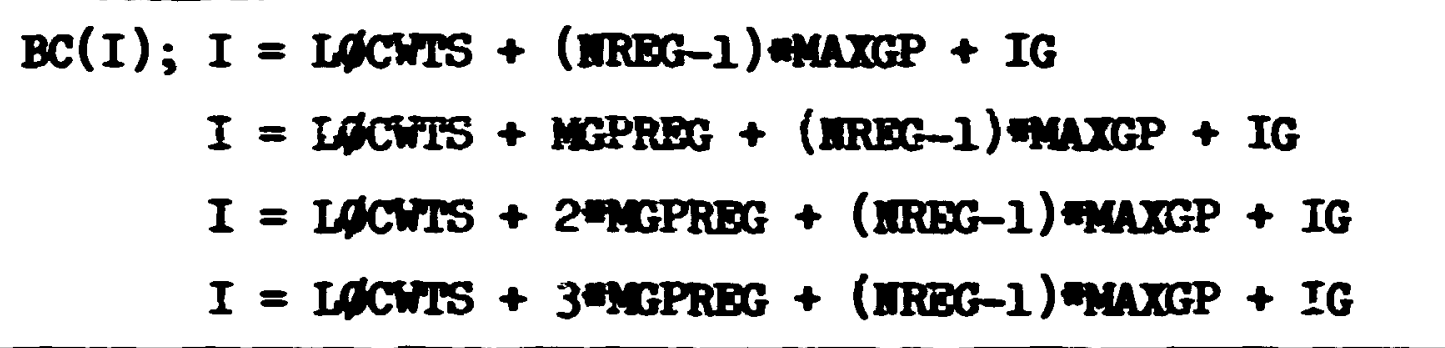 \\
\hline 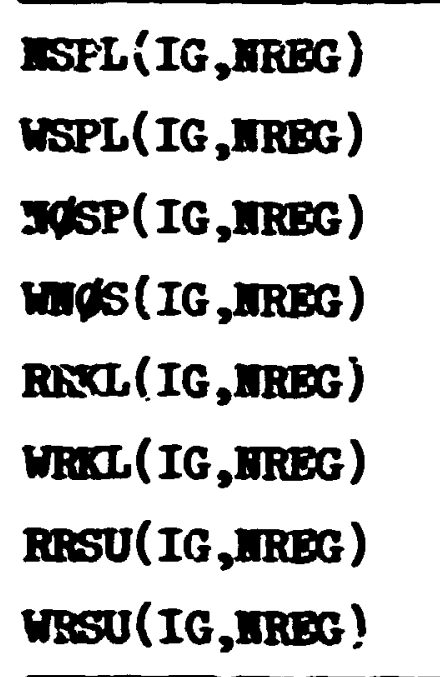 & 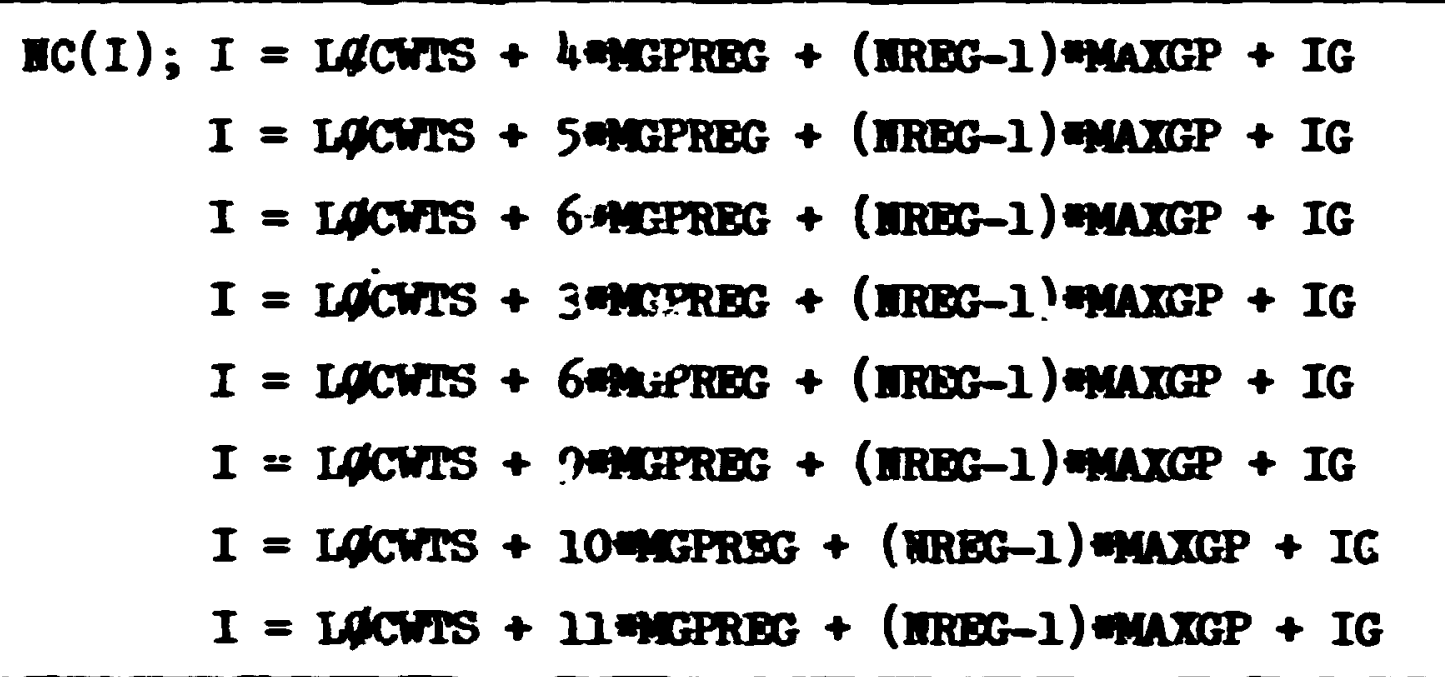 \\
\hline 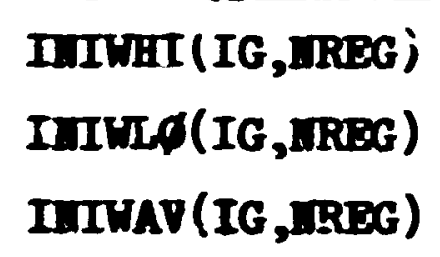 & 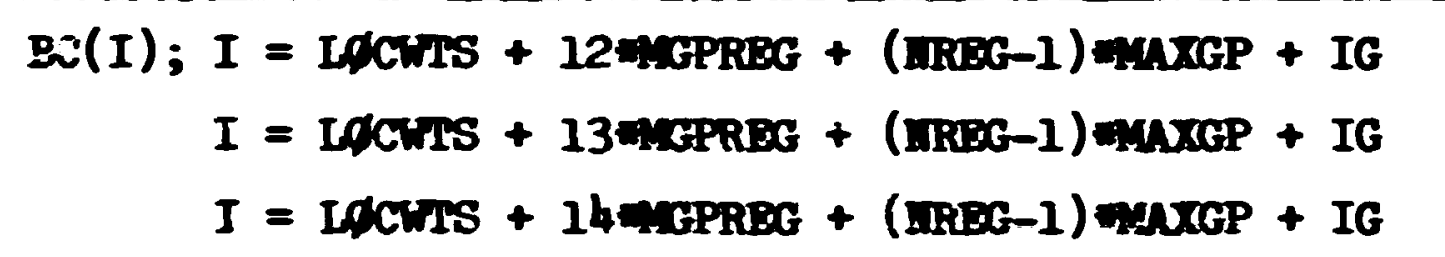 \\
\hline $\begin{array}{l}\text { PALA (IRBG) } \\
\text { DIFLA (IPES) } \\
\text { GIA(IG, WRGG) }\end{array}$ & 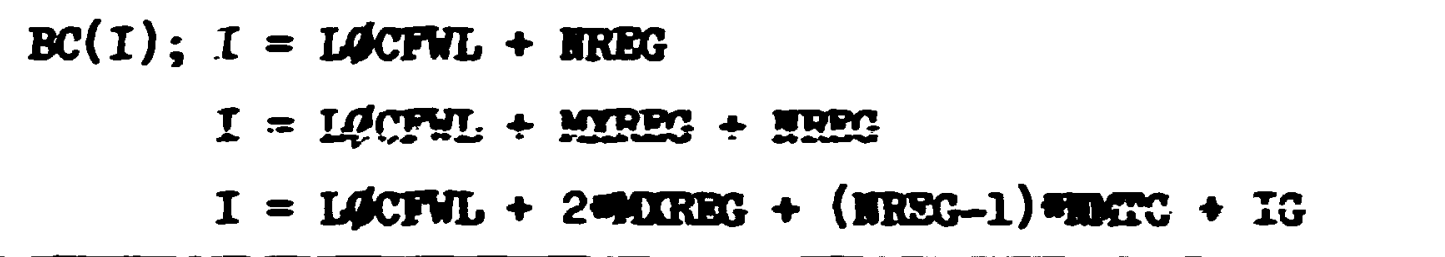 \\
\hline $\operatorname{EPRB}(I G, I R B G)$ & $B C(I) ; I=I$ ACEPR $+($ IREG-I) WITC $+I G$ \\
\hline 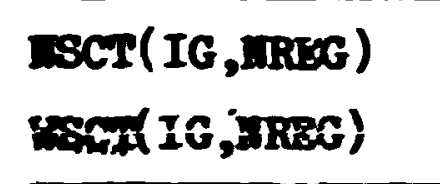 & 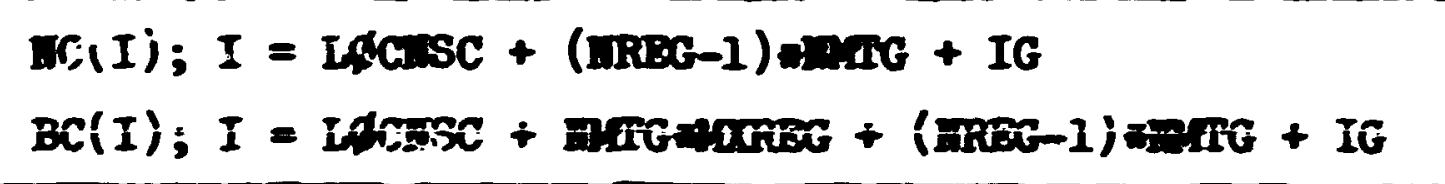 \\
\hline $\begin{array}{l}\text { INLE( IG, IKEG) } \\
\text { WLE( IG, MREG) }\end{array}$ & 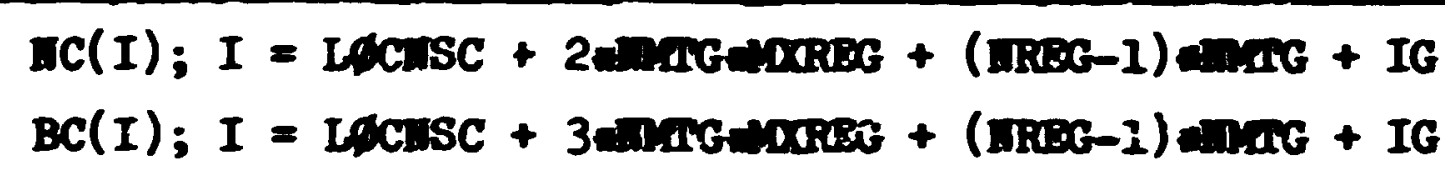 \\
\hline
\end{tabular}


Table IV (cont.)

\begin{tabular}{|c|c|}
\hline $\begin{array}{l}\text { Nuremonic } \\
\text { Variable } \\
\text { Name }\end{array}$ & $\begin{array}{c}\text { Location of Array in Blank Common } \\
(\mathrm{BC}(\mathrm{I}) \text { or } \mathrm{MC}(\mathrm{I}))\end{array}$ \\
\hline $\begin{array}{l}\text { MFIZ } \\
\text { WFIZ }\end{array}$ & 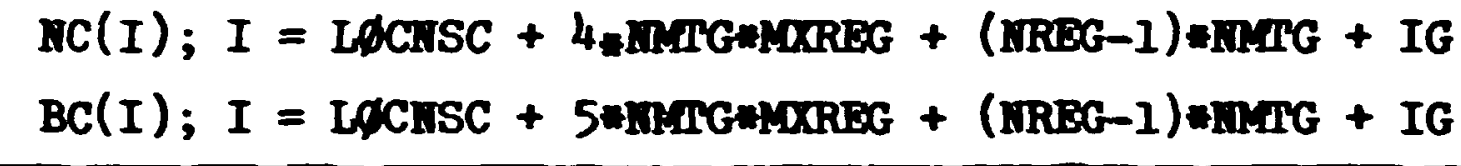 \\
\hline $\begin{array}{l}\text { FGAY } \\
\text { WGAM }\end{array}$ & 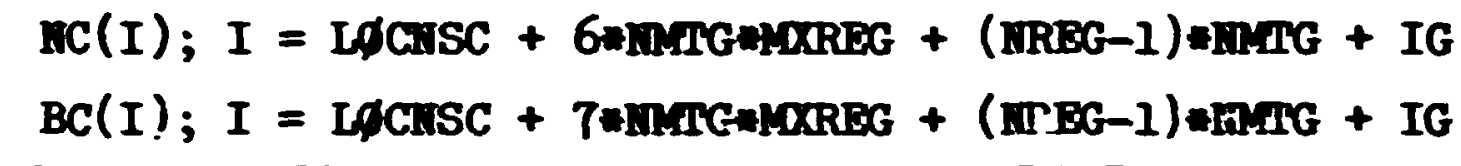 \\
\hline ISCA (INAD) & $\begin{array}{cl}\operatorname{HC}(I) ; & I=\text { IACISC + IMIBD + 8MMAGWWXREG } \\
\text { or } & I=\text { I }\end{array}$ \\
\hline $\begin{array}{l}\text { FISH ( IG , IMED) } \\
\text { FSE (IG , IMED) } \\
\text { GIGI (IG, IMED) }\end{array}$ & 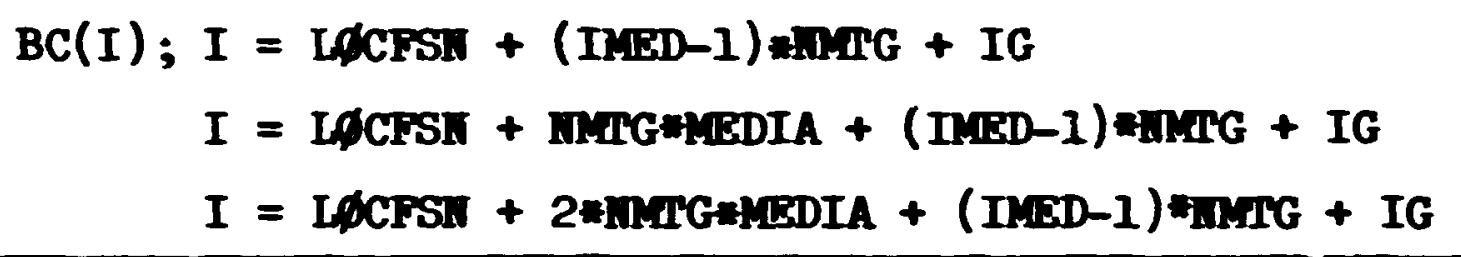 \\
\hline
\end{tabular}




\section{Main Progran}

The main program performs the following functions:

1. Sets the maximum allored size of blank common (all other routines using blenk camon use a dumy dimension of 1);

2. Bnsures that certain labellet comons are loaded in a specified order (which aist agree with the order of these comons in the diagnostic routines using the IfC runction);

3. Loads the junk word $\left(48484848_{16}\right)$ in blank conmon and all labelled camons present in this routine;

4. Sets the two variables used for input and output logical units; and

5. Calls MtRE fo: the actual administration of the jcb. (The size of blank comson is transferred to WRSE as an argument.)

Subroutines calleã: WSE

Functions required: IdC (library runction at Oak Ridge llational Laboratory - output is the absolute address (in 8-bit bytes) of the cell given as the argument)

Variables required: Junk

Variables cinanged: ITdUT ( 10 in wost other routines) IIII (I] in nost other routines).

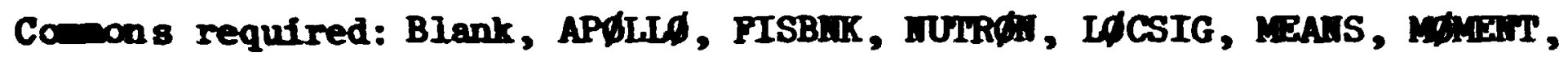
PAL, RESUIT, GEQMC, IGRIAL, PDET, USER, DUEYY.

Helprul Bints:

1. Wote that if a ner cross section, geonetry, or anaiysis package is used, the labelled comons here nay have to be modified correspondingly.

2. The Junk word is the bit pattern that cones closest to beirs output identically as either a fixed or floeting number. It is a]so recognized by subroutine HELPER that the cell has not been usea by the code.

3. The IAC function returns an absolitte address of $a$ variable in bytes, requiring division iy 4 to obtatn the nuber of 4 -byte (32 bit) vords.*

4. To change the size of blank comon only the statenent defining the camor needs to be changed, since the LAC function is used to obtain this value to be transferred to wisE.

See Appendix E for a description or all library routines used in MORSE. 
5. It is recommended that this routine always be compiled and that it be the first routine compiled. This insures that it is loaded first and that the commons it specifies are loaded first, and in the desired order, in the common area.

6. The program size, in bytes, is usually on the order of $150000 \div 4=$ (blank common size in words).

Main Routine

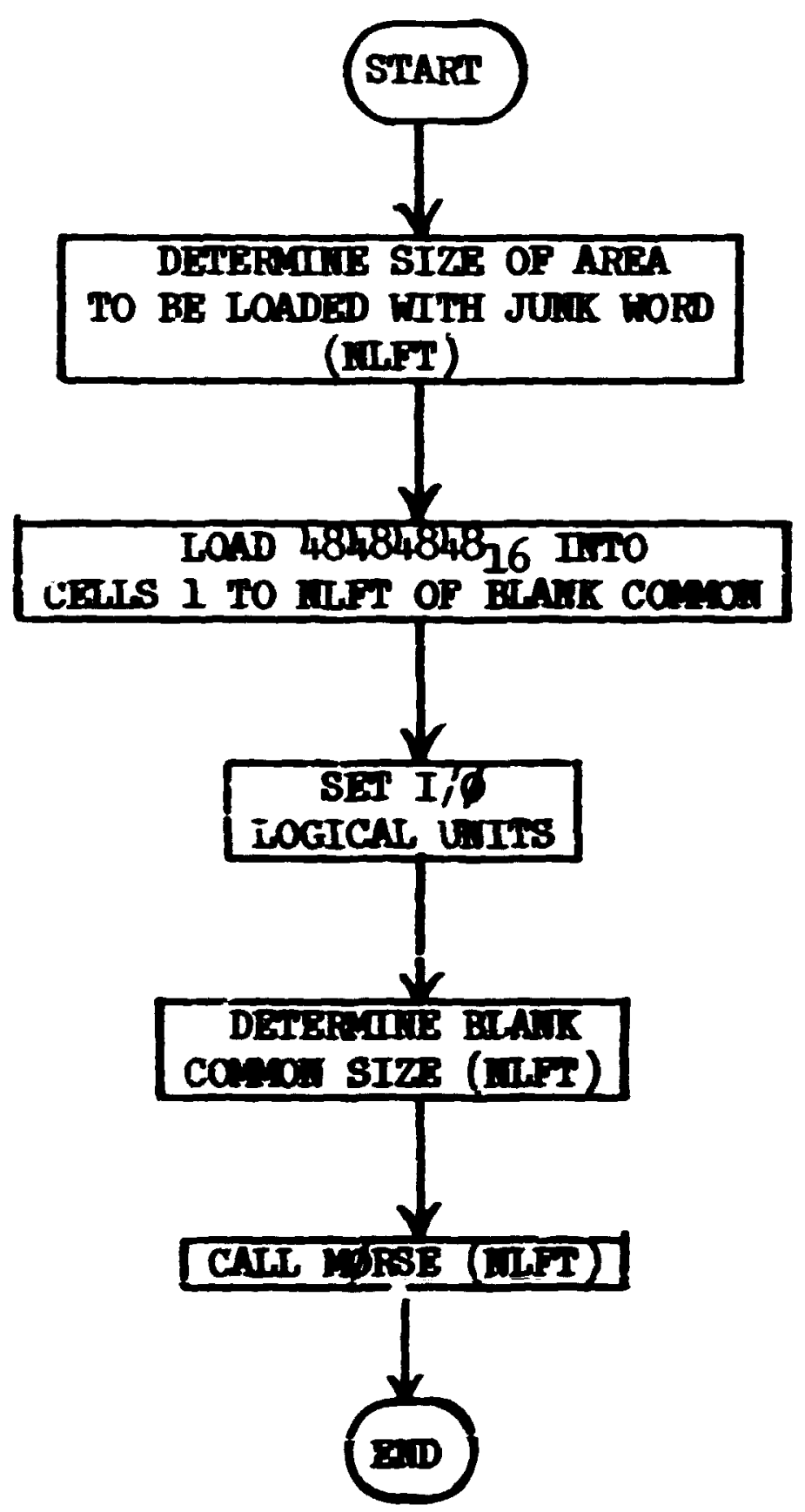




\section{Subroutine NTRE (NLFT)}

WRSE is the executive routine for the valk process and controls the succession of events which comprise the Munte Carlo process. The problem is assumed to consist of MQUT runs, each consisting of NITS batches, and starting out with KSTRT particles in each batch. Thus the functions of are logically broken down into nested loops with the inner loop consisting of the execution of the walk process for each particle. The next loop is for each batch of particles and the outer loop is for eacb run. Several problems may be run in succession by stacking input data.

There is no significant part of the walk process perturmed in MoRSE except for the termination of histories. The bookkeeping of veforecollision parameters, the determination of history terminations, and the ardering of the subroutine calls are the basic functions. The option of terminating a problem by an execution time limit is provided; this option nay only be executed at the end of a batch and the normal termination of a problea occurs in that all end of run processes are completeà.

Called fron: Main program.

Subroutines called: IIPUT, TIMER,BAIKR(-1), BAKKR(2), MSqUR, фLTPT( 1),

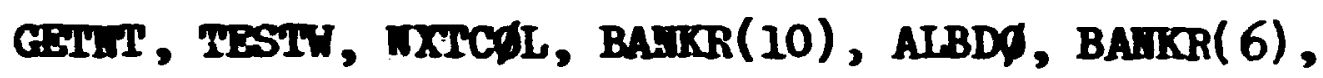
GMAP, FPRdB, GPROB, CQLISH, BAMKR(5), BAIKR(9), BN.KR(-3), ITPTI(2), BAKK(-4), THPT(3), ICIqCK.

Camons required: Blank, APQLI, WUIRd, FISBIK.

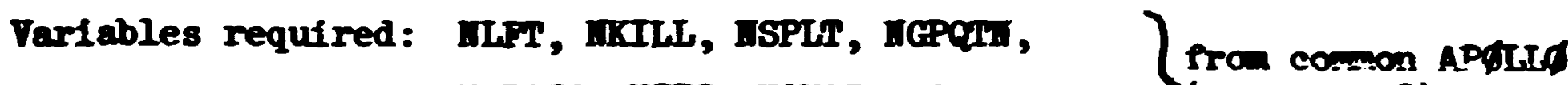

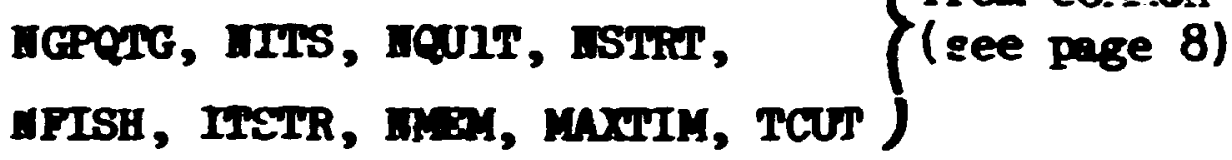

HALB - index indicating that an albedo collision has occurred.

MFISTP - Index indicating that issions are allowed if $>0$. 
Variables changed:

$$
\begin{aligned}
& \operatorname{MDEAD}(I), \operatorname{DEADWT}(I) \text { - counters } \\
& I=1 \text { - Russian roulette kill } \\
& =2 \text { - particle escaped the system } \\
& \text { = } 3 \text { - particle reached energy cutoff } \\
& =4 \text { - particle reached age limit - it was retired. } \\
& \text { IPSCL(I) - counters } \\
& I=5 \text { - number of real collisions } \\
& =6 \text { - number of albedo collisions } \\
& =9 \text { - number of energy deaths } \\
& =10 \text { - number of age terminations. }
\end{aligned}
$$


Subroxtine RSE(NLFi)

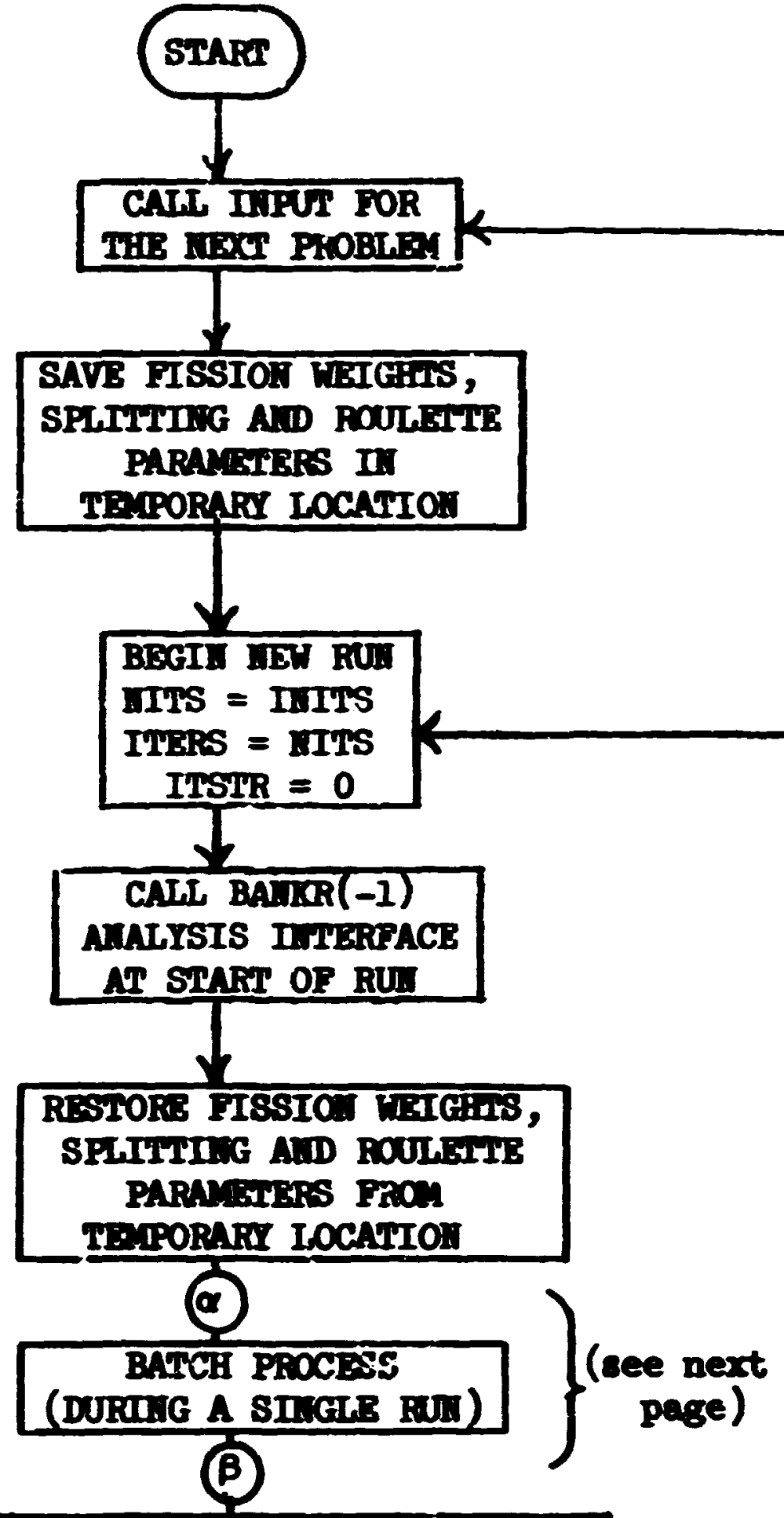

CNI BMR(

AMALISIS I.MBRTACE AT EAD OF RUM

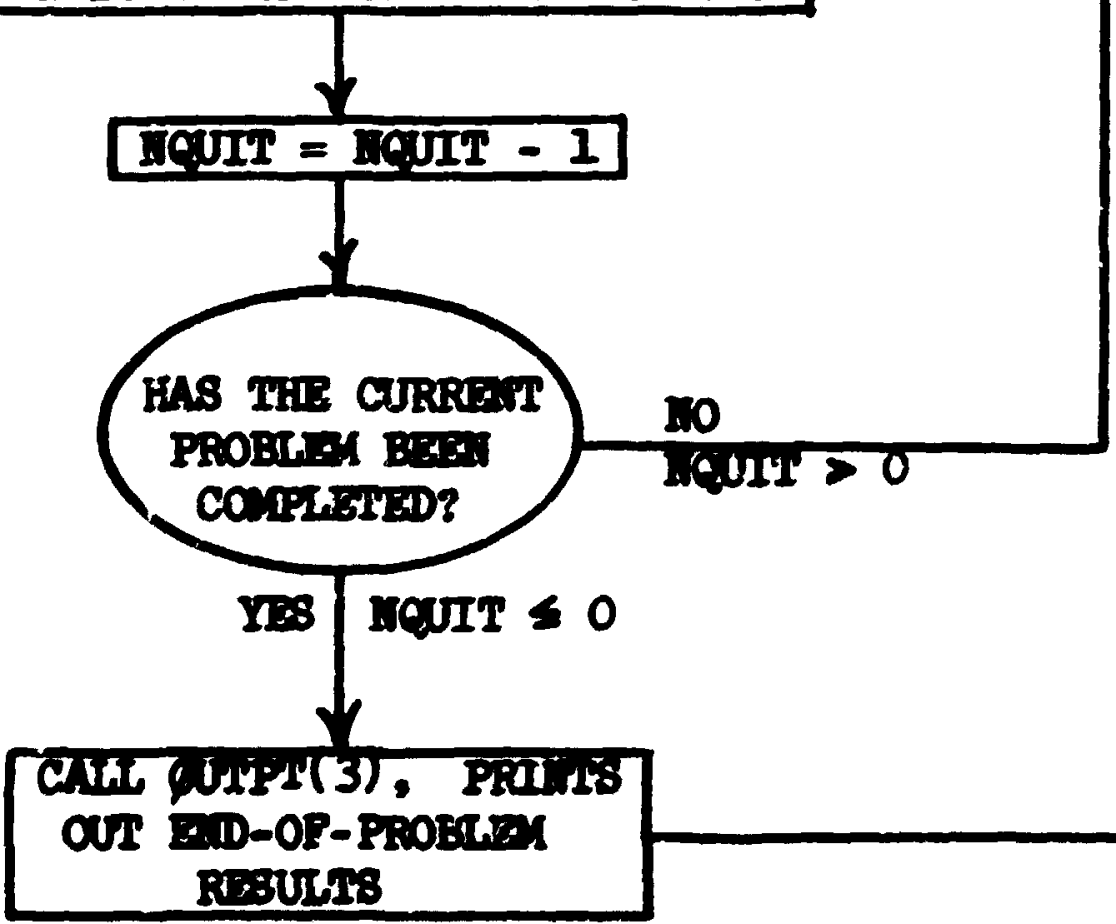




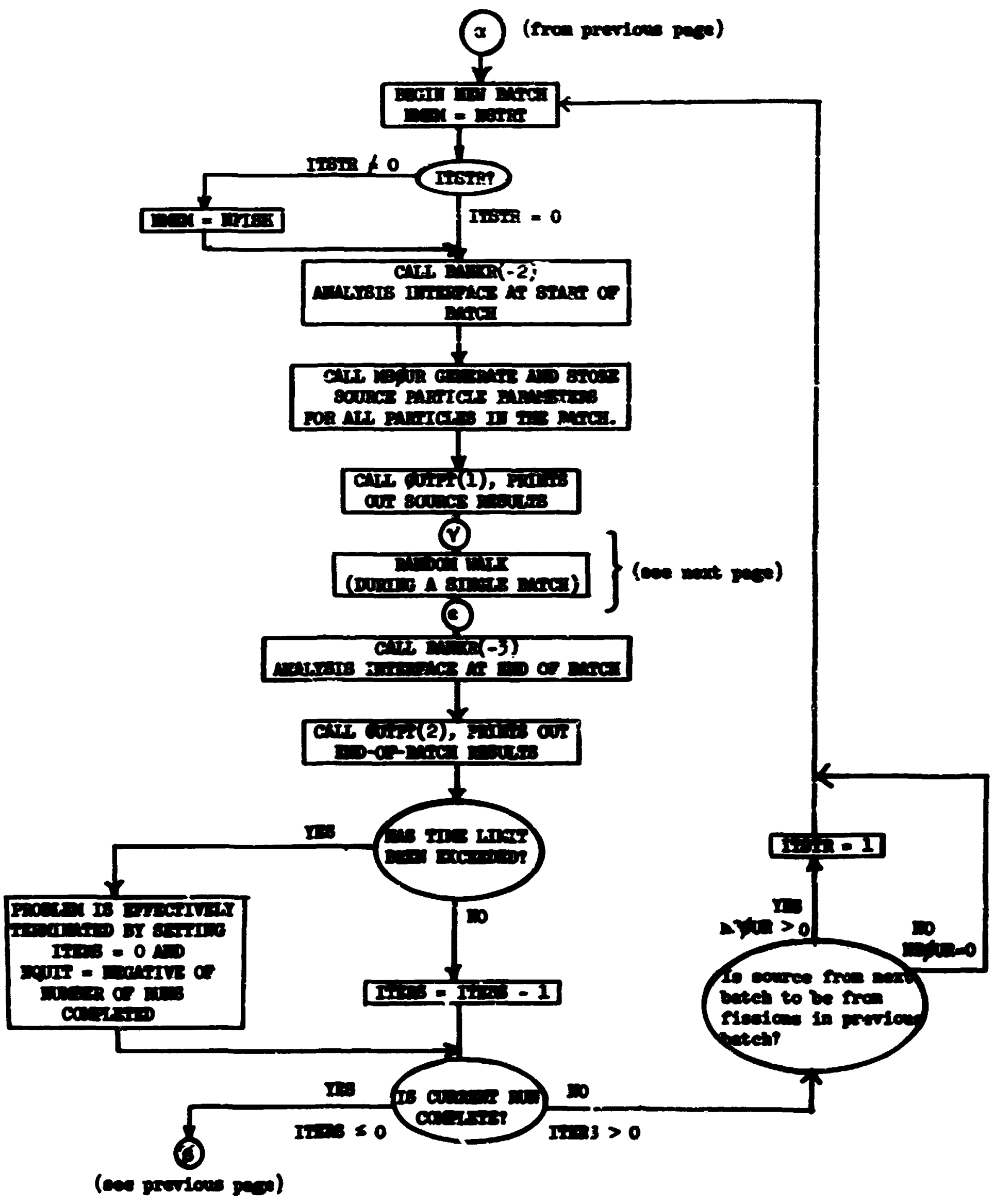




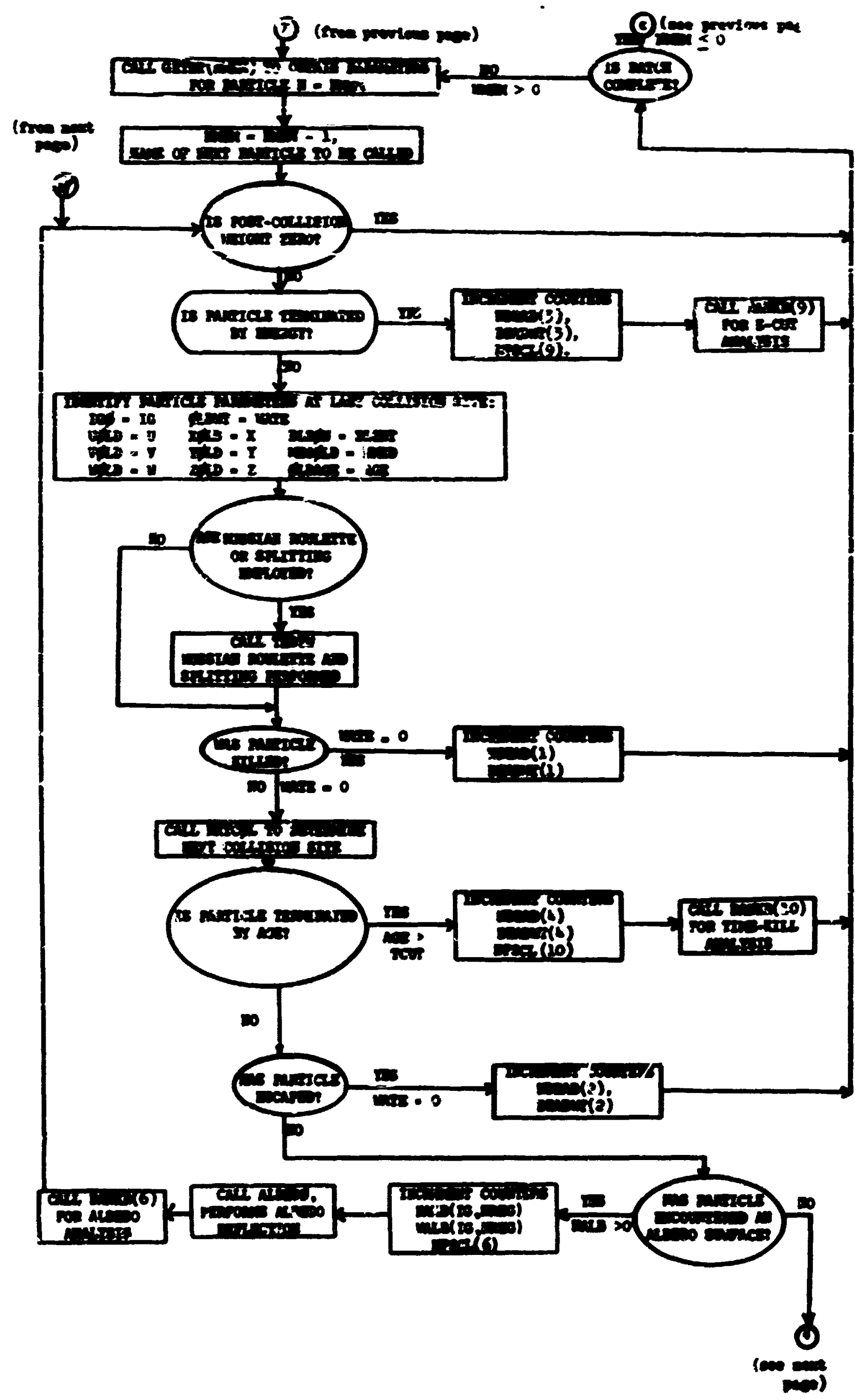


MTSE (Randon Walk, cont.)

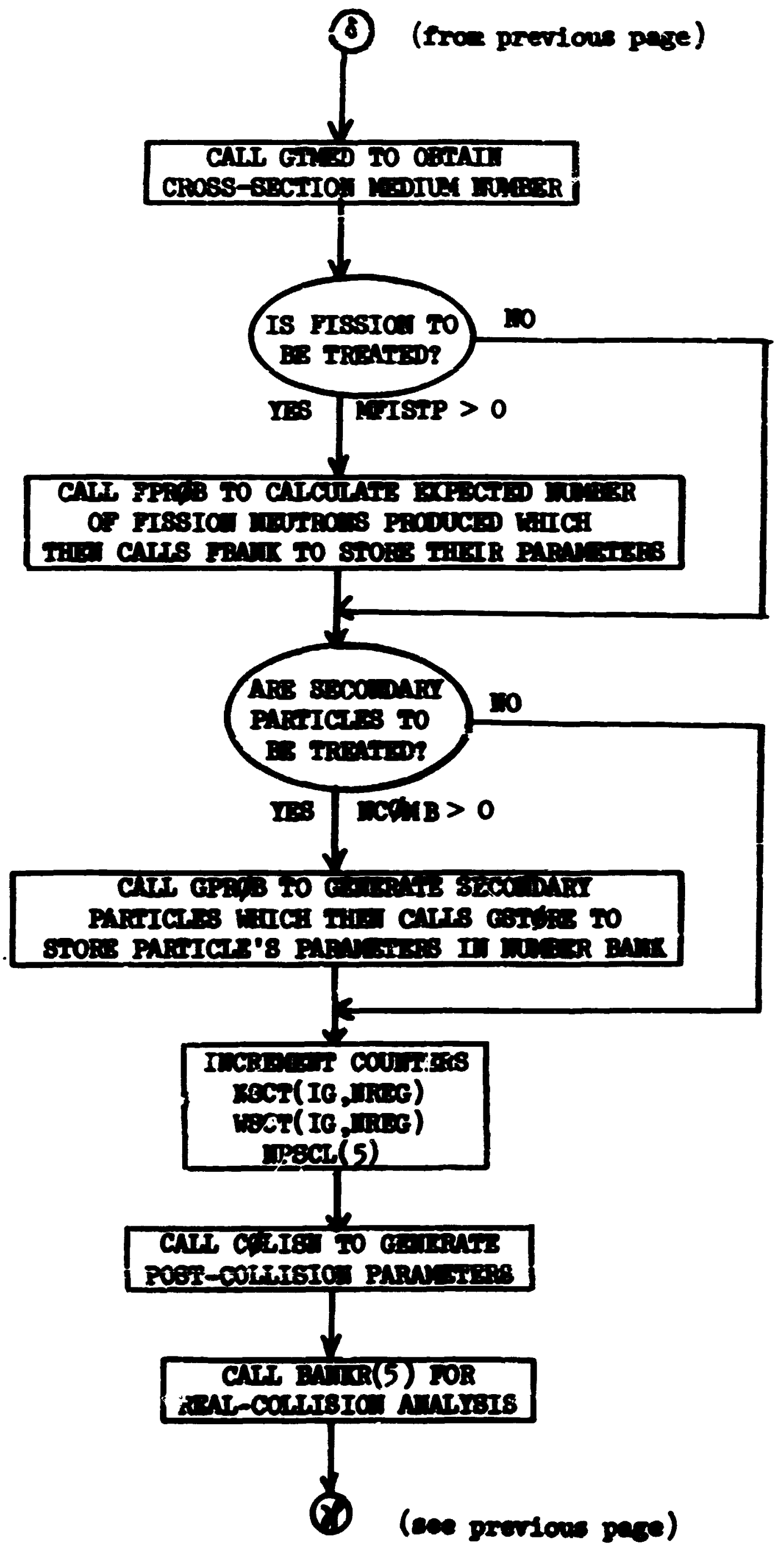


Subroutine Dasrs (A, IN)

Given an array A, DAIB inserts a hollerith string with the day of the week, the month, the day of the month, and the year. It will use as mon as 32 bytes, so $A$ mot be dimensiond at 8 for single precision. IN, on return, is the muber of h-byte words which mast be output.

Typical calling sequence:

DDERIST ARRAY (8)

CALL DATE (ARRAY, MOA)

FRIII 1, (ARRAT(I), I=1, MUN)

1 Fanar ('Trany Is ', 8nk)

producint, if called on $\mathrm{key}$ 30, 1970:

'Tpar is saruboar, mar 30, 1970'.

called rron: IPUP

Boutines calied:

Traex

Intec (Library function at Oak Bidge Hational Laboratory, converts

a 4-uste integer to an BBCDIC string)

InrBCD - same as IndBC except also returns the nuber of bytes in the BBCDIC string

Comcons: DATDAT which contains arrays of BBCDIC characters for months and weekdays, arrays of nubbers of BBCDIC characters and starting points. It is loeded in a Block Data routine with the following values:

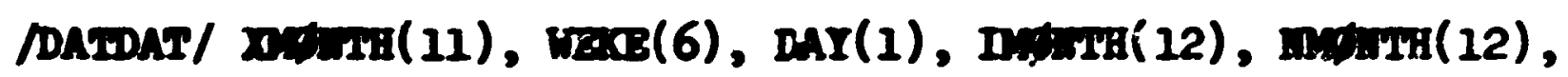

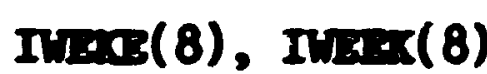




\begin{tabular}{|c|c|c|c|c|c|c|c|}
\hline Index & 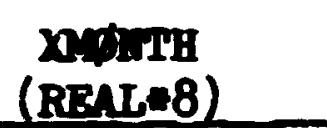 & $\begin{array}{c}\text { NEKT } \\
\text { (RB,L-8) }\end{array}$ & $\begin{array}{c}\mathrm{MY} \\
(\mathrm{RE}: \mathrm{L}-8)\end{array}$ & IIIIH & Drow & IMEKS & IVIS: \\
\hline 1 & JANUERY (I)* & HUH?sU: 1 & DaY, (1) 19 & D 0 & 7 & 0 & 4 \\
\hline 2 & FBBRUARY & MTIUE & & 8 & 8 & 4 & 3 \\
\hline 3 & MARCH (3) & MBDIBS (2) & & 16 & 5 & 8 & 3 \\
\hline 4 & APRIL (3) & IHURS (3) & & 24 & 5 & 12 & 4 \\
\hline 5 & мау (1)лик & FRI(5) & & 32 & 3 & 16 & 6 \\
\hline 6 & JUYAUGU & SATUR (3) & & 36 & 4 & 24 & 5 \\
\hline 7 & STCSgpr & & & 40 & 4 & 32 & 3 \\
\hline 8 & EMBBR (3) & & & 44 & 6 & 40 & 5 \\
\hline 9 & ACTdBER (1) & & & 52 & 9 & & \\
\hline 10 & MVVEABER & & & 64 & 7 & & \\
\hline 11 & DECEABER & & & 72 & 8 & & \\
\hline 12. & & & & 80 & 8 & & \\
\hline
\end{tabular}

(1) denotes 1 blanks. 


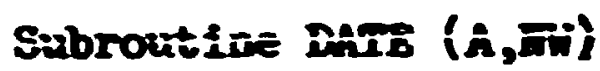

\section{STNRT}<smiles>C1CC2CC12</smiles>

CALL FUGTIO INGST

FOR THB DAY OF NESTK (THSS)

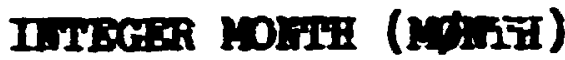

InTBGer DAY OF molHi (IDAT)

IIIPGER MBAR (IMERR)

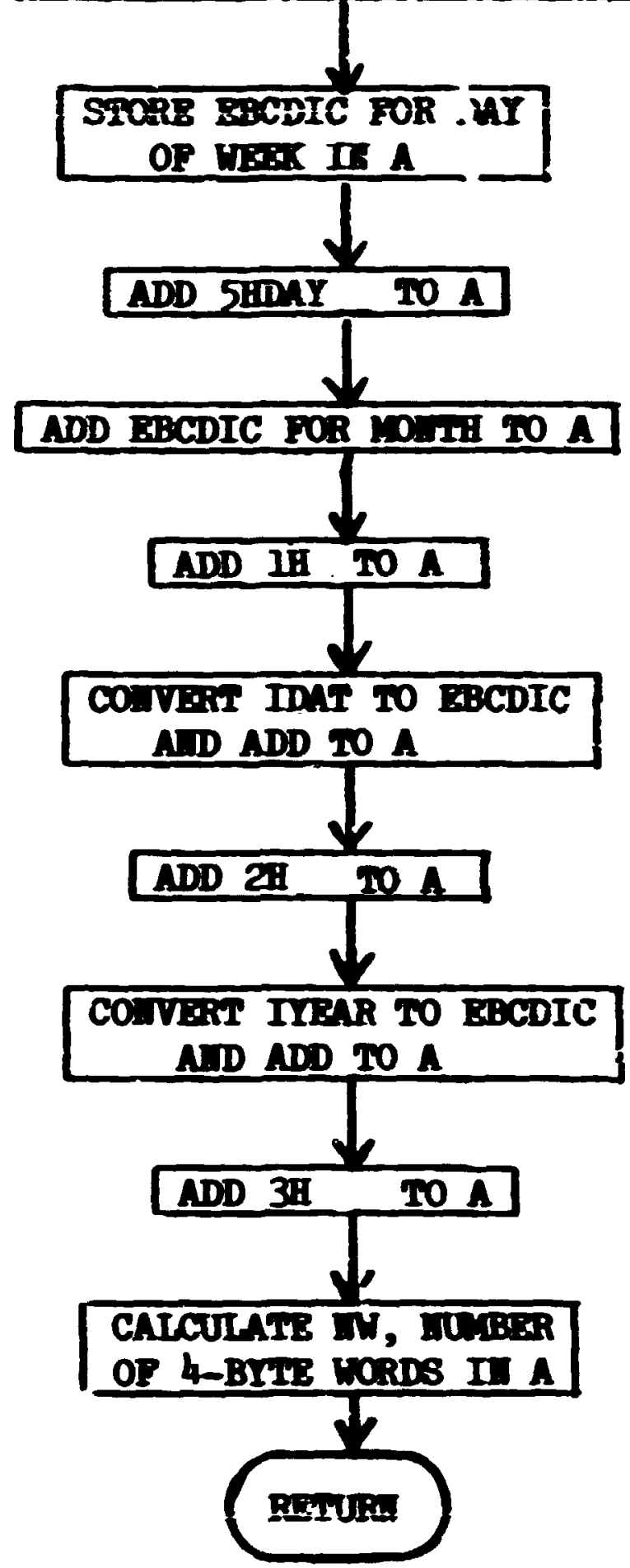




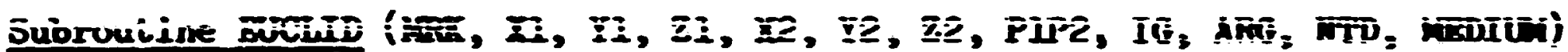

This routine is provided for the user to deterdine the nuber of nean free paths between two points in the systen. It vill eitner return the total numer of mean free peths or vill return the first boundary intersection point and the nuber of mean free paths to that point.

Called ron: GBrism

Subroutines called: Cory, IAde, ISIGIA.

Punctions used: DSORT (library)

Cosmons requirta: Hax.

Variables required:

MPX - Set to 1 upon calling,

X1, II, 21 - coordinates of starsing point,

X2, Y2, 22 - coordinates of end point.

P1P2 - distance between starting and end points,

IG - enerd group index,

WFD = 0 for total mean free paths

\& 0 for intersection points and mean free poths between.

Variables cheaged:

sis = I for a Alght reaching the end point,

= O tor a flight crossing a medil boundary (II $\neq 0$ only),

= -1 for a night escaping the sjoten,

$=-2$ for a flight encountering an internal roid (II \& 0 colly),

$\mathfrak{Z}, \mathbf{M}, \boldsymbol{Z}=$ returns boundary intersection point if IID $\neq 0$,

ARG - negative of muber of nem free paths,

WID - if IID $\neq 0$ on input, WII return as -1 if an escape occurs,

MoIUA - nedile muber of end polnt.

Sigiflchet internal variables:

mir - Nas set by Gand (returned as kix - defined above)

IIIV - internal nas cet to 1 when traveraing an internal roid (nediv 1000).

Linitations: Ho provision is ande in this version for albedo bocendaries. 


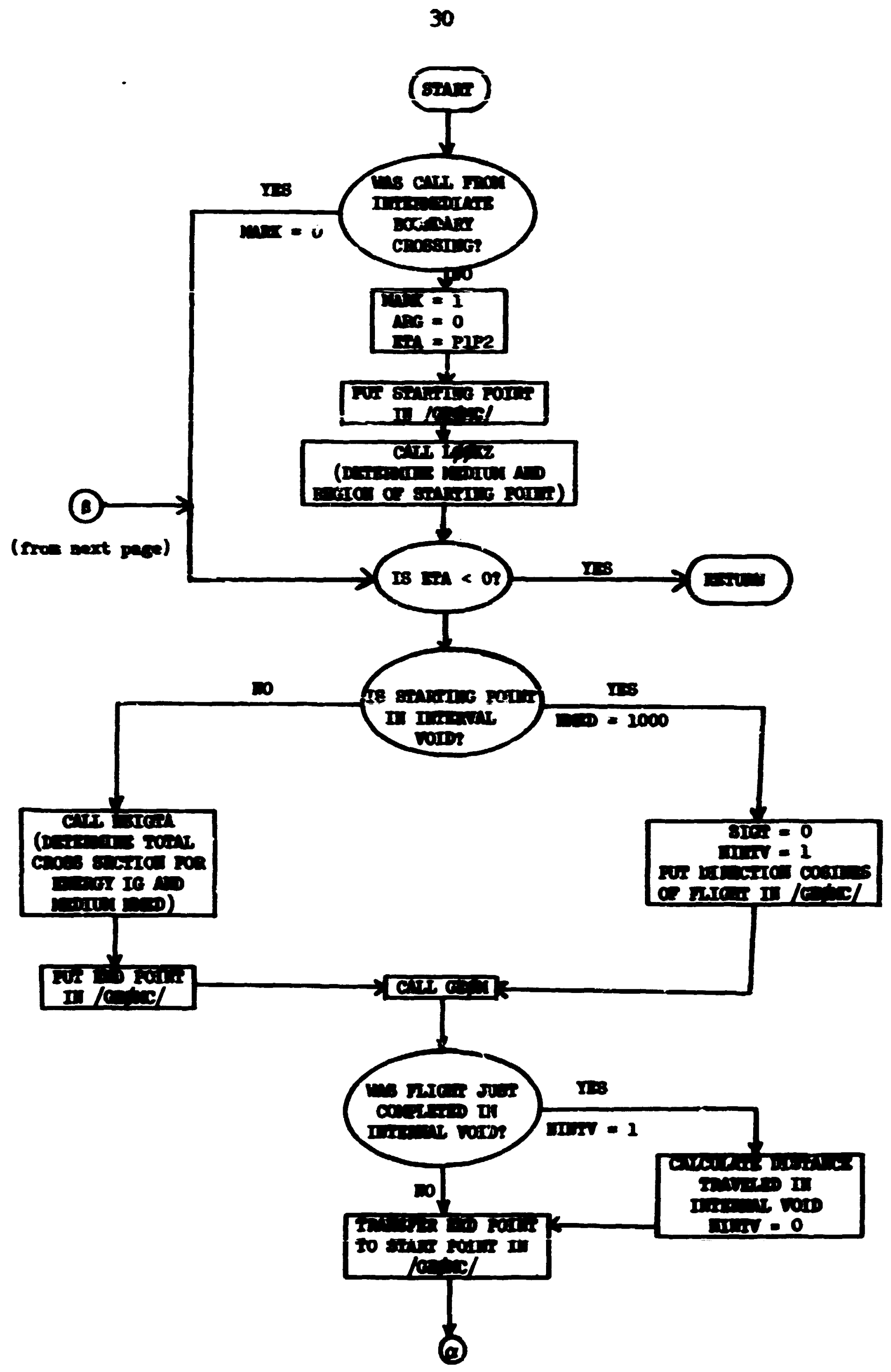




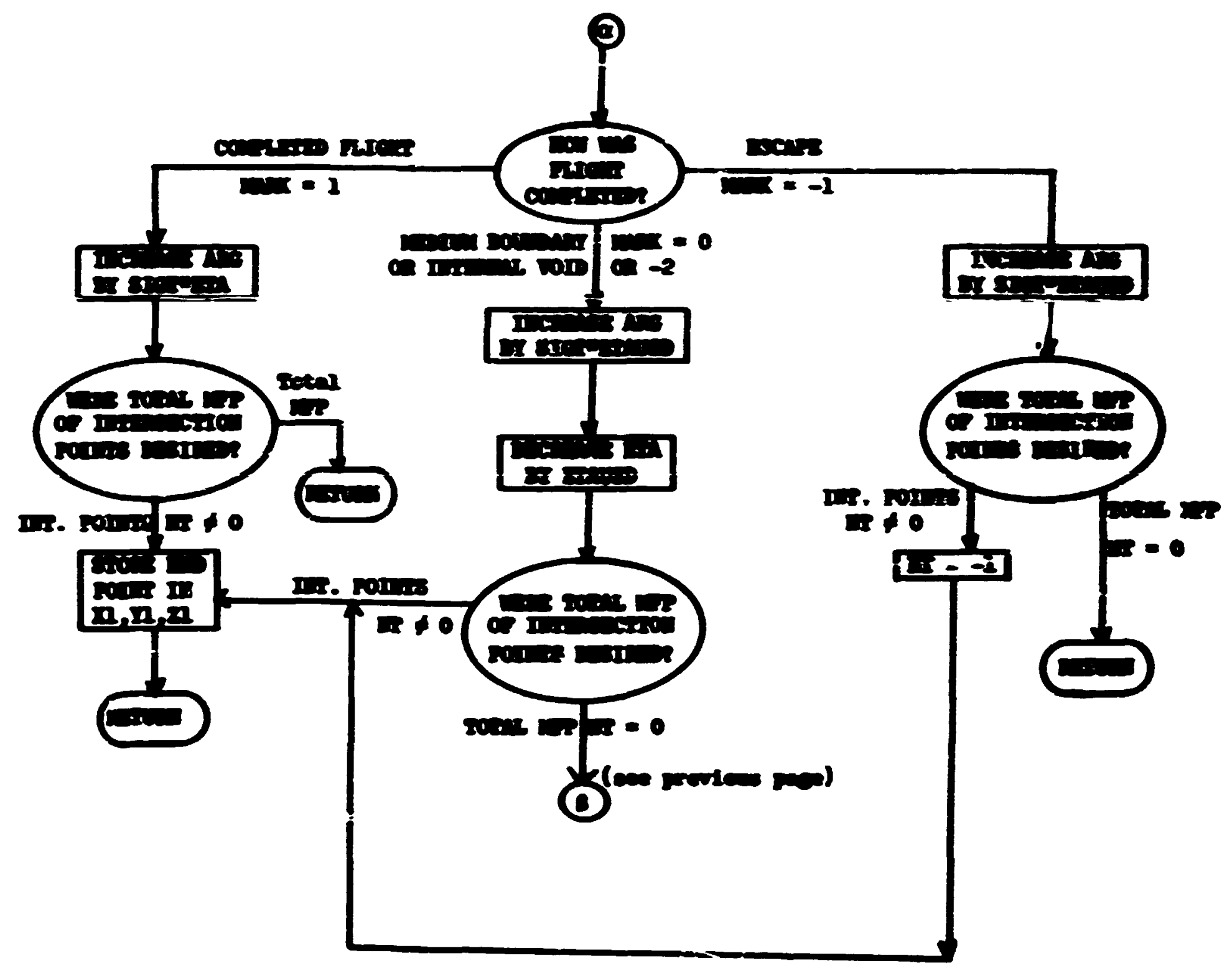




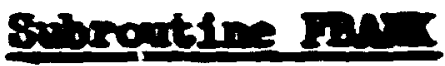

Fissica neutrons are stored by Find in the ares in blaok comon set aide for this purpose. Seven parmeters can be stoced for In'sr neutrons in this Nisaton batk. If it is called as was as 50 times when the beik is Ioll, HitP and BCT are called.

Called rron: Fits.

Sobroutines called: HitP, EUT (Iibrars).

Comom required: Blank, IUTH, APMI, FISE:

Terlables respotred:

IFIS: - location of cell zero of the Nission benk in blent comon, IISA - nuser of neutrons in Nission benk, InsT - main sumber of particles allowed in bank, Shas - welent of fission neatron to be benked,

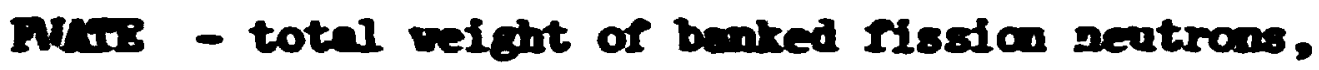

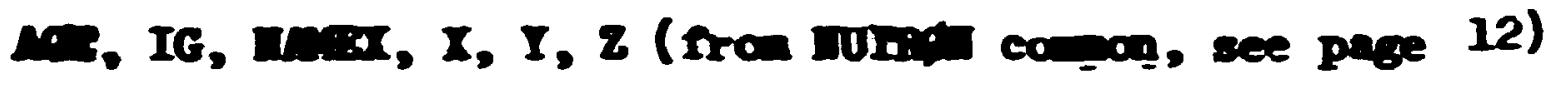

Variables chroged:

IISSI - increvented arter benking,

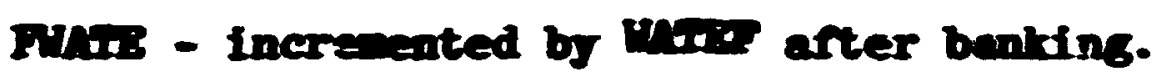

Sigiffent internal varisbles:

DruL - Increnented upos each call when benk is rull. 
Subroutine FBAK

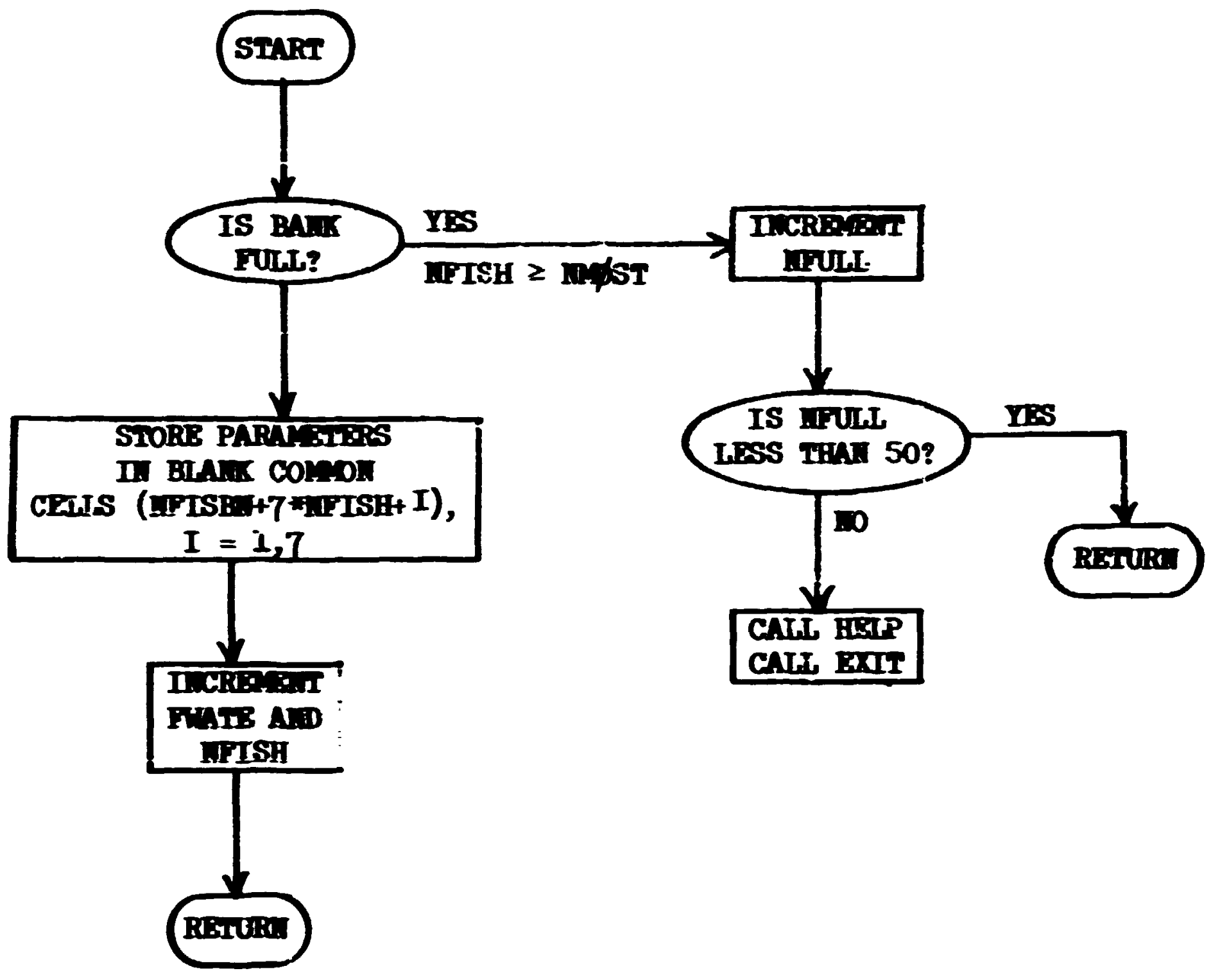




\section{Pando Dr-ber Pactare}

The raxdon nuber package is essentially the DSR package as nodified for the In-360 coputers. Six-byte ( $48 \mathrm{~b}$ it) arithetic is used with a generator (constant mutiplier) equal to $1474496 D_{16}\left(=3277244615_{8}\right)$. If no stertias nuber is given (a value of zero input) the routine uses $3521932 h_{16}$ wich is tulce the gecerator. The trailing zero bit restricts the sienificence of the aritmetic to 47 bits $s 0$ that the paeudo-randan secuence generated by the CDC-1604 pankage may be du 's.cated. (The CDC1604 pacizage must use $3277244615_{8}$ as the senerator and starting number to Are tho sane sequence.)

The following subjecorams are avallable in the package:

Fingur Calling Statement Randon Nuber Cenerated

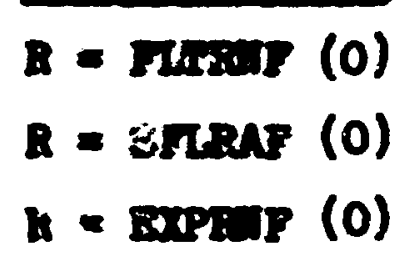

$\operatorname{carr}$ MItim (SI, C,s)

card Potrin (sI, Cis)

ard orso $(x, y, Z)$

$d=\operatorname{lag} \sin (T)$

$R=$ FISEDP $(0)$
Unifarily distributed on the interval $(0,1)$. Iniforily distributed on the ifierval $(-1,1)$. Exponentieliy distribuied: $P(R) d R=e^{-k} d R$ $0 \leq \mathbf{R}<-$

The sibe and cosine of wibre is uniformy distributad on the interral $(0,2 \pi)$. A randon arimathar angle.

The sine and cosine of $\theta$ whare coso is uniforwly distributed on the interesel $(-1,1)$.

A resica polar angle.

An 1 sotropic unit rector. $X=\cos \theta, Y=\cos \phi$ $\sin \theta, Z=\sin \phi \sin \theta$ where $\theta$ is a randon polar angle and is a ranám azinuthal angle.

Maxwellian energy:

$$
P(R) d R=\left(\frac{4}{T^{3} \pi}\right)^{1 / 2} R^{1 / 2} e^{-R / T} d R .
$$

A neutron speed squared from the Hatt flscion spectrum: $P(R) d R=e^{-R / T} \sinh \left(2 / E^{*} E_{f} / T\right)$, where $T=0.965 \times 1.913220092 \times 10^{18}$ and $\mathrm{E}=$ $0.533 \times 1.913220092 \times 10^{18}$ (ref. 8 ). 
(cont.)

Forman Calling Statement

Rendon Huber Generated

CALL RUDIn(R)

Loads $R$ into RADPd $(I), I=2,4$ if $R \neq 0$.

$R$ is read with a 212 format and mast be double precision ( 8 bytes).

CALL FidTr(R)

Loeds RAEDAI(I), I = 2, 4 into $R$.

Wote: The argoments of FLTHW, SFLRA, BCPRT, FISTiF are not used by the routines. 


\section{Subroutine IPR:3}

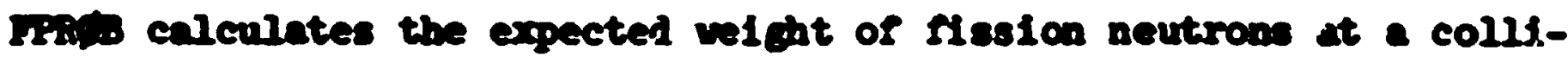
sion point and tiben splits or pleve Ruseien roulette so as to produce the

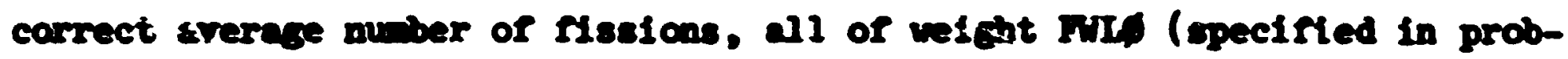
len input for eech region). BBAr is called for each neutron produced, to be stored for processing in the next eneration.

callod tron:

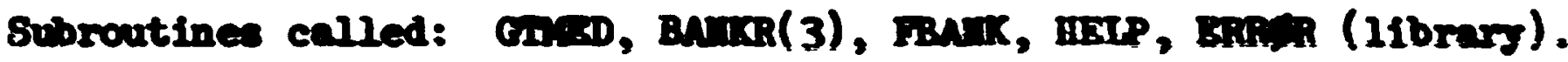

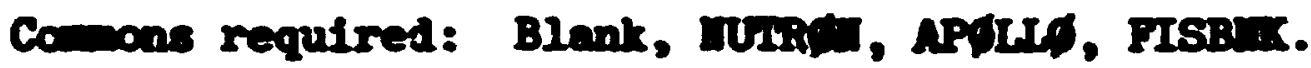

Variables required:

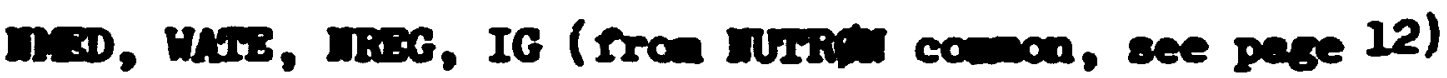

LACrsin - locetion in blenk comon if cell zero of arrav of fission crose sections,

LNGusc - locetion In blank comen of cell sero of scattering counter arrays,

DWD - cross-section mediun of collision point,

wareg - maximn region number,

marc - totel number of enerEs eroups,

FIVI - total of flssion weights from all collisions,

IAChI - location in ulank common of cell zero of array Fritw, IPSCL(3) - Iission counter.

Variables changed:

WATEP - Rission weight transierred to FBAMK

FTrt

IPSCL'́(3)

Significant internal variables:

Fil - current value from array FIT,

ISCT - location in blank coman of (IG, WREG) cell of scattering counter array UFIZ (and later WFIZ). 


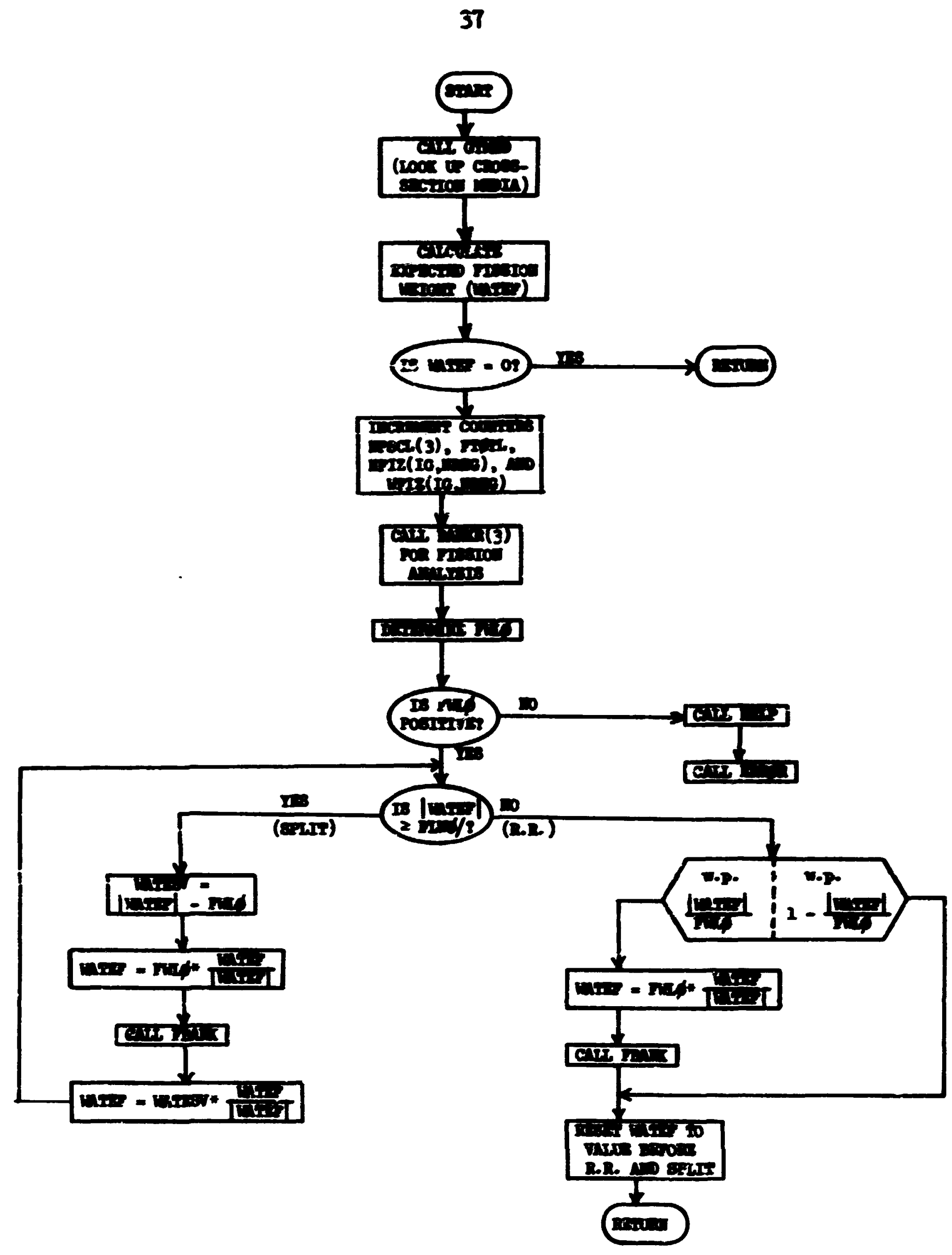




\section{Sompoting Fe lig}

guis motine is ealled by the source executive routine, MSWUR, when the scurce for the present batch is to be telen fron the previous batch Nissions. Its runction is to transfer the neutron paraneters fros. the flseion bank to the neutron bank. If there were no fissions in the previous bateh, it sets a nas, Frints a nessage, and returns.

cerled fro: vitur.

Subroutines called:

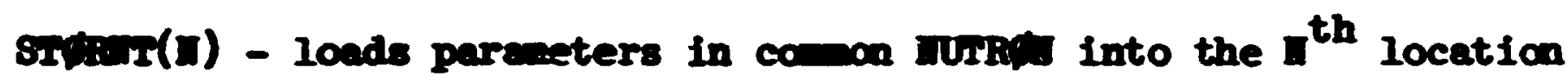
In the neutron bank.

Comons required: Blank, Wuriph, FISBIK, APJILd.

Variables required:

UISA - nuber of fissions promiced in the previous batch,

Irier - set equal to IIISA,

IIrs - nuber of batches requested for the run,

Irtiss - batch counter,

MFISB - location in blank comon of cell zero of the fission bank. Variables chenged:

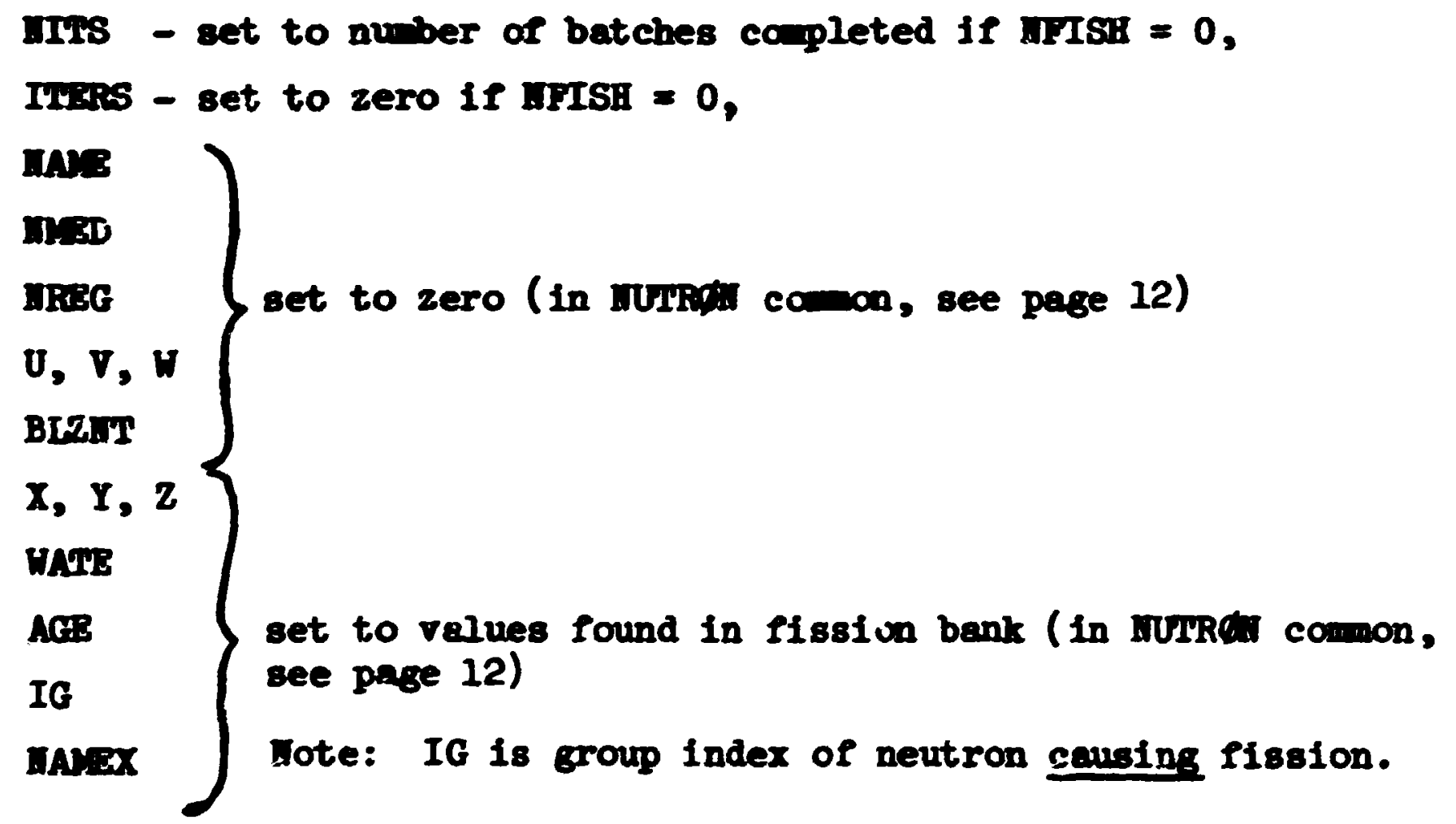


Subroutine FStUR

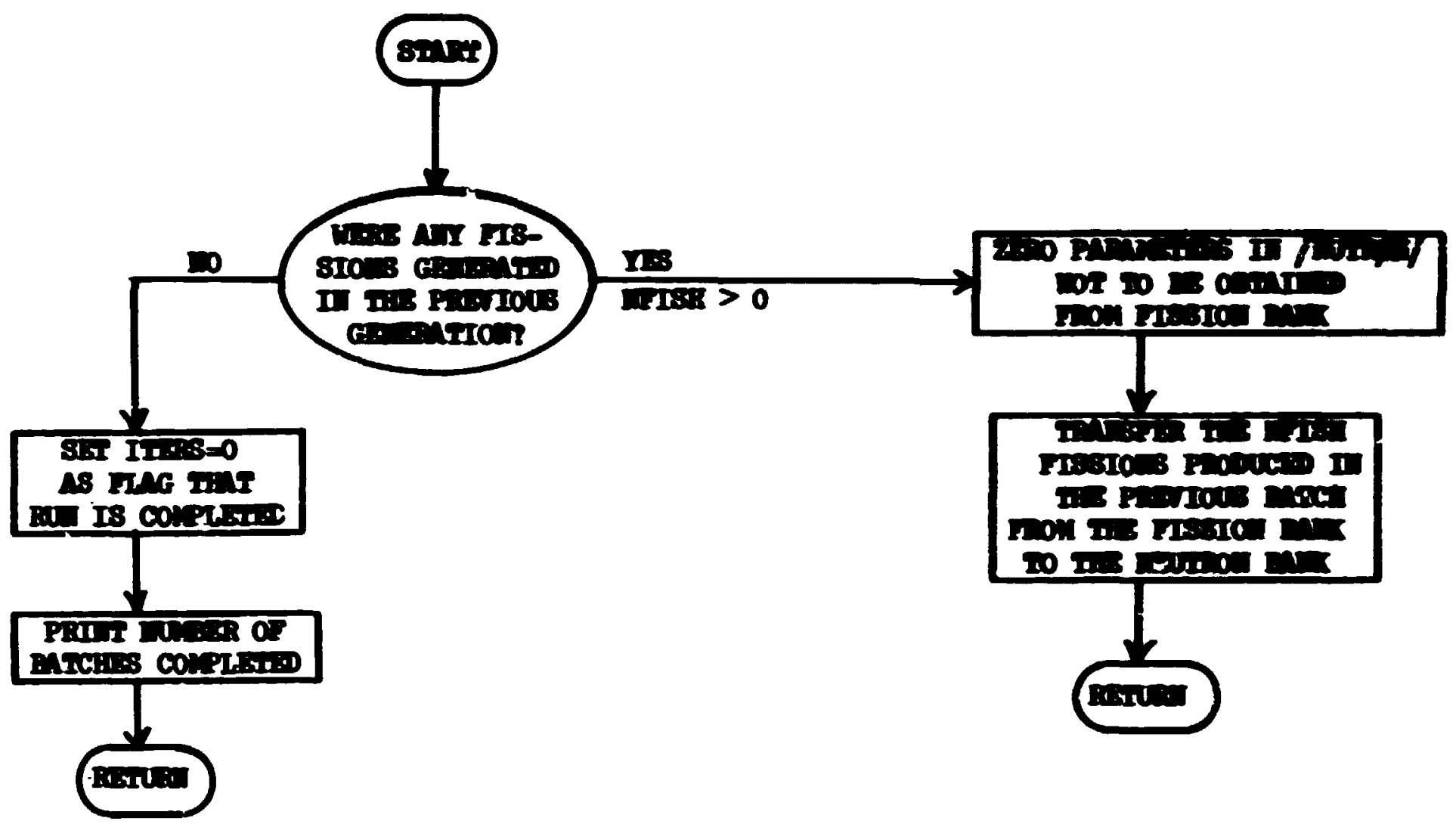




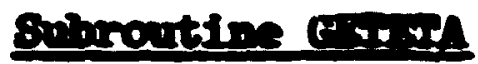

The subroutineG Gim selects Em, the numer of meen-free-paths for the next night, fron an appropriate exponential distribution. Pathleagth stretching based on the exponential transform $9-11$ is included, as vell as an option to select ron a nodified distribution which does not perit a particle to secape sxom the sjstem.

The ubiased flight peth distribution nunction is given by

$$
P_{0}(n)=e^{-n}
$$

where $n$ is the distance traveled in mean-fres paths. Selection of a particular night path BrA from $P_{0}(n)$ is done by the runction BuPiar (in randion nuber package, see page 34 ).

If an external boundary occurs at some distance, ARG mean-free paths from the starting point aloag the night direction, then the probability of escape is $e^{-A R G}$. If it is required that no particle escape, then the distribution function $e^{-\lambda}$ is nornalized over the interval $(0, A R G)$, ana the Night path is selected the nodified distribution

$$
P_{1}(n)=\frac{e^{-n}}{\left(1-e^{-\Lambda R G}\right)}
$$

and the particle's weight is adjusted by the factor

$$
\frac{P_{0}(n)}{P_{1}(n)}=\left(1-e^{-A R G}\right) .
$$

Path-length stretching, wich is a form of biasing (or importance sempling), can be accomplished by selecting from the modified distribution

$$
P_{2}(n)=\frac{1}{\text { BIAS }} e^{-n / B I A S} \text {, }
$$

which produces values of EIA a factor of BIAS times those produced by the unbiased distribution $P_{0}(n)$. Therefore, values of BIAs greater than 
wity will stretch the path length and ralues less then unity ofll shrint the path leagth. The actual selection is acconplished in tern of the distribution nuction for $n^{\prime}=n / B I A S$,

$$
P_{2}\left(n^{\prime}\right)=P_{2}(n)\left|\frac{d n}{d n^{\prime}}\right|=e^{-n^{\prime}}
$$

A selection is sade from $P_{2}\left(n^{\prime}\right)$ which gields ralues of III' and then

$$
\text { BYA = BIAS'BrA' }
$$

If patb-length biasing is useo., then the particle's veight mast be adjusted by the factor

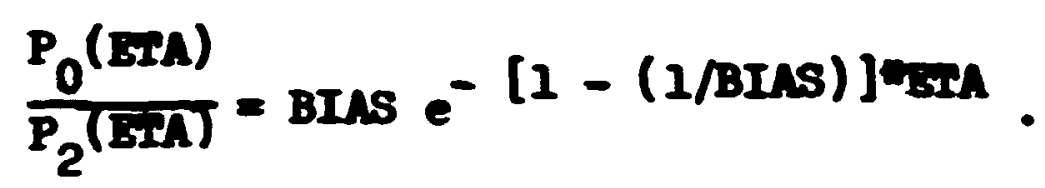

For the combination of path-length stretching and no escape, the nodified distribution is gives by

$$
P_{3}(n)=\frac{e^{-n / B I A S}}{\operatorname{BISS}^{*}\left(1-e^{-\operatorname{RG} / \mathrm{BIAS}}\right)}
$$

with the actual selection of BPA' being ade from the nodified aistribution

$$
P_{3}\left(n^{\prime}\right)=P_{3}(n)\left|\frac{d n}{d n^{\prime}}\right|=\frac{e^{-n^{\prime}}}{\left(1-e^{- \text {ARG/BIAS }}\right)}
$$

where $n=$ BIAS' $^{\prime}$ '. The path-length EFA is then giren by

$$
\text { EHA = BIAS'ENA', }
$$

and the particle's weight altiplied by the factor

$$
\frac{P_{0}(E I A)}{P_{3}(E I A)}=B^{2} A S^{n}\left[1-e^{-\operatorname{EIA}(1-1 / B I A B)}\right]\left(1-e^{-A R G / B I A S)}\right.
$$

The form for the factor BIAS used in this rersion of GritrA is based on the exponential transform and can be expressed as 


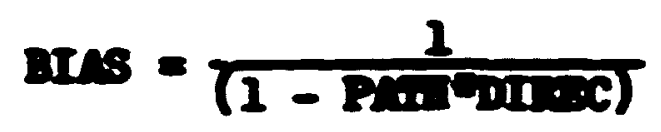

chere

DInx is the coalne of the ande between the nicht direction and the nost inportent direction (celenlated bs the user runction DLIBX),

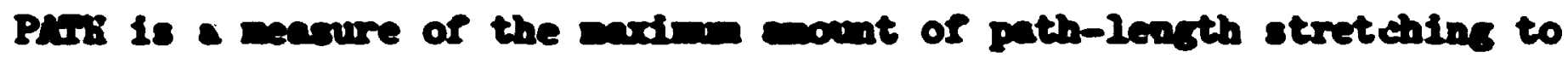
be aplied. A value of zero correcponds to BIAs $=1.0$, and no

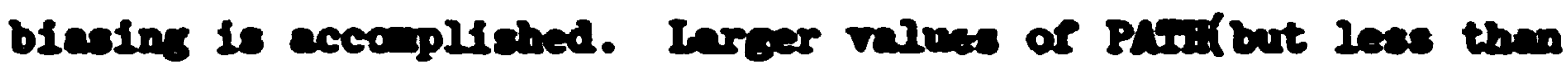
unity) Jield values of BrAs > 1.0 when DIfix > 0 , ad the particle's path length is stretebed accordincly. Conversely. wen DInBC < 0 (the particle is treveling and frow the inportent direction) BIAS $<1.0$ and the track is shori-ned.

Called Irom: Wucti.

Subroutines called: Buchid.

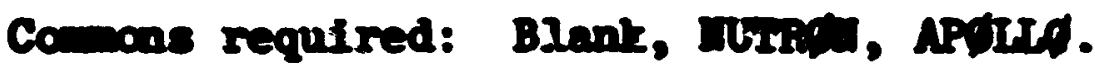

Variables required:

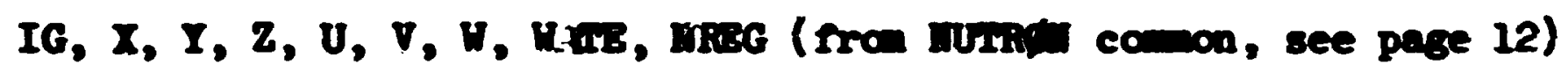
mXeP - nuber of enere coups for velght standards and/or pethlength stretchting parmeters PAut,

polsux - an index for nonleakage biasing,

RAD - the largest overall dinension in the systen,

Pfirl - path-length stretching paraneters (in blank camion).

Variables changed:

EIA - the number of nean-free paths to the next collision,

WAIS - the particle's velght corrected for the biasing exployed during the present filght selection.

Significant internal rariaioles:

ARG - the distance in mean-free paths from the last colliatca site to an external boundary along the present flight direction. 


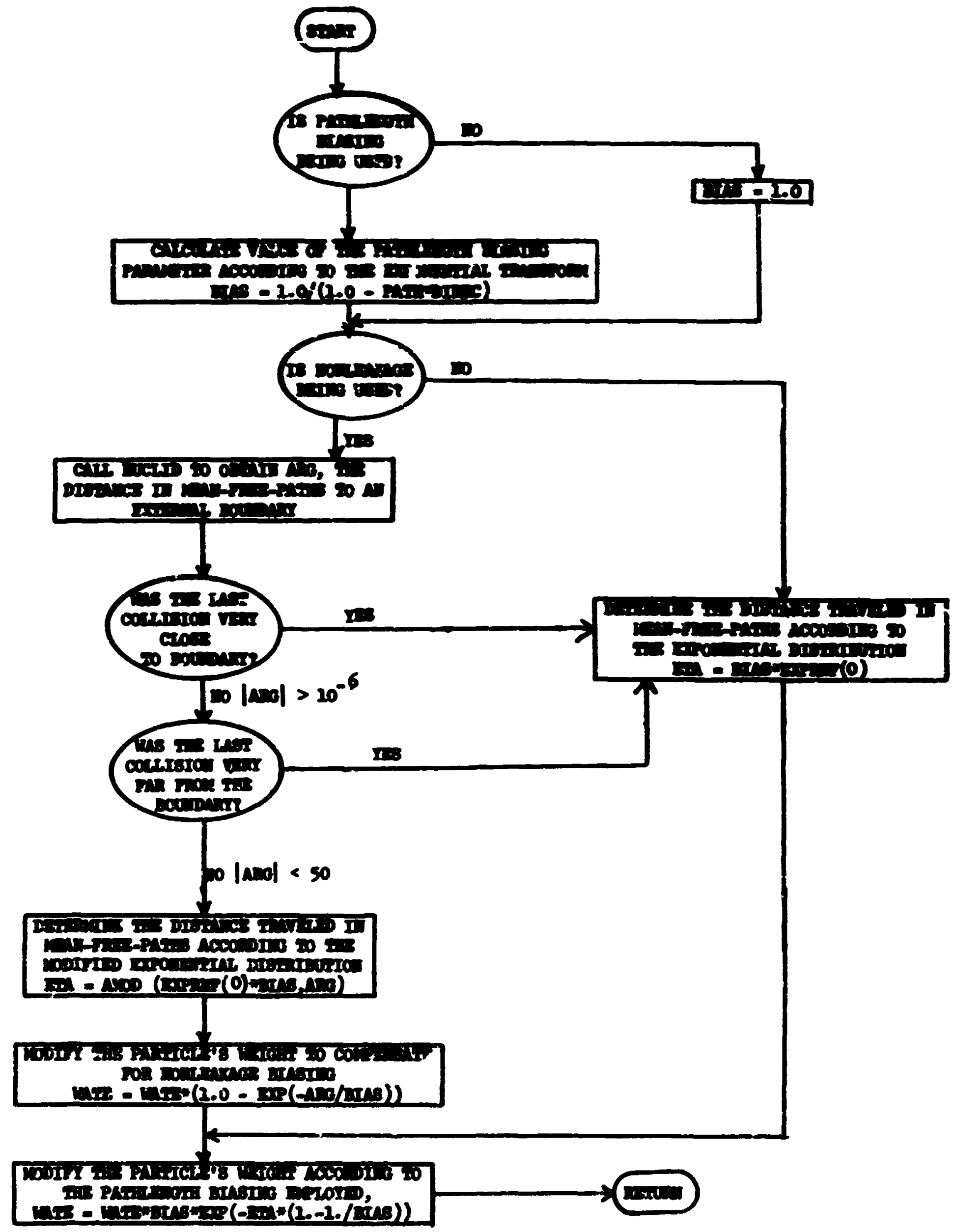


Soroutine Eving(u)

Three entry points are used in this routine. Bntry SBInI saves the adress (in vords) of the first ce' ralable for the neutron bank in blank comon and returns the address of sic last cell it will use. Entry

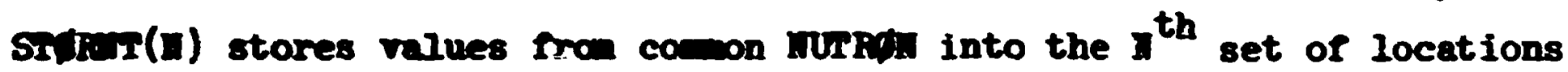
In the noutron bank and Batry $\operatorname{Gin}(I)$ does the reverse; it pichs up variables fron the bank and puts then in comon IUTHal.

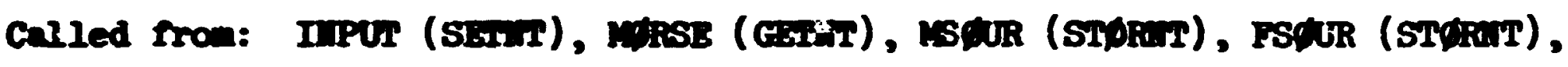
Corps (GSir).

Comons required: Blank, Wurth. The area of blank camon used for the neutron bank is shown in PIG. 3. Hotice that IG, MAN, WNIS, MSD, and IRBG are stored in 2-byte vords (and are therefore linited to $\leq 65535$ ), symolized by a dotted line splitting the noral 4 -byte word.

Variables required:

SBIUT: IIAST

BIST

GSTHY: I

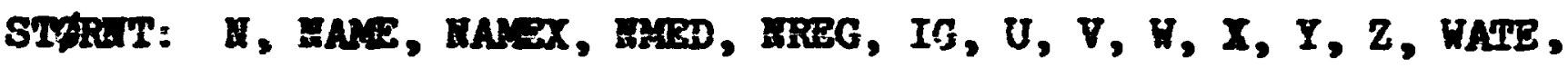

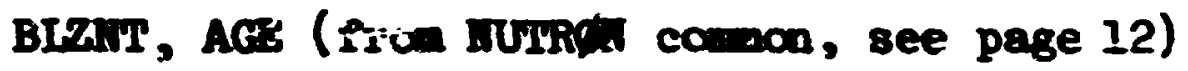

Variables chenged:

SEYWT: BLAST

GEIMT: variables in comon NUTh required by SEIHT above, STORWT: 12 consecutive locations in blank cusmon.

Significant internal variables:

wid - location in biank comon of start of neutron bank. 


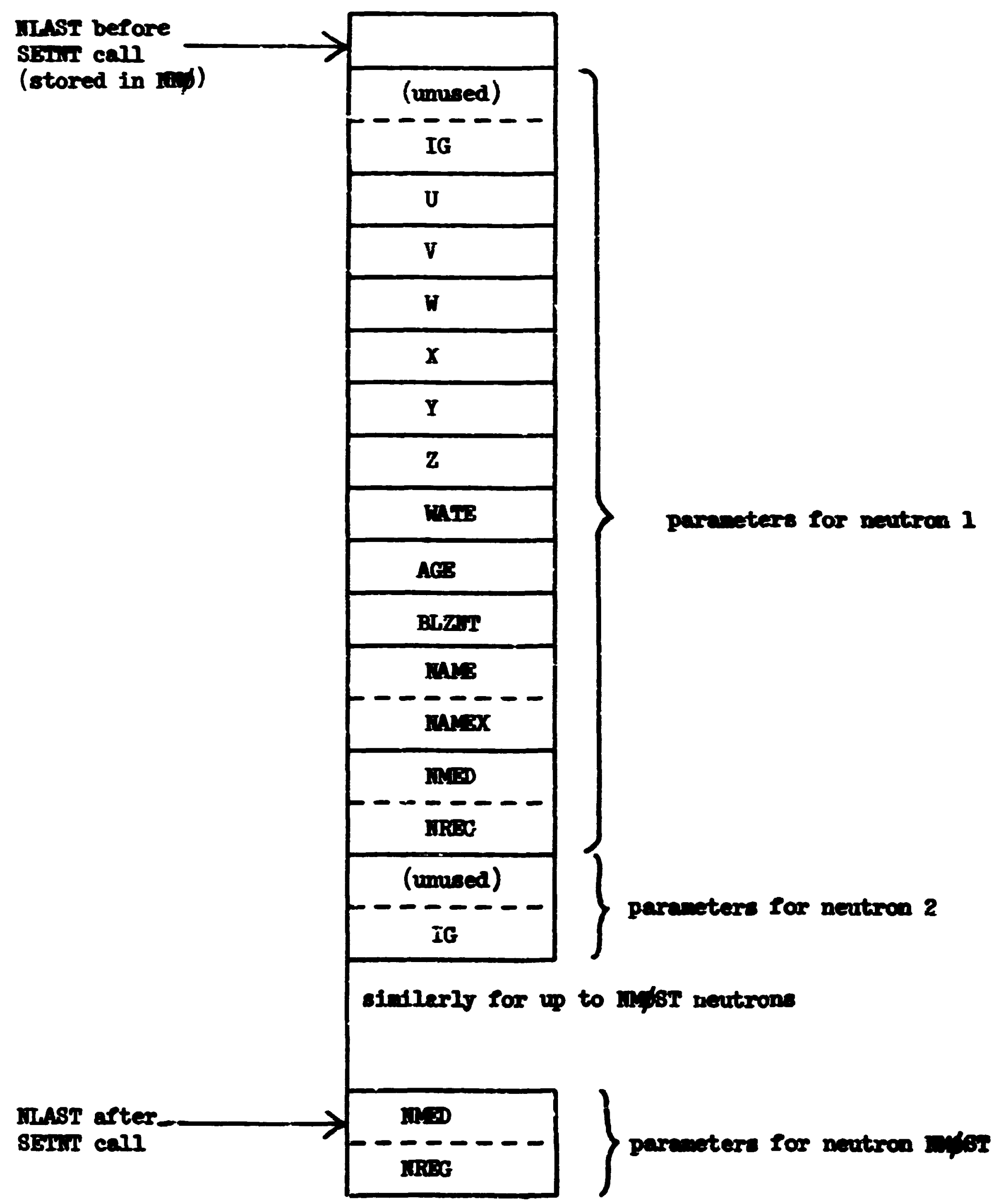

Fig. 3. Lavout of the Neutron Bank in Blank Comion 
Subroutine GETHI(N)
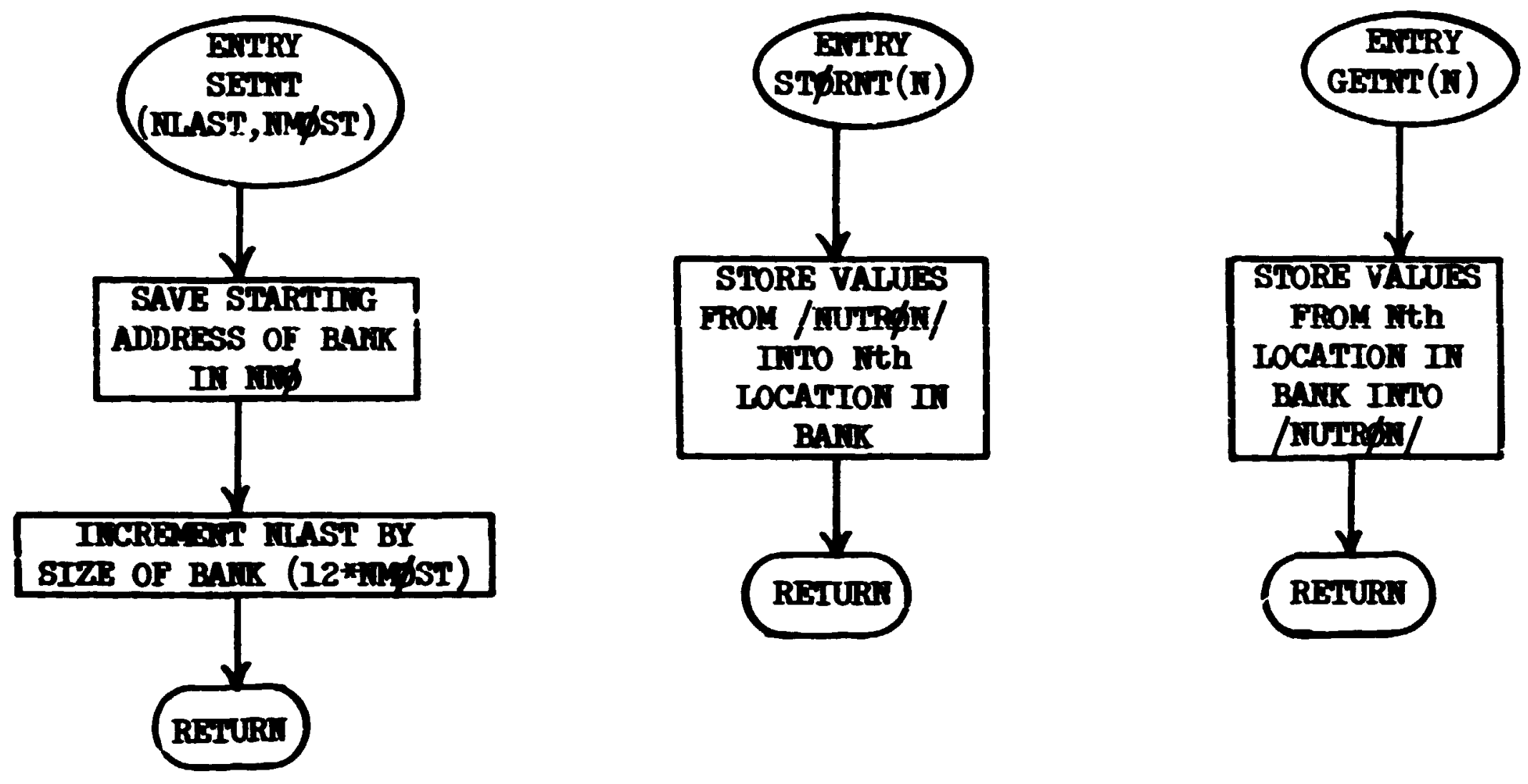
Subroutine GGisT (TSIG, MARK)

The end-of-flipht coordinates are computed assuming the starting medium extends infinitely. The proper data are stored in GEqAC before calling GEV and is restored after the GEdM call. If the flight is starting in interior void (MED $=1000$ ), velocity components (or direction cosines) rather than an end point are given t.o GEdy. If an albedo medium is encomtered the flag AALB is set to the albedo medium number, and then MGRI and GdurfP are called, respectively, to determine the normal to the surface encountered, and to reset certain parax ters for GE to use later in going array from the albedo surface.

Called from: NXrcdL.

Subroutines called: GEM, HoRiL.

Functions used: SQRT (library)

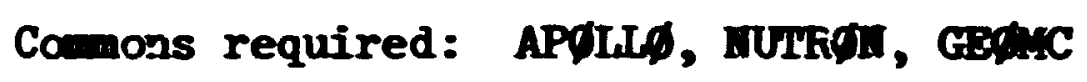

Variaíles required:

$\left.\begin{array}{l}X d L D, Y d L D, Z d L D, ~ M L E D, \\ U, V, W, \text { HREG }\end{array}\right\}$ Trom HOTRda comon, see page 12)

IBLZ $\phi$ - packed worá cortaining block and zone numbers for starting point,

BTATH - distance to be traveled (in $\mathrm{cm}$ ) if the flight remains in the starting medium,

MARK - initial value of flag used by GBAM,

TSIG - totel cross section of starting point medium,

ETA - Plight distance in m.f.p.

Variables changed:

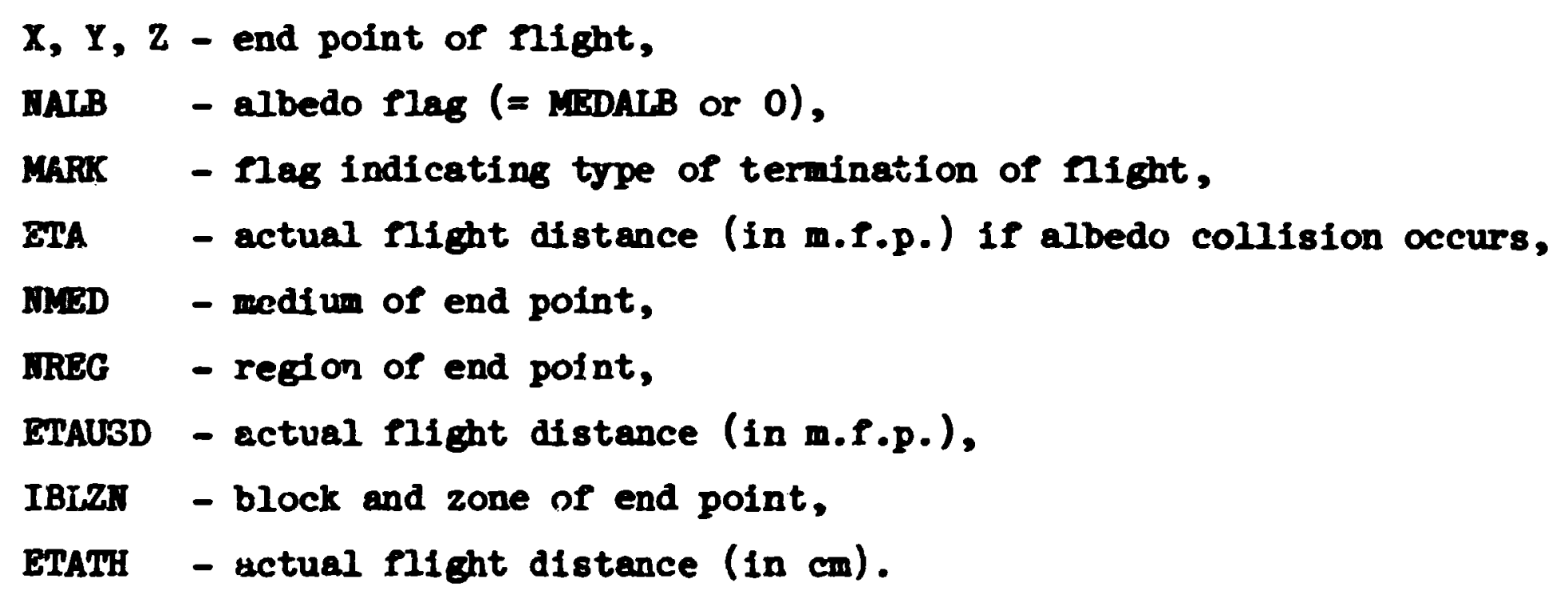




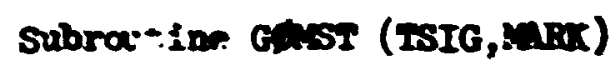

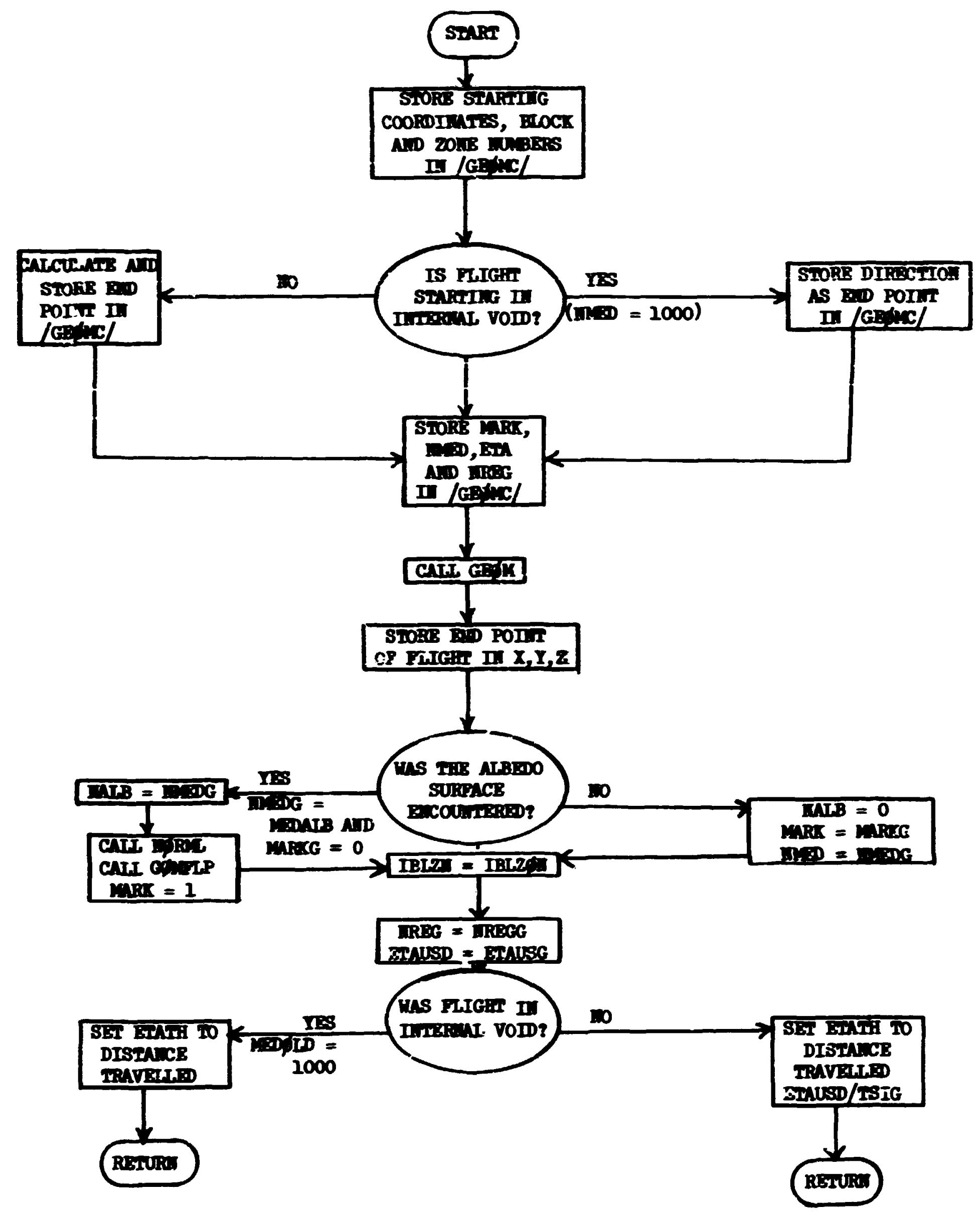


Subroutine GPRDB

This subroutine is the executive routine for the generation and storage of secondary gamma rays (or neutrons for an adjoint, coupled problem). The probability of generating a gamm ray is determined and the resulting gamma-ras weight, HATEG, is compared with input vallies of the desired gama-ray veight, GWL. Russian roulette and splitting are used to produce gamma rays of veight GLL. That is, if the gamma-ray weight is less than the input values, then the gamma ray is killed with probability (GWL-|HATEG|)/GLL and stored with probability (|WATEG|)/GWL. If the gaman-ray weight is greater than the input value, then there are $\mathrm{J}=$ WATEG/GWL gamma rays stcred with veight GW with Russian roulette played with the remaining gamma ray of veight WATEG - J*GLL.

Ancther version of GPRfb which has been found to be more userul in some cases does nut use GuL as a desired gamma weight but instead uses it as the probability of generating a ganma ray. Thus, a random number, if compared with GiL, and, if greater, no gama ray is generated; if less than or equal, then a game ray with weight = HATE*PGE/GUL is stored. This procedure produces gamma rays of varying veights, but the number of garma rays may be controlled easily.

Called from: WpSE

Subroutines called: GAYGEN, GSTORE, HELP, ERRøR.

Functions used: SIGI, ABS (library).

Commons required: Blank, RUTRфI, AF LLD.

Variables required: IG - primary particle energy group,

$$
\begin{aligned}
& \text { IMED - geometry medium, } \\
& \text { WATE - primary particle weight, } \\
& \text { GHL - input weight values for gamma rays, } \\
& \text { NREG - geometry region, } \\
& \text { MMTG - total number of particle groups, } \\
& \text { MXREG - number of regions for which there are } \\
& \text { weight standards. }
\end{aligned}
$$

Significant internal variables:

WATEG - gamma-ray weight.

PGEN - gamma-ray generation probability. 
subroutine GPi,B

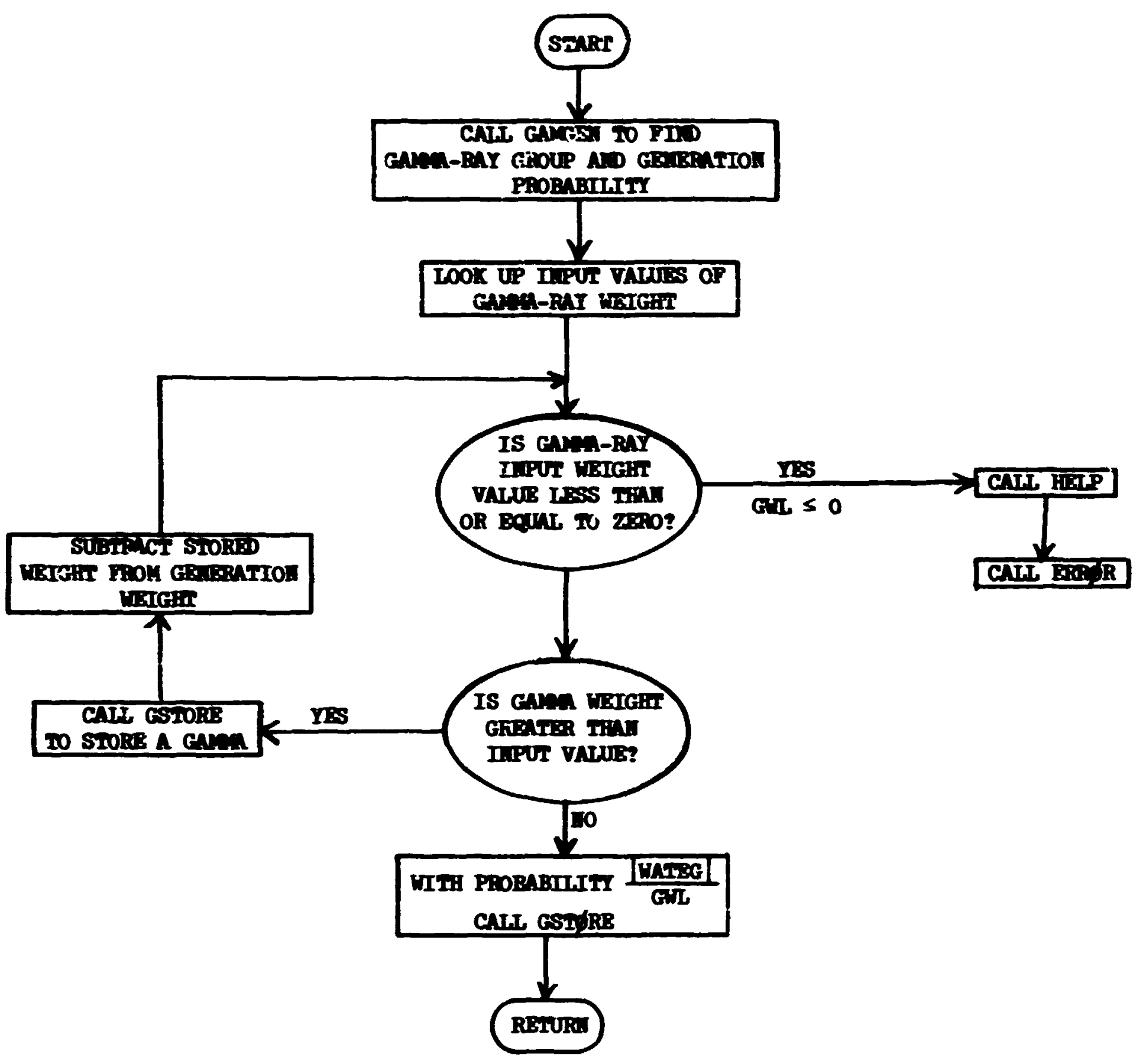


Subroutine GSTQRE (W8G, IGG)

This subroutine checks to see if there is room in th. bank, and if so stores the significant variables for the generfited gama ray (or neutron in an adjoint coupled problem). Since the information in NUTRA common is stored, the current neutron parameters must be saved temporarily and then restored. It is assumed that the gamma ray is emitted uniformly in direction. An option for analyzing the generated gama ray is provided through the BAIKR interface.

Called from: GPR\$B.

Subroutines called: GTIS (U, V, W), STфRINT (MAM), BAIKR (4). Commons required: HUTRQI, APQLIQ.

Variables required:

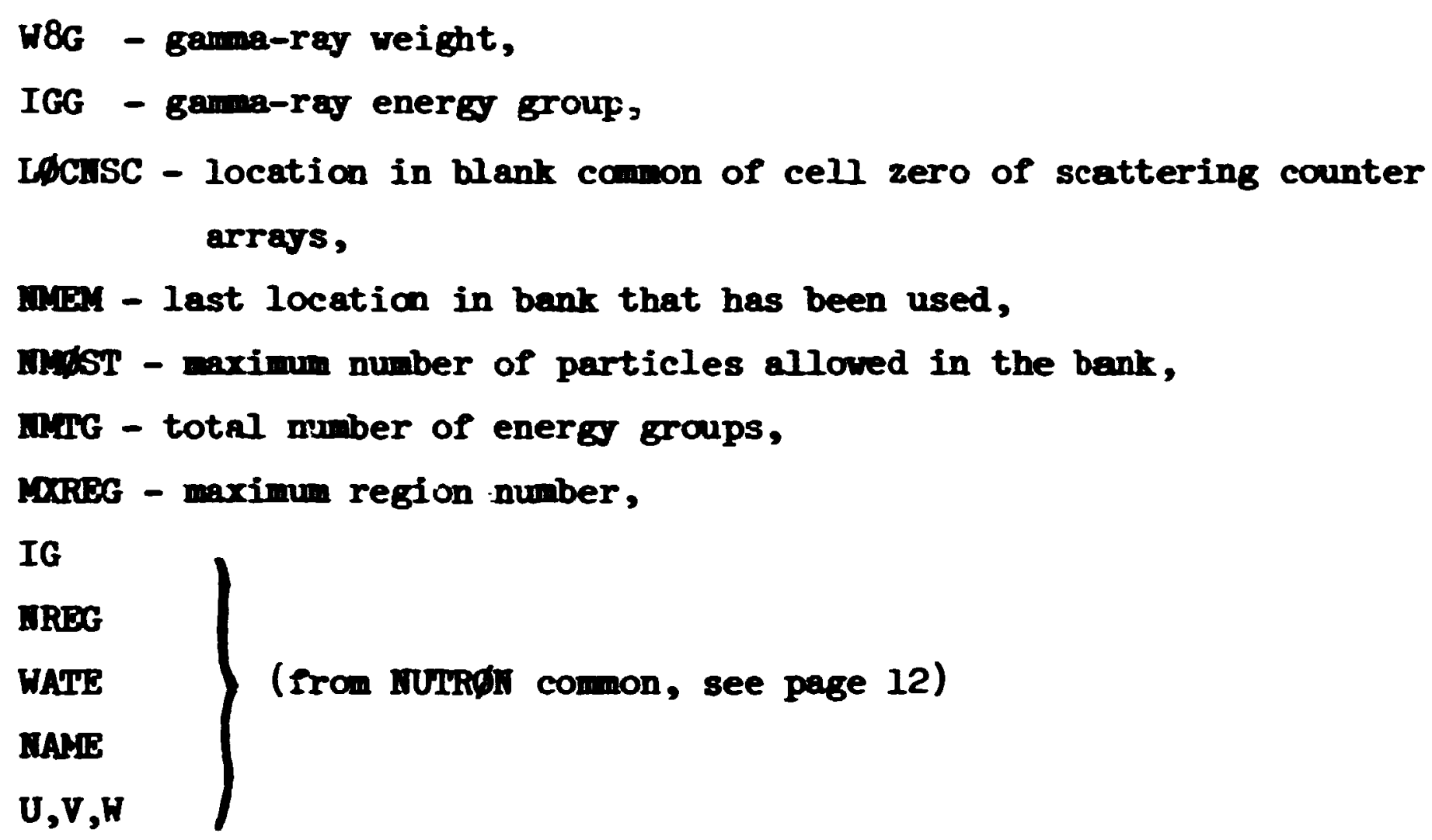

Significant internal variables:

$U, V, W$ - direction cosines of gamma ray.

Limitations: Isotropic gema-ray emission. 
Subroutine GSTQRE

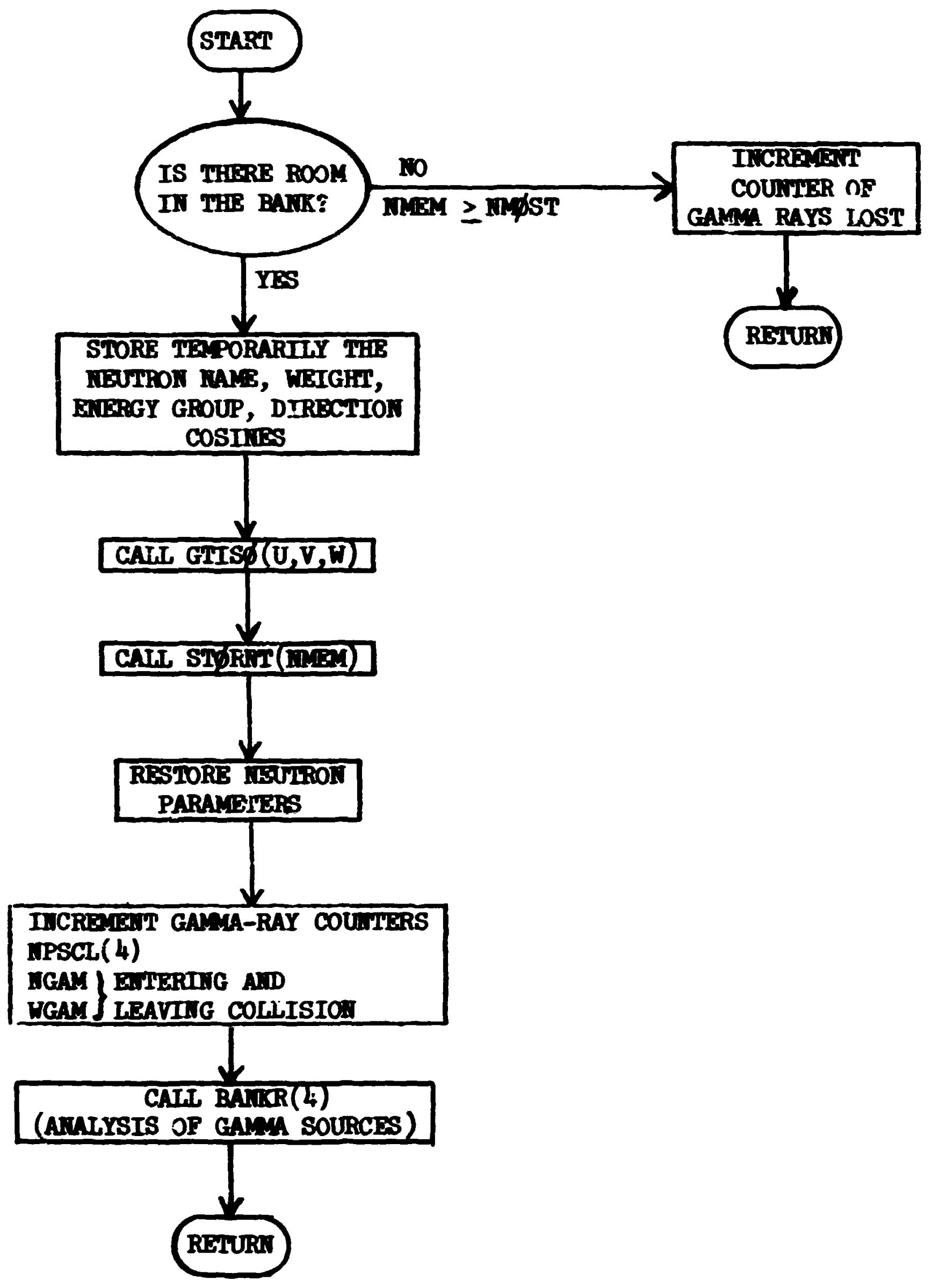




\section{Subroutine INPPI}

The basic functions of subroutine INPUT are to read, from cards, the basic problem description, and to print out this information, to initialize parameters, to perform some initial transformations on basic problem data, and to call other more specialized routines that perform similar initializations. As an example, several group indices must be set differently depending on whether the problem is a neutron only, gamma only, or combined neutron and gamea. If an adjoint problem is being done, many quantities mast be otored differently since all values are input as though a forvard calcula-. tion was being done. For complete details, refer to the flow chart.

Called from: MRSE.

Subroutines called:

DATE - provides BBCDIC siring containing day of week and date, SqRIII - reads cards $E$, source spectra and relative imortarce of source groups, if biasing is desired,

RIDII - stores initial random number,

RUD\%UT - retrieves current random number,

JuIII - reads gecmetry data,

XSEC - reads cross-section data,

SETHT - sets up neutron bank,

EXIT - library,

SCфRIR - user routine for reading analysis data,

GAMCEN - provides gamma-generation probabilities,

FISGET - provides fission-generation probabilities.

Punctions called:

ICQSPA $(A, B, N)$ (Iibrary function at Oak Ridge National Laboratory compares, bit by bit, II bytes of locations $A$ and $B$; returns $z \in r o$ if $A$ and $B$ are identicai)

MODEL - (1jbrary function at Oak Ridge National Laboratory which determines the model of the computer)

Commons required: Blank, GEQNC, BANK, USER, BWKIMC, MUIRQN, APQLID, FISBIK, HQRYAL.

Variables input: (see definitions of variables in common APQID, IUUR申I, USER, pages $8,12,167$. A more detalled listinf of input is given in Appendix C.) 
CARD A (20A4)

Title

(Any character other than a blank or alphameric in columen one wil i terminate the job.)

CARD B (14I5)

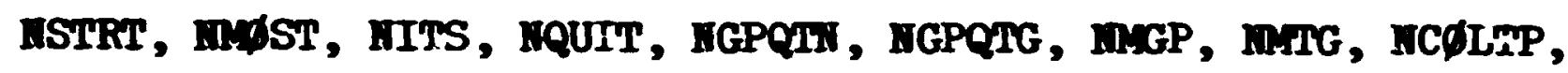
IADJM, MAXTIM, MFIIA, MEDALB

CARD C (4I5,5E10.5)

IS\&UR, HGPFS, ISBIAS,(unused), WISTRT, EB\%TH, EB\%TG, TCUT, VELTH CARD D (TE10.4)

XSTRT, YSTRT, ZSTRT, AGSTRT, UIIIP, VIKP, WIIIP

CARDS El (TE10.4) (skipped if ISqUR >0) (read by SqRII)

FS(I), $I=1$, NGPFS

CARDS E2 (TE10.4) (skipped if IS申UR > 0) (skipped if ISBIAS $\leq 0$ )

(read by $S \phi R T h$ )

$\operatorname{BFS}(I), I=1$, MGPFS

CARDS $F(T E 10.4)$

BaBR (I), I - I, MIIG

CARD G $(2 I 5,5 X, 36 I 1,5 X, 13 I 1)$

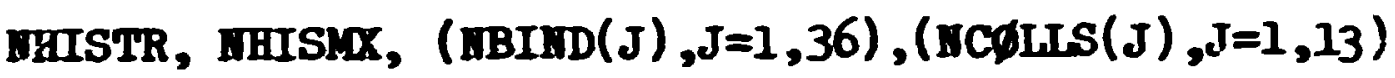

CARE H (Z12)

RAIDTM

CARD I (14I5)

MSPLT, IKILL, IPAST, MWLBAK, IEBIAS, YXXREG, MAXCP

CARDS $J(6 I 5,+\mathrm{E} 10.5)$

IGP1, KDG, MGP2, HRG1, IDRG, MRG2, WHHIH1, WILGH1, WTAVE1, PATH

(read until NGP1 < ?;

CARDS K (TE10.4) (sipipped if IBBIAS $\leq 0$ )

( ( $B P R$ AB (IG,IREG), IG=1, INIG), IRREG=1, MXREG)

CARD I (14I5)

HSQUR, MPISTP, KKCALC, HORTP

CARDS M (7E10.4) (skipped if MITITP $\leq 0)$

(FULO(I), I=1, JCXRGG)

CARI i I (TE10.4) (skipped if MITSTP $\leq 0)$

(FSE (IG,IMED) ,IG $=1$, MMCP) , IMIBD=1, MEDIA)

CAROS $0($ TE10.4) (skipped if NGPQJM or HGPQTG $=0$ ) 
( (GLL)(IG,NREG), IG=1, MMGP or NMG-NMGP), NREG=1, MXREG)

JWIII called for gecoetry data

XSEC called for cross-section data

SCTIII called for analysis data.

Variables changed:

All in common USER - set for use by ankilysis routines,

All in common HoRyal - zeroed,

All in comion Grayc - zeroed,

All in comon MUTRq - filled vith junk vord $\left(48484848_{16}\right)$,

All in common APQLI - except Il, IO, ITINE, ILAST are filled with junk vord,

Maxrng

DFP

IEPQTO

HGPQI

IGPQT2

IGPQT3

INPCQL

ICQIPR

RAIID - set to internal number by RWII if zero is read iu, MAXGP - set to $I$ if 0 is read in,

MXREG - set to $l$ if 0 is read in,

MGPREG - MAXGPWDAREG

LACAIS

INCITL

LACEPR

LACISC

LACPSI

for definitions, see common APLLI, page 8

NGEPA

HLAST

MSIGL

for definitions, see camon APdLd, page 8, and diagram of enerbs group structure, page 11

IIISBI 
Subroctine yipr

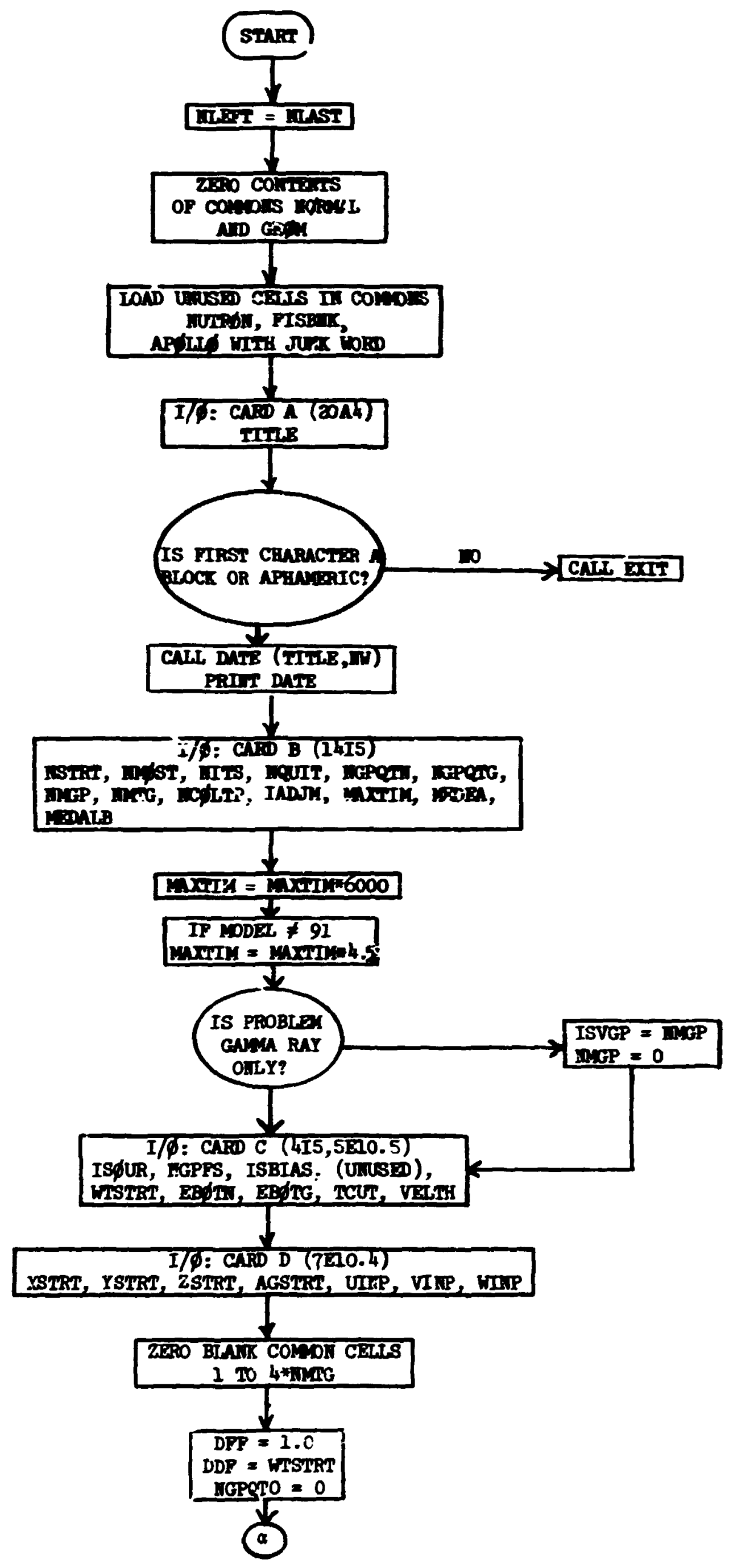



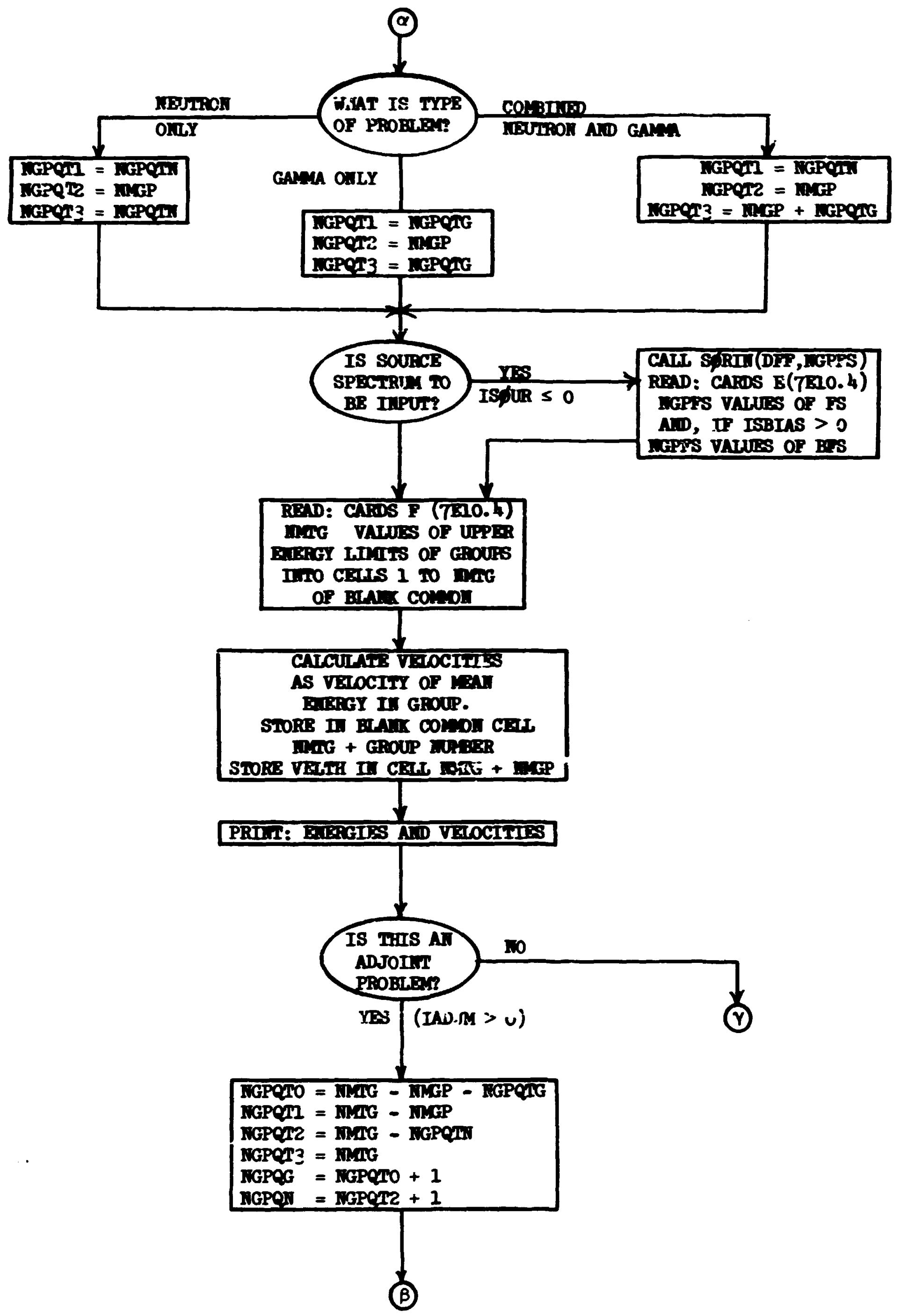


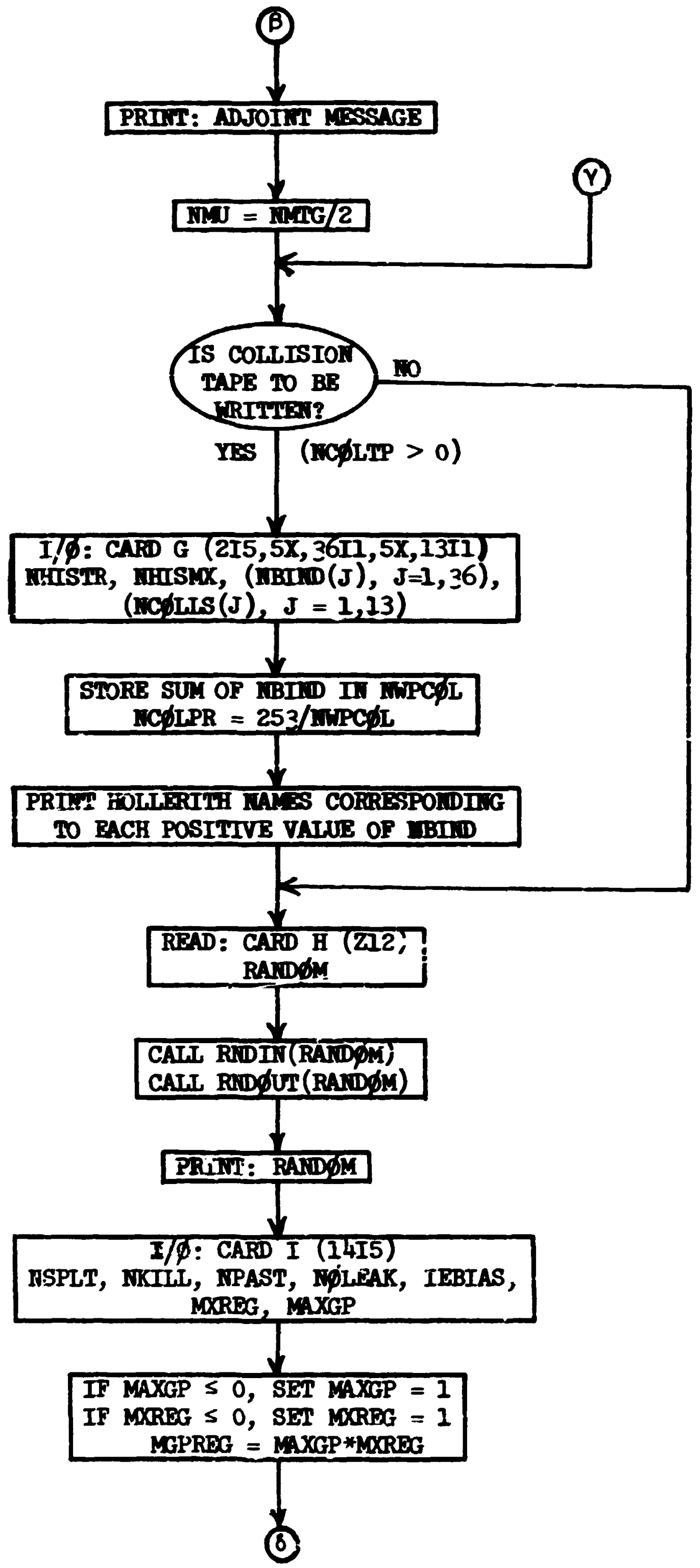




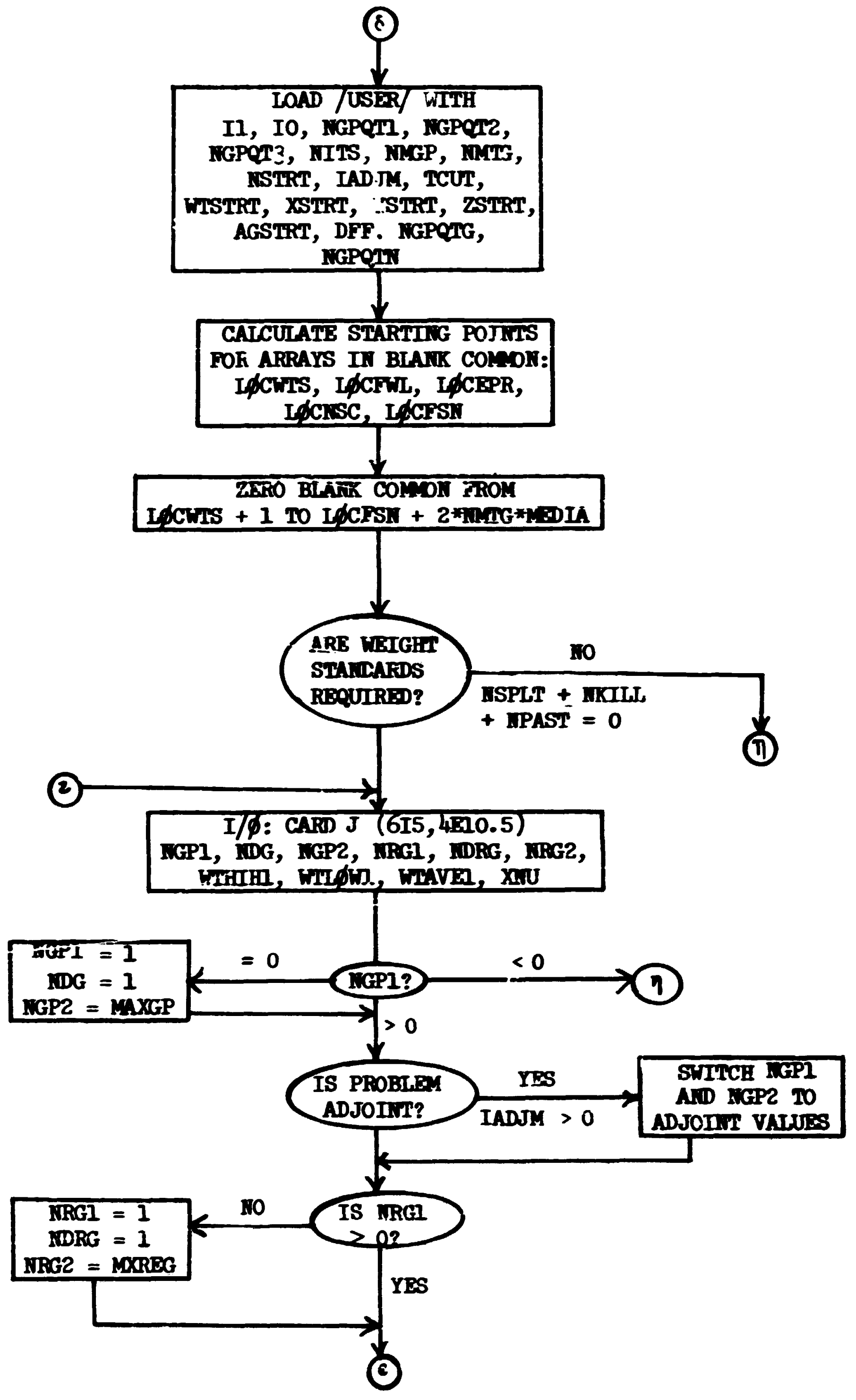



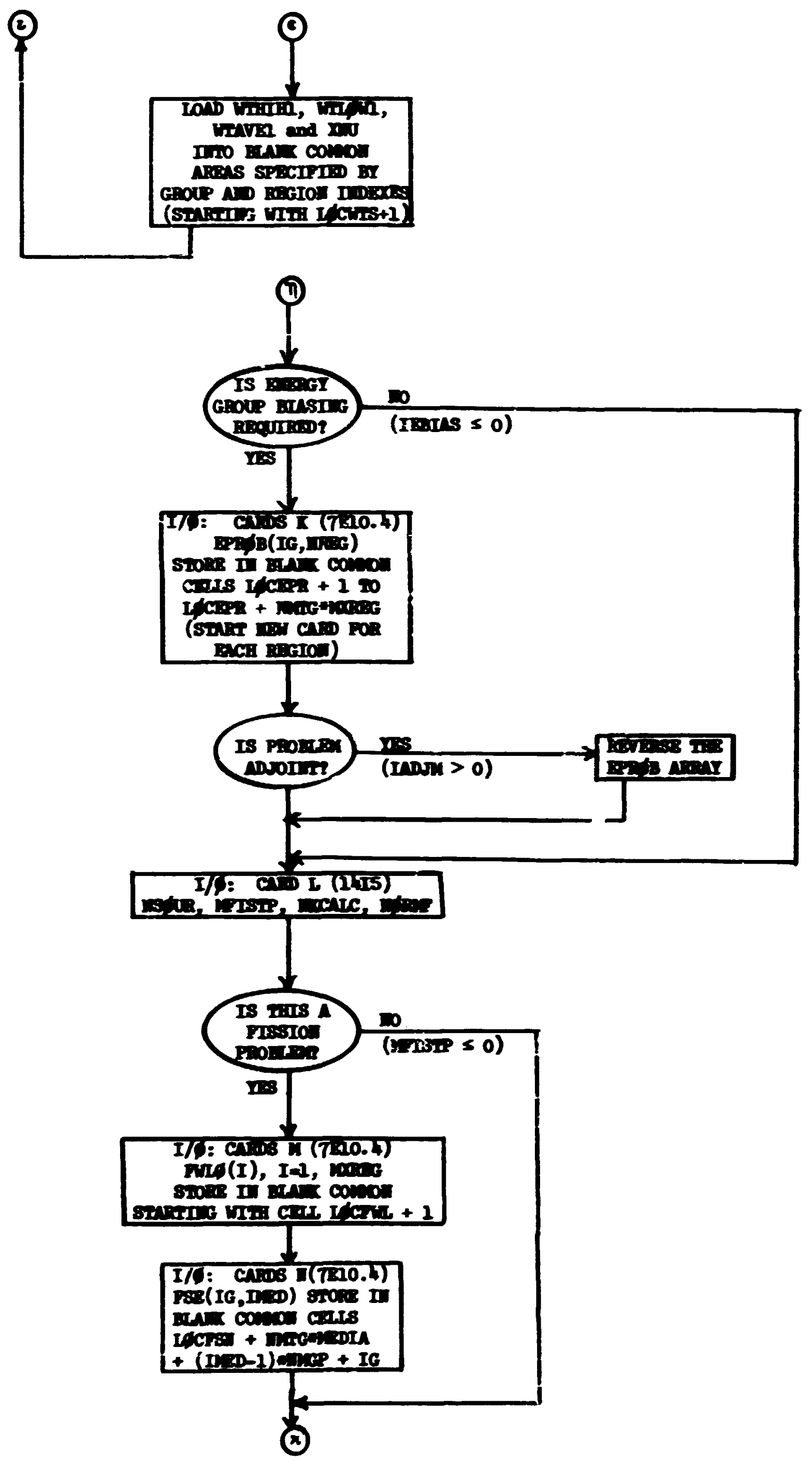


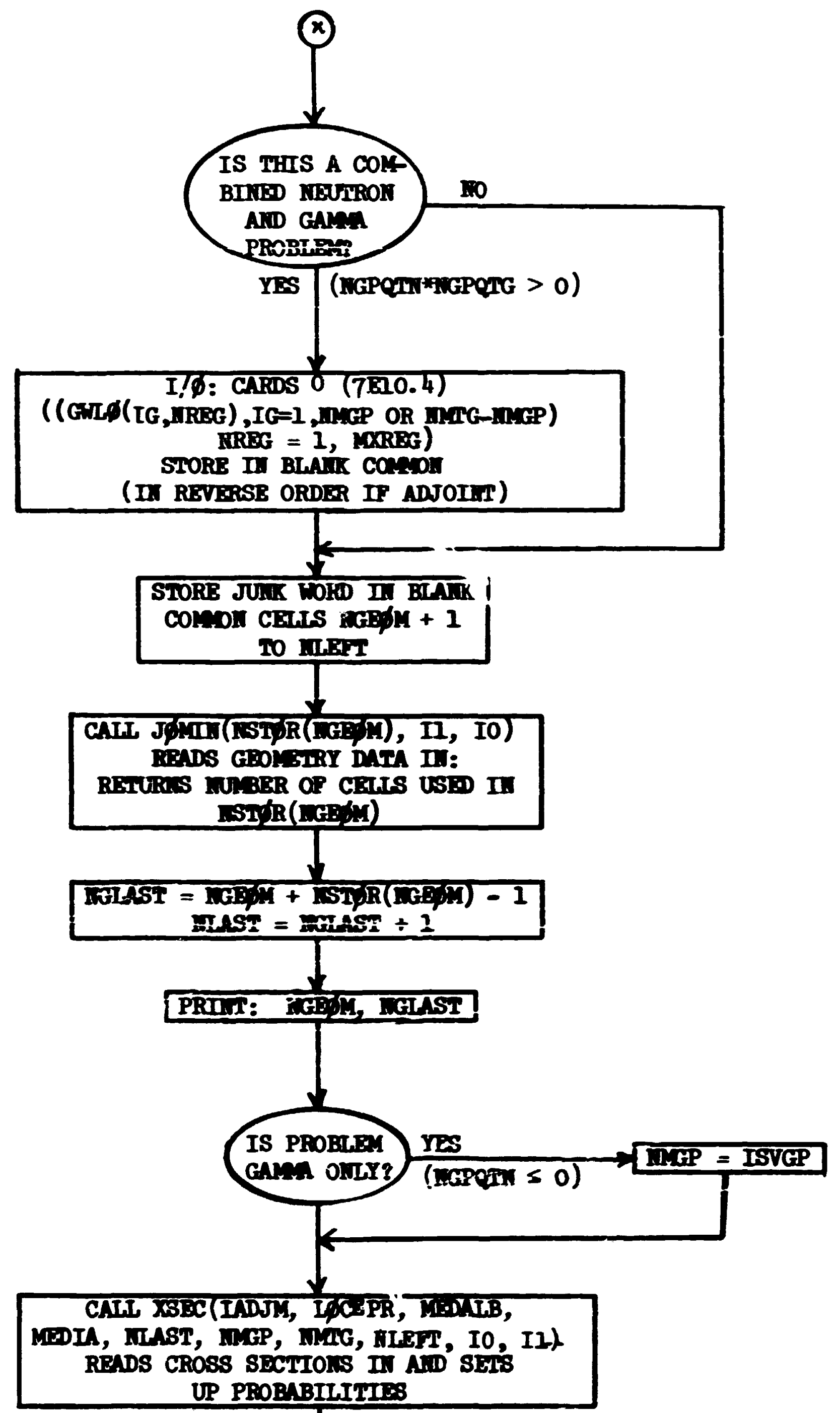




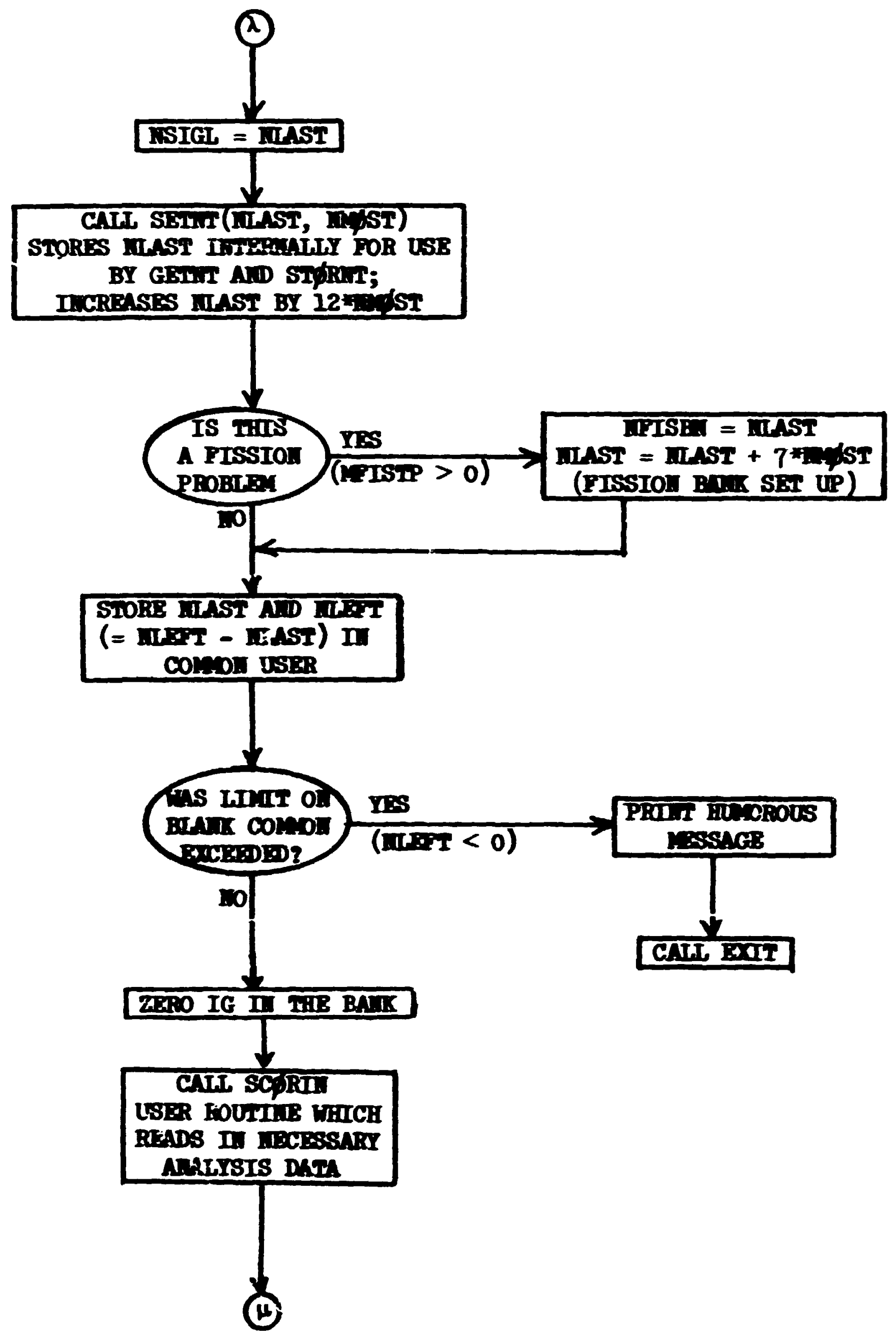




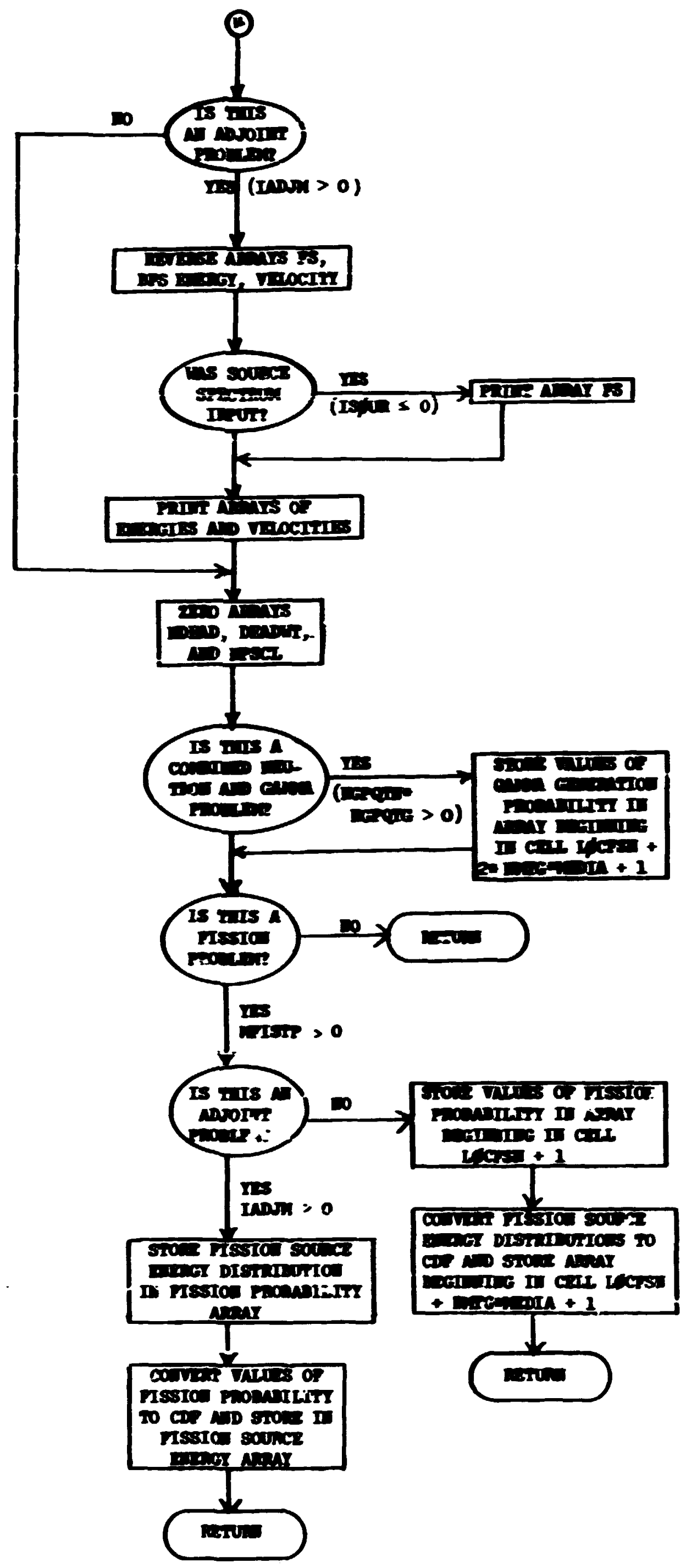




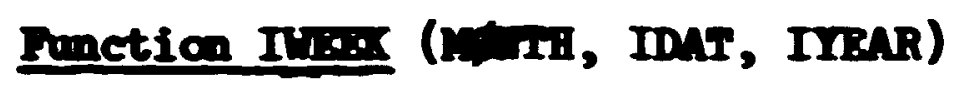

This routine vill lock up the date for you if you don't know it and fill in integer values for MAH, IDAT, and IMRAR (requested vith 50). It also returns, as the function value, an integer from 1 to 7 representing the dey of the veek. If it is given a positive vaiue of Wint, it assumes you have given it a month, day of month, and year and vill not disturb these but will simply deternine the day of the reek. If you stum it (by specifying a year before 1901 or after 2099) IWEEK is returned as zero.

Called by: DArs

Boutines called:

IDAY - library routine at ORIL; the output is two 4-byte words containing 8 BBCDIC characters representing the number of the month, a hyphen, the day of the month, a hophen, and the last two digits of the year. That is, on May 30,1970, the argument for IDAY will return containing the EBCDIC representation of 05-30-70.

Variables required:

WhH $\leq$ - Flag to calculate MrH, IDAT, and IIRAR.

$>0$ - Nug to leave arguments aloce.

Variables sodified:
Wiri - integer representing wanth
IDAT - integer representing dey o: wonth
IrenR - integer representing year
iwsis - integer representing deg of week. 
Punction INETS (F, IH, TDAT,IMBAR)

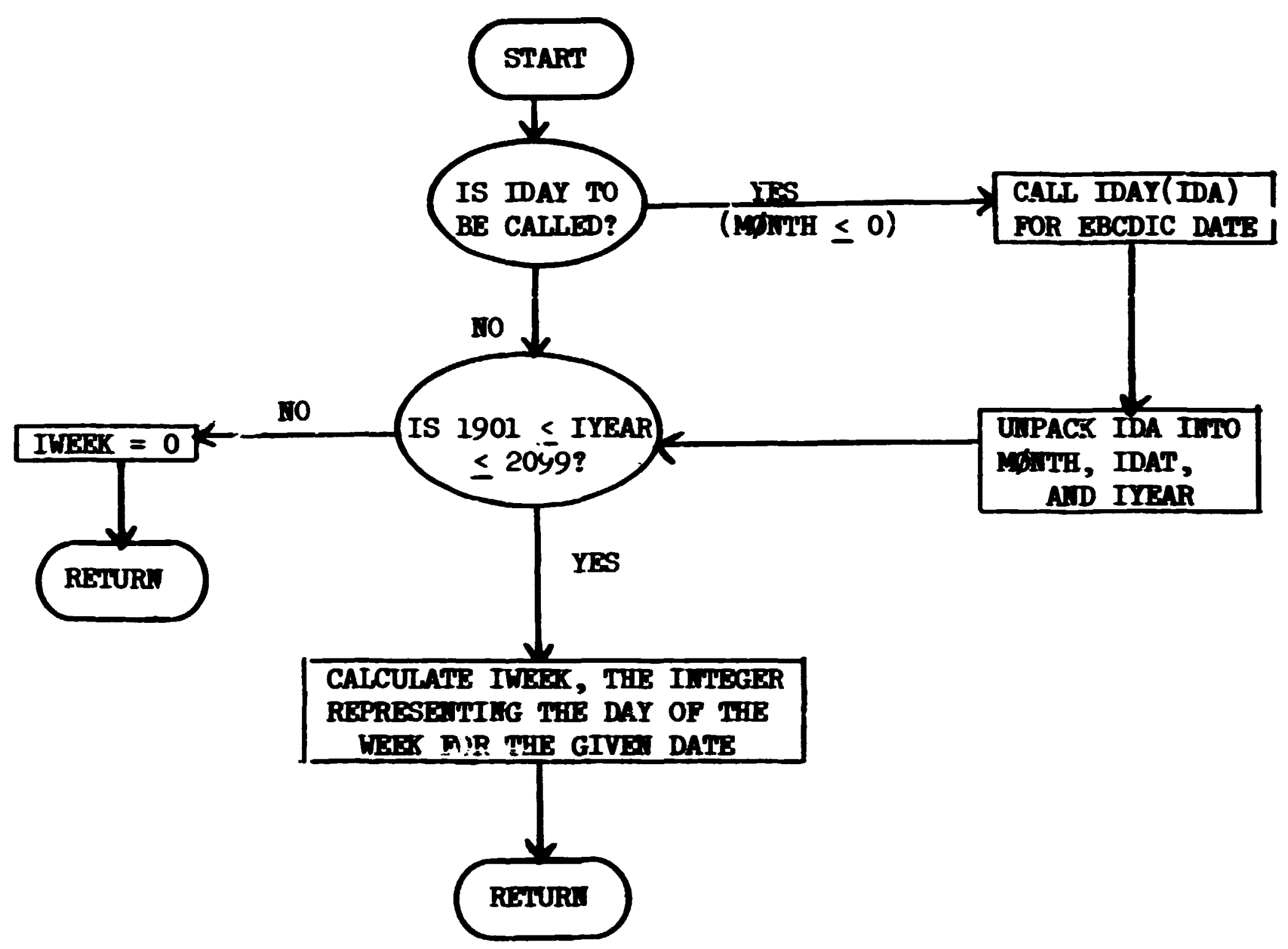




\section{Subroutine isin}

nSW is the executive routine for the generation and storage of the source parameters at the starting of each batch. The source paraneters av be read into IIPUI on caris, zenerated by subrcutine SquRCE or obsained from the fission bank for a mliplying system. For either type cí problea the calculations by subroutine S\&URCE override the fission bank input or the velues read fron curds. If the direction cosines are all input as zero, an isotropic source direction is generated. The group number obtained from the fission bank is the group causing fission and an be used in the selection of the source group for the fission neutrons. FSE in blank comon contains the group distribution for each mediun.

Called from:

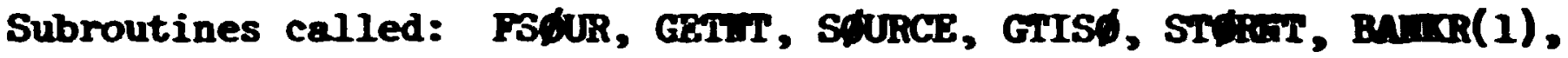
Loterz

Comons required: MUTR, PISBM, APWLD, GEAC

Variables required:

ITSTR - an injes which deternines if the source should be obtained from the previous batch fissions (IISTR $\neq 0$ ) or cenerated by SAURCE or from input data (ITSTR $\approx 0$ )

ISqUR - an index which deternines the options for the enerny distribution of the source. If ISqUR $>0$ the source energies are all generated in enerdy croup IS\$R. If ISWUR $\leq 0$ subroutine INPU calls StRH and the enerE is selected by SAJRCE

MEey - the number of particles to be generated for the batch $=$ WSTART For non-rissioning systems and MFISH for multiplyird systens

ZSTRT, YSTRR, ZSTRT

WTSTRT, ACSTRP

UINP, VINP, ZINP starting parameters input from cards, from comeon APTLID, see page 8

Variables changed:

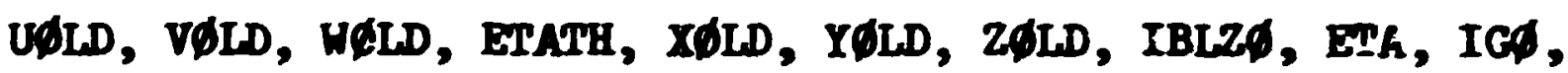
MEDDLD, DIDACE - previous collision parameters are zeroed for the source. GLDWT - previous collision veight set equal to WTSTRT $X, Y, Z$, HATE, AGE, HANGXX, ) IBLZN, NREG, MIED, NAME, parameters set for each particle generated, $U, v, W, I G$ put in MUTRøi common, see fage 12 
IPSCL(1) - counter for number of sources

IEWII - set to name of last particle generated

FIGIL

$\left.\begin{array}{l}\text { FWATE } \\ \text { IFISH }\end{array}\right\}$ zeroed for the next batch 
Subroutine yopiR

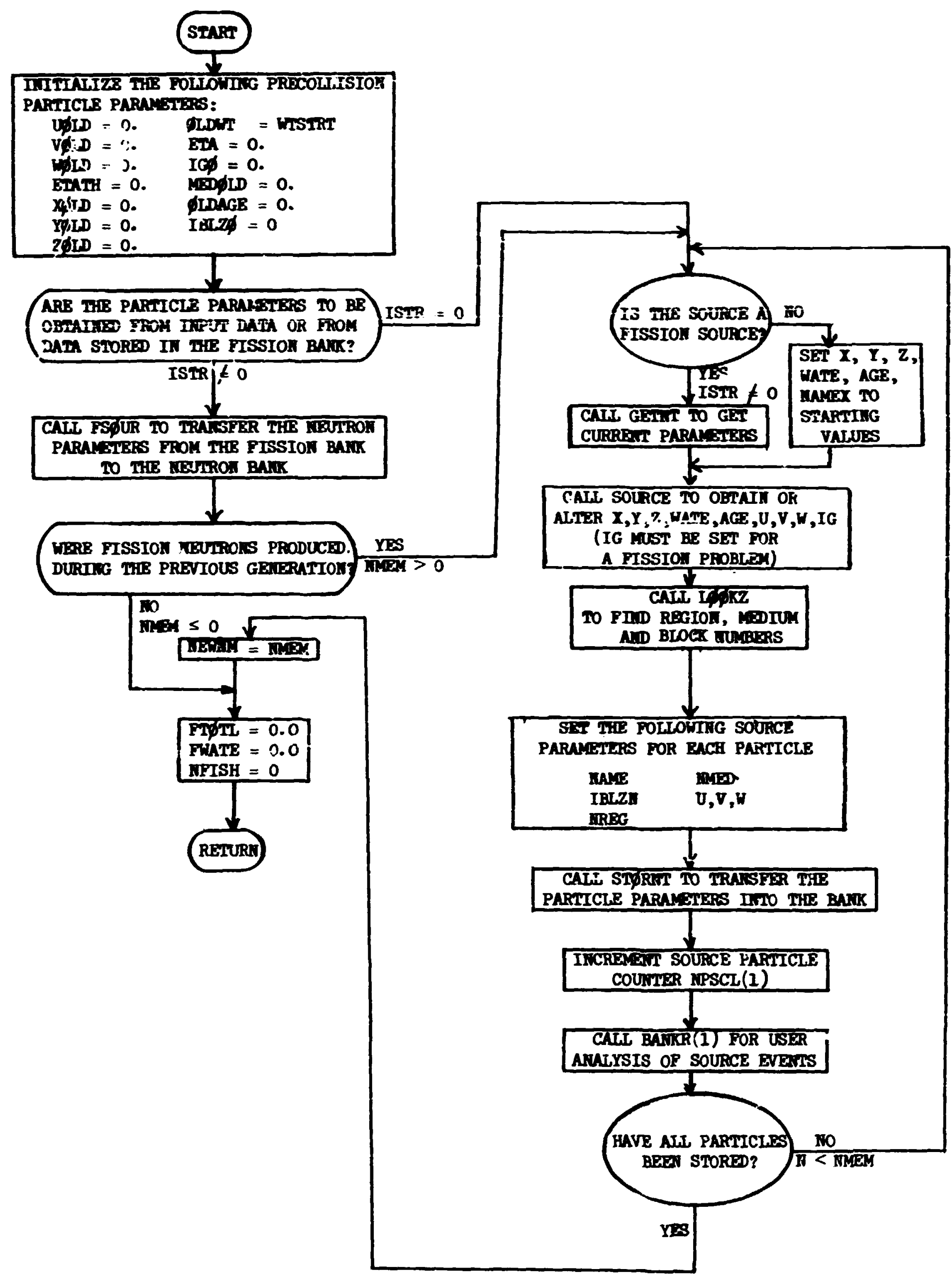




\section{Subroutine NXTC}

This subroutine is called by the main program to determine the spatial coordinates, the block and zone number, particle's age, and nonabsorption probability at the next collision site and at every boundary crossing encountered along the way. The total number of boundary crossings is. recorded as is the number of escapes. If a particle escapes, its weight is set equal to zero and the history vill be terminated by the main program.

\section{Called from: MRSE}

Subroutines called: GETETA, HSIGTA, GRST, BANKR(7), BANKR(8). Commons required: Blank, NUTR

Variables required:

AGE - shronological age of the garticle at the previous collision site, BLZNT - a packed worc containing the block and zone number at the previous collision site,

NMED - the medium number at the previous collision site, XøID. $Y \varnothing L D, Z \emptyset L D$ - spatial coordinates at the previous collision site,

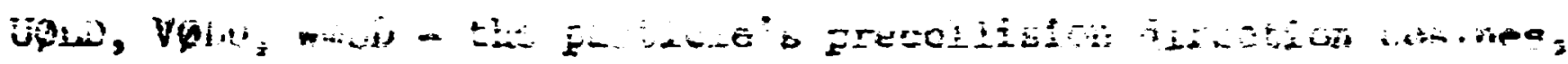

TSIG - totaI cross section.

Variables shanged:

AGE - chronological age at new collision site,

BLZNT - a packed word containing the block and zone number at the new collision site,

NMED - end-of-flight medium,

NPSCL(7) - total uumber of bounuary crossings,

NPSCL( 8 ! - number of escapes,

$X, Y, Z$ - end-of-flight spatial corrdinates,

WATE - weight of particle undergoing flight to the new collision site. Significant internal variables:

MARK - an index which identifies tine type of event at $(X, Y, Z)$; MARK $=0$, normal boundary crossing, MARK $=1$, flight ended within the medium, MARK $=-1$, particle escaped, MARK $=-2$, particle entered an interior void,

ETA - mean-free paths of flight remaining afte: a boundary crossing, 
ETATH - total distance that a particle vould travel if the medium at the starting point was extended indefinitely,

ETAUSD - mean-free paths of flight consumed while traversing a given medium. 


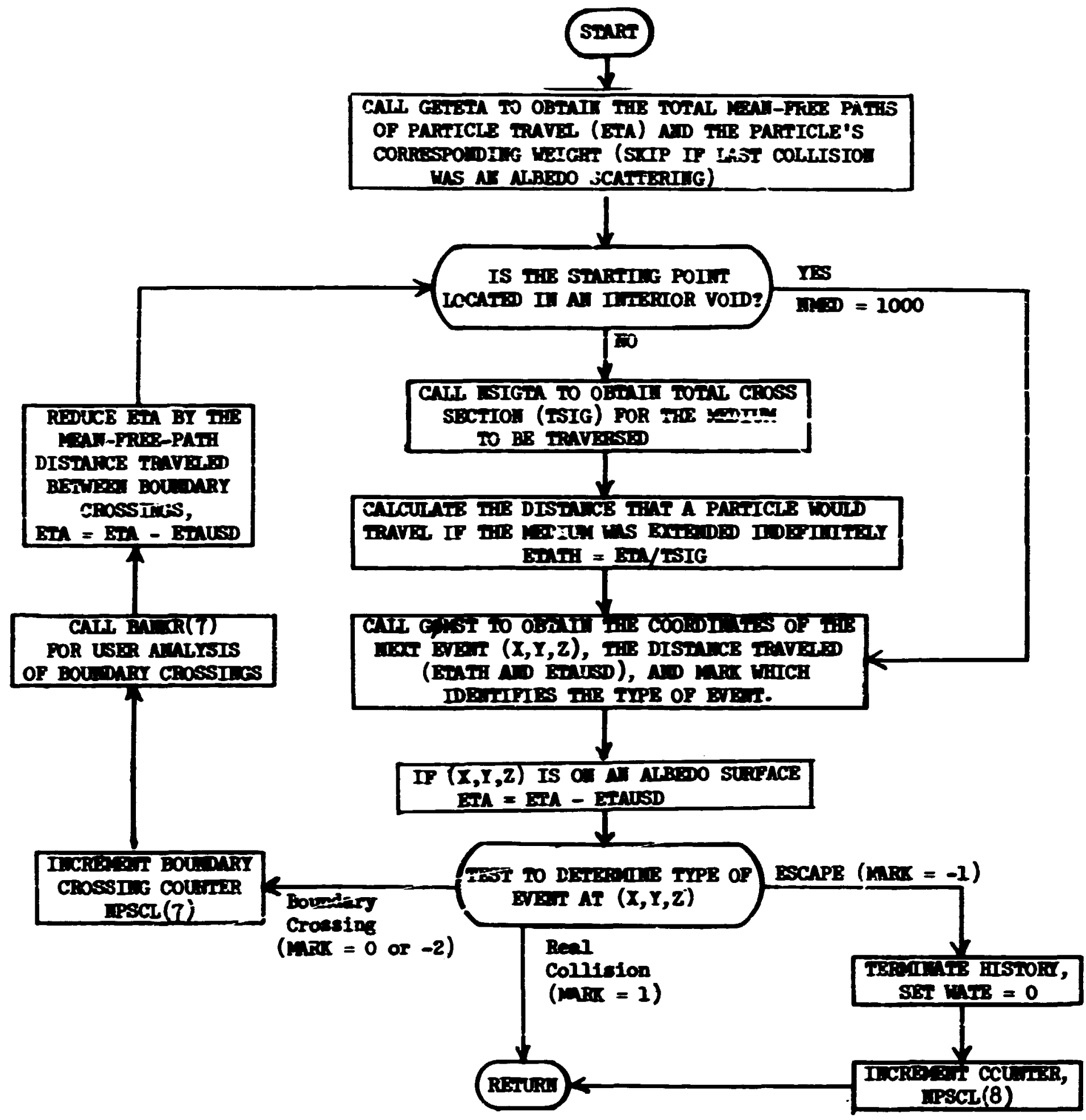




\section{Subroutine ATPT (KKY)}

This routine controls the calculation and output of the average values of the source parameters (beginning of the batch, KEY $=1$ ) and the ccllision counters at the end of each batch (KEY $=2)$. At the end of the run (YISY = 3), results for the numer of scatterings, the vays in wich the particles were terminated, and the counters for splitting and Russian rculette are printed.

For $k$ calculations, the estimpte of $k$ at the end of each batch is output, vith the final value of $k$ and its staidard deviation output at the end of the run.

In addition, the c.p.u. time used is output for each batch.

\section{Called from: MRSE}

Subroutines called: TIMIER, RIDQUT, GEIIT, DUTPT2

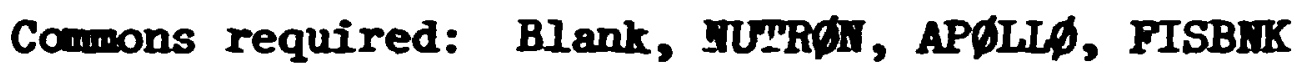

Variables required: (neariy all variabies from NUThl common, see page 12

$$
\text { for definition) }
$$

NITS, ITERS, MIEM, RAFDQM

$\operatorname{IPSCL}(I)$.

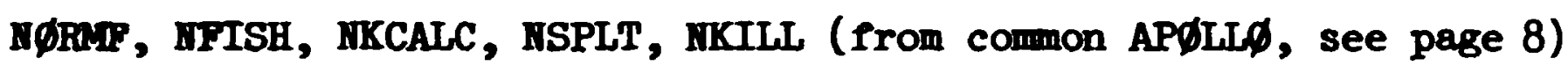

Significant internal variables:

FWKN - a running count of the total number of particles sfarting,

SWATE - the sum of the source particle veights,

FKSUM - running sum of the $k$ values veighted by the number of particles starting the batch,

VARK - running sum of the square of the $k$ values veighted by the number of particles starting the batch,

NITSK - number of batches used for $k$ calculation. 
Subroutine OUIPI (KEI)

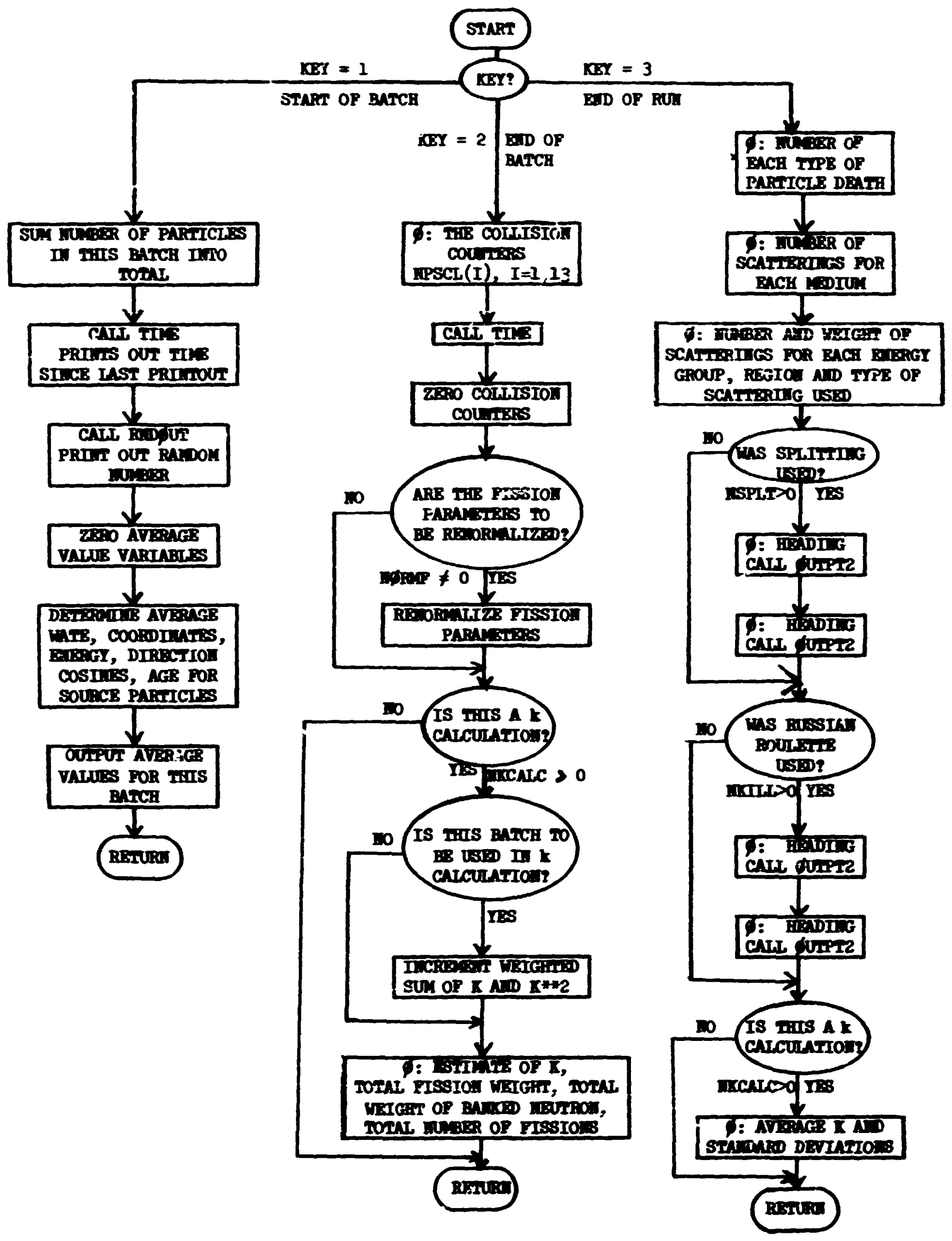


Subroutine QUIPT2 (MI, MII, MAXGP, MXREG, I $\emptyset$ )

This sutroutine is used to output the number (II) and weight (WNI) counters indicating the results of Russian roulette, splitting, and scatterings for the complete problem. The output arrays depend on region and energy group.

Called rron: DUTPT

Variables required: II - the two-dimensional array to be output,

MII - the tric-dimensional array to be output,

MAXGP - the largest group for which Russian roulette and splitting were considered,

MXREG - the number of regions for weight standards,

IØ - logical output tape number.

Subroutine QUTPT2

START<smiles>C1CC1C1CC1</smiles>

OUTPUT: HEADING ALD

REGIOI NUMBERS

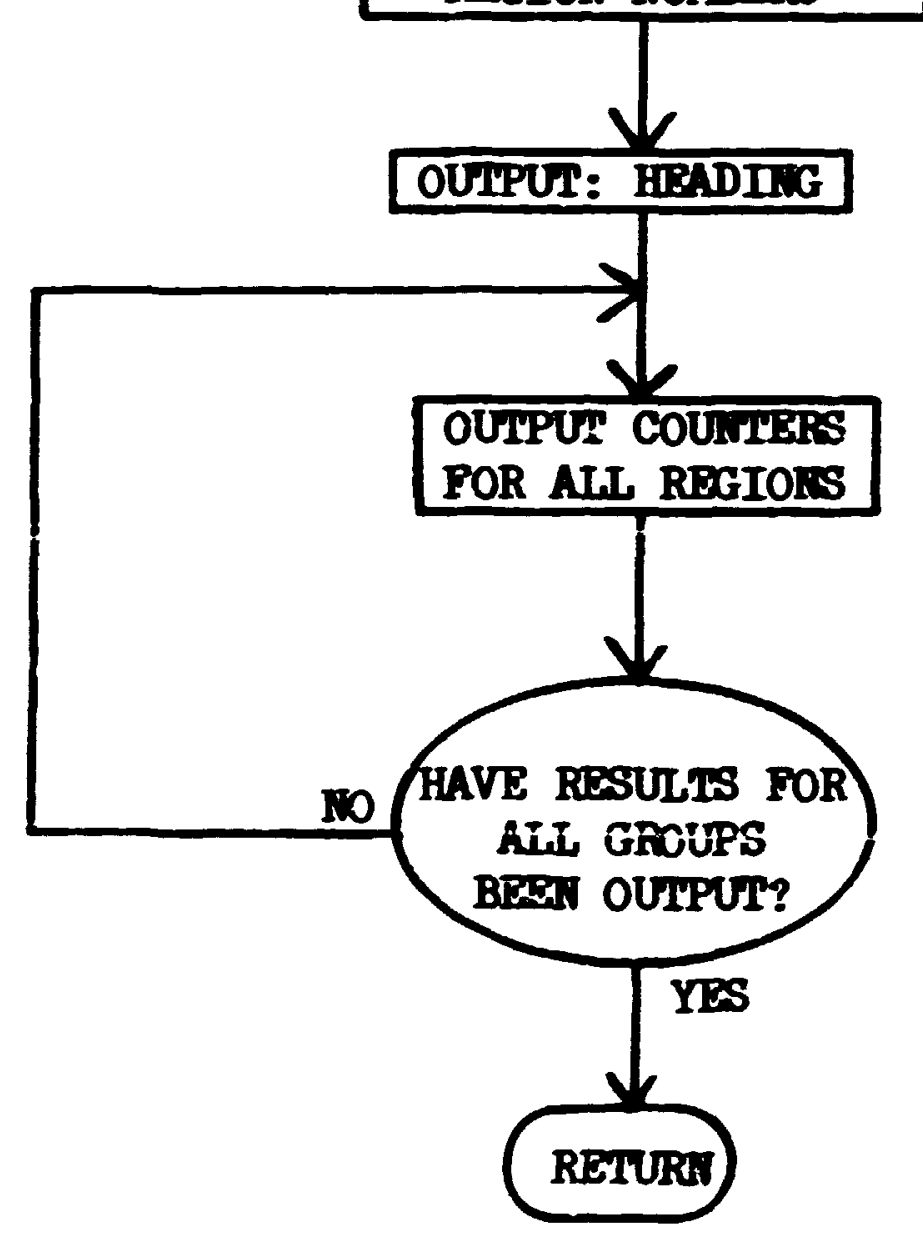


Subroutine S $\phi$ RII (DFF, MGPFS)

The source enerb spectrum (in group form) and, if needed for biasing, the celative importance of source groups, are input and transformed to curulative distribution functions (c.d.f.) by this routine. (Note that the biased spectrum is not input but rather calculated by S\$RIN.)

Forward and adjoint cases are handled automatically. If an adjoint problem is being done, the c.d.f.'s start at 1.0 and decrease with group so they will be in the correct order after INPUT reverses the arrays. RGPFS values of the natural spectrum (referred to as the array FS) and, if requested, the relative importance (referred to as the array BFS) are input into blank common. After PS is input the sumaticns DDF over groups 1 to RGPFS, and DFF over all groups actually being used up to nGPFS are formed. DDF is replaced by (DFF/DDF) *WTTRI for use, in SqURCE, as a veight correction when less than HGPFS groups are being used in the problem. DFP is transferred to comon USER for use by the analysis as a normalization in adjoint probleas. It should be noted that the array FS, as input, is treated as fractions of particles to be emitted in the natural distributions, but, for the adjoint case, should consist of arerages over the group width, not integrals.

Called from: IIIFUT

Punctions used: ABS, MAXO (library)

Commons required: APQLLD

Variables required:

NGPFS - number of values of PS (and BFS) to be read,

NirG - total number of groups in cross sections,

IGPQTI - number of neutron groups,

IGPOTC - numher of gama-ray groups,

FiGP - number of primary particle groups in cross sections,

WISTRT - starting particle weight, as input,

IADJM - positive for adjoint problem, $\leq 0$ for forward, ISBIAS - source bias switch, biasing used if $>0$.

Variables input:

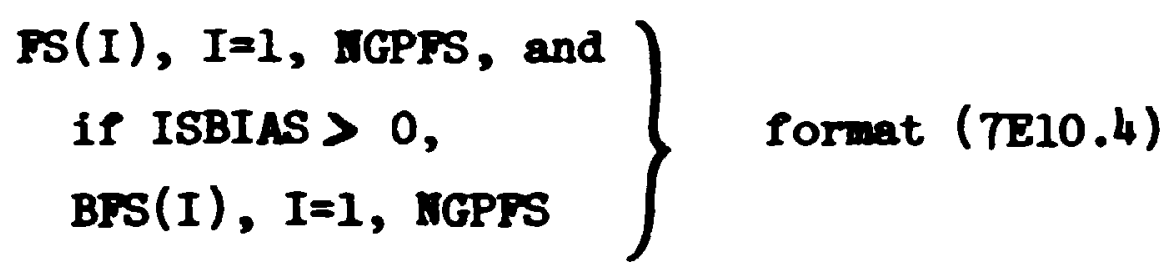


Variables changed:

DFF - sumation of FS over groups being used in problem,

DDF - ratio of DFF to summation of FS over NGPFS groups, times starting veight.

Significant irternal variables:

NGPQT - set to NGPQTI if neutron only or combined problem, set to KGPQTG if gamma only problem,

IGI - set to the largest of NGPFS, NGPQT,, NGPQTG for single particle problem, set to FifP+1 for combined probiem,

HG2 - set to NMGP+NGPQTG for combined problem, irrelevant for single particle type problem. 
Subroutine S,PRI (DFF, MCPFS)

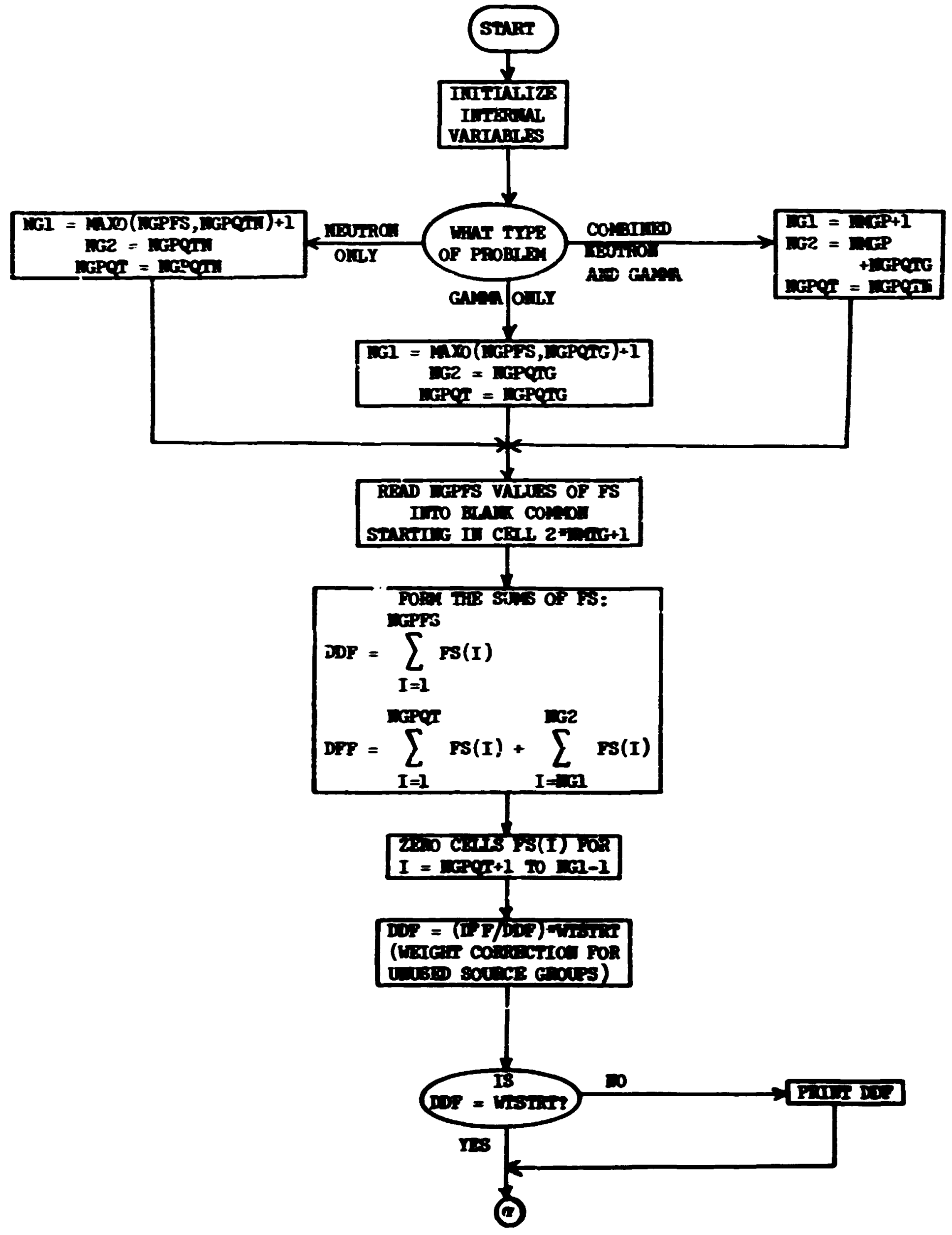




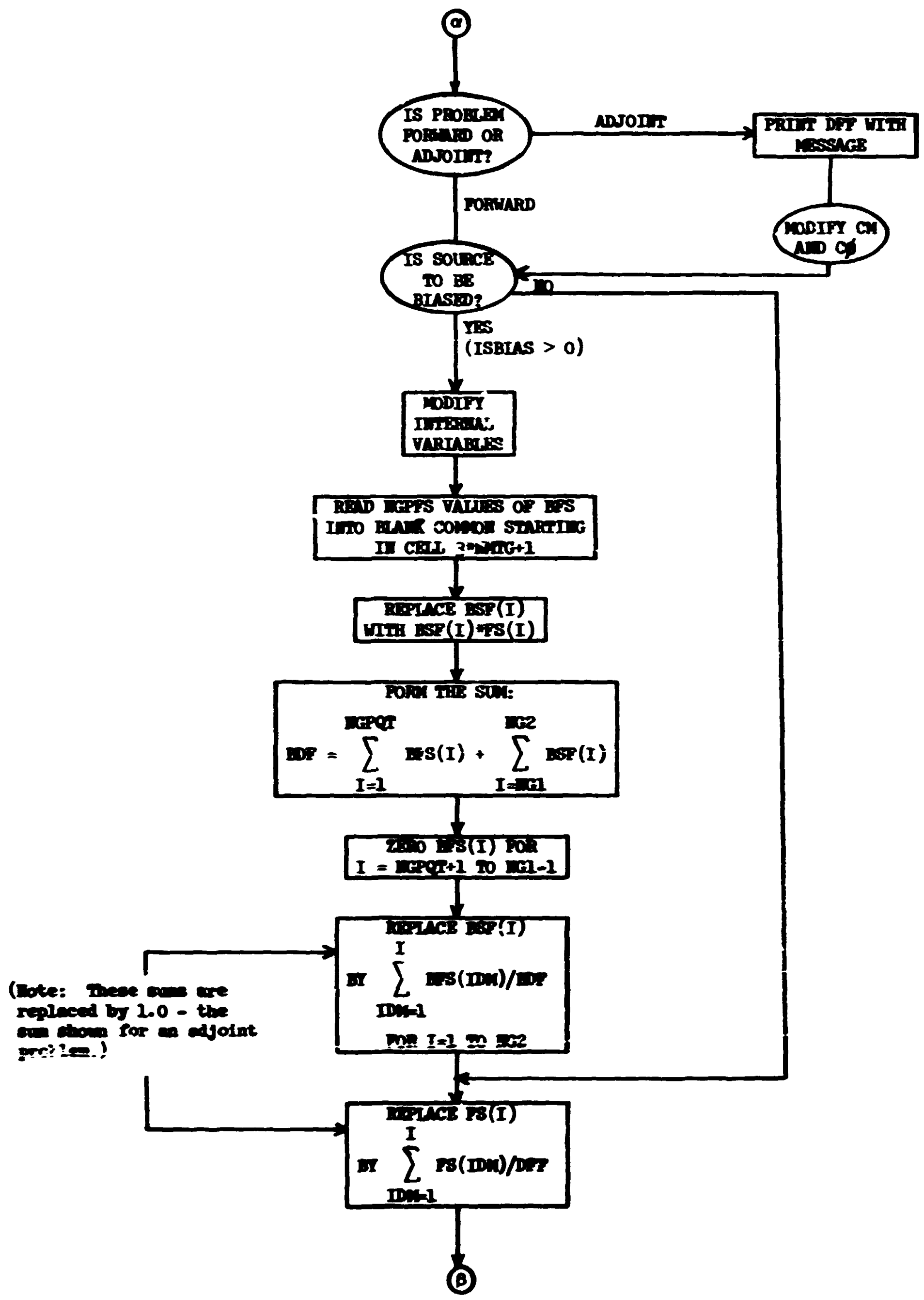




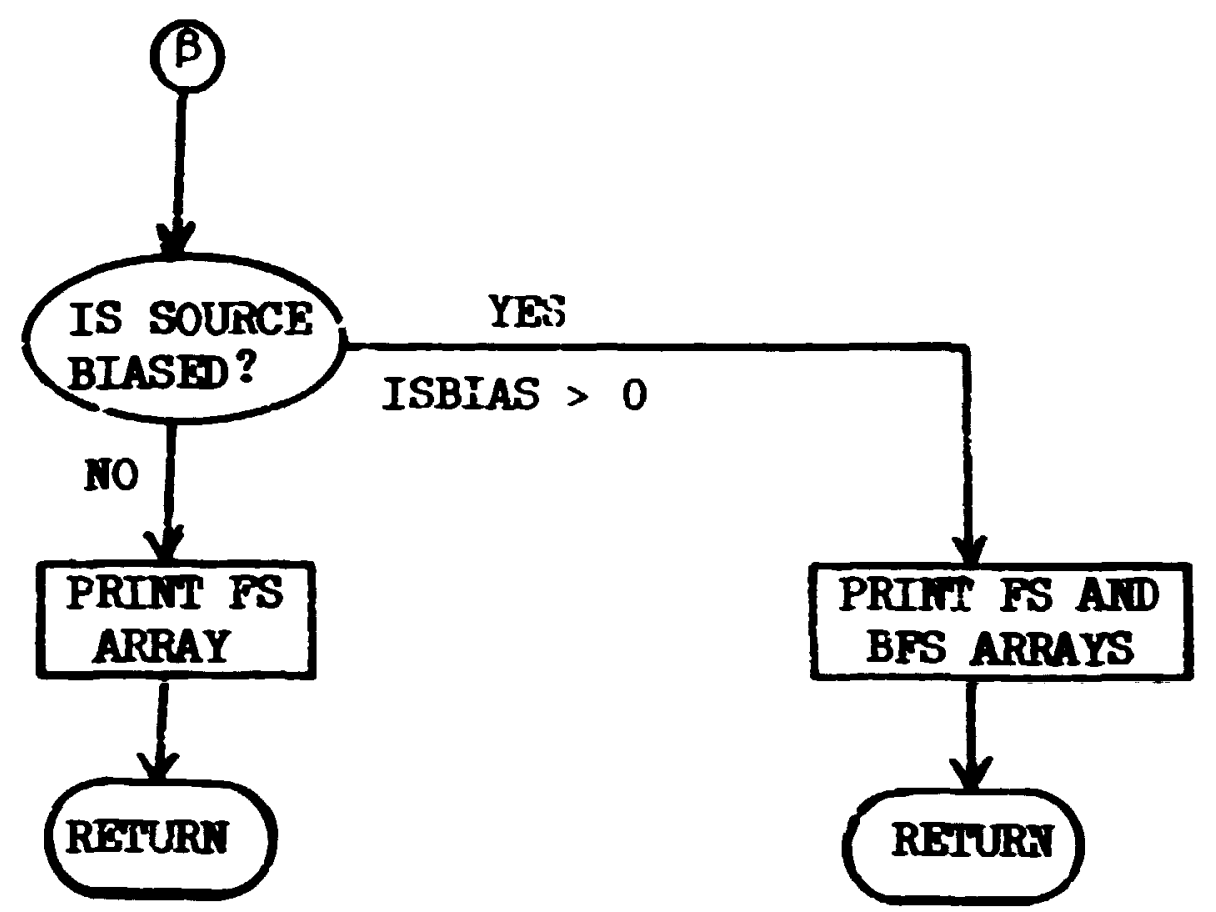


Subroutine TESTW

TESTW is called after a particle is witharawn from the bank and then sfter each coliision. A test is first performed to determine if the Russian roulette and splitting options have been specified. Then a comparison of the particle's weight is made with the Russian rculette weight standara ing to determine if the particle will experience Russian roulette. If the particle is killed, its weight is set equal to zero, and if it survives it assumes a new veight, WTAVE, which is designated by the user.

If Russian roulette is not performed, a comparison of the particle's weight is made with the splitting weight standard WHIR to determine if the particle should be sp]it. If the particle is split, each of the two particles will assume a weight which is half that of the original particls. One of the pair is given $t$ name not in current use, and then placed in the bank. The splitting process is repeated on the remaining particle until the particle's veighi falls below the splitting standard WTHIR.

Called from: MpRSE

Subroutines called: $\operatorname{BAIKR}(11), \operatorname{BANKR}(12), \operatorname{ST} \phi R N T, \operatorname{BANKR}(2)$.

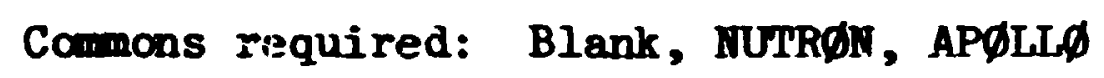

Variabies input:

IG, MAXUP, NKILL, ISPLT,

MMAST, NMEM, NEWM $\}$

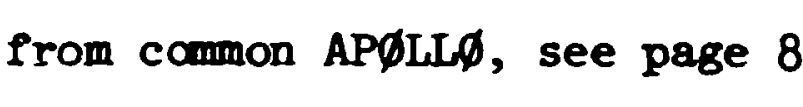

WTHIR .- weight standard for splitting,

WTL $\emptyset R$ - weight standard for Russian roulette,

WTAVE - weight assigned to particle which survives Russian roulette.

Variables changed:

WATE - the weight of the particle after splitting or Russian roulette and just before its next collision,

IMEM - the new number of particles in the bank,

NEWIM - the names of the daughter particles created by splitting. 


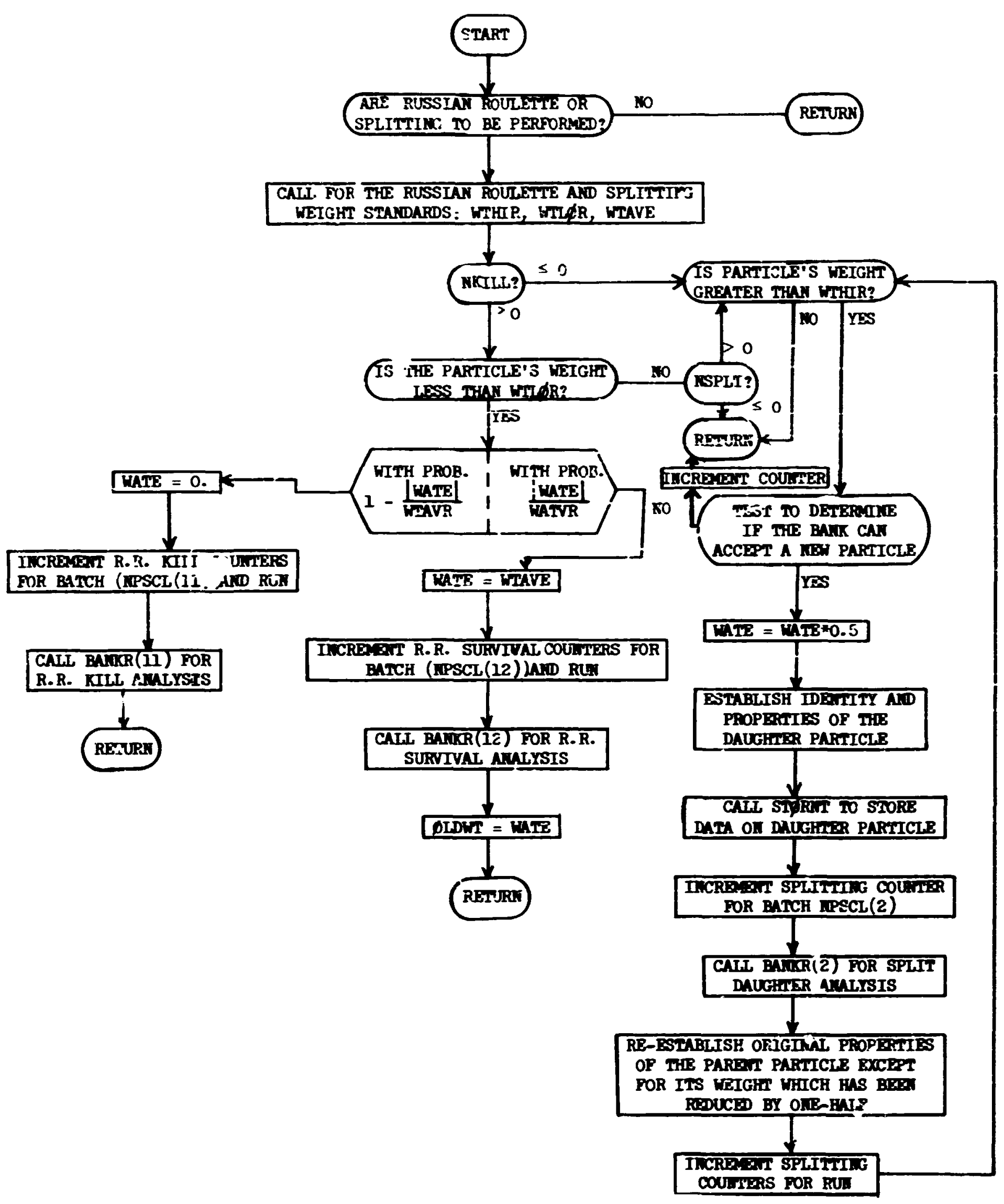


Subroutice TIMER $(\mathrm{L}, \mathrm{A})$

Upon entry to this routine, $L$ is an index having values of $-2,-1$, 0 , or 1 , which spec: 1 y one of the following options:

$\begin{aligned} \frac{\text { L }}{-2} & \text { Intion } \\ -1 & \text { Read global clock } \\ 0 & \text { Read and reset local clock } \\ 1 & \text { Read local clock. }\end{aligned}$

For all except $I=-2$, the appropriate clock reading is converted to an EBCDIC string of up to 39 bytes. If the number of hours is zero, only minutes and seconds are provided. If both the number of hours and minutes are zero, only the number of seconds is provided. If all three are zero, the string is 'LESS THAN ONE SECOND'. The number of 4-byte words necessary to contain the string is returned in $\mathrm{L}$.

Typical Usage:

DIMENSIфN ARRAY (I0)

CHílí TIMER ( $-\hat{c}$, ARRAY)

D I I $=1,10$

LETITIH $=0$

CALL TIMER (LETGGTI, ARRAY)

1 PRITT 2, I, (ARRAY(J), $J=1$, LENGTH)

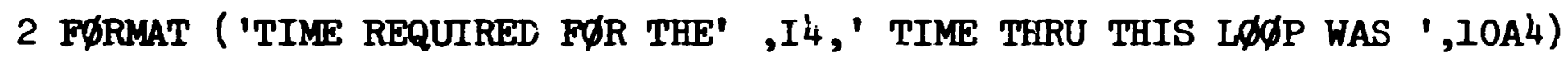
LEIIGTH $=-1$

CALL TDMER (LEITGTH, ARRAY)

PRINT 3, (ARRAY (I), I = 1, LERSMT)

3 FORMAT ('TWTAL TIME FФR THIS CALC. WAS ',10A4)

Called by: MRSE, фUTPT

Routines called:

ICLWCK - ilbrary function at Cak Ridge National Laboratory; returns reading of computer timer (c.p.u. time) in hundredths of seconds. INTBCD - library subroutine at ORNL; converts a 4-byte integer to an EBCDIC string; also returns the length of the string. 
INSERT - library subroutine at ORNL; inssris a string of given length at a specifieu point in anotker string.

Ysriuiles required:

L - see above

Variables modified:

A - see above. 
Subrout.fne TIMER ( $L, A)$

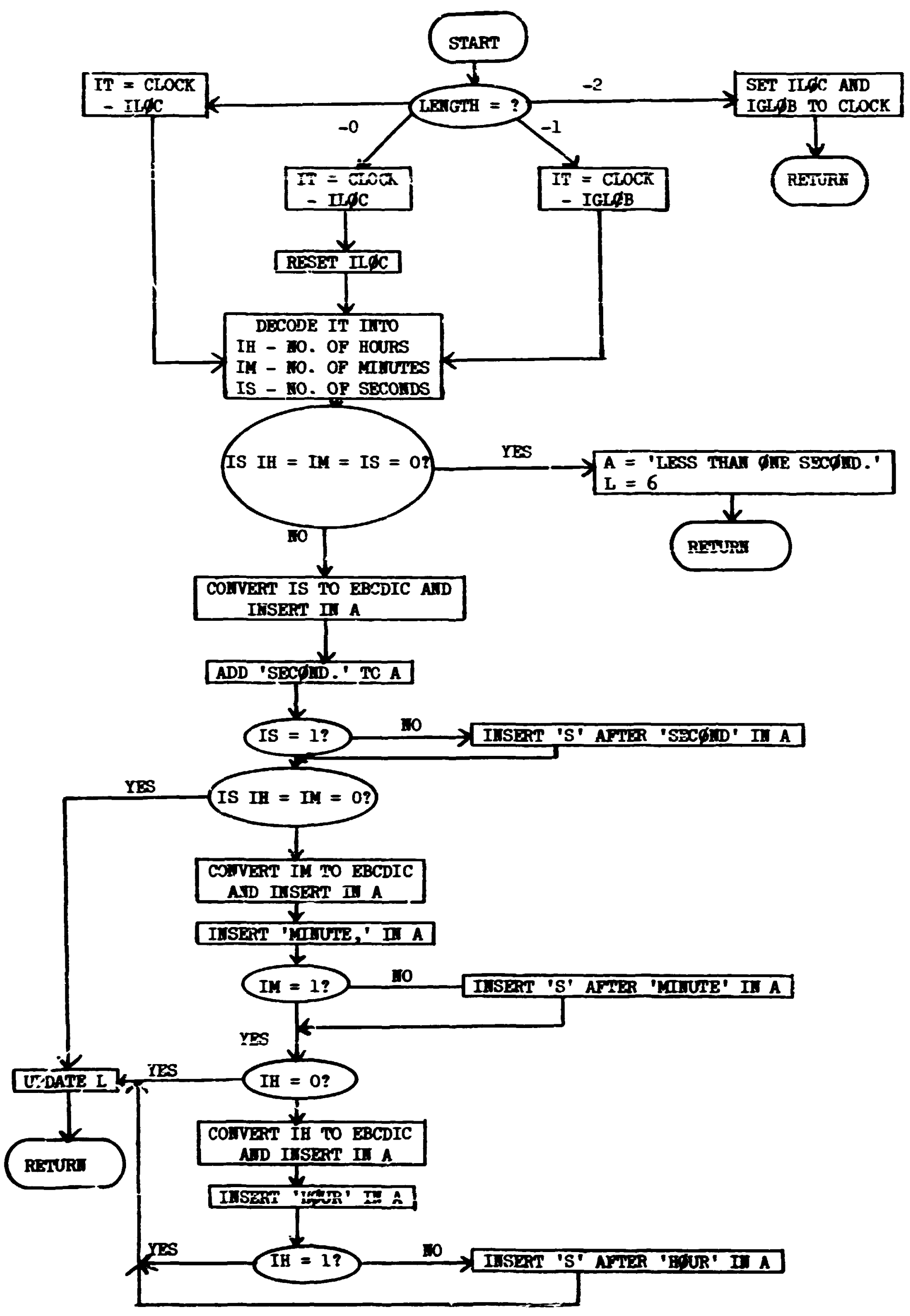




\section{Multigroup Cross-Section Module}

The function of this module in the muitigroup Nonte Carlo code is to read AIISH-type cross sections for media or elements, mix several elements together to sbtain media cross sections, determine group-to-group iransfer probabilities and determine the probabilities and angles of scattering for each group-to-grouis transfer. All variables are flexibly dimensioned, and are part of blank common. Many types of cross sections may be treated, such as neutron only, gemma only, neutron-gana-ray coupled or ganna rays from a neutron gama-ray coupled input. Cross sections for either a forward or adjoint solution may be obtained, and the Legendre coefficients for each group-to-group transfer may be retained for next-flight estimation.

The cross sections are read for one coefficient and one element into a buffer area. Then these eross sections are decomosed into total, fission, and downscatter matrix and stored in temporary arrays so that they may be mixed to form media cross sections. The total and fission cross sections are stored only once for an element, but the downscatter matrix is stored for each coefficient. The cross sections are transposed as stored if an adjoint problem is being solved.

After all cross sections are stored the contribution of each element to the cross section for the media is determined. Also at this time the sum of the downscatter vector for each group is determined for the future calculation of the nonabsorption probability; the gama-production cross section is also determined by summing the transfers to the gana groups. After the cross sections for the medium have been deternined, the nonabsorptinn probability, fission probability, and gama-production probabilities are formed by dividing by the total cross section. The downscatter matrix is converted to a probability table by dividing by the scattering cross section.

The Legendre coefficients for eqch group-to-group transfer are converted to angles and probabilities of scattering at those angles of the use of a generalized Gaussian quadrature using the ainguiar distribution as a weight function. That is, 


$$
\int_{-1}^{+1} f(u) \quad(u) d_{\mu}=\sum_{i=1}^{n} f\left(\mu_{i}\right) \omega_{i},
$$

where

$$
\begin{aligned}
& f(y) \text { is any polynania of order } 2 n-1 \text { or iess, } \\
& w(u) \text { is the angular distribution for } y \text {, the cosine of the saatterine } \\
& \text { angle, } \\
& \mu_{i} \text { is a set of discrete cosines, } \\
& \omega_{i} \text { is the probability of the corresponding cosinc. }
\end{aligned}
$$

Thus, a set of $\mu_{i}$ 's and $p_{i}$ 's that satisfy the equation wust be found. To do this, 2 set of polynomials, $Q_{i}$, which is orthogonal with respect to the angular distribution, is defined such that

$$
\int_{-1}^{1} \varrho_{i}(\mu) Q_{j}(\mu) \omega(\mu) d \mu=\varepsilon_{i j} N_{i} .
$$

where $B_{i}$ is a normalization constant.

The mowents of the angular distribution $M_{i}, i=1,2 n-1$, determine the orthogonal pclynomials, $Q_{i}, i=1, n$. The desired cosines, $\mu_{i}$, are given by the roots of $Q_{n}$,

$$
Q_{n}\left(\mu_{i}\right)=0 \text {, }
$$

and the corresponding probabilities are

$$
\omega_{i}=\left(\sum_{k=1}^{n-1} \frac{Q_{k}^{2}\left(n_{i}\right)}{n_{i}}\right)^{-1} .
$$

In the process of deriving the urtinogonal polynomials, some restrictions on the morents of the angular distribution are attaivicu. These restrictions arise if both the origingl digtribution and the derived point distribution are to be everywhere non-negative. The restrictions are:

1) $\mathbf{x}_{i}>0$ for $i=1, n$. 
This restriction ay be vritten in terns of the determinant of the monents:

$$
\left|\begin{array}{lllll}
1 & M_{1} & M_{2} & \cdots & M_{i} \\
M_{1} & M_{2} & M_{3} & \cdots & M_{i+1} \\
1 & & & & \\
1 & & & & \\
M_{i} & M_{i+1} & M_{i+2} & \cdots & M_{2 i}
\end{array}\right|>0
$$

2) The roots of $Q_{i}(u)$ must all lie in the interval

$$
-1 \leq \mu_{i} \leq 1
$$

It must be exphasized that the restriction arising from the original distribution being ewergwhere positive (or zero) does not restrict the truncated expansion of the distribution to be everyutere positive. That is, monents from a truncated distribution that is not necessarily evergwhere positive are used to derive a discrete distribution vith positive probsbilities.

Other characteristies of this representation are that the information is capact, the angles are clustered where the angular distribution is peaked, and because of the restrictions, cross sections that have blunders in then ere rejected because they produce angles outside the range of -1 to +1 . All of the variables used to locate cross sections occur in comon LACSIG. Definitions of the variables which are set up in subroutine ISEC are given in Table V. An outline of the storage of the cross sections in blank comon is given in Table VI. Other details of the cross-section nodule are given with the description of the various subroutines. A nore detailed description of the theory for the generalfzel Gainssian quadrature is given in Appendix B. 
Table V. Definitions or Variables in Comon LACSIG

\begin{tabular}{|c|c|}
\hline Variable & Definition \\
\hline ISTART & $\begin{array}{l}\text { starting location for the total cross-section rector for the } \\
\text { first nediun }\end{array}$ \\
\hline$J \cdot 3 \cos \theta$ & $\begin{array}{l}\text { starting location for the scattering cross-section vector for } \\
\text { the first nediun }\end{array}$ \\
\hline 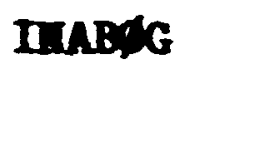 & $\begin{array}{l}\text { starting location for the nonabsorption vector for the first } \\
\text { nediu }\end{array}$ \\
\hline$I \sin 1 / 6$ & $\begin{array}{l}\text { starting location for the gecme-productios fector for the } \\
\text { first nediu }\end{array}$ \\
\hline IFPURG & $\begin{array}{l}\text { starting location ior the } v \text { fission probability vector for } \\
\text { the first nediv }\end{array}$ \\
\hline IPICP & $\begin{array}{l}\text { starting location for the primary-secondary transfer proba- } \\
\text { bility matrix }\end{array}$ \\
\hline IPSPdG & $\begin{array}{l}\text { starting location of the primary downscatter probability } \\
\text { matrix }\end{array}$ \\
\hline IDscdG & $\begin{array}{l}\text { starting location of the secondary dounscatter probability } \\
\text { natrix }\end{array}$ \\
\hline IPRE:G & $\begin{array}{l}\text { starting location of tk prinary scattering angle probability } \\
\text { matrix }\end{array}$ \\
\hline IPRBGG & $\begin{array}{l}\text { starting location of the secondary srattering angle probabil- } \\
\text { ity natrix }\end{array}$ \\
\hline $\begin{array}{l}\text { IScuIG } \\
\text { Isckers } \\
\text { ISPtig }\end{array}$ & $\begin{array}{l}\text { starting location of the prinary scattering angle matrix } \\
\text { starting location of the secondary scattering argie natrix } \\
\text { size of storage needed for each medium, not including Legendre } \\
\text { coefficients }\end{array}$ \\
\hline $\begin{array}{l}\text { ISPORT } \\
\text { IIPBUP } \\
\text { IS IETG }\end{array}$ & $\begin{array}{l}\text { starting location for temorary storage of dornscatter matrix } \\
\text { starting location of input buffer region for the } F_{0} \text { table } \\
\text { starting location for teporary storage of tot:al sross seciion } \\
\text { for elenent } 1\end{array}$ \\
\hline $\begin{array}{l}\text { IAPPGG } \\
\text { IABSTG }\end{array}$ & $\begin{array}{l}\text { starting location for temporary stroage of } v \Sigma_{f} \text { for elenent } 1 \\
\text { starting location for taporary storage of downscatter natrix } \\
\text { for } P_{L} \text { coefficients (primary groups, elenent } 1 \text { ) } \\
\text { total storage required is temorary siorage }\end{array}$ \\
\hline
\end{tabular}


Table V (cont.)

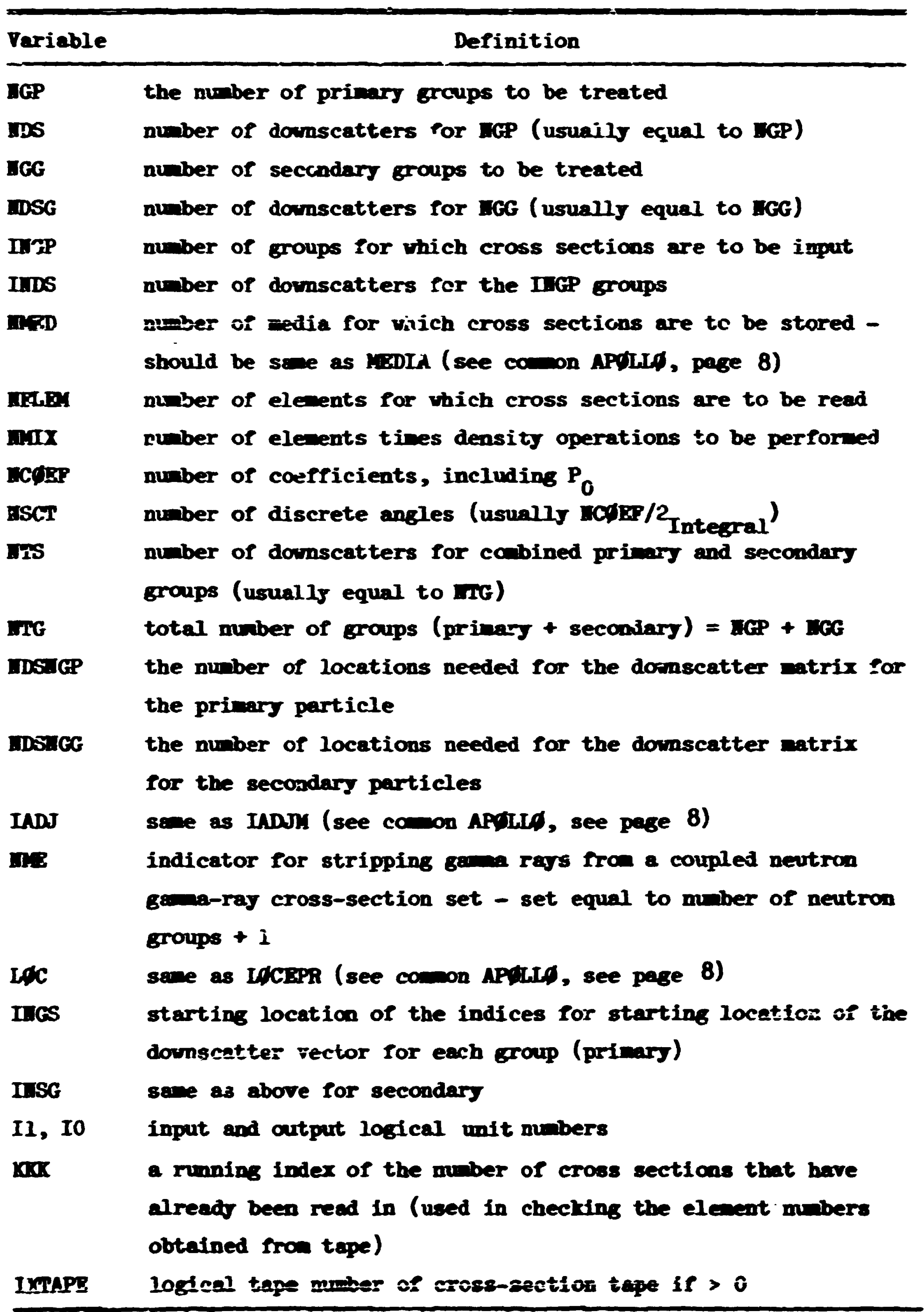


Sable Y. (́cont.)

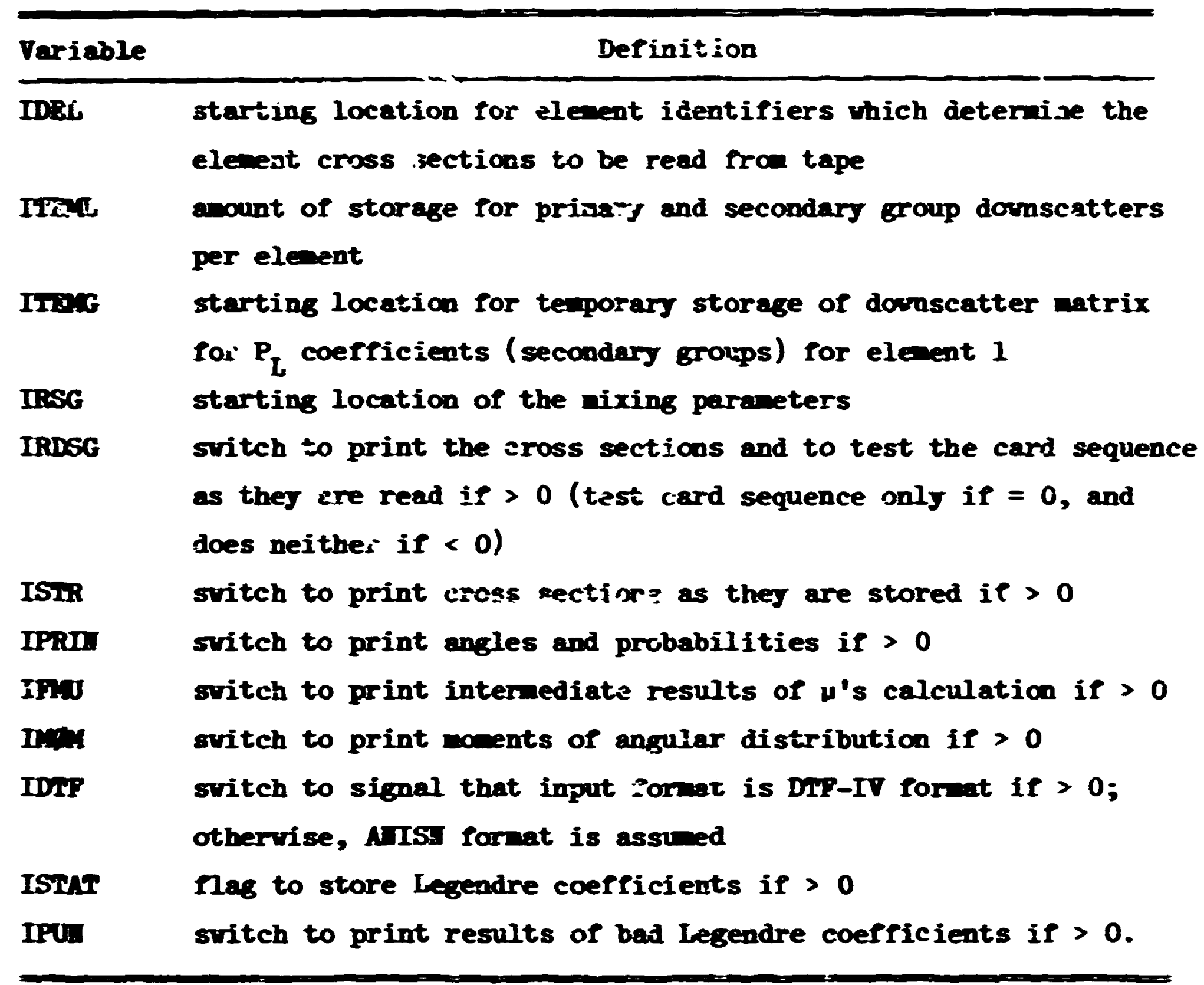

If Legendre coefficients are to be restored, then:

IIFYG - redefined by JuPU as starting location of $P_{1}$ coefficients for secondary group for mediun 1

Irtrsc - redefined by JuPU as total storage required for all medis for each Legendre coerficient

ISPtir - redefined by JIPUT as starting locetion of $P_{1}$ coefficients for primary groups for nediun 1. 
Table VI. Location of Permanent Cross Sections in Blank Coman

\begin{tabular}{|c|c|c|}
\hline Location & Information & Size \\
\hline \multirow{2}{*}{$\begin{array}{l}\text { IFSG = IIAST } \\
\text { IWES }\end{array}$} & $\begin{array}{l}\text { List of } \\
\text { Mixing Tarle }\end{array}$ & 3EnIX \\
\hline & $\begin{array}{l}\text { Index to } \\
\Sigma_{G G} \text { (Primary) }\end{array}$ & IGP \\
\hline INSG & $\begin{array}{l}\text { Index to } \\
\Sigma_{G G}(\text { Secondary })\end{array}$ & IGG \\
\hline IDas & $\begin{array}{l}\text { List of Element } \\
\text { I.D. Nubers }\end{array}$ & $\begin{array}{l}\text { MEDEFTCOEP } \\
\text { if DMAPE > } 0\end{array}$ \\
\hline ISTAFr & $\Sigma_{I}$ & ERG \\
\hline Isceng & $\Sigma_{s}$ & IrG \\
\hline IIAB'G & $\Sigma_{s}$ & Irro \\
\hline IGAB'G & $\Sigma_{Y} / \Sigma_{T}$ & EQP \\
\hline IPPdix & $v \Sigma_{\Gamma} / \Sigma_{T}$ & InS \\
\hline IPLEP & $\Sigma_{n+y}$ & EGP IEG \\
\hline IPSP'G & & $\frac{(\mathrm{DS}+\mathrm{L})(\mathrm{IDS})}{2}$ \\
\hline IDSEIG & $\Sigma_{B^{\prime}+B_{B}}^{Y}$ & $\frac{(I D S G+1)(I D S G)}{2}$ \\
\hline IPRE'G & $P_{8}$ & $\frac{(\operatorname{ms}+i)(\operatorname{ms})}{2} \operatorname{sscT}$ \\
\hline IPRBCE & $P_{E^{t}+B}^{Y}$ & $\frac{(D D S G+1)(D D S G)}{2}$ WSFP \\
\hline Isciug & $A_{8^{\prime}+6}^{I}$ & $\frac{(\operatorname{DS}+1)(\mathrm{DDS})}{2}-\mathrm{NSCT}$ \\
\hline Iscuet & $\overline{A_{g^{\prime}+8}^{Y}}$ & $\frac{(D D S G+1)(\operatorname{DSG})}{2} \mathrm{NSCT}$ \\
\hline $\begin{array}{l}\text { ISPORG } \\
+ \text { ISTAII }\end{array}$ & $\begin{array}{l}\text { Repeat for } \\
\text { next mediu }\end{array}$ & ISPORG \\
\hline
\end{tabular}


Rable vI (cont.)

\begin{tabular}{|c|c|c|}
\hline Location" & Information & Size \\
\hline \multicolumn{3}{|c|}{ If ISTAT >0 } \\
\hline & $\begin{array}{l}P_{1} \text { Coeff. } \\
\text { Primary }\end{array}$ & NDS*IMED \\
\hline \multirow{2}{*}{ INPPdG } & $\begin{array}{l}F_{1} \text { Coeff. } \\
\text { Secondary }\end{array}$ & IDSGAMIED \\
\hline & $\begin{array}{l}\text { Repeat for } \\
P_{L} \text { Coeff. }\end{array}$ & $(\mathrm{NDS}+\mathrm{MDSG}) * \mathrm{MMED}(\mathrm{KCOEF}-2)$ \\
\hline
\end{tabular}

IILAST

Locations are for index of zero.

Total storage is MTG + MEDEISP

where:

$$
\begin{aligned}
& \text { ITG }=\text { IGP + IGG } \\
& \text { ISPQRG = 4HFTG + IGP( } 1+\text { IGG }) \\
& +\left(\frac{2 \pi S C T+1}{2}\right)(\mathrm{NDS}+1)(\mathrm{IDS}) \\
& +\left(\frac{2 \pi S^{2} T+1}{2}\right)(\operatorname{MSG}+1)(\operatorname{MDSG})
\end{aligned}
$$

IX = MELEAAICOEF if IXTAPE > 0

$=0$ otherwise, and

LEG $=($ KDS+IDSG $)$ WWIED $($ ICOEP-1) if ISTAT >0

$=0$ otherwise. 
Subroutine Arind

This routine is called upon encountering an albedo scattering surface and provides the outgoing neutron parameters for the albedo collision.

The sample routine performs specular reflection at the albedo scattering surface. The requiremenis of specular reflection ay be written as

$$
I \cdot \mathbf{I}=-\mathbf{R} \cdot \mathbf{H},
$$

and

$$
I \times \mathbf{H}=\mathbf{R} \times \mathbf{H},
$$

Where $I$ is the incoming neutron direction vector,

$R$ is the reflected neutron direction vector, and

$N$ is the outward normal to the surface $(I \cdot M<0)$.

Manipulation of the above two equations results in

$$
\mathbf{R}=\mathbf{I}-2(I \cdot \mathbf{I}) \mathbf{I}
$$

Called from: WREE.

Comms required: IUTRd, WorkAL.

Variables required: U, V, W ( fron comon NuIRP, see Fage 12)

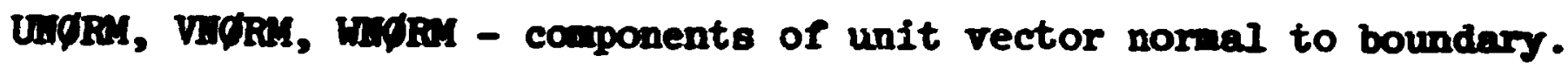

Variables changed: $U, V, U$.

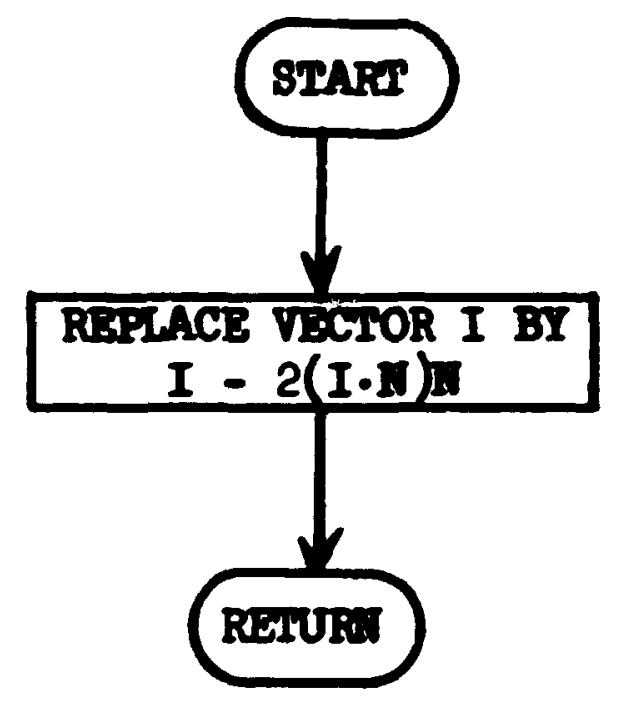


Subroutine MGTES (IGI, JGl, MX)

This is the main executive routine for the generailized caussinn quadrature. First it calls GBFuls which uses the noments of the angular distribution to deternine the recurrence relations which generate the orthogonal polymonials. In 80 doing Grints performs the check for $\mathrm{H}_{1}>0$, which is one of the requirements on the monents. Mext ANGLES calls PIND in an iterative fashion in orcer to calculate the roots of the orthogonal polynomials. FID checks the roots to deternine if the second restriction on the noments, nanely that the roots must lie in the interval $(-1,+1)$, is satisfied. Next AMGLS calcuiates tine weight ractors associated with each root in the Gaussian quadrature. Finally the angles and probabilities which have been calculated are rearranged so that they appear in order of decreasing probability. If the given monents do not satisfy the two requirements, then it is not possible to determine as man angles and weights as initially requested. Horever, NGLEs deternines as many as it can fron the data given.

MOTE: If $2 n+1$ moments are Given (and all are acceptable), then a discrete distribution with $n+1$ scattering angles nay be deternined. If only $2 n$ monents are giren, then there is a certain asount of Ireedon in choosing a $2 n+1-8 t$ monent to crmplete the calculation. In these cases NGLSS 111 compute a value of $\mu_{n+1}$ (and hence of $\mu_{2 n+1}$ ) which is in the midale of the allowed range for $\mu_{n+1}$ and, using this value of $\mu_{n+1}$, conplete the calcular tion of a $(n+1)$-angle distribution.

Called Irom: JuPUI

Subroutines called: GEMUS, FIDD, Q, EXIT, BADYM, XSCHIP. Comons required: MRAT, RESULT, MUIT, LACSIG.

Variables required:

IGI
JGI Indices of group-to-group transfer being
calculated

Disu - number of moments Biten:

$\operatorname{xymar}(I)=M_{1}, i=1, \operatorname{mit}$,

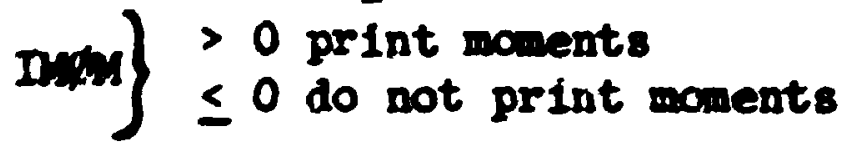

wX - medium number 
IFU. $\leq \frac{1}{>} 0$ do not print error nessages

I0 - output unit number,

nSCT - number of scattering angles expected.

Variables changed:

$\operatorname{PdIr}(I)=X_{i}=$ cosine of scattering angle for $I=1,4 \pi+1$,

Weight $(I)=W_{1}=$ probahility of scattering angle for $I=1, W+1$.

II - number of in values accepted,

$W V$ - number of $\sigma^{2}$ values accepted.

Significant internal variables:

$\operatorname{Var}(I)-\mu_{1}$,
$\operatorname{VAR}(I)-\sigma_{1}^{2}$

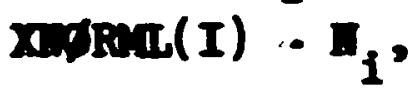

Fin $(I, J)$ - Ith root of $Q_{J}(x)$,

IP $=I V+1=$ numer of angles in discrete distributicn,

IACC $=$ DAW $=$ number of momts accepted.

Output:

$\operatorname{Ixp}(I)=\mu_{1}, I=1$, min.

Indices of roup, nuber of monents accepted lonj if maber accepted

is less than number gived. 
Srbroutine Arars

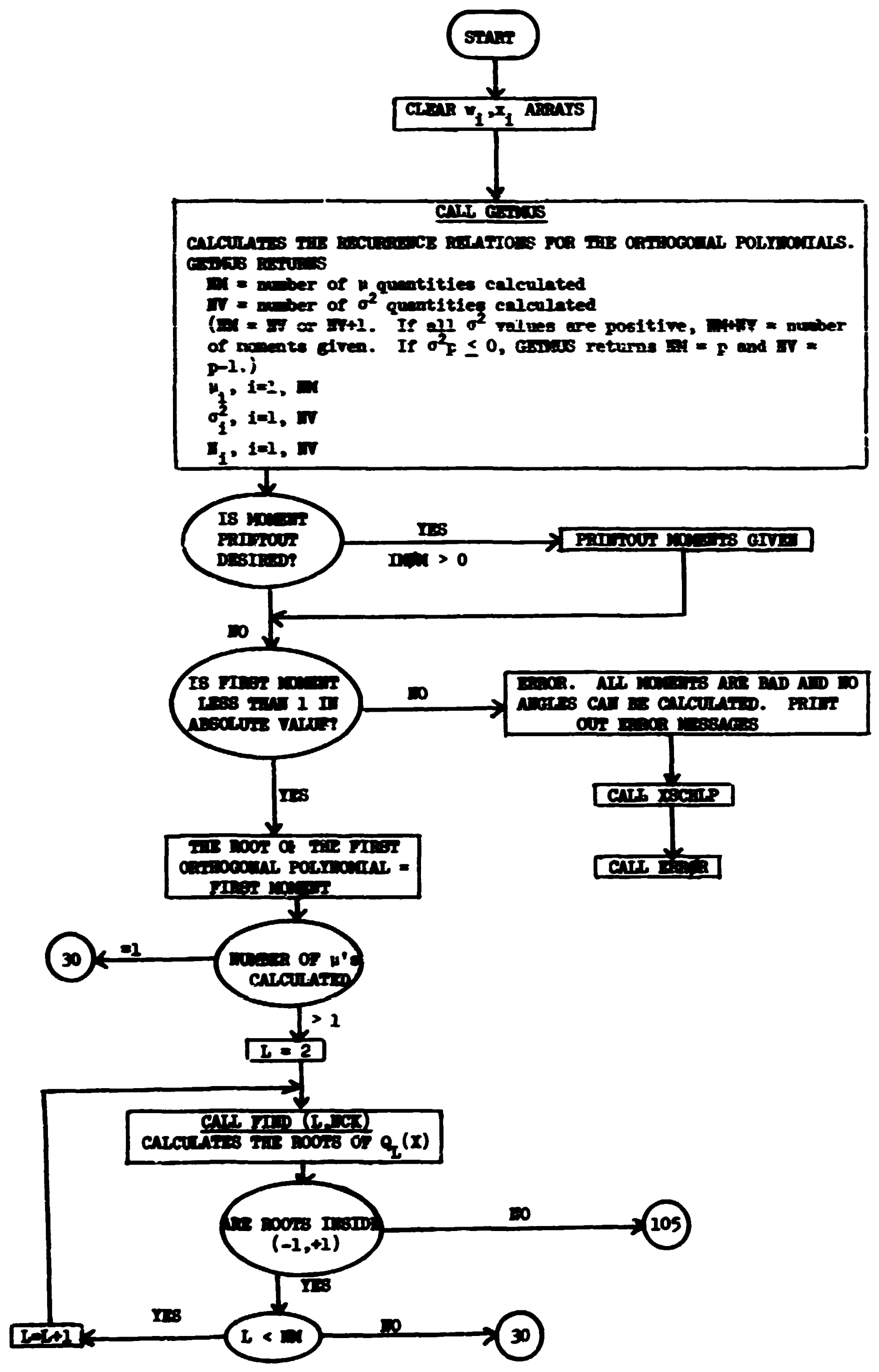




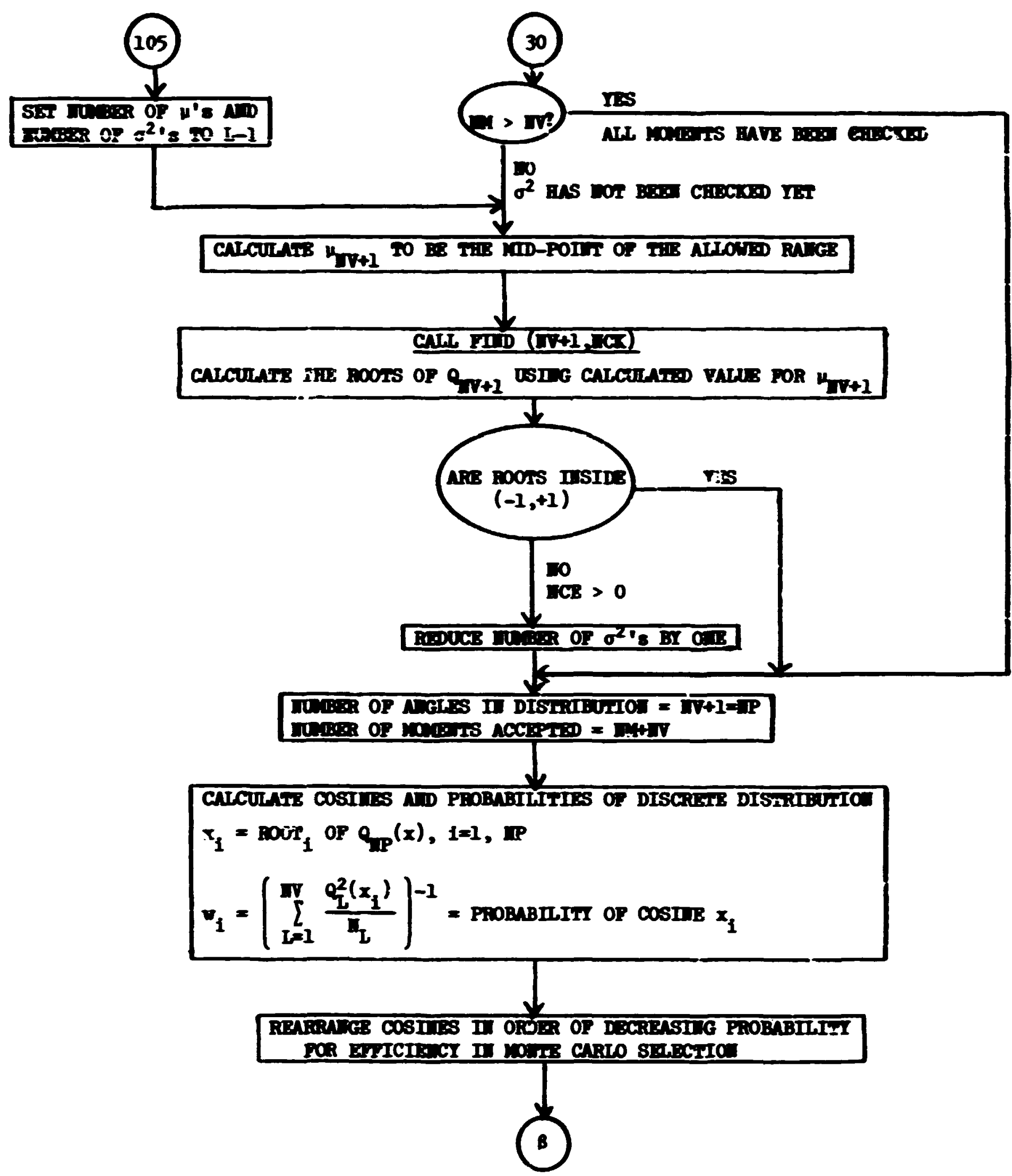




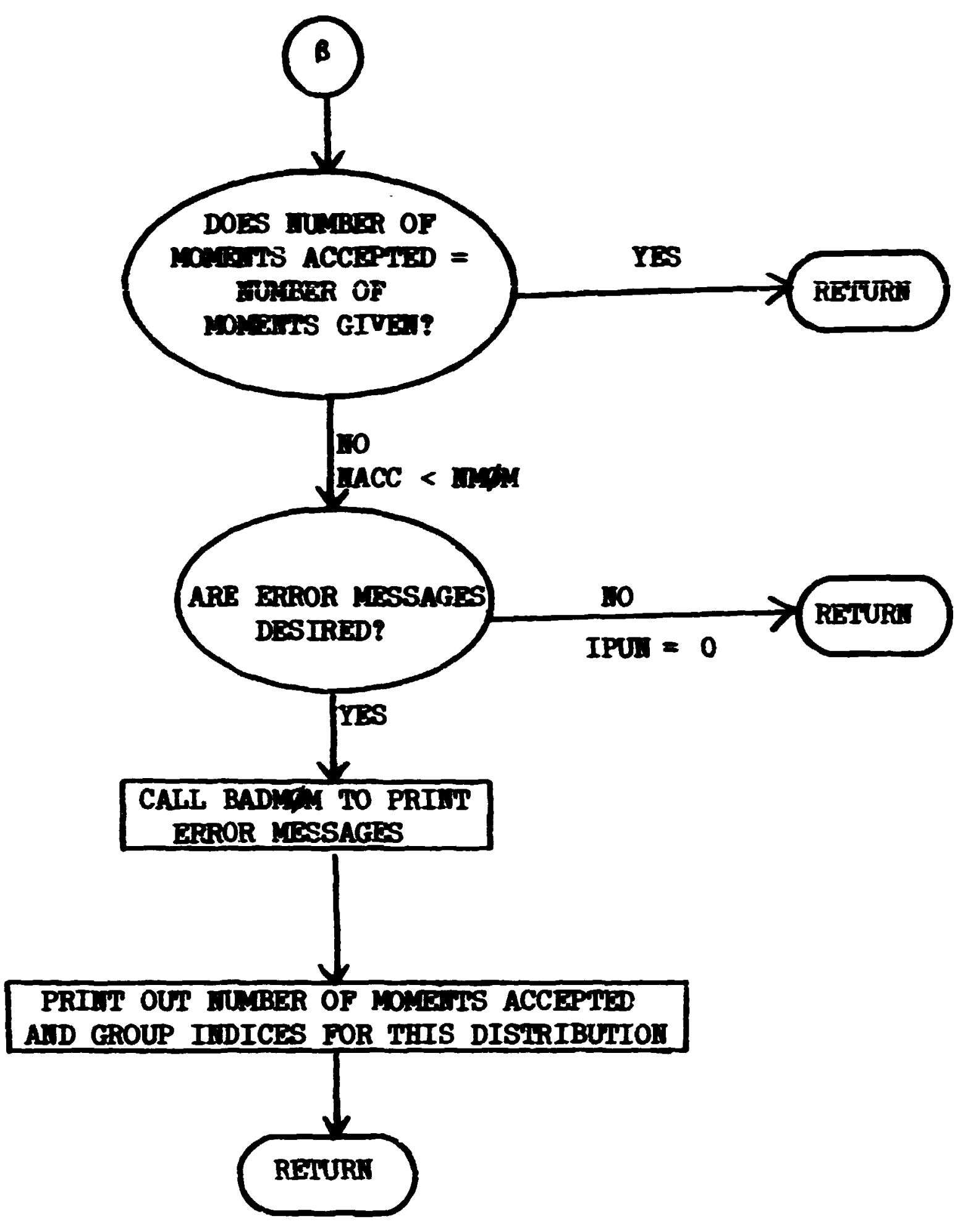




\section{Subroutine Bnpray}

In the erent that a mosent has been refected because it inplied negativity in the angular distribution, BApyty is called to proride a printout to the user giving the value of the quantity rejected, $\mu_{i}$ or $\sigma_{i}^{2}$, of the moment rejected, and of the Legendre coefficient which was rejected. In addition, the allowed linits on these quantities are also printed out.

See mathenatical description for formulas used.

Called Prom: NGLSS.

Subroutines called: Marirr.

Funitions used: $Q$.

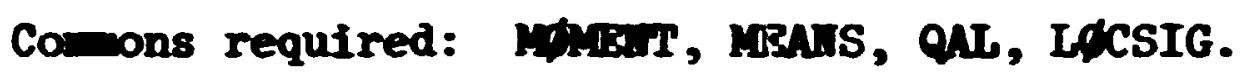

Variables required:

A - number of $\sigma^{2} \cdot s$ accepted,

III - number of $\mu$ 's accepted,

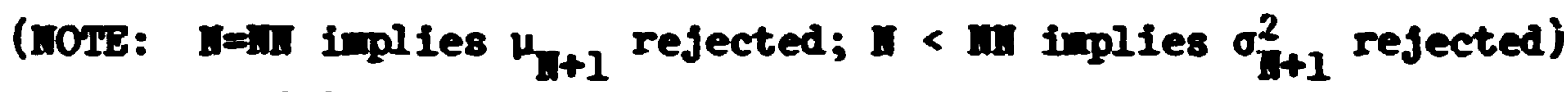

$$
\begin{aligned}
& \operatorname{MU}(I)-\mu_{i}, \\
& \operatorname{VAR}(I)-\sigma_{i}^{2}, \\
& \operatorname{MRU}(I)-M_{i}, \\
& \operatorname{QR}(I)=q_{i}=L_{i} / M_{i-1}, \\
& A(I, K)-a_{i k} .
\end{aligned}
$$

(Wote that $I=1$, but $K=k+1$ )

Significant internal variables:

DI $=\mathrm{H}+\mathrm{III}=$ number of monents accepted,

IBAD $=$ DA+1 = index of noment rejected,

MP1 - I+1,

$\mathbf{m n}=\mathbf{m}-1$.

Output:

$$
\begin{aligned}
& \text { Mr - } \mu^{\max } \text {, } \\
& M B B \text { - } \mu^{m i n} \text {, } \\
& \text { VARI - }\left(\sigma^{2}\right)^{\max } \text {, } \\
& \text { VARB }-\left(\sigma^{2}\right)^{\text {min }} \text {, } \\
& x+x^{\max } \text {, }
\end{aligned}
$$




$$
\begin{aligned}
& \text { MAB - Min } \\
& F P-f^{\text {max }}, \\
& \text { PB }-f^{\text {min }}
\end{aligned}
$$


Subroutine BADrin

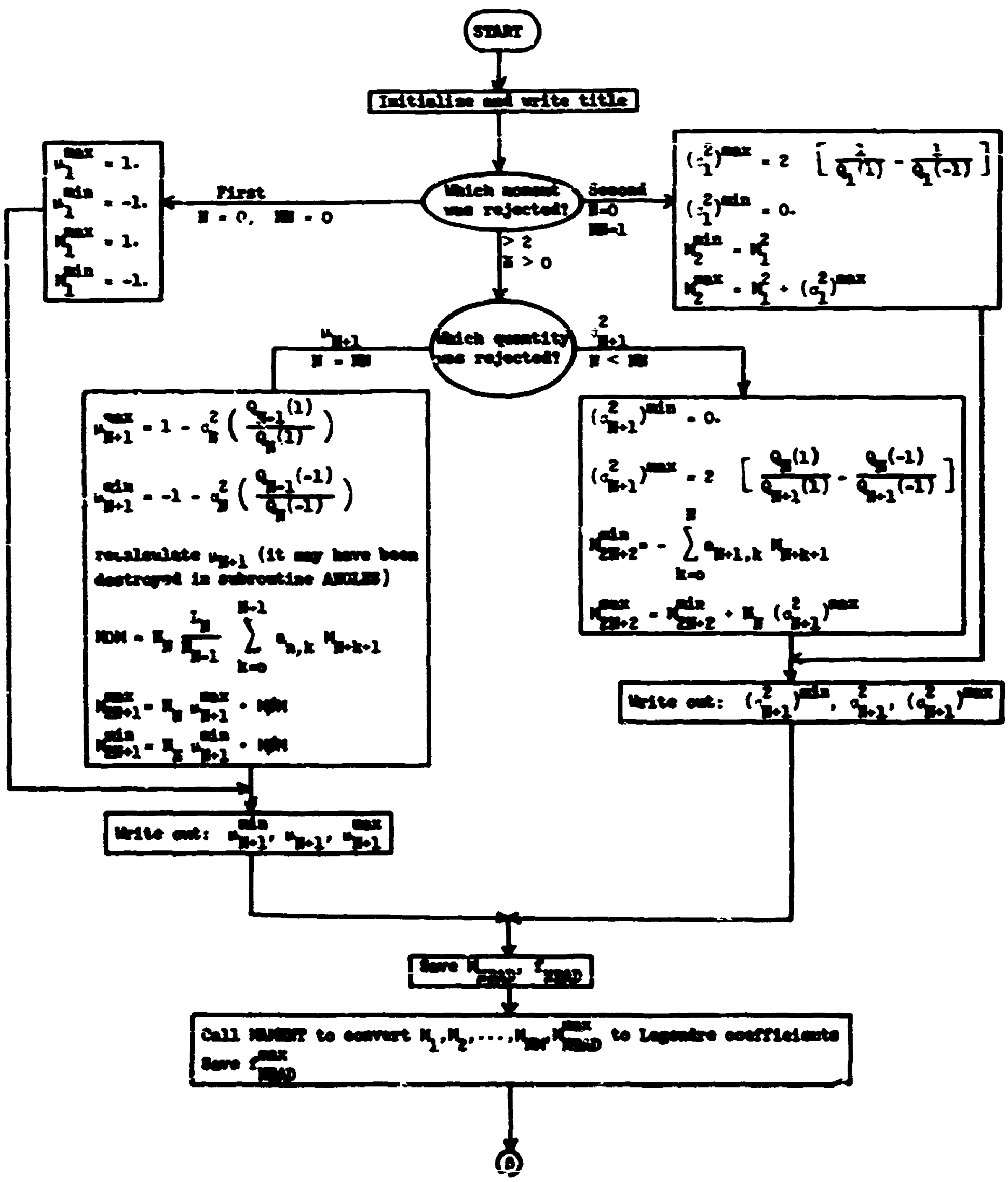


(B)

$\nabla$

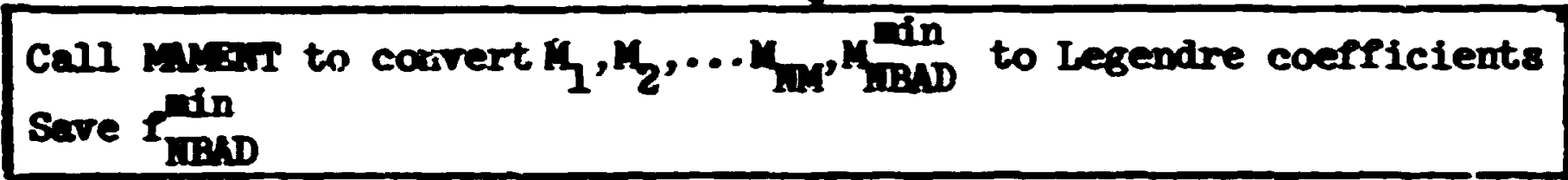

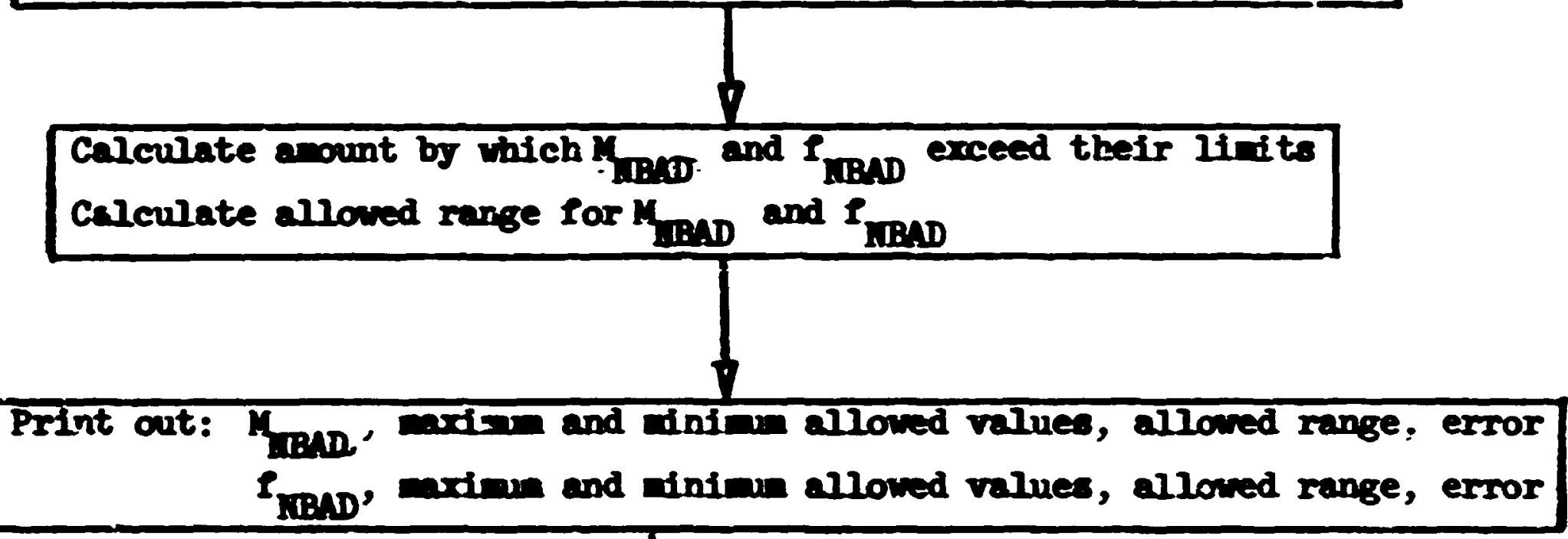

BGIUR. 
Subroutine CQLISI (IG, U, V, W, WATE, IMIND, IREG)

The subroutine is called at each collision and the incoming grcup number, direction cosines, and particle veight are converted into postcollision paraneters. The outgoing group is zelected from the downscatter matrix (the vector corresponding to the incoming group). After determining the outgoing group the cosine of the angle of scattering is jetermined frcm the set of probabilities and angles for the particular group-to-group trensfer. The outgoing direction cosines in the laburatory coordinate system are determined from the incoming directions and the angle of scattering and a uniformly selected azimuthal angle. The particle's weight is altered by the non-absorption probability in lieu of absorption.

As an importance sampling scheme the outgoing group probability distribution may be altered and selection of the outgoing group is made from this biased distribution. If this option is chosen. LфCEPR > subroutine GTIØUT is called.

Called from: MDSE

Subroutines called: GIMED, GII

Functions used: FLTRII, SQRT (1ibrary).

Cammons required: Blank, LøCSIG.

Variables required:

IG - the precollision energy group,

$U, V, W$ - the precollision direction cosines,

WATE - precollision particle veight,

IMED - gecmetry medium of collision,

NREG - eometry region of collision.

(Various indices from common LdCSIG, see page 88 )

Variables changed:

IG - post-collision group,

$U, V, W$ - post-collision direction cosines,

WATE - post-collision weight. 
Significant internal variables:

FTAB - non-absorption probability,

IH - group number = IG for primary particie,

- IG - IGP for secondary farticle,

MADDPG - number of locations letueen sxarting location of siattering angle probabilities for primary and secondizy particles,

$R$ - random number,

IND - location of biasine parameters for GD Jup IG,

RDSK - number of downscatter groups,

FM - cosine of polar angle of scattering,

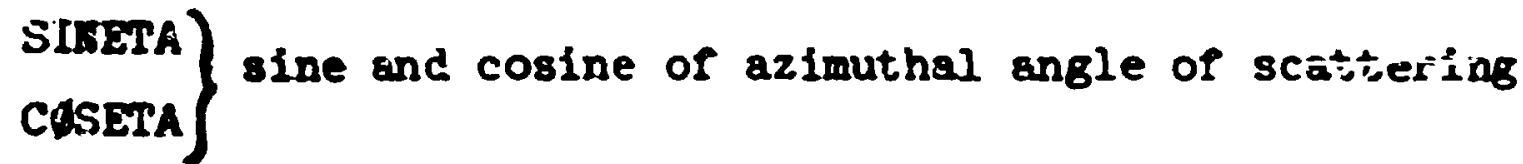

Limitations: number of angles is equal to number of probabilities for each group (assumed in use of MALDPG). 


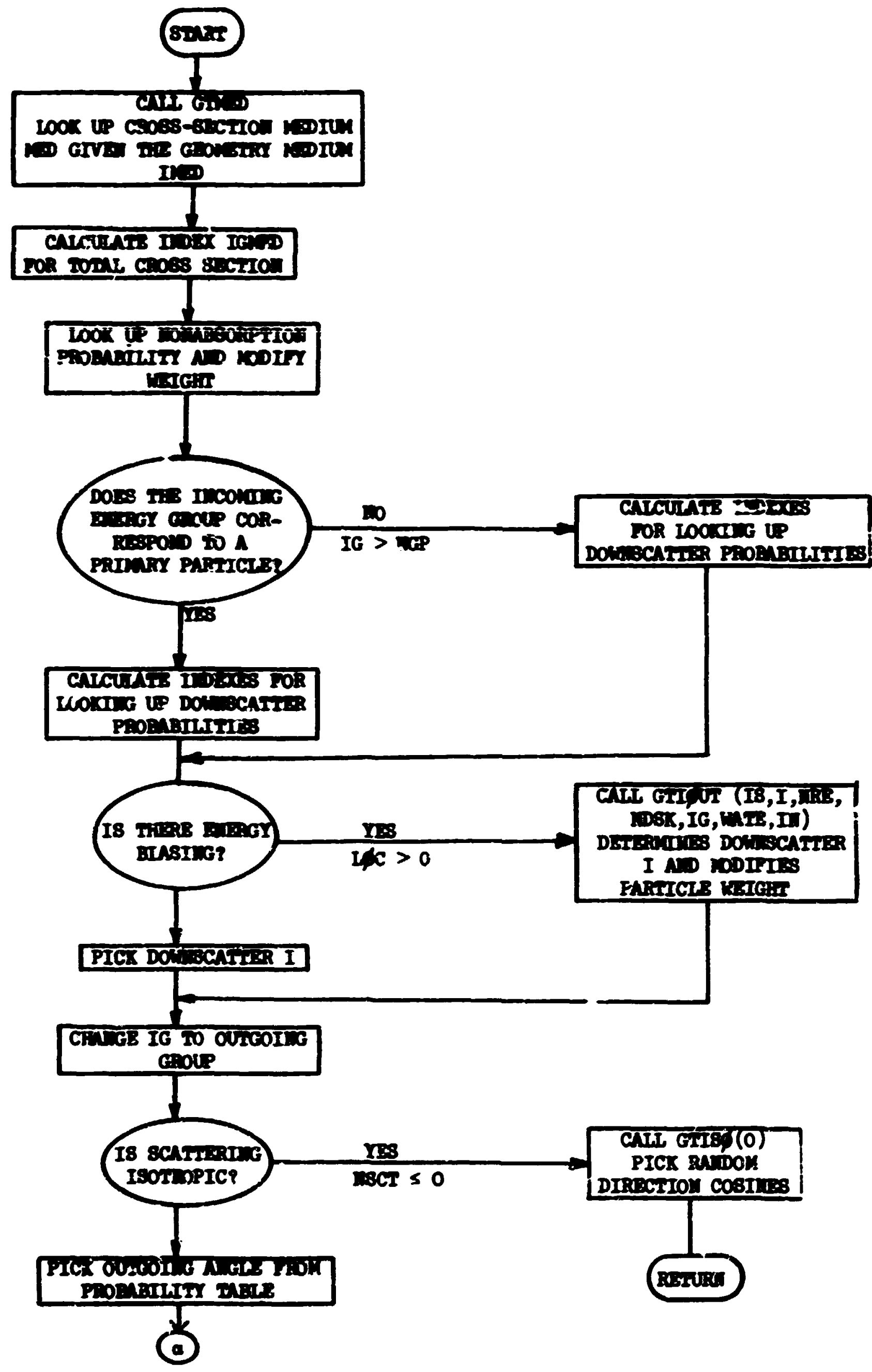




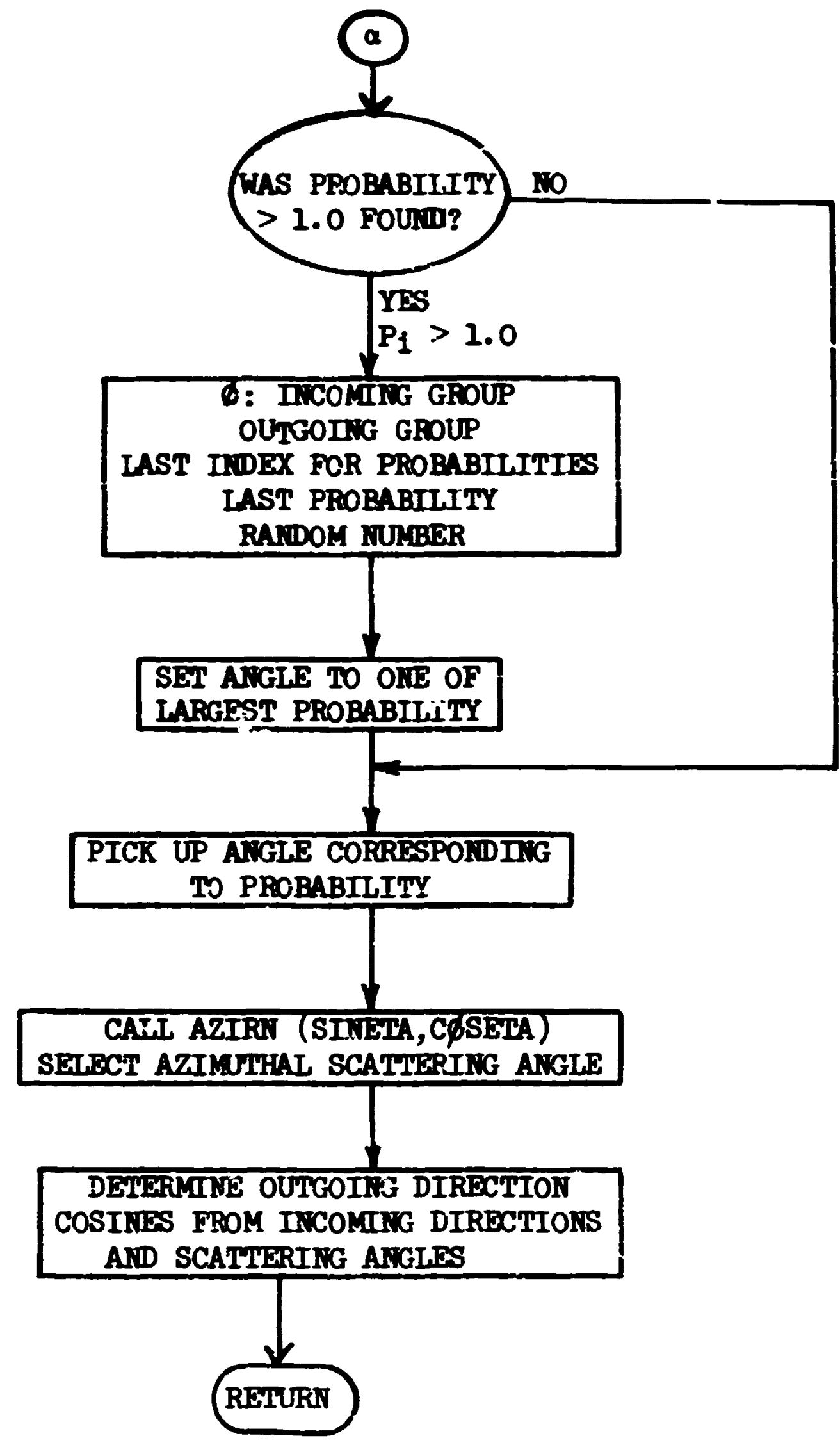


Subroutine FIIID (L, IF)

This subroutine determines if the roots of $Q_{L}(x)$, the Lth order orthogonal polynomiai, lie within the range $(-1,+1)$. If not, a flag, IF, is set to $I$ and the subroutine returns. If the roots lie within the range $(-1,+1)$, then NF $=0$, and the subroutine proceeds to calculate the rcots. The roots, $x_{k}, k=1, L$, ere stored in $R d T(K, L), K=1, L$ in labelled comon RESULT. The roots are in increasing order $\operatorname{RdT}(I, L)<\operatorname{Kr} T(2, \mathrm{~L})<\ldots$ $<\operatorname{Redr}(\mathrm{L}, \mathrm{L})$.

FIn! presumes that the roots of $Q_{L_{-1}}(x)$ have already been calculated and stored in $R$ T $T(K, I-1), K=1, L-1$. Thus it is necsssary to use FIND in a bootstrapping manner. First $\operatorname{RQ}\left(\mathrm{T}(1,1)=M_{1}\right.$, the root of $Q_{1}(x)$, is stored. Then one sequentially calls $\operatorname{FInD}(2, \mathrm{MF}), \operatorname{FIND}(3, \mathrm{NP})$, etc. It is also presumed that the roots of $Q_{L-1}(x)$ are in the interval $(-1,+1)$.

FIID uses the property of orthogonal polynomials that the roots of $Q_{L}$ and $Q_{L-1}$ "interleave." Thus:

1) $Q_{L}$ has no roots above +1 if $Q_{I-1}$ has no roots above +1 and $Q_{L}(+1)$ $>0$. (Remember that $\left.Q_{L}(+\infty)>0.\right)$

2) $Q_{L}$ has no roots below -1 if $Q_{L}(-1)$ differs in sign from $Q_{L}$ (Rfor(1, $L-1)$ ) where $\operatorname{RdT}(1, L-1)$ is the lowest root of $Q_{L-1}(x)$.

3) The Kth root and no other root of $Q_{L}$ lies between the $K-1$ th and the $K+h$ roots of $Q_{I-1}$.

Once the root has been isolated as being between $X \Phi \mathrm{W}=\mathrm{R} \phi \mathrm{T}(\mathrm{K}-1, \mathrm{~L}-\mathrm{I})$ and XUP $=\operatorname{RQ} W(K, L-1)$, it is found by a very simple procedure. The interval $(X \triangle \emptyset W, X U P)$ is bisected by XIRY $=(X I \emptyset W+X U P) / 2$. Then the subiniterval containing the root is determined by the fact that the sign of $q_{1}$ mist change in passing ovor the root. Thus the root lies in (XLGW, XTRY) if sign $\left[Q_{L}(X L \phi W)\right] \neq$ sign $\left[Q_{L}(X T R Y)\right]$ and it lies in (XIRY,XUP) otherwise. XIRY replaces the apprcpriate iimit, XUP or $X \measuredangle \phi W$, and the process is repeated. Each iteration reduces the size of the boundary interval by 2 , or, in other words, increases the accuracy to which the roct is known by one binary bit. Obviously, after as many iterations as the computer word has bits, XTRY will be as close to the root as can be calculated by the computer. 
Called rron: ArguES

Subroutines called: Q

Comons required: RESULT, LACSIG

Variables required:

$L$ - the order of the polynomial whose roots are desired, $\operatorname{RdT}(K, I-1), K=1, I-1$ - the roots of $Q_{L-1}(x)$ in increasing order, IPUI $-\left\{\begin{array}{l}\leq 0 \text { do not print error message } \\ >0 \text { print error message }\end{array}\right.$

Variajies changed:

IIP $-\left\{\begin{array}{l}=0, \text { the roots of } Q_{L}(x) \text { lie in the interval }(-1,+1) \\ =1, \text { the roots of } Q_{L}(x) \text { do not lie in the interval }(-1,+1) .\end{array}\right.$

If $\mathrm{MP}=0$

$F(K, L), K=1, L$ - the roots of $Q_{L}(x)$, in increasing order.

Significant internal variables:

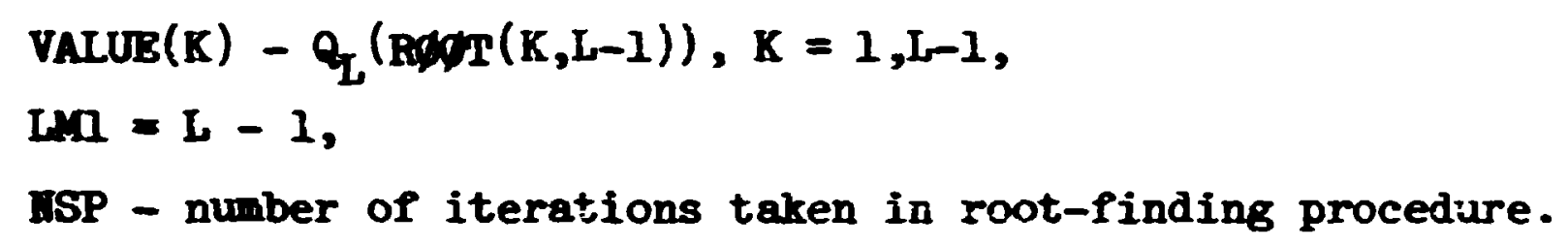

Linitat: $m 8$ : $I \leq 14$. 


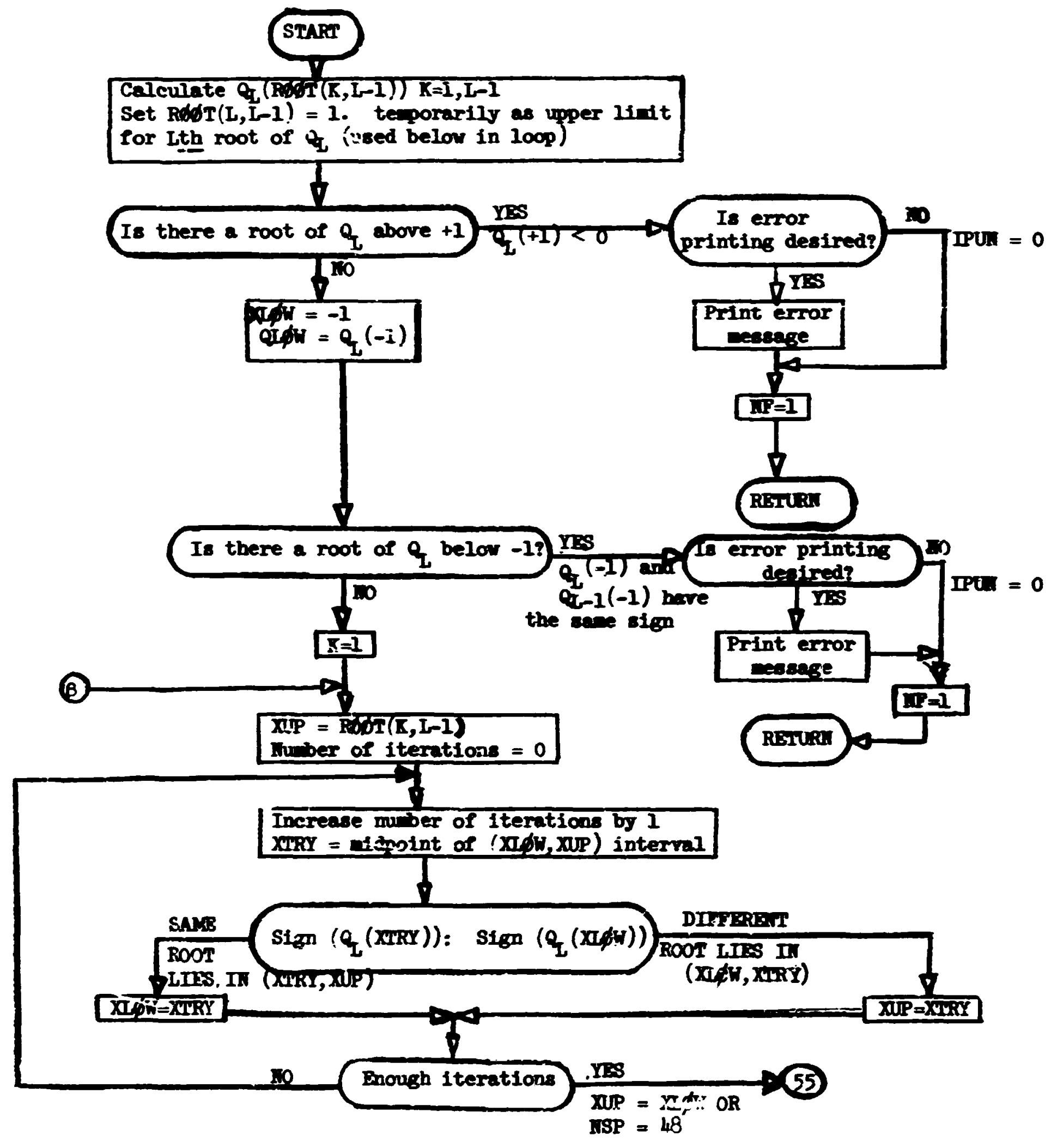




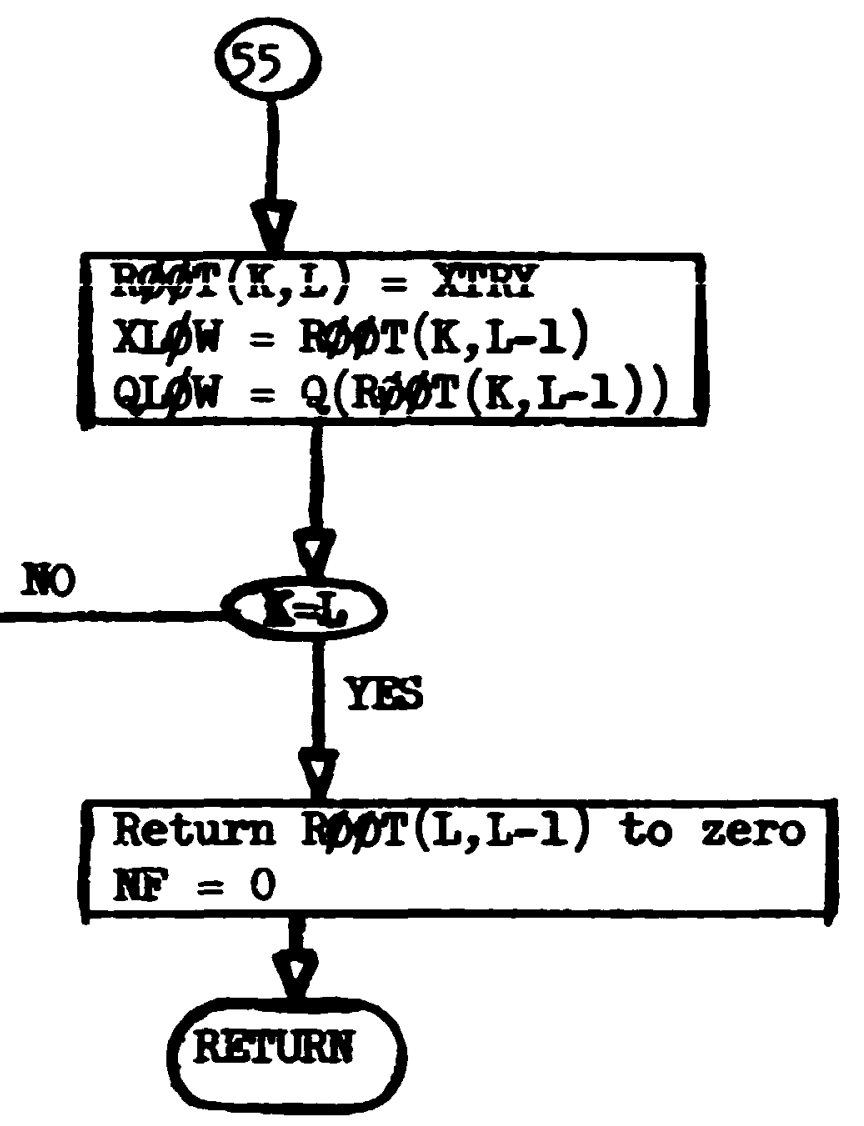


Subroutine FISGE (IG, INED, PNUP)

This subroutine lools up the raive oi $\forall \Sigma_{f}^{\prime} / \Sigma_{T}$ for the current neutron enersy and geonetry medium.

Calles fron: Impirs.

Subroutines called: GheD

Commons required: Blank, IdCSIG.

Variables required: ISPTRG, IFPdRG IG, NESD

( Trow comon IfCSIG, page 83)

Variables changed: PrUP.

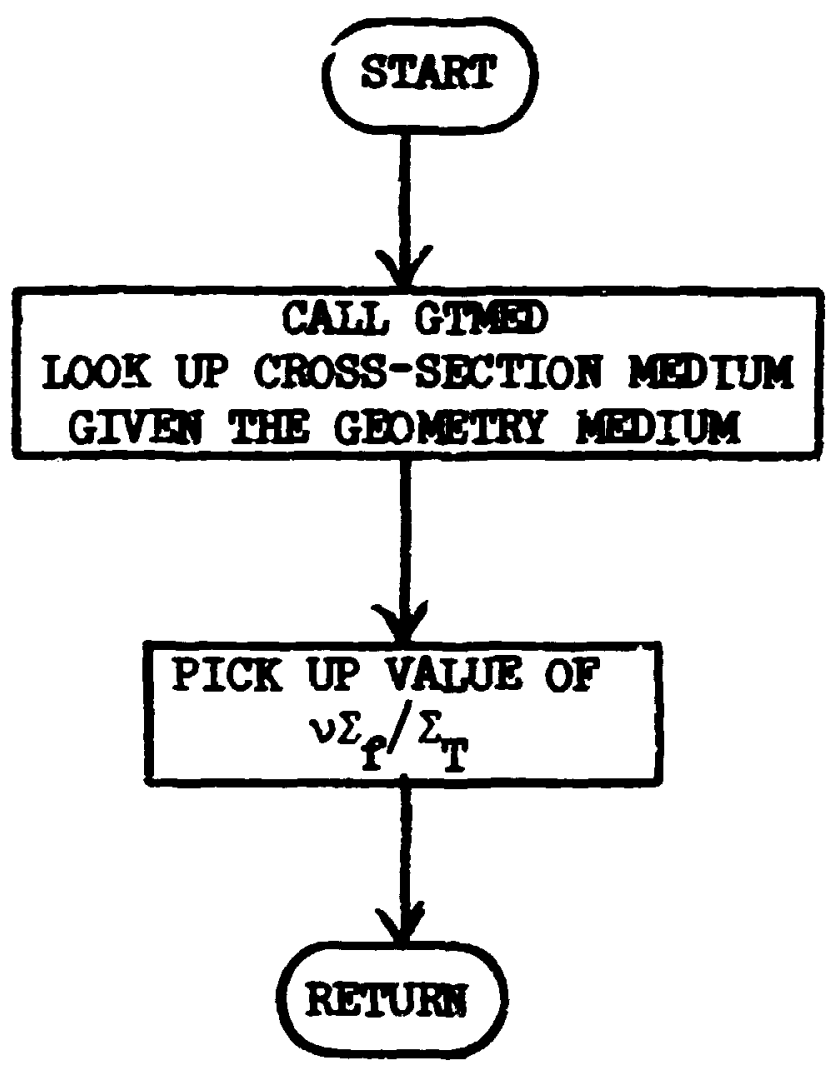


Subroutine GNGE (IG, IED, PGE, ICG)

This subroutine provides the runction of deternining the enersy of the secondary particle to be generated and its probability of generation. for a forvard neutron bema-ray problen, a neutron of energy IG upon suffering a collision in mediu INDD an generate a secondary gana rey of enerG IGG. For an adjoint gameray neutron problea, a gama ray of energs IG Benerates a neutron of energ IGG.

Called rron: GPATB

Subrcutires called: Gtrato

Punctions used: FLIRIF

Commons required: Blank, LACSIo

Variables required: ISPQRS, IFHGP, MGG, IGSB, (Trom camon IACSIÉ, see page 88 )

IG - incoing eneray group, INED - nediun of collision site as provided by the geonetry nodule.

Variables changed: PGEA, IGr. 
Subrout ine GAMEEN

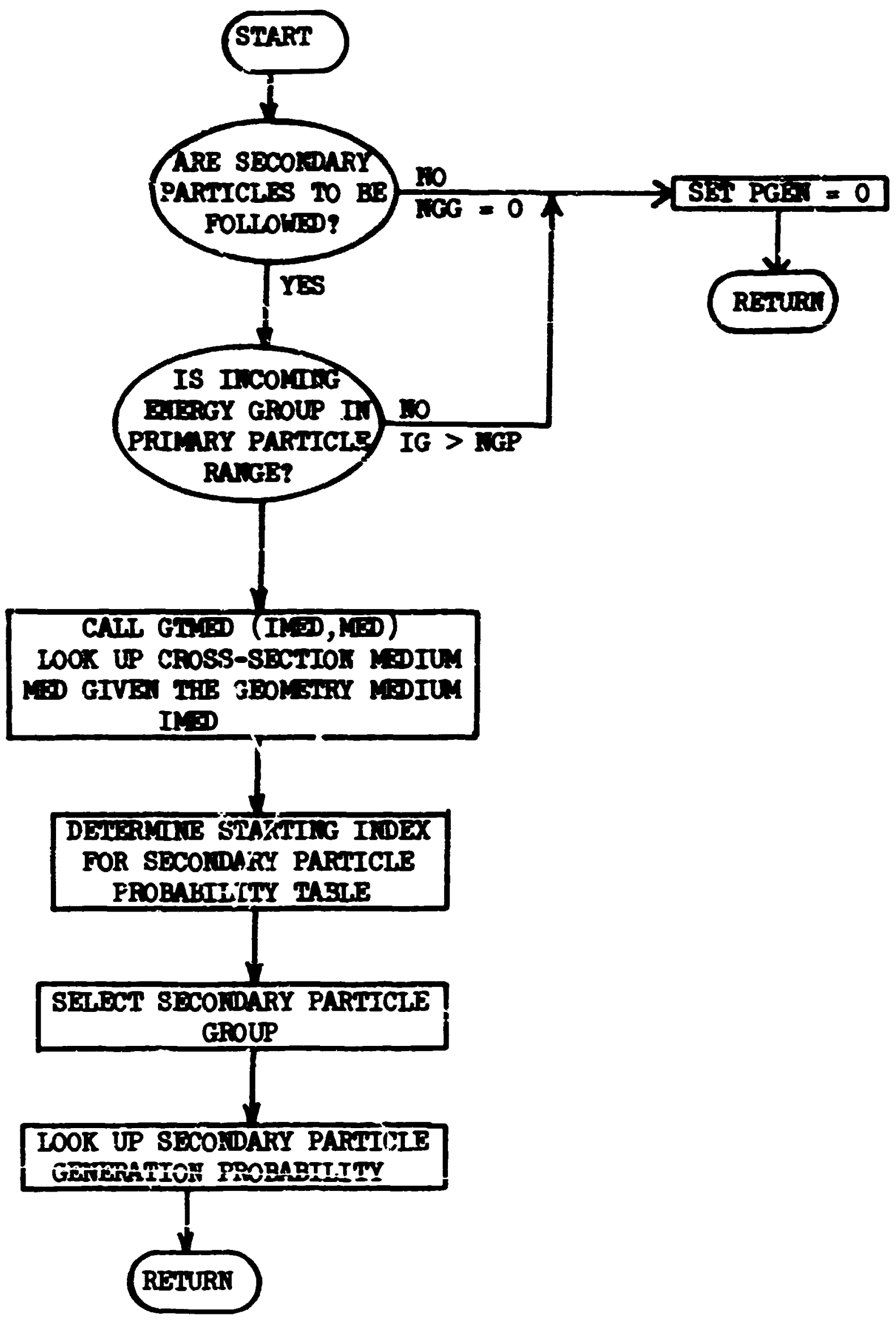




\section{Subroutine cerrus}

This subroutine calculates the quantities $\mu_{i}$ and $\sigma_{i}^{2}$ used in the recurrence ralation for the orthogonal polynowials, $Q_{i}(x)$. It uses as input the nowents, $M_{i}$, of the distribution $f(x)$. GEIMUS also checks to leternine if $\sigma_{1}^{2}>0$. If not, a 198 is set to indicate this.

Let us assume that wity moments are given initially. Then MWM =

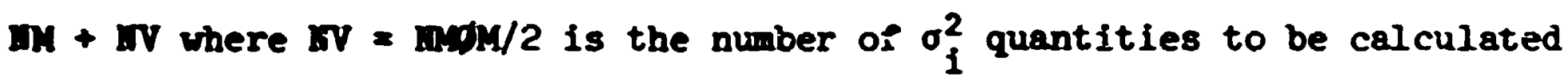
and $M$ is the number of $\mu_{i}$ quentities to be caiculated. $M=M N$ or $M M=$ $\mathbf{N}+1$, depending on whether INWM is even or odd. GETMUS calculates $\mu_{i}=1$, ant and $\sigma_{i}^{2}, 1=1$, av. This is sufficient to determine $Q_{i}(x)$ for $i=0, \mathrm{M}$. If it turns out that some value of $\sigma_{i}^{2}$ is not positive, say $\sigma_{p}^{2} \leq 0$ (this will happen when $n_{p} \leq 0$, a violation of the "nor-aegativity" condition on $f(x)$ ), then the calculation is terminated, a flag is set, and celwus returns with $n=p-1$ and $M X=p$.

The relevant equations are as follows:

The orthogonal polynomials are written

$$
\begin{aligned}
Q_{i}(x) & =\sum_{k=0}^{i} a_{i k} x^{k} \text { yilh } a_{i i}=1 \\
& =\left(x-\mu_{i}\right) Q_{i-1}(x)-\sigma_{i-1}^{2} Q_{i-2}(x) .
\end{aligned}
$$

This lesds tn

$$
a_{i k}=a_{i-1, k-1}-\mu_{i} a_{i-1, k}-\sigma_{i-1}^{2} a_{i-2, k} .
$$

If we define

$$
\begin{aligned}
& \mathrm{v}_{i}=\sum_{k=0}^{i} a_{i k} M_{i+k}, \\
& L_{i}=\sum_{k=0}^{i-1} a_{i-1, k} M_{k+i}, \text { and } \\
& q_{i}=i_{i} / w_{i-1} .
\end{aligned}
$$


Then we have

$$
\begin{aligned}
& u_{i}=q_{i}-q_{i-1}, \text { and } \\
& \sigma_{i}^{2}=N_{i} / N_{i-1} .
\end{aligned}
$$

The calculation proceeds as follows:

Step 1: initial values for quantities for $i=1$ and $\sum$ are set up from explicit formulas from the momerts.

Step 2: set $i=3$.

Step 3: calculate $L_{i}$ from moments and coefficients for $i-1$.

Step 4: caiculate $q_{i}$ from $L_{i}$ and $N_{i-1}$.

Step 5: $\mu_{i}=q_{i}-q_{i-1}$.

Step 6: calculate $a_{i k}, k=0$, i from $k_{i}, \sigma_{i-1}^{2}$, and $a_{i-1, k}$

Step 7: calculate $H_{i}$ from moments and $a_{i, k}$ 's.

Ster 8: $\quad \sigma_{i}^{2}=\mathbb{H}_{i} / \mathbb{N}_{i-1}$.

Step 2: Test $\sigma_{i}^{2}$. If $\sigma_{i}^{2} \leq 0$, terminate the calculation with $n=i-1$ ard set error flag.

Step 10: 1 - iri, return to step 3 .

If Iny is even, the calculetion terminates after step 9 when $1=m / 2$. If $\mathrm{NM}$ M is odd, the calculation terminates at step 5 when $i=(\operatorname{Mis} i / 2)$.

Called from: ANGLES

Commons required: MDLET, MRAIS, QAL, IøCSIG

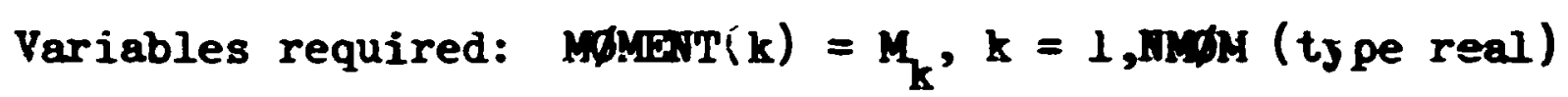

Yariables changed:

$$
\text { IFMU }\left\{\begin{array}{l}
\neq 0 \text { print out } 211 \text { the quantities calculated } \\
\text { by GEMMS } \\
=0 \text { do not print out data except in case } \\
\text { of error }\left(\sigma_{k}^{2} \leq 0\right)
\end{array}\right.
$$

NV - the number of $\sigma^{2}$ 's calculated,

NM - the number of $\mu$ 's calculated,

$M U(I)=\mu_{i}, i=1, \mathbb{M}$ (type real),

$\operatorname{SIG}(I)=\sigma_{i}^{2}, 1=1, \mathbb{N V}$,

$\operatorname{NøRM}(I)=\mathbb{N}_{i}, 1=:, \mathbb{N V}$ (type real). 
Als 0 calculated and put in labelled comiun $Q A L$, although they are not used elsewhere in the program,

$$
\begin{aligned}
& Q(I)=q_{i} \quad i=i, N M \\
& \dot{A}(1, K)=\mu_{i, K-1} \quad i=1, N V ; K=1, i+1 \\
& L(I)=L_{i} \quad i=1, N M .
\end{aligned}
$$

Limitations: $\mathrm{NM} \varnothing \mathrm{M} \leq 27$.

Subroutine GEIMUS

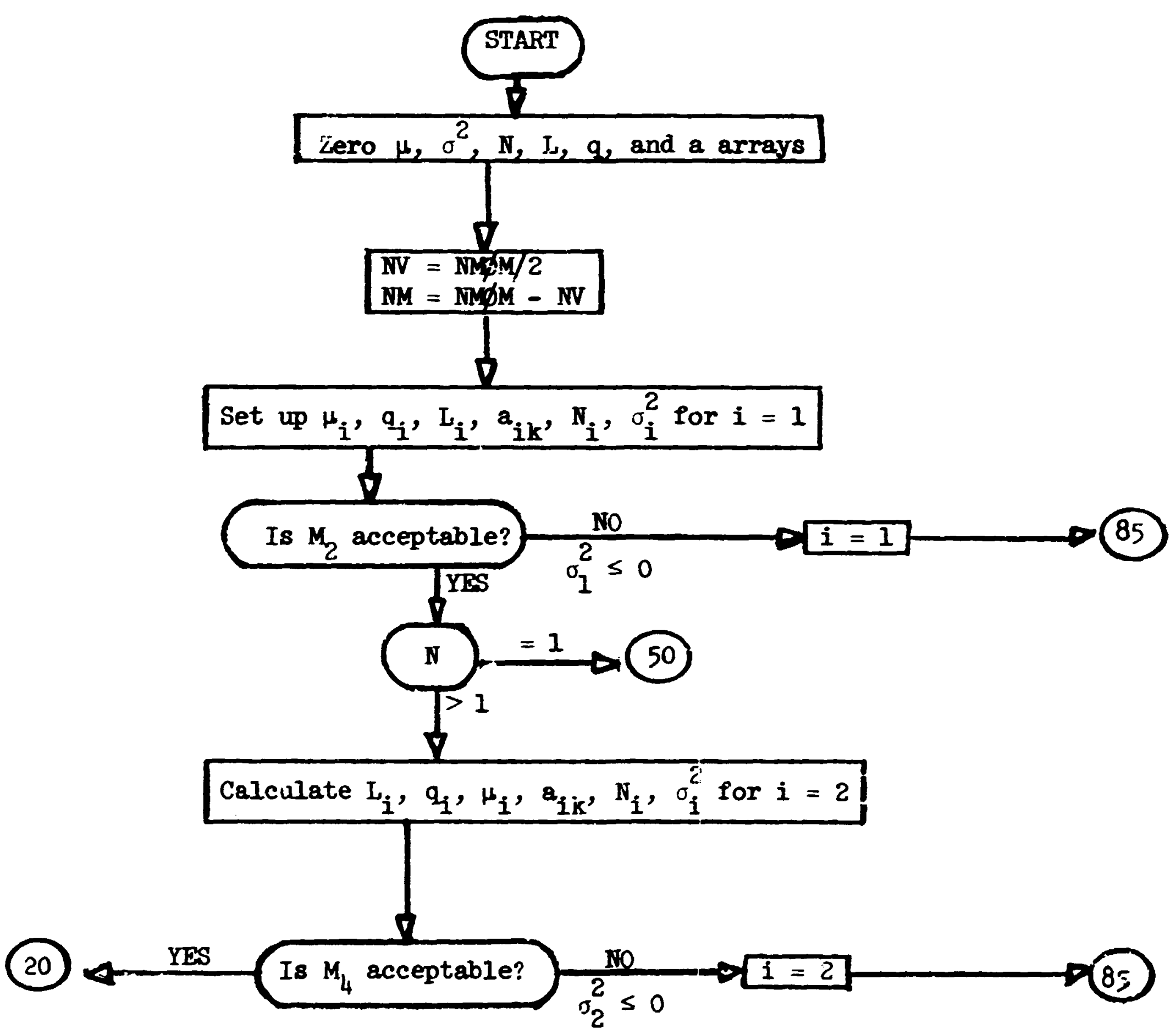



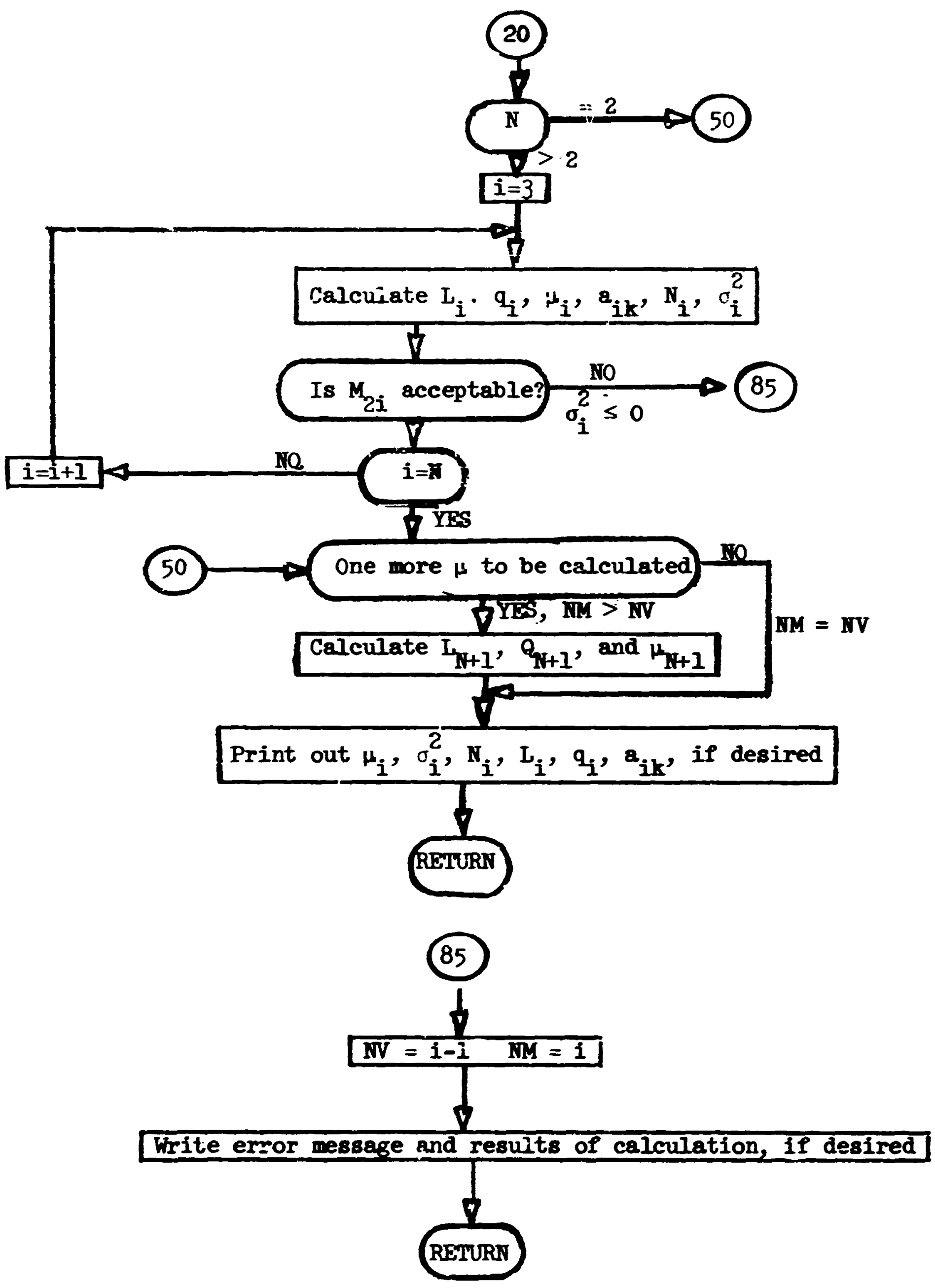
Subrciatine GIIфUT (IS, J, NREG, NDSK, IG, WATE. IND)

This subroutine is called when the selection of the group-to-group transfer is to be biased. Thus, the natural probabilities of scatter from group $I$ to group $J, P(I+J)$, is to be altered by an importance function $V(J)$. Selection of the outgoing group $L$ is made from $P(I \rightarrow J) V(J)$ wilh an associated weight correction of $N /[V(L)]$ where $N=\sum_{J=1}^{N D S K} V(J) P(I \rightarrow J)$.

Called fro 1 : CøLISN

Functions used: FLTRNF

Commons required: Blank

Variables required:

IS - one less than index for within-group scatiering,

NREG - geometrical region of the coliision,

NDSK - number of possible downscatter groups,

IG - incomirg energy group,

WATE - incoming pa ticle weight,

IND - index for the location of importance of within-group scattering.

Variables changed:

$J$ - the number of downscattering groups,

HATE - modified to correct for the biesing.

Significant internal variables:

SBSIG is the normelization $N$ of the biased distribution. 
Sibrcut :ne GTIØUT

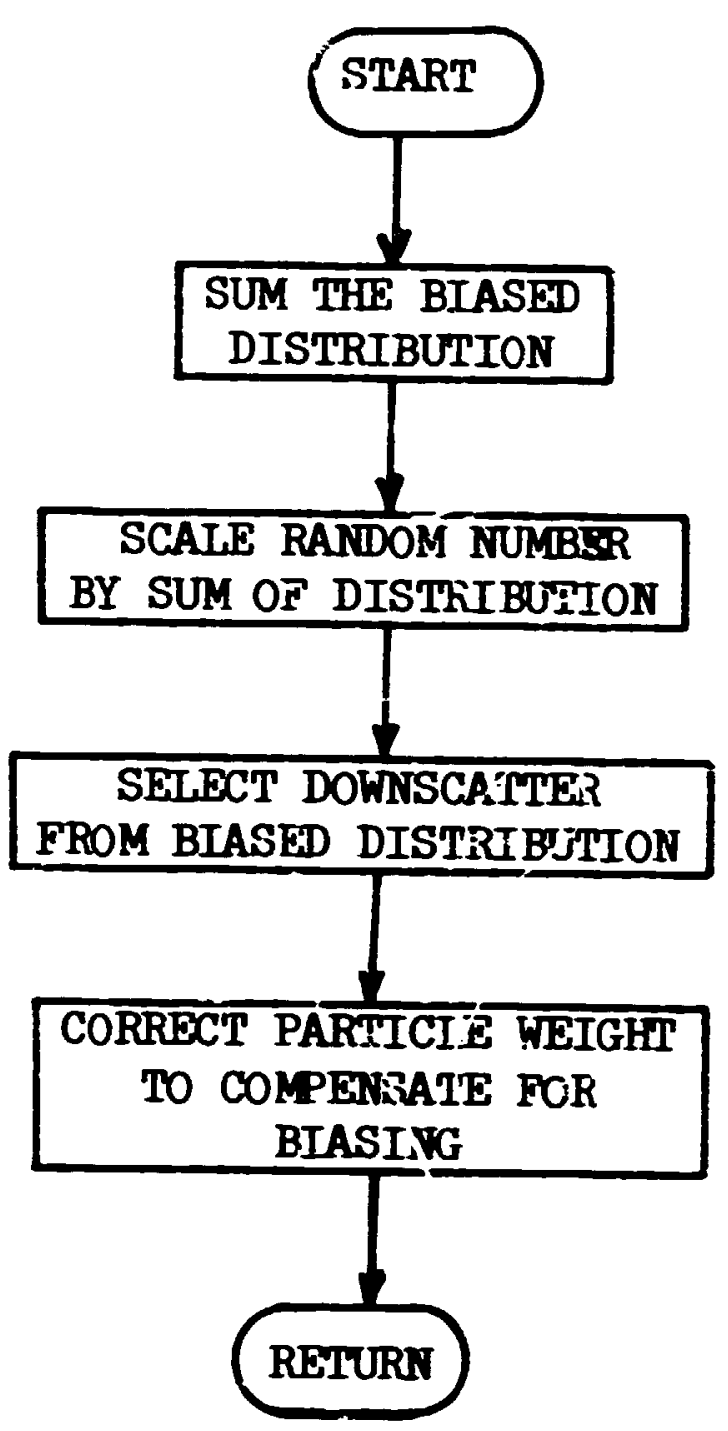




\section{Subroutine JNPUT}

This subroutine is the executive routine for processing the cross sections from the ANISN or DTF-IV formats to the necessary probsibility tables. The major function of this routine is to mix the cross section stored for each element to form media cross sections and tc decompose these cross sections into the individual probability distributions. The Legendre ccefficients for each group-to-group transfer may be restored in a permanent storage area after the discrete angles and probabilities have been

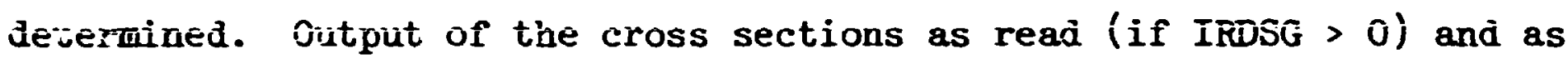
stored (if [PRIN $>0$ ) and the gamma-production cross sections is initiated by this routine. If diagnostic printout of sross-section storage is required a call to XSCHLP ( 1 , 4hJNPT) will give a decimal dump of all ccoss-section storage and commons.

Called from: XSEC

Subroutines called: HEADSG, STQRE, LEGEND, ANGLES

Functions used: IABS (library)

Conmons required: Blank, LфCSIC, MQMENT, MEANS, RESULT

Variabies required: all variables in LDCSIG, see page 88

Variables changed: Blank common from ISTART to MMXSEC.

Input reaà: MIX RH $\emptyset$ times the cross section of the eiement NEL is added NET. to the MIX medium cross section. If HEI is negative, the RH current mixing operation completes the cross section for that medium. There are NMIX of these cards read.

Signilicant internal veriables:

NDSK is the current number of downscatter groups for starting from present location.

Limitations: If cross sections are to be mixed, and then the Legendre coefficients are to be restored, the first element must be mixe.l first. The first element should no: appear in several media since the Legendre coefficients stored for medium 1 may write over the element $i$ cross sections. The seriousness of this limitation is strorgly cependent on the number of groups, coefficients, and elements. 


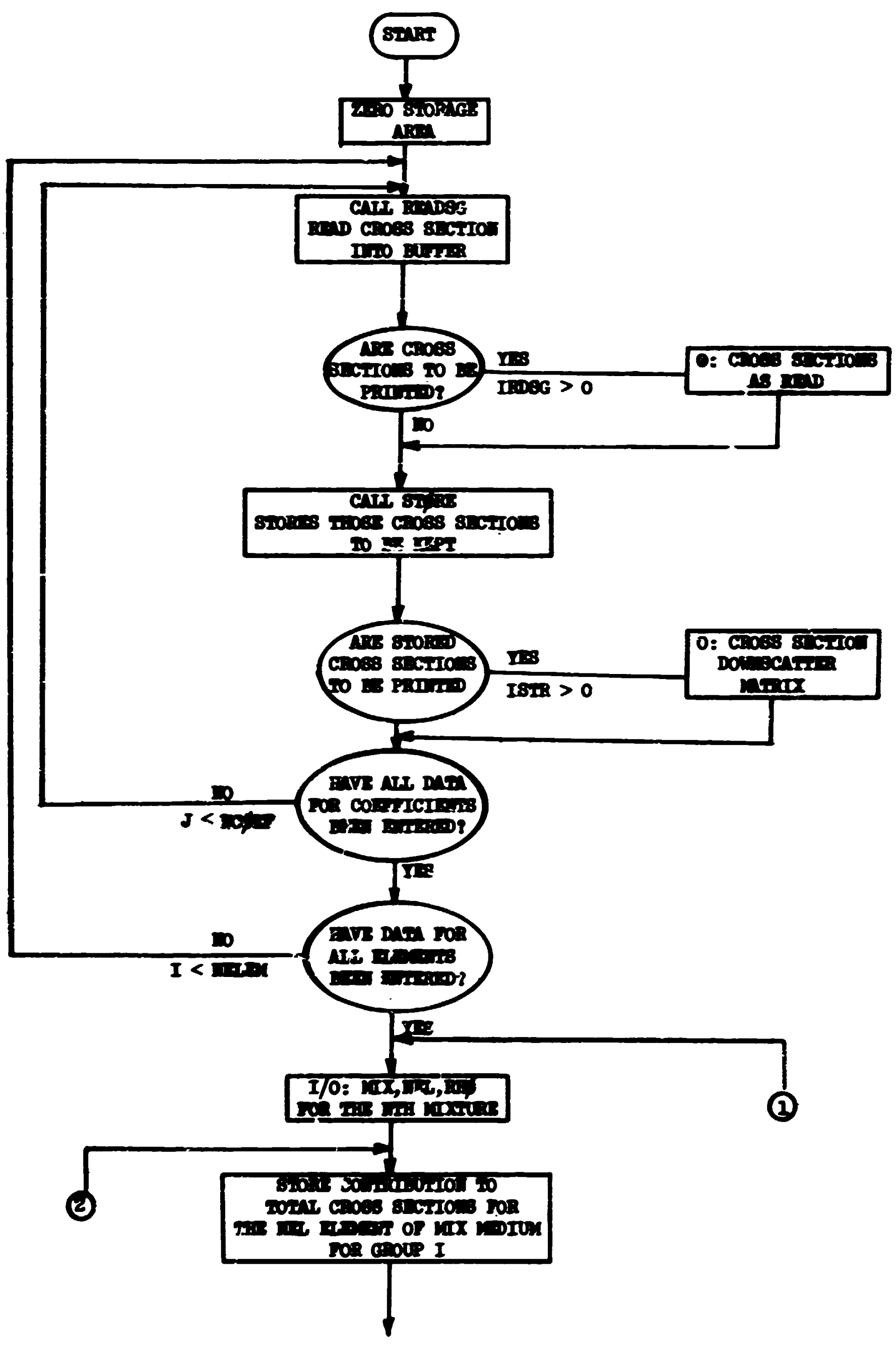




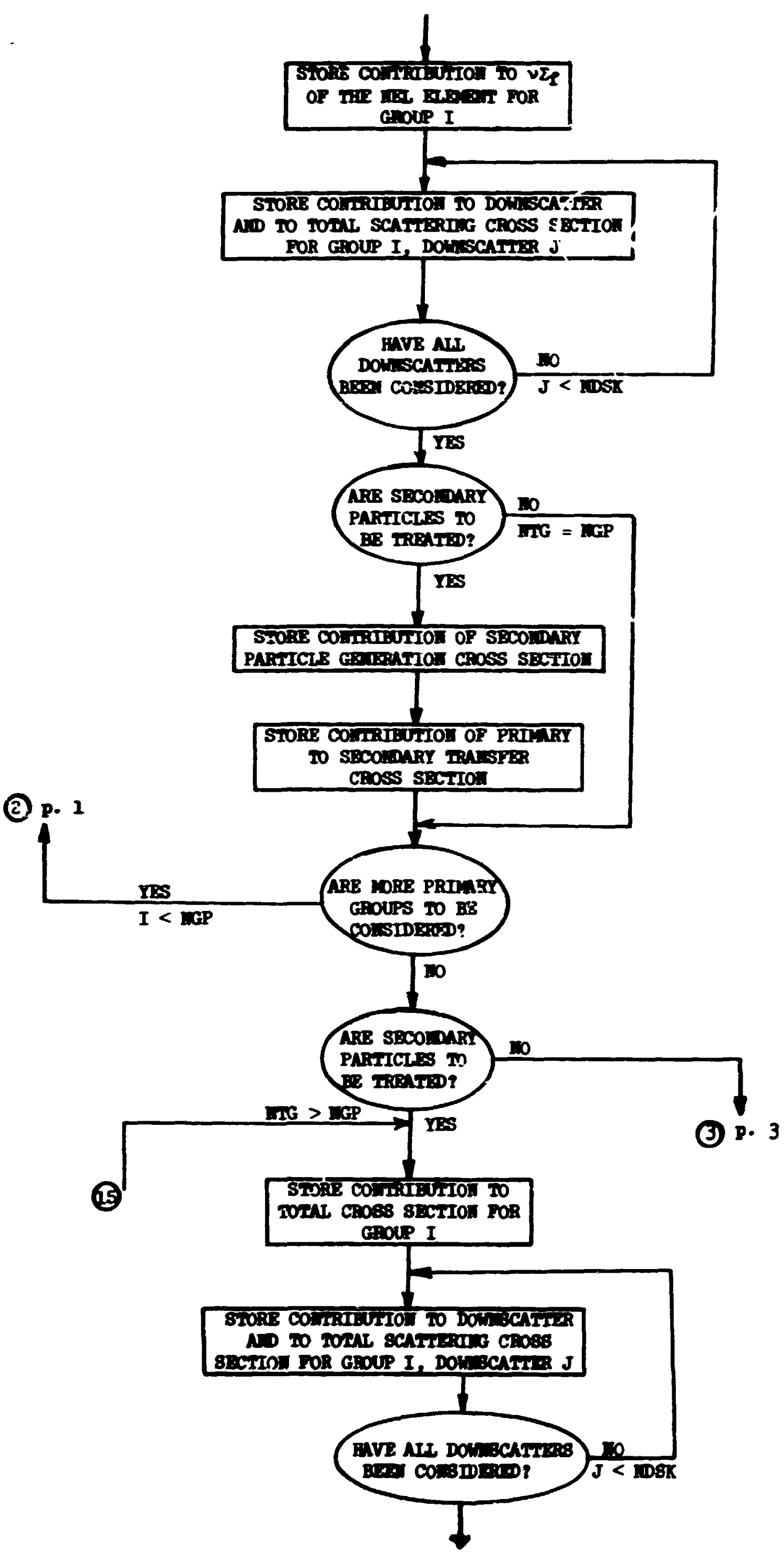




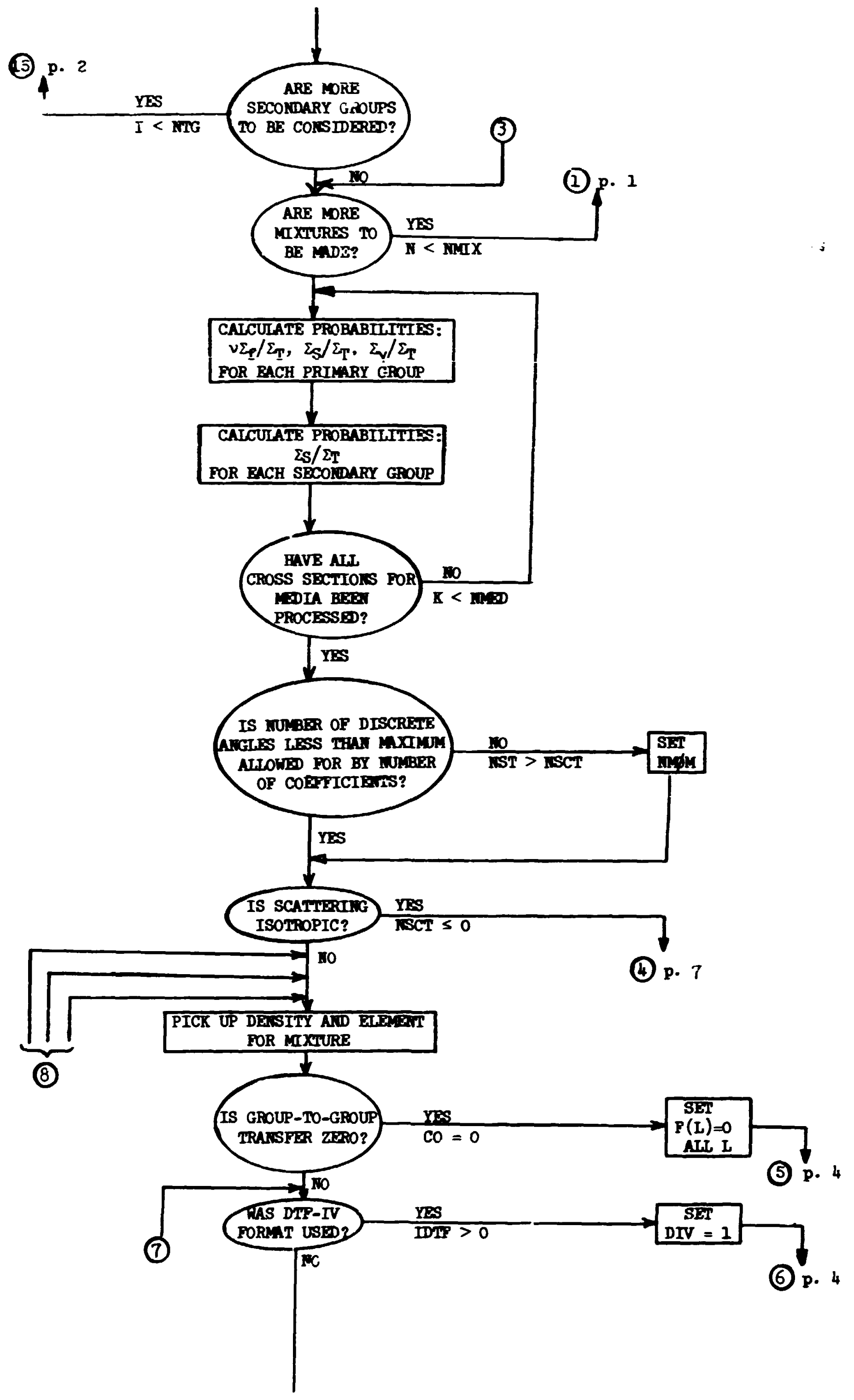




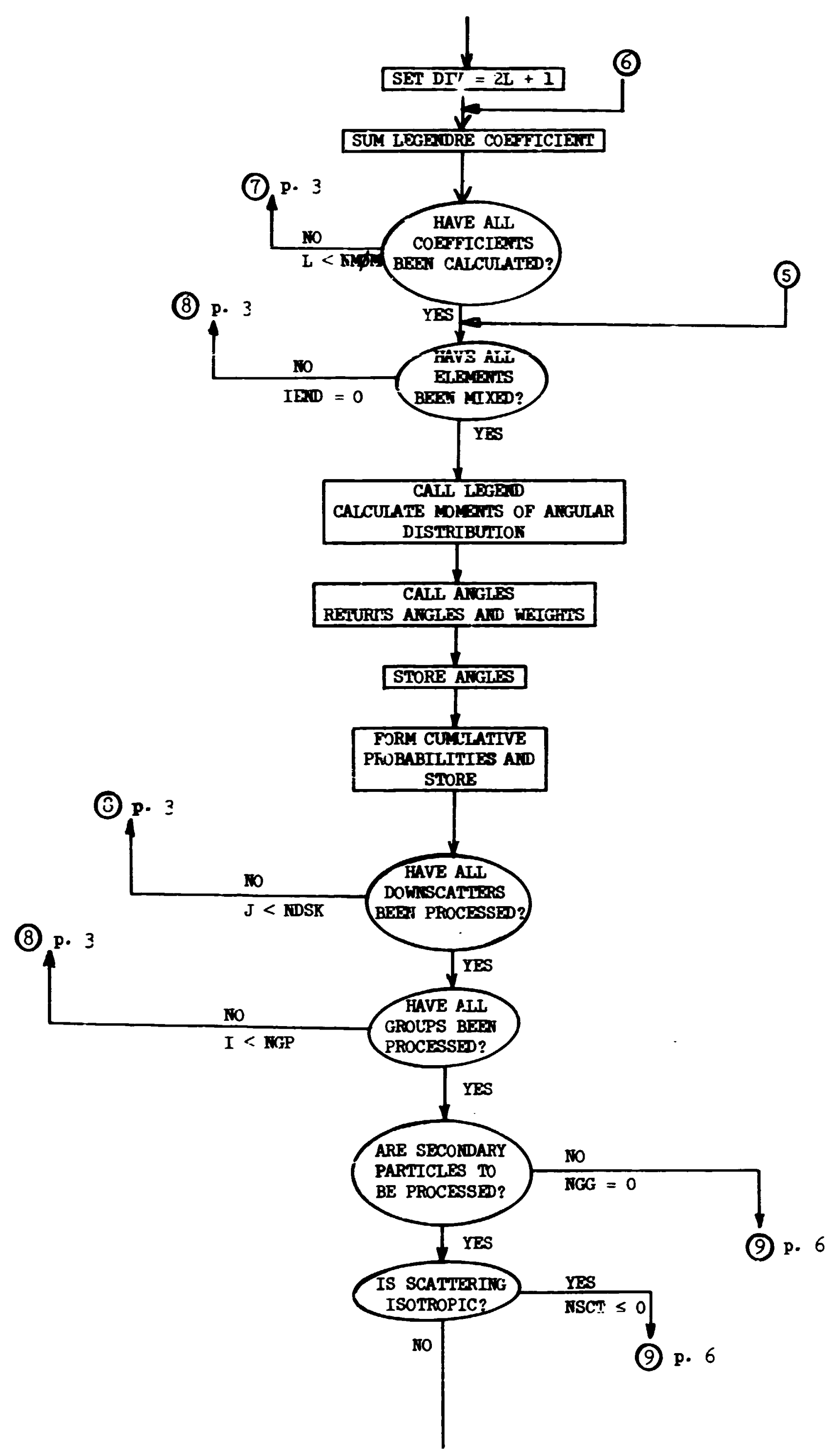




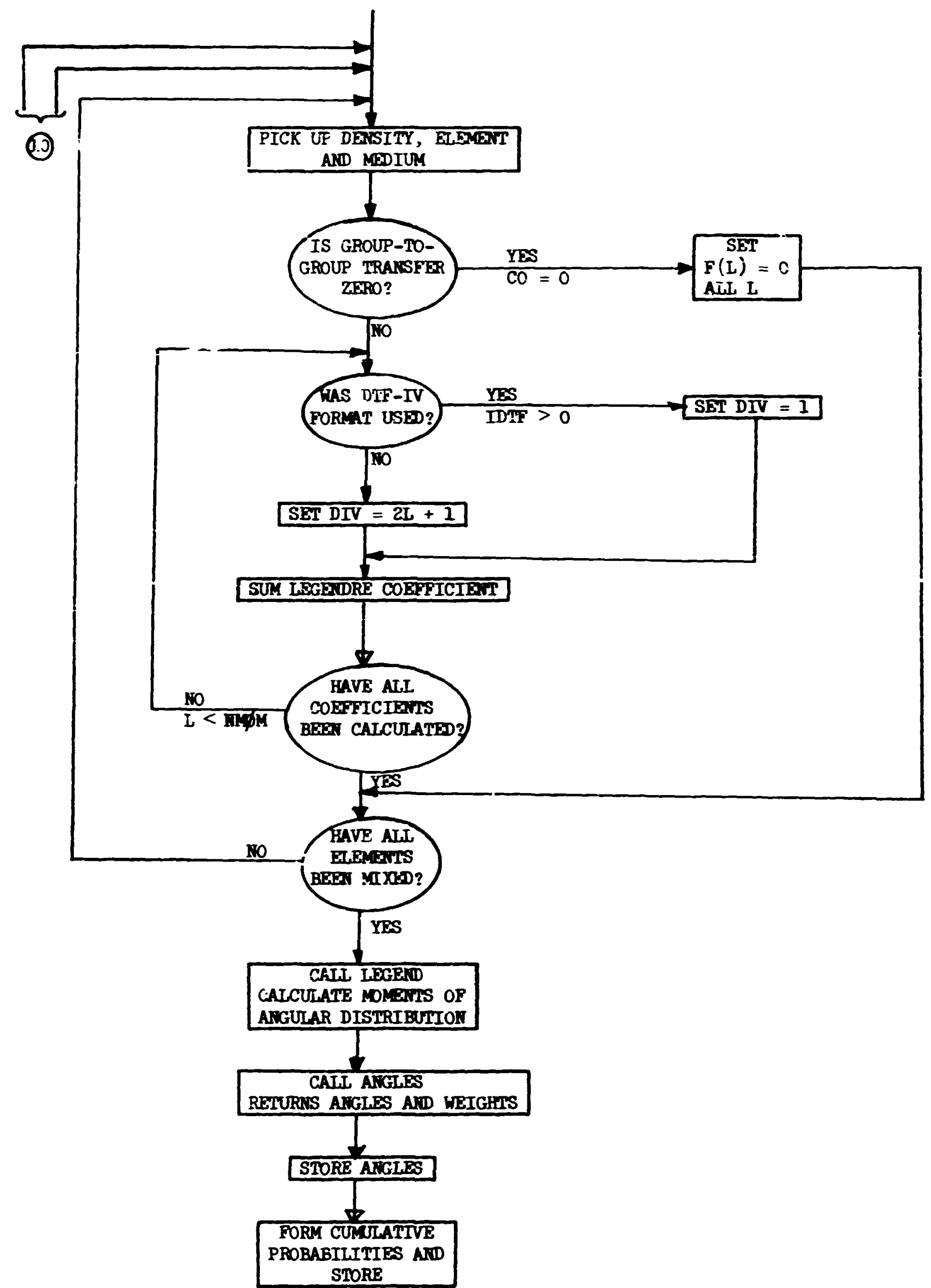




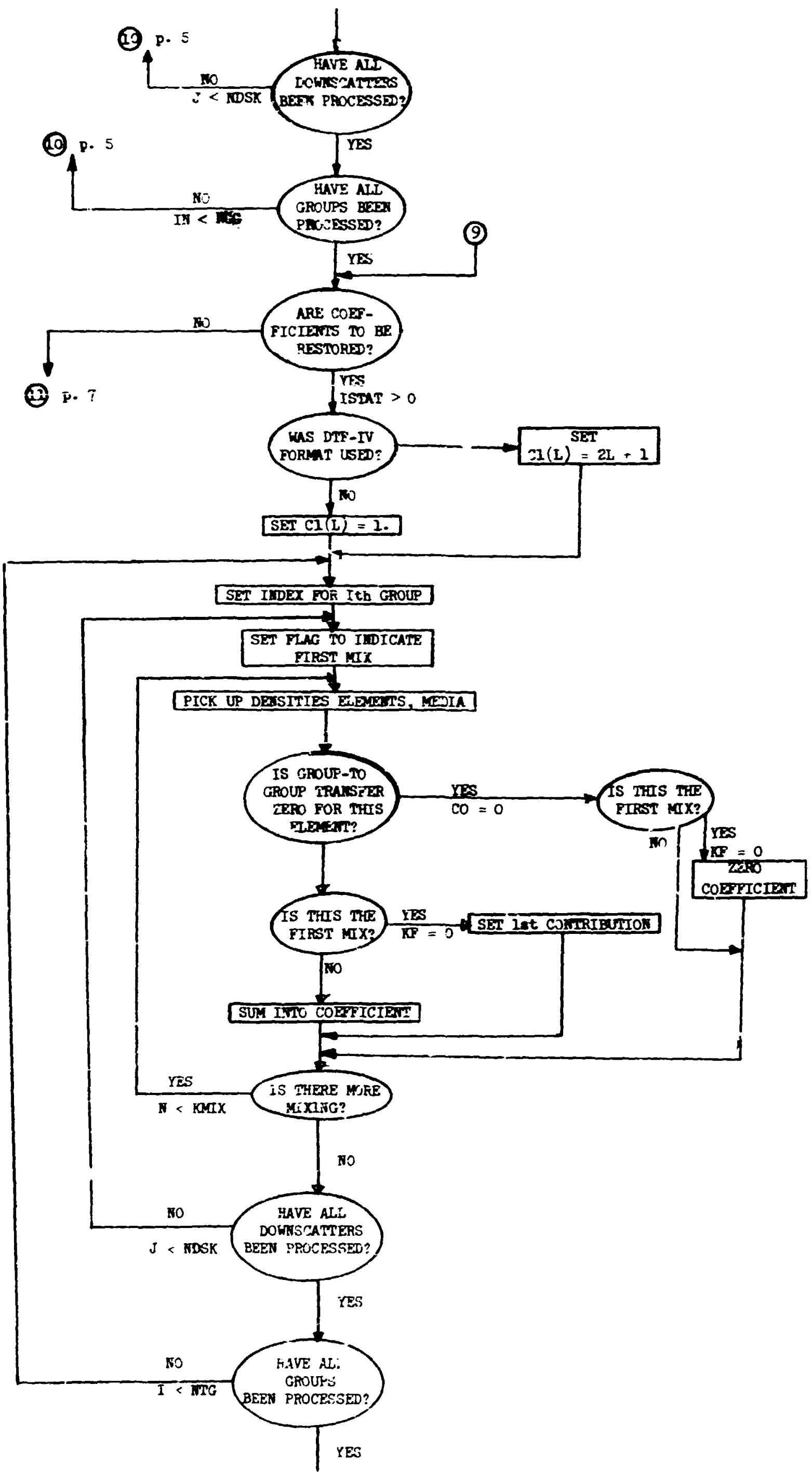


67

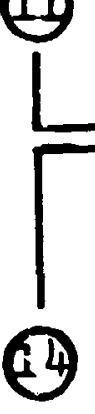

TES

ARE THERE MORE

J2 < NDSK DCWTSCATTERS?

CUMUATIVE DOWNSCATTER

DISTRIBUTION (PRIMARY GPOUP)

ARE THERE

$\mathrm{J}<$ NGP

MORE GROUPS?

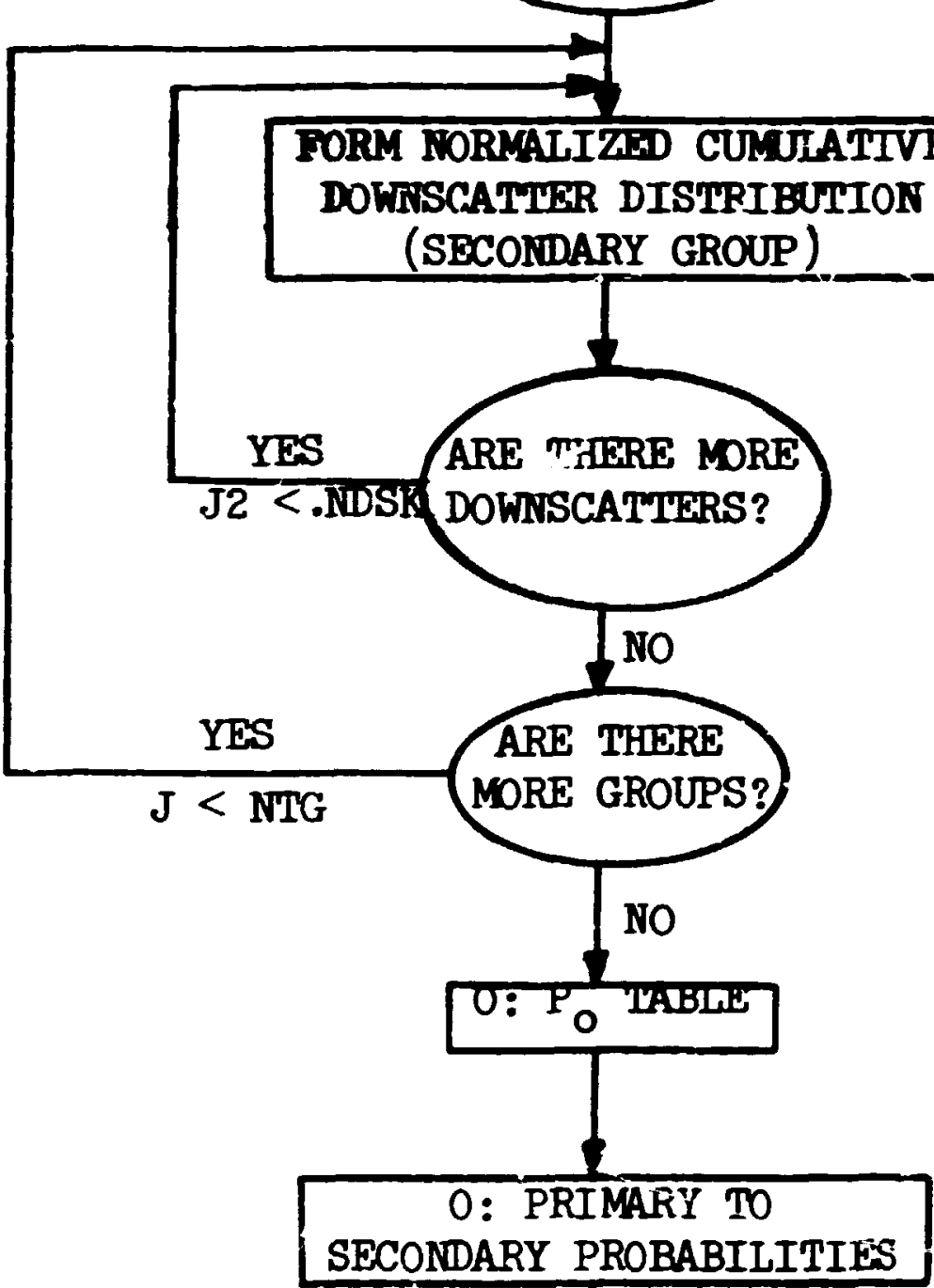




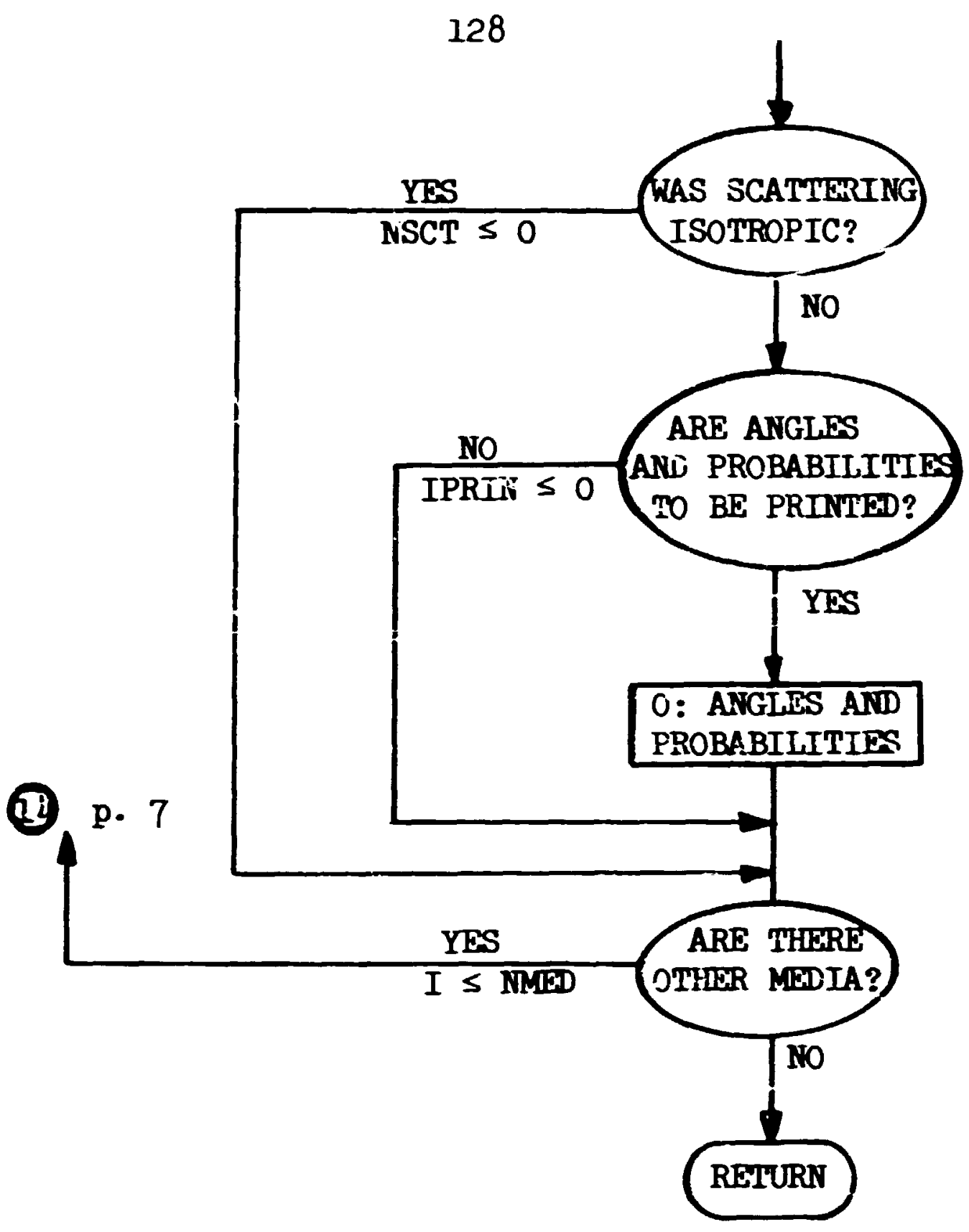




\section{Subroutine LE.GEND}

Subroutine LEGTND converts Legendre coefficients to moments. The ccefficients are given in labelled common MoMENT in the form

$$
f_{\ell}=\int_{-1}^{1} f(\mu) P_{\ell}(\mu) d \mu \ell=I, N F \text { or } f(\mu)=\sum_{\ell=0}^{N F} \frac{2 \ell+1}{2} f_{\ell} P_{\ell}(\mu)\left(f_{0} \equiv 1\right) .
$$

The cutput of LEGEND consists of che moments,

$$
M_{n}=\int_{-1}^{1} \mu^{n} f(\mu) d \mu \quad n=1, N M M \text {. }
$$

Method: If we let

$$
\mathrm{P}_{\mathrm{n}, \ell}^{-1}=\frac{2 \ell+1}{2} \int_{-1}^{1} \mu^{n} \mathrm{P}_{\ell}(\mu) d \mu
$$

Then by using the fundamental recurrence relation for Legendre polynomials we can derive

$$
\begin{aligned}
P_{n, \ell}^{-1} & =\frac{1}{2} \int_{-1}^{1} \mu^{n-1}\left[(2 \ell+1) \mu P_{\ell}(\mu)\right] d \mu \\
& =\frac{1}{2} \int_{-1}^{1} \mu^{n-1}\left[(\ell+1) P_{\ell+1}(\mu)+\ell P_{\ell-1}(\mu)\right] d \mu \\
& =\frac{\ell+1}{2} \int_{-1}^{1} \mu^{n-1} P_{\ell+1}(\mu) d \mu+\frac{\ell}{2} \int_{-1}^{1} \mu^{n-1} P_{\ell-1}(\mu) d \mu \\
& =\frac{\ell+1}{2 \ell+3} P_{n-1, \ell+1}^{-1}+\frac{\ell}{2 \ell-1} P_{n-1, \hat{l}-1}^{-1} .
\end{aligned}
$$

Since we have trivially $\mathrm{F}_{0, \ell}^{-1}=\delta_{O \ell}$ and $\mathrm{P}_{l \ell}^{-1}=\delta_{i l}$, the ccefficients $\mathrm{P}_{\mathrm{nl}}^{-1}$ may Eäsily be computed. Then 


$$
\begin{aligned}
M_{\mathbf{n}} & =\int_{-1}^{1} \mu^{n} f(\mu) d \\
& =\sum_{\ell=0}^{n} \frac{2 \ell+1}{2}+\int_{\ell}^{1} \mu^{n} P_{\ell}(\mu) d \mu \\
& =\sum_{\ell=0}^{n} P_{n \ell}^{-1} f_{\ell} .
\end{aligned}
$$

Called from: JiPUT

\section{Commons required: MAPHENT}

Variables required:

\section{NMDM}

$F(L), L=1, N M D M$ (Fresurably NF $\geq N M \not M$, no checi is made).

Variables changed: XMDMNTíN), $N=1$, NM (XM.

Signiticant internal variables:

$$
\begin{aligned}
& P I(l)=P_{n-1, l}^{-1} \\
& P 2(l)=F_{n, l}^{-1} \\
& P 10=P_{n-1,0}^{-1} \\
& P 20=P_{n, l}^{-1} .
\end{aligned}
$$

Limitations: $N M \varnothing_{M} \leq 24$. 
Subroutine IBesp

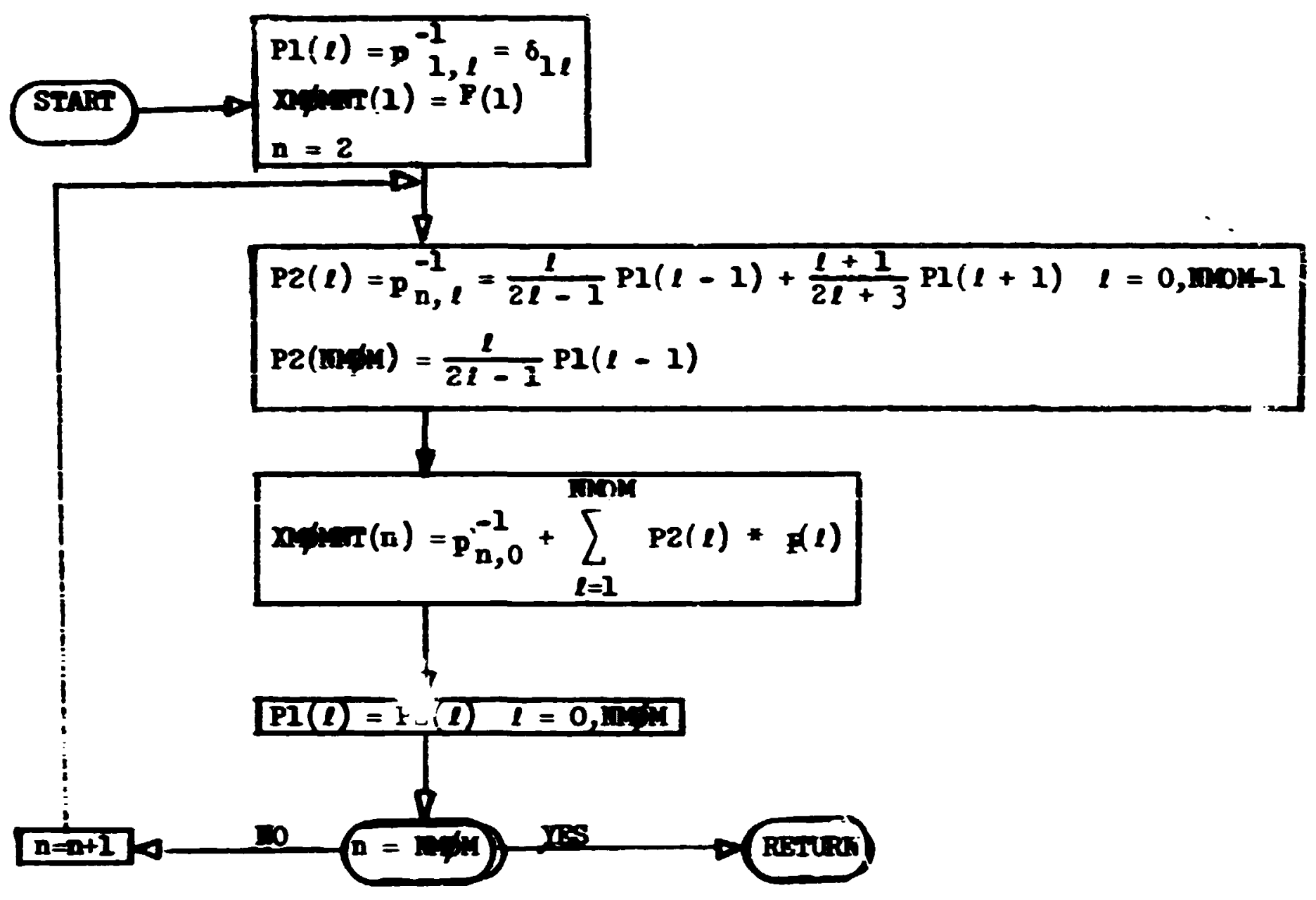

Wote: Recursion relation term involving zeroth order coefficiente ant be barided eqpartely. PIO is PI(0), etc. 


\section{Subroutine MAMENT (NMф)}

This routine converts moments to Legendre oeficients. The moments

$$
M_{n}=\int_{-1}^{1} \mu^{n} f(\mu) d \mu n=1, N M \psi
$$

are given in labelled common $\$$ MENT. The output of the subroutine consists of the sane number of Legendre coefficients stored in labelled common MqMaNT.

Method: $f_{\ell}=\int_{-1}^{i} P_{\ell}(\mu) f(\mu) d \mu$

$$
\begin{aligned}
& =\sum_{n=0}^{i} P_{e, n} \int_{-1}^{I} \mu^{n} f(\mu) d \mu \\
& =\sum_{n=c}^{2} P_{\ell, n} M_{n},
\end{aligned}
$$

where the $\mathrm{P}_{\ell, n}$ are the ccefficients of the $\ell$ th Legendre polynomial,

$$
P_{\ell}(\mu)=\sum_{n=0}^{\ell} P_{\ell, n} \mu^{n} .
$$

Since

$$
\begin{gathered}
P_{\ell}(l)=\frac{(2 \ell-1) \mu P_{\ell-1}(\mu)-(\ell-1) P_{\ell-2}(\mu)}{\ell}, \\
\sum_{n=0}^{\ell} P_{\ell, n} \mu^{n}=\left(\frac{2 \ell-1}{\ell}\right)_{n=0}^{\ell-1} P_{\ell-1, n} \mu^{n+1}-\left(\frac{\ell-1}{\ell}\right)_{n=C}^{\ell-2} P_{\ell-2, n} \mu^{n^{2}} .
\end{gathered}
$$

As this is an identity, we may separately equate the coefficients of each power of $\mu$ giving the reiation

$$
\mathrm{P}_{\ell, \mathrm{n}}=\left(\frac{2 \ell-1}{\ell}\right) \mathrm{P}_{\ell-1, \mathrm{n}-1}-\left(\frac{\ell-1}{\ell}\right) \mathrm{P}_{\ell-2, \mathrm{n}} \text {. }
$$

Since

$$
\begin{aligned}
& P_{0}^{(\mu)}=1 \text { and } P_{1}(\mu)=\mu, \text { we have } \\
& P_{0, n}=\delta_{\text {On }} \text { and } P_{1, n}=\delta_{1 n} .
\end{aligned}
$$


Called from: BADMPY

Commons required: ImpMENT

Variables required: $\operatorname{MM} \varnothing,(\operatorname{MDMN}(\mathrm{N}), \mathrm{N}=1, \mathrm{MM} \phi)$

Variables changed: ( $F(L), I=I, N M \phi)$

Significant internal variables:

$$
\begin{aligned}
& \mathrm{PO}(\mathrm{n})=\mathrm{P}_{\ell-2, n} \\
& P I(n)=P_{\ell-1, n} \\
& \mathrm{P} 2(\mathrm{n})=\mathrm{P}_{\text {ln }} \\
& \mathrm{P} 00=\mathrm{P}_{\ell-2,0} \\
& \mathrm{P} 10=\mathrm{P}_{\ell-1,0} \\
& \mathrm{P} 20=\mathrm{P}_{\ell 0}
\end{aligned}
$$

imitations: $\mathbf{N M} \leq 25$. 


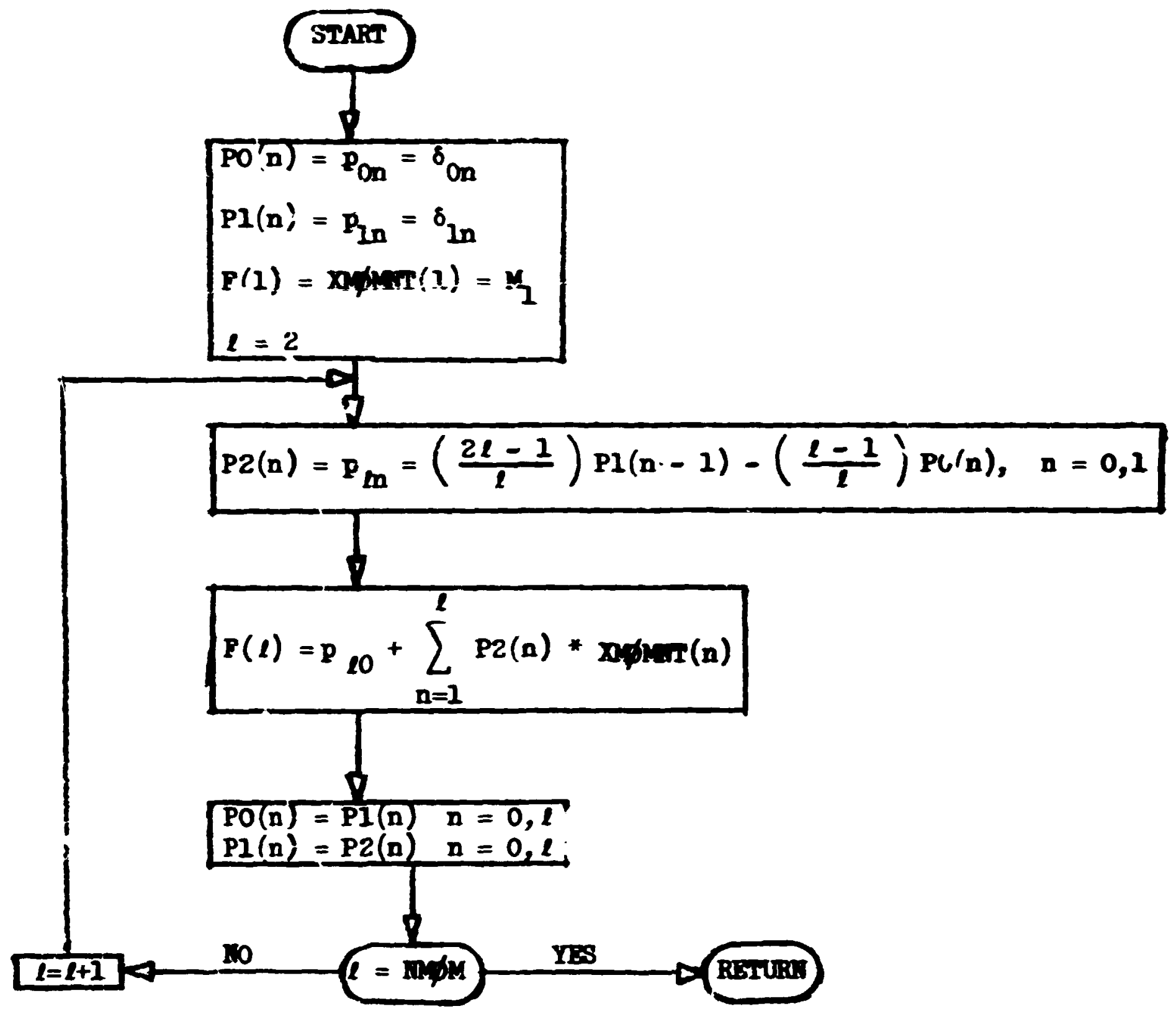

Note: Recureion relations involving zeroth order coerficients ant be bandled separately. POO is PO(0), etc. 
Subroutine NSIGTA (IGA, JMED, TSIG, PNAB)

'the function of this subroutine is to look up the total cross section and non-absorption probabilicy for energy group IGA and geometry medium JMED.

salled from: EUCLID, NXTC $\varnothing \mathrm{L}$, User routines

Subroutines called: GTMED

Commons required: Blank, EфSSIG

Variables required:

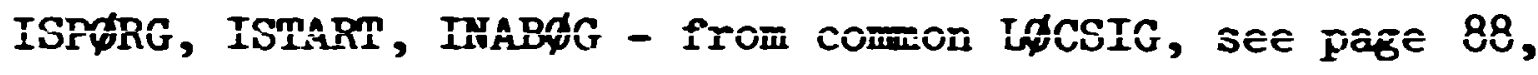

IGA - energy group,

IMED - gecmetry medium.

Variables changed:

TSIG - total cross section,

PNAB - non-absorption probability.

Subroutine NSIGTA

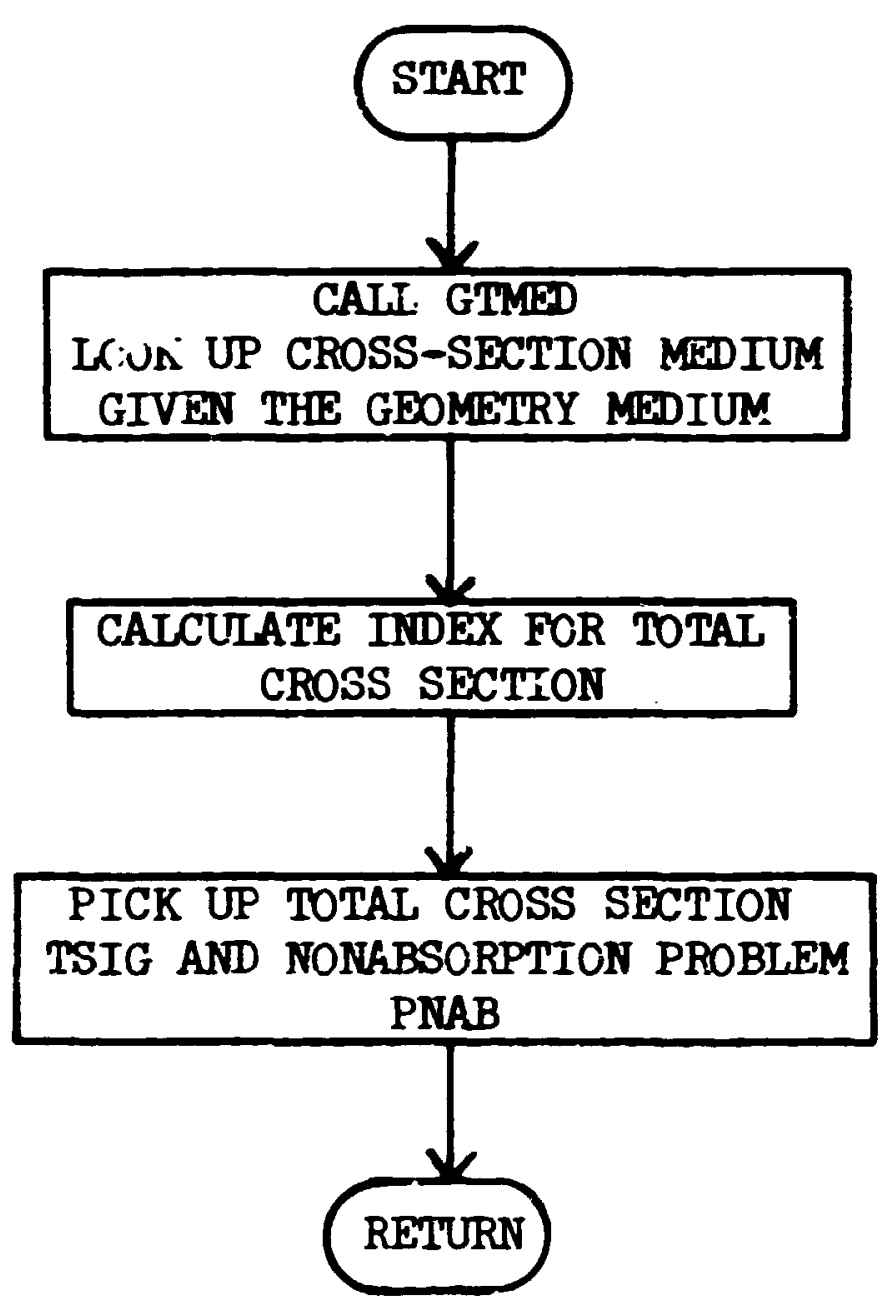


Subroutine PTHETA (IMED, IGQLD, IGQ, THETA, PMU, MMTS)

This routine calculates the probability per steradian of scattering through an angle whose cosine is THETA for an energy transfer from group IG $\varnothing L D$ to other grcups. Use is made of the restored Legendre coefficients with the group-to-group transfer incorporated. Thus, evaluation of

$$
P^{I \rightarrow J}(\theta)=\frac{S}{4 \pi}\left(I+\sum_{\ell=1}^{I+J}(2 \ell+1) f_{\ell}^{I \rightarrow J} P_{\ell}(\theta)\right.
$$

where $P_{S}^{I \rightarrow J}$ is the probebility of scattering from group $I$ to group $J$, $f_{\ell}^{I \rightarrow I}$ is the $\ell$ th Legendre coefficient for scattering from group I to group J,

$P_{\ell}(\theta)$ is the value of the $\ell$ th Legendre polynomial for an aigle whose cosine is $\theta$.

There are NCØEF-1 coefficients restored by JNPUT; i.e., the $\mathrm{P}_{0}$ table is not restored.

It is assumed that within-group scattering is not zero and is calculated for each entry. An option is prcvided for calculating the probability of scattering to all other groups or to a set number of downscaiter groups.

The following recursion relation is used for caiculating the Legendre polynomial:

$$
\text { L } P_{L}(x)=(2 L-1) \times P_{L-1}(x)-(L-1) P_{L-2}(x)
$$

Called from: User routines only.

Subroutines called: GTME!, XSCHLP

Commons required: Blank, LøCSIG

Variables required:

IMED - geometry medium,

IGDLD - the incoming energy group, 
IGQ - the limit of the downscatter for wiich $P(\theta)$ is calculated. That is, $P(\theta)$ is determined for group Io $\varnothing L D$ to IGQ. If IGQ is zero full downscatter is assimed and $P(\theta)$ is determined for IGøLD to NGP,

THETA - sosine of the scattering angle.

Variables required:

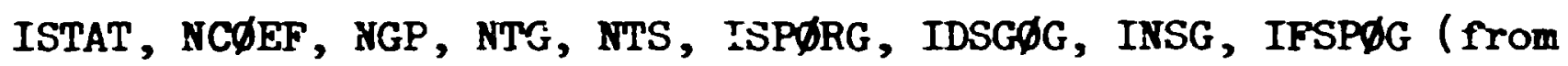
common LøCSIG, ses page 88;

Variajles chamsed:

PMU - the probability of scittering through an argle whose cosine is $\theta$; $P M U$ is dimensioned by IMTG,

NMTG - the total number groups to be considered in the problem.

Significant internal variables:

$P(K)$ - Legendre polynomial of order $K$ evaluated at $\theta$.

Limitations: dimension of 10 for Legendre coefricients. A change in this dimension will allow higher crüer of expansions. 


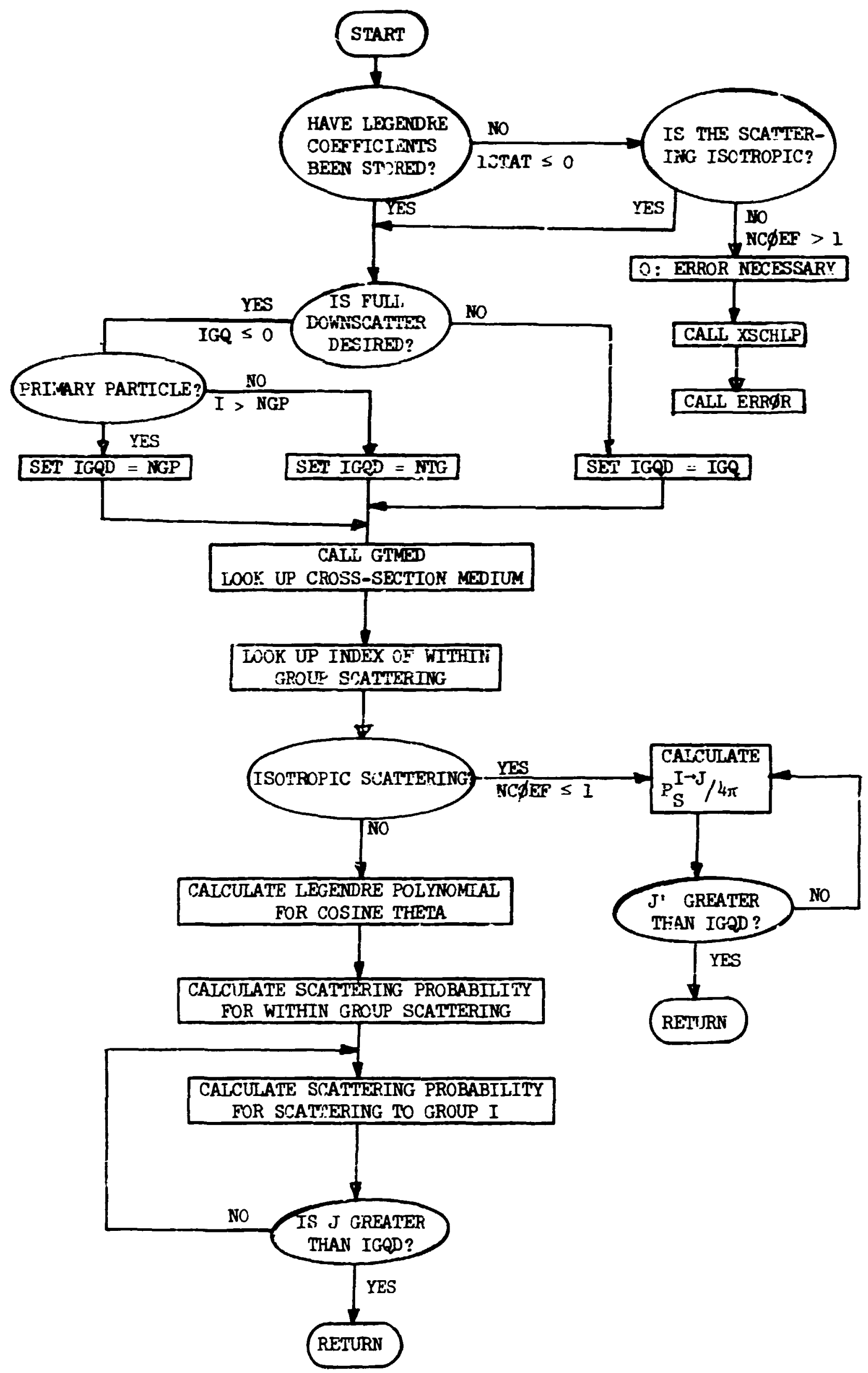


Fingetiun $Q(\underline{H D}, X)$

This function subprogram generates $Q_{N D}(X)$ - the value at $X$ of the orthogonal polynomial, $Q$, of order ND. The recurrence relation for the $Q$ pciynomials is employed to generate the function

$$
\begin{aligned}
& Q_{i}(x)=\left(x-\mu_{i}\right) Q_{i-1}(x)-\sigma_{i-1}^{2} Q_{i-2}(x) \\
& Q_{0}(x)=1 . \\
& Q_{1}(x)=: 6-\mu_{1} .
\end{aligned}
$$

Called from: ANGLES, FIND, BADM

Commons required: MEANS

Variables required:

ND - the degree of the polynomial desired,

$X$ - value of the argument desired,

$\left.\begin{array}{l}\operatorname{XMU}(i)=\mu_{i} \\ \operatorname{VAR}(i)=\sigma_{i}^{2}\end{array}\right\} \quad$ in labeil $\in d$ common MEANS

Variables changed:

$Q$ - the value of the function.

Limitations: $\mathrm{ND} \leq 14$. 
Function $\mathrm{Q}, \mathrm{ND}, \mathrm{X}$ )

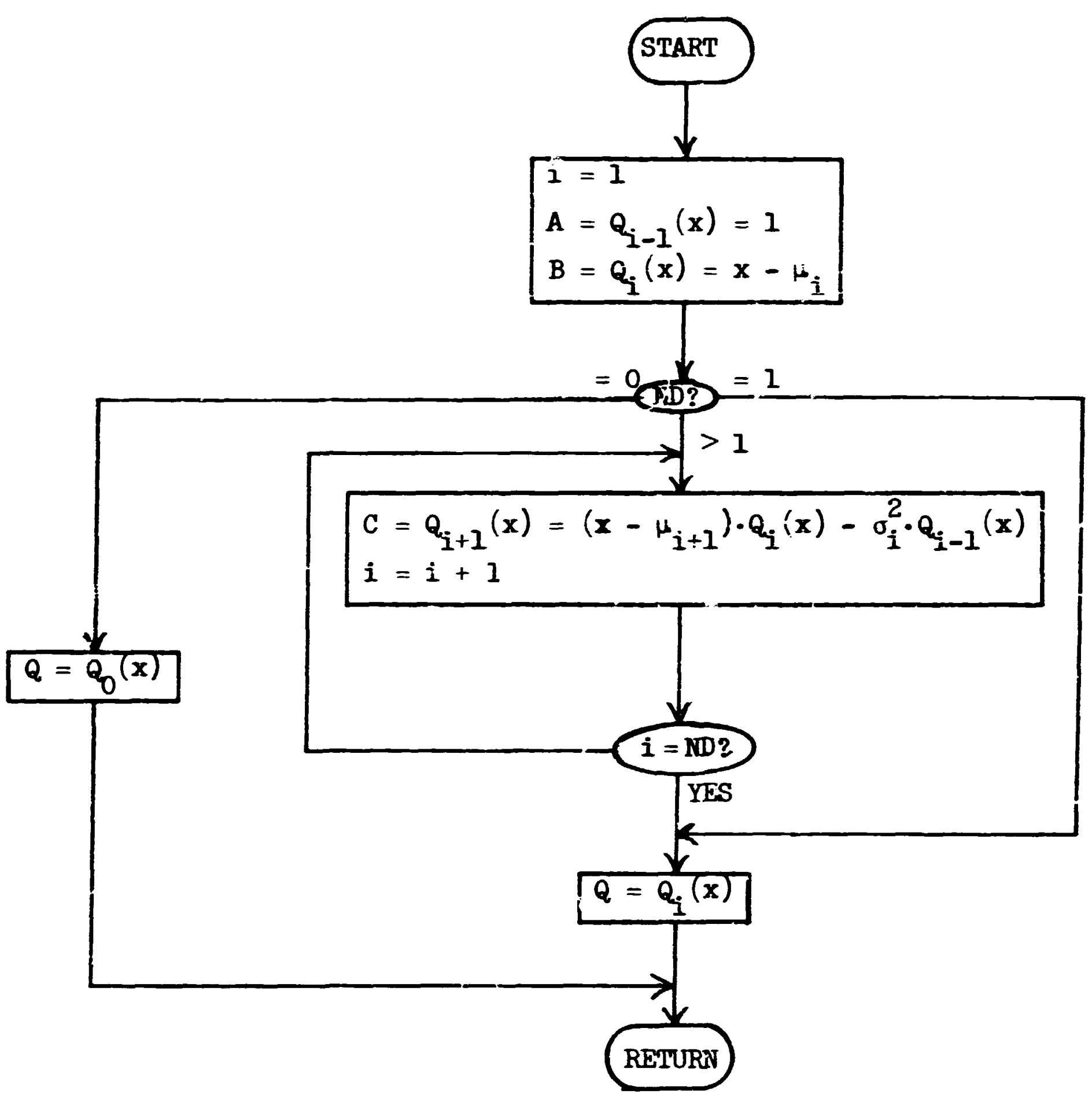




\section{Subroutine READSG}

The purpose of this routine is to read multigroup cross sections and store them in a buffer region of common. If the flag IDTF is greaiei than zero, DTF-IV fursat sross sections may be read; otherwise, the ARISE fcrmat is assumed.

The ANISI cross-section format makes use of the repeat feature; thus, there is a mixture of Hollerith and numbers on the card. This subroutine will therefore be different for various compuiers. On IBM machines each cross-section card is read twice; once for the Hollerith $R$ and once for the eross-section values. For CDC machines decode may be used to separate the Hollerith. DTF-IV format does not permit repeats, and thus the subroutine reads the card numbers directly into the buffer storage region starting at IIPBUF.

If cross sections are read from cards, in the AllSI iurmat, a card sequence check is performed. Three possibilities are taken into account: (1) an energy group number in columns 73-76 and a sequence number for that group in columns 77-80 (format from codes such as GAM or MUG); (2) same as (1) except columns $73-76$ are blank (rormat from codes such as SUPERTOG or XSDRN); (3) solumns 73-76 are blank and colurns 77-80 contain a card sequence number starting at 1 for each set of cross sections (format from AHIST). Non-numeric characters, including blanks, may precede or follow tha sibove sequence numbers without affecting the checks.

If a card is out of order, the card image is printed and the program continues. This test may be removed by setting IRDSG negative. (This also removes the option of printing the cross section as read.)

If IXTAPE $>0$ then cross sections are read from a standard AIIIGI binary cross-section tape. An ideutification record $(4 I 6,6 \mathrm{~A} 8)$ precedes the cross section for each :oefficient. The desired cross section must be required in the order in which they are on tape, and the element identifiers must be the fourth integer in $^{\mathrm{n}}$ the identification record. These identification numbers are required on input card $D$ of the cross-3ection input. 
Called from: JNPUT

Subroutines called:

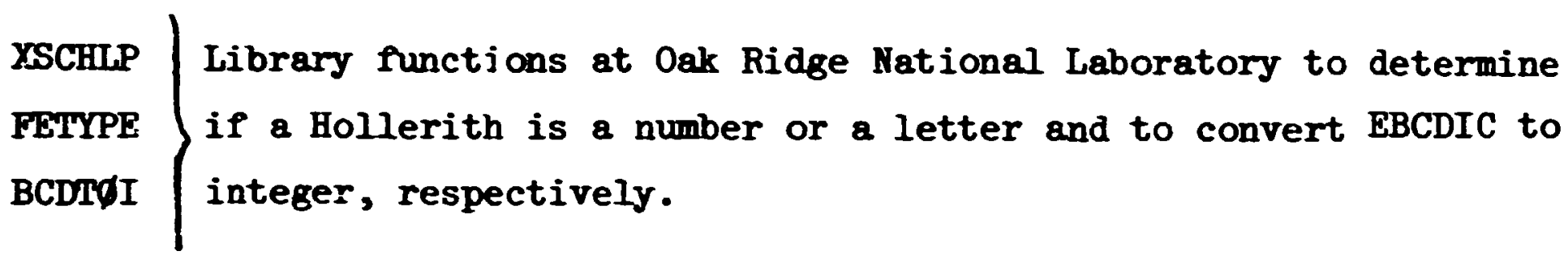

Commons required: Bienk, IACsiG

Variables required: IMPBUP, INGP, INDS, KKK, IXTAPE, IDTF ( from common LACSIG, see page 88)

Input: (IrGPe(INDS+3)) vaiues of cross sections for each call.

Significant internal variables:

$$
\begin{aligned}
& M \text { - number of cross sections for each coefficient, } \\
& \text { IP - number of repeats for a particular cross section. }
\end{aligned}
$$

Limitations: Card formats must be either AIISH or DTF-IV, or a binary tape may be used. 


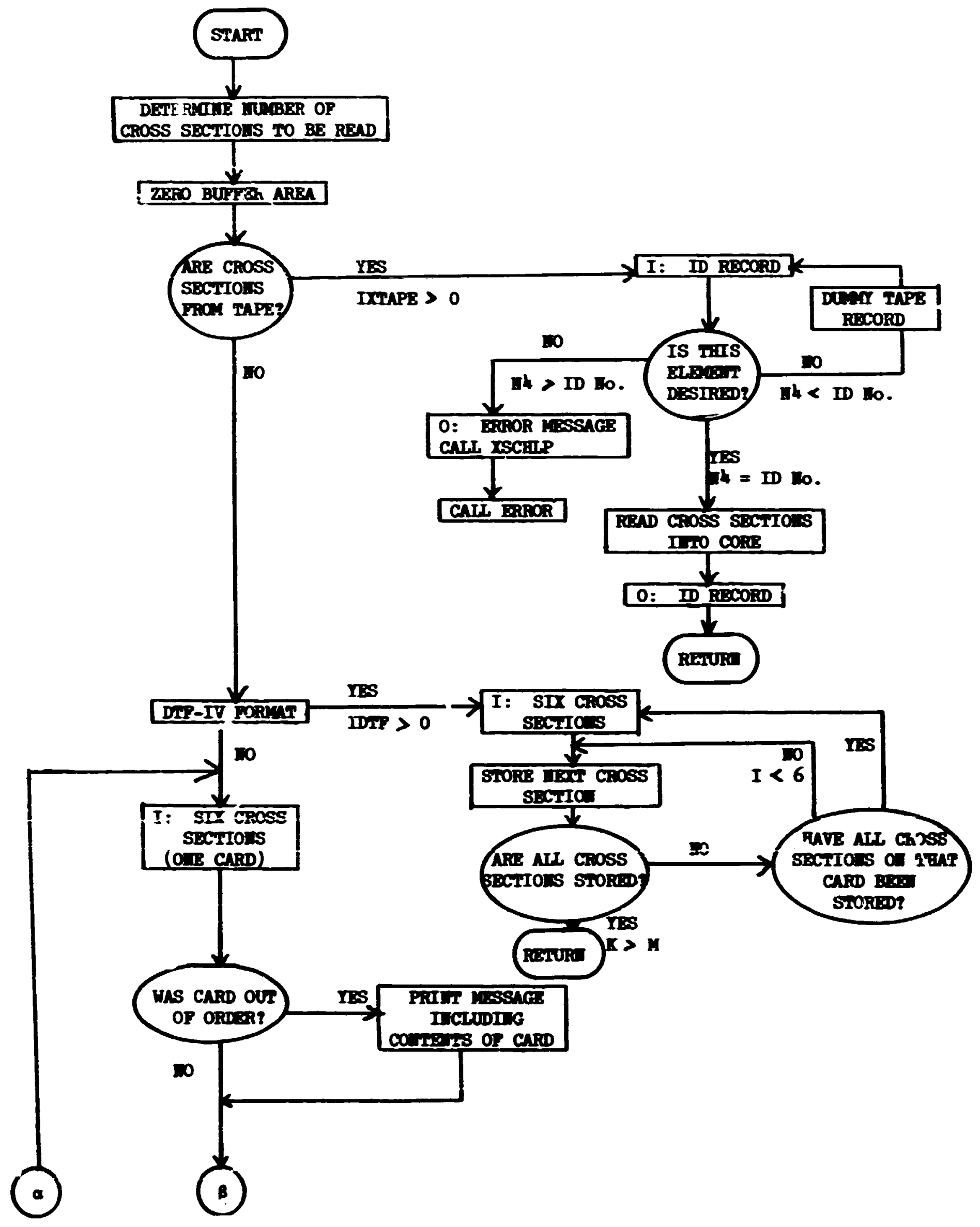




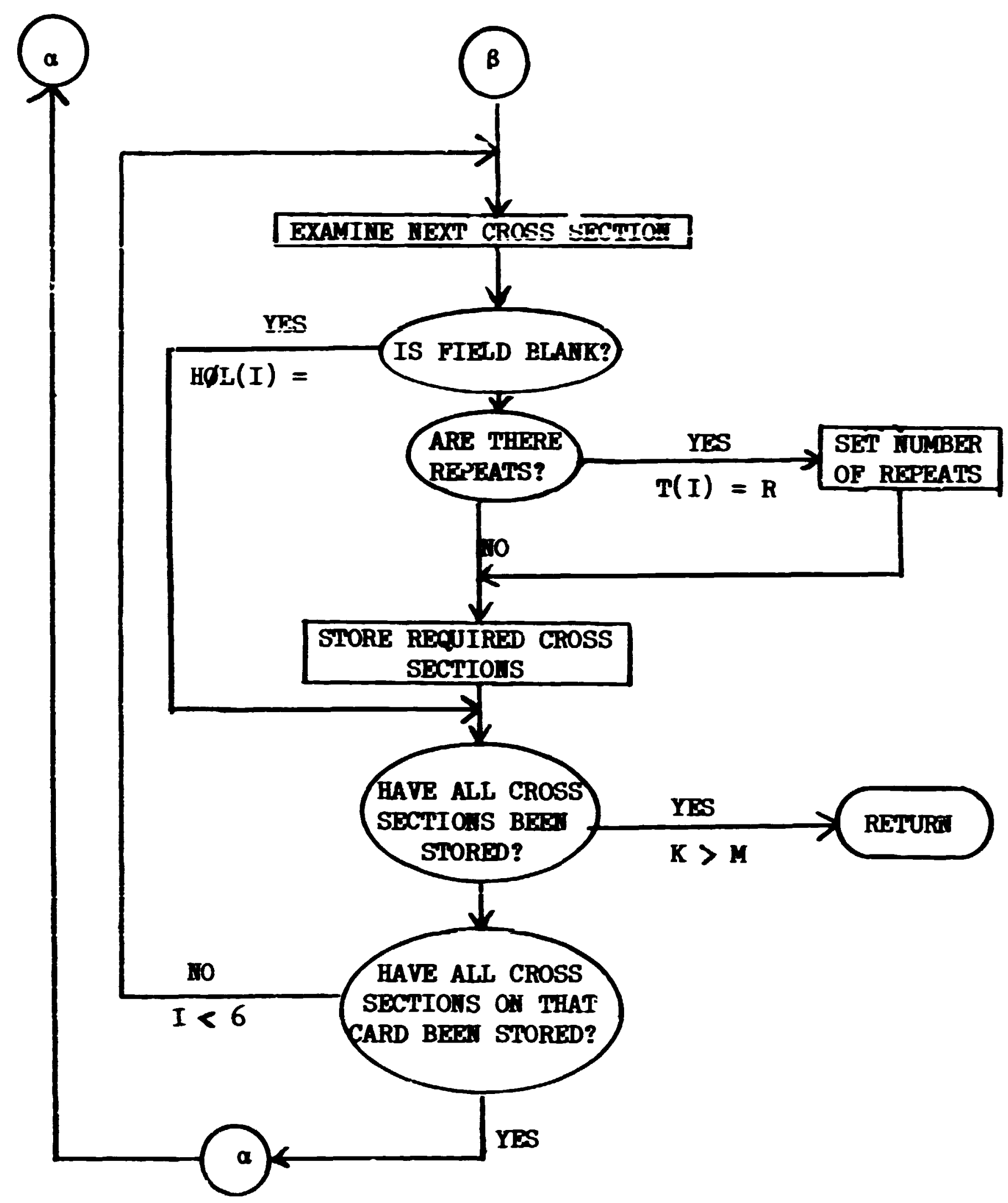


Subroutine STYRE (IE,IC)

The plirpose of subroutine STWRE is to pick uf the cross sections for element IE and coefficient IC from the input buffer region and store the total, fission, and dormscatter matrix in the temporary storage. Only those parts of the input cross sections that are to be reused are stored. That is, the neutrons may be stripped from a coupled neintron-gama set, or the gammas may be stripped from a coupled neutron-gamma set. Also, during the restoring the cross sections are transposed if an adjoint solution is desired.

Called from: JiPT

Commons required: Blank, LdCSIG

vâriables required: IE - element number IC - coefficient number

cross sections in blank common from IIIPBUP to IIPBUF+IIIGPE (INDS+3)

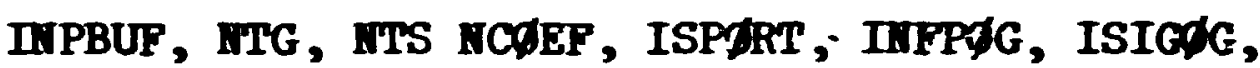
IIIS, IADJ, NME (from comm LACSIG, see page 88).

Variables changed: cross sections in blank coamon from ISPORT to ITWTSG Significant internal variables:

IIDX - starting location of downscatter matrix for the IE elsment and IC coefficient,

IE1 - number of locations to be skipped in the total cross-section array for other elements. 


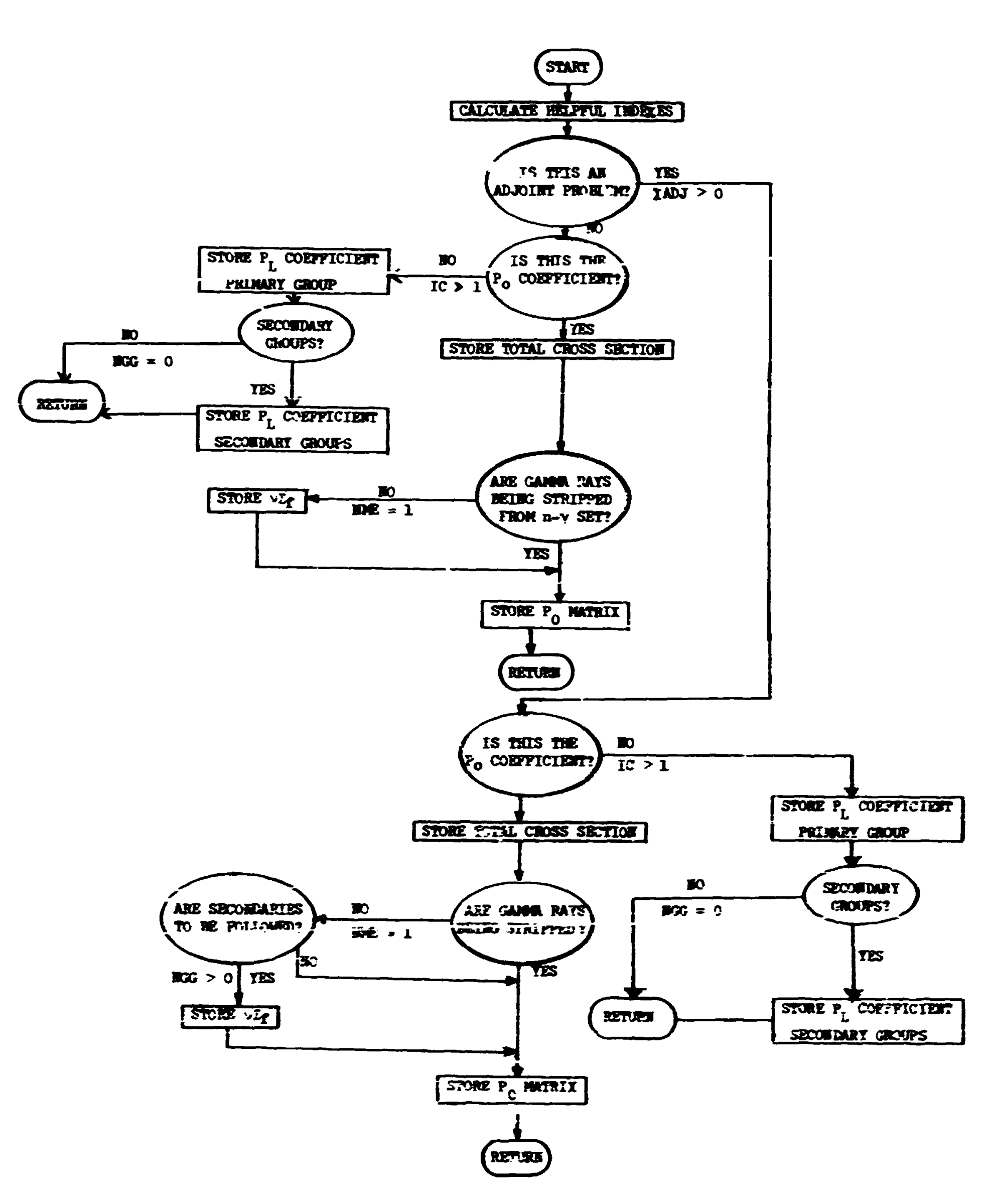




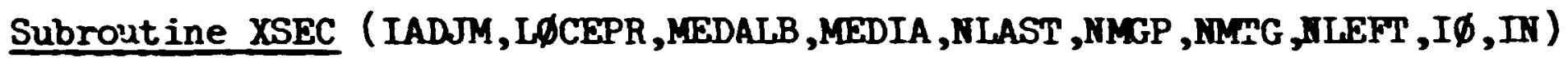

Subroutine XSEC is the primary interface of the cross-section module with the rest of RSE.

The function of XSEC is to read the cross-section information defining the numbe: of groups, coefficients, eiements, media, etc., and to set up the storage locations required. All variables in common LøCSIG are defined in subroutine XSEC. (Three variables are redefined in JNPUT if Legendre coefficients are restored.) After the storage is allocated, subroutine JNPUT is called and is the executive routine for manipulating the cross sections.

The first medium cross sections are stored from ISTART to ISP $\phi R G+$ ISTART; each successive medium requires ISP $\phi R$ G cross sections. The Legendre coefficients are stored behind the media cross sections.

Called from: IIIPUT

Subroutines called: JNPUT, ALBIN, XSCHIP

Commons required: Blank, LøCSIG

Variables required:

IADJM - switch indicating that the problem is an adjoint problem if $>0$,

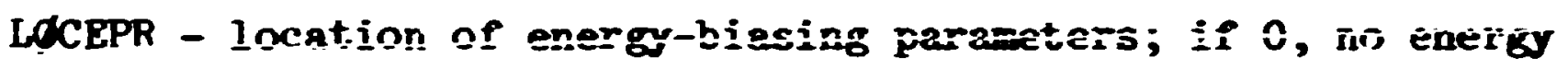
biasing vill be used,

MEDALB - mediun number for the albeio scatterer; MEDALB > 0 signals a combined albedo and normal transport problen; = 0 is flag for normal transfort only and ALBII will not be called; < 0 signals an albedo only problem, normal cross sections will not be read, MEDALB is the albedo nediu,

MEDIA - number of media for wich cross sections are to be read, MLAST - the cell used in blank common before XSEC was called.

Input: There are four cards read by subroutine XSEC. These cards contain: Pirst Card - coment card,

Second Card - IGP, IDC, IGG, IDSG, IMGP, IMDS, MMD, MRLEM, MIIX, MCOETP, NSCT, ISTAT, IXTAPE. Por definitions see comon LCCSIG, page 88.

\footnotetext{
A more detailed description is given in Appendix $C$.
} 
Third Card - IRDSG, ISTR, IFM, IMPM, IPRIN, IPUN, ITTF. For definitions see common IACSIG, page 88 .

Fourth Card (omitted if IXTAPE $\leq 0$ ) - element identifiers of cross sections to be read from tape.

Variables changeà:

MEDALB - set to 7777 if there is no albedo surface in problem,

IIAST - che last cell of permanent storage reguired,

Significant internal variables:

IBC - number of cross sections to be read from tape. 


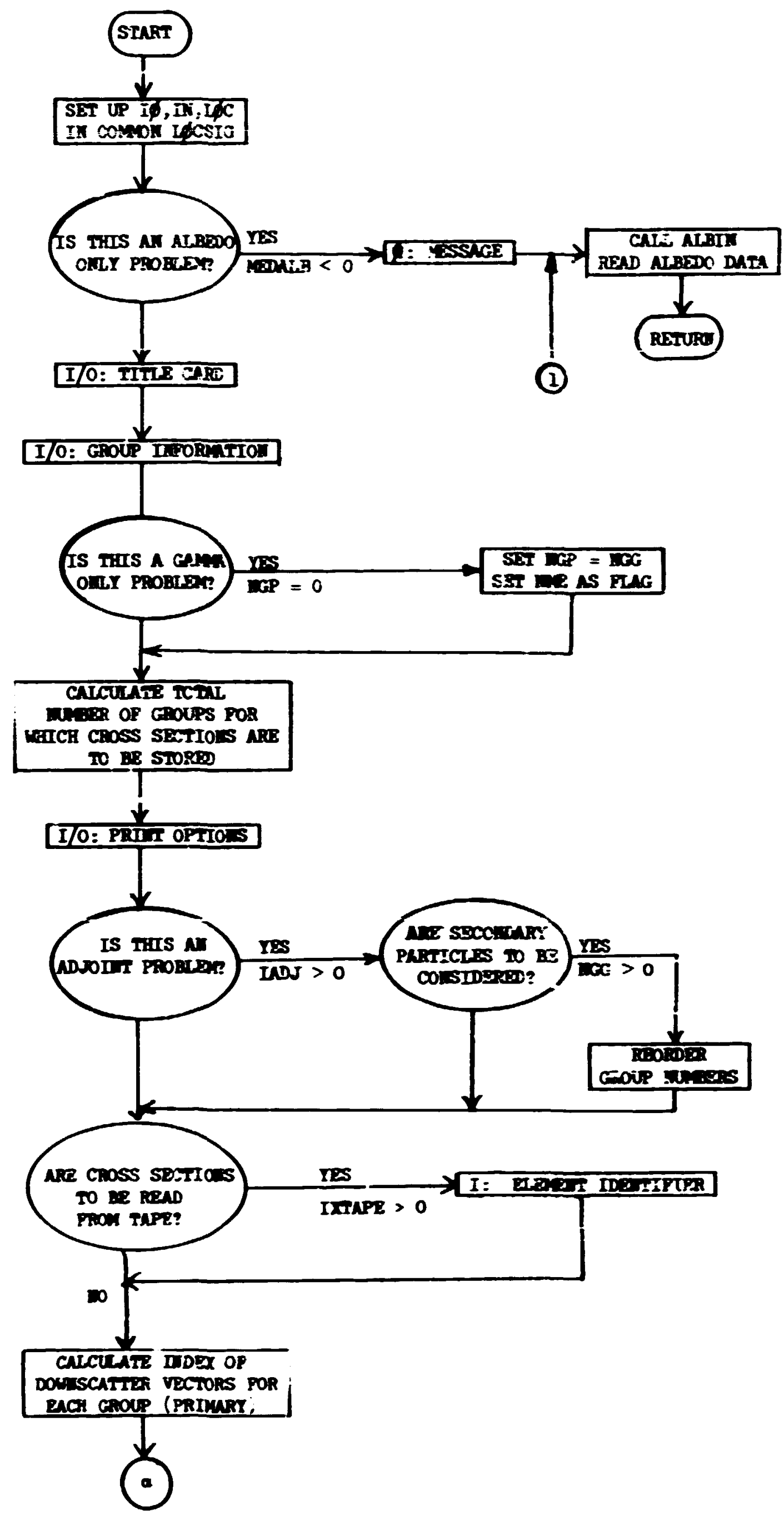




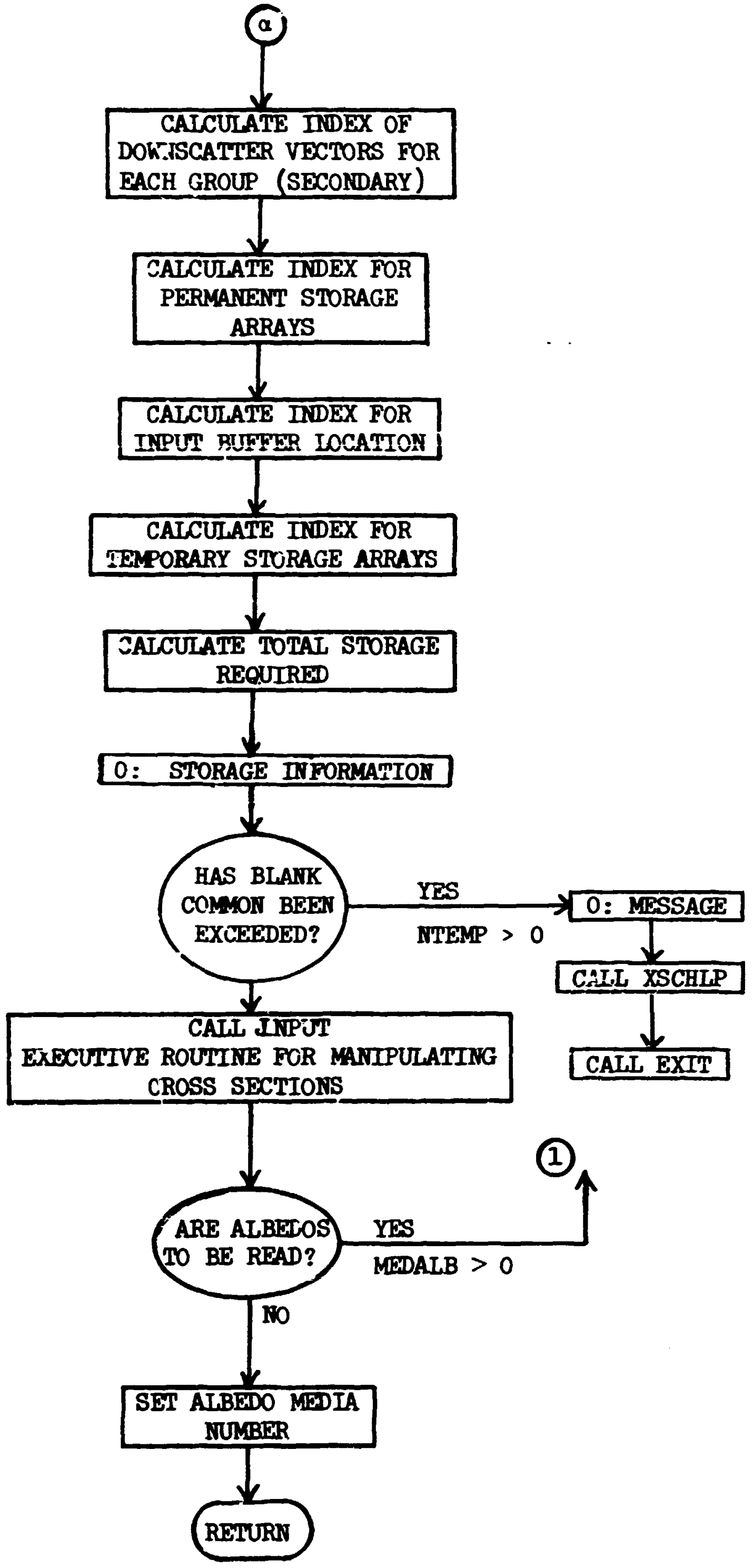




\section{Diagnostic Module}

Frequently in debugsing a problem or in trying to gain further insight into the physics of a problem, it is desirable to dump the contents of certain labelled commons or parts of biank common. This module of MøRSE makes it possible to print out in a readaije format the values of these variables.

The key routine in this module is subroutine HELPER which prints out, in decimal form, any part of a single-precision (4-byte word) array. This routine, along with two machine-language (IBM-360 series) routines, decides whether a number is an integer or a flcating point number and conver's to EBCDIC accordingly. It also recognizes the "junk" word $\left(48484848_{16}\right)$ and outputs the string "NGT USED" in its place. This feature is included because it is nci aiways feasible to depend on the core being zeroed or filled with any particular constant. Selected portions of core are therefore filled with this word, which was selected because it is essertially the same number when treated as an integer or as a floating point number.

A more inclusive dump may be obtained with subroutine HELP which outputs, on request, selected portions of blank common and commons APQLL,, FISBNK, NUTRфK, and ISER.

HELPER is a slight revision of TDUMP. 12 
Subroutine BAKHLP (MANE)

This routine outputs (one paricle to a line) all of the particle bank and, if used, all of the fission bank. If identical lines are encountered, it prints a message giving the number of identical lines. The last line is always printed.

Called from:

HELP - when index IGXBP $<0$.

Subroutines called:

ICduPA (A,D,I) (litrary function at Oas Ridge National Laboratory compares, bit by bit, $N$ bytes of locations $A$ and $B$; returns zero if $A$ and $B$ are identical)

Commons reçuired:

Blank, APфLIф, FISBIK

Variables required:

HSIGL - location in blank common of cell zero of the particle bank, IMST - maximum number of particles alloved for in the bank(s),

I0 - logical unit for output,

MISTP - index indicating that fissions are to be considered if $>0$, WFISBI - location in blank common of cell zers of the fission bank. 
Subroutine BNKHLP (NAME)

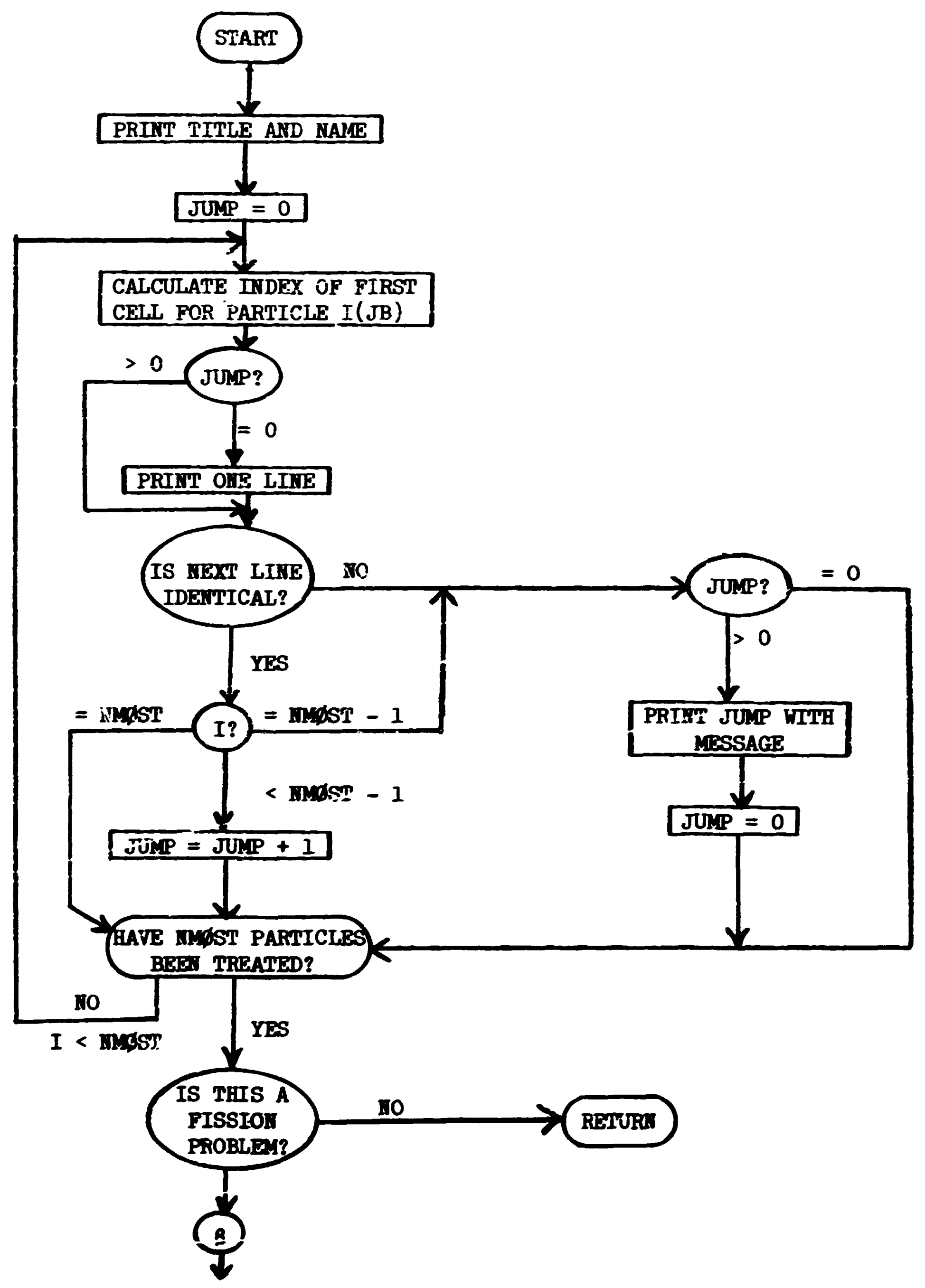




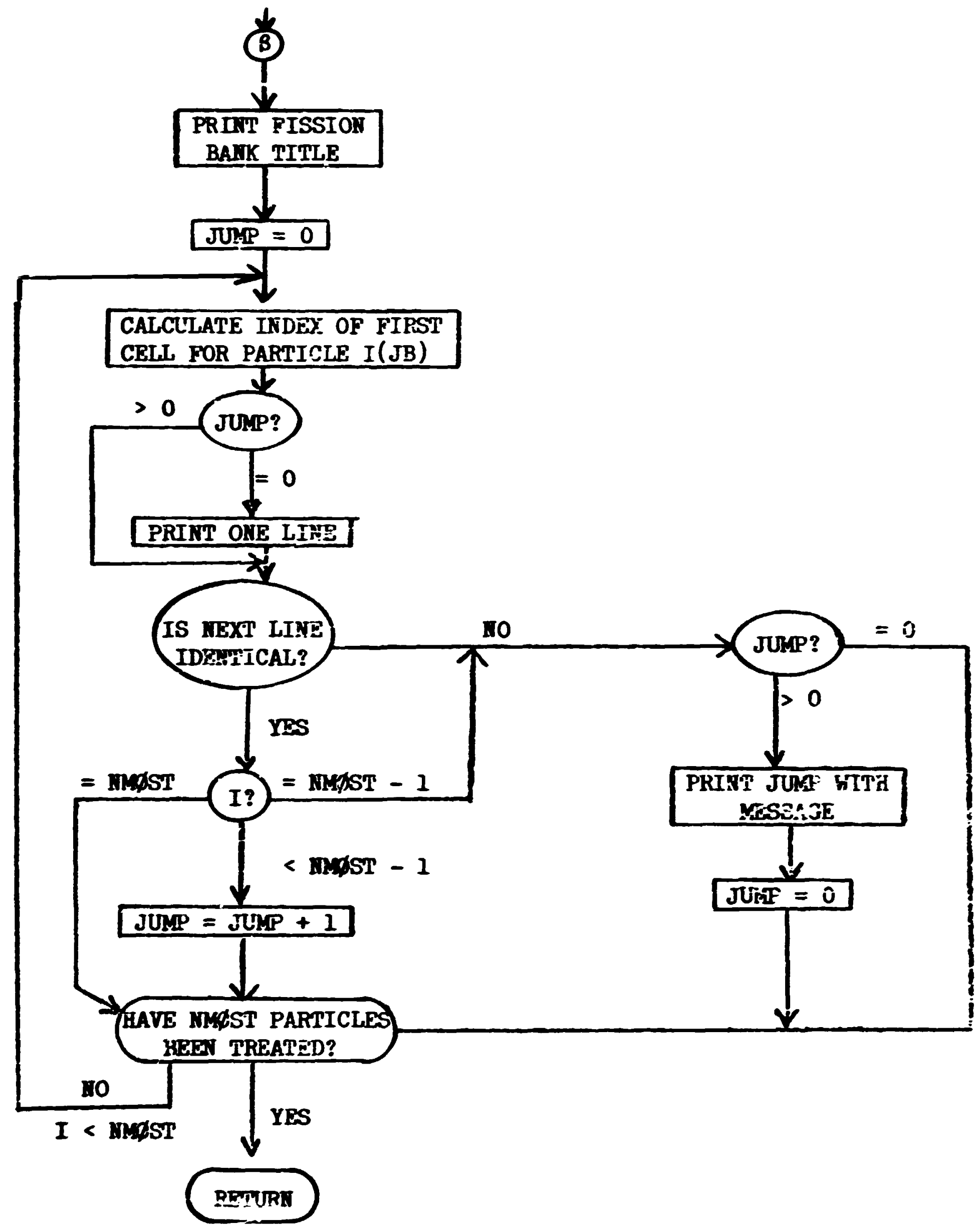


Subroutine HELP (ICALL, IHJIA, ILABP, IGXBP, IUSRP)

This routine is used to output values of selected variables used by the code, at any desired point in the solution of the problem. It vill provide, with setting or the proper switch, prints of:

1) blank comon frum ceil one up to the geometry data storage,

2) first and last eight words of geometry and cross-section data storage areas,

3) first and last 12 words of the neutron bank, or the entire neutron and fission (if used) banks,

4) all the user area in blank common (beyond the neutron and fission banks), and

5) labelled camons APQLL, PISBIK, NUTRQ⿻, and USER.

HELF has been found useful to the vriters of the code in debugging. For this purpose, temporary calls are inserted at points of interest. As the code stands now, calls are made in WRSE just after each problem is completed, and also at a fev points in the code that vill not be reached unless an error occurs.

Called from: MRSE, FBAK, FPR

Subroutines called:

HELPER

BuKHIP - prints ali of the neutron bank and all of the fissiac bank if it is being used.

Function used: IdC

Commons required: Blank, FUTR\%, FISEAK, APQLLף, USER.

Variables required:

ICALL - 4 BBCDIC characters representing location or call,

InUSP - > 0 for print of blank comon,

IIABP - >0 for print of labelled camons,

IGXBP - >0 for print of first and last 8 cells of geometry and cross-section storage, and the first and last 12 cells of the bank,

$<0$ for above print of gecnetry and cross section and also

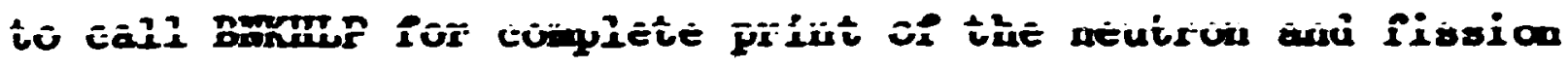
(if used) banks.

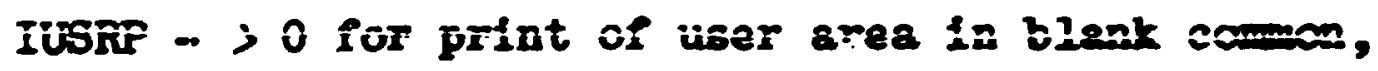


Harg - tutal number of energy groups,

Lforrs - location of cell zero of yeight standards arrays,

MCPREG - product of number of groups and regions for veight standards,

LAcinl - location of cell zero of Ful array,

MABG - nusizer of regions for veight standards,

IfCBPR - location of cell zero of enerEs group bias array,

( = 0 if energy group bias not being used)

LACISC - location of cell zero of scattering counter arrays,

HIA - numer of media in eruss sections,

LACFST - location of cell zero of FISH array,

WGEA - location of cell one of gecoetry data storage,

ISIGI - location of last cell in permanent eross-section storage,

IAST - last cell used by neutron or fission bank,

ILBFT - numer of cells available to user bejond banks. 
Subroutine HET (ICALL, MUAP, ILABP, IGXBP, IUSRP)

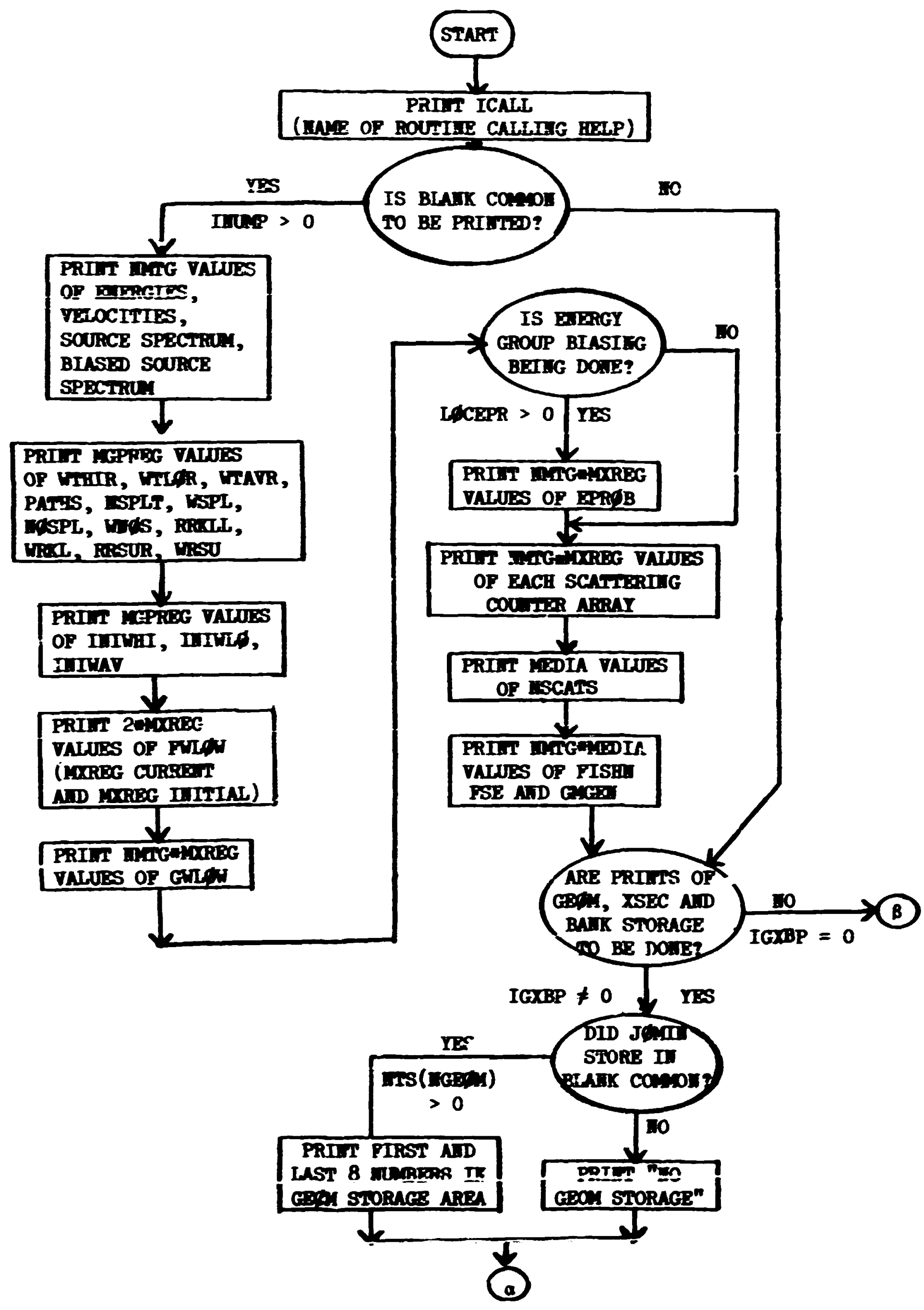




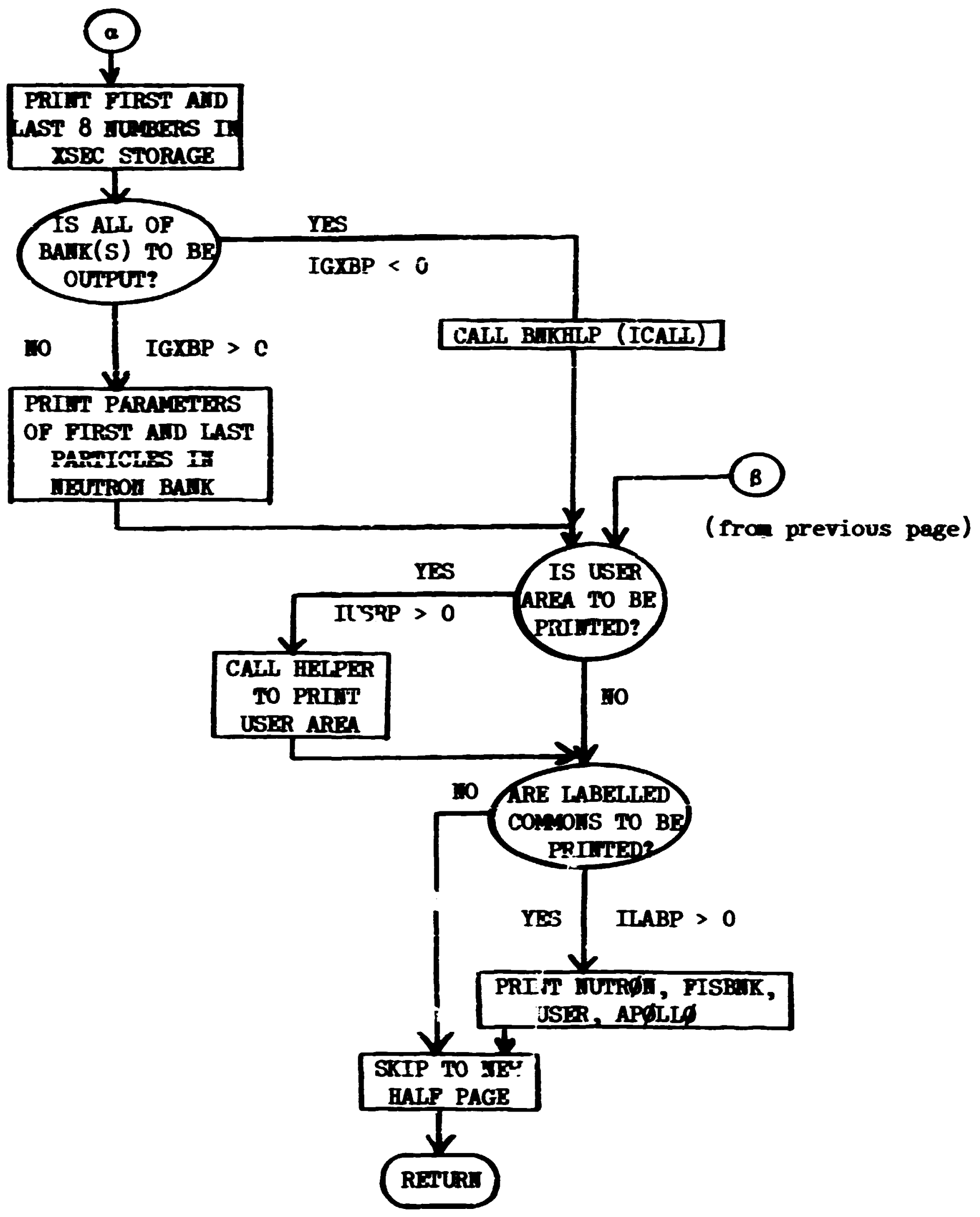


Subroutine HEIPER (A, DII, ILAST, MAYE, IO)

HEIPER enables the user to output, in decinal form, any part of a single-precision (4-byte vord) array at any point in the prograw. The user need not know whether the numbers are integer or floating point. Subers that can be translated as integers in the range $\pm 16^{6}( \pm 16777216)$ vill be printed as such; floating nubers are handled correctly betreen $\pm 1 \epsilon^{-64}\left(210^{-76}\right)$ and $\pm 16^{63}\left(210^{75}\right)$. If the sunk word $\left(48484848_{16}\right)$ is ercountered, "ITS USED" is printed. Hubers are printed eight to a line in an Ell.5 or Ill format and identical lines are replaced by a "REPBATIIG LIIE PATTEF" nessage (except that the last line of an array output is aivays printed.

Called from: HEiP, XSCHIP

Subroutines called:

SUBRT

ICAPA - (library runction at Oak Ridge Hational Laboratory; see B.KKHIP vriteup).

Variables required:

A - first word of array of interest,

IIIT - first 4-byte vori of array $A$ to be output,

IIAST - last 4-byte word of array to be output,

IAVE - 4 hollerith characters to be used as a label, Io - output unit. 
Subrout ine HELPER ( $A$, IITT, ILAST, INE, IO )

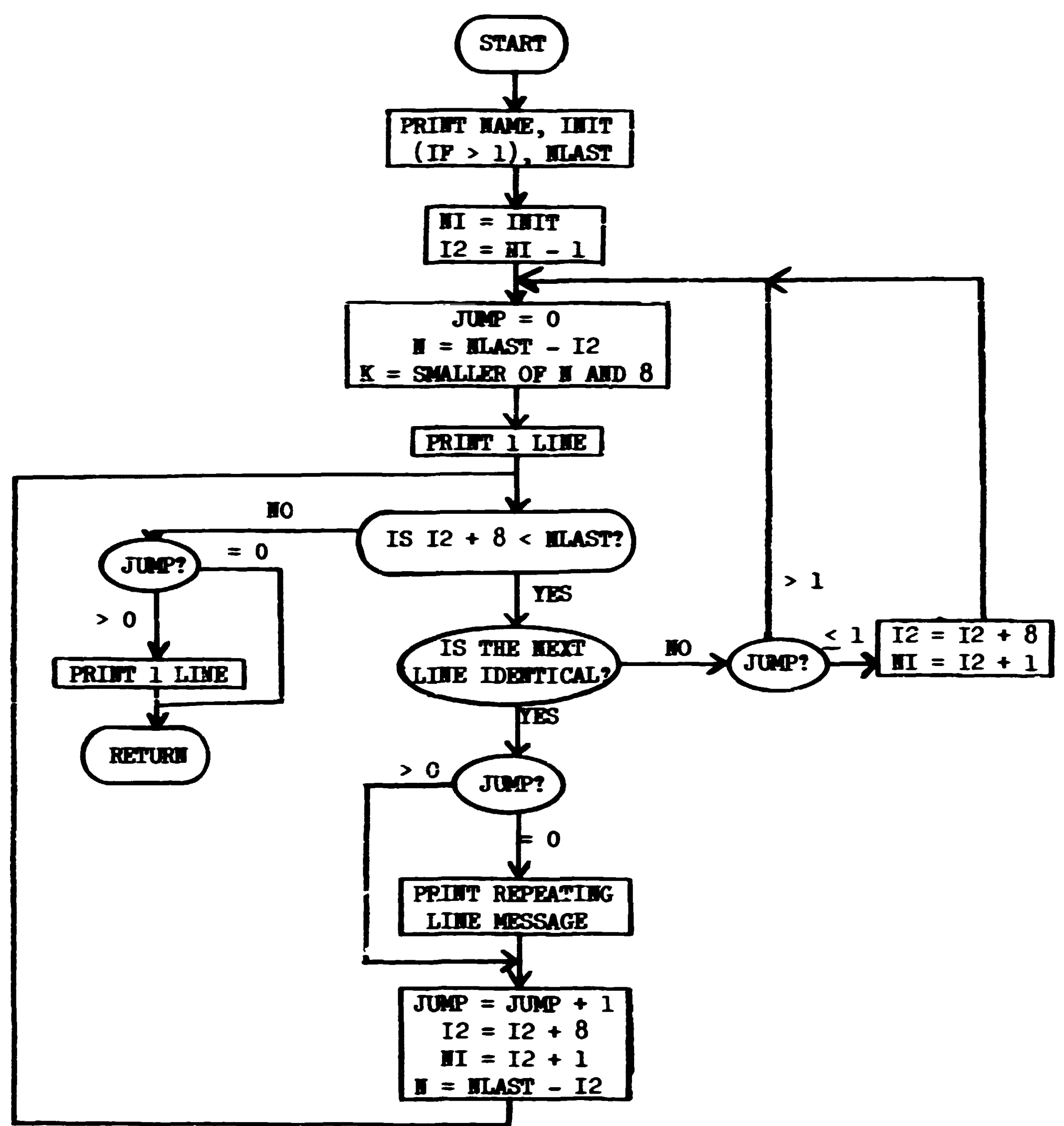


Subroutine SJBNT $(A, N, A)$

SUBRT is an assenbly larguage rcutine cailed by HELPER to perform conversion of a b-byte conputer word to a string of hollerith characters. It tests for unused elenents $\left(48 \div 34848_{i 6}\right)$ returning the string "Wor USED," decides whether the number is an integer or floating point, converts tine number into hollerith if floatiue point, and calls IrIBCD if integer. IrTBCD is cailed io convert ail nubers it receives as intếgers into hollerith and passes control back to SUBRT.

Called from: HETPER

Routines called: IIIECD - library subroutine at OR.L; converts a 4-byte irteger to an BBCUIC string.

Variables required:

A - 4-byte word to be converted,

I - fornat size (HEIPER calls vith $\mathrm{W}=11$ resulting in $I 11$ and 1PE11. 5 formats).

Variables changed:

Al - first vord of 12-byte array for storage of hollerith string. 
Subroutine XSCHLP (IBCDUM, RAYE)

This routine outputs in decimal or integer form the contents of the commons used in the cross-section module, as vell as the contents of the various cross-section arrays ir blank common. (See Table vI for layout of the cross-section area.) This subroutiule waj be called from any location. Called from: READSG, PTHETA, XSEC, and ANGLE (just before error calls). Subroutines called: HFIPER

Punctions used: LQC

Comons required: Blarik, LQCSIF, MEAKS, MMETT, QAL, RESULT.

Variables required:

IBCDUY - contints of blark common are printed if $>0$.

HAYE - a four-character word to indicate the calling program. 
Subroutine XSCHLP (IBCDUA, MNE)

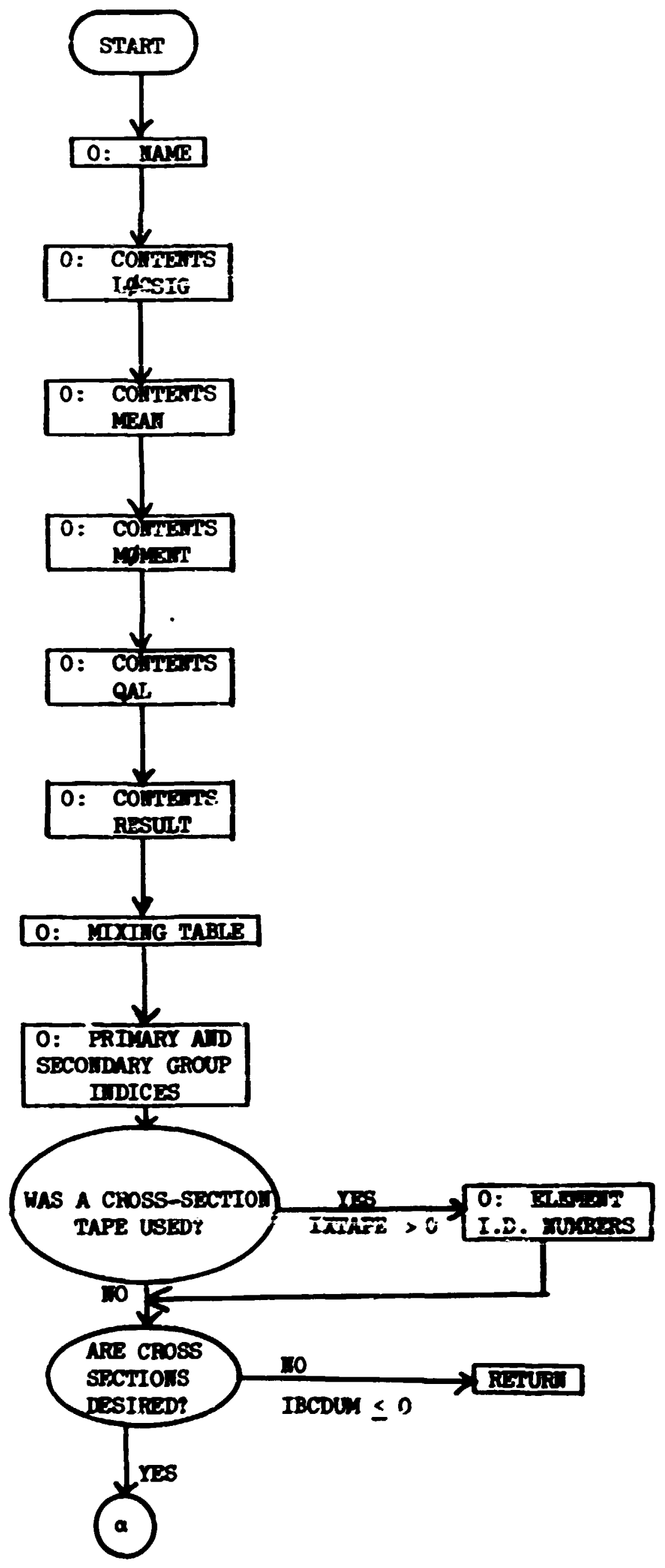




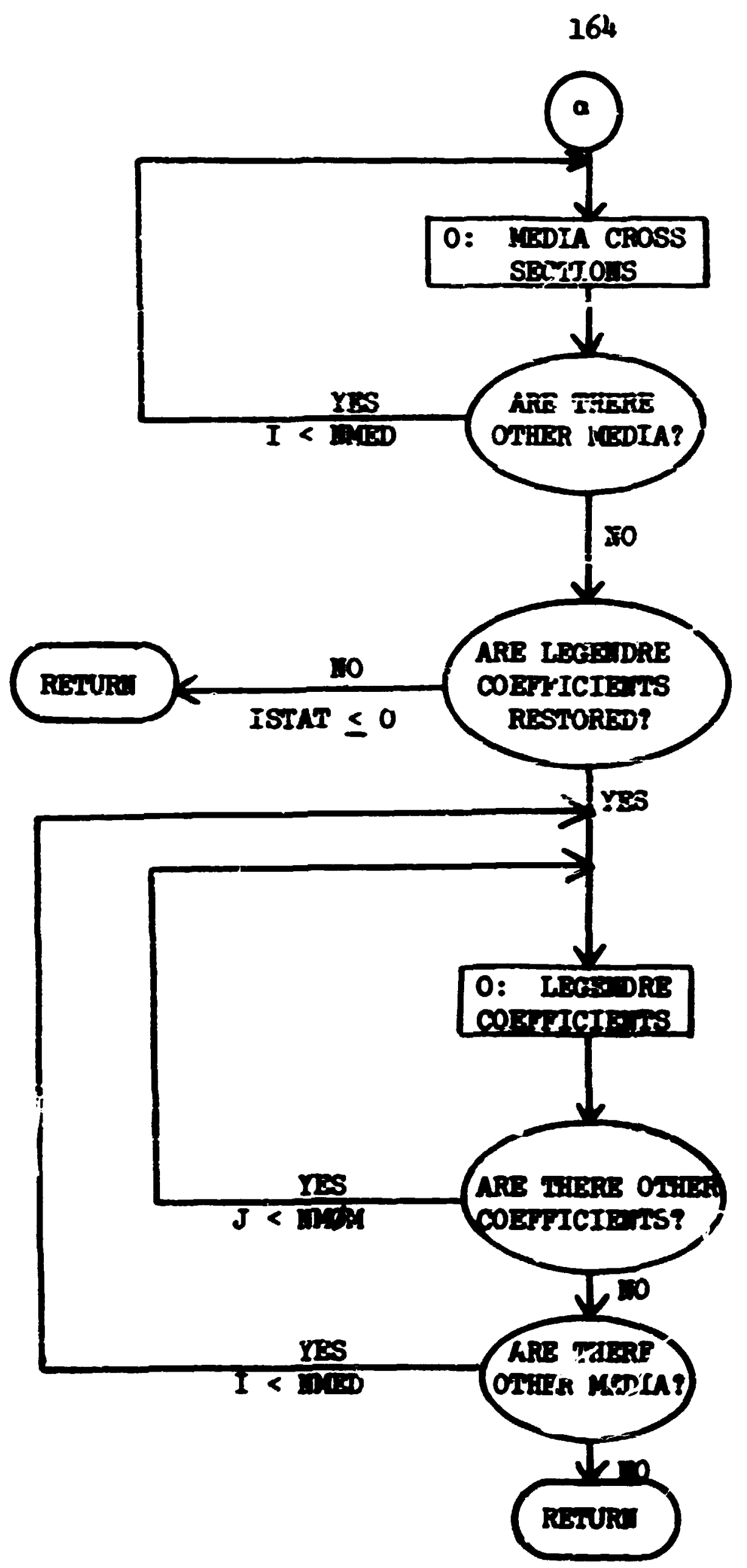




\section{Analrsis Interface and Serple User Routines}

The MOASE interface to user-provided analysis routines is tisough salls to runction DIRBi, and subroutines Giobis, SCWRI, SWURCE, and especially BAHR: Punction DIRBC supplies the dot product between the ieutiss direction rector and tbe nost important direction. It is used by GBIEIA, which deternines the length of the next IIIght, to vary the anout of path-length biasing depending on viether the particle is traveling in an important direction or not. If path-iength biasing is not desired (or if it is desired to blas all paths indegendently of direction), DIREC should return 1.0. FTHED is used to reiate cross-section and geconetry medis. It is called from every rositine needing cross-section dats. These calling routines have araisable the adiu of the point of interest, as specified by the gecnetry data, and need the proper nediun to five the cross-section routines. In wost cases, the geonetry and crose-section nedie are the same, but for special cases such as the infinite honogeneous nedie with a boundary crossing estintor two different nedie for ceif are required. All date required by user-written routines are input bs subroutine SChII wich is celled after the geweral problem specification, gecantry, and crose-section date are laput. Subroutive stuncis is called for each scurce particle (Including neutrons just produced by fission) so that the user no specify the phase space coordinated of each (if it is not desirable to use the constant values specifled ty inpurt cards to the rell routines).

BuId is the prinary interface to tie andyris packege, belos called vith as ang as 17 values of the argunent index to direct the analysis. These argunents and tbels maning are cutlined in Table VII.

It should be noted that not all the BNIAR calls issted in Table VII are ectualiy prooraned in the code (those not progrennad are included as comats); the user as have to add sheas sails for his spectel purposes. Several labelled comons sransfer date for use in the analvele, ad, in adition, the ianed portion of blenk comon is male arailable. Date valch je doternibed by the problem epeciflention (which are not

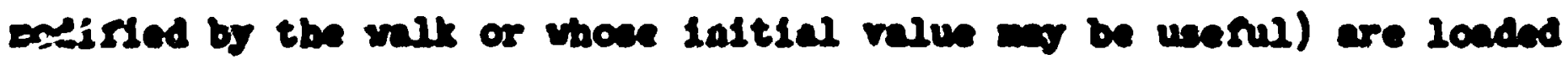

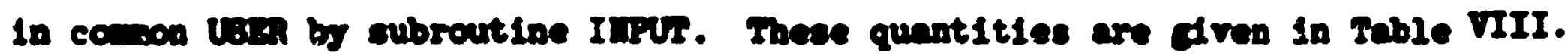


Table VII. BuIR Argurents

\begin{tabular}{|c|c|c|}
\hline $\begin{array}{l}\text { Bump } \\
\text { Argunent }\end{array}$ & Called Pron & Location of call in ralk \\
\hline$-i$ & MSE & $\begin{array}{l}\text { After call to IIPJI - to set paraneters } \\
\text { for nev problem. }\end{array}$ \\
\hline-2 & MISE & $\begin{array}{l}\text { At the beginning of each batch of ISTHT } \\
\text { particles. }\end{array}$ \\
\hline-3 & YTSB & At the end of eacin batci of isTFP particles. \\
\hline-4 & mise & $\begin{array}{l}\text { At the end of each set of nITS batches - } \\
\text { a new problen is about to begin. }\end{array}$ \\
\hline 1 & MStUR & Arter a source event. \\
\hline 2 & TESTI & After a splitting has occurred. \\
\hline 3 & FPA:B & After a fission has occurred. \\
\hline 4 & CSTHR & $\begin{array}{l}\text { After a secondary particle has been } \\
\text { generated. }\end{array}$ \\
\hline 5 & mase & $\begin{array}{l}\text { Arter a real collision has occurred- } \\
\text { post-collision paraneters are avallable. }\end{array}$ \\
\hline 6 & MSEE & $\begin{array}{l}\text { After an albedo cullision has occurred - } \\
\text { post-collision paraneters are arailable. }\end{array}$ \\
\hline 7 & Deret & $\begin{array}{l}\text { Arter a boundary crossize occurs (the } \\
\text { track has encountered a new gecnetry nedium } \\
\text { otber than the albedo or vold nedia). }\end{array}$ \\
\hline 8 & Hared & $\begin{array}{l}\text { After an escape occurs (the geometry has } \\
\text { encountered nediu zero). }\end{array}$ \\
\hline 9 & WESE & $\begin{array}{l}\text { After the post-collisior: eneres group exceeds } \\
\text { the maximan desired. }\end{array}$ \\
\hline 10 & MASE & $\begin{array}{l}\text { Arter the maximin chronological age has } \\
\text { been exceeded. }\end{array}$ \\
\hline 11 & TESTH & Arter a Russian roulette k11l occurs. \\
\hline 12 & TESSH & Arter a Russian roulette survival occurs. \\
\hline 13 & Cstriate & $\begin{array}{l}\text { Arter a secondary particle has been gener- } \\
\text { ated but no roon in the bank is avallable. }\end{array}$ \\
\hline
\end{tabular}


Table VIII. Definition of Variables in Coman USER

\begin{tabular}{|c|c|}
\hline AGSTRT & Initial age (input on card D). \\
\hline WTSTETT & 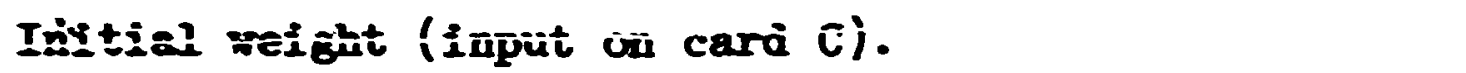 \\
\hline XSTRT & Initial $x$ position (input on card $D$ ). \\
\hline YSTRY & Initial $\mathrm{y}$ positicn (imput on card $\mathrm{D}$ ). \\
\hline ZSTRT & Initial z position (input on card D). \\
\hline DFP & $\begin{array}{l}\text { Mormalization for adjoint problems - caiculated in } \\
\text { SфRIA. }\end{array}$ \\
\hline EBMIN & Lrwer energy boundary of last neutron group. \\
\hline BBQTG & Lover energy boundary of last gama-ray group. \\
\hline TCUT & Age linit (imput on card $c$ ). \\
\hline IO & Logical unit for output. \\
\hline II & Logical unit for input. \\
\hline IADJM & Adjo:nt switeh ( $>0$ for adjoint problem). \\
\hline $\left.\begin{array}{l}\text { IGPQT1 } \\
\text { IGPQT2 } \\
\text { HGPQT3 }\end{array}\right\}$ & $\begin{array}{l}\text { Problem dependen - energy group limits - see flor } \\
\text { chart for subroutine INPUT. }\end{array}$ \\
\hline HGPQTG & Lowest energy gamma-ray group. \\
\hline rGPQIn & Lowest energy neutron group. \\
\hline IITS & Number of batches (imput on card B). \\
\hline MLAST & $\begin{array}{l}\text { Last cell in blank common ussed by either cross-section } \\
\text { package or bank }(8) \text {, whishever is larger. }\end{array}$ \\
\hline HLEFT & Humber of cells in blank common avatlable to use:. \\
\hline MYTP & Number of primary (neutron) groups (input on card B). \\
\hline IITG & Number of total groups (input on curd B). \\
\hline MSTRT? & $\begin{array}{l}\text { Number of source particles for each batch (input on } \\
\text { card B). }\end{array}$ \\
\hline
\end{tabular}


The user will also need camos IUHRta wich contains prior and present collision paraneters (the pre-collision weight is also provided).

All other variables in the walls which way be needed by the user should be transinitted by the prinary interface routine, BNIKR, as arguments in the called routine. See BAIKR writeup for examples.

Sarple User Routines

The problen chosen for this exaple is to calcuiate fluence at up to 20 distances from a point, isotropic source in an infinite mediun. A boundary-crossing estinator is used along with alternating geonetry media 1 and 2 in concentric spherical shells. The information required by the sample analysis routines is passed by comon DET. The variables required are defined in Table IX. Descriptions of each routine including flor charts and listings follow.

A description of the versatile analysis pacirage SAMO is contained in reference 1. Some of the user routines described here are replaced by more general routines in SAMBO; other routines crmplement those in SAMBO. 
Tabie IX. Defiuition of Yeriatles in Coman DET

\begin{tabular}{|c|c|}
\hline Variable & Definition \\
\hline ED & Dumer of detectors. \\
\hline ISCAPE & Counter for boundary crossings beyond the last detector. \\
\hline $\operatorname{RAD}(20$; & $\begin{array}{l}\text { Radii ir cn of the spherical detectors (mist be medis } \\
\text { boundaries). }\end{array}$ \\
\hline $\operatorname{Min}(20)$ & Humber of estinates at each detector. \\
\hline UD(20) & Uncoliided response for the current batch. \\
\hline $\operatorname{sUD}(20)$ & Uncollided response (UD) sumed over batches. \\
\hline SUD2(20) & 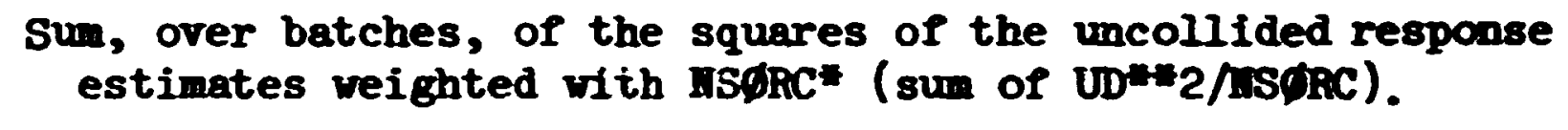 \\
\hline $\mathrm{SD}(20)$ & $\begin{array}{l}\text { Total (uncollided plus collided) response for the current } \\
\text { batch. }\end{array}$ \\
\hline $\operatorname{SSD}(20)$ & Total response (SD) sumed over batches. \\
\hline $\operatorname{SSD2}(20)$ & 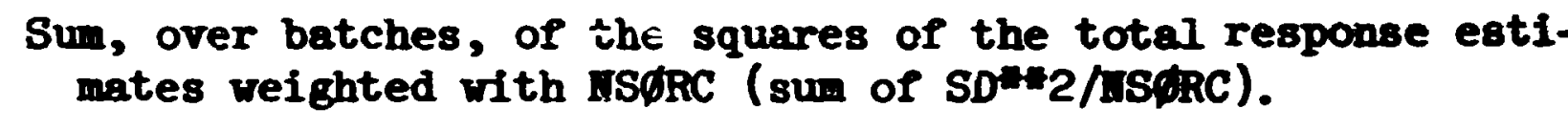 \\
\hline $\operatorname{FDCF}(100)$ & Response function arras. \\
\hline
\end{tabular}

"IS $\varnothing \mathrm{RC}$ is the number of particles starting the batch. 
Subroutine BNIOR (HBiDD)

The Runction of BNKR is to call analjsis and diagnostic subroutines as specified by the user. The particular subroutines called in the analysis codule are deternined by the index IBaKID. In this problem: BNIKR (-4)

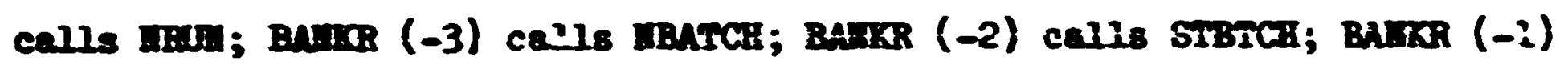
calls STral and HBIP; BAHR (1) calls SMATA; and BAlKR (7) salls BDRXX. $\Delta$ other values of iBuldD result in a return.

A version of BNIKR that urites a collision tape sindiar to that witten by $15 R$ is also araflable.

There are 36 possible variables that ad be sritter on the tape for each of the 13 types of events. The use of the tape-imiting rersion of BuIdR is not encourased but it is provided for that oscasionel circustance vhere it is advantaseous.

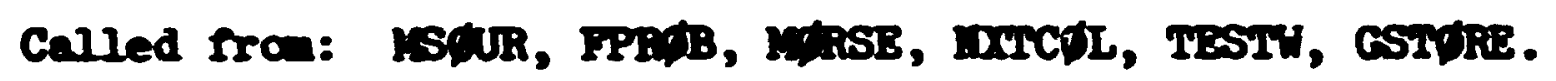

Subroutines called: STRU, STBTCH, BBATCH, IMU, SDAT, BDAR, BEIP. Comons required: APDLIT.

Variables required:

IBUCTD - an index which identifies the tJpe of collision and/or subroutine called (IBWKD $=-4,-3,-2,-1,1,2, \ldots$ i3),

IIIS - nuaber of batches to be run,

IIBAS - number of batches which remain to be grocessed,

HQUIT - nuber of runs remaining plus one (set to negative of the number of runs conpleted, when an execution tine kill occurs),

ania - nuber of particles which renain to be processed in a given batch.

81gnificent internal variables:

BaAT - the batch nuber less one,

ISAVE - the number of particles starting the current batch. 


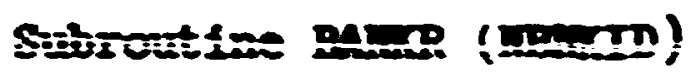

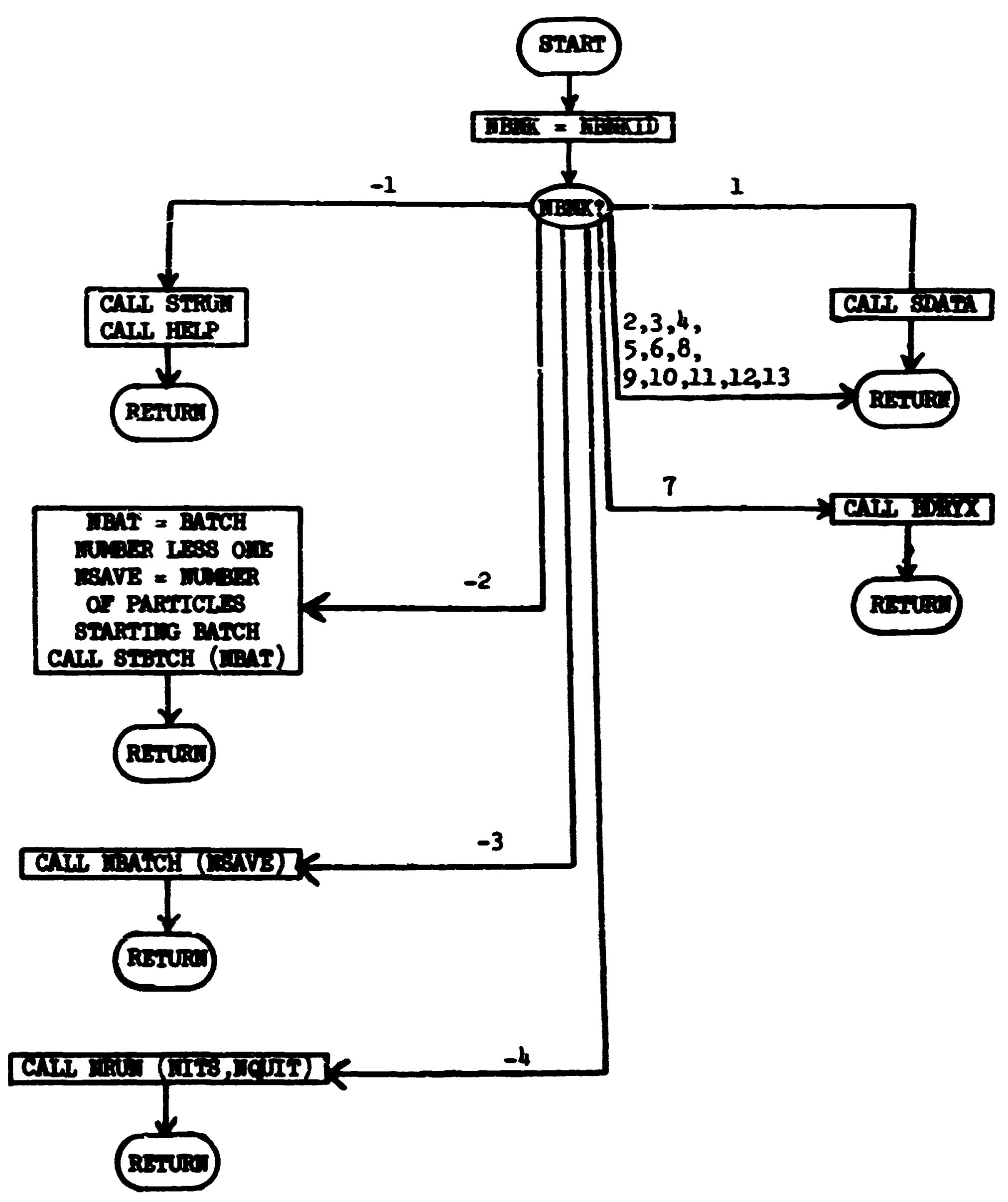




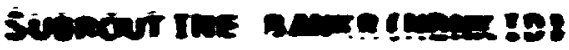

erenc 10

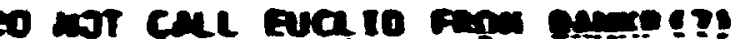

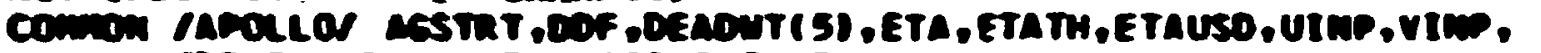

-ī̄ín 20

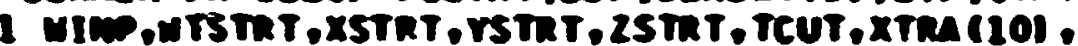

Bumr 30

10.11.

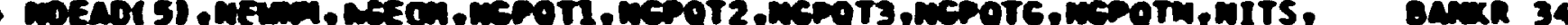

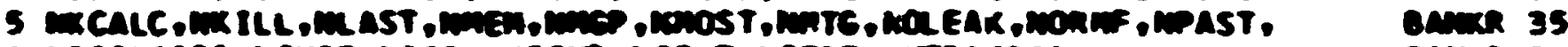

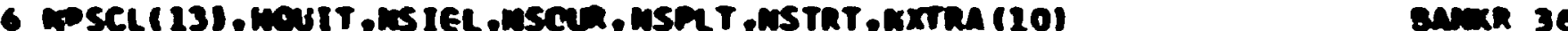

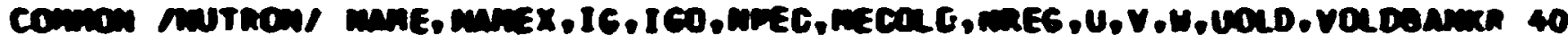

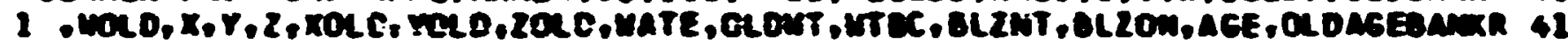
If Imanki 100,10:.140

$100 \operatorname{man}=\operatorname{mon} 65$

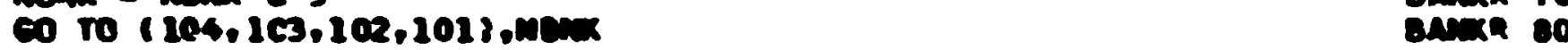

:01 call stming

C

CALL mELP(4HSTRU,2,1,1,1) Return

102 mat $=$ WITS - ITERS

mave = minn

CALL STOTCH(MAT)

C mat is TME BATCH m. Less one RETun

198 CALL matChinsaves

C MSAVE IS TWE Wo. OF particles STARTED IM THE IAST catah neturn

104 call munemits omoutt

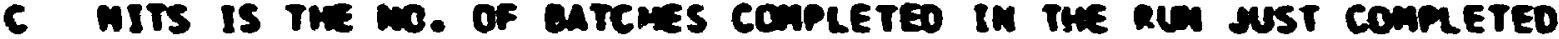

NOUIT.GT. 1 if mone Rous penin

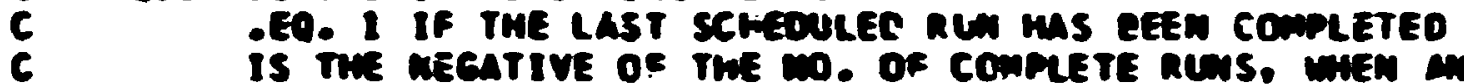

6 is The recative of The wo. OF complete Rons. Wien an minn

140 ec to $12,2,3,4,5,6,7,0,9,10,12,122,10, \mathrm{~mm}$

Banzer 90

$\operatorname{com} 100$

$\operatorname{Banx} 120$

$\operatorname{Bam} 120$

$\sin 130$

$8 \sin 140$

Bain 150

Dan 160

Bamin 170

$\operatorname{com} 180$

$\operatorname{arm} 190$

Bam 200

$\sin 220$

$\operatorname{canx} 220$

BAm 230

$\sin 240$

Bamx 250

OAm 260

Banx 270

$c$
$c$
$c$
$c$
$c$
$c$

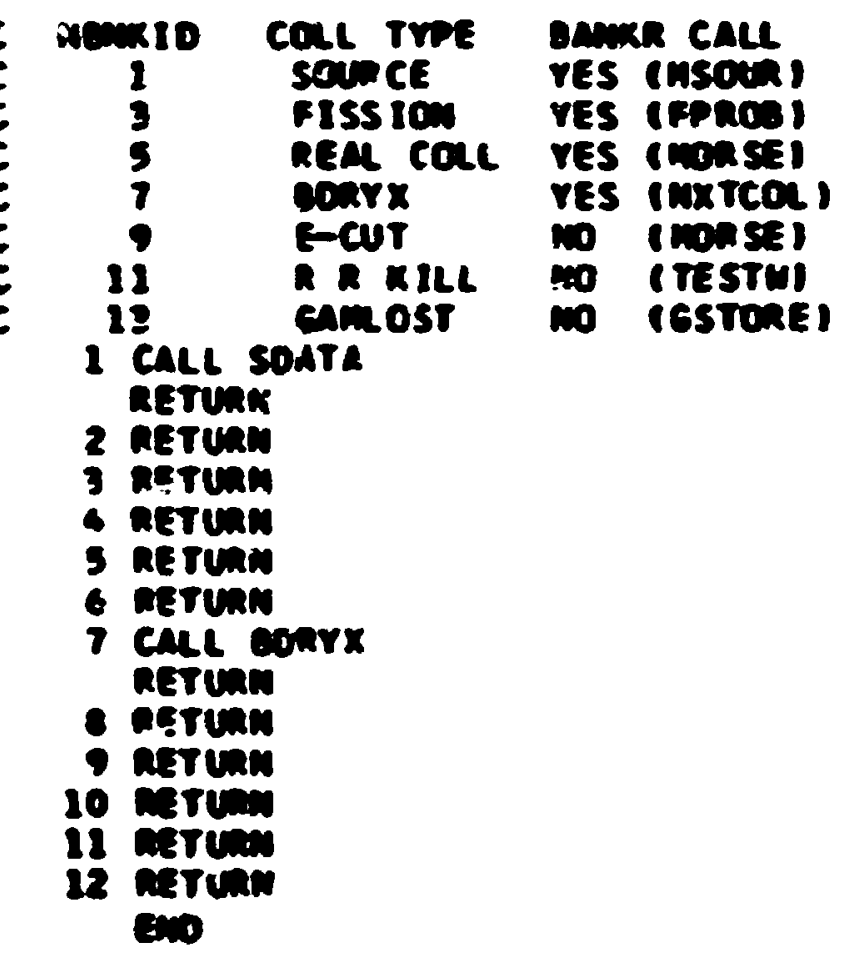

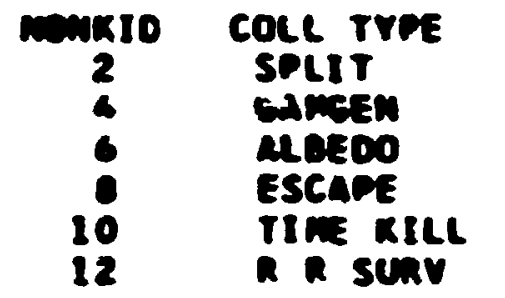

Bamer call sama 200

W ITESTMIOAn 290

m iestoneanm 300

res InIRSEIOAm 320

YES InTCOLank 320

no Imonseisanx 330

R R senv WD itestubien 340

BAmx 350

DAnix 360

$8 \sin 370$

Danm 380

Bamix 390

Bamx 400

BAnx 410

Bamin 420

DAmix 430

$\operatorname{cam} 40$

DAmix 450

$\operatorname{com} 400$

Decm 470

Banx 400

Demix 490

cank 500 


\section{Subroutine BDRYX}

This routine is called whener the particle in the valk encounters a change in geonetry media. If the source-to-collision distance corresponds to a detector position, the reciprocal of the cosine of the angle from the radius vectcr is used as a fluence estimate. The response value for the appropriate exerB group modifies the estimate, which is then stored in the counter for the appropriate detector.

Called from: BAIKR (7)

Subroutines Called: ERRdR (library) ABS (library function)

Comms required: USER, NUTRQA, DET

Variables reçuirid:

$X, Y, Z, U, V, H$, WATE (fron comon NuTht, see page 12)

ID, ISCAPE, III(I), $\operatorname{PDCF}(I), \operatorname{SD}(I), \mathrm{KAD}(I)$ (from common DET, see page 169)

Variables changed: ISCAPE, $\mathrm{HH}, \mathrm{SD}$.

Significant internal variables:

R21 - radial distance to boundary crossing,

R2 - $99 \%$ of R21,

$\mathrm{R} 22$ - 1018 of $\mathrm{R} 21$,

CdS - cosine of angle between particle direction and radius vector, ABCQS - absolute value of CQS,

Ch - fluence estimate,

Coid - response estimate. 
Subroutine BDRYX

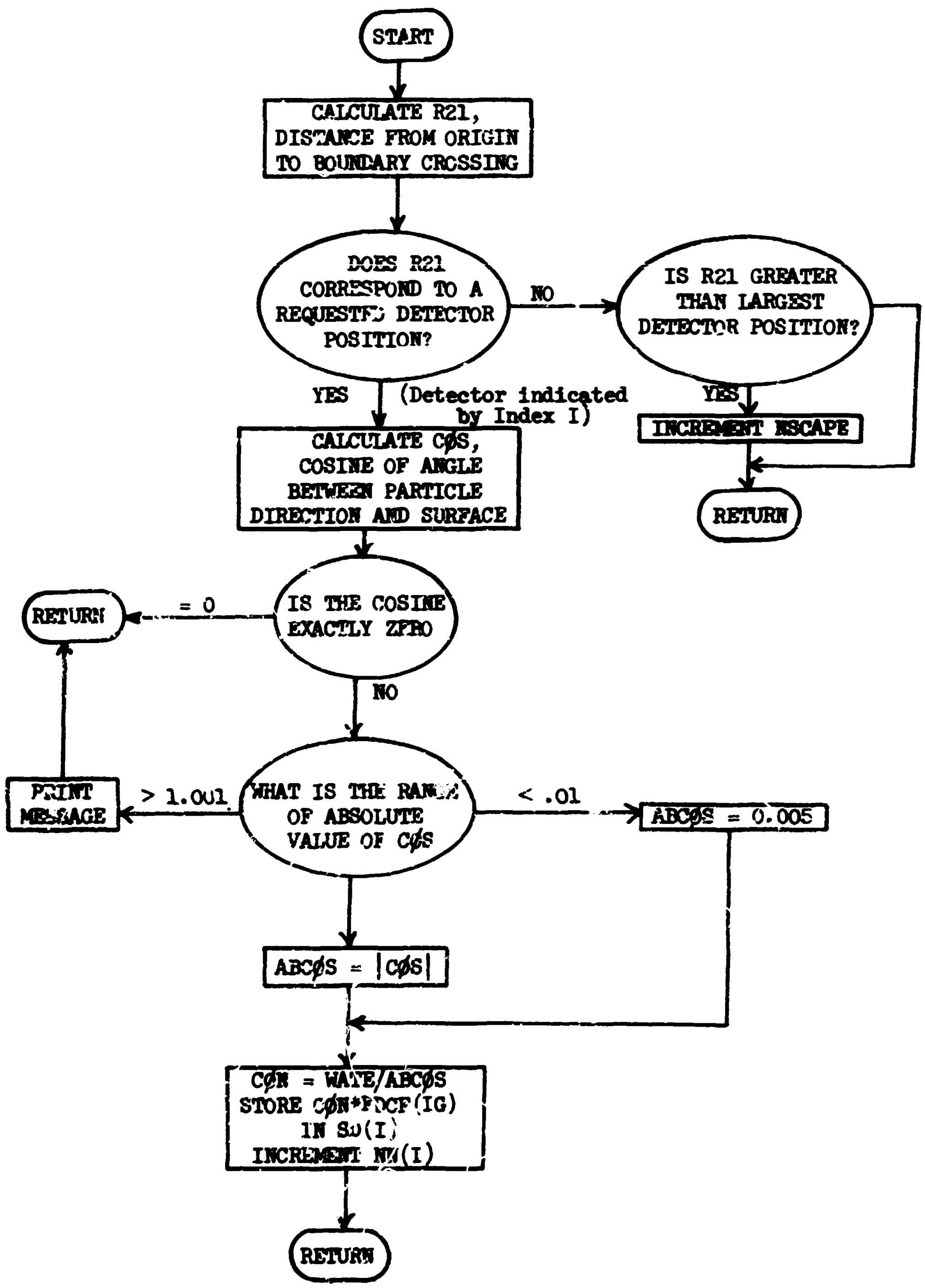


SUBRDUT INE ECRYX

ecarx 10

FCR USE IN SPHER ICAL GEOMETRY OMEY

canrx 20

conrx 30

convx 40

IOENTIFIES DETECTO POSITION MITH a BOAMCARY CROSSIMG AMD TMEN CalCulates ano SUMS OUANTITIES OF INTEREST FCR EAC: BATCh.

conrx so

6 onrx 60

$\operatorname{som} r x 70$

COMMOW /USER/ AGSTRT,WTSTRY,XSTRT,YSTRT,ZSTRT,EFF,EQOTK,EDOTG. octrx 00

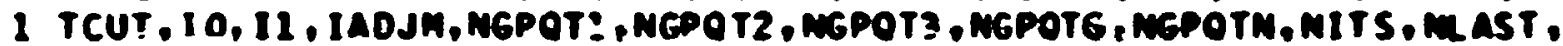
somy or

2 MEFT, NMGP, MMTG,NSTRT

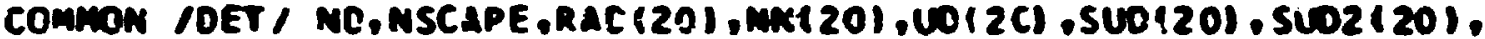

BonYx 12

conrx 90

1 SC(20), SSO 201 , SSO2(20), FOCF : 160)

conrx 92

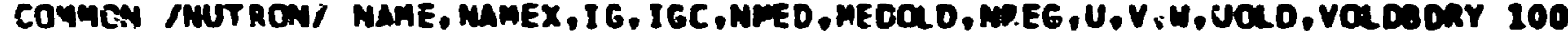

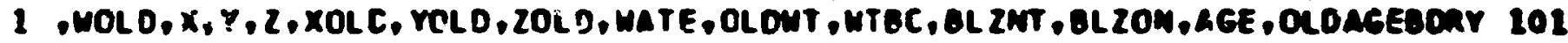

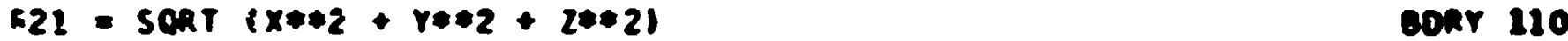

$R=R 22 * 0.99$

$R 22=R 21 * 1.02$

$005 \quad 1=1, M 0$

IF (R2-RAD(II) $15,25.5$

5 CONTIMUE

MSC APE $=$ MSCAPE+ 1

10 RETURN

15 IF (R22-RRD(II) $10.20,20$

20 ERA = UAX + VAY + ME2

COS = ERATP 21 - 1.E-10

If (cos) $30,25,30$

25 MRITE (10.2000)

1000 FORMATC INO, I4H COS=0., RETURMI RETUAN

30 ACCOS=ABS (CCS)

If (AeC OS -1.0091$) 40,40,25$

35 WRITE $(10.1010)$ Aacos

1010 FDF MATIINO. MACOS.6T.1. - E10.4)

CAILL EPROR

40 If (ABCOS-0.02) 45.50 .50

$45 \quad \triangle B C O S=0.005$

50 COW-WATE/ABCOS

$\min (1)=\operatorname{ANM}(1)+1$

COMD = COMAFOCF(16)

SDI $(1)=\operatorname{SD}(1)+\operatorname{COND}$

RETURN

bens 120

ockr 130

ookr 140

ecty 250

ockr 160

bony 173

ookr 180

bont 130

odxy 200

ocar 210

cony 220

oont 230

conr 240

Don:" 250

conr 260

ocar 270

bonr 250

oxy 200

bony 300

cony 310

ecky 320

oony 330

conr 340

oony 350

oont 360

80nY 380

END

eonr 300 
176

Proaction DIm eC (DUnT)

This function provides the dot product of the neutron direction rector and the radius vector. Thus DIREC $=1.0$ for an outgoing neutron and $=$ -1.0 for an Inward going neutron. These value result in marian path stretching and shrinking, respectively, wen used in the calling routine crisis.

Called ron: centra

Function used: Solis (library)

Commons required: wrens

Variables required:

WLD, VID, WID - prior collision direction cosines (at this point they are equal to the current collision values),

XLD, YOLD, 20LD - coorilisates of prior collision site (at this point they are equal to the current collision values?.

Variables changed:

DIBEC - the function value. 
Function DIrec (DUnx)

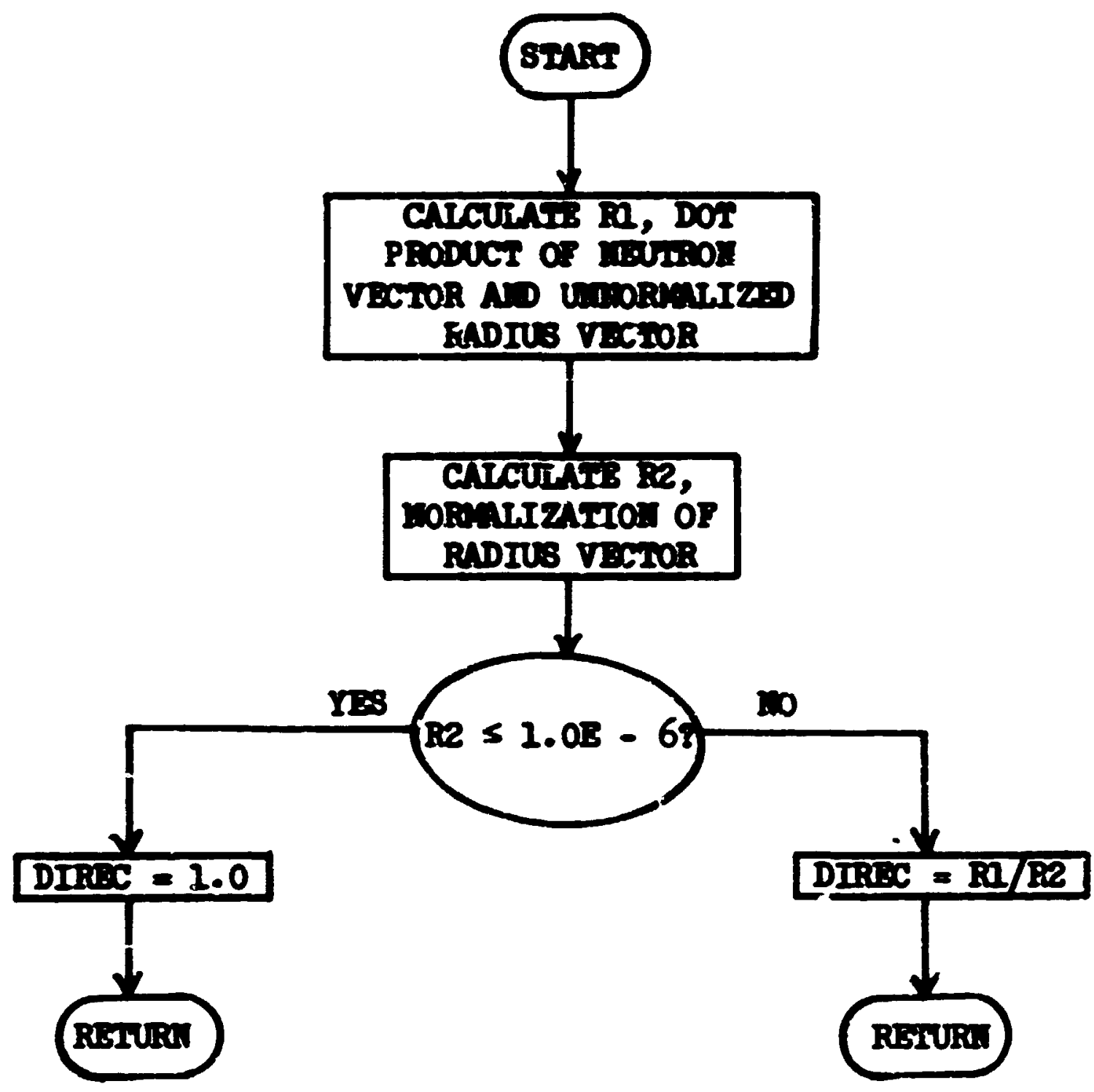




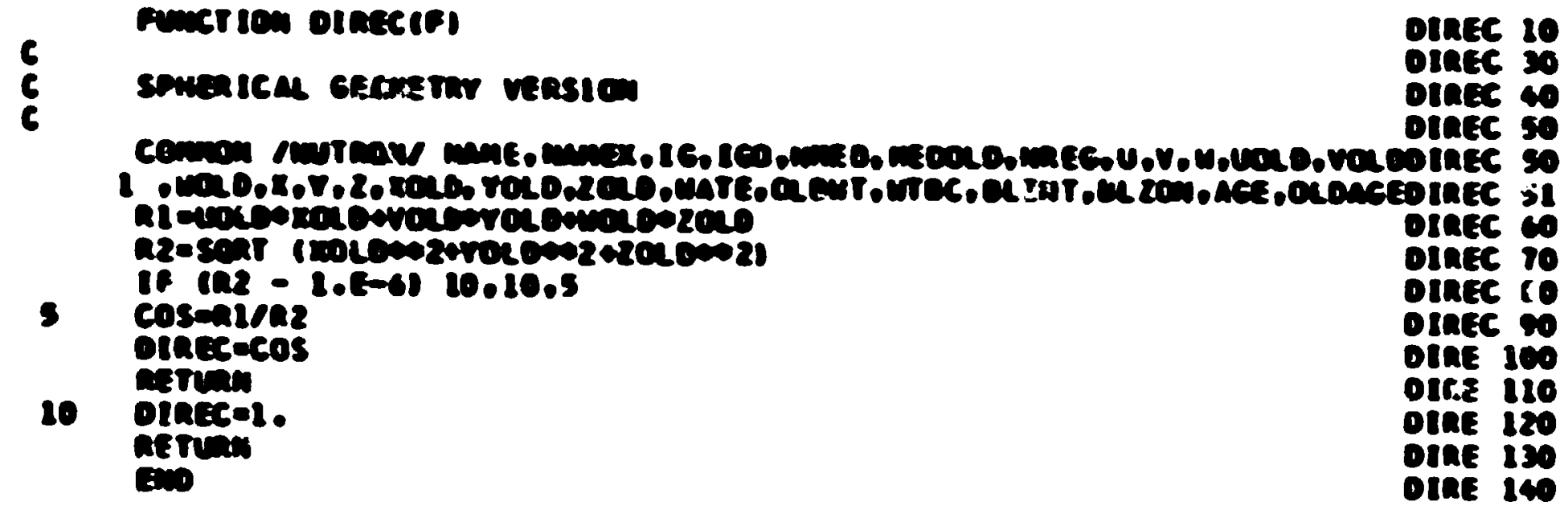




\section{Subroutine GMYED (IDGEGA, MDXSEC)}

This subroutine allows one to equate the cross sections for two different geometric media. Thus, if one uses a bcundary crossing estimator, GED requires that the media on both sides of the boundary differ. Hovever, for a homogeneous problem, the transport needs only one cross section to be stored. For any problem not involving a boundary crossing estimator for a homigeneous system, !DGEDN and MDXSEC may be equivalenced and the subroutine calls remored.

A data siatement set,s the two media numbers that are to have the same cross sections.

Called from: MoRSE, FPRףB, ISIGTA, CфLISN, PTHETA, FISGEN, GAMGEII Variables required: MDGEM, MEDIE, MED2E

Variables changed: MDXSEC

Subroutine GIMED (MDGEGM, MDXSEC)

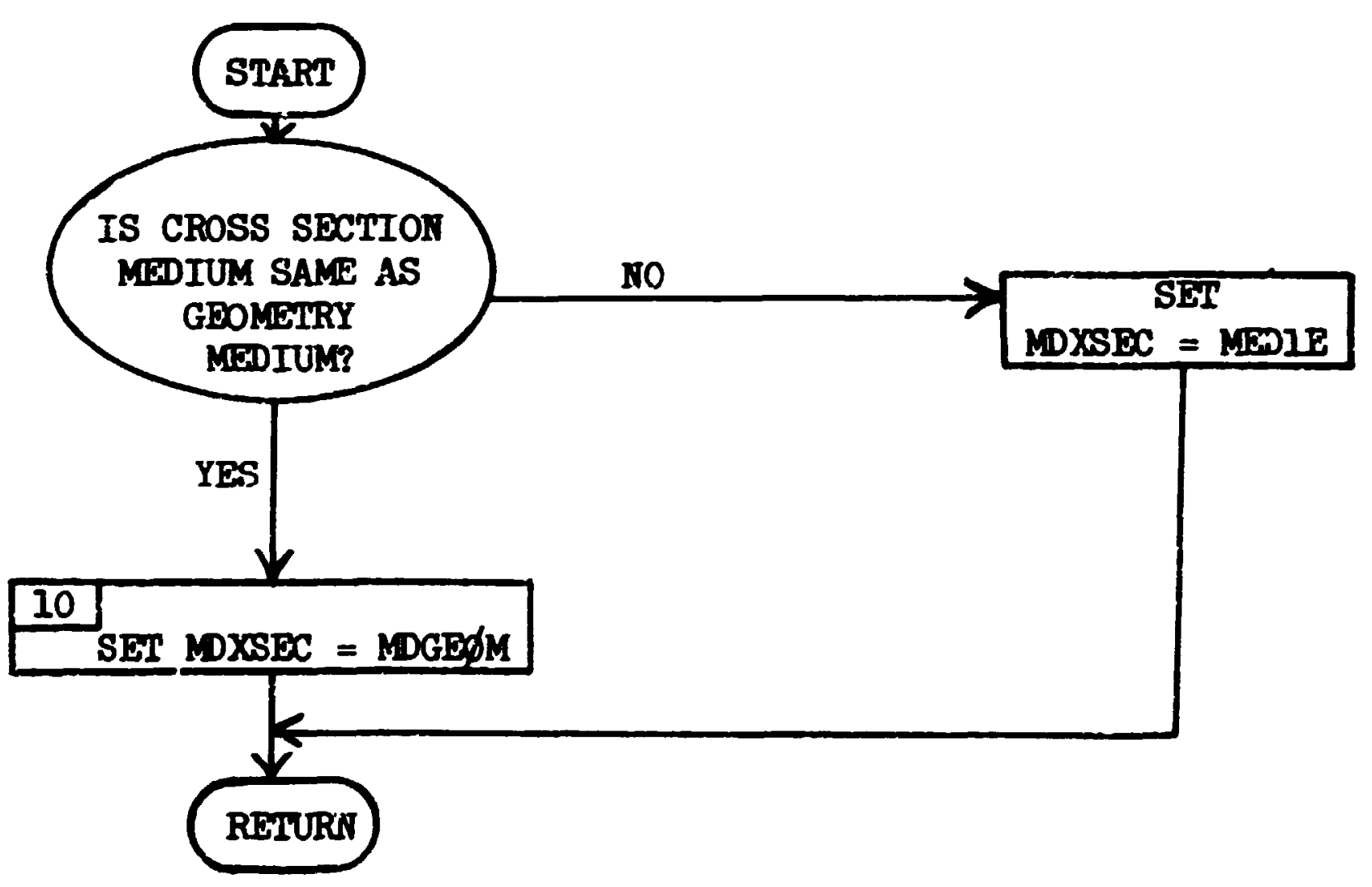


SUarout IWE GTEED(NDGEOM, MOXSEC)

GTMED 10 DATA MEDIE/ I/ . MEOZE/ $2 /$

GTMED 20

IF (MOSEOH - NEOZE) IC.S.1C

5 MOXSEC - MEOLE

RETUNN

13 moxSEC = Magon

RETURN

GTMED 40

GTMED 50

GTME0 60

ENO

GTMED 70

GTMEO 20 
Subroutine ABATCH (NSORC)

This routine is culled at the end of each butch to perform the sums needed for calculation bf batch statistics. Provision is made, although not used in this case, for batches of different sizes. Beceuse of this, the summation of the square of the accumulated estimate is difided by the number of particles starting the batch. (See VARI writeup for statisticai formulae.)

Called fron: BAIKR (-3)

Conmons required: DET

Variables required:

KD, UD(I) , SUD(I) , SUD2(I), SD(I), SSD(I), SSD?(I) (ercon comin DET, see page 169)

IS $\$$ RC - number of particles beginning the batch.

Variables modified: $\operatorname{SUD}(I), \operatorname{SUD} 2(I), \operatorname{SSD}(I), \operatorname{SSD} 2(I)$.

Subroutine NBATCH (IRDPC)

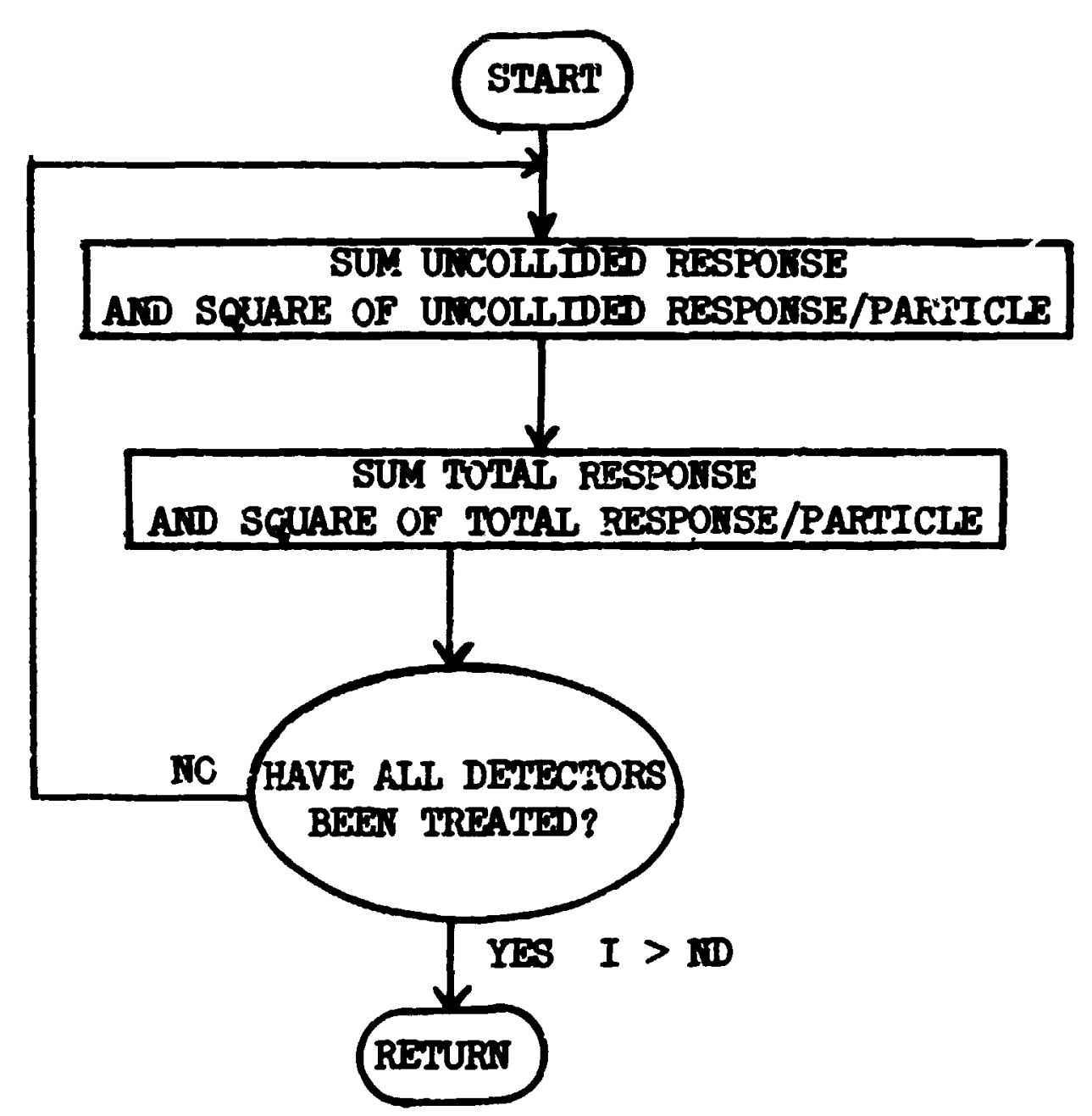




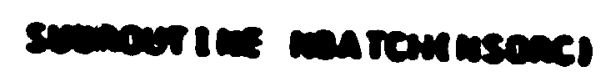

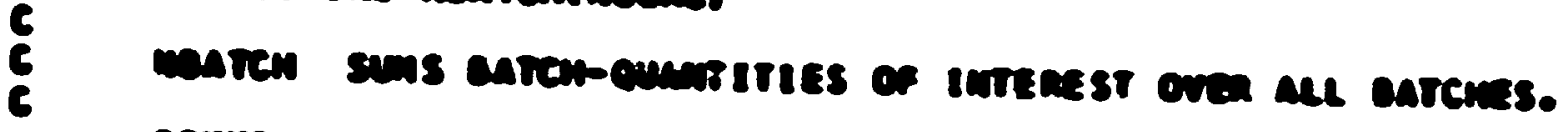

mave 10 min 8 mate is mane क mine of mine 8 minte 5 mine 10 mane os mure of mis 20 mas 180 mor in ming mas 20

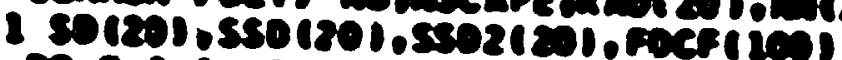
- 5 jelolio

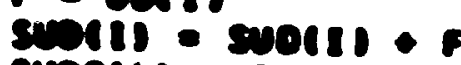

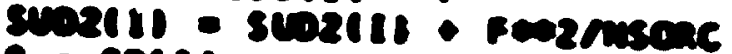
- $\operatorname{sen} 81$

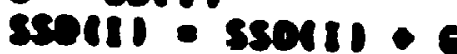

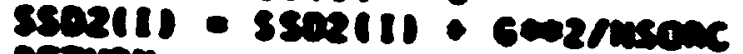
arring 
Subroutine Diur (migus, aquTr)

This routine is cailed at the end of each run (consisting of ununs batches of usitir particles in this case). The calculated quantities are normalized and output, alons witb ractional standard deviations.

Celled rram: Buran(-4)

Subroutines called:

VARI - calculases fractionel standard deviations. Commos required: DES, USER

Variables required:

IRUS - nuber of batsbes completed (note the IITS in camon USER Is the requested nuber of batcbes, not necessarily the actual number completed).

MQUIT - nuber of runs remining plus one, or negative of the number of runs completed when an execution tine kill occurs, MSTRT - nuber of particles per batch, $\mathrm{ID}, \operatorname{SUD}(I), \operatorname{SUD}(I), \operatorname{SSD}(I), \operatorname{SSD} 2(I), M(I), R A(I), \operatorname{MSCAPB}(\mathrm{Tr}$ comon DST, see page 169)

Yariables changed:

$\left.\begin{array}{l}\text { SIm2(I) } \\ \text { SSD2(I) }\end{array}\right\} \begin{aligned} & \text { conrerted to trectionel stenderd deriettans by } \\ & \text { VARl }\end{aligned}$ $\left.\begin{array}{l}\operatorname{SUD}(I) \\ \operatorname{SSD}(I)\end{array}\right\}$ norwalized to unit source particle

Variables output: $\operatorname{RAD}(I), \operatorname{SUD}(I), \operatorname{SUD} 2(I), \operatorname{SSD}(I), \operatorname{SSD} 2(I), \operatorname{PI}(\min (I)$ normalized 2 


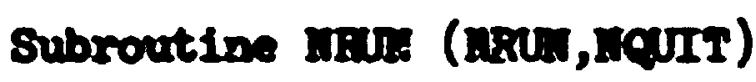

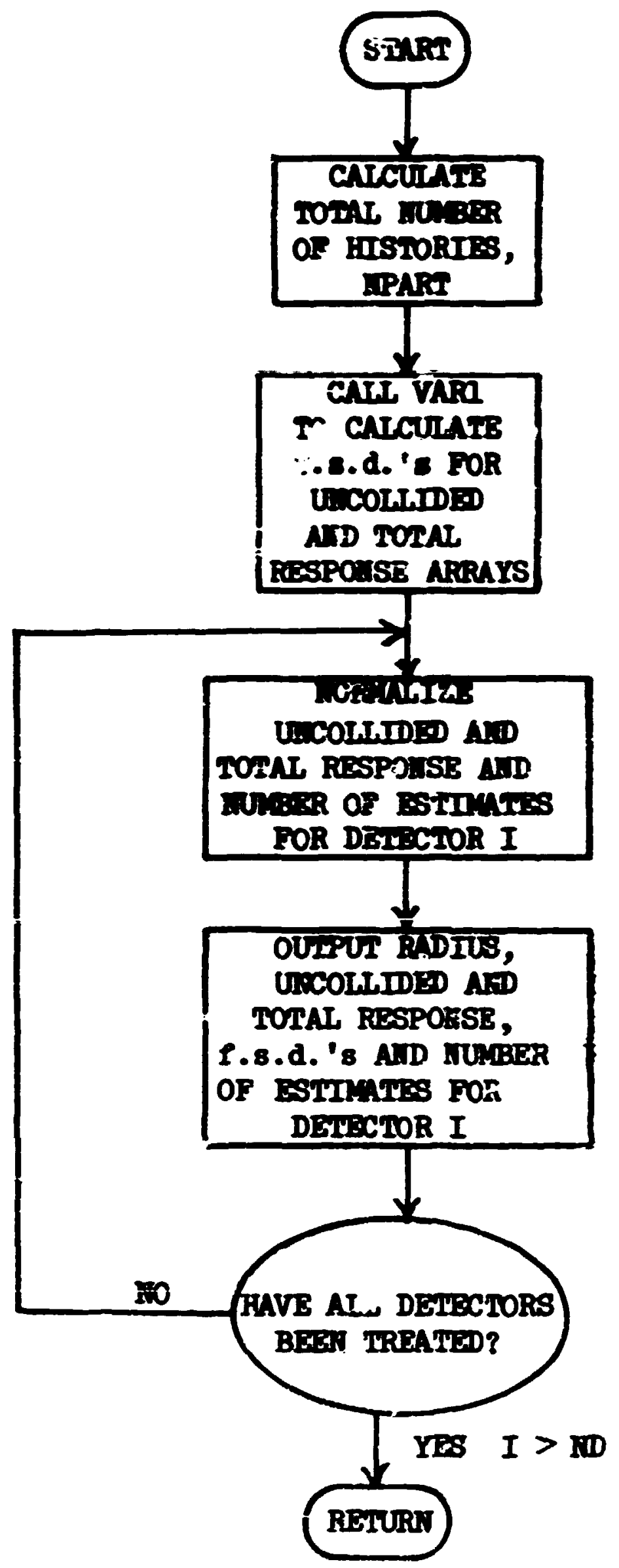





\section{Subroutine SCðRIN}

This rutine is called by subroutine INPUT for the user to input necessary aralysis data. In this sample, a title card, the number and radii of detectors, snd values of the response function are raad in and oircput.

Called from: INPUT

Commons required: USER, DET

Variables input snd output:

KØMENT - 80 hollerith characters,

ND - number of detectors,

$\mathrm{RAD}(\mathrm{I})$ - radii îor $\epsilon a c h$ of ND detectors,

FDCF(I) - NGPQT3 (=NGFQTN in this case) values if the response function. 
Subroutine SCфRTI

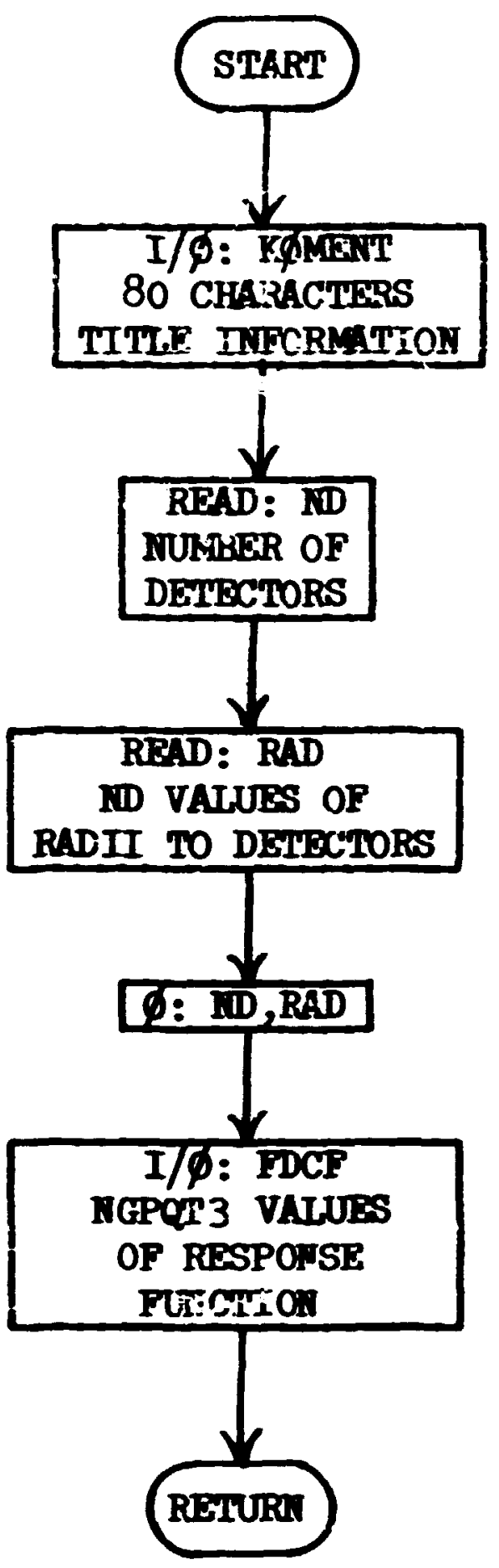


COMMON /USER/ AGSTRT, WTSTRT,XSTRT, YSTRT, 2STRT, DFF, EBOTA,EBOTG,

1 TCUT, I2, 11 , IADJM, NGPOT I, NGPQT2, NGPOT 3 , NGPOTG, NGPQTN, NITS, NLAST,

2 NLEFT, NMGP, NMTG, NSTRT

COMMON IOET/ ND,NSCAPE,RAD(20),NN(20), UD (20), SUD(20), SUD?' 201,

1 SO $(20), S S O(20)$, SSD $2(20)$, FDCF $(100)$

DIMENSION KOMENT (20)

READ (II,1000) KOMENT

$c$
$c$
$c$
$c$
$c$
$c$

FORMAT (20A4)

READ IN PROBLEM OUTPUT PARAMETERS

WHERE

ND = NUMBER OF DETECTORS

REAO $(11,1010)$ ND

1010 FORMAT $(8110)$

$c$
$c$
$c$
$c$

READ IN DETECTOR POSITIONS (MUST CORRESPOND TO MEDIA BOUNDARIESI DETECTOR MUST NOT BE PLACED AT THE LAST MEDIUM BOUNDARY.

SCORI 60

SCORI 61

SCORI 70

SCOR I 80

SCORI 90

SCOR 100

SEOR 110

SCOR 120

SCOR 130

SCOR 140

SCOR 150

SCOR 160

SCOR 170

SCOR 180

SCOR 190

SCOR 200

SCOR 210

SCOR 220

READ (II,1020) (RADII), I=1,ND)

1020 FORMAT (TE20.4)

READ IN NGPOT 3 VALUES OF THE RESPONSE FUNCTIONS

REAC $(11,1020)(F D C F(1), I=1, N G P Q T 3)$

C

WRITE $(12,1030)$ KOMENT

1030 FURMAT (IHI,20A4)

WRITE $(12,1040)$ ND, (RAD $I), I=1, N D)$

1040 FORMAT $(1 \mathrm{HO}, 19$ HNUMBER OF DETECTORS, $14 / 20 \mathrm{H}$

1 (1PSE 14.3))

WRITE (I2,1050) (FOCF (I),I=1,NGPQT3)

1050 FORMAT 11 H2, RESPONSE FUNCTION

RETU.SN

SCOR 230

SCOR 240

SCOR 250

SCOR 260

SCOR 270

SCOR 280

SCOR 290

SCOR 300

SCOR 310

SCOR 320

SCOR 321

SCOR 330

SCOR 340

SCOR 350

ENC

$1.6(1$ PE 14.31$)$

SCOR 360 


\section{Subroutine SDATA}

Cajied by BANKR(1), from MSøUR, for each source collision, this routine calculates uncollided response for each detector.

Calied from: BANKR(1)

Subroutines called: ITSIGTA

Functions required: EXP (library)

Cormons required: USER, DET, NUTRdi

Variables required:

IG - energy group index,

MLRD - nediun number,

TSIG - total cross section providec by HSIGTA,

ID - number of detectors,

RAD(I) - array of detector radii,

NATE - neutron veight,

FDCF(I) - array of response functions

Variables modified:

$L D(I)$ - array of uncollided responses. 
Subroutine SDATA

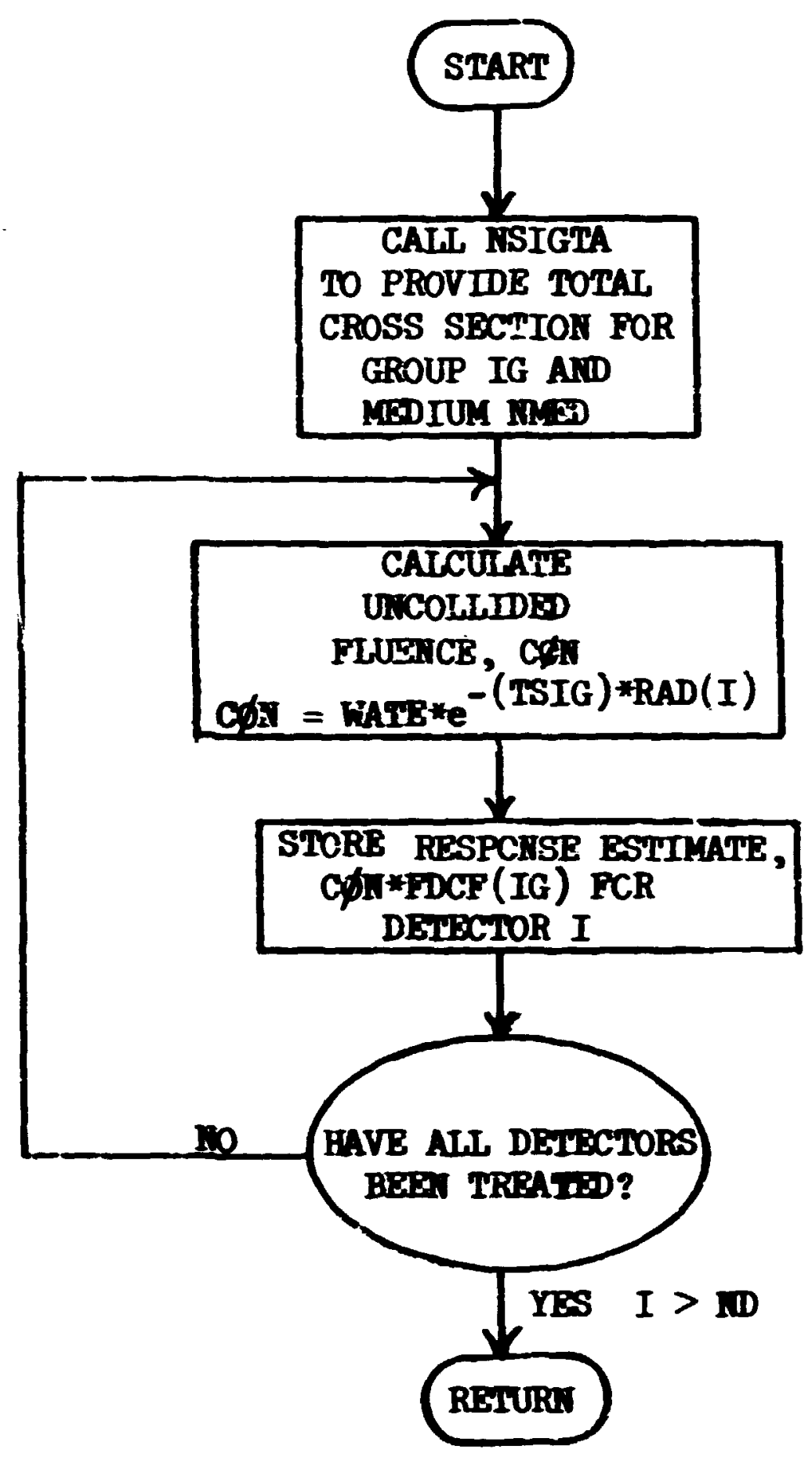




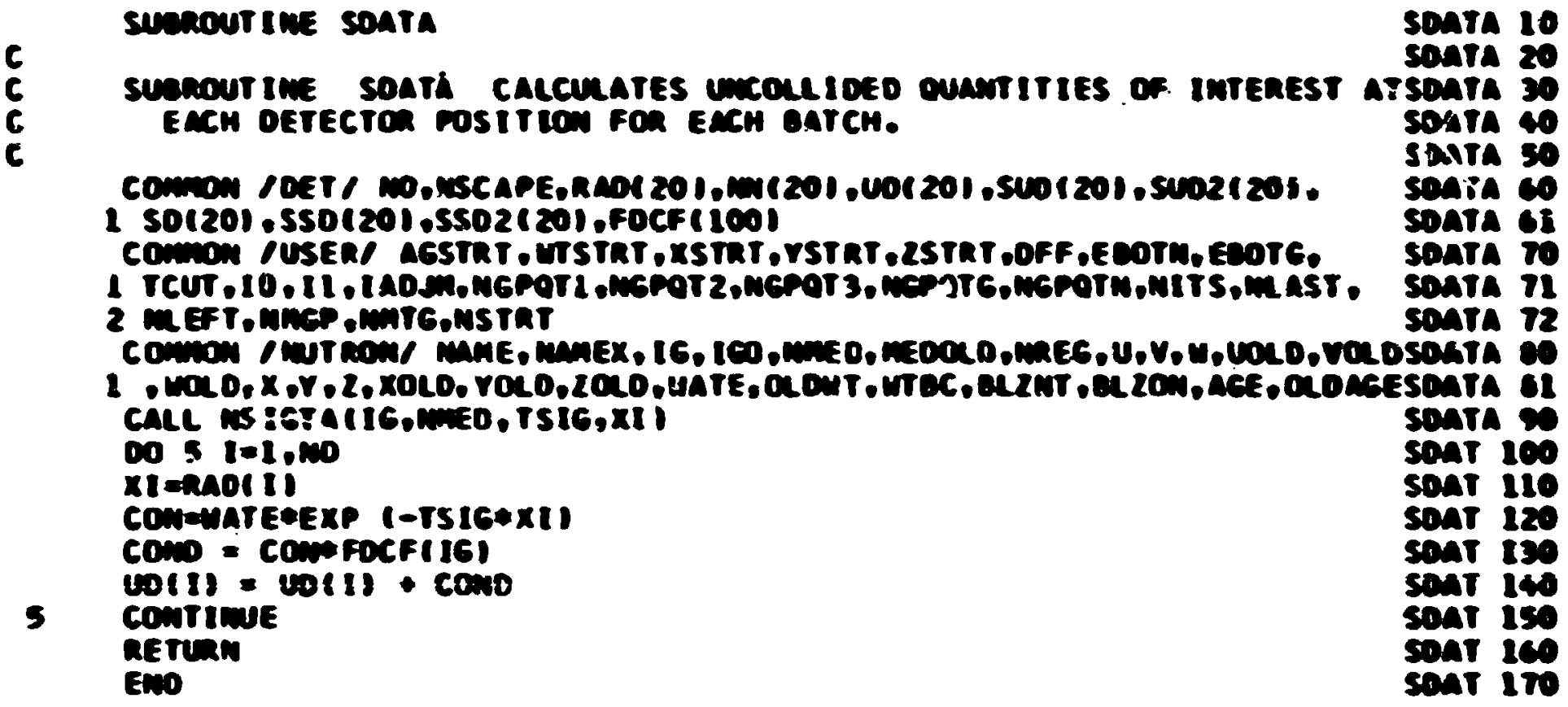


Subroutine SAURCE (IG, U, V, $W, X, Y, Z$, WATE, MRD, AG, IS申UR, ITSTR, IGPQT3, DDP, ISBIAS, NITG)

This subroutine determines the initial paraneters foi all primary particles. If the variabler which are input to MRSE are not altered by SfURCB tben those input parameters are used for every particle. If a fission problem is being considered, the particle group at the time SqURCE is called is the group causing ine fission event and the source energy group for the new particle must be reset. The version of source discussed here merely selects from an input ener spectrü. An option to select from a biased ener $y$ distribution is provided. The veight correction for selecting from the wodified distribution is given by the ratio of the natural probability to the biased probability at the selected energy group.

Called from: MSQUR

Camons required: Blank

Variables required:

ISqUR - a switch which deternines the type of source - see IIPUT, ITSTR - a switch which indicates whether fission is an originai source particle or a jaughter (irrelevant in this problen),

IGPQP3 - total nuber of groups orcr wich the problen is defined, DDF - starting weight corrected for source being defined over different nuber of groups than actually being ised in the problen,

ISBIAS - switch indicating if biased sapling is used for source enersy,

MitG - total numer of groups.

Variables changed:

WITE - particle source veight,

IG - particle eners group.

Significant internal variables:

WT - location of group zero source probability (either biased or unbiesed).

Linitations: This version only seiects an erers roup. 
Subroutine SфURCE ( IG,U,V,W,X,Y,Z, WATE, MIED , AG, IS $\varnothing$ UR, ITSTR RGPQT3,DDF, ISBIAS, MMTG)

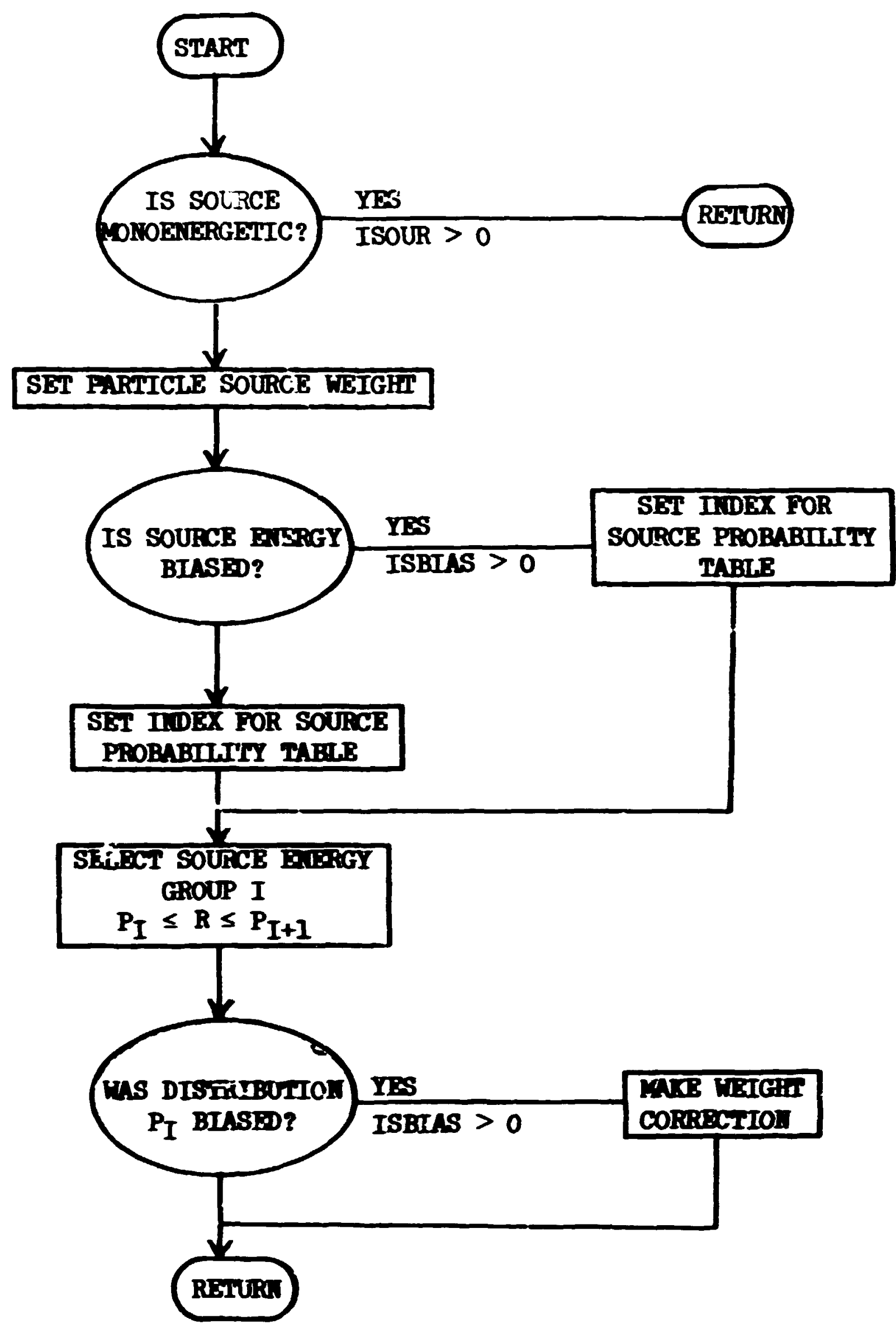




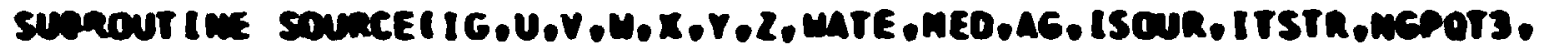
1 DDF. ISEIAS omitGI

$\operatorname{son} 10$ sounc 18 sounc 20

$c$
$c$
$c$
6

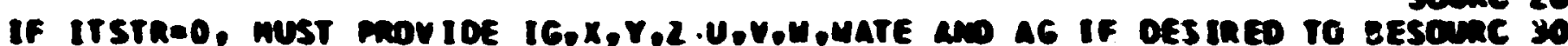

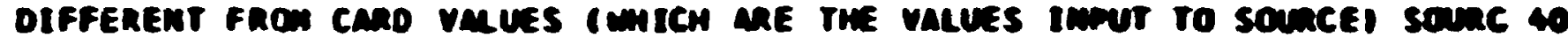

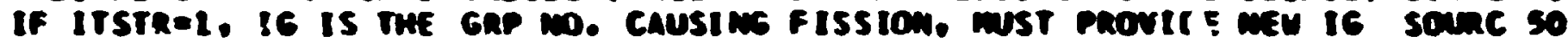

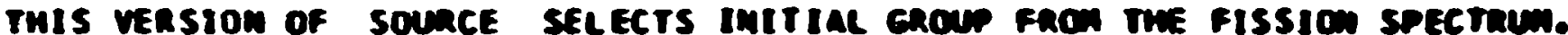

comim nis(1)

IFEISOER $15,5,50$

sounc 70

$\operatorname{son} 6$

$\mathbf{s}$

IF ITSEIASI 10.10.15

sounc so

IF ITSIASI 10.10.15

$\operatorname{son} 200$

$20 \mathrm{MUT}=2$ amis

Co 1020

15 MNT $=3 *$ mmte

$20 \quad n$ Fltantial

0025 I- L.NGOOT3

IF IA.LE.UTSETAWTII Co TO 30

$\operatorname{son} 210$

$\operatorname{son} 120$

$\operatorname{son} 130$

$\operatorname{son} 140$

$\operatorname{sen} 150$

COMTINDE

$30 \quad 16=1$

IF IISOIAS) 50.50 .35

35 IF $\mid 1-1) 50.40 .45$

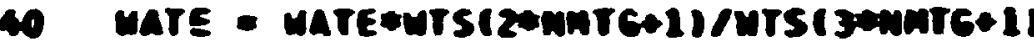

$\operatorname{son} 260$

$\operatorname{son} 170$

$\operatorname{son} 100$

$\operatorname{sov} 180$

$\operatorname{son} 200$

neronn

$\operatorname{sos} 210$

$\operatorname{son} 220$

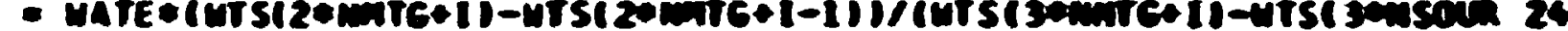
InTG+1-111

So neturn ENo

sock 241

sour 250

sorn 2c0 


\section{Subroutine STBTCH (NBATCH)}

The arrays used to accumulate uncollided and total respczse are zeroed $t_{j}$ this routine. In addition, if NBATCH $=0$ indicating the first batch in a rur is about to begin, all arrays are zeroed which accumulate estimates and squared estimates over batches.

Called erom: MøRSE

Subroutines called: ERR

Commons required: DET, USER

Va:iables requirea:

NBATCH - batch number less one,

ND - number of detectors.

Variables modified:

MSCAPE, $\operatorname{MT}(I), \operatorname{SUD}(I), \operatorname{SUD} 2(I), \operatorname{SSD}(I), \operatorname{SSD} 2(I), \operatorname{SD}(I), \operatorname{UD}(I)$ (from connon LET, see page 169). 
Subroutine STBRCH (NBATCH)

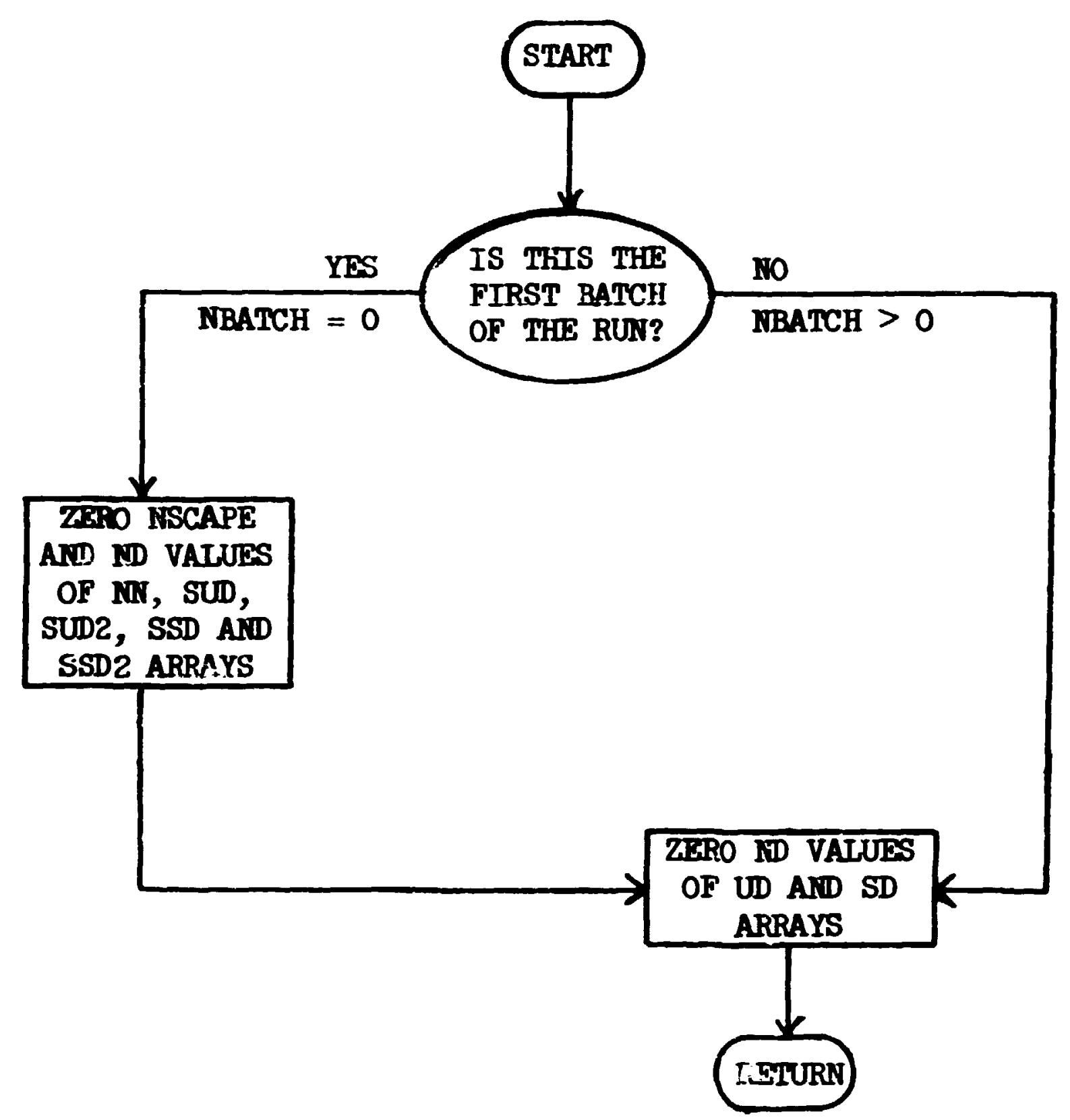


THE FOLLOWING QUANITIES ARE INITIALIZED IN STEATCH

UD(I) = UNCOLL IDED RESPONSE SUMMED OVER A SINGLE BATCH

SUD(I) = SUM OF UNCOLLICED RESPONSE SUMMEO OVER ALL BATCHES

SUD2(I) = SUM OF UD(I)**2

SDI II = TOTAL RESPONSE SUMMED OVER A SINGLE BATCH

SSO(I) = SUM OF TOTAL RESPONSE CVER ALL BATCHES

SSD2 $(I)=$ SUM OF SD $(I) * * 2$

WHERE

I IS THE INDEX FOR DETECTORS (CM)

COMMON /OET / ND,NSCAPE,RAD (20), NN $(20)$, UD $(2 \mathrm{C}), S U D(20), \operatorname{SUD} 2(20)$,

1 SD (20),SSD (20),SSD2 $(20), F D C F(100)$

COMMON IUSER/ AGSTRT, WTSTRT, XSTRT, YSTRT, ZSTRT, CFF, EBOTN, EBCTG,

1 TCUT, I O, II, IADJM.NGPQTI, NGPQT2, NGPQTZ, NGPQTG, NGPOTN, NITS, NLAST,

2 NLEFT, NMGP, NMTG, NSTR T

IF (NEATCH) $5,10,20$

5 CALL ERROR

10 NSCAPE $=0$

DO $15 \quad I=1, N O$

NN $(1)=0$

$\operatorname{SUR}(I)=0.0$

SUD2 (I) $=0.0$

SSD $(I)=0.0$

15 SSD2.(I) $=0.0$

20 DC $25 \quad I=1, N C$

SD(I) $=0.0$

$25 U D(I)=0.0$

PETURN

STBTC 10

STBTC 20

STBTC 30

STBTC 40

STBTC 50

STBTC 60

STBTC 70

STBTC 80

STBTC 90

STBT 100

ST8\% 110

STBT 120

STBT 130

STBT 140

STBT 150

STBT 151

STBT 160

STBT 161

STBT 162

STBT 170

STBT 180

STBT 190

STBT 200

STBT 210

STBT 220

STBT 230

STBT 240

STBT 250

STBT 260

STBT 270

STBT 280

STBT 290

END

STRT 300 
Subroutine STRUI

This routine is called at the beginning of each set of NITS batches and is normally used only for problems like time-dependent fissioning systems. In this sample, it is used to print out the first 50 random numbers for assistance to users trying to duplisate the random number generator. Hote that the starting random number is saved and restored before returning.

Called from: BATKR(-1)

Subröutines called:

Rippour

FiDIII

Functions used: FLTRIF 
Subroutine STRUN

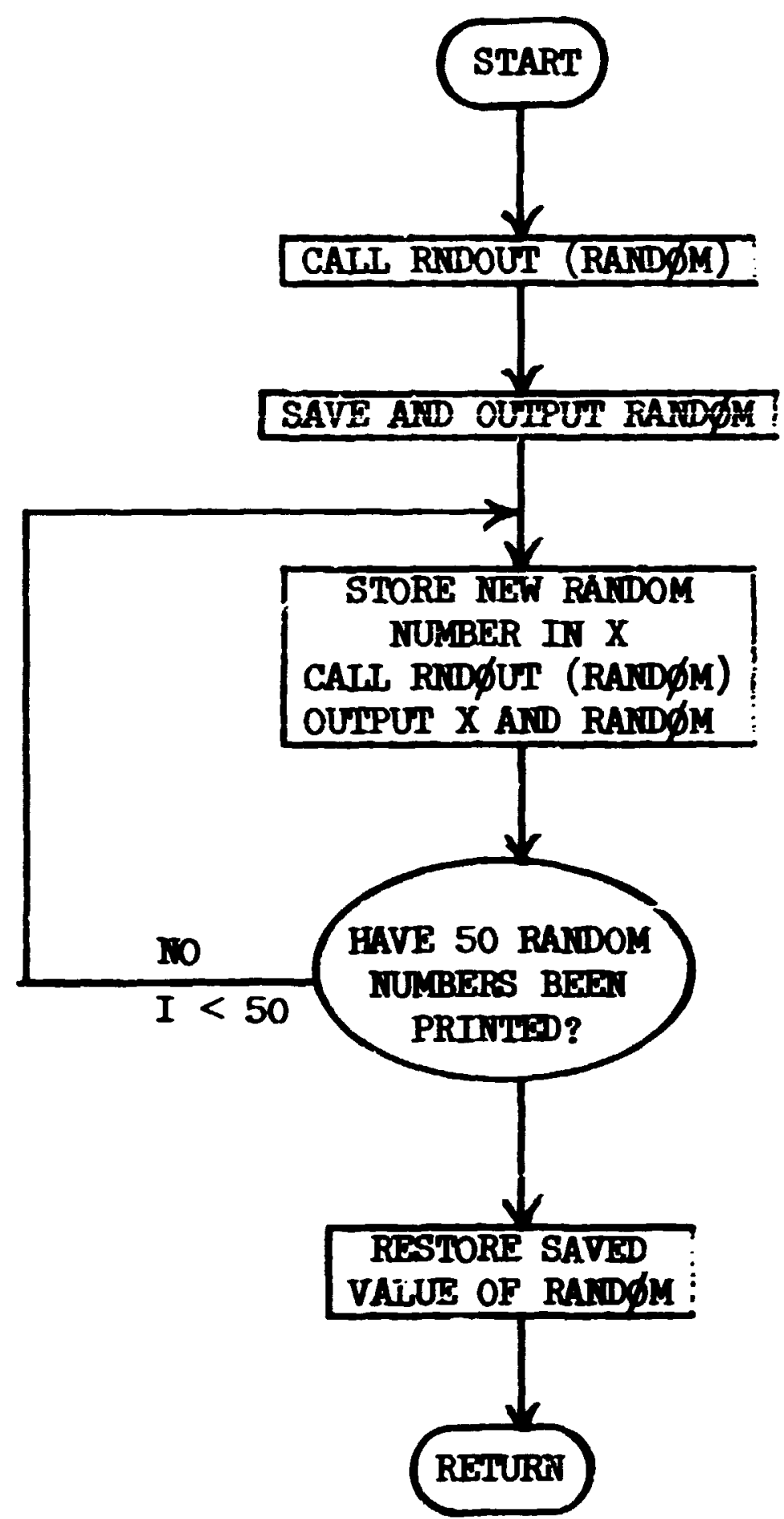


SUanoutime staun

smem 10

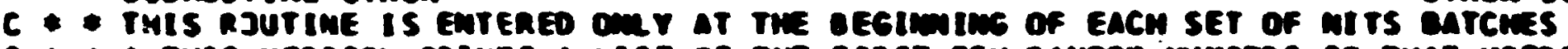

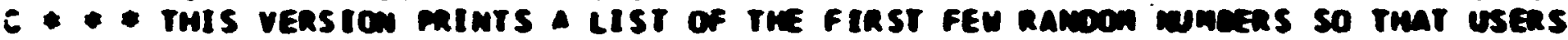

- - of vanious machines mar oune icate the ramoon mumera secuenee staum 40 REAL RAMOCH.RSAVE

CALL RMDOUT (RAKOCH)

RSAVE = RAMDOM

URITE $16.10 \mathrm{CO}$ RMOOM

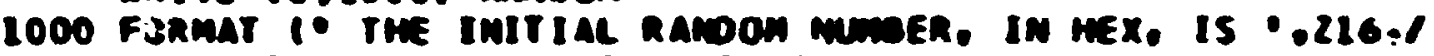

1 - TME MEXT 50 mMBEAs FOLLOW

$005 \quad 1=1.50$

$x=$ Fltrwf $(x)$

CALL RMDOUT IRANOONI

5 WRITE Q6.1005) X.RAMOON

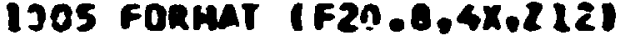

RANOOM a RAVE

CALL PNDIMI RMNOOH

RETUNM

ENO stnem 50

strum 60 staum 70 staum 00 staum 90 STrum 92 STav 100 STRU 210

STAU 120 STRU 130 stay 140 stav 150 STRU 160 sTRe ITC STRU 100 
Subroutine VARI (SX, SX2, H, IBAT, IPART)

This routine calculates rariances and fractional standard deviations (f.s.d.) for batch statistics alloring for unequallv veighted batches. The formula for the variance of the mean is

$$
\sigma_{\frac{2}{x}}=\frac{1}{(N-1)}\left(\frac{1}{n} \sum_{i=1}^{n} n_{i} x_{i}^{2}-\frac{1}{n^{2}}\left(\sum_{i=1}^{N} n_{i} x_{i}\right)^{2}\right),
$$

where $\mathrm{I}$ = number of batches,

$n=$ total number of independent histories,

$n_{i}=$ number of independent histories in the ith batch,

$x_{i}=$ accumulated estimate in the ith batch.

Note that

$$
\begin{aligned}
& n=\sum_{i=1}^{n} n_{i} \\
& x_{i}=\frac{1}{n_{i}} \sum_{j=1}^{n_{i}} x_{i j},
\end{aligned}
$$

where $x_{i j}$ is the estimate from the $j$ th history in the ith batch,

$$
\bar{x}=\frac{1}{n} \sum_{i=1}^{n} n_{i} x_{i},
$$

where $\bar{x}$ is the mean, averaged orer $n$ histories.

The fractional standard deviation is

$$
\text { r.s.d. }=\sqrt{\sigma \frac{2}{x}} / \bar{x} \text {. }
$$

Hote that the routine must be called before the array $s x=n \bar{x})$ is normalized.

Called from: NRUS

Functions required: SQRT, Aã̃ (íibrary functions).

Variables required:

$$
s X(I)-\operatorname{array} \text { of values of } n \bar{x}=\sum_{i=1}^{\pi} n_{i} x_{i},
$$


202

$\operatorname{sxe}(I)-\operatorname{arres}$ of values of $\sum_{i=1}^{M} n_{i} x_{i}^{2}$,

$M$ - number of elenents in SX and SX2,

IBAT $=\mathbf{n}$,

IPART $=n$.

Variables changed:

sX2(I) changed to I.s.d.

Subroutine VARI (SX,SXE, M, KBAT, IPART)

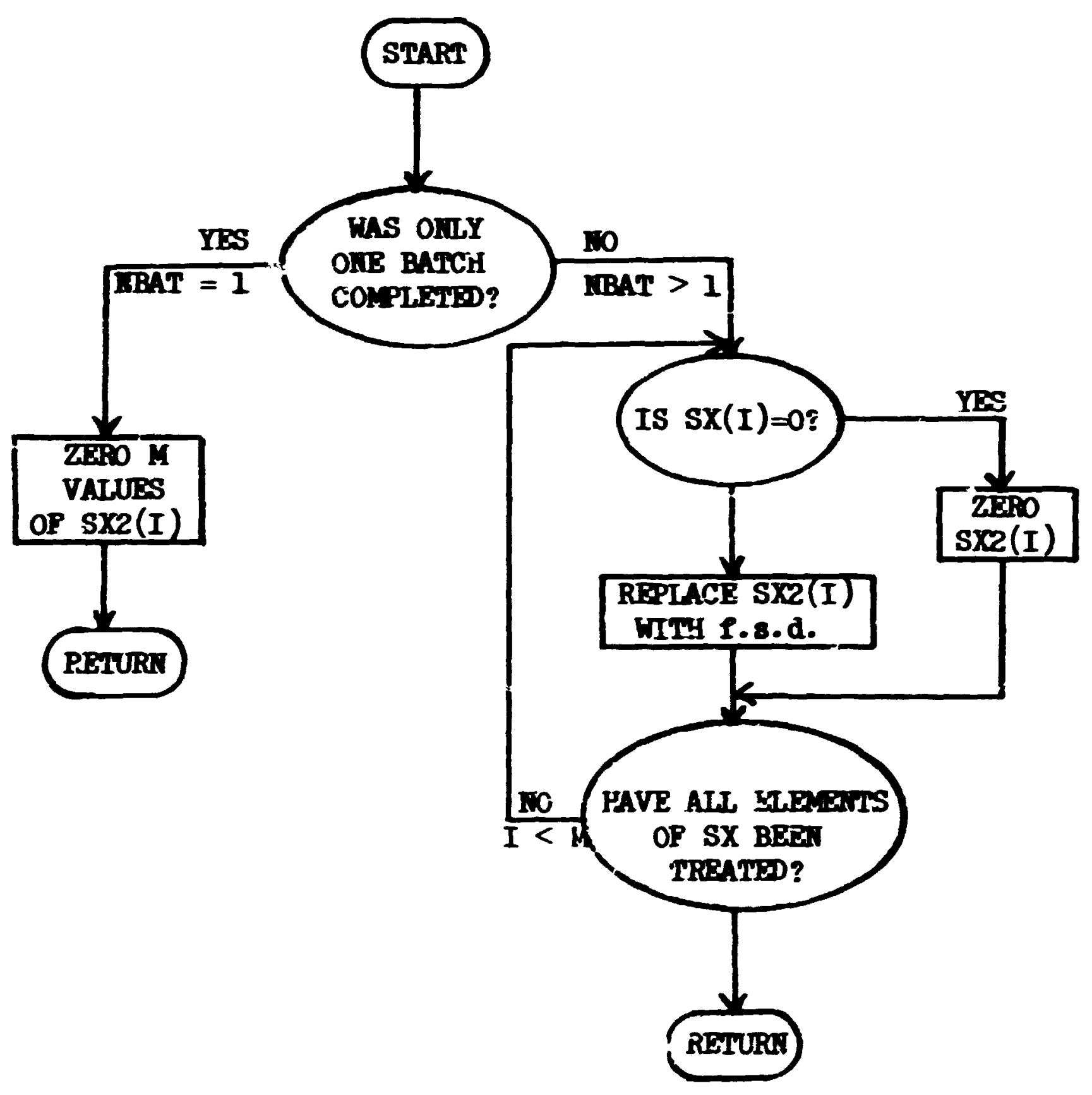




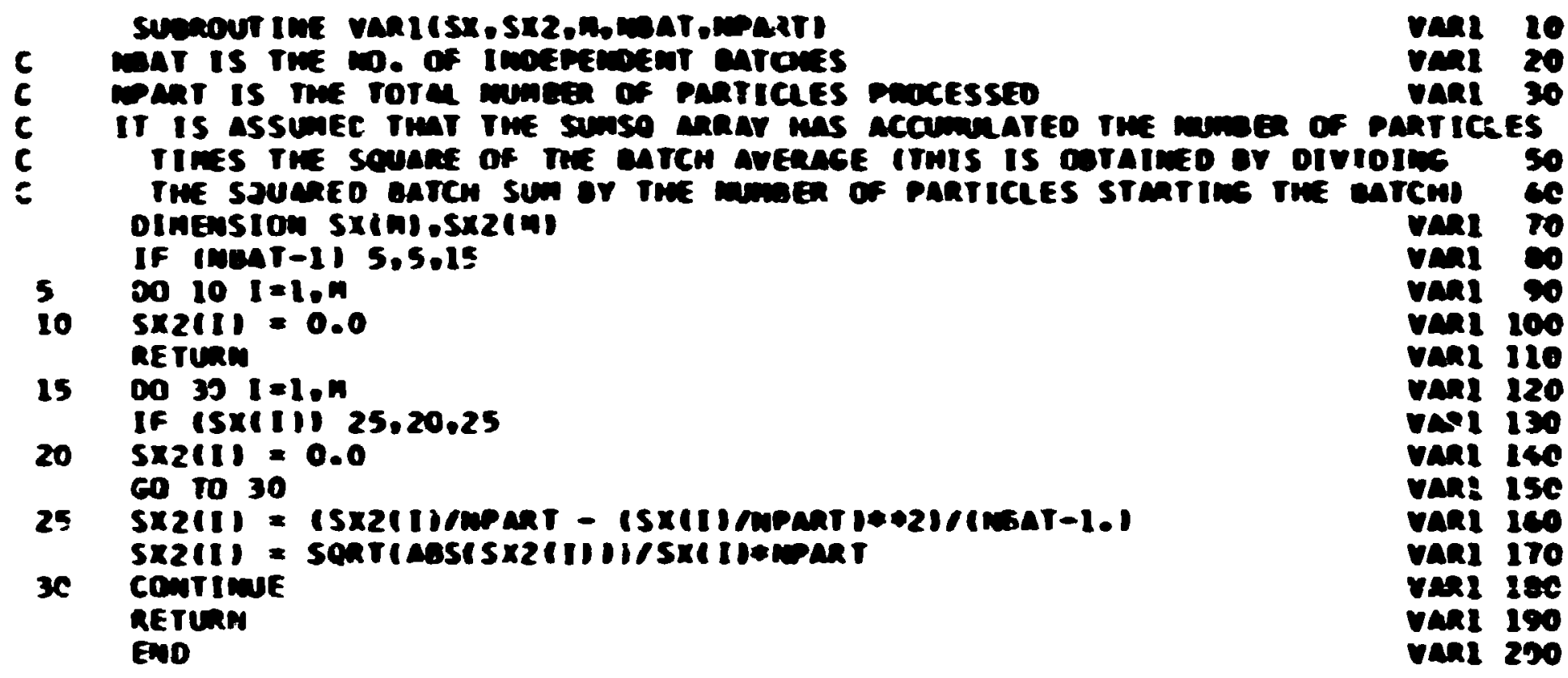




\section{Seple Problen}

The fast-neutron fluence at several radial distances is calculated for a point, isotropic, fission source in an infinite mediu of air. The air was assuned to be made up of only oxygen and nitrogen with a total density of $1.29 \mathrm{~g} / \mathrm{s}$. The special spherical geometry vas used to describe the concentric spherical shells of air surrounding the point source. Although the entire mediun was air, the geometry medium numbers alternate betveen each of the s'jells for use with the boundary-crossing estimator. This estimator requires that each detector lie on a boundary separating tro media. The cross sections for air usec in this calculation vere for 22 neutron groups with five Legendre coefficients used for the angular expansion. Only the top 13 neutron groups vere analyzed. The group structure with the corresponding fraction of particles emitted in each group is given in Table $X$. Splitting, Russian roulecte, and path length stretching vere also implemented.

The problem input and output are listed as follows: 
Table X. Pisston Spectrun in 14-Group Structure

\begin{tabular}{ccc}
\hline Group Ko. & $\begin{array}{c}\text { Energs Linits } \\
(\text { HeV })\end{array}$ & Praction of Source Meutrons \\
\hline 1 & $15.0-12.21$ & $1.5529(-4)^{2}$ \\
2 & $12.21-10.0$ & $8.9338(-4)$ \\
3 & $10.0-8.187$ & $3.4786(-3)$ \\
4 & $8.187-6.36$ & $1.3903(-2 ;$ \\
5 & $6.36-4.966$ & $3.4557(-2)$ \\
6 & $4.966-4.066$ & $3.5047(-2 !$ \\
7 & $4.056-3.012$ & $1.0724(-1)$ \\
8 & $3.012-2.466$ & $8.8963(-2 !$ \\
9 & $2.466-2.350$ & $2.3186(-2)$ \\
10 & $2.350-1.827$ & $1.2030(-1)$ \\
11 & $1.827-1.108$ & $\therefore .1303(-1)$ \\
12 & $1.108-0.5502$ & $1.3837(-1)$ \\
13 & $0.5502-0.1111$ & $1.4036(-1)$ \\
14 & $0.1111-0.3308$ & $1.5489(-2)$ \\
\hline
\end{tabular}

Read as $1.5529 \times 10^{-4}$. 


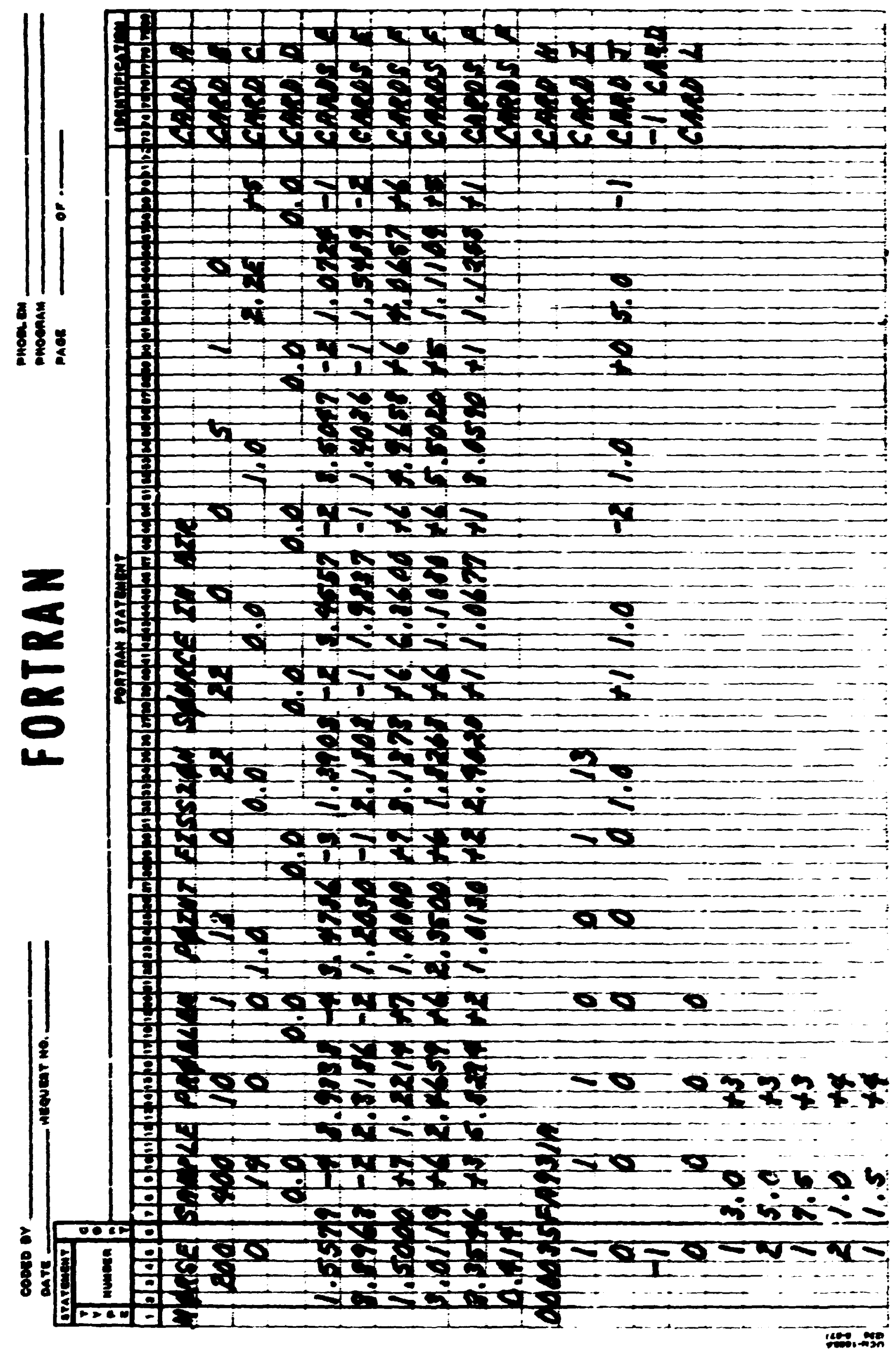




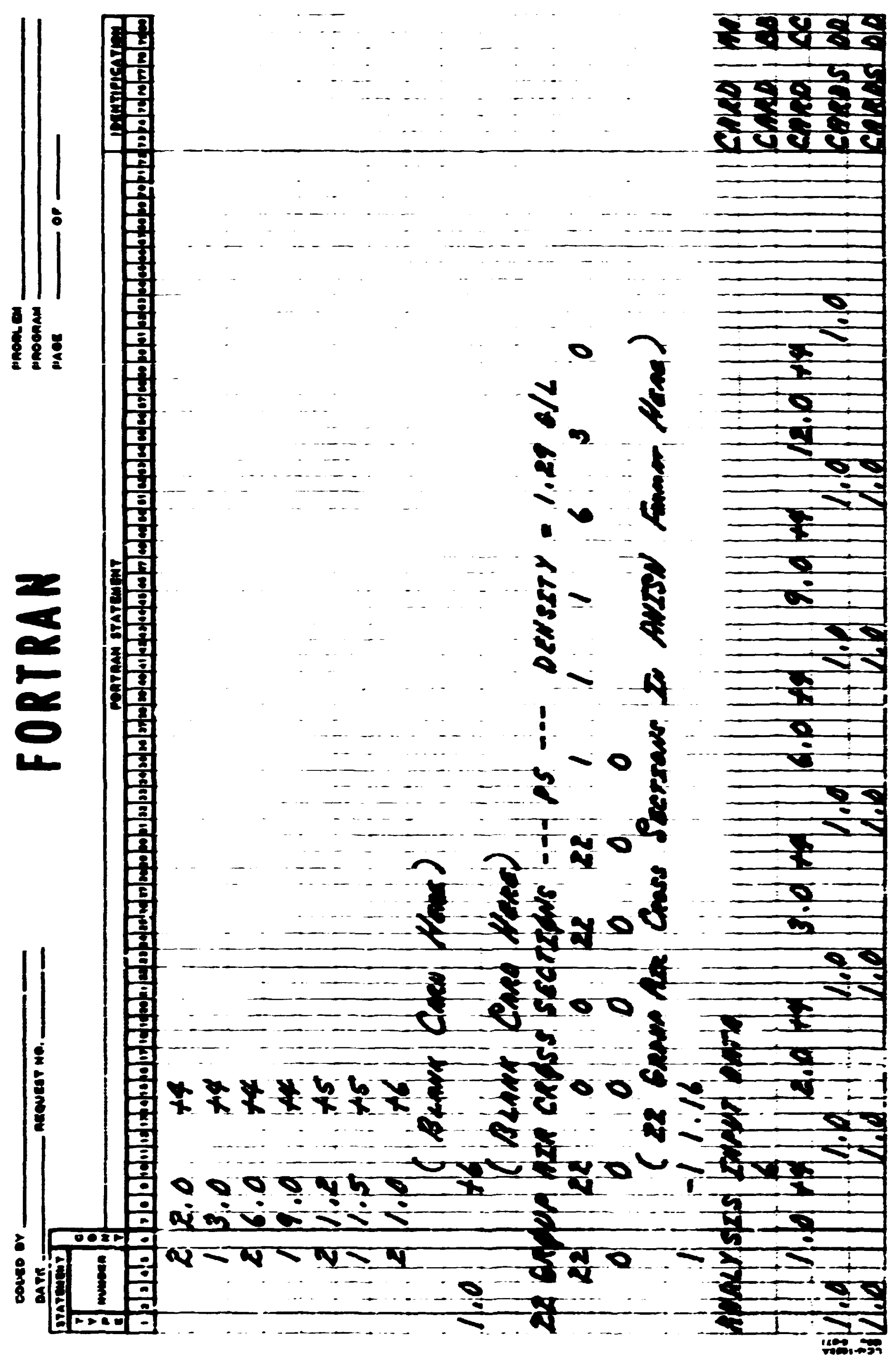


MORSE SAMPLE PROBLEM POINT FISSICN SOURCE IN AIR THIS CASE WAS BEGUN ON TUESDAY, AUGUST 4,1970

\begin{tabular}{|c|c|c|c|c|c|c|c|}
\hline 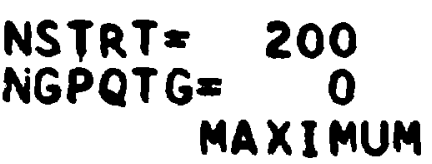 & $\begin{array}{c}\text { NMOSI }= \\
\text { NMGP }= \\
\text { EXECUTION }\end{array}$ & $\begin{array}{l}400 \\
22 \\
\text { TIME }\end{array}$ & $\begin{array}{r}\text { NITS: } \\
\text { NMTG: } \\
5\end{array}$ & $\begin{array}{l}=10 \\
=\quad 22 \\
\text { MINUTES }\end{array}$ & $\begin{array}{l}\text { NQUIT= } \\
\text { NCOLTP= } \\
\text { MEDIA= }\end{array}$ & & $\begin{array}{l}\text { NGPQTN= } \\
\text { IADJM= } \\
\text { MEDALB= }\end{array}$ \\
\hline
\end{tabular}

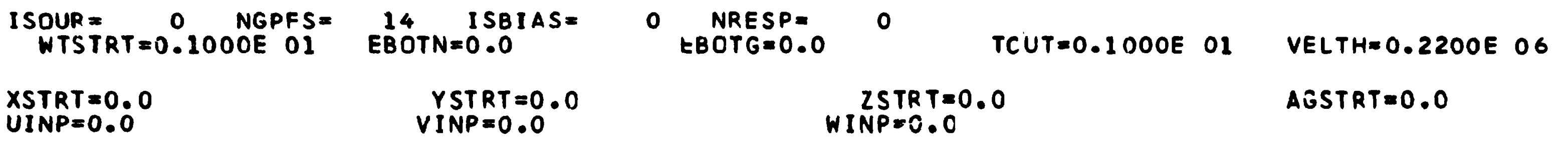

DDF IS DIFFERENT FROM WTSTRT, DDF $=0.98451$ O 00

SPECTRUM OF CUNULATIVE GROUP PROBABILITIES

\begin{tabular}{|c|c|}
\hline $\begin{array}{l}F S(1)=0.1582 E-03 \\
F S(3)=0.4599 E-02 \\
F S(5)=0.5382 E-01 \\
F S(7)=0.1984 E \text { O } \\
F S(9)=0.3123 E \text { O0 } \\
F S(11)=0.6559 E \text { O0 } \\
F S(13)=0.1000 E \text { OI }\end{array}$ & $\begin{array}{l}F S(2)=0.1066 E-02 \\
F S(\quad 4)=0.1872 E-01 \\
\text { FS }(6)=0.8942 E-01 \\
F S(1)=0.2887 E \text { OC } \\
\text { FS }(10)=0.4345 E \text { OO } \\
\text { FS }(12)=0.8574 E \text { OO } \\
\text { FS }(14)=0.0\end{array}$ \\
\hline
\end{tabular}

GROUP PARAMETERS, GROUP NUMBERS GREATER THAN 22 CORRESPONO TO SECONDARY PARTICLES 


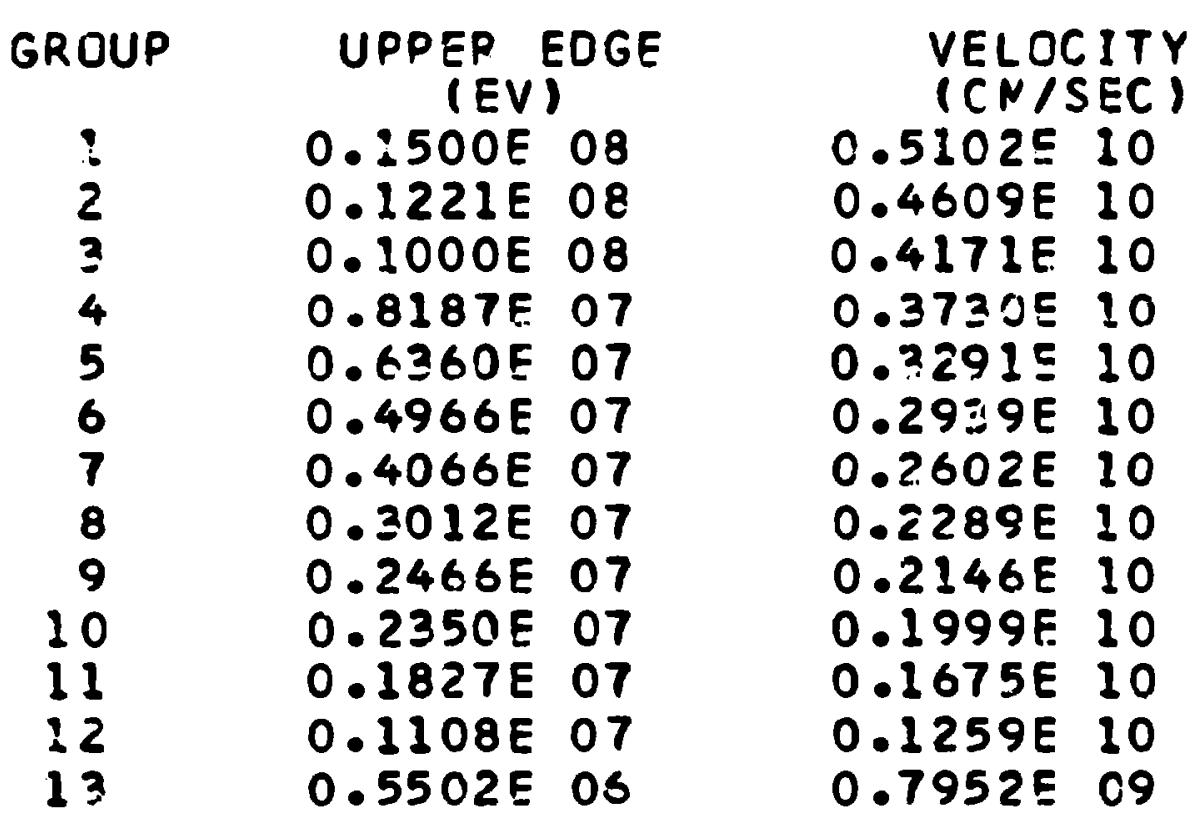

INITIAL RANDOM NUMBER = 000035FA731:

NSPLT $=1$ NKILL= 1 NPAST $=1 \quad$ NOLEAK $=0 \quad$ IEBIAS $=0 \quad$ MXREG $=1 \quad$ MAXGP=13

WEIGHT STANDARDS =OR SPLITTING ANC RUSSIAN ROULETTE AND PATHLENGTH STRETCHING PARAMETERS

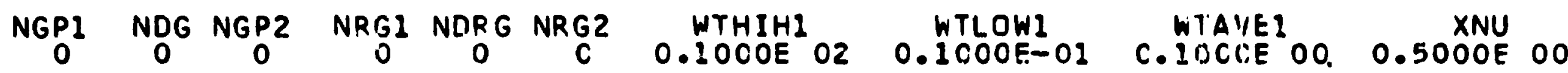

NSOUR $=0 \quad$ MFISTP $=0 \quad$ NKCALC $=0 \quad$ NDRMF $=0$ 
SPHER ICAL GEOM

\begin{tabular}{|c|c|}
\hline $\begin{array}{c}\text { MEDI UM } \\
1 \\
2 \\
1 \\
2 \\
1 \\
2 \\
1 \\
2 \\
2 \\
1 \\
2 \\
1 \\
2 \\
1\end{array}$ & $\begin{array}{r}\text { RACIUS } \\
0.30000 D \\
0.50000 D \\
0.75000 D \\
0.10000 D \\
0.15000 D \\
0.20000 D \\
0.30000 D \\
0.60000 D \\
0.70000 D \\
0.900000 \\
0.120000 \\
0.150000 \\
0.1100000\end{array}$ \\
\hline
\end{tabular}

\section{REGION RADIUS}

I $0.10000 \mathrm{O} 07$

NGEOH $=529, \quad$ NGLAST $=528$ 


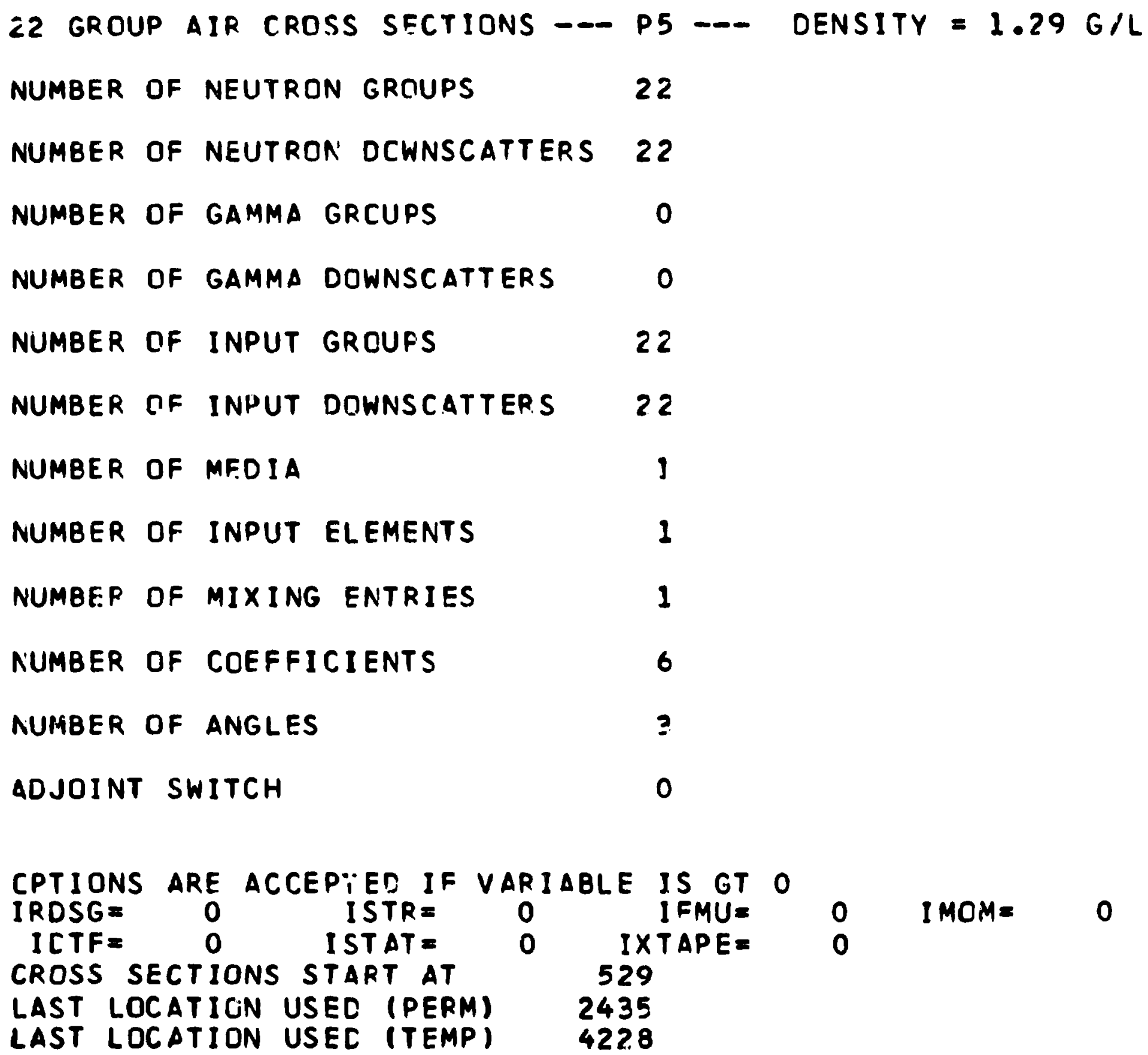

ELEMENT 1 APPEARS IN MEDIA 1 WITH DENSITY 1.16 COE 00 


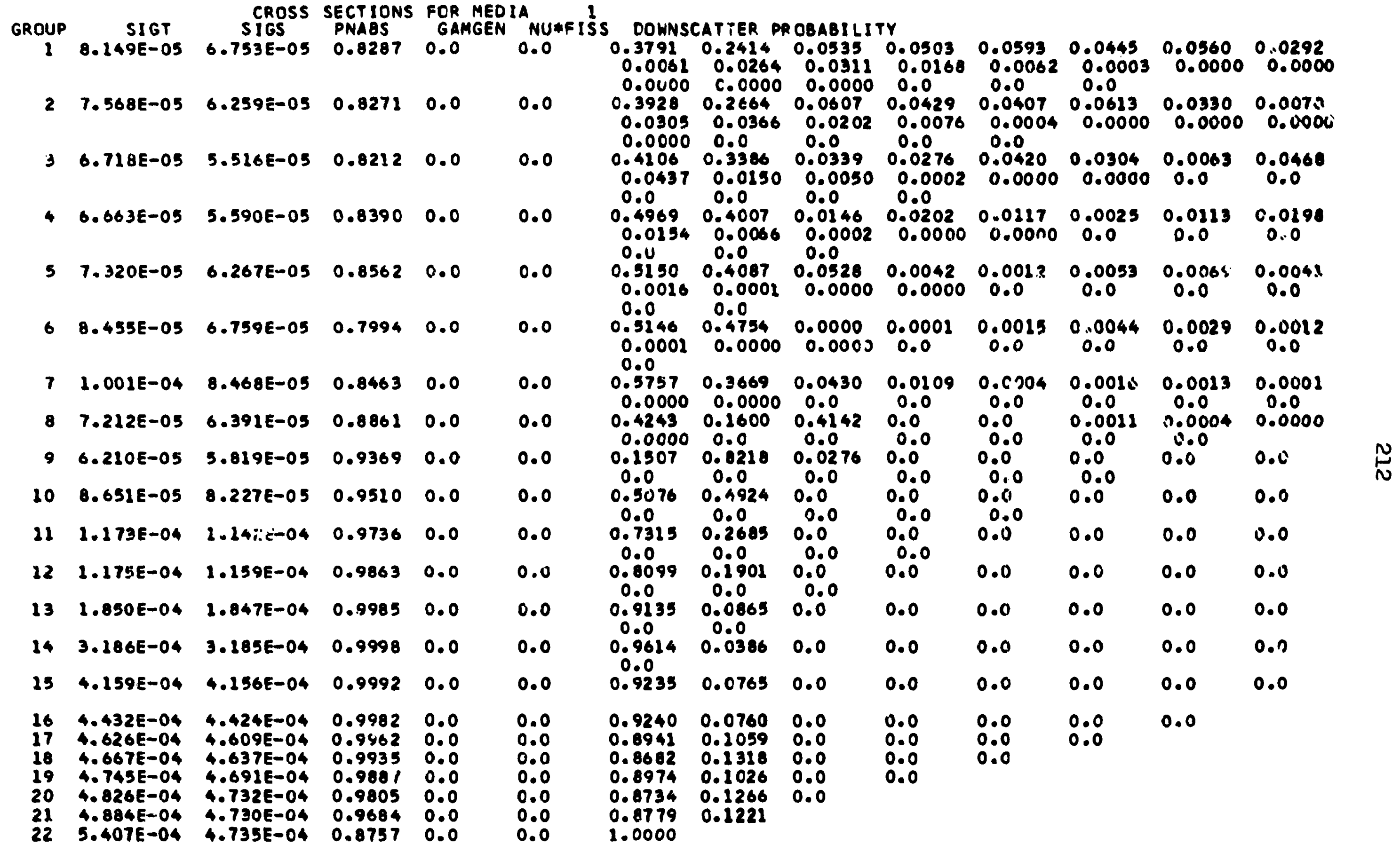

BANKS START AN 


\section{ANALYSIS INPUT DATA}

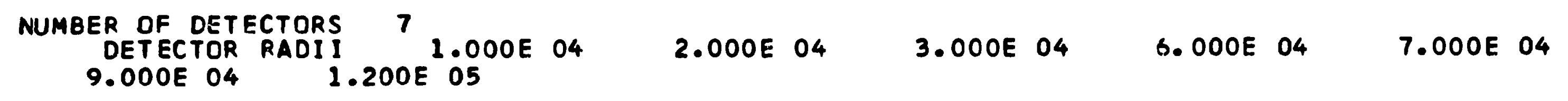

RESPONSE FUACTION

$1.000 E$ OO

$1.000 E$ OO

$1.000 E$ OO
$1.000 E 00$

$1.000 E$ OO

$1.000 E 00$

$1.000 E$ OO
$1.000 E \quad 00$

$1.000 E$ OO
$1.000 E \quad 00$ $1.000 E 00$
$1.000 E 00$

1. OOOE OO 
TIME REOUIRED FOR INPPUT HAS S SECONOS.

YOU ARE USING THE DERAUL VERSION OF STRUN HHICH DOES NGTHING.

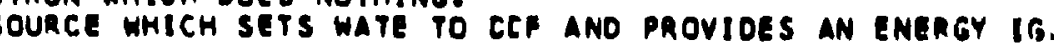

\section{mostakt gatch}

RANDOMAC AFRE 7812412

SOURCE DATA.

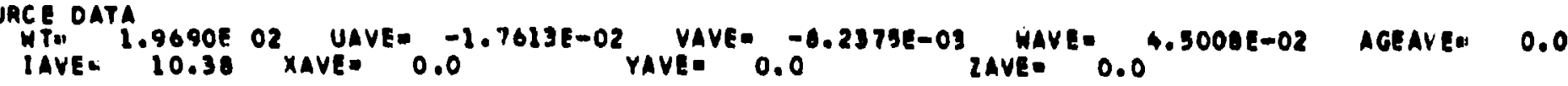

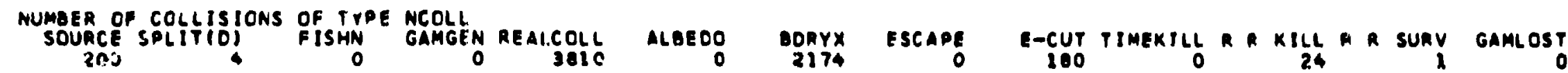

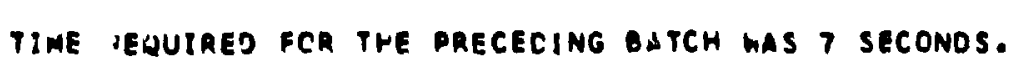

*astart oatch 2

RANDOM-A0902979ESSA

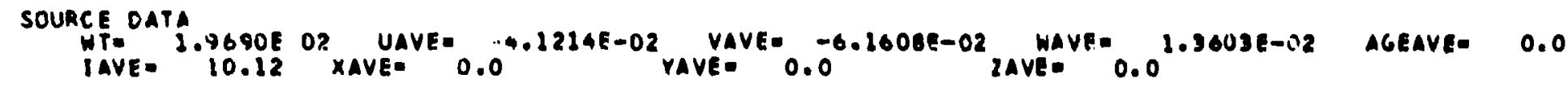

NUMBER OF COLLISICAS OF TYPE NCOLL
SOURCE SPLITIOI
200

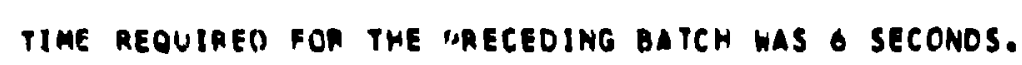

MHASTART BATCH 3

RANDOMAC BEDABQGTTZM

SOURCE DAT
IAVE-

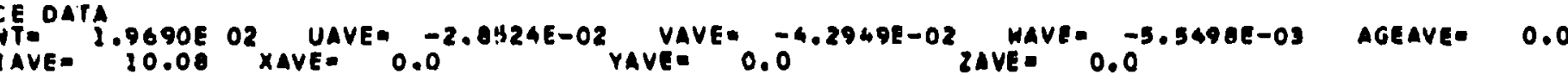

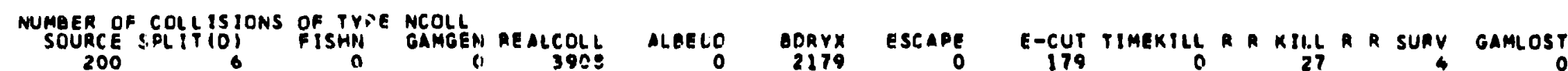
TIME REQUIRED FOR THE PNECEC:NG BATCH WAS 7 SECONOS.

ME*START BATCH \&

RANOOMO $28089 A E 380 E 2$

SOURCE OAT

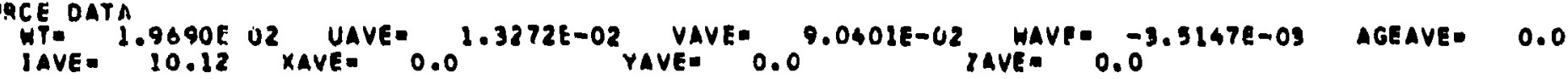

MUMBFR OF COLLISIONS OF TYPE NCFLL
SOURCE SPLITIO)
200

TIME REQUIRI: O FOR THE HRECECING BATCK WAS 7 SECONDS.

manstart batch s

RANDOMAB4032CC $3611 \mathrm{~A}$

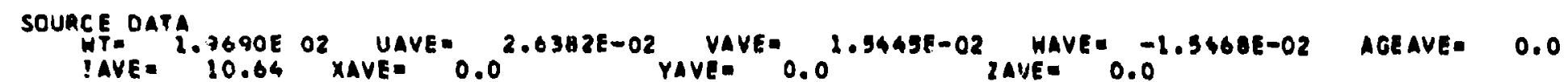

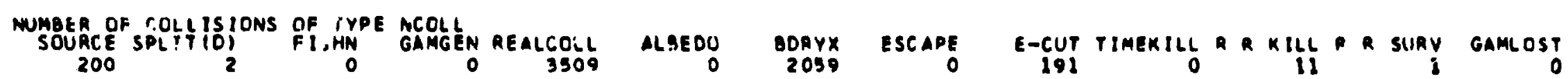


TIME REQUiRed sor the PRECECING batch was o SECONDS.

- wistart batch o

RANDOM-A2E2102437E2

SOURCE DATA

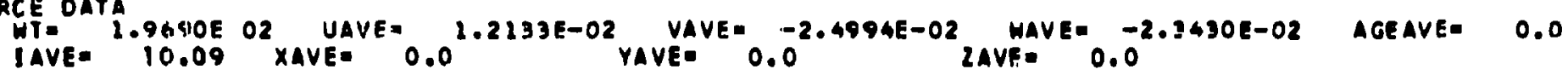

NUMBER OF COLLISIONS OF TYPE NCOLL
SOURCE SPLITIDI
200

200 REOUIRED FOR THE PRECECING OATCH WAS, SECONOS.

-amstart oatch $t$

RANDOM=423C $\triangle D C C E C C A$

SOL TCE DATA

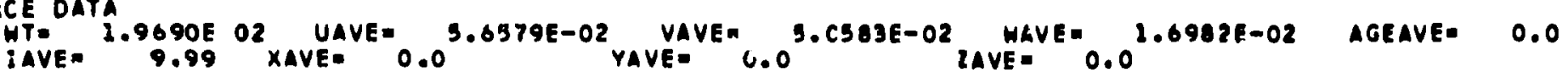

RUMGER OF COLLISIONS OF TYPE MCOLLL
SOURCE SPLITIOI
200

TIME REQUIREO for the PRECECING BatCh WAS 1 SECCNOS.

***start batch o

RANDOM-BL 9?B628586A

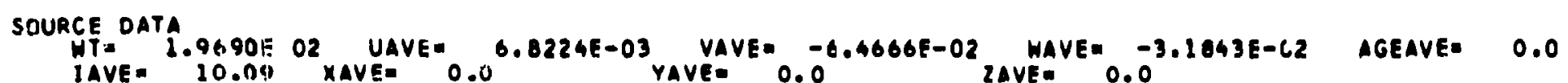

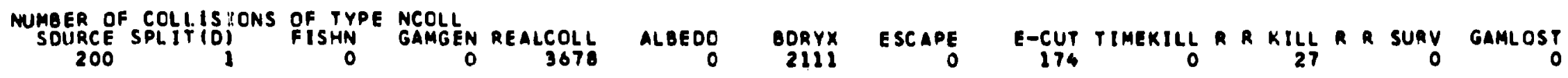

TIME REQUIRED FOR THE PRECEOING BATCH WAS 6 SECONDS.

*astakt oatch o

RANOOM=9LEBT3SOOEAA

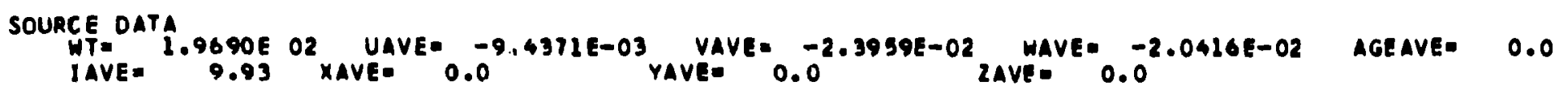

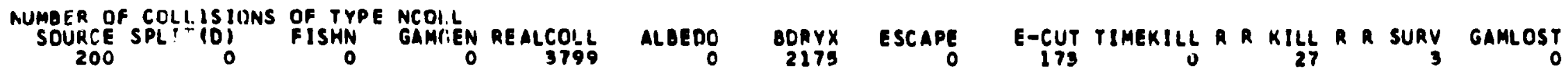

TIME REQUIRED for the PRECEDING oATCH WAS 7 SECONOS.

ow*STaRT batch 10 RanDom=106B29364432

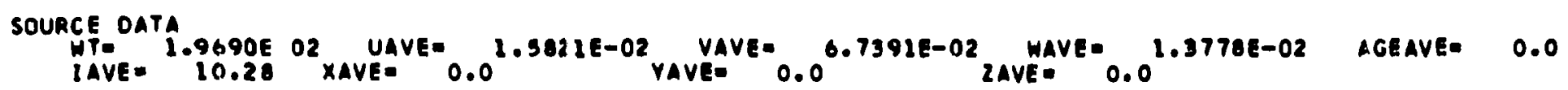

NUMAER OF COLLLSIONS OFF TYPE MCOLL
SOURC: SPLITIOI
200

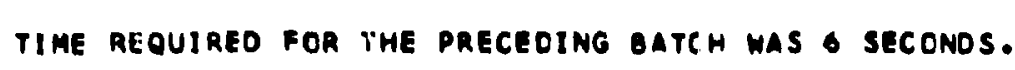




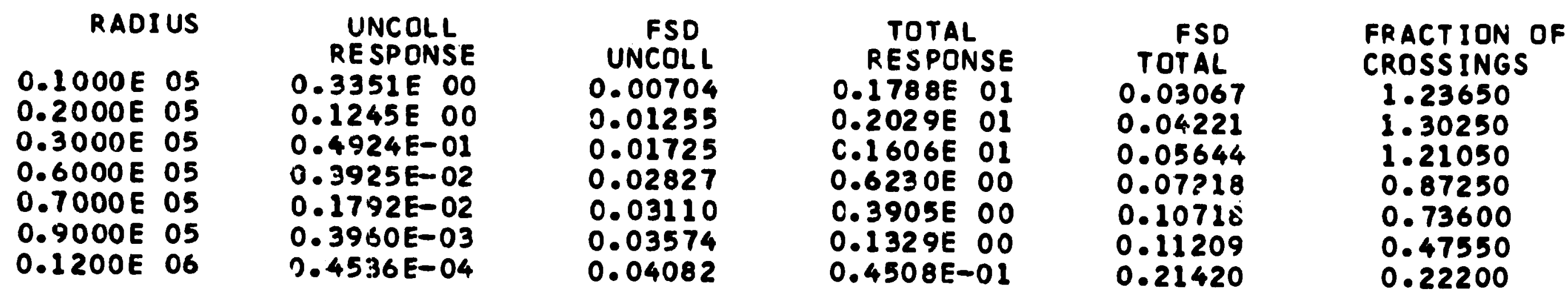

TIME REQUIRED FOR THE PRECEDING 10 BATCHES WAS 1 MINUTE, 16 SECONDS.

NEUTRON DEATHS

KILLED BY RUSSIAN POUI.ETTE

ESCAPED

REACHED ENERGY CUTOFF

REACHED TIME CUTOFF

NUMBER OF SCATTERINGS

MEDIUM

1
NUMBER

38010
NUMBER

WE IGHT

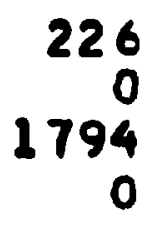

$$
\begin{array}{ll}
0.15116 E & 01 \\
0.0 & \\
0.13430 E & 04 \\
0.0 &
\end{array}
$$


REAL SCATTERING COUNTERS

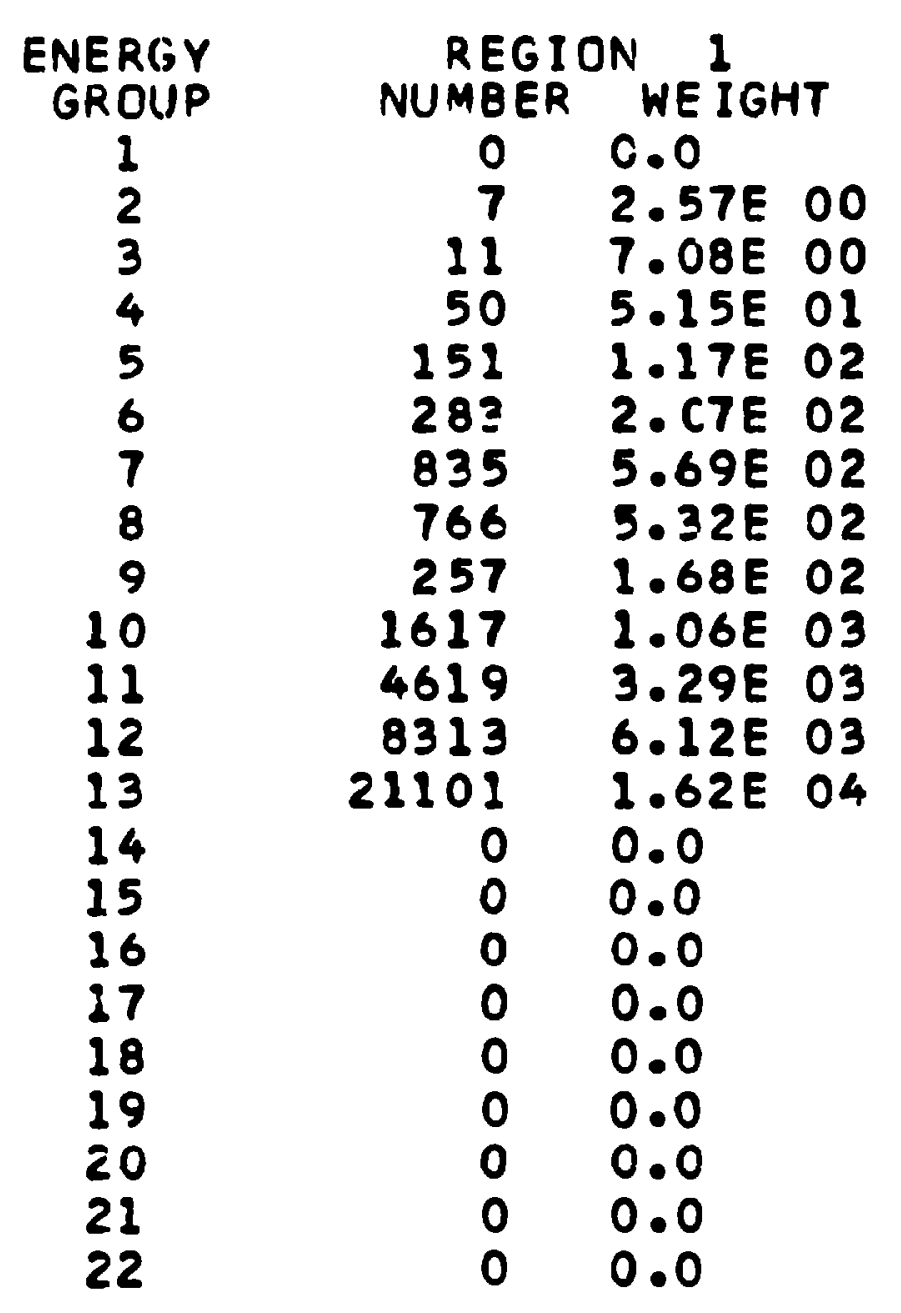


AUMBER OF SPLITTINGS

\begin{tabular}{|c|c|c|}
\hline $\begin{array}{c}\text { ENERGY } \\
\text { GROUP } \\
1 \\
2 \\
3 \\
4 \\
4 \\
5 \\
6 \\
7 \\
8 \\
9 \\
10 \\
11 \\
12 \\
13\end{array}$ & $\begin{array}{c}\text { REGIC } \\
\text { NUMBER } \\
0 \\
0 \\
0 \\
0 \\
0 \\
0 \\
0 \\
0 \\
0 \\
0 \\
0 \\
2 \\
18\end{array}$ & $\begin{array}{l}\text { JN I } \\
\text { WEIGHT } \\
0.0 \\
0.0 \\
0.0 \\
0.0 \\
0.0 \\
0.0 \\
0.0 \\
0.0 \\
0.0 \\
0.0 \\
0.0 \\
1.39 E \quad 01 \\
1.09 F \quad 02\end{array}$ \\
\hline
\end{tabular}

NUMBER OF SPLITTINGS PREVENTED BY LACK IT ROOM

\begin{tabular}{|c|c|c|}
\hline $\begin{array}{c}\text { ENERGY } \\
\text { GR OUP } \\
1 \\
2 \\
3 \\
4 \\
5 \\
6 \\
7 \\
8 \\
9 \\
10 \\
11 \\
12 \\
13\end{array}$ & $\begin{array}{cc}\text { REGION } \\
\text { NUMBER } \\
0 & 0 \\
0 & 0 \\
0 & 0 \\
0 & 0 \\
0 & 0 \\
0 & 0 \\
0 & 0 \\
0 & 0 \\
0 & 0 \\
0 & 0 \\
0 & 0 \\
0 & 0 \\
0 & 0\end{array}$ & $\begin{array}{l}N \text { WEIGHT } \\
0.0 \\
0.0 \\
0.0 \\
0.0 \\
0.0 \\
0.0 \\
0.0 \\
0.0 \\
0.0 \\
0.0 \\
0.0 \\
0.0 \\
0.0\end{array}$ \\
\hline
\end{tabular}


NUPIBER OF RUSSIAN RCULETTE KILLS

\begin{tabular}{ccl} 
ENERGY & \multicolumn{2}{c}{ REGION } \\
GRUUP & NUMBEQ WEIGHT \\
1 & 0 & 0.0 \\
2 & 0 & 0.0 \\
3 & 0 & 0.0 \\
4 & 0 & 0.0 \\
4 & 1 & $4.165-0 ?$ \\
5 & 0 & 0.0 \\
6 & 5 & $3.17 E-02$ \\
7 & 7 & $3.41 E-02$ \\
8 & $i$ & $4.59 E-02$ \\
9 & 6 & $4.03 E-02$ \\
10 & 34 & $2.10 E-01$ \\
11 & $4 ?$ & $2.82 E-01$ \\
12 & 129 & $9.04 E-01$
\end{tabular}

NUMBER OF FUSSIAN RCULETTE SURVIVALS

$\begin{array}{ccl}\text { ENERGY } & \text { REGION } \\ \text { GROUP } & \text { NIMBER } & \text { WEIGHT } \\ ? & 0 & 0.0 \\ 2 & 0 & 0.0 \\ 3 & 0 & 0.0 \\ 4 & 0 & 0.0 \\ 5 & 0 & 0.0 \\ 6 & 0 & 0.0 \\ 7 & 1 & 9.98 E-03 \\ 8 & 0 & 0.0 \\ 9 & 0 & 0.0 \\ 10 & 0 & 0.0 \\ 11 & 1 & 9.94 E-0 ? \\ i 2 & 3 & 2.24 E- \\ 13 & 10 & 8.4 ! E \cdots 02\end{array}$

TOTAL CPL TIME FOR THIS PROELEM WAS 1.28 MINUTES.

\$\$\$\$\$\$\$\$\$\$ MCRSE SAMPLE PIOBLEM ****************** 


\section{Geometry Module}

MORSE uses the geometry packages that are used with $05 R$ with minor changes. That is, there are spherical, slab, cylindrical, and general three-dimensional geometry packages that can be used. There are several descriptions of the various geometry routines in the $05 \mathrm{R}$ manual ${ }^{6}$ and in the helpful hints for 05R iser's marial.?

Changes were made to all of the GEOM packages to allow for albedc, scattering from any material surface and for variable input-output logical units. The GFOM packages are available in double precision for the IBM-360.

The geometry packages may be replaced with any special-purpose gecmetry routines the user might write. The three main functions of the geometry package are performed by the three subroutines discussed below.

Subroutine JQWIN (NADD, INTAPE, I ITAPE)

This subroutine reads geometry input and NADD is the rirst location in blank common that way ce used for input storage. In the special geometry packages blank common is not. used, so NADD is not incremented; otherwise, IADD must be incremented by the storage required by geometry data.

\section{Subroutine Lø\$KZ $(Y, Y, Z)$}

This subroutine determines the block and zone number, medium, and region for the point $X, Y, Z$. This routine is called from MS $\emptyset U R$ to determine the starting region and medium for source particles.

\section{Subroutine GEQM}

This is the main executive routine in that it determines the end point of a flight given the starting point, direction cosines or a tentative end point, and the number of mean free paths (or physical distance in any desired units) the particle will travel. It is called from GØMST ana the information is transferred through common GEØMC (see Table XI). In the more complicated geometry packages there are many routines that assist subroutine GEQM in determining the collision point.

To facilitate the use of the various geometry packages, a trief descri.ption of each is included here, and the input instructions for each are given in Appendix D. 
Table XI . Definition:s of Variables in Common GEQMC As Found in Subroutine GEWM

\begin{tabular}{|c|c|}
\hline Variable & Definition \\
\hline $\mathrm{X} 2, \mathrm{Y} 2, \mathrm{Z} 2$ & $\begin{array}{l}\text { Coordinates at tentative end-of-flight or if the trajec- } \\
\text { tory is in an internal void; X2, } \mathrm{Y} 2, \mathrm{Z2} \text { are the direction } \\
\text { cosines of the t:-ajectory. }\end{array}$ \\
\hline $\mathrm{XI}, \mathrm{Y} 1, \mathrm{Zl}$ & Starting coordinates for the parti $=1 \mathrm{e}$. \\
\hline ETA & $\begin{array}{l}\text { Number of mean free paths to be traversed if flight goes } \\
\text { to } \mathrm{X} 2, \mathrm{Y} 2, \mathrm{Z} 2 \text {. }\end{array}$ \\
\hline ETAUSD & $\begin{array}{l}\text { Number of mean free paths actiaily traversed after the } \\
\text { call to GEQM. }\end{array}$ \\
\hline IE:Z & $\begin{array}{l}\text { An index to the wedium number for the special geometry } \\
\text { packages. For GENERAL GE } \$ \text { M, IBLZ is a packed word giving } \\
\text { the block and zone of the end of Plight. }\end{array}$ \\
\hline IBZN & A dumay variakle. \\
\hline MARK & $\begin{array}{l}\text { A flag set by GEQM indiating the results of the trajec- } \\
\text { tcry calculation. } \\
=1 \text { for completed flight. } \\
=0 \text { for boundary crossing. } \\
=-1 \text { for escape. } \\
=-2 \text { for el iering an internal void. }\end{array}$ \\
\hline NMED & $\begin{array}{l}\text { Medium number at ent nf flight or of medium about to be } \\
\text { entered at a boundary crossing. }\end{array}$ \\
\hline NFEG & $\begin{array}{l}\text { Region number at end of flight; not set at boundary } \\
\text { crossings. }\end{array}$ \\
\hline
\end{tabular}


SPHERICAI GEQM

Spherical GEM is used to dessribe up to 20 concentric spheres cestered at $X=Y=Z=0$. Internal voils may be used in ary location and media numbers need not be ordered with increasing radii; however, regions must be numbered consecutively from the center. The medium and regions are bounded by the outer radius input. For example, the first region is interior to the surface of radius $R_{1}$. External (pure absorber) voids are not alloved except outside the maximum raciius.

Subroutine GQMFLP sets the medium inciax IBIZ and region number FREG to the values appropriate to the medium re-enterea after a reflection. Subroutine HDRML calculates the direction cosines of the normal to the spherical surface.

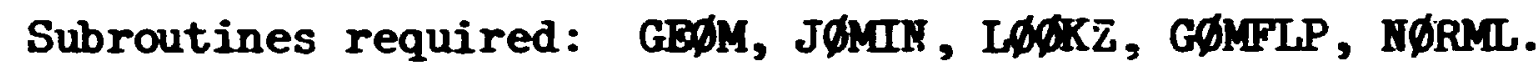

Input instructions are given ori page $D-1$.

Taken from references $t$ and 7 . 


\section{SLAB GEQR}

SLAB GEM can be used whenever there are rectangular paralielepipeds with normals to mediur and region bourdaries parallel to the 7 axis. A finite width and height are allowed. A maximum of 20 medium and region boundaries may be used with internal roius (medium 1000) allowed, but external voids (medium 0) are not permitted inside the system. Media may be numbered in any order but regions must be numbered consecutively with the region of lowest $\mathrm{Z}$ being region 1 . The media and regions may have different internal boundaries but the external boundary must be the same. Subroutines GQMPLP and HøRM provide the same functions for SLAB GEdM as for SPHERTCÁ GEQM.

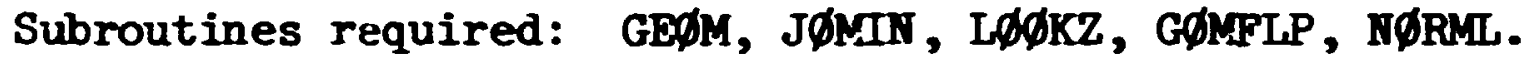

Input instructions are given on page D-2.

Orfinally written by A. A. Betz. 


\section{CYLIMDRICAL GEATH}

CYLIMDRICAL GEQM may be used to describe a series of concentric cylindrical cylindrical surfaces with up to 20 heights and 20 radial boundaries. The radial boundaries may be different for each height interval and internal voids (mediur 1000) are allowed. Negative heights, i.e., $\mathrm{Z}<0$, are not allowed. Hedia and regions numbers may be used in any order.

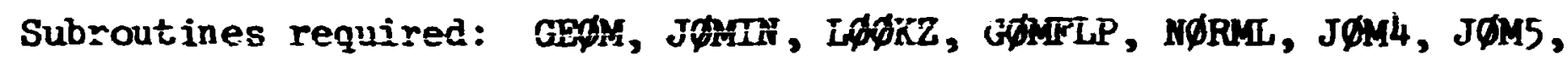
JW6, JWM, JøMO.

Input instructions are given on page $D-3$.

Originally witten by K. D. Franz and K. Morrison. 


\section{GETEKAL GEQMW}

The general three-dimensional geometry package has been described in detail elsewhere. The only limitation of geometry detail that may be treated is that surfaces must be describable by quadratic surfaces.

The description of the system must include a rectangular parallelepiped whose faces are parallel to the $X Y, Y Z$, and $X Z$ coordinate planes. This parallelepiped is then divided into zones with planes that extend across the entire system. The zones are divided into blocks with planes parallel to coordinate axes bui which extend only across the individual zones. Each block is then divided into sectors by quadratic surfaces with the sector defined by whether the volume is positive or negative with respect to the quajratic surfaces. Bach sector may contain only one medium; therefore, if a medium cannot be described by a single quadratic surface, it, mast be divided into several sectors.

Besides material boundaries, internal (medium 1000) and external (medium 0) voids may be used. If an external void is interior to the system it behaves as a perfect absorber since the particle is assumed to have escaped upon entering.

Region geometry may also be described for use in importance sampling. The block and zora boundaries for region geometry must be identical with the material boundaries. A description of geom input is given on page D-4. A code, PICTURE ${ }^{13}$, has been written to aid in debusging both material and region geometry input.

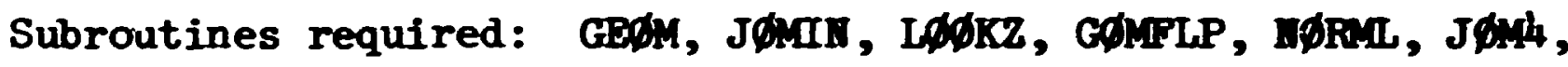

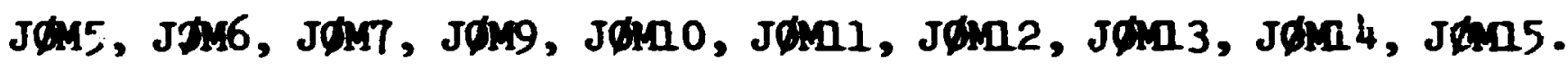

Functions required: J\$M8, Jqu6, JQM7, LهC.

Pigure 4 shows the hierarchy of subroutines for GBIERAL GEDM. Detailed descriptions of the various routines are given in references 6 and 7 ; however, some changes have been mede since those reports were written. The two main changes insorporated in Subroutines GArifP and IORLL are discussed.

\footnotetext{
Discussion t,aken from references 6 and 7 .
} 

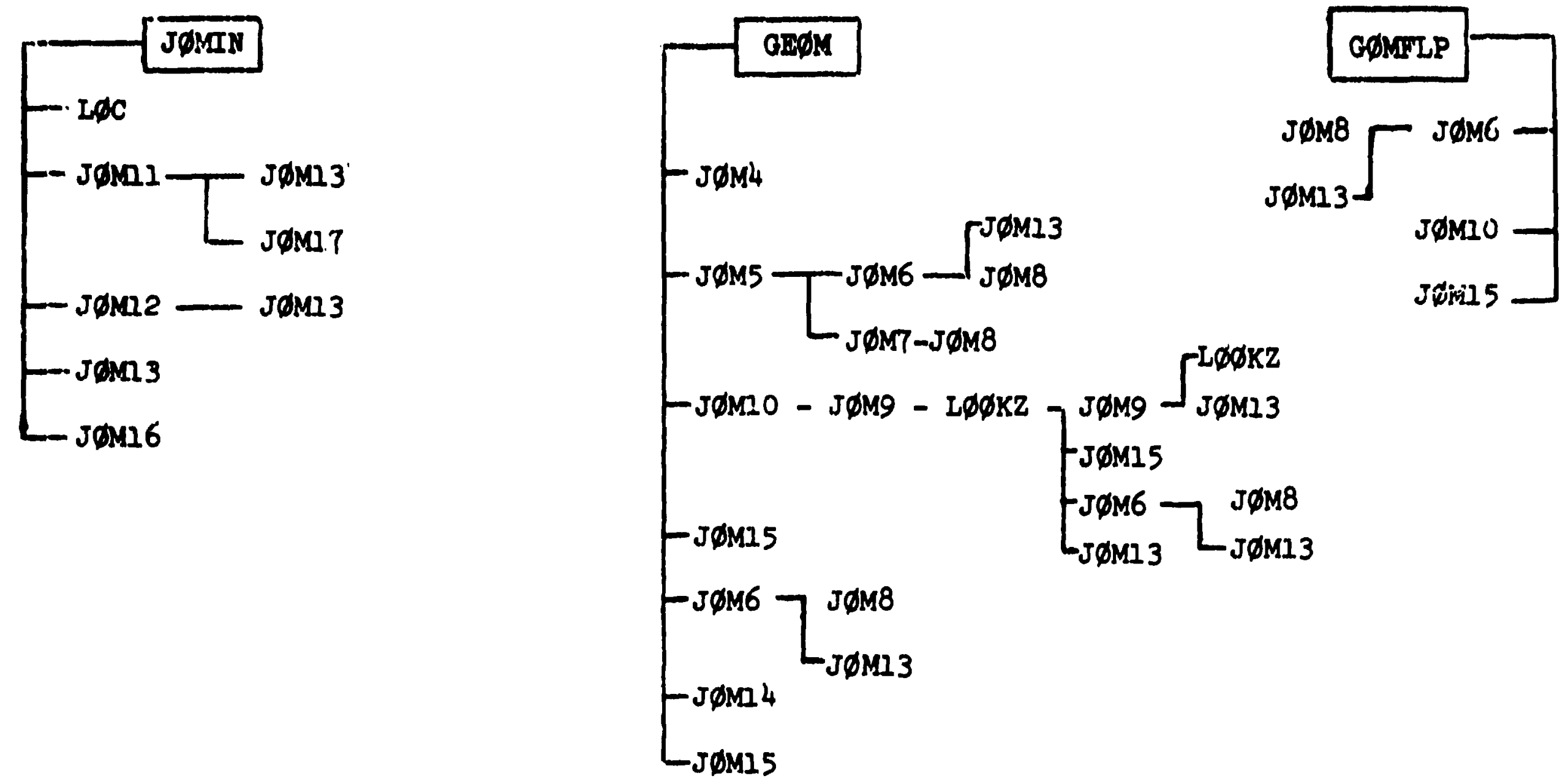

F16. 4. Hierarchy of Subroutines in GENERAL GEDM 


\section{Changes to Geometry Packages}

In order to implement the albedo option, it was necessary to make a minor change to the general GE 6 M package (and to all other special geometry packages). Previously a perticle was always traced through the geometry to a collision point inside a medium. In the albedo option a particle is tracked to the bouriary of the albetu medium where it undergoes a collision and departs in a different direction. In addition, the scattering and other routines needed to know the normal direction to the surface of the aibedo meaium and the region in which the aibedo scattering occurred. To accomplish this, tro subroutines were written and added to the GEDM package. These are GQAFLP, which prepares GE $\varnothing M$ for the particle to reverse direction on its next flight, and NøRML, which calculates the normal to the albedo surface. One change was made to subroutine GEXM to implement this: at block boundary crossings the variable NCUE, indicating which boundary was crossed, is saved in NCUESV located in labelled common GEQMn. The storing of NCURSV is made at FORTRAN statement 7 and the previous statement 7 becomes the next statement in the program.

In addition to the above modification, two other changes to the general GECy package, which is described in reference 7 , have been made. The first consists of putting several additional variables in labelled common for greater ease in examining dunps while debugging. The second change was made only to the IBM-3( ) version of GEWM. This involved changing the logical unit numbers used for the standard imput and output units to

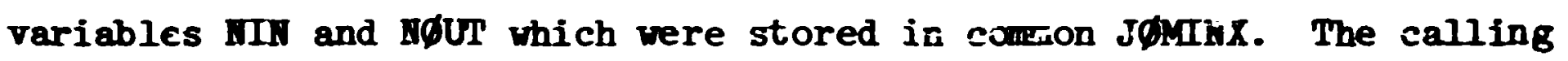
sequence for JWMII was changed to CALL JWMII (ADDR, NII, NDUT) so that the user could convey the desired logical unit numbers to the GEDM subroutines. Additional Paraneters in Labelled Comon

In JOMS and JOM6, the GEAK56 comon added the parameter RBG which is a packed word that describes the present position of the particle with respect to the quadria surfaces in the block. A "1" indicates the particle is on the positive side of the surface, a " 0 " the negative side. The surfaces are in the ordier fin wich they yere mentioned in the block description, starting at the last bit in the word and working back. 
In JゆM7 a new labelled common, GEØM70, was added to contain the variables $P, Q, f(0), f(I),\left(Q^{2}-P F_{0}\right), u, v, w, A u, B v, C w,(A u+D v+E w),(B v+F w)$ used in calculating intersections with the quadric surfaces.

In JQM9 and L $\varnothing \mathrm{KZ}$, the parameters in their calling sequence were changed to $\mathrm{Xl}, \mathrm{Yl}$, and $\mathrm{Zl}$. Then the statements

$$
\begin{aligned}
& \mathrm{X} \text { NE }=\mathrm{XI} \\
& \mathrm{Y} \emptyset \mathrm{NE}=\mathrm{Y} 1 \\
& \mathrm{Z} \text { } \mathrm{NE}=\mathrm{ZI}
\end{aligned}
$$

were added at the start of the program. Finally, XфNE, Y $\emptyset \mathrm{NE}$, and $\mathrm{Z} \emptyset \mathrm{NE}$ were added to the labelled common GEQM39. The error message "YOU ARE LOST," indicating that a point is located outside the system, has been modified to print out the coordinates of the offending juint. 
Subroutine GQMFLP (General GEQM)

The purpose of this subroutine is to prepare GEM for the fact that an albedo-scattered particle is about to reverse direction while at a boundary. The indicators specifying that the particle has crossed the boundary and is entering the new medium must be flipped to indicate that the particle is reentering its original medium. It also calls JW6 to obtain the region number of the albedo-scattering site and stores this in NREG in GEA:tC cominon.

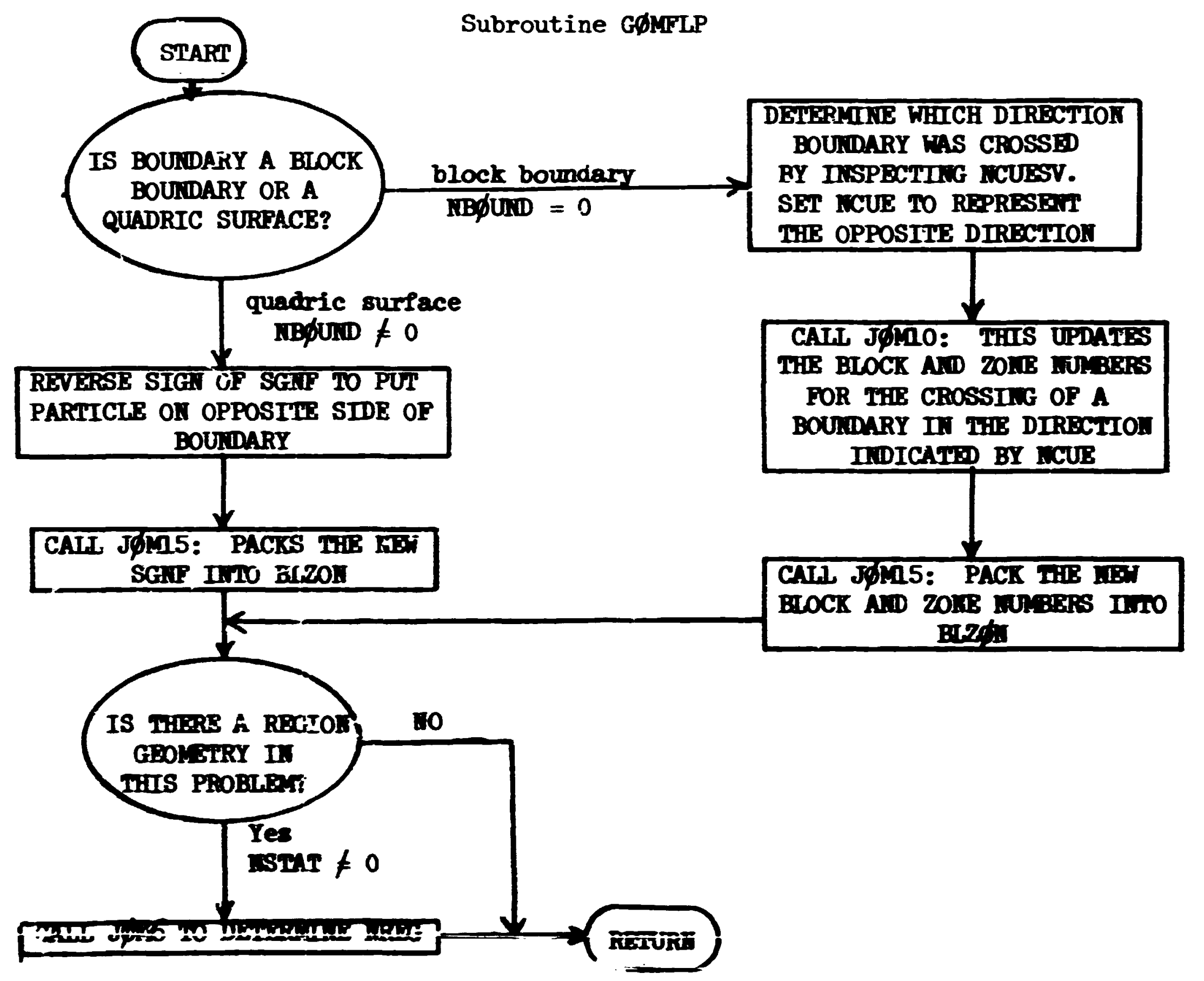


Subroutine HQREI (Genera) GEQM)

Subroutine $n \phi_{R M C}$. Jetermines the $n_{L}$ mal to the albedo surface. The nor-

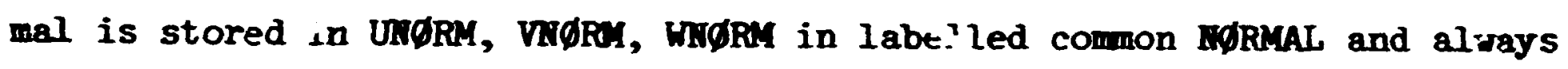
points out of the albedo medium.

Subroutine NARAL

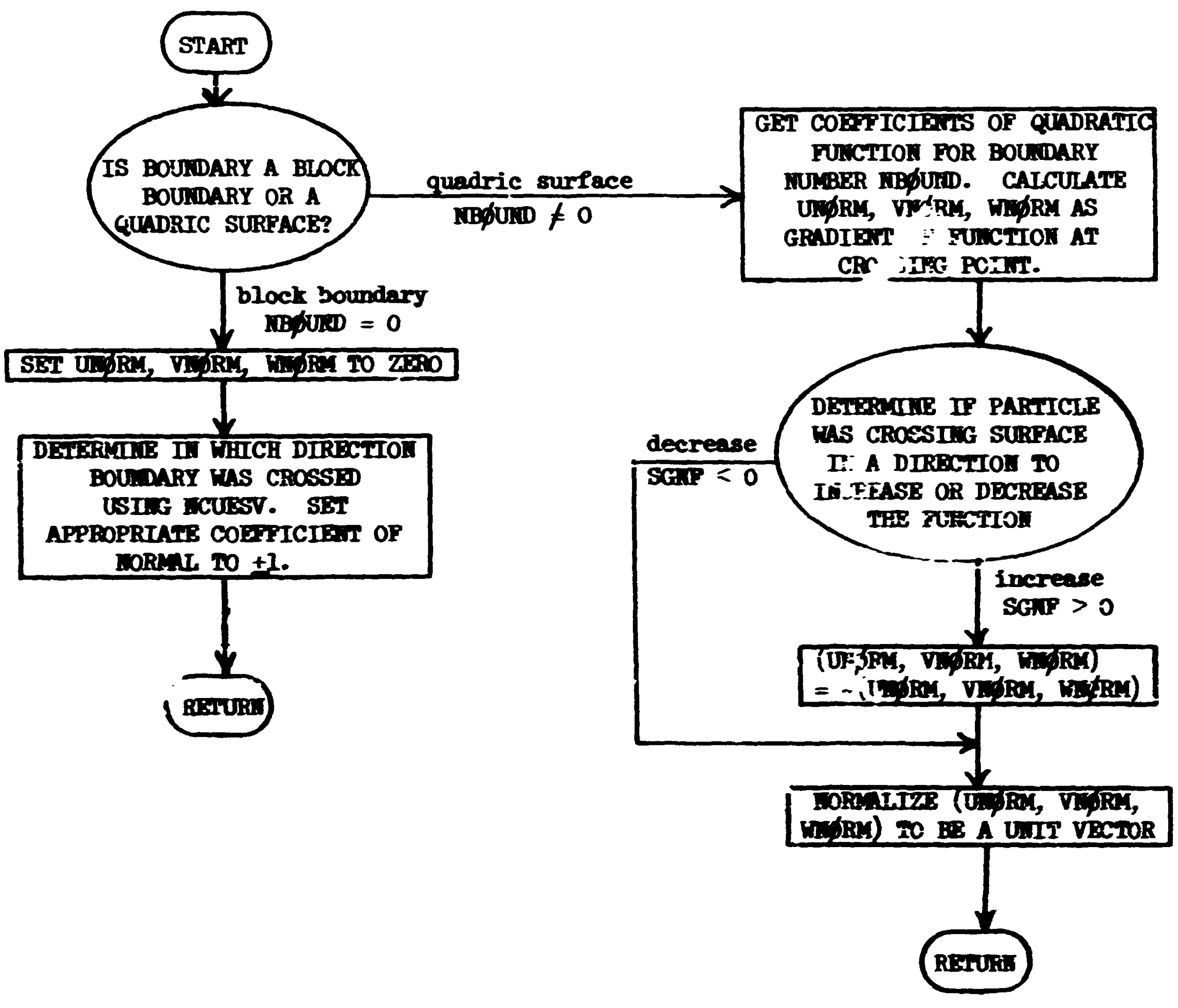




\section{REFEREICES}

1. V. R. Cain, "SAMBO, A Collision Aralysis Package for Multigroup Monte Carlo Codes" (to be published).

2. Radiation Shielding Information Center, Oar Ridge National Laboratory, Oak Ridge, Tenressee.

3. K. D. Lathrop, "DTP-IV, A PORTRAN-IV Prozran For Solving the Multigroup Transport Equation vi'h Anisotropic Scattering," LA-3373, Los Alemos Scientific Laboratory (1965).

4. W. W. Engle, Jr., "A User's Manual for ArIS,," K-1693 (196?).

5. F. R. Mnatt, F. J. Muckenthaler, and P. M. Sterens, "Developaent of Tro-Dimensional Discrete Ordinates Transport Theory for Radiation Shielding," CrC-II.9-952 (1969).

6. D. C. Irving, R. H. Preestone, Jr., and P. B. K. Kan, "GJR, A reneral Purpose Monte Carlo Neutron Transport Code," ORIL-3622 (1965).

7. D. C. Irving, V. R. Cain, and P. M. Preestone, Jr., "An Alplification of Selected Portions of the ISR Monte Carlo Code 'Jser' = Manual," OR.I-TM-2601 (1969).

8. I. Cranberg et 르., Phys. Rev. 103, 662 (1956).

9. P. H. Clark and I. A. Betz, "Importance Sapling Devices fcr Selecting Track Lengths and Directions After Scatter in $15 R, "$ ORI-TH-1484 (1966).

10. F. H. Clark, "The Exponential Transform as an Importance-Sampling Device - A Review," ORIL-RSIC-14 (1966).

11. V. R. Cain, E. A. Straker, and G. Thayer, "Monte Carlo Path Length Selection Routines Based on Some Specific Forms of the Importance Function," ORIL-TH-1967 (1969). 
12. T. J. Tyrrell, "TDURP - A Translation Routine For Use in Truping PORTRAY ATrays," ORIL-CF-TO-7-8 (1970).

13. D. C. Irving and G. W. Jorrison, "PICTURE: An Aid in Debugging GEOM Input Data," ORoLL-TH-2892 (1970). 


\section{APPETIIX A}

The Many Integral Forms of the Bcltzmann Transport Equation and its Adjoint

The purpose here is to derive a complete set of forward and adjoint integral transport equations in energy-group notatior and to relate these equations to the Monte Carlo procedures used in the MRSE code.

The Boltzmann Transport Ezuation

The derivation begins with the general time-dependent integro-differentia] form of the Boltzinann transport equation, the derivation of which can be regarded as a bookkeeping process that sets the net storage of particles within a differential element of phase space ( $\mathrm{d} \overline{\mathbf{r}} \mathrm{dE} d \bar{\Omega}$ ) equal to the particle gains minus particle losses in ( $\overline{\mathrm{r}} \mathrm{dE} d \bar{\Omega})$ and leads to the following familiar and useful form:

$$
\begin{aligned}
& \frac{1}{\nabla} \frac{\partial}{\partial t} \phi(\bar{r}, E, \bar{\Omega}, t)+\nabla \cdot \phi(\bar{r}, E, \bar{\Omega}, t)+\varepsilon_{t}(\bar{r}, E) \phi(\bar{r}, E, \bar{\Omega}, t) \\
& \quad=s(\bar{r}, E, \bar{\Omega}, t)+\iint d E^{\prime} d \bar{\Omega}^{\prime} \Sigma_{s}\left(\bar{r}, E^{\prime}+E, \bar{\Omega}^{\prime}+\bar{\Omega}\right) \phi\left(\bar{r}, E^{\prime}, \bar{\Omega}^{\prime}, t\right)
\end{aligned}
$$

where

$(\bar{r}, E, \bar{\Omega}, t)$ denotes the general seven-dimensional phase spsce, $\overline{\mathbf{r}}=$ position variable,

$E=$ the particle's kinetic energy,

$v=$ the particle's speed corresponding to its kinetic energy $E$,

$\bar{\Omega}=a$ unit vector which describes the particle's direction of motion,

$t=$ time variable,

$\phi(\bar{r}, E, \bar{\Omega}, t)=$ the time-dependent angular flux,

$\phi(\bar{r}, E, \bar{\Omega}, t) d E d \bar{\Omega}=$ the number of particles that cross a unit area normal to the $\bar{\Omega}$ direction per unit time at the space point $\bar{r}$ and $t i m e t$ with energies in $d B$ about $E$ and with directions that lie within the differential solid angle $d \bar{\Omega}$ about the unit vector $\bar{Z}$,

$\frac{1}{\bar{v}} \frac{\partial}{\partial t} \phi(\bar{r}, E, \bar{\Omega}, t) d E d \bar{\Omega}=$ net storage (gains minus 10sses) per unit voluse and time at the space point $\vec{r}$ and time $t$ of particles with energies in $d E$ about $E$ and with directions which lie in $d \bar{\Omega}$ about $\bar{~}$. 
$\bar{D} \cdot \nabla \phi(\bar{r}, E, \bar{D}, t)$ aBd $\bar{D}=$ net conrective $108 \mathrm{~s}$ per unit rolwe and time at the space point $\vec{F}$ and $t i n e t$ of particles with energies in $d E$ about $B$ and directions which ile ir. ab about $\bar{D}$,

$\Sigma_{t}(\bar{r}, B)=$ the total cross section at the spece point $\bar{r}$ for particles of energs $B$,

$E_{t}(\bar{r}, E) \&(\bar{r}, B, \bar{R}, t)$ dBd $=$ collision loss per unit volueve and tine at the space point $\bar{F}$ and tine $t$ of particles with energies in dE about $B$ and directions wich lie in about $\bar{D}$, $\Sigma_{8}\left(\bar{r}, E^{\prime}+B, \bar{D}^{\prime}+\bar{L}\right) \mathrm{d} B d \bar{\alpha}$ = the differential scattering cross section wich desertbes the probability per unit patb that a particle with an initivi eners $E^{\prime}$ and an initial direction $\bar{D}^{\prime}$ undergoes a scattering collision at $\bar{Y}$ unich places it into a direction that lies in a about $\bar{Q}$ vith a new enero in as about $E$.

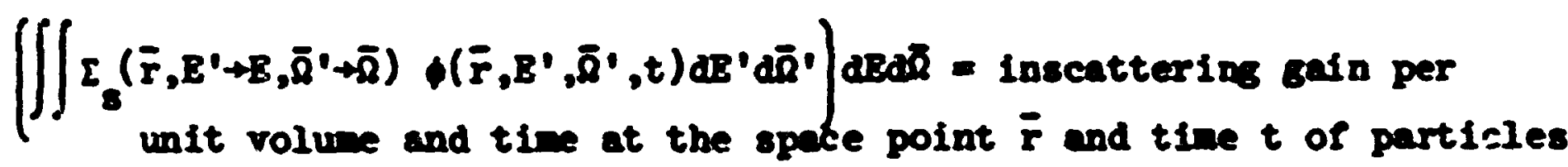
with energiea in ds about $B$ and directions which lie in about $\overline{\mathbf{a}}$

$S(\bar{r}, E, \bar{Q}, t) d B d \bar{Q}=$ source particles eaitted per unit rolume and tise at the space point $\bar{Y}$ and $t i n e t$ with energies in $A B$ about $B$ and directions wich lie in ab about $\bar{D}$.

An effect of inte; sst such as biologdeal dose, enercy deposition, or particle: Hux (denoted by $\lambda$ ) for a given problex can be expreased in terms of the flux fleld $\odot(\bar{r}, E, \bar{d}, t)$ and an approprlate reoponse function $P^{\bullet}(\bar{r}, E, \bar{R}, t)$ due to a unit angular flux and is given by:

$$
\lambda=\iiint P^{\dagger}(\bar{r}, E, \bar{\delta}, t) \Delta(\bar{r}, E, \bar{\Omega}, t) d \bar{r} d B d \bar{d} d t .
$$

Consistent with the mise code, the enered dependence of Equation (1) wil' be represented in terms of enerw eroups which are defined such that: $\Delta E_{B}=$-nercy width of the sth soup, $B=1$ corresponds to the hichest enerw group, $B$ = G corresponds to the lowest enered croup, with the obvicus constral at that

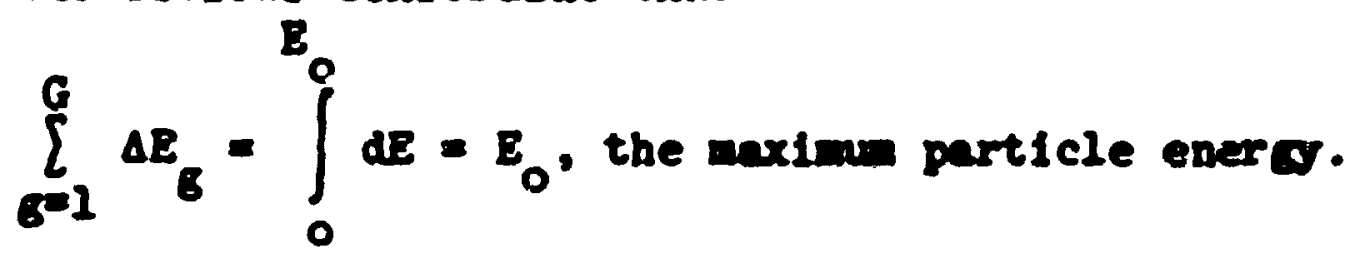

A "group" form of Bquation (I) is obtained by integreting each ter with respect to the enercy variable over the enercy interval $4 B_{B}$ : 


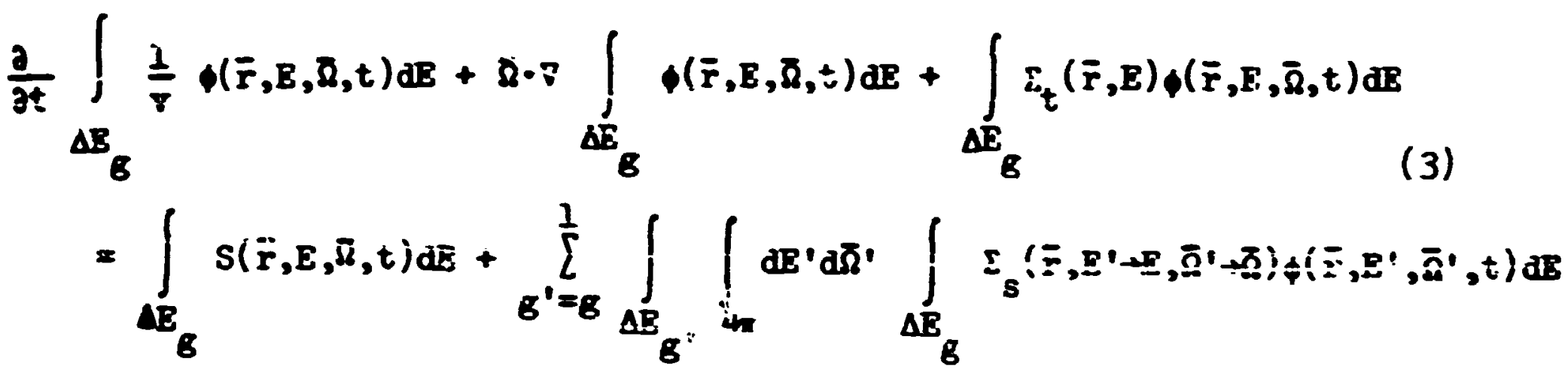

Equation (3) provides the formal basis for the following group parameters: $\phi_{B}(\bar{r}, \bar{\Omega}, t)=$ time-dependent group angular Plux,

$$
=\int_{\Delta E_{B}} \phi(\bar{r}, E, \bar{\Omega}, t) d E=
$$

$\Sigma_{t}^{b}(\bar{r})=$ enerby-averaged tot/2l cross section for the gth group,

$$
\equiv \frac{\int_{\Delta} \sum_{t}(\bar{r}, E) \phi(\bar{r}, E, \bar{\Omega}, t) d E}{\int_{B}(\bar{r}, E, \bar{\Omega}, t) d E}
$$

$\nabla_{B}=$ energy-avereged particie speed for the gth group,

$$
\equiv \frac{\int_{B E} \phi(\bar{r}, \bar{E}, \bar{E}, \hat{i}) d z}{\int_{\Delta E} \frac{1}{\nabla} \phi(\bar{r}, \bar{E}, \bar{Q}, t) d E}
$$

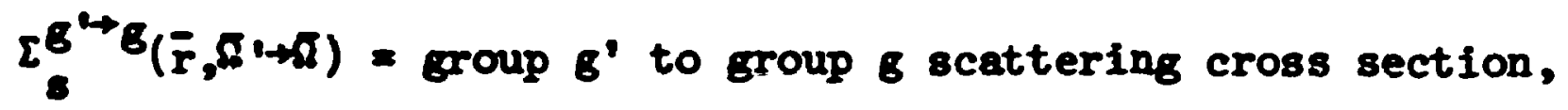

$$
\equiv \frac{\int_{\Delta E} \int_{\Omega^{\prime}} \int_{B_{B}} \sum_{B^{\prime}}\left(\bar{r}, E^{\prime}+E, \bar{\Omega}^{\prime}+\bar{\alpha}\right) \phi\left(\bar{r}, E^{\prime}, \bar{\Omega}^{\prime}, t\right) d E^{\prime} d E}{\int_{\Delta E_{B^{\prime}}} \phi\left(\bar{r}, E^{\prime}, \bar{\Omega}^{\prime}, t\right) d E^{\prime}}
$$

$s_{B}(\bar{r}, A, t)=$ distribution of source particles for the gth group,

$$
\equiv \int_{\Delta E_{B}} S(\bar{r}, E, \bar{Q}, t) d E .
$$

\footnotetext{
These paranetere will be referred to as forvard-weigisted group paremeters.
} 
The group form of the Boltzmann equation expressed in terms of the aforedefined group perameters is giren by

$$
\begin{aligned}
& \frac{I}{v_{B}} \frac{\partial}{\partial t} \phi_{g}(\bar{r}, \bar{\Omega}, t)+\bar{\Omega} \cdot \nabla \phi_{g}(\bar{r}, \bar{\Omega}, t)+\varepsilon_{t}^{G}(\bar{r}) \phi_{g}(\bar{r}, \bar{\Omega}, t) \\
& =S_{B}(\bar{r}, \bar{\Omega}, t)+\sum_{\bar{B}^{\prime}=\bar{B}}^{I} \int_{4 \pi} d \bar{\Omega}^{\prime} \Sigma_{s}^{B^{\prime}+B^{\prime}}\left(\bar{r}, \bar{\Omega}^{\prime}+\bar{\Omega}\right) \phi_{g^{\prime}}\left(\bar{r}, \bar{\Omega}^{\prime}, t\right),
\end{aligned}
$$

where the sumnation over energy groups could be expanded over all $B^{\prime}$ to allow for upscattering -- not usually considered important in shieiding problems.

\section{Integral Flux Density Equation}

The transformation of Equation (9) into an integral form is now considered. To accomplish this, the combination of the convection and storage terms are first expressed in terms of the spatial variable $R$ which relates a fixed point in space $(\bar{r})$ to an arbitrary point $\left(\bar{r}^{\prime}\right)$, as show in Fig. A.l.

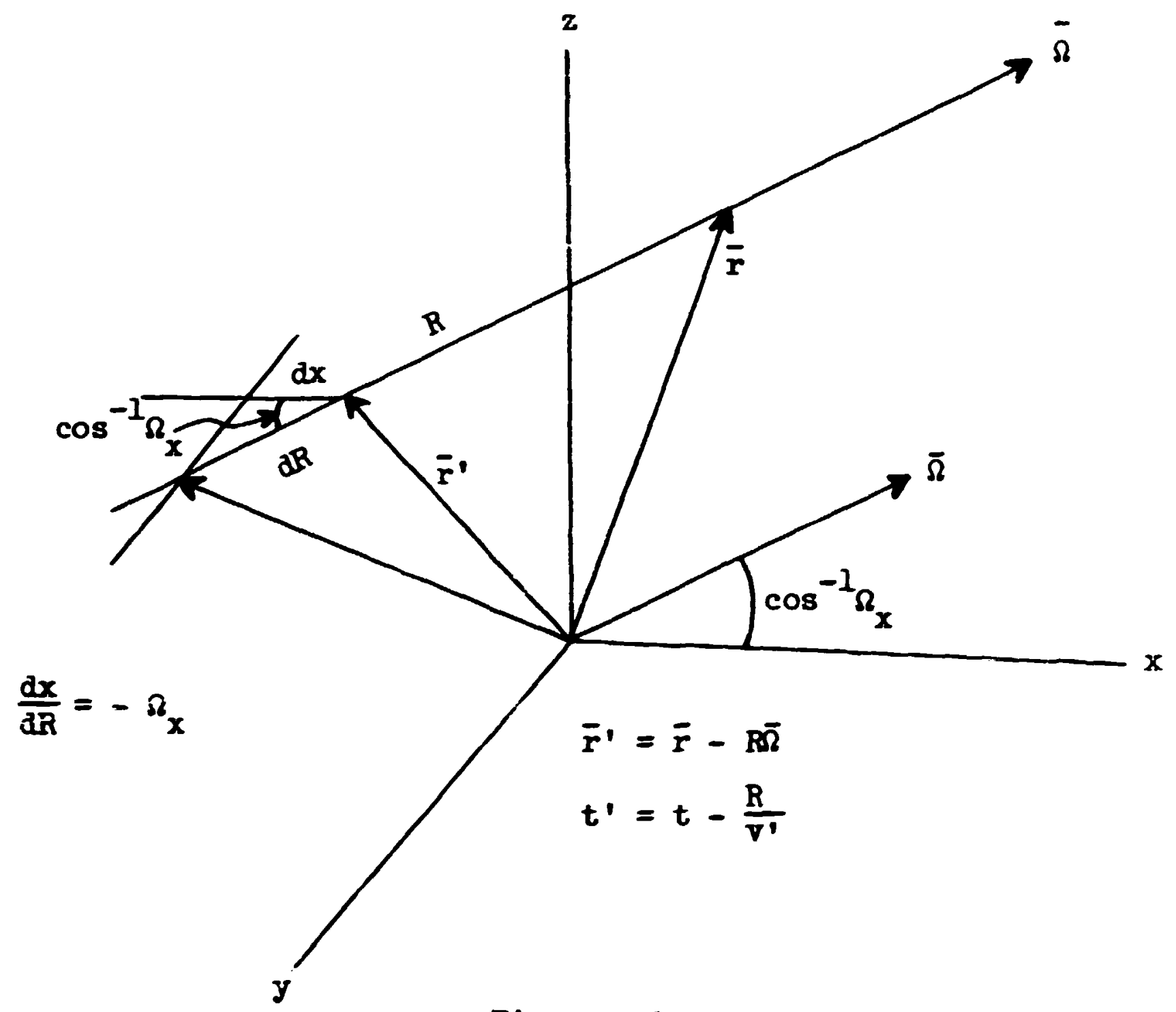

Figure A.1 
The total derivative of the angular flux with respect to $R$ is given by

$$
\frac{d}{d R} \phi\left(\bar{r}^{\prime}, E, \bar{\Omega}, t^{s}\right)=\frac{\partial x}{\partial R} \frac{\partial \phi}{\partial x}+\frac{\partial y}{\partial R} \frac{\partial \phi}{\partial y}+\frac{\partial z}{\partial R} \frac{\partial \phi}{\partial z}+\frac{\partial t}{\partial R} \frac{\partial \phi}{\partial t}
$$

which, according to Fig. A.l and noting that the particle's speed ( $v$ ) is equal to ( $-d R / d t)$ can be rewritten as

$$
\begin{aligned}
\frac{d}{d R} \phi\left(\bar{r}^{\prime}, E, \bar{\Omega}, t^{\prime}\right) & =-\Omega_{x} \frac{\partial \dot{\phi}}{\partial x}-\Omega_{y} \frac{\partial \phi}{\partial y}-\Omega_{z} \frac{\partial \hat{\psi}}{\partial z}-\frac{2}{v} \frac{\partial \phi}{\partial t} \\
& =-\Omega \cdot \nabla \phi\left(\bar{r}^{\prime}, E, \Omega, t^{\prime}\right)-\frac{1}{v} \frac{\partial \phi}{\partial t} .
\end{aligned}
$$

Equation (10) can be expressed in group notation as

$$
-\frac{d}{d R} \phi_{G}\left(\bar{r}^{\prime}, \bar{\Omega}, t^{\prime}\right)=\frac{1}{\nabla_{G}} \frac{\partial}{\partial t} \phi_{E}\left(\bar{r}^{\prime}, \bar{\Omega}, t^{\prime}\right)+\bar{\Omega} \cdot \nabla \phi_{G}\left(\bar{r}^{\prime}, \bar{\Omega}, t^{\prime}\right) .
$$

Substitution of Eq. (11) into Eq. (8) with $\bar{r} \equiv \bar{r}^{\prime}$ and $t \equiv t^{\prime}$ yields

$$
\begin{aligned}
& -\frac{d}{d R} \phi_{g}\left(\bar{r}^{\prime}, \bar{\Omega}, t^{\prime}\right)+\Sigma_{t}^{g}\left(\bar{r}^{\prime}\right) \phi_{g}\left(\bar{r}^{\prime}, \bar{\Omega}, t^{\prime}\right)=s_{g}\left(\bar{r}^{\prime}, \bar{\Omega}, t\right) \\
& +\sum_{g^{\prime}=g}^{1} \int_{4 \pi} d \bar{\Omega}^{\prime} \Sigma_{s}^{g^{\prime}+g^{\prime}}\left(\bar{r}^{\prime}, \bar{\Omega}^{\prime}+\bar{\Omega}\right) \phi_{g^{\prime}}\left(\bar{r}^{\prime}, \bar{\Omega}, t^{\prime}\right) .
\end{aligned}
$$

The integrating factor

$$
e^{-\int_{0}^{R} \Sigma_{t}^{g}\left(\bar{r}-R^{\prime} \bar{\Omega}\right) d R^{\prime}}
$$

is introduced in the following manner:

$$
\begin{aligned}
& \frac{d}{d R}\left[\phi_{g}\left(\bar{r}^{\prime}, \bar{\Omega}, t^{\prime}\right) e^{-\int_{0}^{R} \varepsilon_{t}^{g}\left(\bar{r}-R^{\prime} \bar{\Omega}\right) d R^{\prime}}\right)=-e^{-\int_{0}^{R} \varepsilon_{t}^{g}\left(\bar{r}-R^{\prime} \bar{\Omega}\right) \dot{d} R^{\prime}} \\
& \times\left(-\frac{d \phi_{g}}{d R}+\Sigma_{t}^{g}\left(\bar{r}^{\prime}\right) \phi_{g}\left(\bar{r}^{\prime}, \bar{\Omega}, t^{\prime}\right)\right) .
\end{aligned}
$$


Using Eq. (23), Bq. (12) can be rewritten as

$$
\begin{aligned}
& -\frac{d}{d R}\left(\phi_{G}\left(\bar{r}^{\prime}, \bar{\Omega}, t^{\prime}\right) e^{-\int_{0}^{R} \Sigma_{t}^{G}\left(\bar{r}-R^{\prime} \bar{\Omega}\right) d R^{\prime}}\right)=e^{-\int_{0}^{R} \Sigma_{t}^{G}\left(\bar{r}-R^{\prime} \bar{\Omega}\right) d R^{\prime}}
\end{aligned}
$$

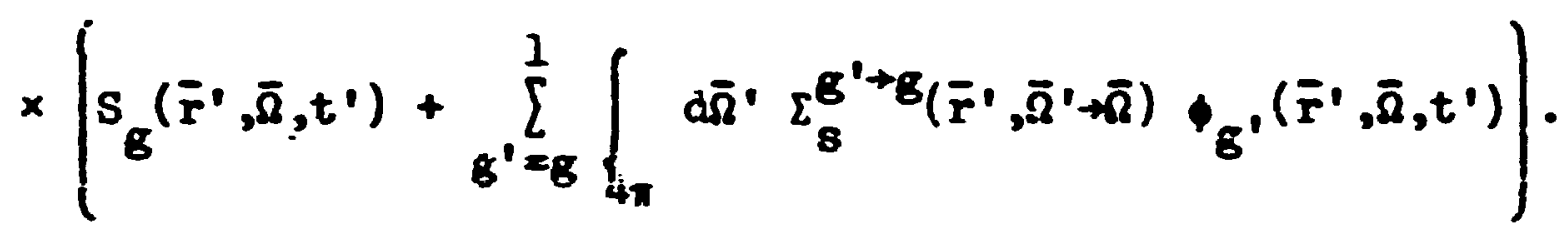

Wultiply Bq. (14) by $d R$ and integrate $(R=0$ to $R=\infty)$; then

$$
\begin{aligned}
& -\int_{0}^{\infty} \varepsilon_{t}^{\mathbf{g}}\left(\bar{r}-R^{\prime} \bar{\Omega}\right) d R^{\prime} \\
& \phi_{B}(\bar{r}, \bar{\Omega}, t)-\phi_{B}\left(\infty, \bar{\Omega}, t_{\infty}\right) e \\
& =\int_{0}^{\infty} d R e^{-\int_{0}^{R} \varepsilon_{t}^{g}\left(\bar{r}-R^{\prime} \bar{\Omega}\right) d R^{\prime}}\left(S_{B}\left(\bar{r}-R \bar{\Omega}, \bar{\Omega}, t-\frac{R}{v}\right)\right. \\
& \left.+\sum_{B^{\prime}=g}^{l} \int_{4 \pi} \bar{\Omega} \cdot \Sigma_{s}^{g^{\prime}+g}\left(\bar{r}-\bar{B}, \bar{\Omega}{ }^{\prime}+\bar{\Omega}\right) \phi_{g^{\prime}}\left(\bar{r}^{\prime}, \bar{\Omega} \cdot, t^{\prime}\right)\right)
\end{aligned}
$$

Require that

$$
\left[\phi_{B}\left(\infty, \bar{\Omega}, t_{\infty}\right) e^{-\int_{0}^{\infty} \Sigma_{t}^{B}\left(\bar{r}-R^{\prime} \bar{\Omega}\right) d R^{\prime}}\right]=0,
$$

and introduce the "optical thickness"

$$
B_{B}(\bar{r}, R, \bar{\Omega}) \equiv \int_{0}^{R} \Sigma_{t}^{B}\left(\bar{r}-R^{\prime} \bar{\Omega}\right) d R^{\prime},
$$

and Eq. (15) besceues

$$
\begin{aligned}
& \varphi_{B}(\bar{r}, \bar{\Omega}, t)=\int_{0}^{\infty} d R e^{-B_{B}(\bar{r}, R, \bar{\Omega})} \int S_{B}(\bar{r}-R \bar{R}, \bar{\Omega}, t-R / v)
\end{aligned}
$$

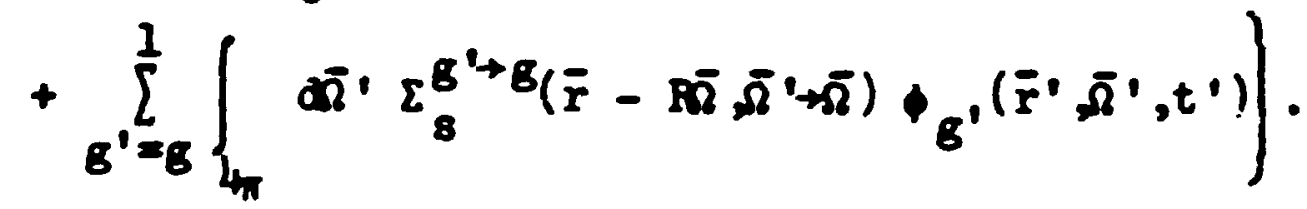


Bquation (18) will be referred to as the "Integral Flux Density Bquation."

An effect of interst $\lambda$ in sroup notation can be expressed as

$$
\lambda_{B}=\iiint_{B} P_{B}^{\phi}(\bar{r}, \bar{\Omega}, t) \phi_{g}(\bar{r}, \bar{\Omega}, t) d \bar{r} d \bar{\Omega} d t,
$$

where

$P_{B}^{\phi}(\vec{r}, \bar{\Omega}, t)=$ the response function of the effect of interest due to a ur.it angular group flux (group $B, \bar{r}, \bar{\Omega}$, tine $t$ ),

$$
=\frac{\int_{\Delta E} P(\bar{r}, E, \bar{\Omega}, t) \phi(\bar{r}, E, \bar{\Omega}, t) d E}{\int_{\Delta E}(\bar{r}, E, \bar{\Omega}, t) d E}
$$

$\lambda_{g}=$ that portion of the effect of interest associated with the gth enerey brour.

The $\lambda_{g}$ are $s 0$ defined that the total effect of interest $i$ is given the summation

$$
\lambda=\sum_{g=1}^{G} \lambda_{8} .
$$

\section{Integrel Brent Density Equation}

The "event density" ${ }_{G}(\bar{r}, \bar{l}, t)$ describes the density of particles going into a collision and is related to the group angular flux in the fullowing manner :

$$
\varphi_{B}(\bar{r}, \bar{\Omega}, t) \equiv \Sigma_{t}^{\bar{B}}(\bar{r}) \varphi_{B}(\bar{r}, \bar{\Omega}, t)
$$

where

$\varphi_{G}(\bar{r}, \bar{\Omega}, t) d \bar{\Omega}=$ the number of collision events per unit volume and tive at the space point $\overrightarrow{\mathbf{r}}$ and time $t$ experienced by particles having energies within the gth enerso group and directions in a about $\bar{a}$. The defining equation for the event density is obtained by multiplying both sides of Eq. (18) by the group total cross section $\Sigma_{t}^{8}(\bar{r})$ and identifying the product $\Sigma_{t}^{B}(\bar{r}) \phi_{B}(\bar{r}, \bar{\Omega}, t)$ as the event density $\downarrow_{B}(\bar{r}, \bar{\Omega}, t)$ : 


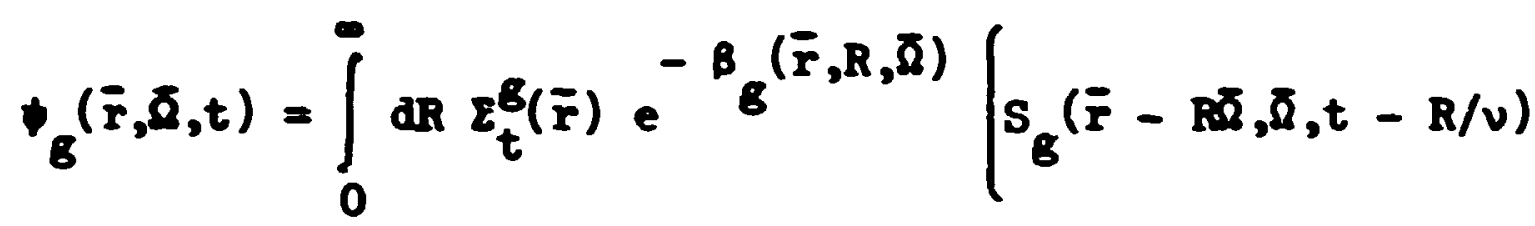

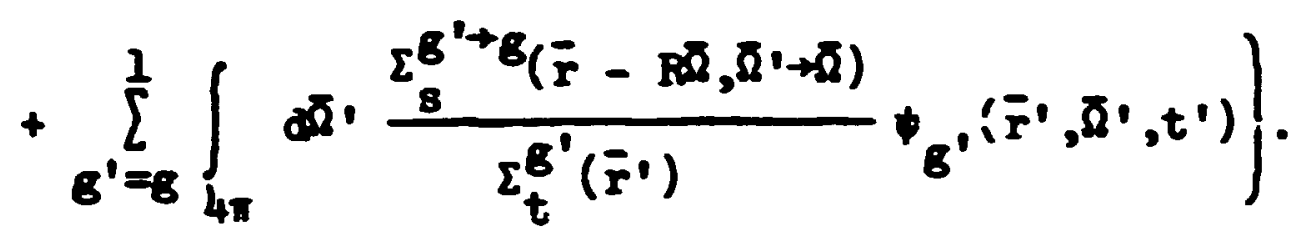

Equation (22) will be referred to as the "Integral Event Density Equation."

The effect of interest $\lambda_{g}$ can be expressed in terms of the event density; consider Bq. (19) rewritten as

$$
\begin{aligned}
\lambda_{B} & =\iiint \frac{P_{g}^{\phi}(\bar{r}, \bar{\Omega}, t)}{\Sigma_{t}^{g}(\bar{r})} \Sigma_{t}^{g}(\bar{r}) \phi(\bar{r}, \bar{\Omega}, t) d \bar{r} d \bar{\Omega} d t \\
& =\iiint P_{g}^{\phi}(\bar{r}, \bar{\Omega}, t) \downarrow_{B}(\bar{r}, \bar{\Omega}, t) d \bar{r} d \bar{l} d t,
\end{aligned}
$$

where

$$
\begin{aligned}
& P_{B}^{+}(\bar{r}, \bar{\Omega}, t)=\text { the response runction of the effect of interest due to a } \\
& \text { particle wich experiences an erent at (group } B, \bar{r}, \bar{\Omega}, t \text { ine } t \text { ), } \\
& P_{B}^{+}(\bar{r}, \bar{\Omega}, t)=P_{B}^{t}(\bar{r}, \bar{\Omega}, t) / \Sigma_{t}^{B}(\bar{r}) \\
& \text { or } \\
& P_{B}^{\phi}(\bar{r}, \bar{\Omega}, t)=\varepsilon_{t}^{B}(\bar{r}) P_{B}^{t}(\bar{r}, \bar{\Omega}, t) .
\end{aligned}
$$

\section{Interral Buergent Particle Densfty Equation}

Define the ewergent particle density $x_{B}(\bar{r}, \bar{\Omega}, t)$ as the density of partiales leaving a source or gerging from a real collision with phase space coordinates (group $B, \bar{r}, \bar{\Omega}, t$ ),

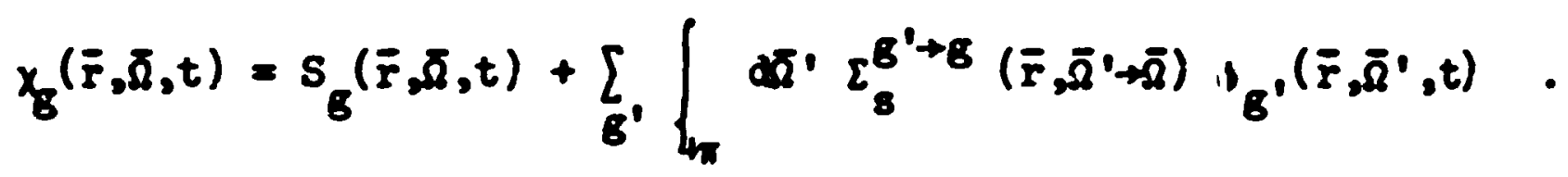

Then Eq. (18) can be written as 


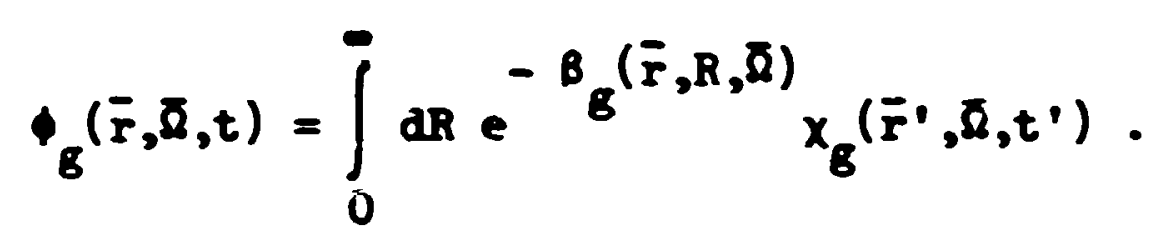

The "Integral Buergent Particle Density Equation" is obtained by substituting $\mathrm{Bq}$. (26) into Bq. (25):

$$
\begin{aligned}
& x_{g}(\bar{r}, \bar{\Omega}, t)
\end{aligned}
$$

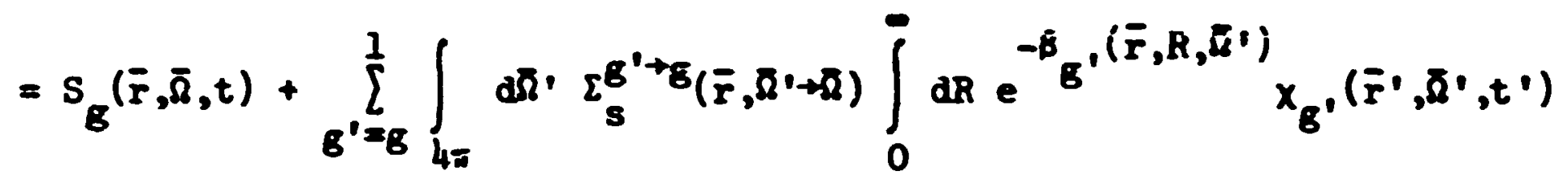

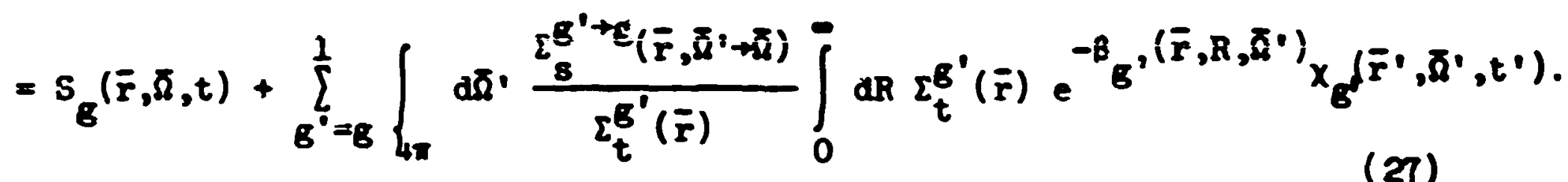

The effect of interest $\lambda_{B} \operatorname{can}$ also be expressed in terms of the emergent particle density

$$
\lambda_{B}=\iiint P_{B}^{X}(\bar{r}, \bar{\Omega}, t) x_{B}(\bar{r}, \bar{\Omega}, t) d \bar{r} d \bar{\delta} d t .
$$

The response runction $P_{B}^{X}(\bar{Y}, \bar{\Omega}, t)$ is obtained by considering a particle which energes rrom a coilision at $\overline{\mathbf{F}}$ uth phase space coordinates (Group $G, \bar{b}$, tine $t$ ). This particle vill experience an event in dR about $\bar{r}^{\prime}=\bar{r}+i \bar{d}$ at time $t^{\prime}=t+R i^{\prime}$ with the probability

$$
\Sigma_{t}^{8}\left(\bar{r}^{\prime}\right) e^{-\int_{0}^{R} \Sigma_{t}^{8}\left(\bar{r}+R^{\prime} \bar{R}\right) d R^{\prime}} d R,
$$

and the contribution of this event is the response nuction $P_{G}^{t}\left(\bar{r}^{\prime}, \bar{\Omega}, t^{\bullet}\right)$. The sum of all such contributions to the effect of interest is given by

$$
\begin{aligned}
& -\int_{0}^{p} \varepsilon_{t}^{G}\left(\bar{r}+R^{\prime} \bar{q}\right) d R^{\prime} \\
& \int_{0}^{\infty} d R \varepsilon_{t}^{B}\left(\bar{r}^{\prime}\right) e^{0} \quad P_{G}^{t}\left(\bar{r}^{\prime}, \bar{D}, t^{0}\right) \text {, }
\end{aligned}
$$

and should be the sane as a response runction $P_{E}^{X}(\bar{r}, \bar{h}, t)$ which is based on energent particle density. This leads to the fallowing relationahip:

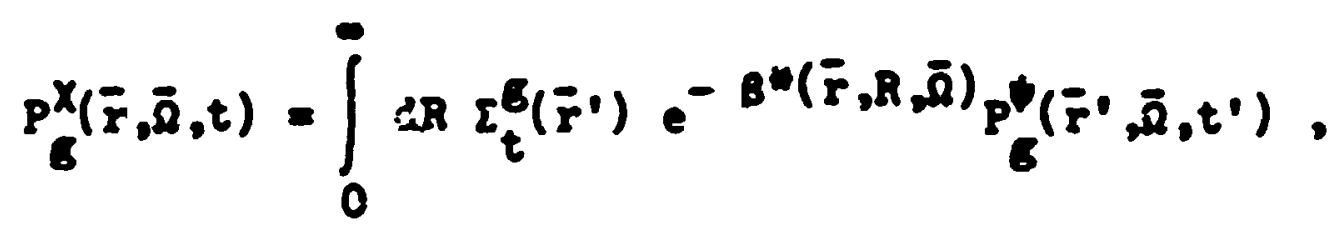


vhare

$\mathrm{P}_{\mathrm{G}}^{X}(\bar{r}, \bar{D}, t) \equiv$ the response rumction (of the effect of interest due to a particle which energes from a collision having the phase space coordinates (Group $B, \bar{r}, \bar{d}, t i n e t$ )

$B *(\bar{r}, R, \bar{R}) \equiv \int_{0}^{R} \varepsilon_{t}^{B}\left(\bar{r}+R^{\prime} \bar{\Omega}\right) d R^{\prime}$.

It is noted that $\beta_{B}^{N}(\bar{r}, R, \bar{\Omega})$ differs from the optical thickness $B_{B}(\bar{r}, R, \bar{\Omega})$ as defined by $\mathrm{Bq} .(17)$ in that the integration is performed in the positive $\overline{2}$ direction and as such $\beta_{B}^{*}(\bar{r}, R, \bar{\Omega})$ is the adjofint of $B_{B}(\bar{r}, R, \bar{R}) \cdot F_{G}^{X}(\bar{r}, \bar{\Omega}, t)$ can also be expressed in terms of $P_{B}^{4}(\bar{r}, \bar{\Omega}, t)$ by substituting Eq. (25) into Bq. (29), Jielding

$$
P_{g}^{X}(\bar{r}, \overline{\bar{L}}, t)=\int_{0}^{\infty} d R e^{-B_{B}^{*}(\bar{r}, R, \bar{\Omega})} P_{B}^{\dot{\phi}}\left(\bar{r}^{\prime}, \bar{\Omega}, t^{\prime}\right) .
$$

\section{Operater Dotation and Sumary of the Forrard Equations}

Desine the transport intigral operator

$$
T_{B}(\bar{r} \cdot \bar{r}, \bar{\Omega}) \equiv \int_{0}^{\infty} d R \varepsilon_{t}^{B}(\bar{r}) e^{-B_{B}(\bar{r}, R, \bar{\Omega})},
$$

and the colisision Integral operator

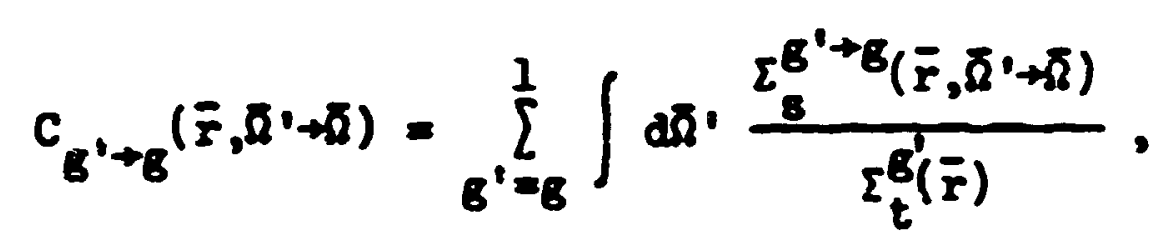

wich can be rearitten as

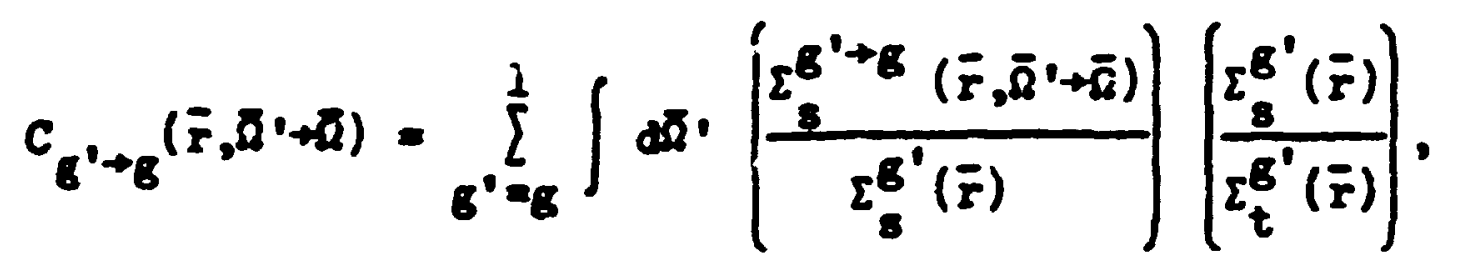

where

$$
\Sigma_{B}^{B^{\prime}}(\bar{r})=\sum_{B} d \bar{Q} \Sigma_{B}^{B^{\prime}+8}\left(\bar{r}, \bar{\Omega}^{\prime}+\bar{Q}\right)
$$


In Eq. $(34),\left[\Sigma_{s}^{B^{\prime}+B}(\bar{r}, \bar{\Omega} \cdot+\sqrt{\Omega}) / \Sigma_{s}^{B^{\prime}}(\bar{r})\right]$ is a normalized probability density function from which the selection of a new energs group and direction can be accouplished and $\left[\Sigma_{S}^{B^{\prime}}(\bar{r}) / \Sigma_{t}^{g^{\prime}}(\bar{r})\right]$ is the nonabsorption probability.

Using the transport and collision integral operators, Eq. (22) can be rewritten as

$$
\psi_{g}(\bar{r}, \bar{\Omega}, t)=T_{g}\left(\bar{r}^{\prime} \rightarrow \bar{r}, \bar{\Omega}\right) s_{g}\left(\bar{r}^{\prime}, \bar{\Omega}, t^{\prime}\right)+C_{g^{\prime} \rightarrow g}\left(\bar{r}^{\prime}, \bar{\Omega}^{\prime}+\bar{\Omega}^{\prime}\right) \psi_{g}\left(\bar{r}^{\prime}, \bar{\Omega}^{\prime}, t^{\prime}\right)
$$

The term $T_{g}\left(\bar{r}^{\prime}, \bar{r}, \bar{\Omega}\right) S_{g}\left(\bar{r}^{\prime}, \bar{\Omega}, t^{\prime}\right)$ can be identified as the "first collision source" and denoted by

$$
S_{c}^{g}\left(\bar{r}_{j}, \bar{l}, t\right) \equiv T_{g}\left(\bar{r}^{\prime}+\bar{r}, \bar{\Omega}\right) S_{g}\left(\bar{r}^{\prime}, \bar{\Omega}, t^{\prime}\right),
$$

and the "Integral Event Density Equation" becomes

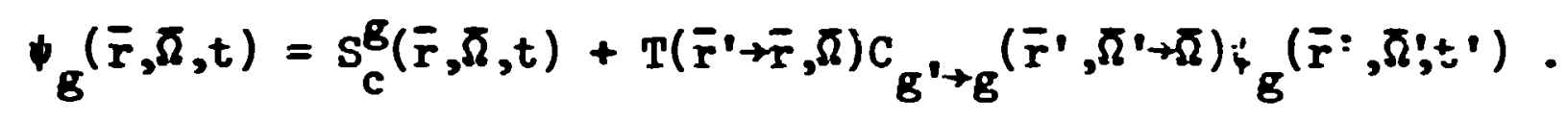

Using the relationship $\psi_{g}(\bar{r}, \bar{\Omega}, t)=\Sigma_{t}^{\mathcal{g}}(\bar{r}) \phi_{B}(\bar{r}, \bar{\Omega}, t), \mathrm{Eq} .(30)$ can be transformed into the "Integral Flux Density Equation:"

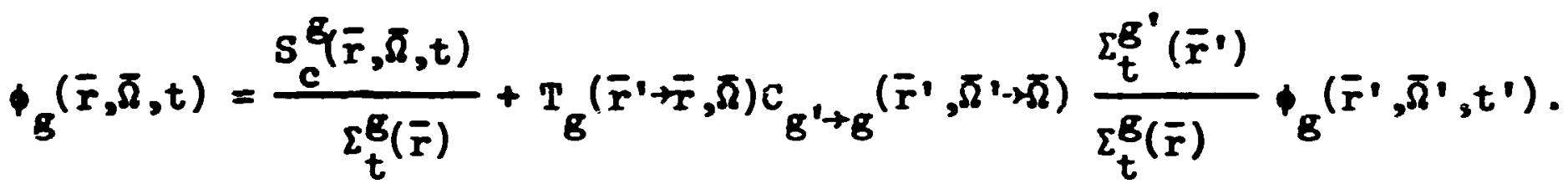

Finally, the integral operators are introduced into Eq. (28) and the following form for the "Integral Bergent Particle Density Equation" is obtained:

$$
x_{B}(\bar{r}, \bar{\Omega}, t)=S_{B}(\bar{r}, \bar{\Omega}, t)+C_{B^{\prime} \rightarrow g}\left(\bar{r}, \bar{\Omega}^{\prime}+\bar{\Omega}\right) T_{g^{\prime}}\left(\bar{r}^{\prime}+\bar{r}, \bar{\Omega}^{\prime}\right) x_{B^{\prime}}\left(\bar{r}^{\prime}, \bar{\Omega}^{\prime}, t^{\prime}\right)
$$

An examination of Equations (38), (39), and (40) would reveal that either the "Integral Event Density Equation" or the "Integral Emergent Particle Density Equation" would provide a reasonable basis for a Monte Carlo random walk. Equation (40) was selected for the MRSE code since the source particles would be introduced according to the natural distrirution rather than the distribution of eirst collisions. However, it is noted that after the introduction of the source particle, the subsequent 
randon wali can be regarded in terms ce either Eg. (38) or Eq. (40) with the particle's weight at a collisign site being the weight befure collistor (ITBC) or the vel git after collision (WATE), respectively.

The rancion valk based on the "Integral Emergent Particle Density Equation" voujd introduce a particle into the system according to the source runct:or. The particle travels to the sice of its first col_isio. as deteraliaed by the transport kernel. Its weight is modified by the non-absorption probability and a new enerky group and fifigt direction are sisiected frow the coliision kernel. The transport and collision kernels are applied successively determining the particle's energent phase sace coordinates corresponding to the second, third, ete., collision sites until the randon walk is terminated due to the reduction of the particie's velght belor sane cut.-off value or because the particle escapes fron that portion of phase spece associated with a particular problem (for exarple, escape $\mathrm{n}$ on the system, slowing dorn below an enersy cutoff, or exceeding some arbitrarily specified age cut-off).

\section{Rendar Walk Procedure}

The actual implesientation of the randon valk procedure is accomplished by aproxinating the integrals inplied in the colision and transport integral operators by the sum

$$
x_{G}(\bar{r}, \bar{\Omega}, t)=\sum_{n=0}^{\infty} x_{B}^{n}(\bar{r}, \bar{\Omega}, t),
$$

where

$$
\begin{aligned}
& x_{B}^{E}(\bar{r}, \bar{\Omega}, t) d \bar{\Omega}=\text { the energent particle density of particles emerging } \\
& \text { from 1ts sth collision and having phase space coordinates (group } \\
& B, \bar{r}, d \Omega \text { about } \bar{\Omega}, t i m e t) \text {, } \\
& x_{g}^{0}(\bar{r}, \bar{\Omega}, t)=s_{g}(\bar{r}, \bar{\Omega}, t) \text {. } \\
& \left.x_{B^{\prime}}^{n_{1}} \bar{r}, \bar{\Omega}, t\right)=\ddot{u}_{B^{\prime}+B^{\prime}}\left(\bar{r}, \bar{\Omega}^{\prime}-\bar{\Omega}\right) T_{B^{\prime}}\left(\bar{r} \leftarrow \bar{r}, \bar{\Omega}^{\prime}\right) x_{B}^{n-1}\left(\bar{r}^{\prime}, \bar{\Omega}^{\prime}, t^{\prime}\right) .
\end{aligned}
$$

Thus, the source cosrinates (Group $\sigma_{0}, \bar{r}_{0}, \bar{\Omega}_{0}$, time $t_{0}$ ) are selected irom $\varepsilon_{g}(\bar{r}, \bar{L}, t)$ end a Iight Aistance $R$ is picked $\Sigma_{t}^{\delta_{0}}(\bar{r}) e^{-B_{B_{0}}}\left(\bar{r}, R, \Omega_{0}\right)$ to deternine the site for the first collisica $\bar{r}_{1}$ and the partisle's age $t_{1}=t_{0}+R / r_{B_{0}}$. The probability of scettering $18 \varepsilon_{8}^{b_{0}}\left(\bar{r}_{1}\right) / \varepsilon_{t}^{E_{0}}\left(\bar{r}_{1}\right)$. All particles are forced to scatter and the? $r$ weight is modified wh this probabllity. A new group $g_{1}$ is selected according to the distribution 


$$
\frac{\int_{i \pi} d \bar{\Omega} \sum_{s}^{B 0+B}\left(\bar{r}_{P} \bar{\Omega}_{0}+\bar{\Omega}\right)}{\sum_{s}^{B_{0}}\left(\bar{r}_{1}\right)}
$$

and then a nev direction $\bar{\Omega}$ is determined fram

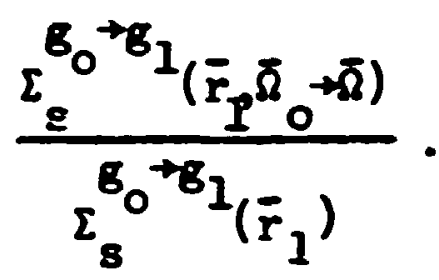

The process is repeated until the particle history is terminated. Contrivutions to the quantity of interest are estinated at appropriate points in the random valk (boundary crossings, before or arter real collisions, etc.) using the particle's WATS and the estinator $P_{B}^{X}(\bar{r}, \bar{D}, t)$.

Derivation of the Adioint Interro-Differ sntial Bo2tzann Fransport Bountion

Consider a (as yet unspecified) runction $(\bar{r}, E, \bar{\Omega}, t)$ which exists over the sane phase spase and satisfies the same kind of boundary conditions satisfied by the forward angular flux $\phi(\bar{r}, E, \bar{D}, t)$. Further, let an operator 0 be defined such that the following integral relationship is satisfied:

$$
\begin{aligned}
& \iiint \int \psi^{*}(\bar{r}, E, \bar{\Omega}, t) 0 \phi(\bar{r}, E, \bar{\Omega}, t) d \bar{r} d B d \bar{R} d t \\
& =\iiint \int(\bar{r}, E, \bar{\Omega}, t) O^{*}(\bar{r}, E, \bar{\Omega}, t) \mathrm{d} \overline{\mathrm{r}} \mathrm{dBd} \bar{\Omega} \mathrm{C} t+\text { (Boundary Terss) . }
\end{aligned}
$$

The $O *$ operator will be referred to as the adjoint operator to the corresponding forward oferator 0 .

Multiply each term of the Boltzmann trarsport equation, Eq. (1), by the furction $(\bar{r}, E, \bar{\Omega}, t)$ and integrate the resultant equation (term by term) over all phase space:

$$
\begin{aligned}
& \iiint \int(\bar{r}, \mathbb{E}, \bar{\Omega}, t) \frac{1}{\bar{v}} \frac{\partial}{\partial t}(\bar{r}, E, \bar{\Omega}, t) d \bar{r} d B d \bar{\Omega} d t+\iiint \int \phi^{*}(\bar{r}, E, \bar{\Omega}, t) \\
& \times \nabla \cdot \bar{\Omega} \phi(\bar{r}, E, \bar{\Omega}, t) d \bar{r} d E d \bar{\Omega} d t+\iiint \int \phi^{*}(\bar{r}, E, \bar{\Omega}, t) \varepsilon_{t}(\bar{r}, E) \\
& \times \phi(\bar{r}, E, \bar{Q}, t) d \bar{r} d E d \bar{\Omega} d t=\iiint \int_{j} \overline{\bar{r}}(\bar{r}, E, \bar{\Omega}, t) s(\bar{r}, E, \bar{\Omega}, t) d \bar{r} d E d \bar{\Omega} d t \\
& +\iiint \phi^{*}(\bar{r}, E, \bar{\Omega}, t) \iint \Sigma_{B}\left(\bar{r}, E^{\prime}+E_{,}, \bar{\Omega}^{\prime}+\bar{\Omega}\right) \phi\left(\bar{r}, E^{\prime}, \bar{\Omega}^{\prime}, t\right) d E^{\prime} d \bar{\Omega}^{\prime} d \bar{r} d E d \bar{\Omega} d t \text {. }
\end{aligned}
$$

It can be show that the following adjolnt relationships are true for the conditions associated with a particle transport problea: 


$$
\begin{aligned}
& \iiint \int \psi(\bar{r}, B, \bar{L}, t) \frac{1}{V} \frac{\partial}{\partial t} \phi(\bar{r}, \bar{z}, \bar{\Omega}, t) d \bar{r} d B d \bar{\delta} d t=-\iiint \int \phi(\bar{r}, E, \bar{L}, t) \frac{1}{r} \frac{\partial}{\partial t}
\end{aligned}
$$

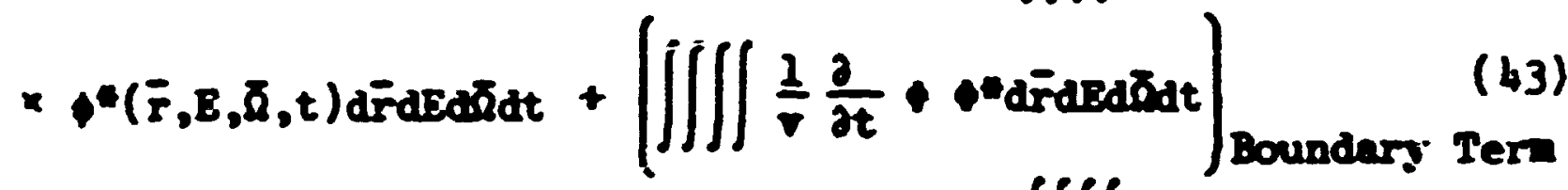

$$
\begin{aligned}
& \iiint \int \psi(\bar{r}, E, \bar{I}, t) \Sigma_{t}(\bar{r}, E) \vartheta(\bar{r}, E, \bar{L} t) d \bar{r} d E d \bar{d} d t=\iiint \int \phi(\bar{r}, E, \bar{L}, t) \\
& \times \Sigma_{t}(\bar{r}, E) \cdot(\bar{r}, E, \bar{\Omega}, t) d \bar{d} d E d \bar{d} d t, \\
& \iiint \int \varphi^{*}(\bar{r}, E, \bar{\Omega}, t) \nabla \cdot \bar{\Omega} \phi(\bar{r}, E, \bar{\Omega}, t) d \bar{r} d B d \bar{d} d t=-\iiint \int \varphi(\bar{r}, E, \bar{D}, t)
\end{aligned}
$$

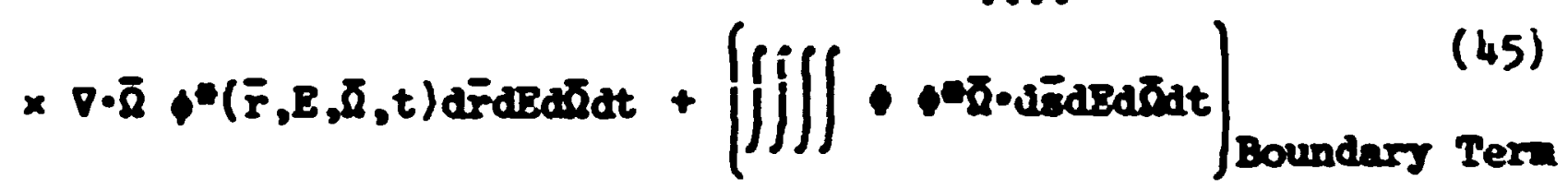

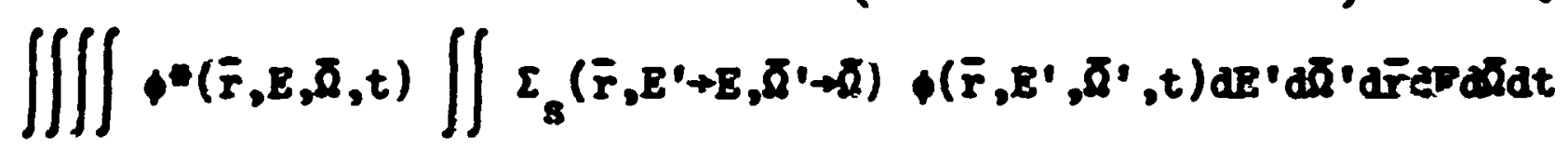

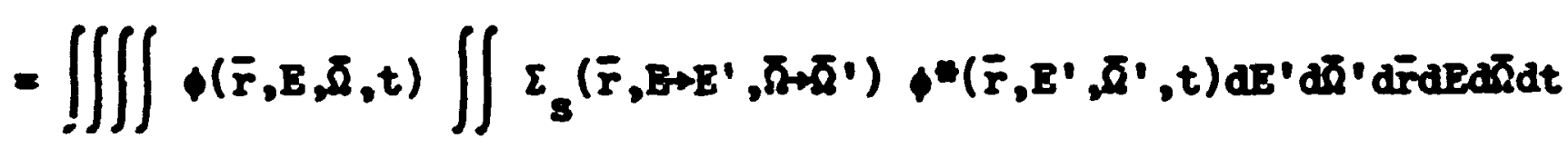

The boundary terms wich occur in Bquations (43) and (45) may be made to vanish while conforning to the natural characteristics of the system under anairsis. For example, the extent of the time domain can be aefined such that initial and final values of $\rightarrow$ and/or " are zero land the boundary ters of Eq. (43) vanishes]. Also, the surface within which the spatial domain of phase space is contained can be so located that the combination [* *i] is zero everynthere on that surfase [and the boundary term of Eq. (45) vanfshes]. For most Monte Carlo analyses, the elimination of the boundary terns in no way restricts the gererality of the solution obtained.

Using the adjoint relationships given by Equations (43) through (46), and presining that the boundary terms vanish, $\mathrm{Eq}$. (42) can be rewritten as

$$
\begin{aligned}
& -\iiint \int \phi(\bar{r}, E, \bar{\Omega}, t) \frac{1}{v} \frac{\partial}{\partial t} \phi^{*}(\bar{r}, E, \bar{\Omega}, t) d \bar{r} d E d \bar{\Omega} d t-\iiint \int \phi(\bar{r}, E, \bar{\Omega}, t) \\
& \times \nabla \cdot \bar{\Omega} \phi^{*}(\bar{r}, E, \bar{\Omega}, t) d \bar{r} d E d \bar{\Omega} d t+\iiint \int \phi(\bar{r}, E, \bar{\Omega}, t) \Sigma_{t}(\bar{r}, E) \phi^{*}(\bar{r}, E, \bar{\Omega}, t) d \bar{r} d E d \bar{\Omega} d t \\
& =\iiint \int \phi(\bar{r}, E, \bar{\Omega}, t) S^{\pi}(\bar{r}, E, \bar{\Omega}, t) d \bar{r} d E d \bar{\Omega} d t+\iiint \int \phi(\bar{r}, E, \bar{\Omega}, t) \\
& \times \iint \Sigma_{s}\left(\bar{r}, E+E^{\prime}, \bar{\Omega}+\bar{\Omega}^{\prime}\right) \phi^{*}\left(\bar{r}, E^{\prime}, \bar{\Omega}^{\prime}, t\right) d E^{\prime} d \bar{r}^{\prime} d \bar{r} d E d \bar{\Omega} d t \text {, }
\end{aligned}
$$


Where the sdjoint source tern $S^{*}(\bar{r}, E, \bar{\Omega}, t)$ is defined suci that

$$
\begin{aligned}
\iiint \int & (\bar{r}, E, \bar{\Omega}, t) S^{*}(\bar{r}, B, \bar{\Omega}, t) d \bar{r} d E d \bar{\Omega} d t \\
& =\iiint \int_{j} \phi^{*}(\bar{r}, E, \bar{\Omega}, t) s(\bar{r}, E, \bar{\Omega}, t) d \bar{r} \bar{\alpha} E d \bar{\Omega} d t .
\end{aligned}
$$

Hoting that the forvard $P I u x \phi(\bar{r}, E, \bar{L}, t)$ can be factored from each tem, Bq. (47) can be rearranged as follons:

$$
\begin{aligned}
& \iiint \int \phi(\overline{\mathbf{r}}, \mathbb{B}, \bar{\Omega}, t)\left(-\frac{1}{\nabla} \frac{\partial}{\partial t} \cdot(\overline{\mathbf{r}}, \mathbf{E}, \bar{\Omega}, t)-\nabla \cdot \bar{\Omega}-(\overline{\mathbf{r}}, \mathbf{E}, \bar{\Omega}, t)\right. \\
& +\Sigma_{t}(\bar{r}, E)-(\bar{r}, E, \bar{\Omega}, t)-S^{*}(\bar{\Sigma}, E, \bar{\Omega}, t)-\iint \Sigma_{s}\left(\bar{r}, E \rightarrow B^{\prime}, \bar{\Omega}^{\prime}+\bar{\Omega}^{\prime}\right) \\
& \left.x \phi^{\prime}\left(\bar{r}, E^{\prime}, \bar{\Omega}^{\prime}, t\right) d E^{\prime} d \bar{\Omega}^{\prime}\right) d \bar{r} d B d \bar{\Omega} d t=0 \text {. }
\end{aligned}
$$

It is required that the forward angular flux $\phi(\bar{r}, E, \bar{\Omega}, t)$ correspond to non-trivial phrsical situations, i.e., $\phi(\bar{r}, E, \bar{\Omega}, t)>0$ over at least some portion of phase space. The observation is made that $*(\bar{r}, \bar{B}, \bar{\Omega}, t)$ is still essentially undefined and that many functions $*(\bar{r}, E, \bar{\Omega}, t)$ probably satisfy Eq. (49). At this point, $\psi(\bar{r}, E, \bar{\Omega}, t)$ j.s defined to be that function which satisfies the following equation:

$$
\begin{aligned}
& {\left[-\frac{1}{\nabla} \frac{\partial}{\partial t} *(\bar{r}, E, \bar{\Omega}, t)-\nabla \cdot \bar{\Omega} \psi(\bar{r}, E, \bar{\Omega}, t)+\varepsilon_{t}(\bar{r}, E) \phi^{*}(\bar{r}, E, \bar{\Omega}, t)-S^{*}(\bar{r}, E, \bar{\Omega}, t)\right.} \\
& \left.-\iint \Sigma_{s}\left(\bar{r}, E^{\prime} \rightarrow E^{\prime}, \bar{\Omega} \rightarrow \bar{\Omega}^{\prime}\right) \psi^{*}\left(\bar{r}, E^{\prime}, \bar{\Omega}^{\prime}, t\right) d Z^{\prime} d \bar{\Omega}^{\prime}\right)=0 \text {. }
\end{aligned}
$$

This condition also satisfies Ea. (49) exactly and provides the following ( $(\bar{r}, E, \bar{\Omega}, t)$-defining integro-differential equation:

$$
\begin{aligned}
-\frac{1}{y} \frac{\partial}{\partial t} \phi^{*}(\bar{r}, E, \bar{\Omega}, t)-\nabla \cdot \bar{\Omega} \phi^{*}(\bar{r}, E, \bar{\Omega}, t)+\varepsilon_{t}(\bar{r}, E) \phi^{*}(\bar{r}, E, \bar{\Omega}, t) \\
\quad=S^{*}(\bar{r}, E, \bar{\Omega}, t)+\iint_{s} \sum_{s}\left(\bar{r}, E^{\prime} \rightarrow E^{\prime}, \bar{\Omega} \rightarrow \bar{\Omega}^{\prime}\right) \phi^{*}\left(\bar{E}, E^{\prime}, \bar{\Omega}^{\prime}, t\right) d E^{\prime} d \bar{\Omega}{ }^{\prime},
\end{aligned}
$$

whicn is commonly cal'ed the "Adjoint Integro-Differential Boltzmann Equation." However, it will not be the practice here to refer to the function $\phi(\bar{r}, \bar{E}, \bar{\Omega}, t)$ 
as the adjoint flux; consistent terninology will be introduced later in this section.

At this point, two procedures for defining and calculating group adjoint fluxes are considered. One nethod involves integrating each term of $\mathrm{Bq}$. (50) over the energy interval $\Delta E_{B}$, wich leads to the following group equations:

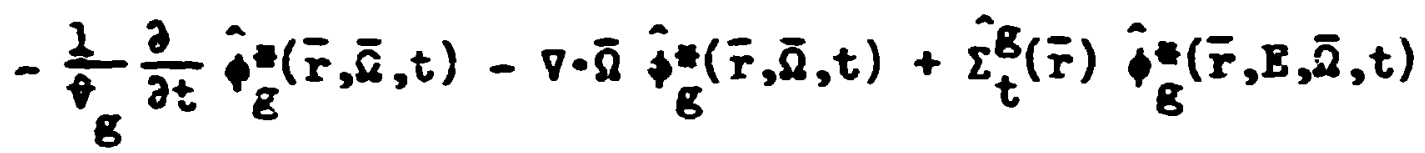

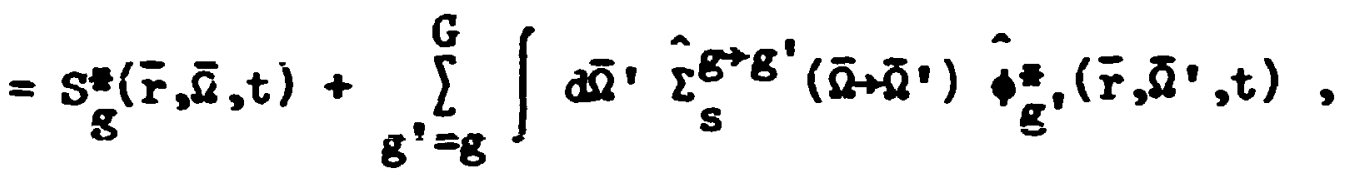

$$
\begin{aligned}
& g=1,2, \ldots G
\end{aligned}
$$

where

$$
\begin{aligned}
& \hat{\phi}_{B}^{*}(\bar{r}, \bar{\Omega}, t)=\frac{1}{\Delta B_{B}} \int_{\Delta E} f_{B}(\bar{r}, E, \bar{\Omega}, t) d E, \\
& \hat{v}_{B}=\frac{\int_{B} \phi^{*}(\bar{r}, E, \bar{\Omega}, t) d E}{\int_{\Delta E} \frac{1}{v} \psi^{*}(\bar{r}, E, \bar{\Omega}, t) d E}
\end{aligned}
$$

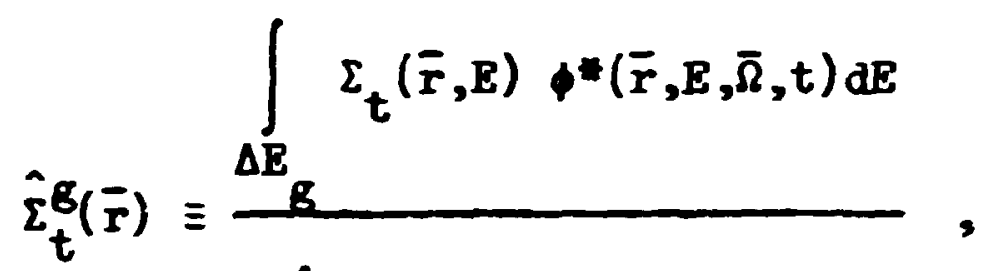

$$
\begin{aligned}
& \int_{\Delta \mathrm{E}} \phi^{E}(\bar{r}, E, \bar{\Omega}, t) d E
\end{aligned}
$$

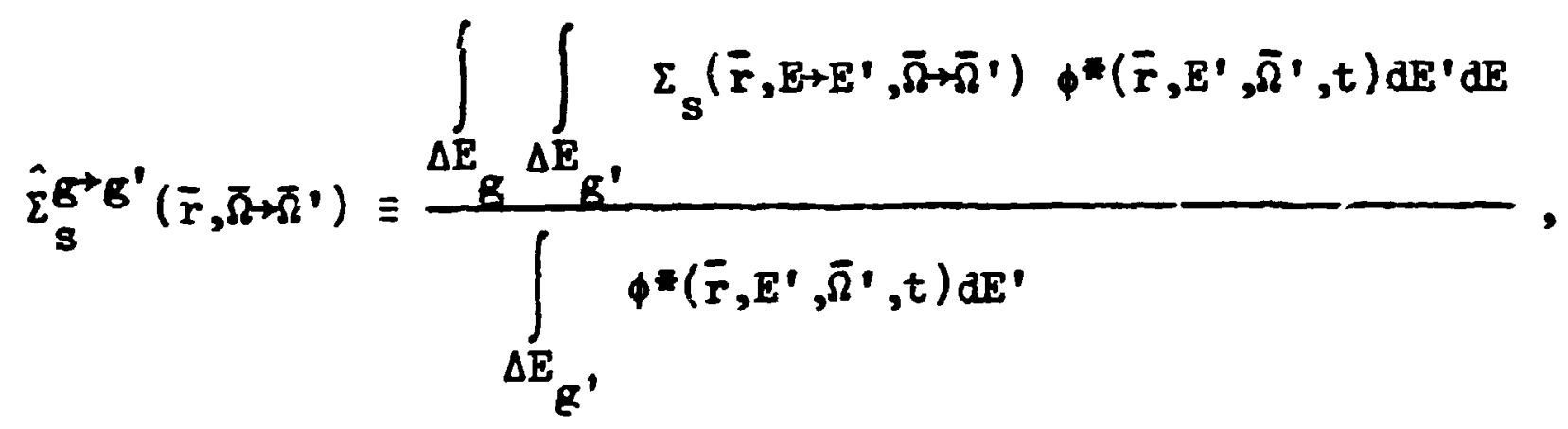

$$
\begin{aligned}
& \hat{S}_{B}^{*}(\bar{r}, \bar{\Omega}, t)=\frac{1}{\Delta E_{B}} \int_{\Delta \mathbb{E}_{B}} S^{*}(\bar{r}, E, \bar{\Omega}, t) d E .
\end{aligned}
$$


The $\hat{L}_{t}^{B}(\bar{r}), \hat{\Sigma}_{s}^{B+B^{\prime}}\left(\bar{r}, \bar{\Omega}+\bar{\Omega}{ }^{\prime}\right)$, and $\hat{v}_{B}$ are adjoint weighted group paraneters and their use in the solution of Eq. (51) provides group adjoint fluxes defined by $\mathrm{Eq}$. (52) where $(\bar{r}, E, \bar{R}, t)$ represents the solution of $\mathrm{Bq} .(50)$.

Another approach for defining group adjoint fluxes is to directly devise the equation which is adjoint to the group form of the Boltzmann equation [Bq. (9)]. The group adjoint equation so obtained is given by

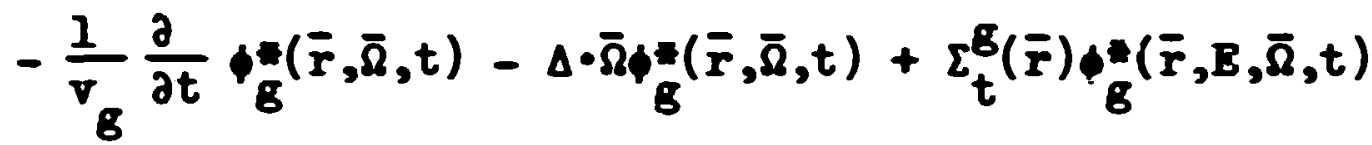

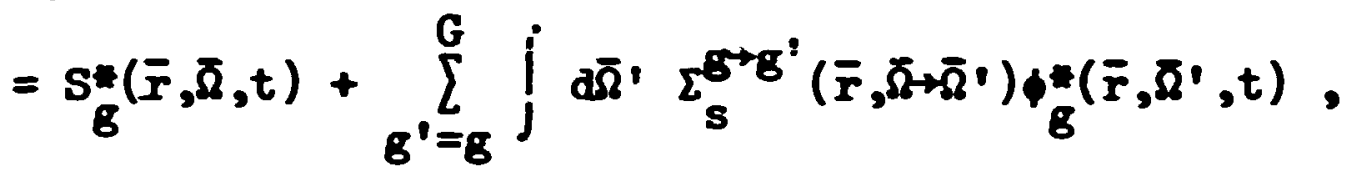

$$
\begin{aligned}
& 8=1,2, \ldots G \text {. }
\end{aligned}
$$

where $v_{B}, \Sigma_{t}^{g}(\bar{r})$ are forvard weighted group parameters identified to those which occur in Eq. (9) and the matrix $\Sigma_{s}^{b+g^{\prime}}\left(\bar{r}, \bar{\Omega}_{\bar{\Omega}}+\bar{\Omega}^{\prime}\right)$ is simply the transposition of the forward weighted group-to-group differential scattering proessection matrix.

The group adjoint fluxes ${ }_{B}(\bar{r}, \bar{\Omega}, t)$ wich represent the solution of $\mathrm{Bq}$. (57) are adjoint to the group fluxes ${ }_{B}$ and do not necessarily assure the same values as the group adjoint fluxes $\hat{\phi}_{B}^{*}(\bar{r}, \bar{\Omega}, t), i . e .$,

$$
\Phi_{B}^{*}(\bar{r}, \bar{\Omega}, t) \neq \frac{1}{\Delta E_{B}} \int_{\Delta E} \phi_{B}(\bar{r}, E, \bar{\Omega}, t) d E
$$

This follows since $\Sigma_{t}^{G}(\bar{r}), \Sigma_{s}^{b+g^{\prime}}\left(\bar{r}, \bar{\Omega}^{\prime}+\bar{\Omega}^{\prime}\right)$, and $v_{g}$ are, in general, different from the adjoint weighted values. Usually forward veignted sroup parameters, as implied by Eq. (57), are used in MORSE. However, other weighting schemes, such as adjoint or adjoint and forward, deserve consideration when cross-section weighting is a problem. When a sufficiently fine group structure is employed, the group parameters become less sensitive to the weighting scheme and the corresponding eroup adjoint fluxes are also nearly the same.

The derivation of Eq. (57) is not presented here because of its similarity with the previcus derivation of $\mathrm{Eq}$. (50); the integrals over energs are simply replaced by appropriate group summations. 


\section{Interral Point-Value Boustion}

Bquation (57) is now transformed into an integral form following essentially the sane procedures used with the forvard equations. As shown in F16. A.2, let $\overline{\mathbf{r}}^{\prime}=\overline{\mathbf{r}}+\mathrm{B}_{\bar{\Omega}}$ rather than $\overline{\mathbf{r}}^{\prime}=\overline{\mathbf{r}}-\mathrm{R} \overline{\mathrm{R}}$ as vas the convention with

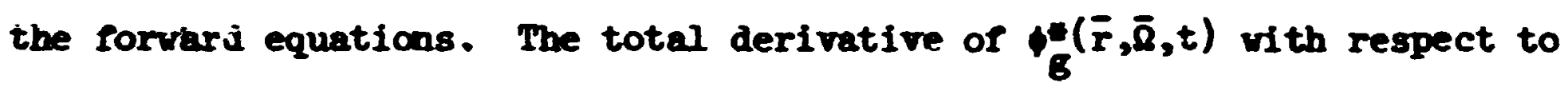
$R$ is given by

$$
\frac{d}{d R} \phi_{B}^{*}\left(r^{\prime}, \Omega, t^{\prime}\right)=\frac{1}{\nabla_{B}} \frac{\partial}{\partial t} \phi_{B}^{*}\left(r^{\prime}, \Omega, t^{\prime}\right)+\nabla \cdot \delta \phi_{B}^{*}\left(r^{\prime}, \Omega, t^{\prime}\right) \text {. }
$$

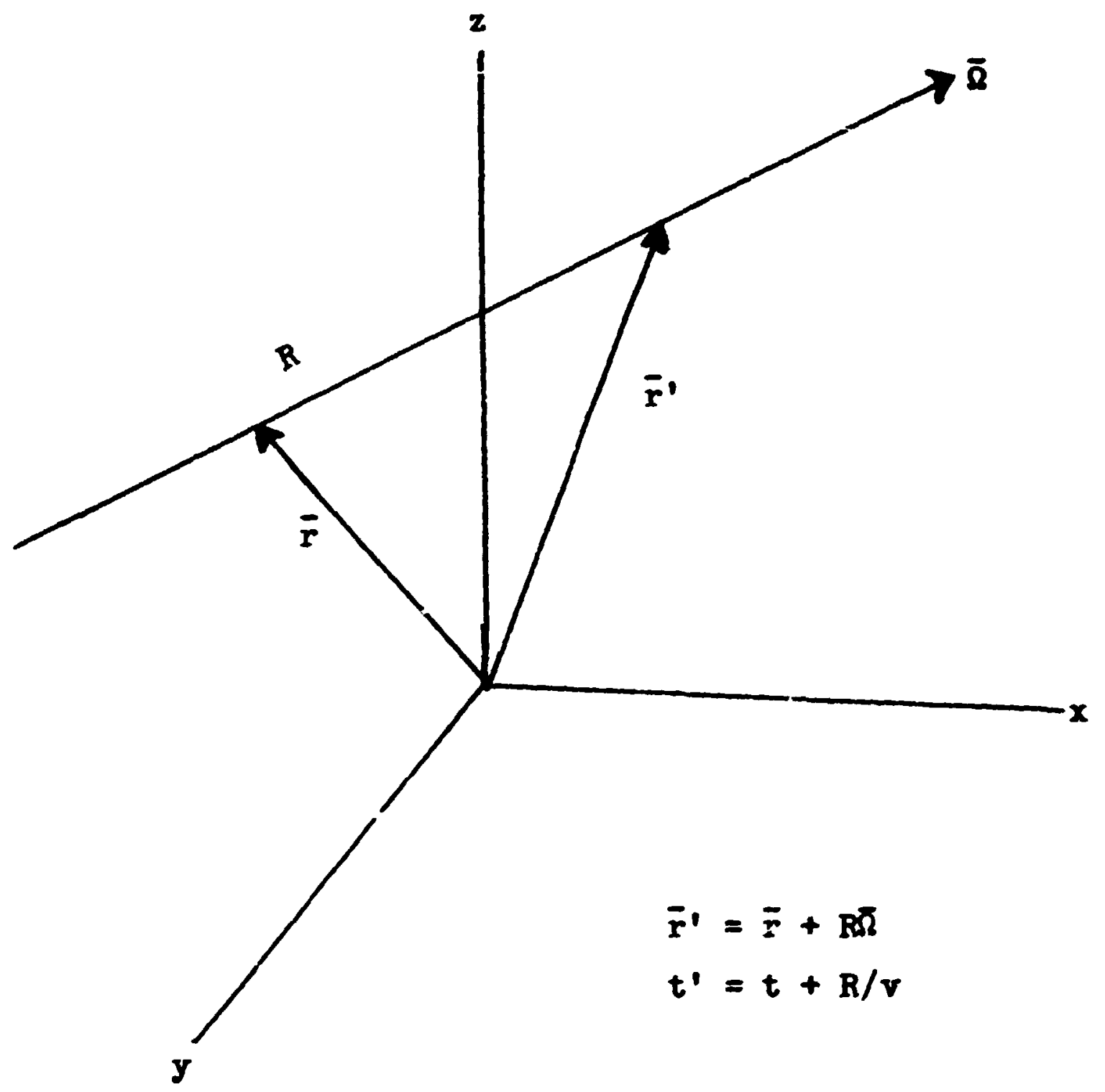

Pigure A.2 
Use of the integrating factor $e$

$$
-\int_{0}^{R} \Sigma_{i}^{B}(\bar{x}+R \cdot \bar{Q}) d R^{\prime}
$$

relatj conship:

$$
\begin{aligned}
& -\int_{0}^{R} \varepsilon_{t}^{\varepsilon}(\bar{r}+R \cdot \bar{R}) d R \cdot \\
& \frac{d}{d R}(\varphi)\left(\bar{E}, \bar{R}_{3}, t^{\prime}\right)=
\end{aligned}
$$

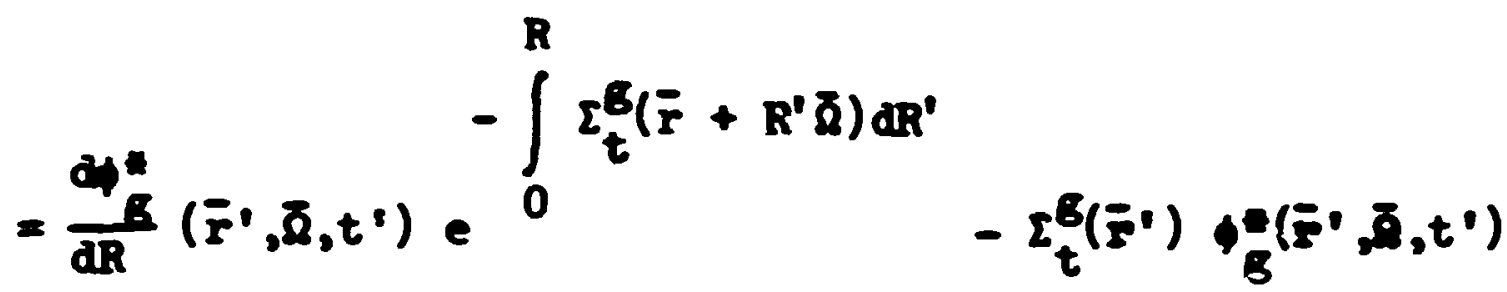

$$
\begin{aligned}
& x e^{-\int_{0}^{R} \varepsilon_{t}^{6}\left(\bar{r}+R^{\prime} \bar{z}\right) d R^{\prime}}=e^{-\int_{0}^{F_{t}^{\prime}} \Sigma_{t}^{6}\left(\bar{r}^{\prime}+R^{\prime} \bar{Q}\right) d R^{\prime}} \\
& \times\left(\frac{d}{d R} \phi_{B}^{m}\left(\bar{r}^{\prime}, \bar{R}^{\prime}, t^{\prime}\right)-\varepsilon_{t}^{B}\left(\bar{r}^{\prime}\right) \phi_{B}^{\prime}\left(\bar{r}^{\prime}, \bar{\Omega}, t^{\prime}\right)\right) .
\end{aligned}
$$

Equation (59), together with Eq. (58), can be arranged to give

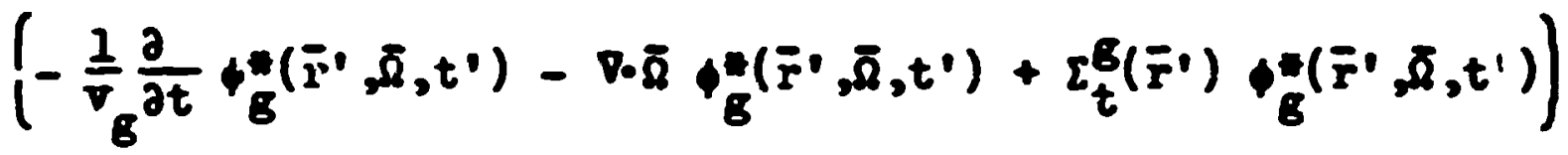

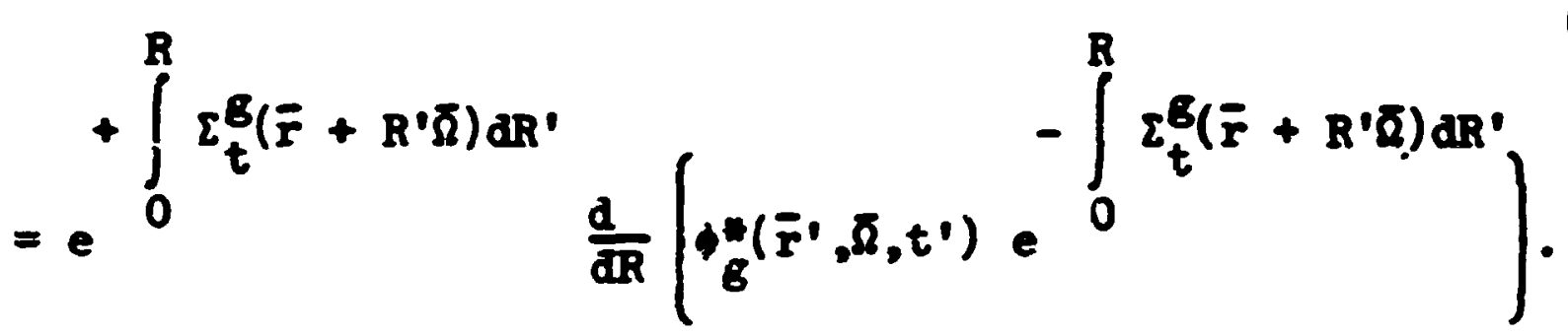

It is noted that Eq. $(60)$ is identically the left-hand side of $\mathrm{Eq}$. (57) which can now be rewritten as

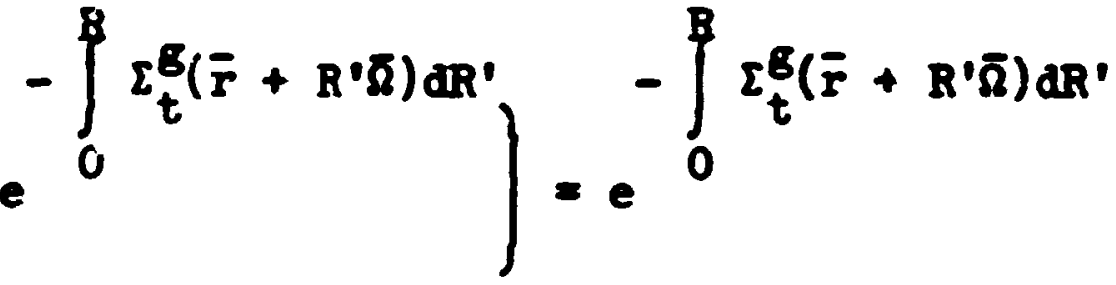

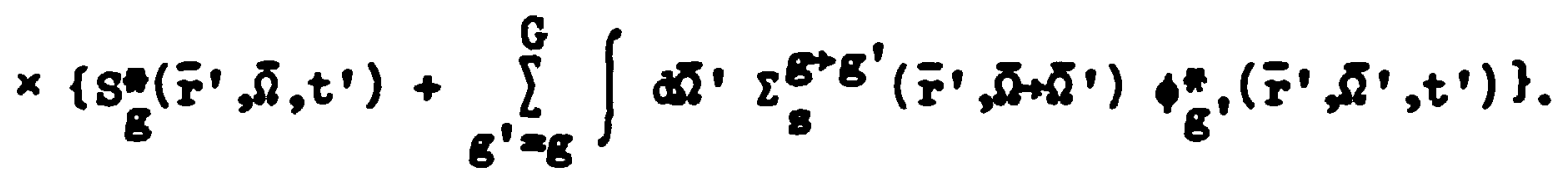


Integrate $\mathrm{Bq}$. (61) Tros $R=0$ to $R=\infty$ and assune that

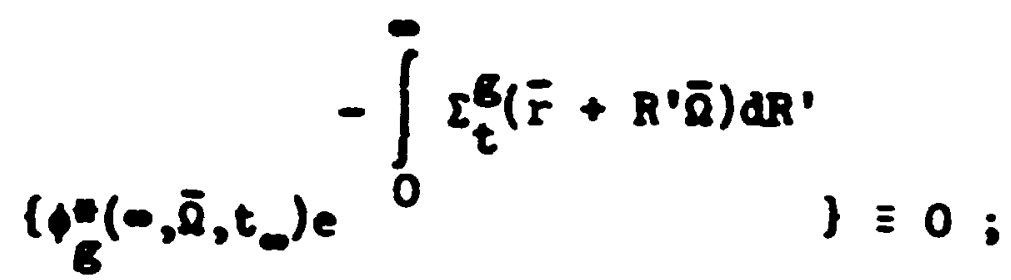

then the following integral expression for $f_{G}(\bar{r}, \bar{D}, t)$ is obtained:

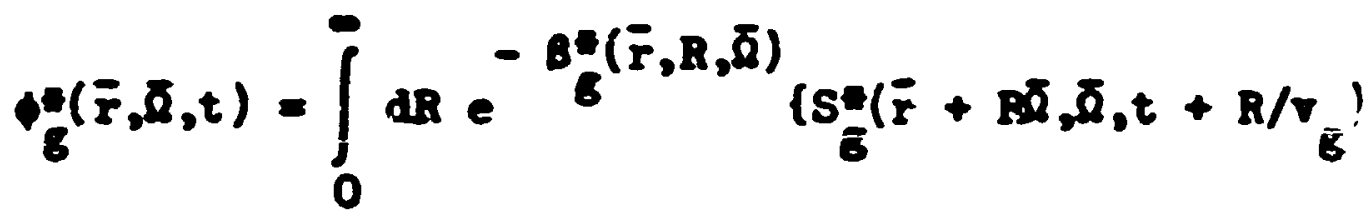

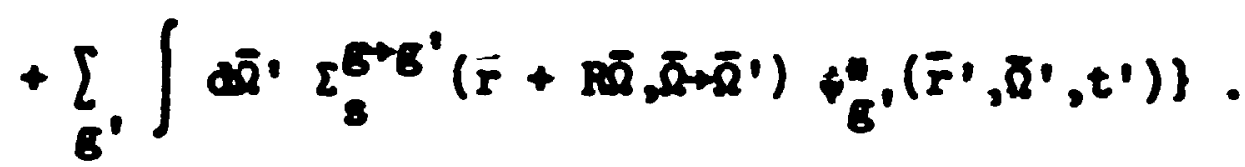

Equation (63) contains the adjoint optical thicluess $B_{B}(\bar{r}, R, \bar{R})$ wich was derined earls sr by $\mathrm{Eq} .(30)$ as

$$
B_{B}=(\bar{r}, R, \bar{\Omega}) \equiv \int_{0}^{R} \varepsilon_{t}^{B}\left(\bar{r}+R^{\prime} \bar{\Omega}\right) d R^{\prime}
$$

Bedefine the source term as

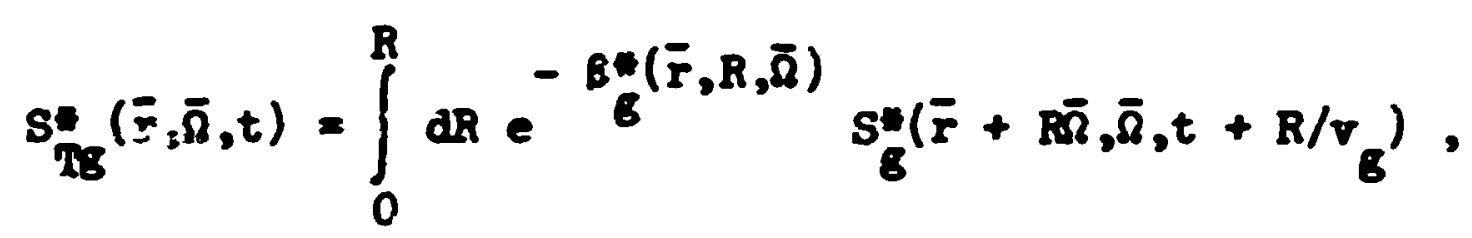

and $\mathrm{Bq}$. (63) can be rewritten as

$$
\begin{aligned}
& \psi_{B}^{*}(\bar{r}, \bar{\Omega}, t)=S_{T g}^{*}(\bar{r}, \bar{\Omega}, t)+\int d R e^{-\beta_{B}^{*}(\bar{r}, \bar{R}, \bar{\Omega})} \sum_{B^{\prime}} \int d \bar{\Omega}^{\prime} \varepsilon_{g}^{G^{+} G^{\prime}}\left(\bar{r} \bar{r}^{\prime}, \bar{\Omega}+\bar{\Omega}^{\prime}\right) \\
& x \phi_{B^{\prime}}\left(\bar{r}^{\prime}, \bar{\Omega} \cdot, t^{\prime}\right)=S_{T_{B}^{*}}(\bar{r}, \bar{\Omega}, t)+\int_{0}^{\infty} d R \Sigma_{t}^{B}(\bar{r}+R) e^{-B_{B}^{*}(\bar{r}, R, \bar{\Omega})} \\
& \times \sum_{B^{\prime}} \int \overline{\Omega^{\prime}} \cdot \frac{\Sigma_{8}^{B^{+} \cdot B^{\prime}}\left(\bar{r}^{\prime}, \bar{\Omega}^{\prime}+\bar{\Omega}^{\prime}\right)}{\Sigma_{t}^{B}\left(\bar{r}^{\prime}\right)} \phi_{B^{\prime}}^{*}\left(\bar{r}^{\prime}, \bar{\Omega}^{\prime}, t^{\prime}\right) \text {, }
\end{aligned}
$$


and in terns of the tranoport and caliision operators, Bq. (65) becomes

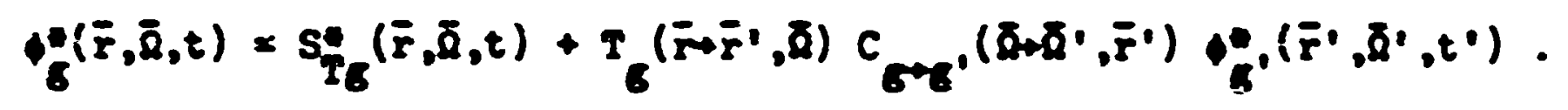

A comparison of Eq. (66) with Equatisns (38), (39), and $(30)$ reveals that the ruction $\theta_{G}(\bar{r}, \bar{l}, t)$ ac defined oy Eq. $(66)$ is adjoint to the carreent particle density $x_{g}(\bar{r}, \bar{h}, t)$ as derined by Bq. $(40)$. Therefure, let $p_{g}(\bar{r}, \bar{q}, t)$ be denoted by $x_{G}(\bar{r}, \bar{L}, \pm)$ and Eq. (66) becomes

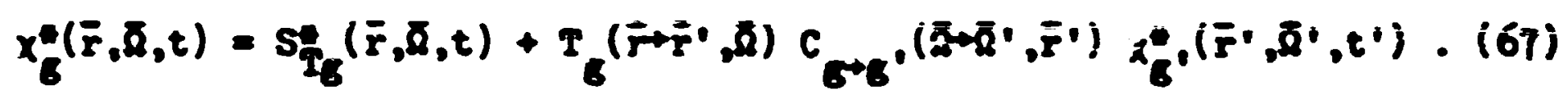

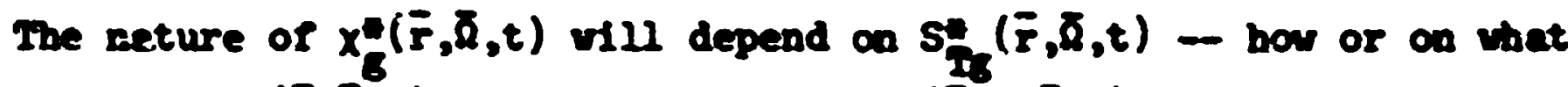
besis should $S_{T E}(\bar{r}, \bar{R}, t)$ be spectified? If $S^{*}(\bar{r}, E, \delta, t)$ is set equal to $P^{\prime}(\bar{r}, B, \bar{\Omega}, t)$ (the response runction of the effect of interest $\lambda$ due to unit arouiar nux), then

$$
\begin{aligned}
& \iiint \int \phi(\bar{r}, E, \bar{\Omega}, t) S=(\bar{r}, E, \bar{R}, \bar{t}) d \bar{r} d E d \bar{l} d t=\iiint \int \phi(\bar{r}, \bar{B}, \bar{\Omega}, t) \\
& \quad \times P^{\phi}(\bar{r}, E, \bar{\Omega}, t) d_{\bar{t}}^{-} d E d \bar{d} d t=\lambda .
\end{aligned}
$$

Accoriling to Eq. (48), the effect of interest $\lambda$ would also be given by

$$
\lambda=\iiint \iint^{*}(\bar{r}, E, \bar{\Omega}, t) S(\bar{r}, E, \bar{\Omega}, t) d \bar{r} d E d \bar{D} d t .
$$

The effect of interest as giren by Eq. (69) can also be expressed in group notation

$$
\begin{aligned}
& \lambda_{E}=\iiint_{B} \hat{B}_{B}(\bar{r}, \bar{\Omega}, t) \hat{S}_{B}(\bar{r}, \bar{\Omega}, t) d \bar{r} d \bar{\Omega} d t \\
& =\iiint g_{B}(\bar{r}, \bar{\Omega}, t) \hat{S}_{B}^{m}(\bar{r}, \bar{\Omega}, t) d \bar{r} \bar{\alpha} \delta d t,
\end{aligned}
$$

where

$$
\begin{aligned}
& \hat{i}_{B}(\bar{x}, \bar{a}, t) \text { is the group adjoint flux corresponding to the adjoint } \\
& \text { weighted group parameters, }
\end{aligned}
$$




$$
\begin{aligned}
& 1-22
\end{aligned}
$$

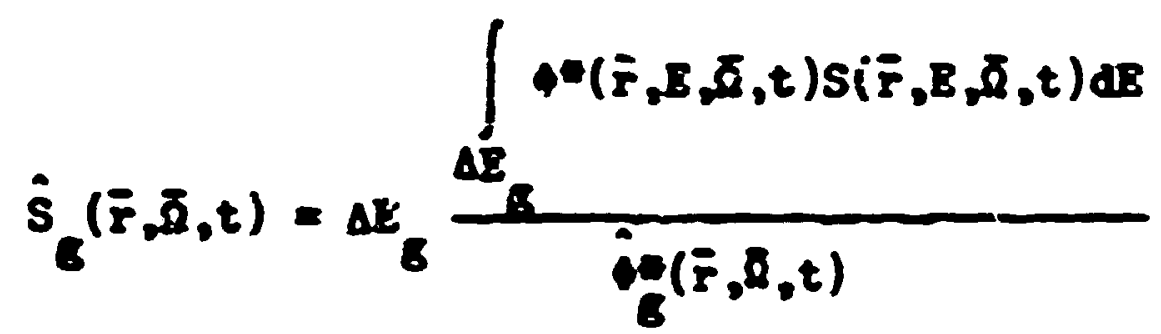

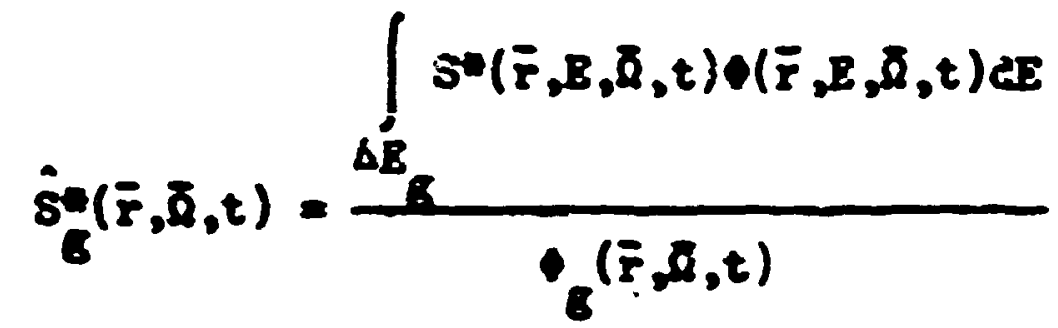

$$
\begin{aligned}
& =\frac{\int_{\Delta B} P^{\hat{T}}(\bar{r}, B, \bar{\Omega}, t) \theta(\bar{r}, B, \bar{\Omega}, t) d B}{P_{B}(\bar{r}, \bar{\Omega}, t)}=P_{B}^{t}(\bar{r}, \bar{\Omega}, t) .
\end{aligned}
$$

Bowever, so noted earlier, usually forrard velghted group paremeters are input to MRSB and tise croup adjoint nuxes $\frac{p}{6}(\overline{5}, \bar{D}, t)$ are calculated. is a direct consequence of the derivation of the $\frac{\|}{B}(\bar{r}, \bar{\Omega}, t)$ defining eqution, Bq. (57), the effect of interest for the eth froup is also edven by

$$
\begin{aligned}
\lambda_{B} & =\iiint_{B}(\bar{r}, \bar{\Omega}, t) s_{B}(\bar{r}, \bar{\Omega}, t) d \bar{r} d \bar{D} d t \\
& =\iiint_{B}(\bar{r}, \bar{\Omega}, t) S_{B}^{*}(\bar{r}, \bar{\Omega}, t) d \bar{r} d \bar{\Omega} d t,
\end{aligned}
$$

where

$$
\begin{aligned}
& S^{*}(\bar{r}, \bar{\Omega}, t) \text { is the group adjoint fux corresponding to the forward } \\
& \text { weighted group parameters, } \\
& S_{B}(\bar{r}, \bar{\Omega}, t)=\int_{\Delta E} S(\bar{r}, E, \bar{\Omega}, t) d E, \\
& S_{B}^{*}(\bar{r}, \bar{\Omega}, t)=P_{B}^{*}(\bar{r}, \bar{\Omega}, t) .
\end{aligned}
$$

'The derivation from this point on will implicitly assume forward weighted group parameters. However, the results can, with slight modificatiou, be made to correspond to the adjoint weighted group parameters. 
Substitution of Bq. (71) into Bq. (64) yields

$$
S_{T_{B}^{*}}(\bar{r}, \bar{Q}, t)=\int A R e^{-\beta^{\hbar}(\bar{r}, R, \bar{\Omega})} P_{B}^{\phi}\left(\bar{r}^{\prime}, \bar{\Omega}, t^{\prime}\right),
$$

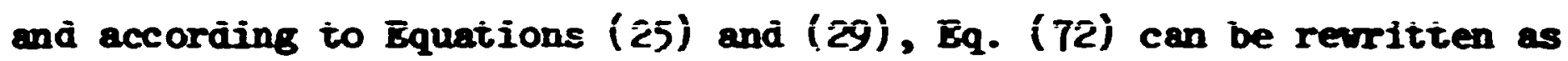
Bquations (73) and (74), respectively:

$$
S_{T_{B}}^{*}(\bar{r}, \bar{\Omega}, t)=\int d R \Sigma_{t}^{B}\left(\bar{x}^{\prime}\right) e^{-\beta_{B}^{\bar{k}(\bar{r}, R, \bar{\Omega})}} P_{E}^{\dagger}\left(\bar{r}^{\prime}, \bar{\Omega}, t^{\prime}\right)
$$

and

$$
S_{T_{B}}(\bar{r}, \bar{L}, t)=P_{B}^{X}(\bar{r}, \bar{\Omega}, t)
$$

Substitution of $\mathrm{Bq}$. (73) into Eq. (67) and Eq. (74) into Bq. (67) Jields the following farsis for the "Integral Point-Value Bquation:"

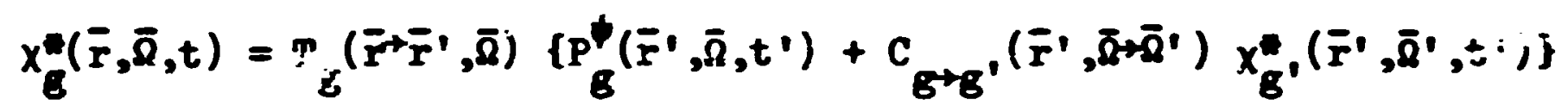

and

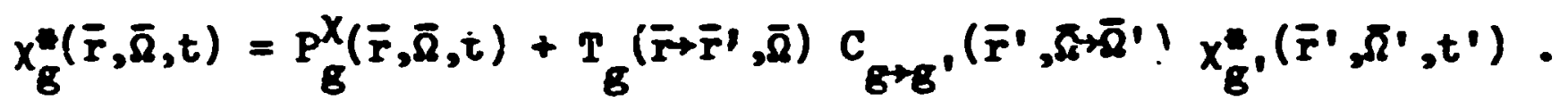

\section{Interrel Event-Valiue Bquation}

At this point let us introduce a value function based on the event density and to relate this cuantity to the point-valus function by considering a particle leuving a collision at $\overline{\mathbf{r}}$ with phase space coordinates (group $8, \bar{\Omega}$, time $t$ ). The value of this particie to the effect of interest is the point-value function $x_{g}^{*}(\bar{r}, \bar{\Omega}, t)$. This perticle will experience an event in dR about $\bar{r}^{\prime}=\bar{r}+R_{\bar{R}}^{\bar{R}}$ with the probability $\left[\Sigma_{t}^{E}\left(\bar{r}^{\prime}\right) e^{-\beta_{\bar{B}}(\bar{r}, R, \bar{\Omega})} d R\right]$ and the value of this event (to the effect of interest) will be referred to as the "event-value" and be denoted by $H_{g}\left(\bar{r}^{\prime}, \bar{\Omega}, t^{\prime}\right)$. That $i s$, the "event-value" $H_{B}\left(\bar{x}^{\prime}, \bar{\Omega}, t^{\prime}\right)$ is defined as the value (to the erfect or intersst) of having an event at $\overline{\mathrm{r}}$ : with an incoming particle which has 
phase space coordinates (group $\bar{B}, \bar{\Omega}$, time $t^{\prime}$ ). The sum of all such contributions to the effect of interest is given by

$$
\int_{0}^{\infty} d R \sum_{t}^{B}\left(\bar{r}^{\prime}\right) e^{-B_{g}^{*}(\bar{r}, R, \bar{\Omega})} W_{g}\left(\bar{r}^{\prime}, \bar{\Omega}, t^{\prime}\right),
$$

and, if the ev unt-value function is properly defined, should equal the point-value function; that is,

$$
x_{g}^{*}(\bar{r}, \bar{\Omega}, t)=\int_{0}^{\infty} \operatorname{dP} \Sigma_{t}^{g}\left(\bar{r}^{\prime}\right) e^{-\beta_{g}^{*}(\bar{r}, R, \bar{\Omega})} W_{g}\left(\bar{r}^{\prime}, \bar{\Omega}, t^{\prime}\right)
$$

or

$$
x_{g}^{*}(\bar{r}, \bar{s}, t)=T_{g}\left(\bar{r} \bar{r}^{\prime}, \bar{\Omega}\right) W_{g^{\prime}}\left(\bar{r}^{\prime}, \bar{\Omega}, t^{\prime}\right)
$$

A comparison of Eq. (78) with Eq. (75) would show that $W_{g}(\bar{r}, \bar{\Omega}, t)$ can be identified as

$$
W_{g}(\bar{r}, \bar{\Omega}, t)=P_{g}^{\psi}(\bar{r}, \bar{\Omega}, t)+C_{g \rightarrow g^{\prime}}\left(\bar{r}, \bar{\Omega}^{\prime} \rightarrow \bar{\Omega}^{\prime}\right) x_{g^{\prime}}^{*}\left(\bar{r}, \bar{\Omega}^{\prime}, t\right),
$$

and substitution of Eq. (78) into Eq. (79) yields the defining equation for the "Event-Value Function"

$$
W_{g}(\bar{r}, \bar{\Omega}, t)=P_{g}^{\psi}(\bar{r}, \bar{\Omega}, t)+C_{g \rightarrow g^{\prime}}\left(\bar{r}, \bar{\Omega}^{\prime} \rightarrow \bar{\Omega}^{\prime}\right) T_{G^{\prime}}\left(\bar{r} \rightarrow \bar{r}^{\prime}, \bar{\Omega}^{\prime}\right) W_{g^{\prime}}\left(\bar{r}^{\prime}, \bar{\Omega}^{\prime}, t^{\prime}\right) .
$$

Equation ( 80 ) will be referred to as the "Integral Event-Value Equation." A comparison of Eq. (80) with Eq. (38) would show that the event-value finction $W_{g}(\bar{r}, \bar{\Omega}, t)$ is adjoint to the event density $\psi_{g}(\bar{r}, \bar{\Omega}, t ;$. Therefore the effect of interest $\lambda_{g}$ is given by

$$
\lambda_{B}=\iiint S_{c}^{g}(\bar{r}, \bar{\Omega}, t) W_{g}(\bar{r}, \bar{\Omega}, t) d \bar{r} d \bar{\Omega} d t .
$$

Integral Emergent Adjuncton Density Equation

The solution of either the point-value equation, Eq. (76), or the event-value equation, Eq. (79), could be accomplished by monte Carlo procedures; however, the random walk would not be the same as that implied by Eq. (40)*. Consider the following altered form of Eq. (76),

The desire in MøRSE is to use the same random walk logic for both forward and adjoint calculations. 


$$
\begin{aligned}
& x_{g}^{*}(\bar{r}, \bar{\Omega}, t)=P_{g}^{\Psi}(\bar{r}, \bar{\Omega}, t)+\int d R \Sigma_{t}^{g}(\bar{r}) e^{-\beta_{B}^{*}(\bar{r}, R, \bar{\Omega})}\left(\frac{\Sigma_{t}^{g}\left(\bar{r}^{\prime}\right)}{\sum_{t}^{g}(\bar{r})}\right)
\end{aligned}
$$

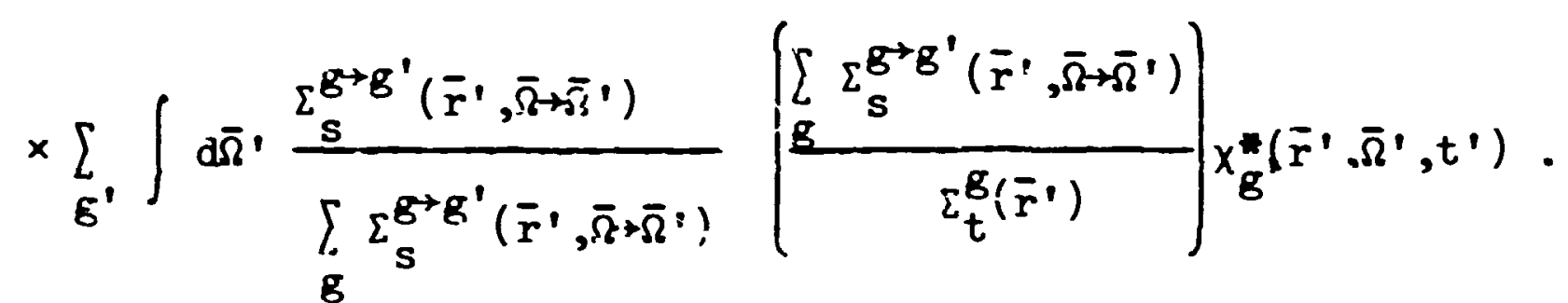

The adaitional weight factor $\left[\underline{\Sigma}_{t}^{g}\left(\bar{r}^{\prime}\right) i \Sigma_{t}^{g}(\bar{r})\right]$ arises since Eq. ( $(6)$ and its altered form (Eq. (82), are actubily slux-like equations, even though $x_{g}^{*}(\bar{r}, \bar{\Omega}, t)$ is adjoint to the emergent particle density $x_{g}(\bar{r}, \bar{\Omega}, t)$.

In a fashion analogous to the forward problem, the following new quantities are defined:

and

$$
H_{g}(\bar{r}, \bar{\Omega}, t) \equiv \Sigma_{t}^{\bar{J}}(\bar{r}) x_{g}^{*}(\bar{r}, \bar{\Omega}, t)
$$

$$
\mathrm{H}_{\mathrm{g}}(\bar{r}, \bar{\Omega}, t)=\mathrm{T}_{\mathrm{g}}\left(\overline{\mathrm{r}}+\overline{\mathrm{r}}^{\prime}, \bar{\Omega}\right) \mathrm{G}_{\mathrm{g}}\left(\overline{\mathbf{r}}^{\prime}, \bar{\Omega}, \mathrm{t}^{\prime}\right) \text {. }
$$

Since $x_{g}(\bar{r}, \bar{\Omega}, t)$ is a flux-like variable, the new variable $H_{g}(\bar{r}, \bar{\Omega}, t)$ can be regerided as an event density and $G_{g}(\bar{r}, \bar{\Omega}, t)$ like an emergent particle dersity. The defining integral equation for $G_{g}(\bar{r}, \bar{\Omega}, t)$ should be the proper basis for an adjoint randoiw walk.

The defining equation for the adjoint event density function $\mathrm{y}_{\mathrm{g}}(\overline{\mathrm{s}}, \bar{\Omega}, t)$ is obtained by considering the following altered form of Eq. (75):

$$
\begin{gathered}
x_{g}^{*(\bar{r}, \bar{\Omega}, t)}=\int d R \Sigma_{t}^{g}\left(\bar{r}^{\prime}\right) e^{-\beta_{g}^{*(\bar{r}, R, \bar{\Omega})}\left[P_{g}^{\psi}\left(\bar{r}, \bar{\Omega}, t^{\prime}\right)\right.} \\
\left.\quad+C_{g \rightarrow g^{\prime}}\left(\bar{r}^{\prime}, \bar{\Omega}^{\prime} \rightarrow \bar{\Omega}^{\prime}\right) x_{g^{\prime}}\left(\bar{r}^{\prime}, \bar{\Omega}^{\prime}, t^{\prime}\right)\right] .
\end{gathered}
$$

Multiply EU. $(\hat{\imath} ;)$ by $\Sigma_{t}^{g}(\bar{r})$ and rearrange as follcws:

$$
\begin{aligned}
& \Sigma_{t}^{g}(\bar{r}) x_{g}^{*}(\bar{r}, \bar{\Omega}, t)=\int d R \Sigma_{t}^{g}(\bar{r}) e^{-\beta_{g}^{*}(\bar{r}, R, \bar{\Omega})}\left[\Sigma_{t}^{g}\left(\bar{r}^{\prime}\right) P_{g}^{\psi}\left(\bar{r}^{\prime}, \bar{\Omega}, t^{\prime}\right)\right. \\
&\left.+\check{C}_{g^{\prime} \rightarrow g^{\prime}}\left(\bar{r}^{\prime}, \bar{\Omega}^{\prime} \rightarrow \bar{\Omega}^{\prime}\right) \Sigma_{t}^{g^{\prime}}\left(\bar{r}^{\prime}\right) x_{g^{\prime}}^{*}\left(\bar{r}^{\prime}, \bar{\Omega}^{\prime}, t^{\prime}\right)\right]
\end{aligned}
$$


where

$$
\check{C}_{g \rightarrow g^{\prime}}\left(\bar{r}^{\prime}, \bar{\Omega}+\bar{s}_{h}^{\prime}\right) \equiv \frac{\Sigma_{t}^{g}\left(\bar{r}^{\prime}\right)}{\sum_{t}^{g^{\prime}}(\bar{r})} c_{g+B^{\prime}}\left(\bar{r}^{\prime}, \bar{\Omega}+\bar{s}^{\prime}\right) .
$$

Noting that:

$$
\begin{aligned}
& H_{g}(\bar{r}, \bar{\Omega}, t)=\Sigma_{t}^{g}(\bar{r}) x_{g}^{*}(\bar{r}, \bar{\Omega}, t), \\
& \int d \bar{R} \Sigma_{t}^{g}(\bar{r}) e^{-\beta_{g}^{*}(\bar{r}, R, \bar{\Omega})}=r_{g}(\bar{r}+\bar{r},, \bar{\Omega}),
\end{aligned}
$$

and

$$
\Sigma_{t}^{\mathrm{g}}(\overline{\mathrm{r}}) \mathrm{P}_{\mathrm{g}}^{\Psi}(\overline{\mathrm{r}}, \bar{\Omega}, t)=\mathrm{P}_{\mathrm{g}}^{\phi}(\overline{\mathrm{r}}, \bar{\Omega}, t),
$$

Eq. ( 86) becomes

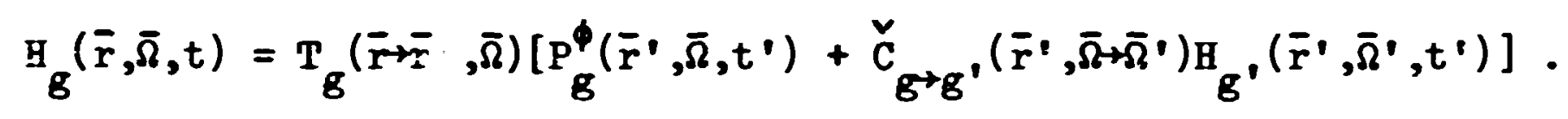

A comparison of Eq. (88) with Eq. (84) reveals that

$$
G_{g}(\bar{r}, \bar{\Omega}, t)=F_{g}^{\phi}(\bar{r}, \bar{\Omega}, t)+\check{C}_{g \rightarrow g^{\prime}}\left(\bar{r}, \bar{\Omega}^{\prime}+\bar{\Omega}^{\prime}\right) H_{g}\left(\bar{r}, \bar{\Omega}^{\prime}, t\right) \cdot
$$

and the subsequent substitution of Eq. (84) into Eq. (39) yieids the following defining equation for the adjoint emergent particle density:

$$
G_{g}(\bar{r}, \bar{\Omega}, t)=P_{g}^{\phi}(\bar{r}, \bar{\Omega}, t)+\check{C}_{\bar{B}^{\prime}+g^{\prime}}\left(\bar{r}, \bar{\Omega}+\bar{\Omega}^{\prime}\right) T_{g^{\prime}}\left(\bar{r} \rightarrow \bar{r}^{\prime}, \bar{\Omega}^{\prime}\right) G_{g^{\prime}}\left(\bar{r}^{\prime}, \bar{\Omega}^{\prime}, t^{\prime}\right) . \text { (90) }
$$

Equation (90) is almost identical with Eq. (40) which defines the forward emergent particle density $x_{B}(\bar{r}, \bar{\Omega}, t)$ and alsc serves as the formal basis for the forward random walk. At this point, let us interpret Eq. (90) in terms of the transport of pseudo-particles called "adjunctons" in the $\left(P^{\prime} \rightarrow P\right)$ direction of phase space. This presents two immediate problems:

1) The transport of the adjunctons from $\bar{r}^{\prime}=\bar{r}+R \bar{\Omega}$ to $\bar{r}$ would be in a direction opposite to the direction vector $\bar{\Omega}$ - therefore, the direction vestor for the adjuncton should be $\hat{\Omega} \equiv-\bar{\Omega}$, and $\overline{\mathbf{r}}^{\prime}=\overline{\mathbf{r}}-\mathrm{R} \dot{\Omega}$. 
2) The collision kernel should be interpreted as describing the $\left(P^{\prime} \rightarrow P\right)$ change in phase space experienced by the adjuncton during its random walk; therefore, let

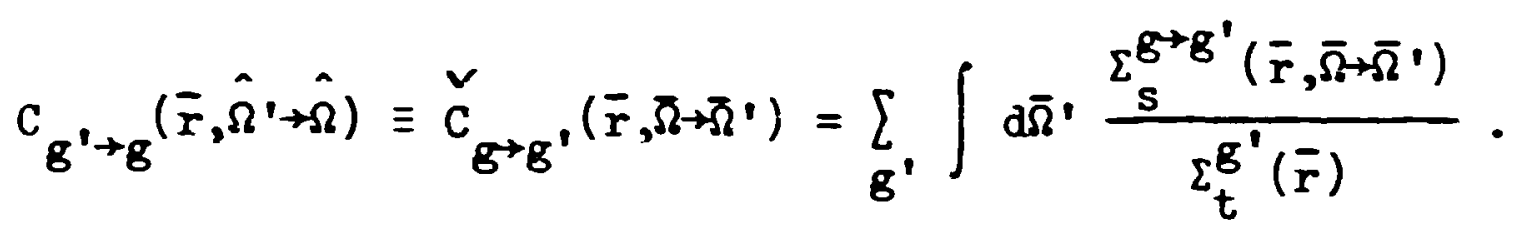

Equation (91) may be rewritten in terms of a noimalized collision kernel and a wétght factor:

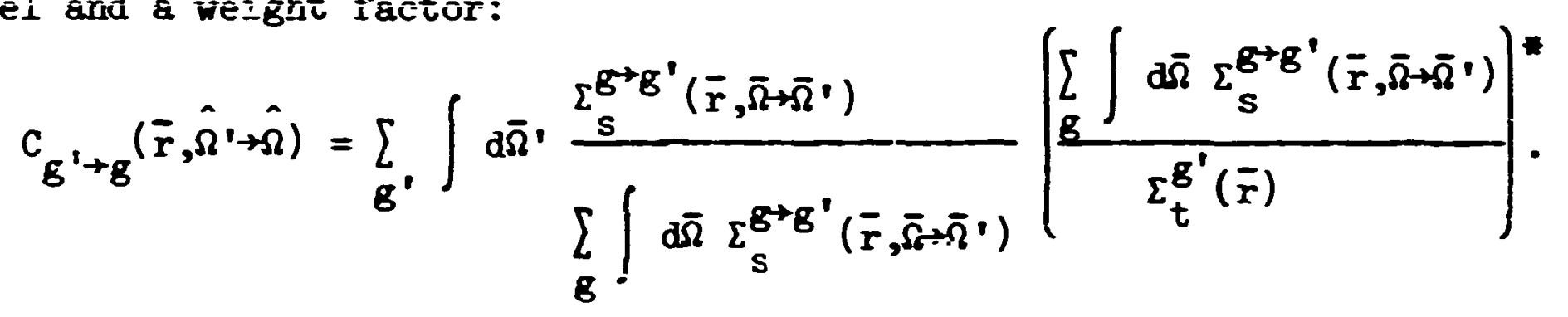

The selection of new phase space coorlinates (group $g, \hat{z}=-\ddot{\Omega}$ ) is made from the normalized kernel and the weight of the adjuncton is modified by the weight factor []* which is no longer a simple non-absorption probability and may assume values in excess of unity. Therefore, there is no "analogue" scattering for adjunctons and the adjuncton's veight may increase at some ccllisions.

Equation (90) can be rewritten as

$$
G_{g}(\bar{r}, \hat{\Omega}, t)=P_{g}^{\phi}(\bar{r}, \hat{\Omega}, t)+C_{g^{\prime} \rightarrow g}\left(\bar{r}, \hat{\Omega}^{\prime} \rightarrow \hat{\Omega}\right) T_{g^{\prime}}\left(\bar{r}^{\prime}+\bar{r}_{,} \hat{\Omega}^{\prime}\right) G_{g^{\prime}}\left(\bar{r}^{\prime}, \hat{\Omega}^{\prime}, t^{\prime}\right),
$$

which now corresponds to the transpcrt of anjunctons and provides the desired basis for the adjoint random walk in the MRSE code. Note that the source of adjunctons is provided by $P_{g}^{\phi}\left(\bar{r}, \hat{S}_{l}, t\right)$ which is related to $P_{g}^{\phi}(\bar{r}, \bar{\Omega}, t)$ as follows:

$$
\mathrm{P}_{\mathrm{B}}^{\phi}(\overline{\mathrm{r}}, \hat{\Omega}, t)=\mathrm{P}_{\mathrm{B}}^{\phi}(\overline{\mathrm{r}}, \bar{\Omega}, \mathrm{t}),
$$

which must be taken into consideration if the response function $P_{B}^{\phi}(\bar{r}, \bar{\Omega}, t)$ has angular dependence -- however, meny physical situations permit an isotropic assumption for the $\bar{\Omega}$-dependence.

A Monte Carlo solution of Eq. (93), the "integral emergent adjunction density equation,"will generate data from which the adjuncton flux $x_{g}^{*}(\bar{r}, \hat{\Omega})$ and 
other quantities of interest can be determined. The general use of $x_{\vec{g}}^{*}(\bar{r}, \hat{\imath})$ must take into eccount the reversal of direstion between adjunctons and real particles, i.e., $\hat{\Omega}=-\bar{\Omega}$. For exemple, consider the various ways of calculating the answer of interest:

$$
\begin{aligned}
& \lambda_{g}=\iiint P_{g}^{\phi}(\bar{r}, \bar{\Omega}, t) \phi_{g}(\bar{r}, \bar{\Omega}, t) d \bar{r} d \bar{\Omega} d t \\
& =\iiint \frac{P_{\bar{B}}^{\phi}(\bar{r}, \bar{\Omega}, t)}{\Sigma_{t}^{g}(\bar{r})} T_{g}\left(\bar{r}^{\prime}+\bar{r}, \bar{\Omega}\right) x_{g}\left(\bar{r}^{\prime}, \bar{\Omega}, t^{\prime} ; j d \bar{r} d \bar{\Omega} d t\right. \\
& \lambda_{g}=\iiint S_{g}(\bar{r}, \bar{\Omega}, t) \chi_{g}^{*}(\bar{r}, \bar{\Omega}, t) d \bar{r} d \bar{\Omega} d t=\iiint_{B}(\bar{r}, \bar{\Omega}, t) \chi_{g}^{*}(\bar{r},-\hat{\Omega}, t) d \bar{r} d \bar{\Omega} d t \\
& \lambda_{B}=\iiint \int_{c}^{\mathcal{G}}(\bar{r}, \bar{\Omega}, t) W_{B}(\bar{r}, \bar{\Omega}, t) d \bar{r} d \bar{\Omega} d t=\iiint S_{c}^{\mathcal{B}}(\bar{r}, \bar{\Omega}, t) W_{B}(\bar{r},-\hat{\Omega}, t) d \bar{r} d \bar{\Omega} d t
\end{aligned}
$$

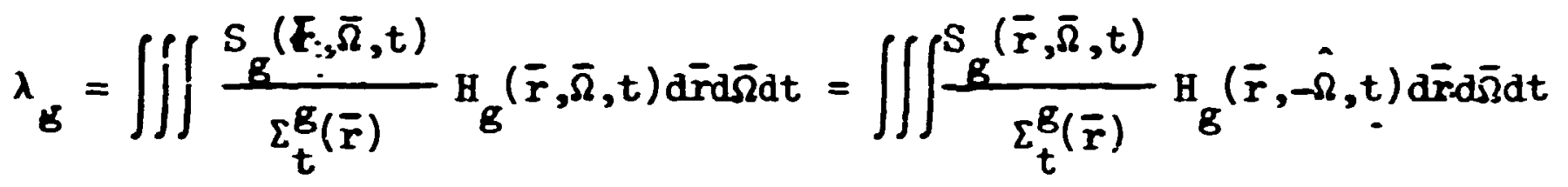

$$
\begin{aligned}
& \lambda_{B}=\iiint \frac{S_{g}(\bar{r}, \bar{\Omega}, t)}{\Sigma_{t}^{g}(\bar{r})} T_{g}\left(\bar{r}^{\prime}-\bar{r}, \bar{S}_{0}\right) G_{g}\left(\bar{r}^{\prime}, \bar{\Omega}, t^{\prime}\right) d \bar{r} d \bar{\Omega} d t \\
& =\iiint \frac{S_{g}(\bar{r}, \bar{\Omega}, t)}{\sum_{t}^{g}(\bar{r})} T_{g}\left(\bar{r}^{\prime}+\bar{r},-\bar{\Omega}\right) G_{g}\left(\bar{r}^{\prime},-\bar{\Omega}, t^{\prime}\right) d \bar{r} \bar{\alpha} \bar{\Omega} d t .
\end{aligned}
$$

Further, if outward boundary crcssings wolla be scored in the forward problem, the corresponding source adjunctons would be introduced in the inward direction. Likevise, adjunctons would be scored for entering a volume fram which the source particles in the forward problem would be emitted. It should te noted that many sources and response furctions are isotropic and the problem of direction reversal need not be considered. 


\section{Multiplying Systems}

The general integral equations in group notation of the previous section are here specialized to the problem ci multipiying systers. In a fissioning system it will be presumed that the source of neutrons for the nth generation comes from fissions which occur uuring the previous generation, the (n-l)st generation. In group nctation and sevendimensional phase space, the source term for the nth generation, $s_{g}^{n}(\bar{r}, \bar{\Omega}, t)$, is given by

$$
S_{g}^{n}(\bar{r}, \bar{\Omega}, t)=\int_{\Delta B_{B}} S^{n}(\bar{r}, E, \bar{\Omega}, t) d E
$$

where

$S^{n}(\bar{r}, E, \bar{\Omega}, t) d E d \bar{\Omega}=$ source particles etitted for the nth generation per init volume and time $a$ s the space point $\bar{r}$ and time $t$ with energies in $d E$ about $E$ and dire ztions which lie in $d \bar{\Omega}$ about $\bar{\Omega}$,

$$
\begin{aligned}
& S^{n}(\bar{r}, E, \bar{\Omega}, i)=\frac{f(E)}{4 \pi} \iint_{4 \pi} d E^{\prime} d \bar{\Omega} \cdot v \Sigma_{f}\left(\bar{r}, E^{\prime}\right) \phi^{n-1}\left(\bar{r}, E^{\prime}, \bar{\Omega}^{\prime}, t\right) \\
& f(E) d E=\text { fraction of fission } x \text { cutrons emitied heving energies in } d E \\
& \text { about } E, \\
& \phi^{n-1}(\bar{r}, E, \bar{\Omega}, t)=\text { anguiar neutron flux for the }(n-1) \text { st generation, } \\
& v \Sigma_{f}\left(\bar{r}, E^{\prime}\right)=\text { fission neutron jield } x \text { macroscopic } f^{\prime} \text { ssion cross section. }
\end{aligned}
$$

Substitution of Eq. (101) into Eq. (100) and expressing the energy integration as a summation over energy groups yields

$$
S_{B}^{n}(\bar{r}, \bar{\Omega}, t)=\frac{f}{4 \pi} \sum_{B^{\prime}=G}^{1} \int_{4 \pi} d \bar{\Omega}^{\prime} v \Sigma_{f}^{g^{\prime}}(\bar{r}) \phi_{g^{\prime}}^{n-1}\left(\bar{r}, \bar{\Omega}^{\prime}, t\right),
$$

The terms generation and betch will be used interchangeably in this section and will refer to the batches of neutrons processed in the MdRSE Monte Carlo calculation. 
where

$$
\begin{aligned}
& f_{B}=\int_{\Delta E} f(E) d E \\
& \nu \Sigma_{f}^{B}(\bar{r})=\frac{\int_{\Delta E} \nu \Sigma_{f}(\bar{r}, E) \phi(\bar{r}, E, \bar{\Omega}, t) d E}{\int_{\Delta E} \phi(\bar{r}, E, \bar{\Omega}, t) d E}
\end{aligned}
$$

Equation (102) can also be expressed in terms of the emergert particle deisity:

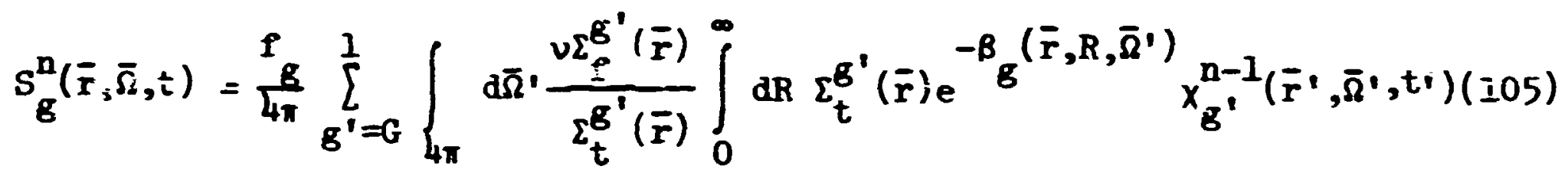

where

$$
\phi_{g^{\prime}}\left(\bar{r}, \bar{\Omega}^{\prime}, t\right)=\int_{0}^{\infty} d R e^{-\beta_{B^{\prime}}\left(\bar{r}, R, \bar{\Omega}^{\prime}\right)} x_{g^{\prime}}\left(\bar{r}^{\prime}, \bar{\Omega}^{\prime}, t^{\prime}\right),
$$

so that for 2 given $S_{g}^{n}(\bar{r}, \bar{\Omega}, t)$, the emergent particle density distribution for the nth generation can be calculated using the following modified form of Eq. (27):

$$
\begin{aligned}
& x_{G}^{n}(\bar{r}, \bar{\Omega}, t)=s_{g}^{n}(\bar{r}, \bar{\Omega}, t)
\end{aligned}
$$

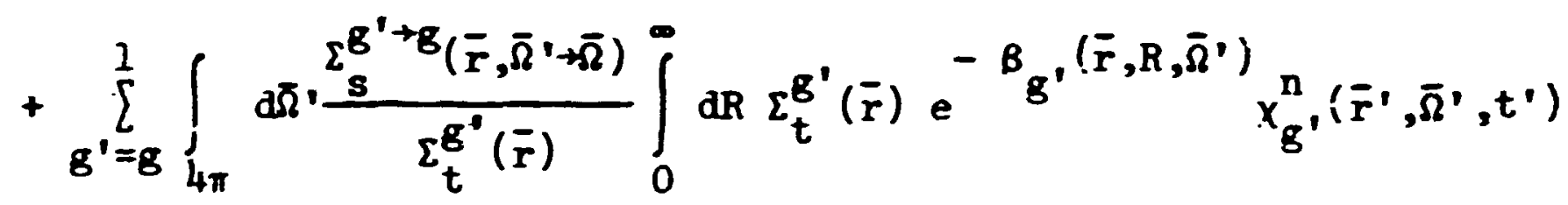

$$
\begin{aligned}
& =S_{B}^{n}(\bar{r}, \bar{\Omega}, t)+C_{B^{\prime}+g^{\prime}}\left(\bar{r}, \bar{\Omega}^{\prime}+\bar{\Omega}\right) T_{G^{\prime}}\left(\bar{r}^{\prime}+\bar{r}, \bar{\Omega}^{\prime}\right) x_{B^{\prime}}^{r_{1}}\left(\bar{r}^{\prime}, \bar{\Omega}^{\prime}, t^{\prime}\right) .
\end{aligned}
$$

Equations (105) and (10?) can be combined and written as an eigenvalue squation in seven-dimensional phase space 


$$
\begin{aligned}
& x_{g}(\bar{r}, \bar{\Omega}, t)=\frac{1}{k} \frac{f}{4 \pi} \sum_{g^{\prime}=G}^{l} \int_{4 \pi} d \bar{\Omega} \cdot \frac{v \Sigma_{f}^{g^{\prime}}(\bar{r})}{\sum_{t}^{g^{\prime}}(\bar{r})} d R \Sigma_{t}^{g^{\prime}}(\bar{r}) e^{-B} e_{g}^{(\bar{r}, R, \bar{\Omega})} x_{g^{\prime}}\left(\bar{r}, \bar{\Omega}, t^{\prime}\right)
\end{aligned}
$$

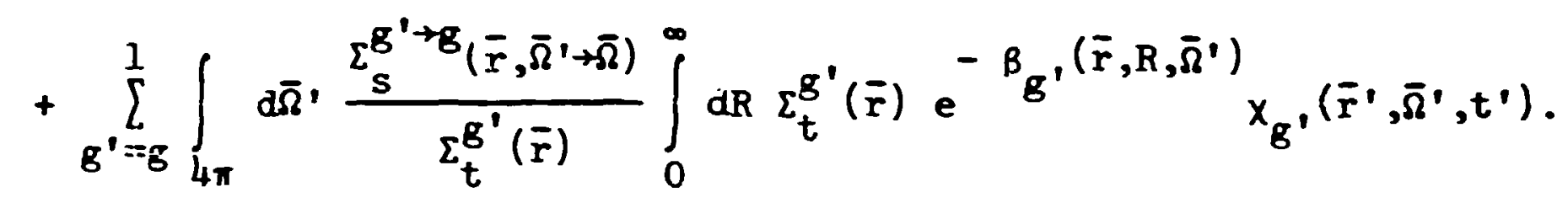

The usual objective in a reactor calculation is to find the eifenfunctions $x_{g}(\bar{r}, \bar{\Omega}, t)$, and the eigenvalue $k$. In MORSE this is accomplished iteratively, each batch being one iteration. The source for the first batch is unknown and must be assumed. From this source ari estimate o: the resulting emergent particle densities, $\chi_{g^{3}}^{1}$ are caiculated from $\mathrm{Bq}$. '.107). The source for the next batch, $\mathrm{s}_{\mathrm{g}}^{2}$, is obtained from $\mathrm{Eq}$. (105) and then estimates of the $x_{g}^{2}$ are obtaired from $\vec{B}_{q}$. (107). After the source has converged (usuaily after a few batches), the $x_{g}^{n}$ are presumed to be a valid estimate of the eigenfunction $x_{B}$ in Eq. $(108)$ and ar estimate of the multiplication factor can be obtained for eash of the succeeding batches.

The mitiplication factor corresponding to the nth generation (or batch) is defined as the ratio of the total proajction of fission neutrons during the nth generation to the total number of source neutrons introduced into the rith generation

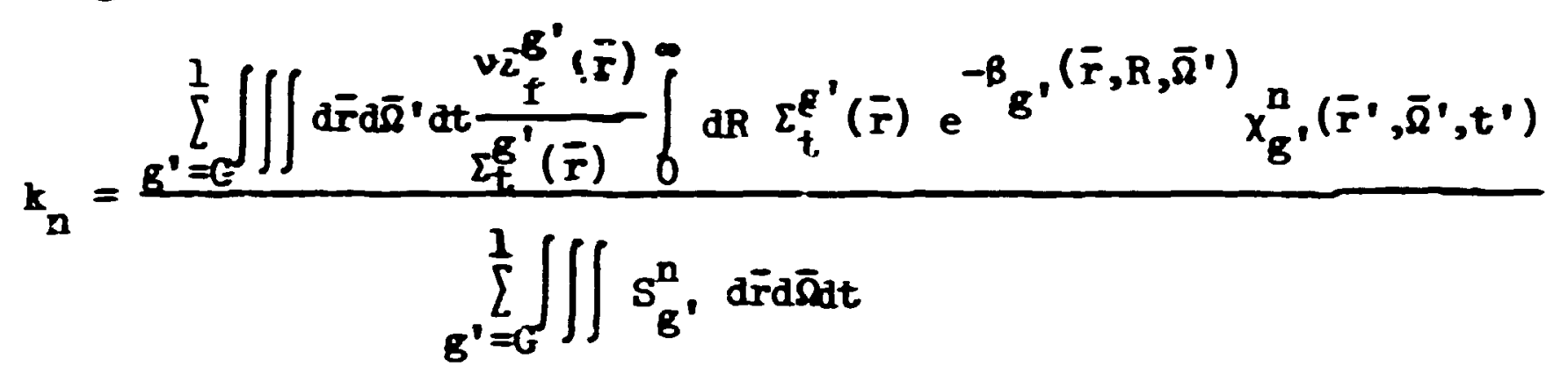

which can also be expressed as the ratio of successive sources

$$
k_{n}=\frac{\sum_{g^{\prime}=G}^{1} \iiint s_{B^{\prime}}^{n+1}(\bar{r}, \bar{\Omega}, t) d \bar{r} d \bar{\Omega} d t}{\sum_{g^{\prime}=G}^{1} \iiint s_{g^{\prime}}^{n}(\bar{r}, \bar{\Omega}, t) d \bar{r} d \bar{\Omega} d t}
$$


The multiplication factor is calculated at the end of each batch and the eigenvalue, $k$, is taken as the mean value if the $k_{n}$ averaged over all the batches calculated after convergence of the eigenfunctions was ac rieved.

Equation (107) is solved by MRSE in the same manner as it would be for non-fissioning systems. The fission event is treated as an absorption and the neutron's weight is modified accordingly, i.e., fissions that occur do not introduce new neutrons into the present generation. The multiplication factor, $k_{n}$, is estimated by sumining the contribution $v \varepsilon_{f}^{G}(\bar{r}) / \varepsilon_{t}^{b}(\bar{r})$. $W_{b}$ at every collision ( $w_{b}$, the neutron's veight before collision, is an estimate of the collision density). At the end of the batch, $k_{n}$ is divided by $\mathbf{B}$, the total starting veight of the batch.

The source for the next batch is not obtained direcily from the individual contributions $\left(v \Sigma_{\mathbf{f}}^{\mathrm{g}} \cdot \mathrm{u}_{b}\right)$. Rather, Russien roulette and splitting are used to discretize these contributions intc ones of equel raide. The splitting and Russian roulette parameters used are deterrined by the input parameter, Fllw, the desired value of a single contribution. To keep the number of neutrons from multip.ying or decreasing indefinitely, Fil is modified from batch to batch such that the number of source neutrons for each batch remains nearly constant. The value of Fuld for the $(n+1)$ st batch is calculated at the completion of the nth batch as follows:

$$
F W \phi_{n+1}=F W \phi_{n} \cdot \overline{\mathbf{k}}_{n} \cdot \frac{\text { (fissior. neutrons produced during the } \mathrm{nth} \text { batch) }}{\text { (source neutrons introduced into the first batch) }}
$$

where $\bar{k}_{n}$ is an accimulative estimatc of $k$ through $n$ batcies. The $\bar{k}_{n}$ modifying factor is requireI since the Futd calculated arter tha nth batch affects the number of source neutrons in the $(n+2)$ nd batch.

The adjoint problem for the fissioning system is solved by MRSE in terms of the random walk of "adjunctons" as described by the integral emerisent adjuncton density equation, Eq. (93), that car: be rerritten in batch notation as

$$
G_{g}^{n}(\bar{r}, \hat{\Omega}, t)=\left[P_{g}^{\phi}(\bar{r}, \hat{\Omega}, t)\right]^{n}+C_{g^{\prime}+g}\left(\bar{r}, \hat{\Omega}^{\prime}+\hat{\Omega}\right) T_{g^{\prime}}\left(\bar{r}^{\prime}+\overline{\bar{x}}^{\prime}, \hat{\Omega}^{\prime}\right) G_{\bar{G}^{\prime}}^{n}\left(\bar{r}^{\prime}, \hat{\Omega}^{\prime}, t^{\prime}\right),
$$




\section{A-33}

with the source of adjunctors being provided by the response function based on flux density, $\mathrm{P}_{\mathrm{g}}^{\phi}$. The effect of interest for the nth genersicion, $\lambda_{g}^{n}$, is the production of fission neutrons due to fissions in group $g$ that appear at the fission site in the next generation according to the group fission spectrum, $f_{f}$, and is given by

$$
\begin{aligned}
& \lambda_{g}^{n-1}=\iiint_{j}\left[P_{R}^{\phi}(\bar{r}, \bar{\Omega}, t)\right]^{n}{ }_{g}^{n-1}(\bar{r}, \bar{\Omega}, t) d \bar{r} d \bar{\Omega} d t
\end{aligned}
$$

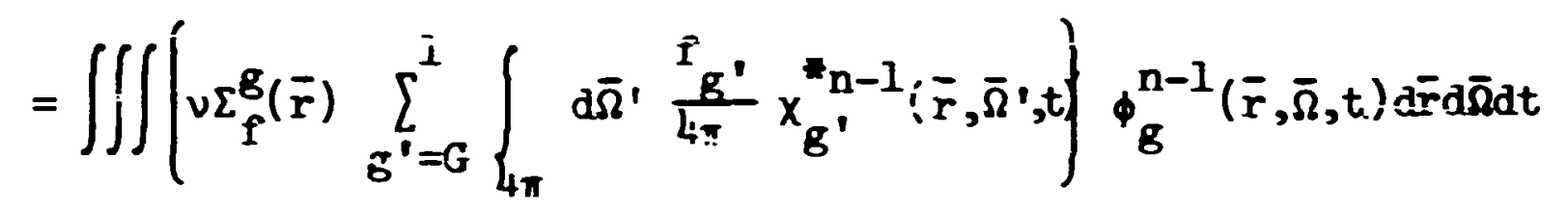

where

$$
\begin{aligned}
& x_{g}^{{ }^{n}} n(\bar{r}, \bar{\Omega}, t)=\text { the value to the effect of interest in the nth generation } \\
& \text { of an emergent neutron with phase space coordinates (group } B \text {, } \\
& \bar{r}, \bar{\Omega}, t \text { ). }
\end{aligned}
$$

From Eq. (113), the source of adjunctons for the nth generation is identified as

$$
\left[P_{g}^{\phi}(\bar{r}, \bar{\varepsilon}, t)\right]^{n}=v \Sigma_{f}^{g}(\bar{r}) \sum_{g^{\prime}=G}^{l} \int_{4 \pi} d \bar{\Omega}^{\prime} \frac{P_{g^{\prime}}}{4 \pi} x_{g^{\prime}}^{k_{n}-1}\left(\bar{r}, \bar{\Omega}^{\prime}, t\right)
$$

Noting that according to Equations (83) and (84)

$$
x_{g}^{m n}(\bar{r}, \bar{\Omega}, t)=\frac{1}{\sum_{t}^{g}(\bar{r})} T_{g}\left(\bar{r}^{\prime}+\bar{r}, \bar{\Omega}\right) G_{B}^{n}\left(\bar{r}^{\prime}, \bar{\Omega}, t^{\prime}\right)
$$

Eq. (114) can be rewritten as

$$
\begin{aligned}
& {\left[P_{g}^{P}(\bar{r}, \bar{\Omega}, t)\right]^{n}=v \Sigma_{f}^{g}(\bar{r}) \sum_{g^{\prime}=G}^{I} \int_{4 \pi} d \bar{\Omega}^{\prime} \frac{P_{g^{\prime}}}{4 \pi \Sigma_{t}^{g^{\prime}(\bar{r})}} T_{g^{\prime}}\left(\bar{r} \mapsto \bar{r}, \bar{\Omega}^{\prime}\right) G_{g^{\prime}}^{n-1}\left(\bar{r}{ }^{\prime}, \bar{\Omega}^{\prime}, t^{\prime}\right)} \\
& =\frac{v \Sigma_{p^{g}(\bar{r})}}{4 \pi} \sum_{B^{\prime}=G}^{I} \int_{4 \pi} d \bar{\Omega}^{\prime} f_{g^{\prime}} \int_{0}^{\infty} d R e^{-\beta_{B^{\prime}}\left(\bar{r}, R^{R}, \bar{\Omega}^{\prime}\right)} G_{G^{\prime}}^{n-1}\left(\bar{r}^{\prime}, \bar{\Omega}^{\prime}, t^{\prime}\right)
\end{aligned}
$$


lloting that the fission process is indeperdient of the incident neutron's direction and that the fission neutrons are emitted isotropically

$$
\left[P_{g}^{\phi}(\ddot{r}, \bar{r}, t)\right]^{n} \equiv\left[P_{g}^{\phi}(\bar{r}, \hat{\Omega}, t)\right]^{n}
$$

and Eq. (116) can be used in confunction with Eq. (112), i.e., $\bar{\Omega}$ replaced by $\hat{\Omega}$.

Equation (116) can be rewritten as

$$
\begin{aligned}
& {\left[P_{g}^{\phi}(\bar{r}, \hat{\Omega}, t)\right]^{n}=\frac{1}{4 \pi} \frac{v \Sigma_{f}^{g}(\bar{r})}{v \Sigma_{f^{\prime}}(\bar{r})} \sum_{g^{\prime}=G}^{l} \int_{4 \pi} d \hat{\Omega}^{\prime}\left[f_{g^{\prime}} v \Sigma_{f}(\bar{r})\right] \int_{0}^{\infty} d R e^{-B_{g}\left(\bar{r}, P_{i}, \hat{\Omega}^{\prime}\right)}} \\
& \times G_{g^{\prime}}^{n-1}\left(\bar{r}^{\prime}, \hat{\Omega}^{\prime}, t^{\prime}\right)
\end{aligned}
$$

where

$$
\begin{aligned}
& v \Sigma_{f}(\bar{r})=\sum_{g=G}^{l} v \Sigma_{f^{g}}^{g}(\bar{r}), \\
& \frac{v \Sigma_{f}^{g}(\bar{r})}{v \Sigma_{f}(\bar{r})}=\text { energy distribution of adjunctons energing from an adjoint } \\
& \quad \text { fission, } \\
& {\left[f_{g^{\prime}} v \Sigma_{f}(\bar{r})\right]=\text { the } g^{\prime} \text { th group cross section for adjoint, fission. }}
\end{aligned}
$$

It is noted that the integral emergent particl. density equation, Eq. (107), is identical in form with the integral emergent adjuncton density equation, Eq. (112), so that essentially* the same randor walk. procedures can apply to the solution of the forward and adjoint fissioning systems. The adjoint source, $\bar{B}_{\mathfrak{q}}$. (118), differs from the forwar source, Eq. (105), only in that the fission cross section and the group fission spectrum have charged their roles. The adjoint-fission group cross section is $\left[f_{g} \cdot v \Sigma_{f}(\bar{r})\right]$ and the energy distribution of the edjunctons emerging from an adjoint fission is $\nu \Sigma_{f}^{g}(\bar{r}) / \nu \Sigma_{f}(\bar{r})$.

The same differences will. exist between the forward and adjoint collision kernels here as was the case for non-fissioning systems. 
The adjoint solution is started by assuming sume arbitrary initial source, $\left[P^{\phi}(\vec{r}, \hat{\Omega}, t)\right]^{l}$, and calculating the $G_{g}^{+}(\bar{r}, \widehat{s} b t)$ using Ea.(112). A new source term, $\left[P_{g}^{\phi}\left(\frac{G}{r}, \widehat{\Omega}\right)\right]^{2}$ is thon calculated from Eq. (118) and the next estimate, $G_{\sigma}^{2}(\bar{r}, \hat{\Omega})$ is calculated using Eq. (112). This procedure continues until, as in the forward case, the source has converged and the $G_{g}^{n}$ 's are presured to be an estimate of the eigenfunctions $G_{g}$. Then for each succeeding barch, the following estimate is made for the eigenvalue $k$ :

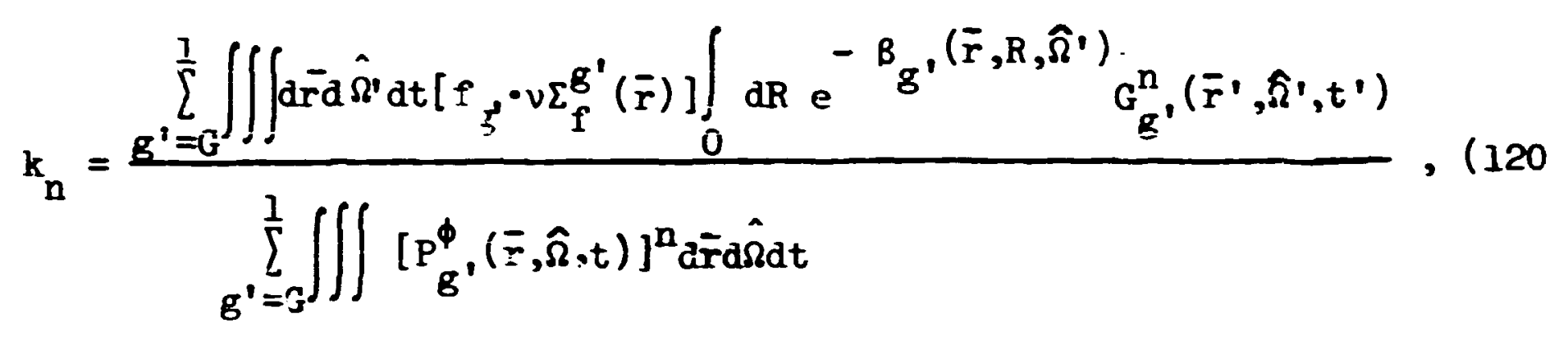

and the eigenvalue, $k$, is taiken as the mean value of the $k_{n}$ averaged over all the batihes calculated after convergence of the eigenfunctions was achieved -- exactly the same procedure used in the forward calculation. 


\section{APPENDIX B}

\section{Generalized Gsussian Quadrature}

\section{General Statement of the Problem and Its Solution}

Given $\omega(x), a \leq x \leq b$, such that $\omega(x) \geq 0$ (Resiriction $I$ ).

Problem: find $\left\{x_{i}, w_{i}\right\}$ for $i=1, n$ so that:

$$
\int_{a}^{t} f(x) \omega^{\prime}(x) d x=\sum_{i=1}^{n} f\left(x_{i}\right) \cdot \omega_{i} \quad \text { (Restriction II) }
$$

holds for all. $f(x)$ where $f(x)$ is a polynomial of degree $2 n-1$ or less.

Solution: Determine a set of polynomials $Q_{i}(x)(i=1, n)$ orthogonal with respect, to $\omega(x)$. That is

$$
\int_{a}^{b} Q_{i}(x) Q_{j}(x) \omega(x) d x=\delta_{i j} N_{i}
$$

where $\delta_{i j}$ is the Kronecker delta and $N_{i}$ is a normalization constant. Then $\left\{x_{i}\right\}_{i=1}^{n}$ are given by the roots of $Q_{n}(x), Q_{n}\left(x_{i}\right)=0$, and

$$
\omega_{i}=\left(\sum_{i=1}^{n-1} Q_{i}^{2}\left(x_{i}\right) / N_{i}\right)^{-1} \text {. }
$$

Note: Since the functions $1, x, x^{2}, \ldots, x^{2 n-1}$ are independent and form a basis for: the space of all polynomials of degree $2 n-1$ or less, it is equivalent to Restriction II to require that

$$
M_{v}=\int_{a}^{b} x^{\nu} \omega(x) d x=\sum_{i=1}^{n} x_{i}^{v} \cdot \omega_{i} \text { for } v=0,2 n-1 \text {. }
$$

In other words, the problem is that of finding a discrete distribution.

$$
\omega^{*}(x)=\sum_{i=1}^{r_{1}} \omega_{i} \delta\left(x-x_{i}\right)
$$


having its rirst $2 n$ moments, $\left\{M_{v}\right\}_{v=0}^{2 n-1}$ identical to those of the original distribution $\omega(x)$.

It is then possible to relixx the non-negativity restriction, $\omega(x) \geq 0$, and in fact to state that $\left(\mathbf{y}^{\prime}(x)\right.$ ueed not be completely specified but only its first zn moments be given. Restriction $I$ then becomes two restrictions on the moments:

$$
I_{a}: \quad\left|c_{i}\right| \geq 0 \quad i=1, n-i
$$

where $\left|C_{i}\right|$ is the Gram determinant

$$
\left|C_{i}\right|=\left|\begin{array}{l}
M_{0} M_{1} \\
M_{2} M_{2}
\end{array}\right|,\left|C_{2}\right|=\left|\begin{array}{ll}
M_{0} M_{1} M_{2} \\
M_{1} M_{2} M_{3} \\
M_{2} M_{3} M_{4}
\end{array}\right|, \ldots,\left|C_{n-1}\right|=\left|\begin{array}{lll}
M_{0} M_{1} M_{2} & \ldots & M_{n-1} \\
M_{1} M_{2} & \ldots & M_{n} \\
\cdot \\
M_{n-1} M_{n} & \cdots & M_{2 n-2}
\end{array}\right|
$$

and

$\left.I_{b}\right)$ The roots of $Q_{n}(x)$ lie inside the interval $[a, b]$, i.e., $a \leq x_{i} \leq b$ whenever $Q_{n}\left(x_{i}\right)=0$.

\section{Equivalence of Moments and Legendre Coefficierts}

W. shall use the following form or the Legencire expansion of an angular distribution:

$$
f(\mu)=\sum_{\ell=0}^{\infty} \frac{2 \ell+1}{2} \mathrm{f}_{\ell} \mathrm{P}_{\ell}(\mu)
$$

From this it follows that

$$
f_{\ell}=\int_{-1}^{1} f(\mu) P_{\ell}(\mu) d \mu \text { and } f_{0} \equiv 1 .
$$

The moments of the distribution are defined by

$$
M_{n}=\int_{-1}^{1} \mu^{n} f(\mu) d \mu \text {. }
$$


If the Legendre polynomials are writien

$$
P_{\ell}(\mu)=\sum_{n=0}^{\ell} p_{\ell n} \mu^{n},
$$

[the $p_{\ell n}$ 's may be derived easily from the recurrence relation for $P_{\ell}(\mu)$ ]. Then it follows simply from Equation (2) that

$$
f_{\ell}=\sum_{n=0}^{\ell} F_{\ell n} \int_{-1}^{1} f(\mu) H^{n} d_{\mu}=\sum_{n=0}^{\ell} F_{\ell n} M_{n} .
$$

Likewise

$$
M_{n}=\int_{-1}^{1} \mu^{n} f(\mu) d \mu=\sum_{\ell=0}^{\infty} \frac{2 \ell+1}{2} f_{\ell} \int_{-1}^{1} \mu^{n} P_{\ell}(\mu) d \mu
$$

From the orihngonality property ve know that $P_{\ell}(\mu)$ is orthcgonal to any polynomial of degree less than $\ell$. Hence

$$
\int_{-1}^{1} \mu^{n} P_{\ell}(\mu) d \mu=0 \text { for } \ell>n \text {. }
$$

Then

$$
M_{n}=\sum_{l=0}^{n} \frac{2 l+1}{2} f_{\ell} p_{n l}^{-1}
$$

where

$$
p_{n \ell}^{-1}=\int_{-1}^{1} \mu^{n} P_{\ell}(\mu) d \mu
$$

are the coefficients for a Legendre expansion of $\mu^{n}$, that is,

$$
\mu^{n}=\sum_{\ell=0}^{n} \frac{2 \ell+1}{2} \bar{p}_{n \ell}^{-1} P_{\ell}(\mu) .
$$

[The Legendre polynomial recurrence relation may also be used to derive recurrence relations for the $p_{n \ell}^{-1}$.] 



$$
s_{i}(x)=\sum_{k=0}^{i} s_{i k} Q_{k}(x)
$$

It follows that

$$
E\left[s_{i}(x) Q_{j}(x)\right]=0 \quad \text { for } i<j
$$

Let us presume that we have obtained the first $i$ polynomials and are attempting to derive $Q_{i+1}(x)$. Due to our normalization condition $\left(a_{i}=1\right.$ ) we nave

$$
Q_{i+1}(x)=x^{i+1}+R_{i}(x)
$$

where

$$
\begin{aligned}
R_{i}(x) & =\sum_{k=0}^{i} a_{i+1, k} x^{k} . \\
Q_{i+1}(x) & =x \cdot x^{i}+R_{i}(x) \\
& =x \cdot\left[Q_{i}(x)-R_{i-1}(x)\right]+R_{i}(x) \\
& =x Q_{i}(x)+\left[R_{i}(x)-x R_{i-1}(x)\right] .
\end{aligned}
$$

The term $R_{i}(x)-x R_{i-1}(x)$ is a polynomial of order $i$ ani may be expanded in ierms of the $Q^{\prime} s$. Thus

$$
Q_{i+1}(x)=x Q_{i}(x)+\sum_{k=0}^{\dot{j}} d_{i k} Q_{k}(x) .
$$

For $j \leq i-2$ we can use the orthogonality relation

$$
\begin{aligned}
E\left[Q_{i+1}(x) Q_{j}(x)\right] & =0=E\left[x Q_{i}(x) Q_{j}(x)\right]+\sum_{k=0}^{i} a_{i !} E\left[Q_{k}(x) Q_{j}(x)\right] \\
& =E\left[Q_{i}(x)\left(x Q_{j}(x)\right)\right]+d_{i j} N_{j} \\
& =d_{i j} N_{j},
\end{aligned}
$$


since $x Q_{j}(x)$ is a polynomial of order $\leq i-1$ and is orthogonal to $Q_{i}(x)$. Since $I_{j}>0$ ve must have $d_{i j}=0$.

If ve write

$$
u_{i+1}=-d_{i, i}
$$

and

$$
\sigma_{i}^{2}=-d_{i, i-1},
$$

then Eq. (11) reduces to

$$
Q_{i+2}(x)=\left(x-\mu_{i+1}\right) Q_{i}(x)-\sigma_{i}^{2} Q_{i-1}(x) .
$$

This equation is the basic recurrence relation for our polynomials. We have

$$
\begin{aligned}
& E\left[Q_{i+1}(x) Q_{i-1}(x)\right]=0 \\
& \quad=E\left[x Q_{i}(x) Q_{i-1}(x)\right]-\mu_{i+1} E\left[Q_{i}(x) Q_{i-1}(x)\right]-\sigma_{i}^{2} E\left[Q_{i-1}^{2}(x)\right] \\
& =E\left[Q_{i}(x)\left(x Q_{i-1}(x)\right)\right]-\sigma_{i}^{2} \mathbb{N}_{i-1} \\
& =E\left[Q_{i}(x)\left\{Q_{i}(x)-\sum_{k=0}^{i-1} d_{i-1, k} Q_{k}(x)\right\}\right]-\sigma_{i}^{2} \mathbb{N}_{i-1} \\
& =E\left[Q_{i}^{2}(x)\right]-\sigma_{i}^{2} \mathbb{N}_{i-1} \\
& =\mathbb{N}_{i}-\sigma_{i}^{2} \mathbb{N}_{i-1} .
\end{aligned}
$$

This is easily solved for

$$
\sigma_{i}^{2}=\mathbf{N}_{i} / \mathbf{N}_{i-1} .
$$

If we return tc Equation (10), it is easy to see that

$$
\begin{aligned}
N_{i} & =E\left[Q_{i}(:) Q_{i}(x)\right]=E\left[Q_{i}(x) x^{i}\right]+E\left[Q_{i}(x) R_{i-1}(x)\right] \\
& =E\left[Q_{i}(x) x^{i}\right]=\sum_{x=0}^{i} a_{i k} \int_{a}^{b} x^{k} x^{i} d x=\sum_{k=0}^{i} a_{i k} M_{k+i} .
\end{aligned}
$$


Likevise ve vill define

$$
\begin{aligned}
L_{i+1} & =E\left[Q_{i}(x) x^{i+1}\right] \\
& =\sum_{k=0}^{i} a_{i, k} M_{k+i+1}
\end{aligned}
$$

Then the final orthogonality relation used in defining $Q_{i+1}(x)$ gives us

$$
\begin{aligned}
E\left[Q_{i+1}(x) Q_{i}(x)\right] & =0 \\
& =E\left[Q_{i+1}(x) x^{i}\right]+E\left[Q_{i+1}(x) R_{i-1}(x)\right] \\
& =E\left[x Q_{i}(x) x^{i}\right]-\mu_{i+1} E\left[Q_{i}(x) x^{i}\right]-\sigma_{i}^{2} E\left[Q_{i-1}(x) x^{i}\right] \\
& =L_{i+1}-\mu_{i+1} H_{i}-\sigma_{i}^{2} L_{i}
\end{aligned}
$$

or

$$
\begin{aligned}
\mu_{i+1} & =\frac{L_{i+1}}{N_{i}}-\sigma_{i}^{2} \frac{L_{i}}{N_{i}} \\
& =\frac{L_{i+1}}{N_{i}}-\frac{L_{i}}{N_{i-1}} .
\end{aligned}
$$

The coefficients $a_{i k}$ may be obtained from the recurrerce relation, Eq. (12), by taking the coefficient of $x^{k}$ on both sides of the equation. This gives

$$
a_{i+1, k}=a_{i, k-1}-\mu_{i+1} a_{i, k}-\sigma_{i}^{2} a_{i-1, k}
$$

To recapitulate, one uses moments through $M_{2 i}$ and the values of $a_{i k}$ from $Q_{i}(x)$ to calculate $\mathbb{N}_{i}(E q .14) . N_{i}$, along with the previously determined

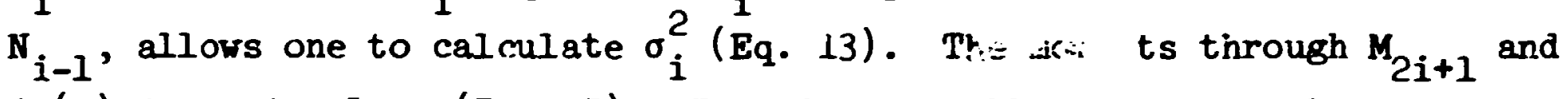
$Q_{i}(x)$ determine $L_{i+1}(E q .15)$. This in turn allows the calculation of $\mu_{i+1}(E q .16)$. Witb $\sigma_{i}^{2}$ and $\mu_{i+1}$ the recurrence relation (Eq. 12) determines $Q_{i+1}(x)$. In sum the moments $M_{0}, M_{1}, \ldots, M_{2 n-1}$ of $w(x)$ allow the determination of the orthogonal polynomials $Q_{0}(x), Q_{1}(x), \ldots, Q_{n}(x)$. 
This is subject only to the restriction $M_{i}>0, i=0, n$. Aithough it is far irom obvious, this restriction may be written in simple closed form as:

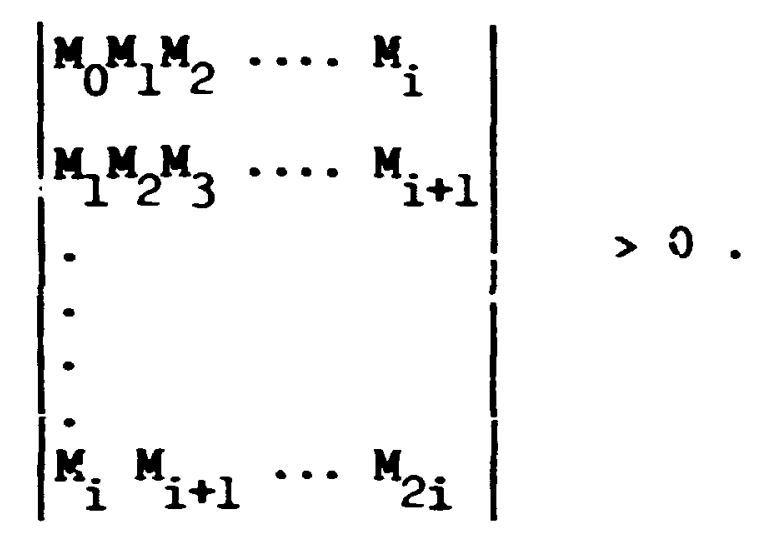

Froperties of the Roots of the Orthogonal Polynomials

The roots of the orthogonal polynomials have two useful properties wich we shall prove.

Lemme I: $Q_{n}(x)$ has $n$ distinct, reai roots which "interlezve" with the roots of $Q_{n-i}(x)$; that is, between any two adjacent roots of $Q_{n-1}(x)$ there is one and only one root of $Q_{n}(x)$, and furthersore there is one root of $Q_{n}(x)$ greater than the largest root of $Q_{n-1}(x)$ and one smaller than the least root of $Q_{n-1}(x)$. Likewise there is one and only one root of $Q_{n-1}(x)$ between any two adjacent roots of $Q_{n}(x)$.

Proof: We assume the Lemma to he true for $Q_{n-1}$ and $Q_{n-2}$. Let $x_{1}>x_{c}>\ldots>x_{n-1}$ be the roots of $Q_{n-1}$. Then it follows that the sequence $Q_{n-2}\left(x_{1}\right), Q_{n-2}\left(x_{2}\right), \ldots, Q_{n-2}\left(x_{n-1}\right)$ alternates in sign. Since

$$
\begin{aligned}
Q_{n}\left(x_{i}\right) & =\left(x_{i}-\mu_{n}\right) Q_{n-1}\left(x_{i}\right)-\sigma_{n-1}^{2} Q_{n-2}\left(x_{i}\right) \\
& =-\sigma_{n-1}^{2} Q_{n-2}\left(x_{i}\right) .
\end{aligned}
$$

The sequence $Q_{n}\left(x_{i}\right), Q_{n}\left(x_{2}\right), \ldots, Q_{n}\left(x_{n-1}\right)$ also alternates in sign. This establishes that there is at least one root of $Q_{n}$ between any two roots of $Q_{n-1}$. Because the $Q_{i}$ 's are normalizen to $a_{i i}=1$, they are all positive at $+\infty$ and alternate in sign at $-\infty . Q_{n-2}$ has no root between $x_{1}$ and $+\infty$; hence $Q_{n-2}\left(x_{1}\right)>0$. But $\sigma_{n-1}^{2}>0$ (because $N_{n-1}>0$ and $N_{n-2}>0$ ); therefore, $Q_{n}\left(x_{1}\right)<0$ and $Q_{n}$ must have at least one root greater than $x_{1}$. 
Similar reasoning ieads to the conclusion that $Q_{n-2}\left(x_{n-1}\right), Q_{n-2}(x \rightarrow-\infty)$, and $Q_{n}(x \rightarrow-\infty)$ have the same sign wile $Q_{n}\left(x_{n-1}\right)$ is of the opposite sign. Thus $Q_{n}$ must have at least one root between $x_{n-i}$ and $\rightarrow$. Since this gives us $n$ intervals where $Q_{n}$ must have "at least ore" root, it is clear that $Q_{n}$ has $n$ distinct roots which interleave with the rocts of $Q_{n-1}$

The proof by induction may be comileted by using similar arguments to show that one of the two roots of $Q_{2}(x)$ lies above the singie root $c i$ $Q_{I}(x)$ and cone below it.

Lemma II: The $n$ roots of $Q_{n}(x)$ lie in the interval $(a, b)$.

Proof: Assume that $Q_{n}(x)$ has only s changes of sign in the interval $(a, b)$ at the point,s $x_{1}, x_{2}, \ldots, x_{s}$. i.et

$$
\theta(x)=\left(x-x_{1}\right)\left(x-x_{2}\right)\left(x-x_{3}\right) \ldots\left(x-x_{s}\right)
$$

then $\theta(x) Q_{n}(x)$ does not change sign in the interval $(a, b)$. It follows that *

$$
E\left[\theta(x) Q_{n}(x)\right]=\int_{a}^{b} \theta(x) Q_{n}(x) \omega(x) d x \neq 0 .
$$

However, $\theta(x)$ is a poiynomial of order $s=2$. Since $\chi_{n}(x)$ is orthogonal to all polynomials of order less than $n$, we must have $s=n$, thus proving the assertion.

The Meaning of the Two Restrictions Which Replace the Non-Negativity Requirement, $\omega(x) \geq 0$

In the foregcing development, knowledge of the entire function $\omega(x)$ is never required. Instead, all that is needed are the ooments, $M_{0}, M_{1}$, ...., $M_{2 n-1}$, of $w(x)$. The generalized quadrature thus develcped is thereby valid for the whole class of functions having those moments. Since the moments are equivalent to the Legendre coefficients, $f_{0}, f_{1}, \ldots$, $f_{2 n-1}$, this class is cumprised of all functions having the same truncated

This step relies on the requirement that $\omega(x)$ be non-negative. We wish to relax this restriction somewhat but not completely. Since Lenma II expresses a property which will be essential to the use of this development as a Monte Carlo selection technique, we will use this property as one of the requirements for a "well-behaved" $\omega(x)$ with which we shall replace the non-negativity restriction. 
Legendre expansion; that is,

$$
\omega(x) \forall \omega^{*}(x)=\sum_{\ell=0}^{2 n-1} \frac{2 \ell+1}{2} \approx_{\ell} P_{\ell}(x)
$$

In particular, the discrete distribution derived by this technique is itself one function from this class.

It is not required that all functions of this class be non-negative; in fact, there are infinitely many which are not. It is not even required that the truncated Legendre expansion $\omega^{*}(x)$ be non-negative. Howevei, it is essential that at least one function in this class be non-negative. The restrictions

1) $\mathrm{N}_{i}>0, i=1, \ldots, \mathrm{n}$ and

2) $Q_{n}(x)$ has $n$ roots in the interval $(-1,+1)$

express exactly this requirement. Then it follows that $\omega^{*}(x)$ is the truncated expansion of some unspecified non-negative function. The failure cf either of irose two conditions expresses the fact that the given moments (or Legendre coetficients) are not those of any everywhere positive function. Generation of the Generalized Gaussian Quadrature

We are given $q(x), a \leq x \leq b$, [or rather, ve are given the moments of $\omega(x)]$ and we are atfempting to find a set of points, $x_{i}$, and associated weights, $\omega_{i}$, so that, for any arbitrary polynomial, $f(x)$, of order $2 n-1$ or less,

$$
\Sigma[f(x)]=\int_{a}^{b} f(x) \omega(x) d x=\sum_{i=1}^{n} f\left(x_{i}\right) \cdot \omega_{i} .
$$

By simple division $\cong$ polynomials,

$$
f(x)=q_{n-1}(x) Q_{n}(x)+r_{n-1}(x)
$$

where $a_{n-1}(x)$ and $r_{n-1}(x)$ are polynomials of order $n-1$ or less.

$$
\begin{aligned}
E[f(x)] & =E\left[q_{n-1}(x) Q_{n}(x ;]+E\left[r_{n-1}(x)\right]\right. \\
& =E\left[r_{n-1}(x)\right] \text { from the orthogonality property of } Q_{n} .
\end{aligned}
$$


However, we want

$$
\begin{aligned}
E[f(x)] & =\sum_{i=1}^{n} f\left(x_{i}\right) \cdot w_{i}=\sum_{i=1}^{n} q_{n-1}\left(x_{i}\right) Q_{n}\left(x_{i}\right) \cdot w_{i}+\sum_{i=1}^{n} r_{n-1}\left(x_{i}\right) \cdot w_{i} \\
& =\sum_{i=1}^{n} q_{n-1}\left(x_{i}\right) Q_{n}\left(x_{i}\right) \cdot w_{i}+E\left[r_{n-1}(x)\right] .
\end{aligned}
$$

By subtracting Eq. (18) from Eq. (19), we find that we must require, for all polynomials, $q_{n-1}(x)$, that

$$
\sum_{i=1}^{n} q_{n-1}\left(x_{i}\right) Q_{n}\left(x_{i}\right) \cdot \omega_{i}=0 \text {. }
$$

This condition can only be met if

$Q_{n}\left(x_{i}\right)=0$, that is, the desired points, $x_{i}$, are the roots of $Q_{n}(x)$

Now we still must pick the weights, $\omega_{i}$, so that

$$
E\left[r_{n-1}(x)\right]=\sum_{i=1}^{n} r_{n-1}\left(x_{i}\right) \cdot \omega_{i}
$$

where $r_{n-1}(x)$ is an arbitrary polynomial of order $n-1$ or less. Since $r_{n-1}$ may be exparided as a linear sum of the orthogonal polynomials, $Q_{0}$, $Q_{1}, \ldots, Q_{n-1}$, it is sufficient to require

$$
E\left[Q_{k}(x)\right]=\sum_{i=1}^{n} Q_{k}\left(x_{i}\right) \cdot w_{i} \text { for } k=0,1, \ldots, n-1 \text {. }
$$

However,

$$
E\left[Q_{k}(x)\right]=E\left[Q_{k}(x) Q_{0}(x)\right]=N_{0} \delta_{k o}
$$

Thus we mist have

$$
\sum_{i=1}^{n} Q_{k}\left(x_{i}\right) \cdot w_{i}=N_{0} \delta_{k 0} \text { for } k=0,1, \ldots, n-1
$$


Multiplying $E_{q} .(23)$ by $\left[Q_{k}\left(x_{j}\right) / N_{\mathfrak{q}}\right]$ and summing over $k$, we find

$$
\begin{gathered}
\sum_{k=0}^{n-1} \frac{Q_{k}\left(x_{j}\right)}{N_{j}} \sum_{i=1}^{\bar{j}} Q_{k}\left(x_{i}\right) \cdot w_{i}=\sum_{i=1}^{r_{1}} \omega_{i}\left\{\sum_{k=0}^{n-1} \frac{Q_{k}\left(x_{j}\right) Q_{k}\left(x_{j}\right)}{N_{k}}\right\} \\
=\sum_{k=0}^{n-1} \frac{Q_{k}\left(x_{j}\right)}{N_{j}} N_{0} \delta_{k 0}=\frac{Q_{0}\left(x_{j}\right)}{N_{0}} N_{0}=1 .
\end{gathered}
$$

Introducing the function

$$
D_{n-1}(x, y)=\sum_{k=0}^{n-1} \frac{Q_{k}(x) Q_{k}(y)}{N_{k}},
$$

we can write $\mathrm{Eq} .(24)$ as

$$
\sum_{i=1}^{n} \omega_{i} D_{n-1}\left(x_{j}, x_{i}\right)=1
$$

To proceed further we must establish the Christoffel-Darboux identit:

$$
\begin{aligned}
& \frac{Q_{n}(x) Q_{n-1}(y)-Q_{n-1}(x) Q_{n}(y)}{N_{n-1}(x-y)} \\
& =\frac{\left[\left(x-\mu_{n}\right) Q_{n-1}(x)-\sigma_{n-1}^{2} Q_{n-2}(x)\right] Q_{n-1}(y)-Q_{n-1}(x)\left[\left(y-\mu_{n}\right) Q_{n-1}(y)-\sigma_{n-1}^{2} Q_{n-2}(y)\right]}{N_{n-1}(x-y)} \\
& =\frac{(x-y) Q_{n-1}(x) Q_{n-1}(y)+\sigma_{n-1}^{2}\left[Q_{n-1}(x) Q_{n-2}(y)-Q_{n-2}(x) Q_{n-1}(y)\right]}{N_{n-1}(x-y)} \\
& =\frac{Q_{n-1}(x) Q_{n-1}(y)}{N_{n-1}}+\frac{Q_{n-1}(x) Q_{n-2}(y)-Q_{n-2}(x) Q_{n-1}(y)}{N_{n-1}(x-y)} \cdot \frac{N_{n-1}}{N_{n-2}} \\
& =\frac{Q_{n-1}(x) Q_{n-1}(y)}{N_{n-1}}+\frac{Q_{n-1}(x) Q_{n-2}(y)-Q_{n-2}(x) Q_{n-1}(y)}{N_{n-2}(x-y)} \\
& =\frac{Q_{n-1}(x) Q_{n-1}(y)}{N_{n-1}}+\frac{Q_{n-2}(x) Q_{n-2}(y)}{N_{n-2}}+\frac{Q_{n-2}(x) Q_{n-3}(y)-Q_{n-2}(x) Q_{n-2}(y)}{N_{n-2}(x-y)}
\end{aligned}
$$


B-13

$$
\begin{aligned}
& \sum_{k=1}^{n-1} \frac{Q_{k}(x) Q_{k}(y)}{N_{k}}+\frac{Q_{1}(x) Q_{0}(y)-Q_{0}(x) Q_{1}(y)}{N_{0}(x-y)} \\
= & \sum_{k=1}^{n-1} \frac{Q_{k}(x) Q_{k}(y)}{N_{k}}+\frac{\left(x-\mu_{1}\right)-\left(y-\mu_{1}\right)}{N_{0}(x-y)} \\
= & \sum_{k=1}^{n-1} \frac{Q_{k}(x) Q_{k}(y)}{N_{k}}+\frac{1}{N_{0}}=\sum_{k=1}^{n-1} \frac{Q_{k}(x) Q_{k}(y)}{N_{k}}+\frac{Q_{0}(x) Q_{n}(y)}{N_{0}} \\
= & \sum_{k=0}^{n-1} \frac{Q_{k}(x) Q_{k}(y)}{N_{k}}=D_{n-1}(x, y) .
\end{aligned}
$$

Therefore

$$
D_{n-1}\left(x_{j}, x_{i}\right)=\frac{Q_{n}\left(x_{j}\right) Q_{n-1}\left(x_{j}\right)-Q_{n-1}\left(x_{j}\right) Q_{n}\left(x_{i}\right)}{i_{n-1}\left(x_{j}-x_{i}\right)}
$$

For $i \neq j$ and $Q_{n}\left(x_{j}\right)=Q_{n}\left(x_{i}\right)=0$,

$$
n_{i-i}\left(x_{j}, x_{i}\right)=0
$$

Therefore, returning to Eq. (25),

$$
\sum_{i=1}^{n} w_{i} D_{n-1}\left(x_{j}, x_{i}\right)=\omega_{j} D_{n-1}\left(x_{j}, x_{j}\right)=1
$$

or

$$
\omega_{j}=\left\{D_{n-1}\left(x_{j}, x_{j}\right)\right]^{-1}=\left(\sum_{k=0}^{n-1} \frac{Q_{k}^{2}\left(x_{j}\right)}{\mathbb{N}_{k}}\right)^{-1}
$$


Limits of $\mu_{i}$ and $\sigma_{i}^{2}$

In the calculations leading to the generalized Gaussian quaìrature we obtained two restrictions which had to be satisfied in order to have a positive distribution located on the interval $(-1,+1)$. These restrictions were:

1) $\mathbf{N}_{i}>0$.

2) All the roots of $Q_{i}(x)$ lie in the interval $(-1,+1)$.

Let us determine first what limitations these two restrictions place on the quantities $\mu_{i}, \sigma_{i}^{2}$. Consider first the effect of adding an infinitesimal amount $\Delta \mu$ to $y_{i}$. We have

$$
Q_{i}(x)=\left(x-\mu_{i}\right) Q_{i-1}(x)-\sigma_{i-1}^{2} Q_{i-2}(x)
$$

and

$$
Q_{i}^{*}(x)=\left(x-\mu_{i}-\Delta \mu\right) Q_{i-1}(x)-\sigma_{i-1}^{2} Q_{i-2}(x)=\psi_{i}(x)-\Delta \mu Q_{i-1}(x) .
$$

If $Q_{i}$ has a rooi at $x_{0}$, then $Q_{i}^{*}$ will have a root at $x_{0}+\Delta x_{0}$

$$
Q_{i}^{*}\left(x_{0}+\Delta x_{0}\right)=0=Q_{i}\left(x_{0}+\Delta x_{0}\right)-\Delta \mu Q_{i-1}\left(x_{0}+\Delta x_{0}\right)
$$

If we expand the right-hand side and keen only first order terms

$$
0=Q_{i}\left(x_{U}\right)+\Delta x_{0} Q_{i}^{!}\left(x_{0}\right)-\Delta \mu Q_{i-1}\left(x_{0}\right)=\Delta x_{0} Q_{i}^{!}\left(x_{0}\right)-\Delta \mu Q_{i-1}\left(x_{c}\right)
$$

or

$$
\Delta x_{C}=\frac{Q_{i-1}\left(x_{0}\right)}{Q_{i}^{\prime}\left(x_{0}\right)} \Delta \mu
$$

Since $Q_{i}(x)$ is positive as $x$ approashes $+\infty$, then $Q_{i}^{\prime}\left(x_{0}\right)>0$ at $x_{0}$ equal to the largest root of $Q_{i}$. At successively smaller roots of $Q_{i}$ the sign of $Q_{i}^{\prime}(x)$ alternates from positive tc naive. $Q_{i-1}(x)$ is similarly positive at +o. Also, it has no rocts greater than the largest root of $Q_{i}$. Therefore $Q_{i-1}(x)>0$ at the largest root of $Q_{i}$. Because the roots of $Q_{i-1}$ "interleave" with the roots of $Q_{i}$, the sign of $Q_{i-1}(x)$ must 
alternate at successive roots of $Q_{i}(x)$. Therefore, at aii roots of $Q_{i}(x)$ we must have:

$$
\frac{Q_{i-1}(x)}{Q !(x)}>0
$$

or, going back to Equation (29)

$$
\frac{d x_{0}}{d j_{i}}>0
$$

Therefore, as $\mu_{i}$ is increased, the roots of $Q_{i}(x)$ shift to the right, and, as $\mu_{i} i s$ decreased, the roots shift downard. If $\mu_{i}$ is steadily increased, the largest root of $Q_{i}$ will eventually equal 1 . This point is determined by

$$
Q_{i}(I)=0=\left(I-\mu_{i}\right) Q_{i-1}(I)-\sigma_{i-1}^{2} Q_{i-2}(i)
$$

or

$$
\mu_{i}=1-\sigma_{i-1}^{2} \frac{Q_{i-2}(1)}{Q_{i-1}(1)} \text {. }
$$

This is clearly the maximum value of $\mu_{i}$, which will generate positivity in the interval $(-1,+1)$. Likevise there is a minimum value at which the lowest root of $Q_{i}$ occurs at $x=-1$.

$$
Q_{i}(-1)=0=\left(-1-\mu_{i}\right) Q_{i-1}(-1)-c_{i-1}^{2} Q_{i-2}(-1)
$$

or

$$
\mu_{i}^{\min }=-1-g_{i-1}^{2} \frac{Q_{i-2}(-1)}{Q_{i-1}(-1)} .
$$

Note that

$$
a_{i}=\frac{Q_{i-2}(1)}{Q_{i-1}(1)}>0,
$$

die to the positivity of the functions as they approach +o and that

$$
B_{i}=-\frac{Q_{i-2}(-1)}{Q_{i-1}(-1)}>0,
$$


due to their alternation in sigr at $\rightarrow$. Since $\sigma_{i-1}^{2}>0$, we have the following picture on a $\mu_{i}$-axis

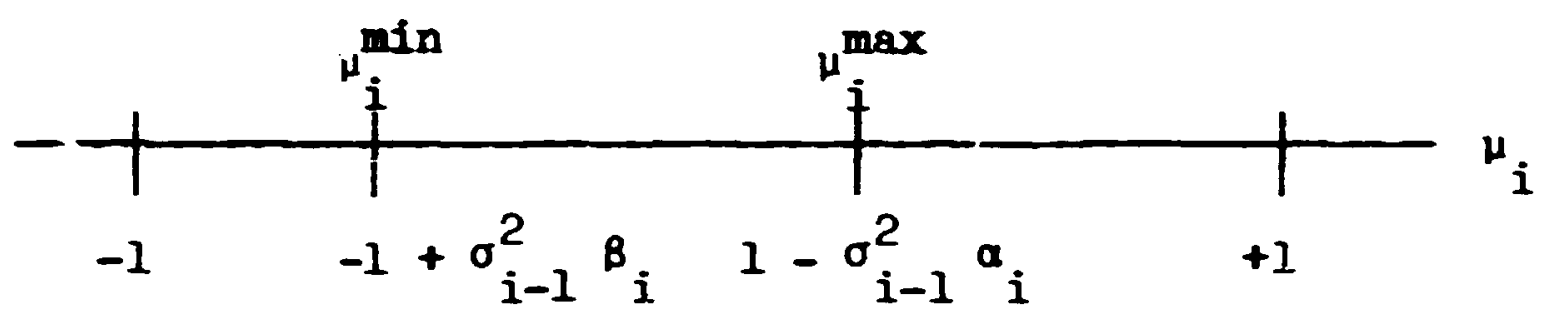

Now that we have upper and lower limits for $\mu_{i}$, what can we say about $\sigma_{i}^{2}$ ? Since $\sigma_{i}^{2}=N_{i} / K_{i-1}$, restriction $I$ implies that $\sigma_{i}^{2}>0$. We can obtain an upper limit to $\sigma_{i}$ by setting $\mu_{i+1}^{\min }=\mu_{i+1}^{\max }$. For larger values of $\sigma_{i}^{i}$, $\mu_{i+1}^{\min }>\mu_{i+1}^{\max }$, which means that there is no value of $\mu_{i+1}$ which will allow all the roots of $Q_{i+1}(x)$ to lie inside $(-1,+1)$. Thus

$$
\begin{aligned}
& 1-\left(\sigma_{i}^{2}\right)_{\max } \frac{Q_{i-1}(+1)}{Q_{i}(+1)}=-1-\left(\sigma_{i}^{2}\right) \max \frac{Q_{i-1}(-1)}{Q_{i}(-1)} \\
& 2=\left(\sigma_{i}^{2}\right)_{\max }\left[\frac{Q_{i-1}(+1)}{Q_{i}(+1)}-\frac{Q_{i-1}(-1)}{Q_{i}(-1)}\right] \\
& \left(\sigma_{i}^{2}\right)_{\max }=2 /\left[\frac{Q_{i-1}(+1)}{Q_{i}(+1)}-\frac{Q_{i-1}(-j)}{Q_{i}(-1)}\right]
\end{aligned}
$$

We can work back $f_{i}$ sm the limits on $\mu_{i}$ and $\sigma_{i}^{2}$ to obtain limits on the moments.

$$
\begin{gathered}
\sigma_{i}^{2}=N_{i} / N_{i-1} \\
H_{i}=\sum_{k=0}^{i} a_{i k} M_{k+i}=M_{2 i}+\sum_{k=0}^{i-1} a_{i k} M_{k+i} \quad \text { since } a_{i i}=1 .
\end{gathered}
$$

Therefore

$$
0<\sigma_{i}^{2}<2 /\left[\frac{Q_{1-1}(+1)}{Q_{1}(+1)}-\frac{Q_{1-1}(-1)}{Q_{1}(-1)}\right]
$$


implies

$$
\begin{aligned}
& -\sum_{k=0}^{i-1} a_{i k} M_{k+i}<M_{2 i}<\frac{2 N_{i-1}}{\left[\frac{Q_{i-1}(+1)}{Q_{i}(+1)}-\frac{\beta_{i-1}(-1)}{Q_{i}(-1)}\right]}-\sum_{k=0}^{i-1} a_{i k} M_{k+i} \\
& \mu_{i+1}=\frac{L_{i+1}}{N_{i}}-\frac{L_{i}}{N_{i-1}} \\
& L_{i+1}=\sum_{k=0}^{i} a_{i k} M_{k+i+1}=M_{2 i+1}+\sum_{k=0}^{i-1} a_{i k} M_{k+i+1} \\
& \mu_{i+1}^{\max }=1-\sigma_{i}^{2} \frac{Q_{i-1}(1)}{Q_{i}(1)} ; \text { therefore, } \\
& L_{i+1}^{\max }=N_{i}-N_{i} \sigma_{i}^{2} \frac{Q_{i-j}(1)}{Q_{i}(1)}+\frac{N_{i} L_{i}}{N_{i-1}}=N_{i}\left(1-\sigma_{i}^{2} \frac{Q_{i-1}(1)}{Q_{i}(1)}\right)+L_{i} \sigma_{i}^{2} \\
& M_{2 i+1}<N_{i}\left(1-\sigma_{i}^{2} \frac{Q_{i-1}(1)}{Q_{i}(1)}\right)+L_{i} \sigma_{i}^{2}-\sum_{k=0}^{i-1} a_{i k} M_{k+i+1}
\end{aligned}
$$

also

$$
M_{2 i+1}>N_{i}\left(-1-\sigma_{i}^{2} \frac{Q_{i-1}(-1)}{Q_{i}(-1)}\right)+L_{i} \sigma_{i}^{2}-\sum_{k=0}^{i-1} a_{i k} M_{k+i+1} \text {. }
$$

To ottain the limits on the Legendre coefficients, take the set of moments already determined $M_{1}, M_{2}, \ldots, M_{2 i-1}$ combined with $M_{2 i}^{\max }$ and convert from roments to Legendre coefficients. This gives $f_{2 i}^{\max }$. When $M_{1}, M_{2}, \ldots, M_{2 i-1}$ are combined with $M_{2 i}^{m i n}$ and converted, one obtains $f_{2 i}^{m i n}$. 


\section{APPENDIX C}

\section{MORSE Input Instructions}

There are five subroutines that read information for a complete MøRSE run. A description of the formats and variable definitions is given in this appendix.

The input read by Subroutine INPUT is as follows:

CARD A (20A4)

Title card.

(Any character other than a biank or alphameric in column one will terminate the job.)

CARD B (15I5)

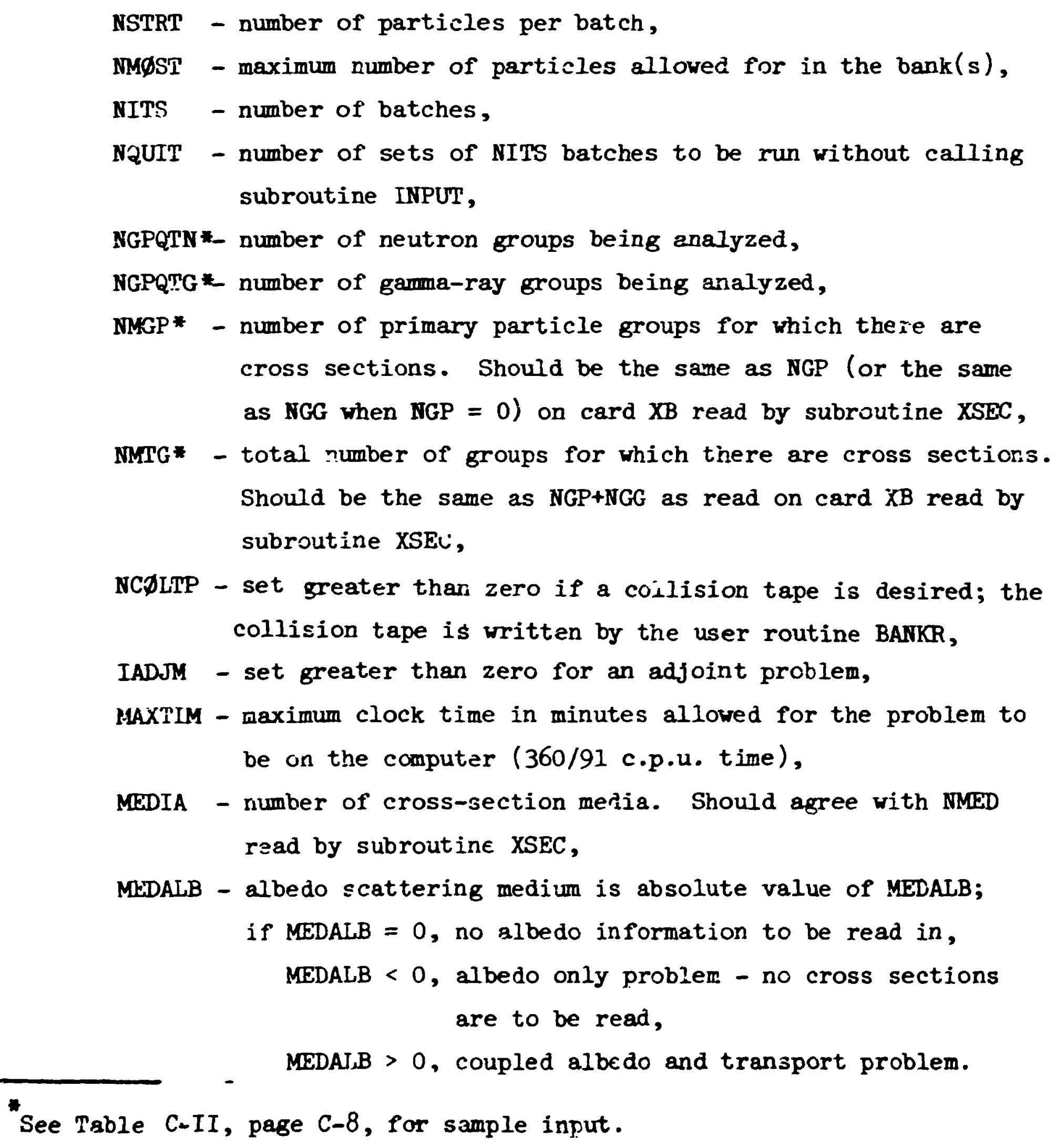
cross sections. Should be the same as NGP (or the same as NGG when NGP $=0$ ) on card XB read by subroutine XSEC, Should be the same as NGP+NGG as read on card $X B$ read by subroutine XSEC,

NCDLTP - set greater than zero if a coilision tape is desired; the collision tape is written by the user routine BANKR,

IADJM - set greater than zero for an adjoint problem,

MAXTIM - maximum clock time in minutes allowed for the problem to be on the computer (360/91 c.p.u. time),

MEDIA - number of cross-section meria. Should agree with IMED read by subroutine XSEC,

MEDALB - albedo scattering medium is absolute value of MELALB; if $M E D A L B=0$, no albedo information to be read in, MEDALB < 0 , albedo only problem - no cross sections are to be read, MEDAI,B > 0 , coupled albedo and transport problem.

See Table C-II, page C-8, for sample inp̣ut. 
CARD C (4I5, 5E10.5)

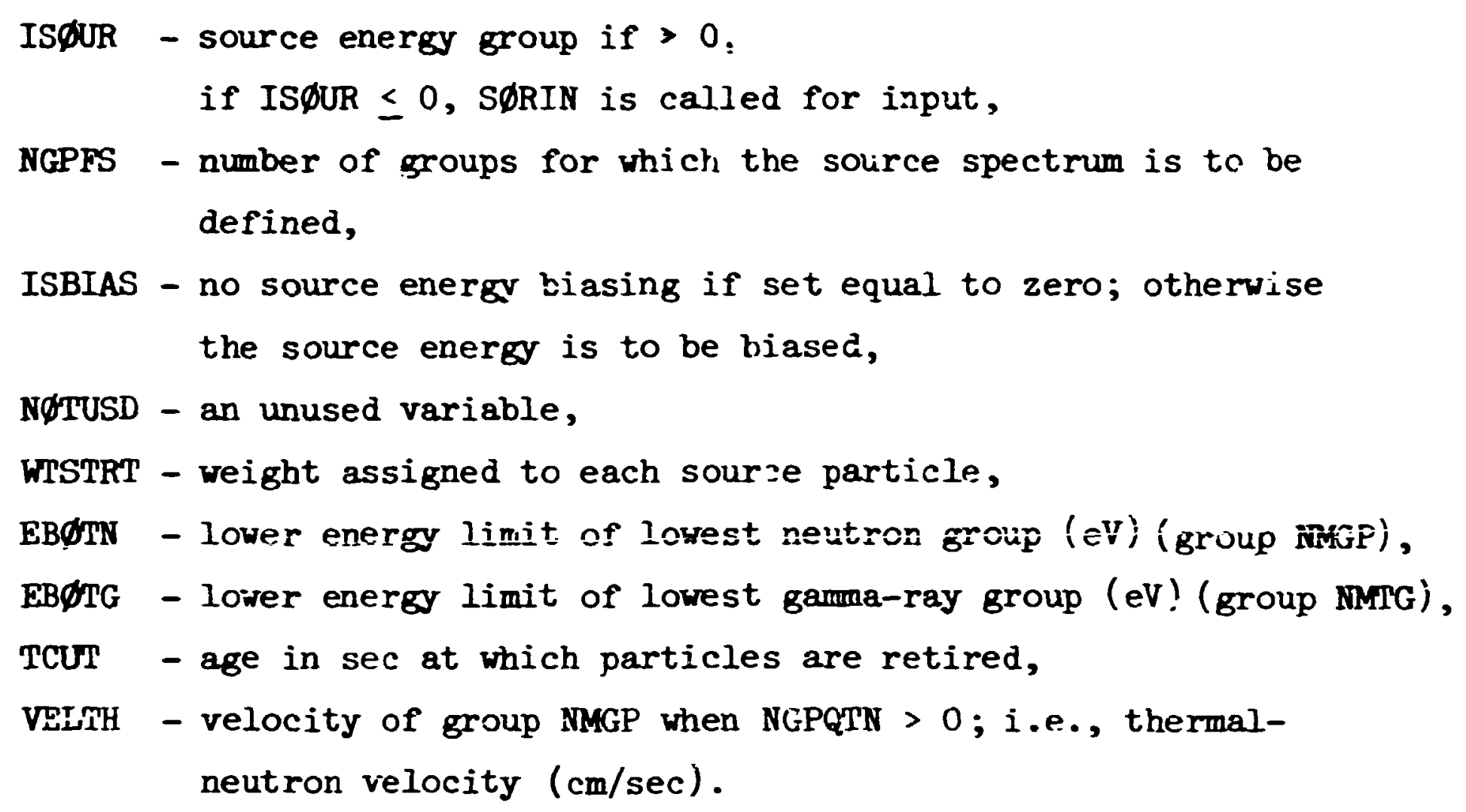

CARD D

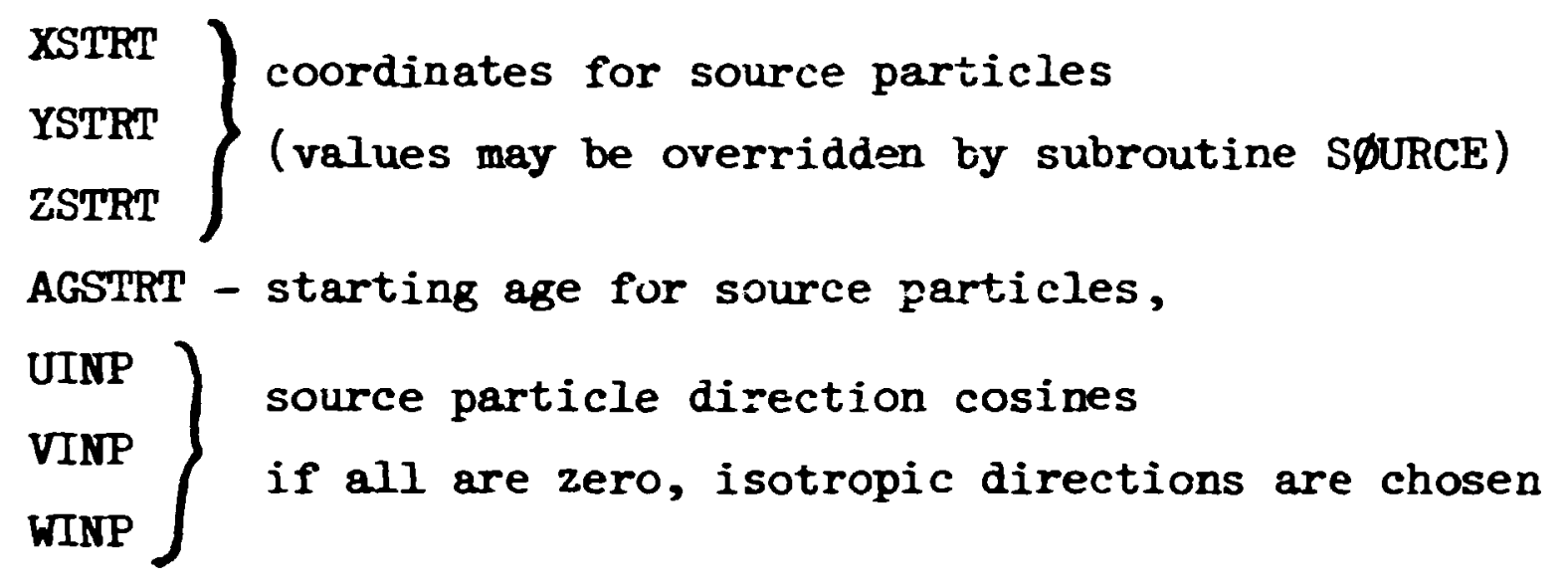

If IS $\phi U R$ on card $C$ is $\leq 0$, subroutine $S \emptyset R i N$ will be called for the input, of source data. For the sample problem $\varepsilon$ input spect.rum with biasing parameters is input.

CARDS El ( $7 \mathrm{E} 10.4$ )

NGPFS values of FS( $(I)$, the fraction of source particles in group

$I$, are required.

CARDS E2(TE10.4)

If ISEIAS > 0 , NGPFS values of BFS(I), the relative importance of a source in group I, are required.

Reminder: Cards E1 and E2 are not needed if IS $\varnothing$ UR $>0$. 
CARUS F (TEIO.4)

NMTG values of ENER, the energies (in eV) at the upper eage of tr:e energy group boundaries.

Note: The lower energies of Eroups NMGP and INTS vere read on Card $C$.

If a collision tape is desirea ( $N C \emptyset L_{\alpha}^{\pi} P>0$ ) on cara 5 , include Card

G; otherwise omit.

CARD G $(2 I 5,5 X, 36 I 1,13 I 1)$

NHISTR - logical tape number for the first collision tape,

NHISWX - the highest logical number trat a collision tape may be assigned,

$\operatorname{NBIND}(J), I=1,36$ - an index 20 indicate the collision parameters

to be writien or tape (see Table C-I for aefinition of parameters).

$\operatorname{NC} \varnothing \operatorname{LiS}(J), J=1,13$ - an index to indicate the types of ccllisions

to be put on tape (BAHKR arguments 1-13, page $166 \mathrm{fcr}$ definition).

CARD H (Z12)

RANDdM - starting random number.

CARD I (14I5)

NSPLT - index indicating that splitting is allowed if >0,

NKILL - index indicating that Russian rouiette is allowed if $>0$,

NPAST - index indicating that exponential transform is allowed if > 0 ,

NØLEAK - index indicating that no:i-leakage is allowed if $>0$,

IEBIAS - index indicating that energy biasing is allowed if $>0$,

MXREG - maximum number of regions for wich there are weight standards and exponential transform variables ( vill be set to one if $\leq 0$ ),

MAXGP - maximum number of groups for which there are weight standards ard exponential transform variables (will be set to cne if $\leq 0$ ).

If (NSPLT + NKILL + NFAST) $=0$, omit cards $\mathrm{J}$.

CARD J $(6 I 5,4 E 10.5)$ (see p. 41 of rer. 6)

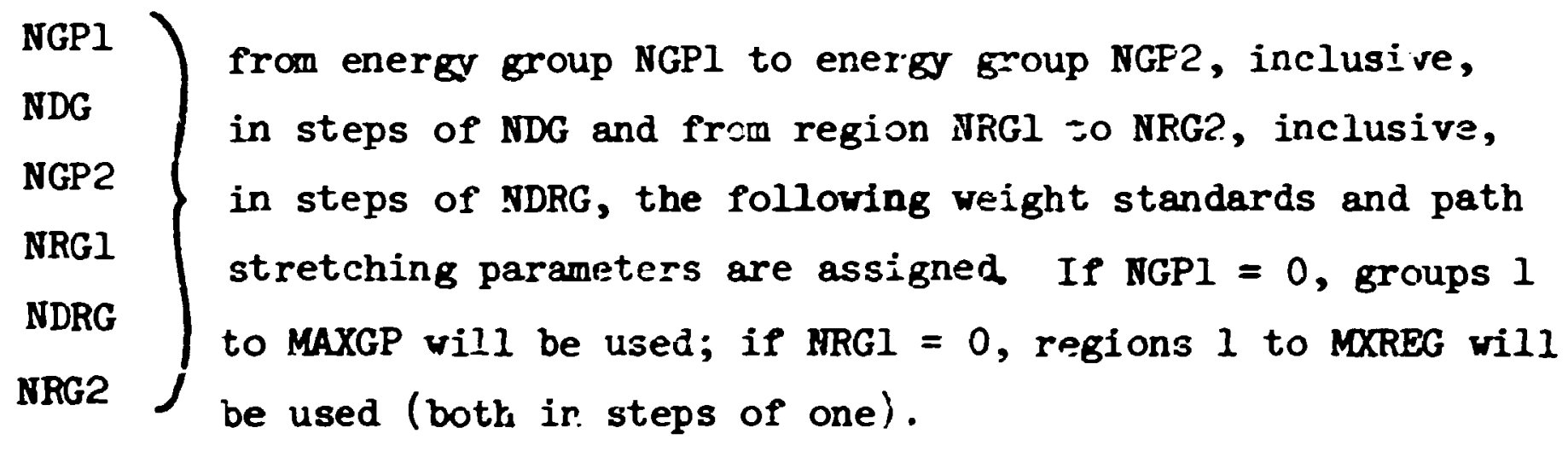


WTHIHl - weight above which splitting will occur,

WLIWl - weight below which Russian roulette is played,

WTAVEl - weight given those particles surviving Russian roulette,

PATH - path length stretching parameters for use in exponential transform (usually $0 \leq$ PATH < 1 ).

The above information is repeated until data for all groups and regions are input. If either NGPI or NRGI equai zero, $t h \equiv$ values will be stored for all MAXGP and MXREG.

End cards $J$ with negative value of NGPI (ex., -1 in columns 4 and 5 ). The following cards are omitted if IEBIAS $\leq 0$

CAR̃LS $K($ TEIO.4 $)$

( (EPRфB (IG,AREG), IG = 1, NMTG), NREG = 1, MXREG)

Values of the relative energy importance of particles leaving a collision in region NREG. Input for each region must start on a new card.

CARD L (14I5)

$$
\begin{aligned}
& \text { NSøUR - set } \leq 0 \text { for a fixed source problem; otherwise the source } \\
& \text { is from fissions generated in a previous batch, } \\
& \text { MFISTP - index for fission problem, if } \leq 0 \mathrm{nc} \text {; fissions are } \\
& \text { allowed, } \\
& \text { NKCALC - the number of the first batch to be included in the csti- } \\
& \text { mate of } k \text {; if } \leq 0 \text { no estimate of } k \text { is made, } \\
& \text { NøRMF - the weight standards and fission weights are unchanged } \\
& \text { if } \leq 0 \text {; otherwise fission weights will be multiplied, } \\
& \text { at the end of each batch, by the latest estimate of } k \\
& \text { and the waight standards are multiplied by the ratio of } \\
& \text { fission weights produced in previous batch to the average } \\
& \text { starting weight for the previous batch. For time-dependent } \\
& \text { decaying systems, N } \emptyset \mathrm{RMF} \text { should be > } 0 .
\end{aligned}
$$

If MFISTP $\leq 0$, omit cards $M$ and $N$ CARDS $M(T E 10.4)$

(FWL $\emptyset(I), I=1$, MXREG) valuts of the weight to be assigned to fission neutrons.

CARDS N (TE10.4)

(FSE (IG,IMED), IG=1, N:MGP), IMED=1, MEDIA) the fraction of fission_ induced source particles in group IG and medium IMFD.

Note: Input for each meiium must start on a new card. For a combined froblem, the following cards must be included; omit for a pure neutron or gamma-ray problem. 
CARDS 0 (7E10.5)

( (GWLð (IG,NREG) IG = 1, HGPQTN or NGPQTG), NREG = I, MYREG) values of the weight to be assigned to the secondary particles being generated. NGPQTN groups are read for each region in a forward problem and NGPQTG for an adjoint. Input for each region must start on $a$ new card.

Geometry input data

Read by subroutine JøMIN and the speciric input depends on t2- seometry packages used (see ref. 1, pp. 49-53 and p. 18C, and see Appendix D).

\section{XSEC input data}

Read by subroutine XSEC.

CARD XA (20A4)

Title card for cross sections.

CARD XB (16I5;

NGP* - the number of primary groups for which there are cross sections to be stored. Should be same as NMGP un card B,

NDS - numider of downscatters for MGP (usuaj ? $\mathrm{j}$ NGP),

NGG* - number of secondary groups fir which there are cross sections to be stored,

NDSG - number of downscatters for NGG (usually NGG),

INGP* - total number of groups for which cross sections are to be input,

INDS - number of downsatters for the INGP uroups (usually INGP),

NMED - number of media for which cross sections are to te stored should te same es MEDIA on card $B$,

NELEM - number of elements for which cross sections are to be read,

NMIX - number of mixing operations (elements times density operations; to be performed (must be $\geq 1$ ),

NCØEF - numiver of coefficients, including $\mathrm{P}_{0}$,

NSCT - number of discrete angles (usually NCøEF/2 ) Jntegral'

ISTAT - flag to store Lagendre coefficients if greater than zero,

IXTAPE - logical tape unit of binary cross-section tape, set $=\hat{\theta}$ if cross sections are from cards.

CARD XC (16I5)

IRDSG - switch to print the cross sections as they are read if $>\dot{v}$, ISTR - switch to print cross sections as they are stored if $>0$,

\footnotetext{
*ee Table C-II, page C-8, for sample input.
} 
IFMU - switch to print intermedicte results of $\mu$ 's es.lculation if $>0$,

IMDM - switch to print moments of on gular distribution if $>0$,

IPRIN - switch to print angles and probabilities if > 0 ,

IPUN - switch to print results of bad Legendre coefíicients if $>0$,

IDTF - switch to signal that input format is DIF-IV format if

> 0; otherwise, ANISN format is assumed.

CARD XD (16I5)

Element identifiers for cross-section tape, omit if IXTAPE $\leq 0$.

Element identifiers must be in same order as elements are on tape.

The fulloring cards are read by subroutine READSG.

CARD XE

ANISN format if IDTF $\leq 0$; otherwise DTF-IV forwat.

Cross sections for INGP groups with INLS downscatters for NELEM

e] ements each with NCWF coefficients.

The mixing cards are read $h_{j}$ subroutine JNPUT.

CARDS XF ( PT5, E10.5)

Nind $X$ (see card $X B$ ) cards are required.

15: - medium number,

KB - element number occurring in medium KM (negative value indicates last mixing operation for that medium).

$\mathrm{RH} \emptyset$ - density of element $\mathrm{K}$ in medium $\mathrm{KM}$.

Analysis intut data

Read by subrcutine SCQRIR.

For the sample problem, the following cards are required:

CARD SA (2OA4)

analysis title card

CARD SB (IIC)

ND - number of detectors.

CARD SC $(T E 10.4)$

$\operatorname{RAD}(I) I=1$, ND - detector radii.

CARD SD (TE,10.4)

FDCF(I) I = 1, NGPQTN - response function for neutrons groups

1. through NGPQTN, see Card B. 


$$
\text { C-? }
$$

Table C-I. Variables That May Be Written on Tape

\begin{tabular}{|c|c|c|c|}
\hline$J$ & Variable* & $J$ & Variable \\
\hline 1 & NC\$LL & 10 & WTBC \\
\hline 2 & NAME & 20 & ETAUSD \\
\hline 3 & IG & $2 i$ & ETA \\
\hline 4 & $U$ & 22 & $A G E$ \\
\hline 5 & $\mathbf{V}$ & 23 & $\emptyset$ LDAGE \\
\hline 6 & $\mathbf{w}$ & 24 & INREG \\
\hline 7 & $\mathbf{x}$ & 25 & NMED \\
\hline 8 & $\mathbf{Y}$ & 26 & NAMEX \\
\hline 9 & $\mathrm{Z}$ & 27 & KATEF \\
\hline 10 & WATE & 28 & BLZNT \\
\hline 11 & $I s \phi$ & 29 & BLZØN \\
\hline 12 & UQLD & 30 & VEL(IG) \\
\hline 13 & VØLD & 31 & $\operatorname{VSI}(\operatorname{Ig} \phi)$ \\
\hline 14 & WI:D & 32 & TSIG \\
\hline 15 & $X \not \Lambda I D$ & 33 & PNAB \\
\hline 16 & YØLD & 34 & NXPKA \\
\hline 17 & $2 \emptyset \mathrm{L}$ & 35 & EXTRAI \\
\hline 18 & $\emptyset$ LDWT & 36 & EXIPRÁ2 \\
\hline
\end{tabular}


Table C-II. Sample Group Input Numbers for Some Representetive Problems*

\begin{tabular}{|c|c|c|c|c|c|c|}
\hline \multirow[b]{2}{*}{$\begin{array}{l}\text { Incut } \\
\text { Variable }\end{array}$} & \multicolumn{6}{|c|}{ Troblem Type } \\
\hline & 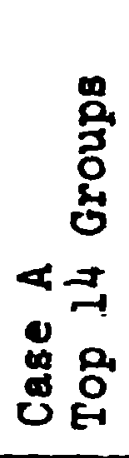 & 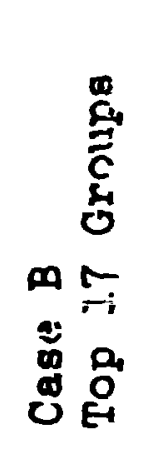 & 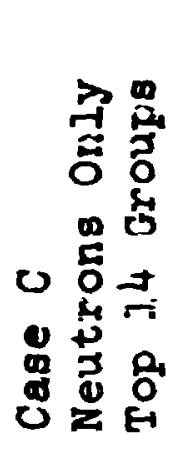 & 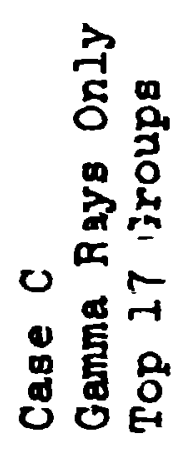 & 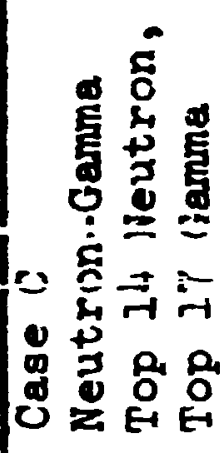 & \\
\hline HGPQT & 14 & 0 & 14 & 0 & 14 & 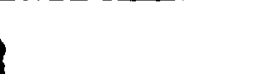 \\
\hline HGPOTG & 0 & 17 & 0 & 17 & 17 & CARD B \\
\hline MMGP & 22 & 18 & 22 & 18 & 22 & Variohles \\
\hline FIATG & 22 & 18 & 22 & 18 & 40 & \\
\hline IGPP & 22 & 18 & 22 & 0 & 22 & \\
\hline IGG & 0 & 0 & 0 & 18 & 18 & CARD XB \\
\hline IMGP & 22 & 18 & 40 & 40 & 40 & Variables \\
\hline
\end{tabular}

For cross sections with full downscatter NDS $=$ NGP, NDSG $=$ NGG and INDS = INGP. 
APPETDIX D

\section{Geometry Inout Instructions}

\section{SPHERICAL GEQM}

CARD GA (I5, D10.5)

MED - medium number interior to $R(>0)$

$R$ - outer radius of sphere or spherical shell containing MED.

Repeat CAPD GA for all rauii ( $\leq 20)$ in increasing order.

End CARD GA input with blank card.

CARD GB (D10.5)

$R$ - region radius of sphere or spherical shell containing regions. Region numbers are assigned in consecutive order starting with 1 , and $R$ must be in increasing order.

Repeat CARD GB for the number of regions $(\leq 20)$.

End CARD GB input with blank card. If no regions are desired, a blank caru mist be used to signal no region geometry.

Taken from ref. 6. 


$$
\text { D-2 }
$$

SIAB GBdM

CARD GA (IS, DI0.5)

MED - nedive vith $Z$ as lover bound (>0)

Z - Iover limit or mediue MED.

Repeat CARD GA for ol? zoimiaries with the last card containing $M B D=0$ and the boundary of the sjstem.

CARD GB (D10.5)

Z - lower limit of region boundary. Region numbers are assigned in consecutive order starting with 1 and $Z$ must be in increasing order.

Repeat CARD UB for all region boundaries.

Fnd CARD GB input vith a blank card. If no region geometry is Jesired a blank card is required.

CARD GC (4D10.5)

XI - lower boundary of system in X direction.

XU - upper boundary of system in X direction.

YL - lower boundary of system in $Y$ direction.

YU - upper boundary of syster in Y direction. 


\section{CYLIIDRICAL GEQY}

CARD GA (I5, 5X, A8)

IREGIM - flag to indicate material media $(=1)$ or both region and naterial media $(=2)$.

SEX - sex of programer.

CARD GB (E10.5)

R - radii of the cylindrical shells describing the material media in ascending order.

Repeat CARD GB until all radii have been input.

End CARD GB input vith a blank card.

CARD GC (E1C.5, 12I5/8I5)

H - upper height of medi= $M(I)(>0)$.

Cylinders assumed to start at $\mathrm{H}=0$.

M(I) - media for the cylindrical shells for this height.

Repeat CARD GC until all height intervals have been input.

Fnd CARD GC input with a blank card or if there are more than 12 radial intervais, 2 blank cards.

CARD GD (B10.5) onit if WREGM = 1

RG - radii of the cylindrical shells describing the region geometry in ascending order.

Repeat CARD GD until ali region geometry has been input.

End CARD GD vith a blank card.

CARD GE (E10.5, 12I5/8I5) onit if IREGII = 1

HG - upper height of regin MO(I).

WG(I) - region numbers for the cylindrical shells for this height.

Repeat CARD GE until all height intervals have been input.

Bnd CARD GE input with a blank card or if there are wore than 12 radial intervals, 2 blank cards. 
GB.ERAL GBQA

CARL GA (i5, 5X, A6, IX, AT) Holleritin left adjusted

NSTAT - flag to indicate material media only if 1 and both region and alerial media if 2.

SEX - z:a zi the frogramer. (Select one from MALE, PQALE, o:

blank indicating lincertain.;

STATUS - marital status of programer.

CARD GB (2A4, A3, SíDI0.5, Ai)

DUAY(3) - hollerith characters not used.

FII(I) - zone boundaries in increasing order along the X-axis.

$B C D(I)$ - flag to inaicate end of input if blenk, even means to continue.

Repeat CARD GB if wore than five boundaries along the $Y$ axis are needed. CARU GC - same as CARD GB except for $Y$ axis.

Repeat CARD GC if zore than fire boundaries along the $Y$ axis are needec: CARD GD - same as CARD GB cicept for $Z$ axis.

Repeat CARD GD if more than five boundaries along the $Z$ axis are needed.

CARD GE (A4, A2, 3I5)

BCDl - hollerith ZONE

BCD2 - dumens

IXZ10 - integers wich specify the zone as being the ExZaroti

Frxwo - zone in the $X$ direction, MYaroth zone in the $Y$

IZZW - direction, and FZZwoth zone in the $\mathrm{Z}$ direction.

CARD GP(2A4, A3, 5(D10.5, Al)

DayY(3) - hollerith characters not used.

FIII(I) - block boundaries in increasing order along the axis.

$B C D(I)$ - flag to indicate end of input if blank, comma mears to continue.

Repeat CARD GF if more than five boundaries along the $X$ axis are needed. CARI GC - same as CARD GP except for $Y$ axis.

Repeat CARD GG if more than five boundaries along the $\ddot{i}$ axis are needed. CARD GF - same as CARD GP except for 2 axis. 
Repeat CARD GH if more than five boundaries along the $Z$ axis are needed.

CARDS GI to 60 describe the geometry for a block and must be included for each biock in the zone.

CARD GI (A4, A2, 3IS)

BCDI - hollerith BLOC

$B C D$ ?

IXBND - integers which specify the bluck as being the

FYBTD - FXBDDth in the $X$ direction, the FYBXDth in the

IZBU - Y direction, and the MZBUD in the $Z$ direction.

GARD RI (3AL, I I

HAY2 - bollerith MEDI

$\operatorname{Dar}(\varepsilon)$ - du: uy

IIP(I) - a list of nedia sector by sector in the block

$B C D(I)$ - 7 ag to indicate end of input if blank, a coma means to con inue.

Continuation vith $12(\mathrm{I} 5, \mathrm{Al})$ format is pernissible.

CAPD CX $\left(3 A^{4}, 10(I 5, A I)\right)$

MAY2 - hol lerith SURP

DuA(2) - dung

U.P(I) - a list of quadratic surfaces apper.ring in the block.

numbers mist appear in the order the surfaces are described on CARD GQ.

$B C D(I)$ - flag to indicate end of input of blank, a coma reans to continue.

Continuation of CARD GK in $12(15,41)$ format is permissible.

CARD GL (A4, AÊ, 1813)

S1 - hollerith SECT

DUA - dum

IFD(I) - the designstion of each sector which describes the position of the sector relative to quadratic surfaces.

+1 : sector is on positive side of surface,

-i: sector is on negative side of surface,

ô: surface is not needed to define sector. 
There must be a CARD GL for each sector and references to quadratic surfaces must be in sane order as they ere listed on CARD GQ.

CARD G $(3 \mathrm{AH}, \operatorname{Ir}(\mathrm{I5}, \mathrm{Al})$

IAMP - hollerith REGI

$\operatorname{DUA}(2)$ - dumy

IIPP(I) - a list of regions sector by sector in the block.

$B C D(I)$ - flag to indicate end of input if blank, a coma means to continue.

Contiruation with $12(15, \mathrm{Al})$ format is permissible.

CĀ̄̃ G. (3A4, $10(i 5, A 1))$

INYE - hollerith SURP

Dut(2) - dumy

I.P(I) sane as for CARD GK except for region input instead of $B C D$ (I) naterial input.

CARD $C O(A 4, A 2,1813)$

S1 - ho:lerith SECT

DUM - duraty

I.D(I) - sane as for CNRD GL except for region input instead of material input.

Repeat CARDS GI to Gd for each block.

CARD GP (I5, 16A4, A2)

NOBD - total number of quadratic surfaces in the entire system. DUA(I) - hollerith characters ignored by the code. (Helpful in identifying input at $\mathrm{a}$ later time.)

CARD GQ $: 4(D 10.5, A 4,1 X, A I))$

$C \emptyset F(J)$ - coefficient of the term

$\operatorname{BCDI}(J)$ - hollerith indicating which term of the equation. XSQ, YSQ, ZSQ, XZ, YX, YZ, XY, ZX, YZ, X, Y, Z, or

blank are the possibilities.

$\operatorname{BCD} 2(J)$ - a flag which indicates the quadratic equation coxtinues. Any non-blank character ends the field. The next function must start on new card.

Repeat CARDS GQ until all surfaces have been described.

A sample of the input is shown in Figure D.1. 


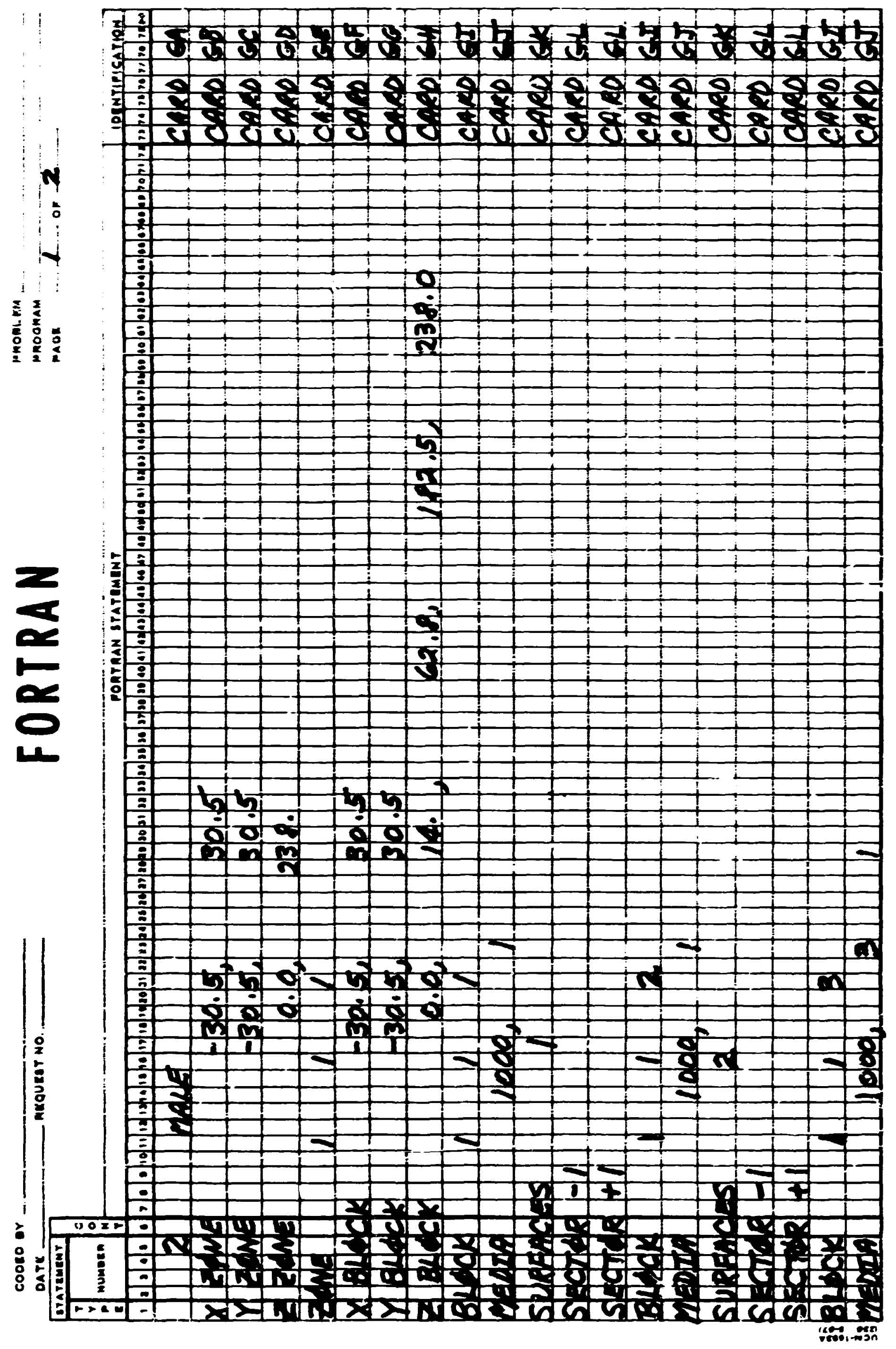




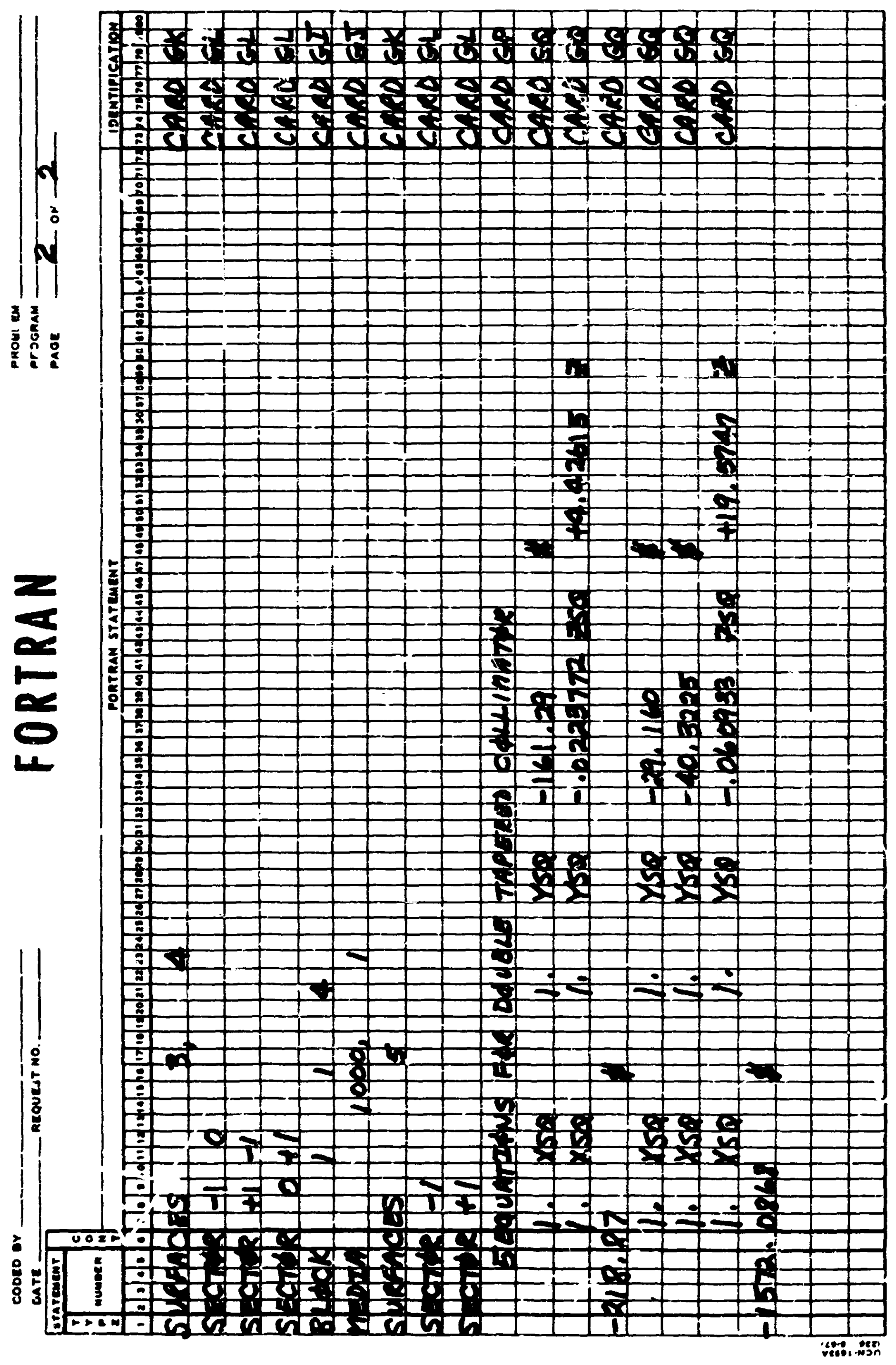


APPENDIX E

\section{Library Subroutines ind Functions}

The following subroutines ard functions are library routines at 0ak Ridge National Laboratory and are not provided with MORSE.

\begin{tabular}{|c|c|c|}
\hline $\begin{array}{l}\text { Subroutine or } \\
\text { Function } \\
\end{array}$ & Called From & Purpose \\
\hline LDC & $\begin{array}{l}\text { Main, XSCHLP, } \\
\text { HELP }\end{array}$ & $\begin{array}{l}\text { Determine absolute address of cell given } \\
\text { as argument. }\end{array}$ \\
\hline INT $\emptyset \mathrm{BC}$ & DATE & Converts integer to $\mathrm{BBCDIC}$. \\
\hline INTBCD & $\begin{array}{l}\text { DATE, TTMER, } \\
\text { SUBFT }\end{array}$ & $\begin{array}{l}\text { Converts integer to EBCDIC and returns } \\
\text { number of bytes in EBCDIC string. }\end{array}$ \\
\hline FETYP:' & READSG & $\begin{array}{l}\text { Determines if a character is a number or } \\
\text { a letter. }\end{array}$ \\
\hline $\mathrm{BCDI} \varnothing \mathrm{I}$ & READSG & Converts EBCDIC to integer. \\
\hline ICXMPA & $\begin{array}{l}\text { INPUT, BNn'HLP, } \\
\text { HELPER }\end{array}$ & $\begin{array}{l}\text { Comcares bit by bit } N \text { bytes of two } \\
\text { variables; returns zero if the two } \\
\text { variables are identical. }\end{array}$ \\
\hline MØDEL & INPUT & $\begin{array}{l}\text { Determines whether the problem is being } \\
\text { executed on the IBM- } 360 \text { model } 75 \text { or } 91 \text {. }\end{array}$ \\
\hline IDAY & IWEEK & $\begin{array}{l}\text { Determines number of the month, day, } \\
\text { and year. }\end{array}$ \\
\hline ICL $\varnothing \mathrm{CK}$ & TIMER, MфRSE & Determines c.p.u. time. \\
\hline INSEPT & TIMER & $\begin{array}{l}\text { Inserts a string of given length at a } \\
\text { specified point in another string of } \\
\text { characters. }\end{array}$ \\
\hline
\end{tabular}

There are several uses of these library routines. One is to provide the time, day or the week, and year that the job is being executed. A second use, provided by Subroutine TIMER, is ir. determining the amount of c.p.u. time used per batch and for input and output. To obviate several of these library subroutines, dumby subroutines TIMER and DATE may be used. A third use is in the diagnostic module. The absolute iocation of varjables in commons, the determination of a repeating array, a "not used" feature, and an integer or floeting point output are the features 
of the diagnostic module that require these special routines. If similar routines are not available, other user-written routines can be supplied for XSHLP, BNKHLP, and HELP.

Severai other uses of these routines are made, but they are relatively unimportant. MDDEL is used to scale MAXPIM, depending on the machine on which the job is being executed. ICAPA is used by INPUT to terminate a job when a non-biank or alphanumeric character appears in the first column of Card A. READSG has an option of checking for seçuence errors in the cross-section caràs. While none of these features are necessary to the operation of Mrise, they have proven to be quite useful. 